\title{
Enantioselective Intermolecular Excited-State Photoreactions Using a Chiral Ir Triplet Sensitizer: Separating Association from Energy Transfer in Asymmetric Photocatalysis
}

Jian Zheng, ${ }^{\dagger}$ Wesley B. Swords,,${ }^{\dagger \star}$ Hoimin Jung,,${ }^{\S}$ Kazimer L. Skubi, ${ }^{\dagger}$ Jesse B. Kidd, ${ }^{\dagger}$ Gerald J. Meyer, ${ }^{\ddagger}$ Mu-Hyun Baik, $,{ }^{\rrbracket}, * *$ Tehshik P. Yoon ${ }^{\dagger, *}$

$\dagger$ Department of Chemistry, University of Wisconsin-Madison, 1101 University Avenue, Madison, Wisconsin 53706, United States

$\$$ Department of Chemistry, University of North Carolina at Chapel Hill, Murray Hall 2202B, Chapel Hill, North Carolina 27599-3290, United States

$\S$ Department of Chemistry, Korea Advanced Institute of Science and Technology (KAIST), Daejeon 34141, Republic of Korea

ๆ Center for Catalytic Hydrocarbon Functionalizations, Institute for Basic Science (IBS), Daejeon 34141, Republic of Korea 


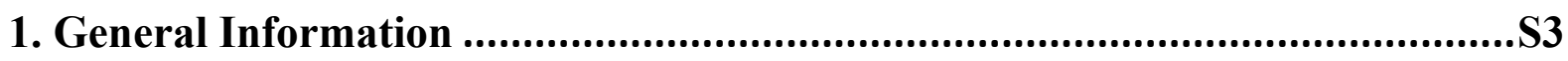

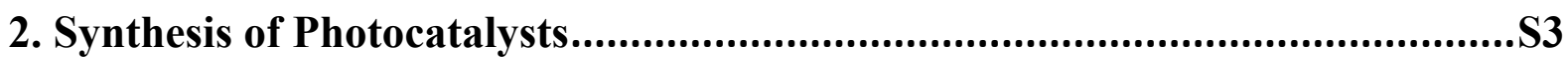

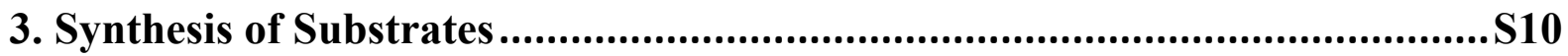

4. Photosensitized [2+2] Cycloadditions...............................................................S14

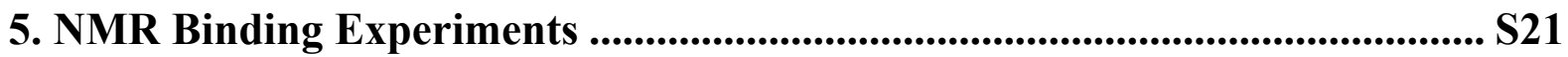

6. Room Temperature Stern-Volmer Quenching Experiments.............................. S23

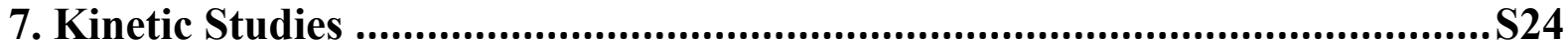

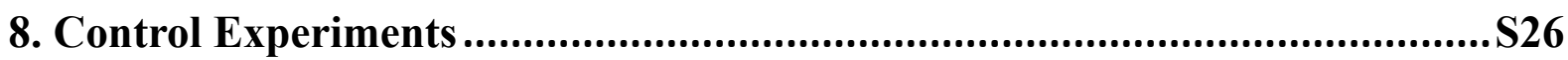

9. Transient Absorption Spectroscopy .................................................................S26

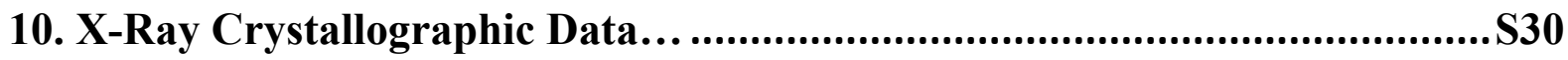

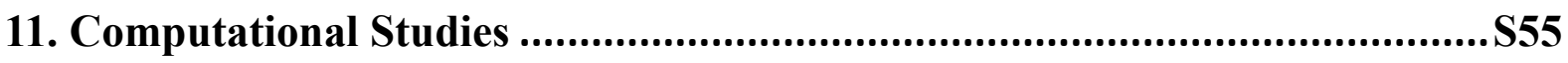

12. Cyclic Voltammetry Data................................................S113

13. Quantum yield of the parent reaction........................................S114

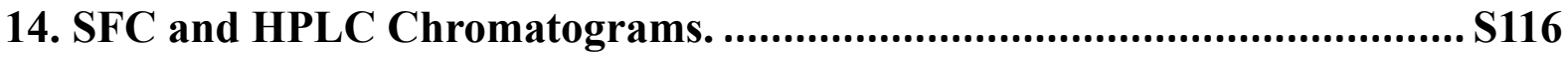

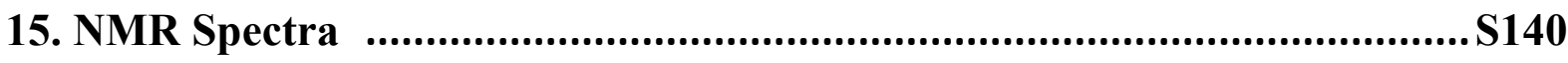

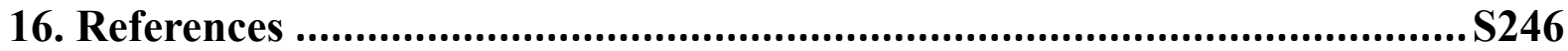




\section{General Information}

All reagents were purchased from commercial suppliers and used without additional purification unless otherwise noted. Flash column chromatography was carried out with Purasil $60 \AA$ silica gel (230-400 mesh). ${ }^{1} \mathrm{H},{ }^{13} \mathrm{C}$, and ${ }^{19} \mathrm{~F}$ NMR spectra were obtained using a Bruker Avance-400, Avance-500, or Avance-600 spectrometer with DCH, Prodigy, BBFO+, or TCI-F probes. ${ }^{1} \mathrm{H}$ spectra were internally referenced to tetramethyl silane or the residual protio-solvent peak in $\mathrm{CDCl}_{3}, \mathrm{CD}_{3} \mathrm{OD}$, DMSO- $d_{6}$, or acetone- $d_{6}$. Enantiomeric excesses were determined for isolated, purified material using either chiral SFC (Waters/TharInvestigator) or chiral HPLC (Waters Alliance), Daicel CHIRALCEL ${ }^{\circledR}$ columns and Chromasolv ${ }^{\circledR}$-grade solvents. IR spectra were obtained using a Bruker Alpha Platinum spectrometer (powder or thin film). Melting points were obtained using a Stanford Research Systems DigiMeltapparatus, and are uncorrected. Optical rotations were measured using a Rudolph Research Autopol III polarimeter at room temperature. UV-Vis emission spectra were acquired on ISS PC1 photon-counting fluorometer. The spectrometers used for this work are supported by the NSF (CHE-1048642, CHE-0342998, CHE-9208463), NIH (S10 OD012245, S10 RR13866-01), and a generous gift from Paul J. and Margaret M. Bender. Mass spectrometry was performed with a Thermo Q Exactive ${ }^{\mathrm{TM}}$ Plus. This instrumentation is supported by the NIH (1S10 OD020022-1) and the University of Wisconsin.

\section{Synthesis of Photocatalysts}

\section{a. Synthesis of Ligands}<smiles>Fc1cc(Br)cc(OCCOc2ccccc2)c1F</smiles><smiles>FC(F)(F)c1ccc(Cl)nc1</smiles>

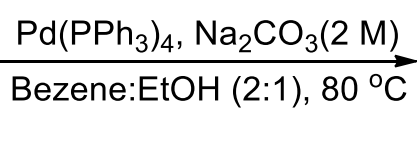<smiles>Fc1cc(-c2ccc(C(F)(F)F)cn2)cc(F)c1F</smiles>

Phenylpyridine (ppy) ligands were prepared based on a known procedure. ${ }^{1}$ A $50 \mathrm{~mL}$ flask was charged with chloropyridine (5.00 mmol, 1.0 equiv.) and arylboronic acid (5.50 mmol, 1.1 equiv.), followed by $\mathrm{Pd}\left(\mathrm{PPh}_{3}\right)_{4}(0.125 \mathrm{mmol}, 0.025$ equiv. $)$. Benzene $(6.7 \mathrm{~mL})$ was added, followed by $2 \mathrm{M}$ aq. $\mathrm{Na}_{2} \mathrm{CO}_{3}$ (10.0 mmol, 2.0 equiv.) and $\mathrm{EtOH}(1.25 \mathrm{~mL})$. Then the mixture was heated at $80{ }^{\circ} \mathrm{C}$ under $\mathrm{N}_{2}$ and maintained at this temperature for $2 \mathrm{~d}$. The reaction was then cooled to room temperature and diluted by $6 \mathrm{~mL} \mathrm{H}_{2} \mathrm{O}$. The crude was extracted by EtOAc (10 mL X 3). Then the organic layers were combined, dried with $\mathrm{MgSO}_{4}$, filtered and concentrated, and the residue was further purified by flash column chromatography (EtOAc:Hexanes $=1: 10)$ to afford the pure product.

5-(Trifluoromethyl)-2-(3,4,5-trifluorophenyl)pyridine (A1): Prepared as described before. Spectral

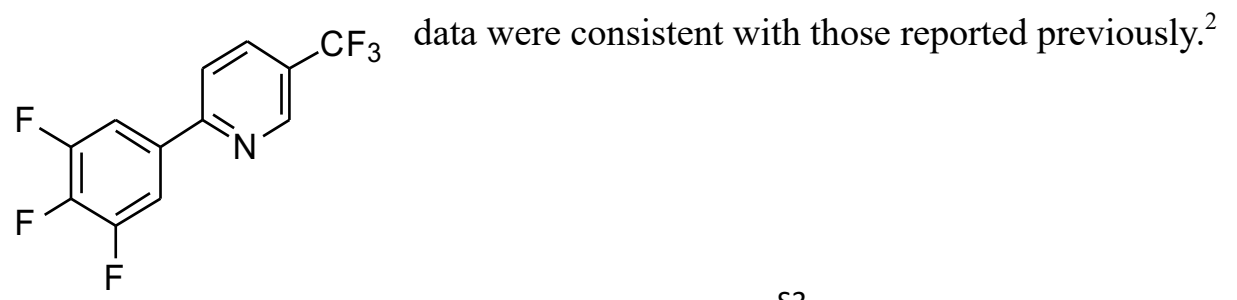


2-(2,5-Difluorophenyl)-5-(trifluoromethyl)pyridine (A2): $1.12 \mathrm{~g}, 86 \%$ yield, white solid; ${ }^{1} \mathrm{H}$ NMR<smiles>Fc1ccc(F)c(-c2ccc(C(F)(F)F)cn2)c1</smiles>
$\left(400 \mathrm{MHz}, \mathrm{CDCl}_{3}\right) \delta 8.98(\mathrm{~s}, 1 \mathrm{H}), 8.05-7.94(\mathrm{~m}, 2 \mathrm{H}), 7.82$ (ddd, $J=9.1,6.1$, $3.0 \mathrm{~Hz}, 1 \mathrm{H}), 7.22-7.08(\mathrm{~m}, 2 \mathrm{H}) .{ }^{13} \mathrm{C} \mathrm{NMR}\left(126 \mathrm{MHz}, \mathrm{CDCl}_{3}\right) \delta 160.00(\mathrm{dd}, J$ $=243.0,2.2 \mathrm{~Hz}), 156.75(\mathrm{dd}, J=246.9,2.4 \mathrm{~Hz}), 155.47-155.24(\mathrm{~m}), 146.64$ (q, $J=4.0 \mathrm{~Hz}), 133.85(\mathrm{q}, J=3.1 \mathrm{~Hz}), 127.24(\mathrm{dd}, J=13.4,7.9 \mathrm{~Hz}), 125.60(\mathrm{q}$, $J=33.1 \mathrm{~Hz}), 123.92$ (d, $J=11.8 \mathrm{~Hz}), 123.49$ (q, $J=272.2 \mathrm{~Hz}), 118.04$ (dd, $J=$ 24.4, 9.1 Hz), $117.69(\mathrm{dd}, J=26.2,8.4 \mathrm{~Hz}), 117.28(\mathrm{dd}, J=25.7,3.0 \mathrm{~Hz}) .{ }^{19} \mathrm{~F}$ $\operatorname{NMR}\left(377 \mathrm{MHz}, \mathrm{CDCl}_{3}\right) \delta-62.41(\mathrm{~s}, 3 \mathrm{~F}),-117.92(\mathrm{~d}, J=18.2 \mathrm{~Hz}, 1 \mathrm{~F}),-122.23(\mathrm{~d}, J=18.2 \mathrm{~Hz}, 1 \mathrm{~F})$. IR (thin film): $3080,2927,1604,1569,1500,1475,1326,1129,817,757 \mathrm{~cm}^{-1}$; HRMS (ESI): $[\mathrm{M}+\mathrm{H}]^{+}$ calculated for $\mathrm{C}_{12} \mathrm{H}_{7} \mathrm{~F}_{5} \mathrm{~N}^{+} \mathrm{m} / z 260.0493$, found $\mathrm{m} / z$ 260.0490; $\mathrm{mp}=65.2-65.4{ }^{\circ} \mathrm{C}$.

2-(2-Fluoro-4-(trifluoromethyl)phenyl)-5-(trifluoromethyl)pyridine (A3): Prepared as described<smiles>Fc1cc(C(F)(F)F)ccc1-c1ccc(C(F)(F)F)cn1</smiles>
before. Spectral data were consistent with those reported previously. ${ }^{2}$

2-(3,5-Difluorophenyl)-5-(trifluoromethyl)pyridine (A4): $1.2 \mathrm{~g}, 93 \%$ yield, pale yellow solid; ${ }^{1} \mathrm{H}$<smiles>Fc1cc(F)cc(-c2ccc(C(F)(F)F)cn2)c1</smiles>
$\mathrm{NMR}\left(500 \mathrm{MHz}, \mathrm{CDCl}_{3}\right) \delta 8.97-8.93(\mathrm{~m}, 1 \mathrm{H}), 8.02(\mathrm{dd}, J=8.4,2.0 \mathrm{~Hz}$, $1 \mathrm{H}), 7.81(\mathrm{~d}, J=8.3 \mathrm{~Hz}, 1 \mathrm{H}), 7.63-7.56(\mathrm{~m}, 2 \mathrm{H}), 6.92(\mathrm{tt}, J=8.6,2.3 \mathrm{~Hz}$, $1 \mathrm{H}) .{ }^{13} \mathrm{C}$ NMR $\left(126 \mathrm{MHz}, \mathrm{CDCl}_{3}\right) \delta 163.48(\mathrm{dd}, J=248.8,12.6 \mathrm{~Hz})$, $158.07-157.85(\mathrm{~m}), 146.80(\mathrm{q}, J=3.9 \mathrm{~Hz}), 141.11(\mathrm{t}, J=9.3 \mathrm{~Hz}), 134.32$ (q, $J=3.5 \mathrm{~Hz}), 125.94(\mathrm{q}, J=33.2 \mathrm{~Hz}), 123.48(\mathrm{q}, J=272.3 \mathrm{~Hz}), 120.02-$ $119.98(\mathrm{~s}), 110.40-110.01(\mathrm{~m}), 105.33(\mathrm{t}, J=25.4 \mathrm{~Hz}) \cdot{ }^{19} \mathrm{~F}$ NMR $\left(377 \mathrm{MHz}, \mathrm{CDCl}_{3}\right) \delta-62.38(\mathrm{~s}$, 3F),-108.75 (s, 2F). IR (thin film): 3101, 1598, 1570, 1323, 1125, $839 \mathrm{~cm}^{-1}$; HRMS (ESI): $[\mathrm{M}+\mathrm{H}]^{+}$ calculated for $\mathrm{C}_{12} \mathrm{H}_{7} \mathrm{~F}_{5} \mathrm{~N}^{+}$requires $m / z 260.0493$, found $m / z 260.0491 ; \mathrm{mp}=52-54{ }^{\circ} \mathrm{C}$.
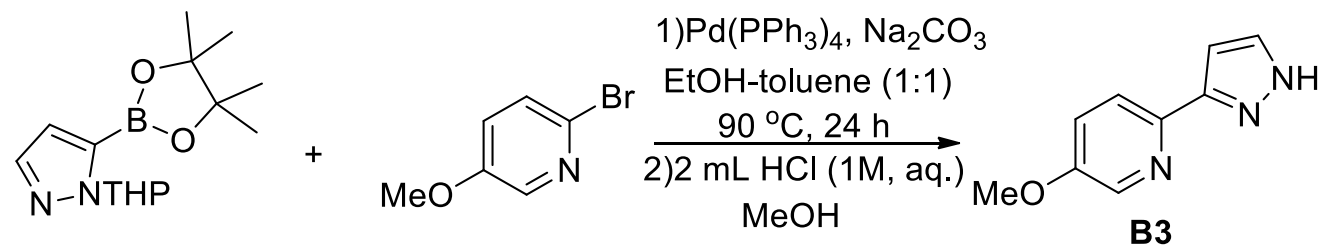

Pyrazolpyridine (pzpy) ligands were prepared based on a known procedure. ${ }^{3}$ A $25 \mathrm{~mL}$ flask was charged with 2-bromo-5-methoxypyridine (1.20 mmol, 1.2 equiv.) and 1-(tetrahydro-2 $H$-pyran-2-yl)-5-(4,4,5,5-tetramethyl-1,3,2-dioxaborolan-2-yl)-1 $H$-pyrazole (1.0 mmol, 1.0 equiv.), followed by $\mathrm{Pd}\left(\mathrm{PPh}_{3}\right)_{4}(0.025 \mathrm{mmol}, 0.025$ equiv. $)$. Toluene $(1.0 \mathrm{~mL})$ was added, followed by $1.5 \mathrm{~mL} 2 \mathrm{M}$ aq. $\mathrm{Na}_{2} \mathrm{CO}_{3}(3 \mathrm{mmol}, 3.0$ equiv.) and $\mathrm{EtOH}(1.0 \mathrm{~mL})$. Then the mixture was heated at $90{ }^{\circ} \mathrm{C}$ under $\mathrm{N}_{2}$ and maintained at this temperature for $24 \mathrm{~h}$. The reaction was then cooled to $0{ }^{\circ} \mathrm{C}$ and diluted with $6 \mathrm{~mL} \mathrm{H}_{2} \mathrm{O}$. The crude was extracted into EtOAc $(10 \mathrm{~mL} \mathrm{X} \mathrm{3)}$ at RT. Then the organic layers were combined and concentrated, and the residue was dissolved in $2 \mathrm{~mL} \mathrm{MeOH}$, followed by 2 
$\mathrm{mL} \mathrm{HCl}(1 \mathrm{M}$, aq.). After stirring for $5 \mathrm{~h}$ at $\mathrm{RT}$, the crude was quenched with $3 \mathrm{~mL} \mathrm{NaOH}$ (2 M, aq.). The reaction was then diluted with $6 \mathrm{~mL} \mathrm{H}_{2} \mathrm{O}$ and the crude was extracted into EtOAc (10 $\mathrm{mL} \mathrm{X} \mathrm{3).}$ Then the organic layers were combined, dried with $\mathrm{MgSO}_{4}$, filtered and concentrated, and the residue was further purified by flash column chromatography (EtOAc:Hexanes $=1: 1$ ) to afford the pure product.

2-(1H-Pyrazol-3-yl)-5-(trifluoromethyl)pyridine (B2): Prepared as described before. Spectral data<smiles>FC(F)(F)c1ccc(-c2cc[nH]n2)nc1</smiles>
were consistent with those reported previously. ${ }^{3}$

B2

5-Methoxy-2-(1 H-pyrazol-3-yl)pyridine (B3): (Note: the ligand appears to have fewer than the<smiles>COc1ccc(-c2cc[nH]n2)nc1</smiles>

B3 expected number of signals in the ${ }^{13} \mathrm{C}$ NMR. This phenomenon is consistent with previously-reported results. ${ }^{2}$ We found that the $\mathrm{HCl}$ salt gave better data.): $143.4 \mathrm{mg}, 82 \%$ yield, white solid; ${ }^{1} \mathrm{H}$ NMR (500 MHz, $\left.\mathrm{CDCl}_{3}\right) \delta 8.39$ $(\mathrm{d}, J=2.8 \mathrm{~Hz}, 1 \mathrm{H}), 7.70(\mathrm{~d}, J=8.7 \mathrm{~Hz}, 1 \mathrm{H}), 7.65(\mathrm{~d}, J=1.9 \mathrm{~Hz}, 1 \mathrm{H}), 7.25$ (dd, $J=8.7,2.9 \mathrm{~Hz}, 1 \mathrm{H}), 6.71(\mathrm{~d}, J=1.9 \mathrm{~Hz}, 1 \mathrm{H}), 3.89(\mathrm{~s}, 3 \mathrm{H}) .{ }^{1} \mathrm{H}$ NMR

$\left(\right.$ B3· HCl, $\left.500 \mathrm{MHz}, \mathrm{DMSO}-d_{6}\right) \delta 9.68(\mathrm{~s}, 2 \mathrm{H}), 8.35(\mathrm{~d}, J=2.8 \mathrm{~Hz}, 1 \mathrm{H}), 8.23(\mathrm{~d}, J=8.9 \mathrm{~Hz}, 1 \mathrm{H}), 8.02$ - $7.95(\mathrm{~m}, 1 \mathrm{H}), 7.95-7.91(\mathrm{~m}, 1 \mathrm{H}), 7.16(\mathrm{~s}, 1 \mathrm{H}), 3.97(\mathrm{~s}, 3 \mathrm{H}) .{ }^{13} \mathrm{C}$ NMR $(\mathbf{B 3} \cdot \mathbf{H C l}, 126 \mathrm{MHz}$, DMSO- $\left.d_{6}\right) \delta 156.56,143.61,140.00,132.41,131.18,129.30,124.13,105.30,57.41$. IR (thin film): 3171, 2943, 2840, 1571, 1486, 1459, 1272, 1230, 1026, 836, $786 \mathrm{~cm}^{-1}$; HRMS (ESI): $[\mathrm{M}+\mathrm{H}]^{+}$ calculated for $\mathrm{C}_{9} \mathrm{H}_{10} \mathrm{~N}_{3} \mathrm{O}^{+}$requires $m / z$ 176.0818, found $\mathrm{m} / \mathrm{z} 176.0817$; $\mathrm{mp}=105-107^{\circ} \mathrm{C}$;

(S)-2-(4-Isopropyl-4,5-dihydrothiazol-2-yl)phenol ('PrHBT):Prepared as described before and the<smiles>CC(C)[C@H]1CSC(c2ccccc2O)=N1</smiles>
spectral data were consistent with those reported previously. ${ }^{4}$ 


\section{b. Synthesis of Iridium Complexes}
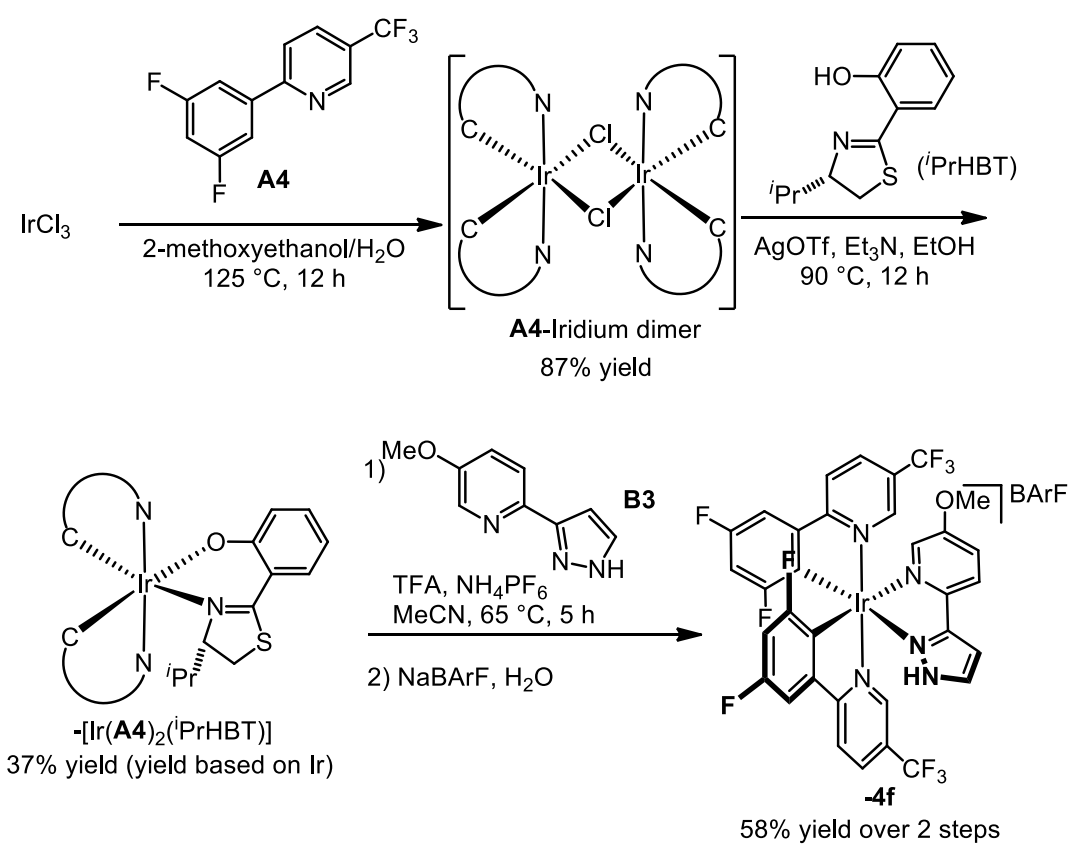

The iridium complexes were prepared following the procedure reported by our group. ${ }^{2}$

Step 1: A flask was charged with a stir bar, $\mathrm{IrCl}_{3} \cdot \mathrm{xH}_{2} \mathrm{O}$ (2.00 mmol, 1.0 equiv.), ppy ligand (2.25 mmol, 2.25 equiv. $)$ and $36 \mathrm{~mL}$ co-solvent $\left(\mathrm{V}_{2 \text {-methoxyethanol: }} \mathrm{V}_{\mathrm{H} 2 \mathrm{O}}=2: 1\right)$. The solution was heated at $125{ }^{\circ} \mathrm{C}$ under $\mathrm{N}_{2}$ overnight. After cooling to room temperature, $100 \mathrm{~mL} \mathrm{H}_{2} \mathrm{O}$ was added, resulting in precipitation. The resulting solid was isolated by suction filtration on a frit and rinsed with $\mathrm{H}_{2} \mathrm{O}$ and hexanes before drying under vacuum. The crude iridium dimer was carried on to the following steps without further purification.

Step 2: The iridium dimer ( $0.6 \mathrm{mmol}, 1.0$ equiv.) was added to a round-bottomed flask, along with EtOH $(60 \mathrm{~mL}),{ }^{i} \operatorname{PrHBT}$ (2.2 equiv.), $\operatorname{AgOTf}\left(2.2\right.$ equiv.) and $\mathrm{Et}_{3} \mathrm{~N}$ (10.0 equiv.). The mixture was heated at $90{ }^{\circ} \mathrm{C}$ under $\mathrm{N}_{2}$ overnight. After cooling to RT, the solvent was removed in vacuo and the diastereomers were separated by flash column chromatography to afford the pure product, which was assigned as the $(S), \Lambda$-diastereomer by analogy to the compounds reported before. ${ }^{2}$

Step 3: A round-bottomed flask was charged with the $(S), \Lambda$-iridium complex ( $0.08 \mathrm{mmol}, 1$ equiv.), $\mathrm{NH}_{4} \mathrm{PF}_{6}$ (0.40 mmol, 5.0 equiv.), pyridyl pyrazole ligand ( $0.20 \mathrm{mmol}, 2.5$ equiv.) and dry $\mathrm{MeCN}(8$ $\mathrm{mL})$, then placed under a nitrogen atmosphere. Trifluoroacetic acid $(0.40 \mathrm{mmol}, 5.0$ equiv. $)$ was added by syringe, and the solution was heated at $65{ }^{\circ} \mathrm{C}$ in the dark. After $5 \mathrm{~h}$, the mixture was cooled to RT and concentrated. The crude residue was purified by flash column chromatography on silica gel $\left(\mathrm{CH}_{2} \mathrm{Cl}_{2}\right)$ to afford the pure $\mathrm{PF}_{6}$ salt, which was carried on without further characterization. This material was dissolved in $1 \mathrm{~mL} \mathrm{CH}_{2} \mathrm{Cl}_{2}$ and $\mathrm{NaBArF}$ (1.0 equiv.) was added. After stirring for $5 \mathrm{~min}$ at room temperature, the mixture was washed with $\mathrm{H}_{2} \mathrm{O}(3 \mathrm{~mL} \mathrm{X} \mathrm{3)}$. The final photocatalyst was purified by flash column chromatography $\left(\mathrm{CH}_{2} \mathrm{Cl}_{2}\right){ }^{5}$ 
(S), $\Lambda$-[ $\left[\mathrm{Ir}(\mathrm{A2})_{2}\left({ }^{\mathrm{i}} \mathrm{PrHBT}\right)\right]: 342.7 \mathrm{mg}, 46 \%$ yield, yellow solid; ${ }^{1} \mathrm{H}$ NMR $\left(400 \mathrm{MHz}, \mathrm{CDCl}_{3}\right) \delta 9.37$ (s,

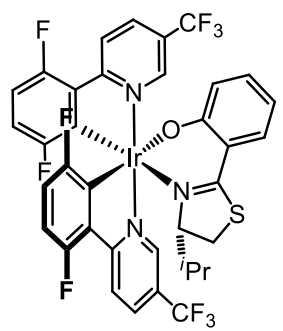
$1 \mathrm{H}), 8.67(\mathrm{~s}, 1 \mathrm{H}), 8.42(\mathrm{dd}, J=8.7,2.8 \mathrm{~Hz}, 1 \mathrm{H}), 8.34$ (dd, $J=8.7,3.9 \mathrm{~Hz}, 1 \mathrm{H})$, $7.98(\mathrm{dd}, J=8.7,1.5 \mathrm{~Hz}, 1 \mathrm{H}), 7.84(\mathrm{~d}, J=8.8 \mathrm{~Hz}, 1 \mathrm{H}), 7.43(\mathrm{dd}, J=8.2,1.6 \mathrm{~Hz}$, $1 \mathrm{H}), 7.15(\mathrm{ddd}, J=8.6,6.9,1.7 \mathrm{~Hz}, 1 \mathrm{H}), 6.68(\mathrm{dd}, J=8.6,0.9 \mathrm{~Hz}, 1 \mathrm{H}), 6.66-$ $6.53(\mathrm{~m}, 2 \mathrm{H}), 6.50-6.31(\mathrm{~m}, 3 \mathrm{H}), 4.37(\mathrm{~d}, J=10.3 \mathrm{~Hz}, 1 \mathrm{H}), 3.35(\mathrm{dd}, J=11.7$, $9.7 \mathrm{~Hz}, 1 \mathrm{H}), 3.06(\mathrm{dd}, J=11.7,1.3 \mathrm{~Hz}, 1 \mathrm{H}), 0.57-0.45(\mathrm{~m}, 1 \mathrm{H}), 0.36(\mathrm{~d}, J=6.9$ $\mathrm{Hz}, 3 \mathrm{H}), 0.33(\mathrm{~d}, J=6.9 \mathrm{~Hz}, 3 \mathrm{H}) .{ }^{19} \mathrm{~F} \mathrm{NMR}\left(377 \mathrm{MHz}, \mathrm{CDCl}_{3}\right) \delta-62.47(\mathrm{~s}, 3 \mathrm{~F})$, $-62.80(\mathrm{~s}, 3 \mathrm{~F}),-109.17(\mathrm{~d}, J=22.1 \mathrm{~Hz}, 1 \mathrm{~F}),-112.62(\mathrm{~d}, J=21.6 \mathrm{~Hz}, 1 \mathrm{~F})$, -117.89 (d, $J=21.6 \mathrm{~Hz}, 1 \mathrm{~F},-118.26$ (d, $J=22.1 \mathrm{~Hz}, 1 \mathrm{~F}$ ). IR (thin film): 2960, 1605, 1553, 1523 , 1329, 1139, 1092, 924, $848 \mathrm{~cm}^{-1}$; HRMS (ESI): $[\mathrm{M}+\mathrm{H}]^{+}$calculated for $\mathrm{C}_{36} \mathrm{H}_{25} \mathrm{~F}_{10} \mathrm{IrN}_{3} \mathrm{OS}^{+}$requires $\mathrm{m} / z$ 930.1182; found $m / z$ 930.1190; $[\alpha]_{22}^{\mathrm{D}}+478.9^{\circ}\left(c 0.73, \mathrm{CH}_{2} \mathrm{Cl}_{2}\right)$.

$(S), \Lambda$-[Ir( $\left.(\mathbf{A 3})_{2}\left({ }^{i} \operatorname{PrHBT}\right)\right]$ : Prepared as described before. Spectral data were consistent with the

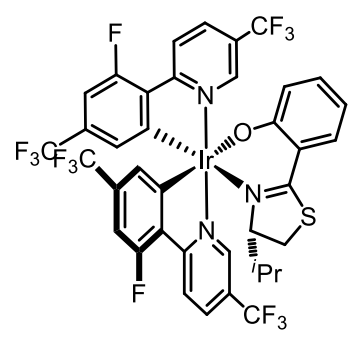
previous report. ${ }^{2}$

$(S), \Lambda$-[Ir(A4) $\left.)_{2}\left({ }^{\mathrm{i}} \mathrm{PrHBT}\right)\right]: 273.6 \mathrm{mg}, 37 \%$ yield, yellow solid; ${ }^{1} \mathrm{H}$ NMR $\left(400 \mathrm{MHz}, \mathrm{CDCl}_{3}\right) \delta 9.35(\mathrm{~s}$,

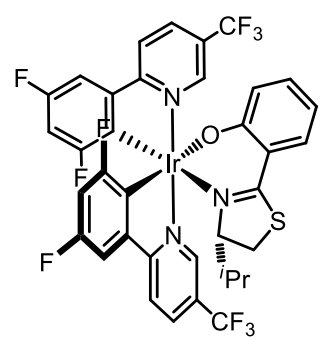
$1 \mathrm{H}), 8.65(\mathrm{~d}, J=0.5 \mathrm{~Hz}, 1 \mathrm{H}), 7.99(\mathrm{dd}, J=8.6,1.8 \mathrm{~Hz}, 1 \mathrm{H}), 7.89(\mathrm{~d}, J=8.5 \mathrm{~Hz}$, $1 \mathrm{H}), 7.85(\mathrm{dd}, J=8.7,1.6 \mathrm{~Hz}, 1 \mathrm{H}), 7.79(\mathrm{~d}, J=8.6 \mathrm{~Hz}, 1 \mathrm{H}), 7.44(\mathrm{dd}, J=8.2$, $1.7 \mathrm{~Hz}, 1 \mathrm{H}), 7.34$ (dd, $J=9.0,2.3 \mathrm{~Hz}, 1 \mathrm{H}), 7.22(\mathrm{dd}, J=9.2,2.3 \mathrm{~Hz}, 1 \mathrm{H}), 7.15$ (ddd, $J=8.6,6.9,1.7 \mathrm{~Hz}, 1 \mathrm{H}), 6.67(\mathrm{dd}, J=8.6,1.0 \mathrm{~Hz}, 1 \mathrm{H}), 6.42-6.31$ (m, $2 \mathrm{H}), 6.25(\mathrm{td}, J=9.5,2.3 \mathrm{~Hz}, 1 \mathrm{H}), 4.40-4.34(\mathrm{~m}, 1 \mathrm{H}), 3.35(\mathrm{dd}, J=11.7,9.8$ $\mathrm{Hz}, 1 \mathrm{H}), 3.06$ (dd, $J=11.8,1.4 \mathrm{~Hz}, 1 \mathrm{H}), 0.52-0.39$ (m, 1H), 0.35 (d, $J=6.8$ $\mathrm{Hz}, 3 \mathrm{H}), 0.31(\mathrm{~d}, J=6.8 \mathrm{~Hz}, 3 \mathrm{H}) .{ }^{19} \mathrm{~F}$ NMR $\left(377 \mathrm{MHz}, \mathrm{CDCl}_{3}\right) \delta-62.39(\mathrm{~s}, 3 \mathrm{~F})$, -62.73 (s, 3F), -100.53 (d, $J=5.8 \mathrm{~Hz}, 1 \mathrm{~F}),-103.91$ (d, $J=6.4 \mathrm{~Hz}, 1 \mathrm{~F}),-119.68$ (d, $J=6.3 \mathrm{~Hz}, 1 \mathrm{~F})$, -120.07 (d, $J=5.9 \mathrm{~Hz}, 1 \mathrm{~F}$ ).IR (thin film): $3068,2960,1606,1556,1524,1464,1435,1323,1134$, 1085, 991, 844, $743 \mathrm{~cm}^{-1}$; HRMS (ESI): $[\mathrm{M}+\mathrm{H}]^{+}$calculated for $\mathrm{C}_{36} \mathrm{H}_{25} \mathrm{~F}_{10} \mathrm{IrN}_{3} \mathrm{OS}^{+}$requires $\mathrm{m} / \mathrm{z}$ 930.1182; found $m / z$ 930.1182; $[\alpha]_{22}^{\mathrm{D}}:+360^{\circ}\left(c 1.15, \mathrm{CH}_{2} \mathrm{Cl}_{2}\right)$.

$\Lambda-\mathbf{4 a}$ : Prepared as described before. Spectral data match the previous report. ${ }^{2}$

$\Lambda$-4b:116.4 mg, 78\% yield, pale yellow solid; ${ }^{1} \mathrm{H}$ NMR (400 MHz, $\left.\mathrm{CDCl}_{3}\right) \delta 11.15(\mathrm{~s}, 1 \mathrm{H}), 8.53-$

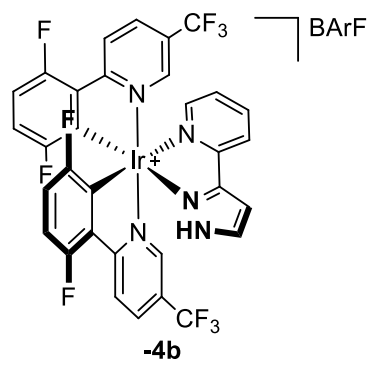

$8.38(\mathrm{~m}, 2 \mathrm{H}), 7.99$ (t, $J=9.1 \mathrm{~Hz}, 2 \mathrm{H}), 7.94-7.85(\mathrm{~m}, 2 \mathrm{H}), 7.77$ (d, $J=7.9$ $\mathrm{Hz}, 1 \mathrm{H}), 7.71(\mathrm{~s}, 8 \mathrm{H}), 7.54(\mathrm{~s}, 1 \mathrm{H}), 7.46(\mathrm{~m}, 5 \mathrm{H}), 7.43-7.35(\mathrm{~m}, 2 \mathrm{H}), 6.92$ $-6.78(\mathrm{~m}, 2 \mathrm{H}), 6.71-6.58(\mathrm{~m}, 3 \mathrm{H}) .{ }^{19} \mathrm{~F}$ NMR $\left(377 \mathrm{MHz}, \mathrm{CDCl}_{3}\right) \delta-62.50$ (s, 24F), -63.30 (s, 3F), -63.34 (s, 3F), -109.69 (d, $J=21.4 \mathrm{~Hz}, 1 \mathrm{~F})$, $-110.11(\mathrm{~d}, J=21.5 \mathrm{~Hz}, 1 \mathrm{~F}),-115.17(\mathrm{~d}, J=21.4 \mathrm{~Hz}, 1 \mathrm{~F}),-115.31(\mathrm{~d}, J=$ $21.5 \mathrm{~Hz}, 1 \mathrm{~F})$. IR (thin film): 2928, 1613, 1455, 1355, 1329, 1275, 1119, 675 $\mathrm{cm}^{-1}$; HRMS (ESI): $[\mathrm{M}]^{+}$calculated for $\mathrm{C}_{32} \mathrm{H}_{17} \mathrm{~F}_{10} \mathrm{IrN}_{5}{ }^{+}$requires $\mathrm{m} / z$ 
854.0948; found $m / z$ 854.0951; $[\alpha]_{22}^{\mathrm{D}}+124.8^{\circ}\left(c 0.66, \mathrm{CH}_{2} \mathrm{Cl}_{2}\right)$.

$\Lambda-\mathbf{4 c}$ : Prepared as described before. Spectral data match the previous report. ${ }^{2}$

$\Lambda$-4d:70.2 mg, 25\% yield, yellow solid; ${ }^{1} \mathrm{H}$ NMR (400 MHz, $\left.\mathrm{CDCl}_{3}\right) \delta 10.87(\mathrm{~s}, 1 \mathrm{H}), 8.03-7.97(\mathrm{~m}$,

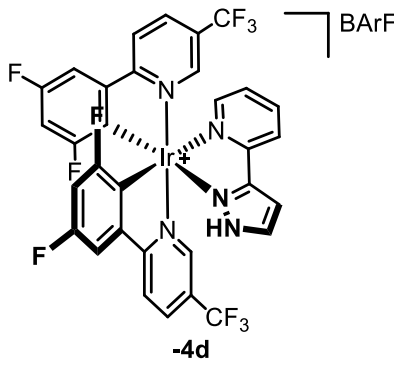
$2 \mathrm{H}), 7.96-7.89(\mathrm{~m}, 3 \mathrm{H}), 7.86(\mathrm{td}, J=7.8,1.5 \mathrm{~Hz}, 1 \mathrm{H}), 7.74(\mathrm{~d}, J=8.1$ $\mathrm{Hz}, 1 \mathrm{H}), 7.70(\mathrm{~s}, 8 \mathrm{H}), 7.52(\mathrm{~s}, 1 \mathrm{H}), 7.48-7.43(\mathrm{~m}, 5 \mathrm{H}), 7.43-7.34(\mathrm{~m}$, $4 \mathrm{H}), 6.66(\mathrm{~s}, 1 \mathrm{H}), 6.58-6.48(\mathrm{~m}, 1 \mathrm{H}) .{ }^{19} \mathrm{~F} \mathrm{NMR}\left(377 \mathrm{MHz}, \mathrm{CDCl}_{3}\right) \delta$ -62.49 (s, 24F), $-63.22(\mathrm{~s}, 3 \mathrm{~F}),-63.26(\mathrm{~s}, 3 \mathrm{~F}),-100.97$ (d, $J=6.8 \mathrm{~Hz}, 1 \mathrm{~F})$, $-101.36(\mathrm{~d}, J=7.0 \mathrm{~Hz}, 1 \mathrm{~F}),-115.14(\mathrm{~d}, J=6.9 \mathrm{~Hz}, 1 \mathrm{~F}),-115.24(\mathrm{~d}, J=$ $6.8 \mathrm{~Hz}, 1 \mathrm{~F})$. IR (thin film): 1613, 1392, 1354, 1327.37, 1273, 1118, 996, $889,673 \mathrm{~cm}^{-1}$; HRMS (ESI): [M] $]^{+}$calculated for $\mathrm{C}_{32} \mathrm{H}_{17} \mathrm{~F}_{10} \mathrm{IrN}_{5}{ }^{+}$requires $m / z$ 854.0948; found $m / z$ 854.0940; $[\alpha]_{22}^{\mathrm{D}}:+130^{\circ}\left(c 0.75, \mathrm{CH}_{2} \mathrm{Cl}_{2}\right)$.

$\Lambda$-4e: $142.7 \mathrm{mg},>99 \%$ yield, yellow solid; ${ }^{1} \mathrm{H}$ NMR (400 MHz, $\left.\mathrm{CDCl}_{3}\right) \delta 10.51(\mathrm{~s}, 1 \mathrm{H}), 8.12-8.01$

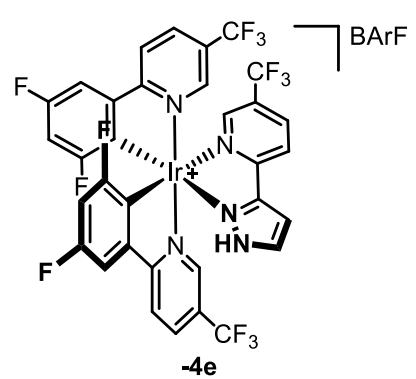
(m, 4H), $7.99-7.94(\mathrm{~m}, 2 \mathrm{H}), 7.76-7.65(\mathrm{~m}, 9 \mathrm{H}), 7.46(\mathrm{~s}, 4 \mathrm{H}), 7.44-$ $7.42(\mathrm{~m}, 2 \mathrm{H}), 7.41(\mathrm{~s}, 1 \mathrm{H}), 7.38-7.36(\mathrm{~m}, 1 \mathrm{H}), 7.35(\mathrm{~s}, 1 \mathrm{H}), 6.63-6.49$ $(\mathrm{m}, 3 \mathrm{H}) .{ }^{19} \mathrm{~F}$ NMR $\left(377 \mathrm{MHz}, \mathrm{CDCl}_{3}\right) \delta-62.51(\mathrm{~s}, 24 \mathrm{~F}),-63.00(\mathrm{~s}, 3 \mathrm{~F})$, $-63.26(\mathrm{~s}, 3 \mathrm{~F}),-63.37(\mathrm{~s}, 3 \mathrm{~F}),-101.17$ (d, $J=7.4 \mathrm{~Hz}, 1 \mathrm{~F}),-101.19$ (d, $J=$ $6.8 \mathrm{~Hz}, 1 \mathrm{~F}),-114.04$ (d, $J=7.1 \mathrm{~Hz}, 1 \mathrm{~F}),-114.58$ (d, $J=6.9 \mathrm{~Hz}, 1 \mathrm{~F})$. IR (thin film): 1616, 1355, 1327, 1275, 1116, 888, $673 \mathrm{~cm}^{-1}$; HRMS (ESI): $[\mathrm{M}]^{+}$calculated for $\mathrm{C}_{33} \mathrm{H}_{16} \mathrm{~F}_{13} \mathrm{IrN}_{5}{ }^{+}$requires $\mathrm{m} / \mathrm{z}$ 922.0822; found $\mathrm{m} / \mathrm{z}$ 922.0816; $[\alpha]_{22}^{\mathrm{D}}:+66.7^{\circ}\left(c 0.83, \mathrm{CH}_{2} \mathrm{Cl}_{2}\right)$.

$\Lambda$-4f: $81.2 \mathrm{mg}, 58 \%$ yield, yellow solid; ${ }^{1} \mathrm{H}$ NMR (400 MHz, $\left.\mathrm{CDCl}_{3}\right) \delta 12.29$ (br. s, $\left.1 \mathrm{H}\right), 7.98-7.85$

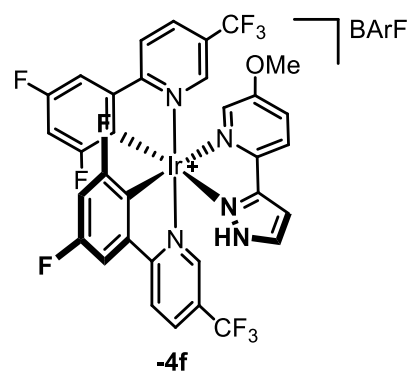
$(\mathrm{m}, 4 \mathrm{H}), 7.75-7.66(\mathrm{~m}, 9 \mathrm{H}), 7.58(\mathrm{~s}, 1 \mathrm{H}), 7.53-7.48(\mathrm{~m}, 3 \mathrm{H}), 7.46(\mathrm{~s}$, $4 \mathrm{H}), 7.38-7.32(\mathrm{~m}, 2 \mathrm{H}), 7.30(\mathrm{dd}, J=8.9,2.7 \mathrm{~Hz}, 1 \mathrm{H}), 6.63(\mathrm{~d}, J=2.8$ $\mathrm{Hz}, 1 \mathrm{H}), 6.50(\mathrm{td}, J=9.1,2.2 \mathrm{~Hz}, 1 \mathrm{H}), 6.41(\mathrm{td}, J=9.1,2.2 \mathrm{~Hz}, 1 \mathrm{H})$, $3.66(\mathrm{~s}, 3 \mathrm{H}) .{ }^{19} \mathrm{~F}$ NMR $\left(377 \mathrm{MHz}, \mathrm{CDCl}_{3}\right) \delta-62.51(\mathrm{~s}, 24 \mathrm{~F}),-63.13(\mathrm{~s}$, $3 \mathrm{~F}),-63.23(\mathrm{~s}, 3 \mathrm{~F}),-101.00(\mathrm{~d}, J=6.8 \mathrm{~Hz}, 1 \mathrm{~F}),-101.25(\mathrm{~d}, J=6.9 \mathrm{~Hz}$, $1 \mathrm{~F}),-115.15$ (d, $J=7.0 \mathrm{~Hz}, 1 \mathrm{~F}),-115.49$ (d, $J=6.8 \mathrm{~Hz}, 1 \mathrm{~F})$. IR (thin film): 2924, 1612, 1571, 1393, 1354, 1327, 1275, 1123, 1092, 889, 842, $674 \mathrm{~cm}^{-1}$; HRMS (ESI): $[\mathrm{M}]^{+}$calculated for $\mathrm{C}_{33} \mathrm{H}_{19} \mathrm{~F}_{10} \mathrm{IrN}_{5} \mathrm{O}^{+}$requires $m / z$ 884.1054; found $m / z$ 884.1052; $[\alpha]_{22}^{\mathrm{D}}:+136^{\circ}\left(c 0.97, \mathrm{CH}_{2} \mathrm{Cl}_{2}\right)$. 
Racemic ( \pm -4f was synthesized according to a literature procedure. ${ }^{1}$ Spectral data were consistent with the enantiopure material prepared by our method.

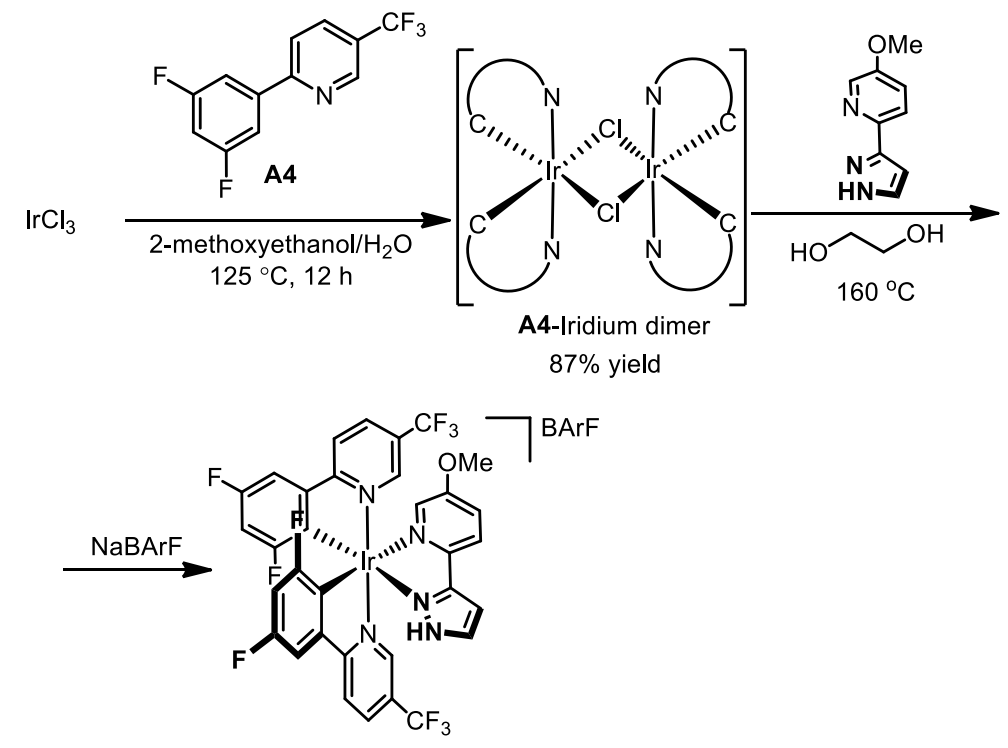

$65 \%$ yield over 2 steps 


\section{Synthesis of Substrates}
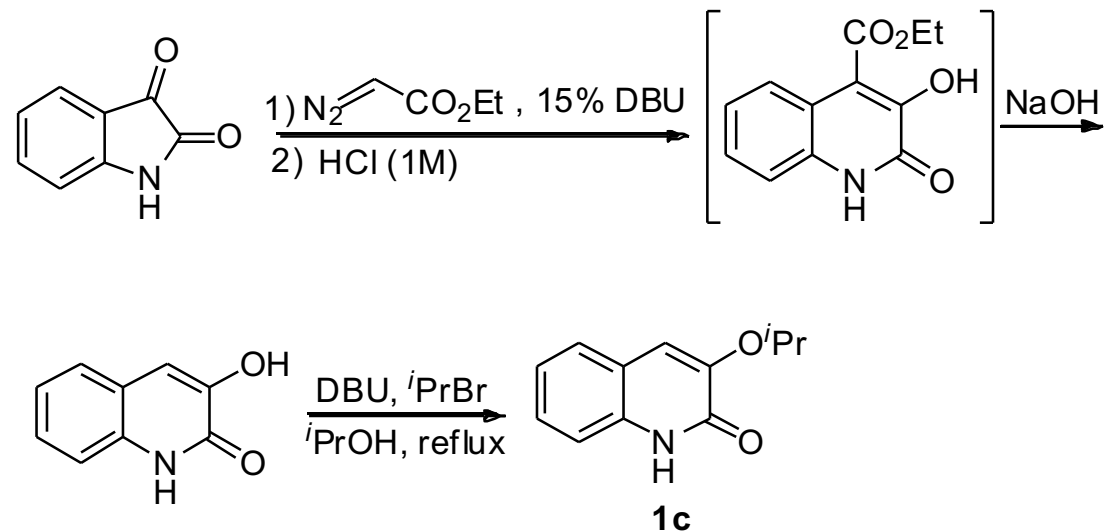

Substituted 3-alkoxyquinolones were prepared by the route shown above, following known procedures $^{2}$ unless otherwise noted. A $25 \mathrm{~mL}$ flask was charged with substituted 3-hydroxyquinolone (2.0 mmol, 1.0 equiv.), ${ }^{i} \mathrm{PrOH}(10 \mathrm{~mL}, 0.25 \mathrm{M}$ with respect to substrate), DBU (3.12 mmol, 1.56 equiv.), and ${ }^{i} \mathrm{PrBr}$ ( $2.5 \mathrm{mmol}, 1.25$ equiv.), then heated at $95{ }^{\circ} \mathrm{C}$ under $\mathrm{N}_{2}$ overnight. Following this period, the mixture was cooled to room temperature and concentrated in vacuo. The crude residue was subjected to flash column chromatography (EtOAc:Hexanes $=2: 1$ ) to afford the 3-O ${ }^{i} \operatorname{Pr}$ quinolone.

3-Butoxyquinolin-2(1H)-one (1a): Prepared as described before and the spectral data were consistent with those reported previously. ${ }^{6}$

3-Methoxyquinolin-2(1H)-one(1b): Prepared as described before ${ }^{7}$ and the spectral data were consistent with those reported previously. ${ }^{8}$

3-Isopropoxyquinolin-2(1H)-one (1c): 71\% yield, white solid; ${ }^{1} \mathrm{H}$ NMR (400 MHz, $\left.\mathrm{CDCl}_{3}\right) \delta 12.74$<smiles>CCCOc1cc2ccccc2[nH]c1=O</smiles>
(s, 1H), $7.52(\mathrm{~d}, J=8.1 \mathrm{~Hz}, 1 \mathrm{H}), 7.45(\mathrm{~d}, J=7.8 \mathrm{~Hz}, 1 \mathrm{H}), 7.37-7.30(\mathrm{~m}, 1 \mathrm{H})$, $7.21-7.13(\mathrm{~m}, 1 \mathrm{H}), 7.02(\mathrm{~s}, 1 \mathrm{H}), 4.68$ (hept, $J=6.1 \mathrm{~Hz}, 1 \mathrm{H}), 1.47$ (d, $J=6.1 \mathrm{~Hz}$, $6 \mathrm{H}) .{ }^{13} \mathrm{C}$ NMR $\left(101 \mathrm{MHz}, \mathrm{CDCl}_{3}\right) \delta 160.53,146.73,133.59,127.25,126.02$, $122.76,120.45,116.15,114.71,71.14,21.63$. IR (thin film): 3170, 3058, 2975, 1657, 1611, 1574, 1432, 1192, 1114, $754 \mathrm{~cm}^{-1}$; HRMS (ESI): $[\mathrm{M}+\mathrm{H}]^{+}$calculated for $\mathrm{C}_{12} \mathrm{H}_{14} \mathrm{NO}_{2}{ }^{+} \mathrm{m} / z$ 204.1019, found $m / z$ 204.1018; $\mathrm{mp}=151-153^{\circ} \mathrm{C}$.

3-Isopropoxy-6-methylquinolin-2(1H)-one (1e): 30\%yield, white solid; ${ }^{1} \mathrm{H}$ NMR (400 MHz, $\mathrm{CDCl}_{3}$ ) Me Oipr $\delta 11.27$ (br. s, $1 \mathrm{H}), 7.29-7.26(\mathrm{~m}, 2 \mathrm{H}), 7.20(\mathrm{dd}, J=8.3,1.4 \mathrm{~Hz}, 1 \mathrm{H}), 6.97$ (s, 1H), 4.69 (hept, $J=6.1 \mathrm{~Hz}, 1 \mathrm{H}), 2.43$ (s, 3H), 1.49 (d, $J=6.1 \mathrm{~Hz}, 6 \mathrm{H})$. ${ }^{13} \mathrm{C}$ NMR $\left(101 \mathrm{MHz}, \mathrm{CDCl}_{3}\right) \delta 159.75,146.85,132.33,131.31,128.64$, $125.95,120.41,115.42,114.39,71.08,21.61,21.00$. IR (thin film): 3158 2979, 1656, 1613, 1579, 1232, 1110, $601 \mathrm{~cm}^{-1}$; HRMS (ESI): $[\mathrm{M}+\mathrm{H}]^{+}$ calculated for $\mathrm{C}_{13} \mathrm{H}_{16} \mathrm{NO}_{2}{ }^{+} \mathrm{m} / z$ 218.1176, found $m / z 218.1173$; $\mathrm{mp}=155-157{ }^{\circ} \mathrm{C}$.

6-Iodo-3-isopropoxyquinolin-2(1H)-one (1f): $63 \%$ yield, white solid; ${ }^{1} \mathrm{H}$ NMR $\left(500 \mathrm{MHz}, \mathrm{CDCl}_{3}\right) \delta$ I $12.00(\mathrm{~s}, 1 \mathrm{H}), 7.83(\mathrm{~d}, J=1.6 \mathrm{~Hz}, 1 \mathrm{H}), 7.63(\mathrm{dd}, J=8.5,1.6 \mathrm{~Hz}, 1 \mathrm{H}), 7.21(\mathrm{~d}$, 
$J=8.6 \mathrm{~Hz}, 1 \mathrm{H}), 6.90(\mathrm{~s}, 1 \mathrm{H}), 4.68$ (hept, $J=6.1 \mathrm{~Hz}, 1 \mathrm{H}), 1.50$ (d, $J=6.1 \mathrm{~Hz}, 6 \mathrm{H}) .{ }^{13} \mathrm{C}$ NMR $(126$ $\left.\mathrm{MHz}, \mathrm{CDCl}_{3}\right) \delta 159.93,147.38,135.64,134.45,132.66,122.69,117.64,112.65,85.77,71.42,21.53$. IR (thin film): 2980, 2904, 1663, 1609, 1568, 1273, 1227, 938, $720 \mathrm{~cm}^{-1}$; HRMS (ESI): $[\mathrm{M}+\mathrm{H}]^{+}$ calculated for $\mathrm{C}_{12} \mathrm{H}_{13} \mathrm{INO}_{2}{ }^{+} \mathrm{m} / z$ 329.9986, found $\mathrm{m} / \mathrm{z} 329.9982 ; \mathrm{mp}=212-214{ }^{\circ} \mathrm{C}$.

6-Bromo-3-isopropoxyquinolin-2(1H)-one (1g): 36\% yield, white solid; ${ }^{1} \mathrm{H}$ NMR (400 $\mathrm{MHz}, \mathrm{CDCl}_{3}$ )<smiles>CCCOc1cc2cc(Br)ccc2[nH]c1=O</smiles>
$\delta 12.27(\mathrm{~s}, 1 \mathrm{H}), 7.61(\mathrm{~d}, J=2.0 \mathrm{~Hz}, 1 \mathrm{H}), 7.46-7.32(\mathrm{~m}, 2 \mathrm{H}), 6.90(\mathrm{~s}, 1 \mathrm{H})$, 4.67 (hept, $J=6.1 \mathrm{~Hz}, 1 \mathrm{H}), 1.48(\mathrm{~d}, J=6.1 \mathrm{~Hz}, 6 \mathrm{H}) .{ }^{13} \mathrm{C}$ NMR $(101 \mathrm{MHz}$, $\left.\mathrm{CDCl}_{3}\right) \delta 160.03,147.56,132.14,130.05,128.25,122.17,117.56,115.54$, 112.83, 71.42, 21.53. IR (thin film): 2982, 2916, 1666, 1612, 1570, 1418, $1270,938,722 \mathrm{~cm}^{-1}$; HRMS (ESI): $[\mathrm{M}+\mathrm{H}]^{+}$calculated for $\mathrm{C}_{12} \mathrm{H}_{13} \mathrm{BrNO}_{2}{ }^{+} \mathrm{m} / \mathrm{z}$ 282.0124, found $\mathrm{m} / \mathrm{z} 282.0125 ; \mathrm{mp}=192-194^{\circ} \mathrm{C}$.

6-Fluoro-3-isopropoxyquinolin-2(1H)-one (1h): $50 \%$ yield, white solid; ${ }^{1} \mathrm{H}$ NMR (500 $\mathrm{MHz}, \mathrm{CDCl}_{3}$ )<smiles>CCCOc1cc2cc(F)ccc2[nH]c1=O</smiles>
$\delta 11.71(\mathrm{~s}, 1 \mathrm{H}), 7.38(\mathrm{dd}, J=8.9,4.7 \mathrm{~Hz}, 1 \mathrm{H}), 7.17(\mathrm{dd}, J=8.9,2.6 \mathrm{~Hz}, 1 \mathrm{H})$, $7.12(\mathrm{td}, J=8.6,2.7 \mathrm{~Hz}, 1 \mathrm{H}), 6.95(\mathrm{~s}, 1 \mathrm{H}), 4.70$ (hept, $J=6.1 \mathrm{~Hz}, 1 \mathrm{H}), 1.51(\mathrm{~d}$, $J=6.1 \mathrm{~Hz}, 6 \mathrm{H}) \cdot{ }^{13} \mathrm{C} \mathrm{NMR}\left(126 \mathrm{MHz}, \mathrm{CDCl}_{3}\right) \delta 159.62(\mathrm{~s}), 158.56(\mathrm{~d}, J=$ $241.2 \mathrm{~Hz}), 147.78$ (s), 129.72 (s), 121.45 (d, $J=9.2 \mathrm{~Hz}), 117.12$ (d, $J=8.7$ $1 \mathrm{~h}$ $\mathrm{Hz}), 115.24(\mathrm{~d}, J=24.5 \mathrm{~Hz}), 113.27(\mathrm{~d}, J=3.1 \mathrm{~Hz}), 111.11(\mathrm{~d}, J=23.3 \mathrm{~Hz})$, 71.38 (s), 21.55 (s). ${ }^{19} \mathrm{~F}$ NMR (377 MHz, $\mathrm{CDCl}_{3}$ ) $\delta-119.91$ (s, 1F). IR (thin film): 2988, 2947, 2866, $1660,1622,1583,1521,1422,1272,950,852,603 \mathrm{~cm}^{-1}$; HRMS (ESI): $[\mathrm{M}+\mathrm{H}]^{+}$calculated for $\mathrm{C}_{12} \mathrm{H}_{13} \mathrm{FNO}_{2}{ }^{+}, m / z 222.0925$, found $m / z 222.0922 ; \mathrm{mp}=158-160{ }^{\circ} \mathrm{C}$.

3-Isopropoxy-6-methoxyquinolin-2(1H)-one (3i): 38\% yield, white solid; ${ }^{1} \mathrm{H}$ NMR (400 $\mathrm{MHz}$,<smiles>CCCOc1cc2cc(OC)ccc2[nH]c1=O</smiles>
$\left.\mathrm{CDCl}_{3}\right) \delta 12.08(\mathrm{~s}, 1 \mathrm{H}), 7.37(\mathrm{~d}, J=8.8,1 \mathrm{H}), 6.99(\mathrm{dd}, J=8.9,2.7 \mathrm{~Hz}, 1 \mathrm{H})$, $6.97(\mathrm{~s}, 1 \mathrm{H}), 6.91(\mathrm{~d}, J=2.6 \mathrm{~Hz}, 1 \mathrm{H}), 4.68$ (hept, $J=6.1 \mathrm{~Hz}, 1 \mathrm{H}), 3.85$ (s, $3 \mathrm{H}), 1.48(\mathrm{~d}, J=6.1 \mathrm{~Hz}, 6 \mathrm{H}) \cdot{ }^{13} \mathrm{C}$ NMR $\left(101 \mathrm{MHz}, \mathrm{CDCl}_{3}\right) \delta 159.64$, $155.42,147.27,127.94,121.22,117.17,116.23,114.26,107.90,71.15$, 55.65, 21.63. IR (thin film): 2978, 2929, 2839, 1654, 1615, 1576, 1501, 1421, 1283, 1216, 1185, 1109, $596 \mathrm{~cm}^{-1}$; HRMS (ESI): $[\mathrm{M}+\mathrm{H}]^{+}$calculated for $\mathrm{C}_{13} \mathrm{H}_{16} \mathrm{NO}_{3}{ }^{+} \mathrm{m} / z$ 234.1125, found $m / z 234.1122 ; \mathrm{mp}=170-172{ }^{\circ} \mathrm{C}$.

7-Bromo-3-isopropoxyquinolin-2(1H)-one (1j): 48\% yield, white solid; ${ }^{1} \mathrm{H}$ NMR (500 $\left.\mathrm{MHz}, \mathrm{CDCl}_{3}\right)$<smiles>CCCOc1cc2ccc(Br)cc2[nH]c1=O</smiles>
$\delta 12.22(\mathrm{~s}, 1 \mathrm{H}), 7.56(\mathrm{~d}, J=1.3 \mathrm{~Hz}, 1 \mathrm{H}), 7.25-7.18(\mathrm{~m}, 2 \mathrm{H}), 6.88(\mathrm{~s}, 1 \mathrm{H})$, 4.60 (hept, $J=6.1 \mathrm{~Hz}, 1 \mathrm{H}), 1.41(\mathrm{~d}, J=6.1 \mathrm{~Hz}, 6 \mathrm{H}) .{ }^{13} \mathrm{C}$ NMR $(126 \mathrm{MHz}$, $\left.\mathrm{CDCl}_{3}\right) \delta 160.23,146.99,134.27,127.34,126.17,120.88,119.39,118.44$, 113.92, 71.44, 21.58. IR (thin film): 2977, 2931, 2839, 1659, 1607, 1565, 1 j $1400,1374,1262,1189,937,872 \mathrm{~cm}^{-1}$; HRMS (ESI): $[\mathrm{M}+\mathrm{H}]^{+}$calculated for $\mathrm{C}_{12} \mathrm{H}_{13} \mathrm{BrNO}_{2}{ }^{+} \mathrm{m} / \mathrm{z} 282.0124$, found $\mathrm{m} / \mathrm{z} 282.0123 ; \mathrm{mp}=225-227^{\circ} \mathrm{C}$.

7-Chloro-3-isopropoxyquinolin-2(1H)-one (1k): 13\% yield, white solid; ${ }^{1} \mathrm{H}$ NMR (500 MHz, $\mathrm{CDCl}_{3}$ )<smiles>CCCOc1cc2ccc(Cl)cc2[nH]c1=O</smiles>
$\delta 11.44(\mathrm{~s}, 1 \mathrm{H}), 7.43-7.39(\mathrm{~m}, 2 \mathrm{H}), 7.18(\mathrm{dd}, J=8.4,1.9 \mathrm{~Hz}, 1 \mathrm{H}), 6.98(\mathrm{~s}$, 1H), 4.70 (hept, $J=6.1 \mathrm{~Hz}, 1 \mathrm{H}), 1.51(\mathrm{~d}, J=6.1 \mathrm{~Hz}, 6 \mathrm{H}) .{ }^{13} \mathrm{C}$ NMR $(126$ 
$\left.\mathrm{MHz}, \mathrm{CDCl}_{3}\right) \delta 159.86,146.90,133.98,133.10,127.32,123.44,119.03,115.19,113.79,71.41,21.58$. IR (thin film): 2978, 2933, 1663, 1609, 1568, 1402, 1376, 1262, 1190, 943, 873, $627 \mathrm{~cm}^{-1}$; HRMS (ESI): $[\mathrm{M}+\mathrm{H}]^{+}$calculated for $\mathrm{C}_{12} \mathrm{H}_{13} \mathrm{ClNO}_{2}{ }^{+} \mathrm{m} / \mathrm{z} 238.0629$, found $\mathrm{m} / \mathrm{z} 238.0627 ; \mathrm{mp}=231-233{ }^{\circ} \mathrm{C}$.

6,7-Difluoro-3-hydroxyquinolin-2(1H)-one (01): 45\% yield, white solid; ${ }^{1} \mathrm{H}$ NMR (500 $\mathrm{MHz}$,<smiles>O=c1[nH]c2cc(F)c(F)cc2cc1O</smiles>

이

DMSO- $\left.d_{6}\right) \delta 12.10(\mathrm{~s}, 1 \mathrm{H}), 9.72(\mathrm{~s}, 1 \mathrm{H}), 7.61(\mathrm{dd}, J=11.3,8.6 \mathrm{~Hz}, 1 \mathrm{H}), 7.19$ $(\mathrm{dd}, J=11.3,7.2 \mathrm{~Hz}, 1 \mathrm{H}), 7.07(\mathrm{~s}, 1 \mathrm{H}) .{ }^{13} \mathrm{C}$ NMR $\left(126 \mathrm{MHz}, \mathrm{DMSO}-d_{6}\right) \delta$ 158.79 (s), 148.51 (dd, $J=244.5,14.7 \mathrm{~Hz}), 147.10$ (d, $J=2.6 \mathrm{~Hz}), 145.74$ (dd, $J=244.5,13.9 \mathrm{~Hz}), 130.63(\mathrm{dd}, J=9.5,0.9 \mathrm{~Hz}), 118.00(\mathrm{dd}, J=7.8,2.4 \mathrm{~Hz})$, $113.56(\mathrm{~d}, J=18.9 \mathrm{~Hz}), 112.03(\mathrm{~s}), 103.45(\mathrm{~d}, J=21.2 \mathrm{~Hz}) .{ }^{19} \mathrm{~F}$ NMR $(377$ MHz, DMSO- $\left.d_{6}\right) \delta-138.74(\mathrm{~d}, J=23.3 \mathrm{~Hz}, 1 \mathrm{~F}),-145.23$ (d, $J=23.3 \mathrm{~Hz}, 1 \mathrm{~F}$ ). IR (thin film): 3074 , 2989, 1629, 1562, 1522, 1368, 1295, 1207, 893, $776 \mathrm{~cm}^{-1}$; HRMS (ESI): $[\mathrm{M}+\mathrm{H}]^{+}$calculated for $\mathrm{C}_{9} \mathrm{H}_{6} \mathrm{~F}_{2} \mathrm{NO}_{2}{ }^{+} m / z 198.0361$, found $m / z 198.0360 ; \mathrm{mp}>260{ }^{\circ} \mathrm{C}$.

6,7-Difluoro-3-isopropoxyquinolin-2(1H)-one (11): 37\% yield, white solid; ${ }^{1} \mathrm{H}$ NMR $(500 \mathrm{MHz}$,<smiles>CCCOc1cc2cc(F)c(F)cc2[nH]c1=O</smiles>
$\left.\mathrm{CDCl}_{3}\right) \delta 11.78(\mathrm{~s}, 1 \mathrm{H}), 7.20-7.15(\mathrm{~m}, 2 \mathrm{H}), 6.83(\mathrm{~s}, 1 \mathrm{H}), 4.59$ (hept, $J=6.1$ $\mathrm{Hz}, 1 \mathrm{H}), 1.41(\mathrm{~d}, J=6.1 \mathrm{~Hz}, 6 \mathrm{H}) \cdot{ }^{13} \mathrm{C} \mathrm{NMR}\left(126 \mathrm{MHz}, \mathrm{CDCl}_{3}\right) \delta 159.89(\mathrm{~s})$, 149.93 (dd, $J=244.6,14.7 \mathrm{~Hz}), 147.00$ (d, $J=2.7 \mathrm{~Hz}), 146.93$ (dd, $J=244.6$, $14.0 \mathrm{~Hz}), 129.79$ (dd, $J=9.3,1.5 \mathrm{~Hz}), 116.81$ (dd, $J=7.3,2.7 \mathrm{~Hz}), 113.29$ (s), $113.25(\mathrm{~d}, J=17.8 \mathrm{~Hz}), 104.24(\mathrm{~d}, J=21.3 \mathrm{~Hz}), 71.50$ (s), 21.55 (s). ${ }^{19} \mathrm{~F}$ NMR $\left(377 \mathrm{MHz}, \mathrm{CDCl}_{3}\right) \delta-136.10(\mathrm{~d}, J=21.9 \mathrm{~Hz}, 1 \mathrm{~F}),-143.24$ (d, $\left.J=21.9 \mathrm{~Hz}, 1 \mathrm{~F}\right)$. IR (thin film): 3061 , 2981, 2937, 1659, 1614, 1577, 1501, 1428, 1208, 1110, 939, $595 \mathrm{~cm}^{-1}$; HRMS (ESI): $[\mathrm{M}+\mathrm{H}]^{+}$ calculated for $\mathrm{C}_{12} \mathrm{H}_{12} \mathrm{~F}_{2} \mathrm{NO}_{2}{ }^{+} \mathrm{m} / z 240.0831$, found $\mathrm{m} / z 240.0828$; $\mathrm{mp}=236-238{ }^{\circ} \mathrm{C}$.<smiles>O=C1Nc2cccc(Cl)c2C1=O</smiles><smiles>O=c1[nH]c2cccc(Cl)c2cc1O</smiles><smiles>CCCOc1cc2c(Cl)cc(C)cc2[nH]c1=O</smiles>

5-Chloro-3-isopropoxyquinolin-2(1H)-one (1m): 33\% yield from 5-chloro-3-hydroxyquinolin-2(1 H) -one ${ }^{7}$, white solid; ${ }^{1} \mathrm{H}$ NMR (500 MHz, $\left.\mathrm{CDCl}_{3}\right) \delta 11.37$ (s, 1H), 7.31 (s, 1H), $7.24-7.19(\mathrm{~m}, 3 \mathrm{H}), 4.70$ (hept, $J=6.1 \mathrm{~Hz}, 1 \mathrm{H}), 1.44(\mathrm{~d}, J=6.1 \mathrm{~Hz}, 6 \mathrm{H}) \cdot{ }^{13} \mathrm{C} \mathrm{NMR}\left(126 \mathrm{MHz}, \mathrm{CDCl}_{3}\right) \delta 159.59,147.67$, 134.23, 130.36, 127.37, 123.39, 118.56, 114.42, 110.29, 71.54, 21.49. IR (thin film): 2980, 2902, $1662,1605,1571,1431,1237,1177,786 \mathrm{~cm}^{-1}$; HRMS (ESI): $[\mathrm{M}+\mathrm{H}]^{+}$calculated for $\mathrm{C}_{12} \mathrm{H}_{13} \mathrm{ClNO}_{2}{ }^{+}$ $\mathrm{m} / \mathrm{z} 238.0629$, found $\mathrm{m} / \mathrm{z} 238.0627 ; \mathrm{mp}=209-211^{\circ} \mathrm{C}$.

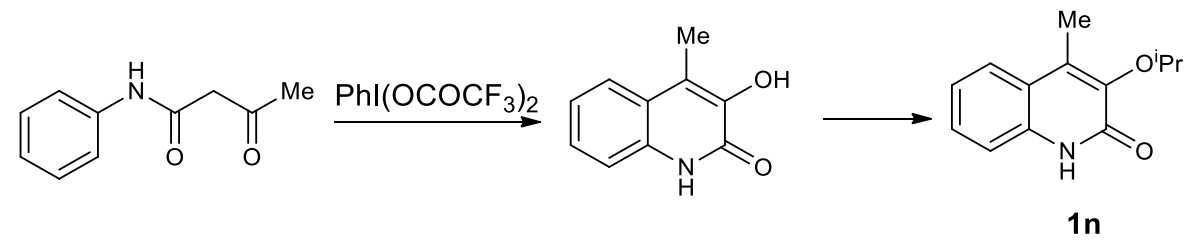

3-Isopropoxy-4-methyl quinolin-2(1H)-one (1n): 34\% yield from 3-hydroxy-4-methylquinolin-<smiles>CCOc1c([N+](=O)[O-])c(=O)[nH]c2ccccc12</smiles2(1H)-one ${ }^{9}$, white solid; ${ }^{1} \mathrm{H}$ NMR $\left(400 \mathrm{MHz}, \mathrm{CDCl}_{3}\right) \delta 12.26(\mathrm{~s}, 1 \mathrm{H}), 7.66(\mathrm{~d}, J=$ $8.4 \mathrm{~Hz}, 1 \mathrm{H}), 7.47-7.39$ (m, 2H), $7.36-7.16$ (m, 1H), 4.97 (hept, $J=6.2 \mathrm{~Hz}, 1 \mathrm{H}$ ), 
$2.47(\mathrm{~s}, 3 \mathrm{H}), 1.38(\mathrm{~d}, J=6.2 \mathrm{~Hz}, 6 \mathrm{H}) .{ }^{13} \mathrm{C} \mathrm{NMR}\left(101 \mathrm{MHz}, \mathrm{CDCl}_{3}\right) \delta 160.87,143.82,135.47,135.17$, 128.36, 124.28, 122.47, 121.23, 115.93, 74.01, 22.74, 12.10. IR (thin film): 2974, 2861, 1656, 1612, 1566, 1501, 1434, 1276, 1208, $743 \mathrm{~cm}^{-1}$; HRMS (ESI): $[\mathrm{M}+\mathrm{H}]^{+}$calculated for $\mathrm{C}_{13} \mathrm{H}_{16} \mathrm{NO}_{2}{ }^{+} \mathrm{m} / \mathrm{z}$ 218.1176, found $m / z 218.1174 ; \mathrm{mp}=155-158^{\circ} \mathrm{C}$.

8-Chloro-3-isopropoxyquinolin-2(1H)-one (10): 4\% yield, white solid; ${ }^{1} \mathrm{H}$ NMR (500 $\mathrm{MHz}, \mathrm{CDCl}_{3}$ )<smiles>CCCOc1cc2cccc(Cl)c2[nH]c1=O</smiles>
$\delta 9.07(\mathrm{~s}, 1 \mathrm{H}), \delta 7.33(\mathrm{dd}, J=7.9,1.2 \mathrm{~Hz}, 1 \mathrm{H}), 7.31(\mathrm{~d}, J=8.0 \mathrm{~Hz}, 1 \mathrm{H}), 7.06(\mathrm{t}, J$ $=7.9 \mathrm{~Hz}, 1 \mathrm{H}), 6.84(\mathrm{~s}, 1 \mathrm{H}), 4.60$ (hept, $J=6.1 \mathrm{~Hz}, 1 \mathrm{H}), 1.39$ (d, $J=6.1 \mathrm{~Hz}, 6 \mathrm{H})$. ${ }^{13} \mathrm{C}$ NMR $\left(126 \mathrm{MHz}, \mathrm{CDCl}_{3}\right) \delta 158.20,147.82,129.58,126.98,125.05,123.06$, $121.70,118.75,112.91,71.42,21.51$. IR (thin film): 3057, 2978, 2932, 1657, $1608,1562,1479,1380,1292,1249,1108,930,835 \mathrm{~cm}^{-1}$; HRMS (ESI): $[\mathrm{M}+\mathrm{H}]^{+}$ calculated for $\mathrm{C}_{12} \mathrm{H}_{13} \mathrm{ClNO}_{2}{ }^{+} \mathrm{m} / z$ 238.0629, found $\mathrm{m} / z$ 238.0626; $\mathrm{mp}=136-138{ }^{\circ} \mathrm{C}$.

8-Fluoro-3-isopropoxyquinolin-2(1H)-one (1p): 29\% yield, white solid; ${ }^{1} \mathrm{H}$ NMR (500 MHz, $\mathrm{CDCl}_{3}$ )<smiles>CCCOc1cc2cccc(F)c2[nH]c1=O</smiles>
$\delta 9.45(\mathrm{~s}, 1 \mathrm{H}), 7.26-7.21(\mathrm{~m}, 1 \mathrm{H}), 7.15-7.07$ (m, 2H), 6.94 (s, 1H), 4.66 (hept, $J$ $=6.1 \mathrm{~Hz}, 1 \mathrm{H}), 1.46(\mathrm{~d}, J=6.1 \mathrm{~Hz}, 6 \mathrm{H}) \cdot{ }^{13} \mathrm{C} \mathrm{NMR}\left(126 \mathrm{MHz}, \mathrm{CDCl}_{3}\right) \delta 158.10(\mathrm{~s})$, 149.02 (d, $J=244.7 \mathrm{~Hz}), 147.96$ (s), 122.55 (d, $J=7.3 \mathrm{~Hz}), 122.33$ (d, $J=2.9 \mathrm{~Hz})$, $122.05(\mathrm{~d}, J=13.4 \mathrm{~Hz}), 121.63(\mathrm{~d}, J=3.6 \mathrm{~Hz}), 112.96(\mathrm{~d}, J=2.7 \mathrm{~Hz}), 112.35$ (d, $J$ $=17.2 \mathrm{~Hz}), 71.34(\mathrm{~s}), 21.51(\mathrm{~s}) .{ }^{19} \mathrm{~F}$ NMR $\left(377 \mathrm{MHz}, \mathrm{CDCl}_{3}\right) \delta-135.99(\mathrm{~s}, 1 \mathrm{~F})$. IR (thin film): 2974, 2484, 1643, 1606, 1576, 1489, 1437, 1257, 1236, 1105, 868, 791, $531 \mathrm{~cm}^{-1}$; HRMS (ESI): $[\mathrm{M}+\mathrm{H}]^{+}$calculated for $\mathrm{C}_{12} \mathrm{H}_{13} \mathrm{FNO}_{2}{ }^{+} \mathrm{m} / \mathrm{z} 222.0925$, found $\mathrm{m} / \mathrm{z} 222.0922 ; \mathrm{mp}=175-176{ }^{\circ} \mathrm{C}$.

3-Isopropoxy-2H-chromen-2-one (1q): 82\% yield from 3-hydroxycoumarin, white solid; ${ }^{1} \mathrm{H}$ NMR<smiles>CCCOc1cc2ccccc2oc1=O</smiles>
$\left(500 \mathrm{MHz}, \mathrm{CDCl}_{3}\right) \delta 7.33-7.27(\mathrm{~m}, 2 \mathrm{H}), 7.22(\mathrm{~d}, J=8.2 \mathrm{~Hz}, 1 \mathrm{H}), 7.17(\mathrm{td}, J=7.5$, $1.2 \mathrm{~Hz}, 1 \mathrm{H}), 6.76(\mathrm{~s}, 1 \mathrm{H}), 4.54$ (hept, $J=6.1 \mathrm{~Hz}, 1 \mathrm{H}), 1.36(\mathrm{~d}, J=6.1 \mathrm{~Hz}, 6 \mathrm{H}) .{ }^{13} \mathrm{C}$ $\operatorname{NMR}\left(126 \mathrm{MHz}, \mathrm{CDCl}_{3}\right) \delta 158.12,149.53,142.67,128.34,126.30,124.60,119.90$, $1 q$ 116.24, 115.13, 71.85, 21.50. IR (thin film): 3068, 2978, 2932, 1728, 1621, 1453, $1295,1134,1107,930,755 \mathrm{~cm}^{-1}$; HRMS (ESI): $[\mathrm{M}+\mathrm{H}]^{+}$calculated for $\mathrm{C}_{12} \mathrm{H}_{13} \mathrm{O}_{3}{ }^{+}$ $\mathrm{m} / \mathrm{z} 205.0859$, found $\mathrm{m} / \mathrm{z} 205.0857 ; \mathrm{mp}=71-73^{\circ} \mathrm{C}$.

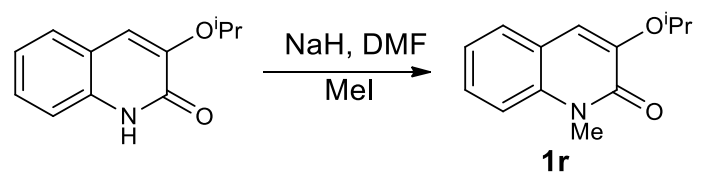

3-Isopropoxy-1-methylquinolin-2(1H)-one(1r) was prepared from 1c following a known method ${ }^{2}$ : 98\% yield, colorless oil; ${ }^{1} \mathrm{H}$ NMR $\left(500 \mathrm{MHz}, \mathrm{CDCl}_{3}\right) \delta 7.47(\mathrm{dd}, J=7.7,1.1 \mathrm{~Hz}, 1 \mathrm{H}), 7.42$ (ddd, $J=$ 8.7, 7.3, $1.4 \mathrm{~Hz}, 1 \mathrm{H}), 7.31$ (d, $J=8.4 \mathrm{~Hz}, 1 \mathrm{H}), 7.22$ (t, $J=7.5 \mathrm{~Hz}, 1 \mathrm{H}), 6.91$ (s, 1H), 4.64 (hept, $J=$ $6.1 \mathrm{~Hz}, 1 \mathrm{H}), 3.77(\mathrm{~s}, 3 \mathrm{H}), 1.44(\mathrm{~d}, J=6.1 \mathrm{~Hz}, 6 \mathrm{H}) .{ }^{13} \mathrm{C} \mathrm{NMR}\left(126 \mathrm{MHz}, \mathrm{CDCl}_{3}\right) \delta 158.77,146.59$, $135.48,127.34,127.09,122.44,120.78,113.80,113.09,71.01,29.98,21.64$. IR (thin film): 2978 , 1650, 1600, 1462, 1238, 1115, $751 \mathrm{~cm}^{-1}$; HRMS (ESI): $[\mathrm{M}+\mathrm{H}]^{+}$calculated for $\mathrm{C}_{13} \mathrm{H}_{16} \mathrm{NO}_{2}{ }^{+} \mathrm{m} / z$ 218.1176, found $\mathrm{m} / \mathrm{z} 218.1172$. 


\section{Photosensitized [2+2] Cycloadditions}

\section{a. Racemic [2+2] Cycloadditions}

A $12 \mathrm{~mL}$ vial was charged with substrate $(0.1 \mathrm{mmol}, 1.0$ equiv. $),( \pm)-\mathbf{4 f}(0.0015 \mathrm{mmol}, 1.5 \mathrm{~mol} \%)$, maleimide $\left(0.5 \mathrm{mmol}, 5\right.$ equiv.) and dry $\mathrm{CH}_{2} \mathrm{Cl}_{2}(5 \mathrm{~mL})$. The mixture was irradiated with a $16 \mathrm{~W}$ blue LED lamp or $15 \mathrm{~W}$ blue LED lamp for 14-24 h at room temperature, then concentrated in vacuo. Purification by silica gel chromatography (EtOAc:Hexanes $=2: 1)$ provided racemic cyclobutane.

\section{b. Enantioselective[2+2] Cycloadditions}

A $25 \mathrm{~mL}$ Schlenk tube was charged with a magnetic stir bar, substrate $(0.2 \mathrm{mmol}, 1.0$ equiv. $),(\Lambda)-\mathbf{4 f}$ (0.003 mmol, $1.5 \mathrm{~mol} \%)$, maleimide ( $1 \mathrm{mmol}, 5$ equiv.) and dry $\mathrm{CH}_{2} \mathrm{Cl}_{2}(10 \mathrm{~mL})$. The mixture was irradiated with a $15 \mathrm{~W}$ blue LED lamp for $24 \mathrm{~h}$ at $-78{ }^{\circ} \mathrm{C}$, then the mixture was concentrated in vacuo. The residue was purified by silica gel column chromatography (EtOAc:Hexanes $=2: 1$ or EtOAc: $\left.\mathrm{CH}_{2} \mathrm{Cl}_{2}=1: 1\right)$ to afford the target [2+2] cycloadduct. In cases where a mixture of diastereomers were formed, these products could not be separated by flash column chromatography. Instead, we found that the minor product could be removed by washing with $\mathrm{CHCl}_{3}$. The resulting residue, which was the major diastereomer, was used for further characterization.

(6aR,6bS,9aS,9bR)-6a-Butoxy-9a,9b-dihydro-5H-pyrrolo[3',4':3,4]cyclobuta[1,2-c]quinoline-6,7,<smiles>C[C@@]12C(=O)Nc3ccccc3[C@H]1[C@@H]1C(=O)NC(=O)[C@H]12</smiles>

$3 a$ 9(6aH,6bH,8H)-trione (3a): $14 \%$ NMR yield, $7 \%$ ee (HPLC, Daicel CHIRALPAK ${ }^{\circledR}$ AD-H, Gradient $5 \%{ }^{i} \mathrm{PrOH}\left(95 \%\right.$ Hexane) to $40 \%{ }^{i} \mathrm{PrOH}(60 \%$ Hexane), $\left.1.0 \mathrm{~mL} / \mathrm{min}, 221 \mathrm{~nm} ; \mathrm{t}_{1}=11.44 \mathrm{~min}, \mathrm{t}_{2}=14.79 \mathrm{~min}\right) ;{ }^{1} \mathrm{H}$ NMR $(500$ $\left.\mathrm{MHz}, \mathrm{CD}_{3} \mathrm{OD}\right) \delta 7.19-7.11(\mathrm{~m}, 2 \mathrm{H}), 6.97(\mathrm{td}, J=7.5,1.0 \mathrm{~Hz}, 1 \mathrm{H}), 6.82(\mathrm{~d}, J=$ $7.9 \mathrm{~Hz}, 1 \mathrm{H}), 3.78(\mathrm{~d}, J=5.6 \mathrm{~Hz}, 1 \mathrm{H}), 3.61(\mathrm{dd}, J=6.5,1.2 \mathrm{~Hz}, 1 \mathrm{H}), 3.38(\mathrm{dt}, J$ $=8.7,6.2 \mathrm{~Hz}, 1 \mathrm{H}), 3.26(\mathrm{dt}, J=8.7,6.7 \mathrm{~Hz}, 1 \mathrm{H}), 2.77(\mathrm{dd}, J=6.4,5.7 \mathrm{~Hz}, 1 \mathrm{H})$, $1.46-1.33(\mathrm{~m}, 2 \mathrm{H}), 1.31-1.17(\mathrm{~m}, 2 \mathrm{H}), 0.77(\mathrm{t}, J=7.4 \mathrm{~Hz}, 3 \mathrm{H}) .{ }^{13} \mathrm{C}$ NMR $\left(126 \mathrm{MHz}, \mathrm{CD}_{3} \mathrm{OD}\right) \delta 179.84,174.84,167.41,135.69,128.66,127.86,123.76$, 121.23, 115.63, 73.16, 64.83, 48.39, 47.18, 41.10, 31.39, 18.75, 12.73. IR (thin film): 3238, 2958, 1773, 1716, 1679, 1598, 1338, 1182, $760 \mathrm{~cm}^{-1}$; HRMS (ESI): $\left[\mathrm{M}+\mathrm{NH}_{4}\right]^{+}$calculated for $\mathrm{C}_{17} \mathrm{H}_{22} \mathrm{~N}_{3} \mathrm{O}_{4}{ }^{+}$ $\mathrm{m} / \mathrm{z} 332.1605$, found $\mathrm{m} / \mathrm{z} 332.1599 ; \mathrm{mp}=233-235^{\circ} \mathrm{C}$.

(6a $R, 6 \mathrm{~b} S, 9 \mathrm{a} S, 9 \mathrm{~b} R)-6 \mathrm{a}-M e t h o x y-9 \mathrm{a}, 9 \mathrm{~b}-d i h y d r o-5 H$-pyrrolo $\left[3^{\prime}, 4^{\prime}: 3,4\right]$ cyclobuta[1,2-c]quinoline-6,<smiles>C[C@@H]1C(=O)NC(=O)[C@@H]2c3ccccc3NC(=O)[C@]12C</smiles>

$3 \mathbf{b}$ 7,9(6aH,6bH,8H)-trione (3b): $30 \%$ NMR yield, $70 \%$ ee (HPLC, Daicel CHIRALPAK ${ }^{\circledR}$ AD-H, Gradient $5 \%{ }^{i} \mathrm{PrOH}$ (95\% Hexane) to $40 \%{ }^{i} \mathrm{PrOH}(60 \%$ Hexane), $\left.1.0 \mathrm{~mL} / \mathrm{min}, 277.4 \mathrm{~nm} ; \mathrm{t}_{1}=13.64 \mathrm{~min}, \mathrm{t}_{2}=15.54 \mathrm{~min}\right) ;{ }^{1} \mathrm{H}$ NMR $(500$ $\left.\mathrm{MHz}, \mathrm{CD}_{3} \mathrm{OD}\right) \delta 7.27-7.21(\mathrm{~m}, 2 \mathrm{H}), 7.07(\mathrm{td}, J=7.6,0.8 \mathrm{~Hz}, 1 \mathrm{H}), 6.92(\mathrm{~d}, J$ $=7.9 \mathrm{~Hz}, 1 \mathrm{H}), 3.93(\mathrm{~d}, J=5.5 \mathrm{~Hz}, 1 \mathrm{H}), 3.70(\mathrm{dd}, J=6.5,1.0 \mathrm{~Hz}, 1 \mathrm{H}), 3.26(\mathrm{~s}$, $3 \mathrm{H}), 2.85(\mathrm{dd}, J=6.3,5.8 \mathrm{~Hz}, 1 \mathrm{H}) .{ }^{13} \mathrm{C} \mathrm{NMR}\left(126 \mathrm{MHz}, \mathrm{CD}_{3} \mathrm{OD}\right) \delta 179.80$, 174.92, 167.08, 135.65, 128.66, 127.84, 123.79, 121.26, 115.65, 73.74, 51.97, 48.20, 46.58, 40.96. IR (thin film): 3231, 3086, 1771, 1713, 1679, 1600, 1494, 1341, 1183, $761 \mathrm{~cm}^{-1}$; HRMS (ESI): $\left[\mathrm{M}+\mathrm{NH}_{4}\right]^{+}$calculated for $\mathrm{C}_{14} \mathrm{H}_{16} \mathrm{~N}_{3} \mathrm{O}_{4}{ }^{+} \mathrm{m} / z$ 290.1135, found $\mathrm{m} / z$ 290.1134; $\mathrm{mp}>260{ }^{\circ} \mathrm{C}$. 
(6a $R, 6 \mathrm{~b} S, 9 \mathrm{a} S, 9 \mathrm{~b} R)$-6a-Isopropoxy-9a,9b-dihydro-5H-pyrrolo[3',4':3,4]cyclobuta[1,2-c]quinoline<smiles>O=C1NC(=O)[C@H]2[C@H]1[C@]1(C(F)(F)F)C(=O)Nc3ccccc3[C@H]21</smiles>

$-6,7,9(6 \mathbf{a H}, 6 \mathbf{b} H, 8 H)$-trione (3c): $82 \%$ yield, white solid, 97\% ee (SFC, Daicel CHIRALPAK $^{\circledR}$ AD-H, isocratic $20 \% \mathrm{MeOH}, 3.0 \mathrm{~mL} / \mathrm{min}, 221 \mathrm{~nm} ; \mathrm{t}_{1}=4.03$ $\left.\min , \mathrm{t}_{2}=5.58 \mathrm{~min}\right) .{ }^{1} \mathrm{H} \mathrm{NMR}\left(400 \mathrm{MHz}, \mathrm{CD}_{3} \mathrm{OD}\right) \delta 7.16(\mathrm{td}, J=7.8,1.4 \mathrm{~Hz}$, $1 \mathrm{H}), 7.12(\mathrm{~d}, J=7.6 \mathrm{~Hz}, 1 \mathrm{H}), 6.97(\mathrm{td}, J=7.6,1.1 \mathrm{~Hz}, 1 \mathrm{H}), 6.85(\mathrm{~d}, J=7.6 \mathrm{~Hz}$, $1 \mathrm{H}), 3.82(\mathrm{~d}, J=5.8 \mathrm{~Hz}, 1 \mathrm{H}), 3.71$ (hept, $J=6.1 \mathrm{~Hz}, 1 \mathrm{H}), 3.58$ (dd, $J=6.5,1.2$ $\mathrm{Hz}, 1 \mathrm{H}), 2.74(\mathrm{dd}, J=6.4,5.9 \mathrm{~Hz}, 1 \mathrm{H}), 1.00(\mathrm{~d}, J=6.1 \mathrm{~Hz}, 3 \mathrm{H}), 0.93(\mathrm{~d}, J=$ $6.1 \mathrm{~Hz}, 3 \mathrm{H}) .{ }^{13} \mathrm{C}$ NMR $\left(126 \mathrm{MHz}, \mathrm{CD}_{3} \mathrm{OD}\right) \delta 179.89,174.92,168.61,135.77$, $128.76,127.91,123.83,121.22,115.71,72.78,69.25,49.32,48.48,40.83$, 22.52, 22.09.IR (thin film): $3233,1719,1668,1595,1387,1120,1086 \mathrm{~cm}^{-1}$; HRMS (ESI): $\left[\mathrm{M}+\mathrm{NH}_{4}\right]^{+}$ calculated for $\mathrm{C}_{16} \mathrm{H}_{20} \mathrm{~N}_{3} \mathrm{O}_{4}{ }^{+} \mathrm{m} / z$ 318.1448, found $\mathrm{m} / z$ 318.1445; mp $=152-154{ }^{\circ} \mathrm{C} ;[\alpha]_{22}^{\mathrm{D}}-91.8^{\circ}$ $\left(c 0.305, \mathrm{CH}_{3} \mathrm{OH}\right)$.

(6a $R, 6 \mathrm{~b} S, 9 \mathrm{a} S, 9 \mathrm{~b} R)-6 \mathrm{a}-I s o p r o p o x y-2-m e t h y l-9 \mathrm{a}, 9 \mathrm{~b}-d i h y d r o-5 H$-pyrrolo[3',4':3,4]cyclobuta[1,2-c]<smiles>Cc1ccc2c(c1)[C@@H]1[C@H](OC(F)(F)F)C(=O)NC(=O)[C@H]1[C@H]2O</smiles>
quinoline-6,7,9(6a $\mathbf{H , 6 b H}, 8 H)$-trione (3e): $>99 \%$ yield, white solid, 97\% ee (SFC, Daicel CHIRALPAK ${ }^{\circledR}$ OJ-H, isocratic $20 \% \mathrm{MeOH}, 3.0 \mathrm{~mL} / \mathrm{min}$, $\left.221 \mathrm{~nm} ; \mathrm{t}_{1}=3.27 \mathrm{~min}, \mathrm{t}_{2}=4.42 \mathrm{~min}\right) .{ }^{1} \mathrm{H}$ NMR $\left(500 \mathrm{MHz}, \mathrm{CD}_{3} \mathrm{OD}\right) \delta 7.10$ $(\mathrm{dd}, J=8.1,1.2 \mathrm{~Hz}, 1 \mathrm{H}), 7.06(\mathrm{~s}, 1 \mathrm{H}), 6.86(\mathrm{~d}, J=8.1 \mathrm{~Hz}, 1 \mathrm{H}), 3.89(\mathrm{~d}, J=$ $5.8 \mathrm{~Hz}, 1 \mathrm{H}), 3.82$ (hept, $J=6.1 \mathrm{~Hz}, 1 \mathrm{H}), 3.69$ (dd, $J=6.5,1.2 \mathrm{~Hz}, 1 \mathrm{H})$, $2.84(\mathrm{dd}, J=6.5,5.8 \mathrm{~Hz}, 1 \mathrm{H}), 2.31(\mathrm{~s}, 3 \mathrm{H}), 1.12(\mathrm{~d}, J=6.1 \mathrm{~Hz}, 3 \mathrm{H}), 1.05$ $(\mathrm{d}, J=6.1 \mathrm{~Hz}, 3 \mathrm{H}) .{ }^{13} \mathrm{C}$ NMR $\left(126 \mathrm{MHz}, \mathrm{CD}_{3} \mathrm{OD}\right) \delta 179.98,174.91$, $168.48,133.72,133.28,129.23,128.20,121.07,115.59,72.76,69.19,49.32,48.56,40.87,22.49$, 22.03, 19.31.IR(thin film): 3207, 2975, 1772, 1711, 1671, 1509, 1335, 1115, 821, $731 \mathrm{~cm}^{-1}$; HRMS (ESI): $\left[\mathrm{M}+\mathrm{NH}_{4}\right]^{+}$calculated for $\mathrm{C}_{17} \mathrm{H}_{22} \mathrm{~N}_{3} \mathrm{O}_{4}{ }^{+} \mathrm{m} / \mathrm{z} 332.1605$, found $\mathrm{m} / \mathrm{z} 332.1601 ; \mathrm{mp}=155-156{ }^{\circ} \mathrm{C}$; $[\alpha]_{22}^{\mathrm{D}}+44^{\mathrm{o}}\left(c 0.25, \mathrm{CH}_{3} \mathrm{OH}\right)$.

(6a $R, 6 \mathrm{~b} S, 9 \mathrm{a} S, 9 \mathrm{~b} R)$-2-Iodo-6a-isopropoxy-9a,9b-dihydro-5H-pyrrolo[3',4':3,4]cyclobuta[1,2-c]qu<smiles>CO[C@H]1[C@H]2C(=O)NC(=O)[C@@H]2[C@H]2c3cc(I)ccc3NC(=O)[C@H]12</smiles>
inoline-6,7,9(6aH, $\mathbf{6 b} \boldsymbol{H}, \mathbf{8 H})$-trione (3f): 67\% yield, 9:1 d.r., white solid, 99\% ee (SFC, Daicel CHIRALPAK ${ }^{\circledR}$ OD-H, isocratic $20 \% \mathrm{MeOH}, 3.0 \mathrm{~mL} / \mathrm{min}$, $\left.221 \mathrm{~nm} ; \mathrm{t}_{1}=10.28 \mathrm{~min}, \mathrm{t}_{2}=11.34 \mathrm{~min}\right) .{ }^{1} \mathrm{H} \mathrm{NMR}\left(500 \mathrm{MHz}, \mathrm{CD}_{3} \mathrm{OD}\right) \delta$ $7.66-7.52(\mathrm{~m}, 2 \mathrm{H}), 6.83-6.71(\mathrm{~m}, 1 \mathrm{H}), 3.95(\mathrm{~d}, J=6.1 \mathrm{~Hz}, 1 \mathrm{H}), 3.83$ (hept, $J=6.1 \mathrm{~Hz}, 1 \mathrm{H}), 3.69$ (dd, $J=6.1,1.0 \mathrm{~Hz}, 1 \mathrm{H}), 2.89$ (t, $J=6.1 \mathrm{~Hz}$, $1 \mathrm{H}), 1.12(\mathrm{~d}, J=6.1 \mathrm{~Hz}, 3 \mathrm{H}), 1.06(\mathrm{~d}, J=6.1 \mathrm{~Hz}, 3 \mathrm{H}) .{ }^{13} \mathrm{C}$ NMR $(126 \mathrm{MHz}$, $\left.\mathrm{CD}_{3} \mathrm{OD}\right) \delta 179.69,174.70,168.37,137.66,136.54,135.78,123.63,117.63$, 85.69, 72.52, 69.36, 49.34, 47.68, 40.66, 22.47, 22.04. IR (thin film): 3057, 2945, 1723,1690, 1609, 1353, 1226, 1067, $867 \mathrm{~cm}^{-1}$; HRMS (ESI): $\left[\mathrm{M}+\mathrm{NH}_{4}\right]^{+}$calculated for $\mathrm{C}_{16} \mathrm{H}_{19} \mathrm{IN}_{3} \mathrm{O}_{4}{ }^{+} \mathrm{m} / z$ 444.0415, found $m / z$ 444.0408; $\mathrm{mp}=230-233{ }^{\circ} \mathrm{C} ;[\alpha]_{22}^{\mathrm{D}}-24^{\circ}\left(c 0.28, \mathrm{CH}_{3} \mathrm{OH}\right)$.

(6a $R, 6 \mathrm{~b} S, 9 \mathrm{a} S, 9 \mathrm{~b} R)$-2-Bromo-6a-isopropoxy-9a,9b-dihydro-5H-pyrrolo [3',4':3,4]cyclobuta[1,2-c]<smiles></smiles>
quinoline-6,7,9(6a $\mathbf{H}, 6 \mathbf{b} \boldsymbol{H}, 8 \mathrm{H})$-trione (3g): 71\% yield, 9:1 d.r., white solid, 95\% ee (SFC, Daicel CHIRALPAK ${ }^{\circledR}$ OD-H, isocratic 20\% MeOH, 3.0 $\left.\mathrm{mL} / \mathrm{min}, 221 \mathrm{~nm} ; \mathrm{t}_{1}=8.23 \mathrm{~min}, \mathrm{t}_{2}=9.00 \mathrm{~min}\right) .{ }^{1} \mathrm{H} \mathrm{NMR}(500 \mathrm{MHz}$, $\left.\mathrm{CD}_{3} \mathrm{OD}\right) \delta 7.44-7.40(\mathrm{~m}, 2 \mathrm{H}), 6.98-6.80(\mathrm{~m}, 1 \mathrm{H}), 3.98(\mathrm{~d}, J=5.8 \mathrm{~Hz}$, 
1H), 3.83 (hept, $J=6.1 \mathrm{~Hz}, 1 \mathrm{H}), 3.71(\mathrm{dd}, J=6.6,1.0 \mathrm{~Hz}, 1 \mathrm{H}), 2.91(\mathrm{t}, J=6.2 \mathrm{~Hz}, 1 \mathrm{H}), 1.11(\mathrm{~d}, J=$ $6.1 \mathrm{~Hz}, 3 \mathrm{H}), 1.06(\mathrm{~d}, J=6.1 \mathrm{~Hz}, 3 \mathrm{H}) .{ }^{13} \mathrm{C} \mathrm{NMR}\left(126 \mathrm{MHz}, \mathrm{CD}_{3} \mathrm{OD}\right) \delta 179.65,174.72,168.37,135.18$, 131.62, 130.61, 123.39, 117.47, 115.71, 72.48, 69.37, 49.34, 47.81, 40.64, 22.49, 22.07.IR(thin film): 3217, 2974, 2928, 1775, 1715, 1677, 1490, 1341, $1182 \mathrm{~cm}^{-1}$; HRMS (ESI): [M+NH$]^{+}$calculated for $\mathrm{C}_{16} \mathrm{H}_{19} \mathrm{BrN}_{3} \mathrm{O}_{4}{ }^{+} m / z$ 396.0553, found $m / z 396.0549 ; \mathrm{mp}=223-225^{\circ} \mathrm{C} ;[\alpha]_{22}^{\mathrm{D}}+43^{\circ}\left(c 0.23, \mathrm{CH}_{3} \mathrm{OH}\right)$.

(6aR,6bS,9aS,9bR)-2-Fluoro-6a-isopropoxy-9a,9b-dihydro-5H-pyrrolo[3',4':3,4]cyclobuta[1,2-c]<smiles>O=C1NC(=O)[C@H]2[C@@H]1[C@H]1c3cc(F)ccc3NC(=O)[C@@]21C(F)(F)F</smiles>
quinoline-6,7,9(6aH,6bH,8H)-trione (3h): 77\% yield, white solid, 90\% ee (SFC, Daicel CHIRALPAK ${ }^{\circledR}$ OJ-H, isocratic $20 \% \mathrm{MeOH}, 3.0 \mathrm{~mL} / \mathrm{min}, 221$ $\left.\mathrm{nm} ; \mathrm{t}_{1}=2.65 \mathrm{~min}, \mathrm{t}_{2}=4.22 \mathrm{~min}\right) .{ }^{1} \mathrm{H}$ NMR $\left(500 \mathrm{MHz}, \mathrm{CD}_{3} \mathrm{OD}\right) \delta 7.08-$ $7.01(\mathrm{~m}, 2 \mathrm{H}), 6.98(\mathrm{dd}, \mathrm{J}=8.3,4.8 \mathrm{~Hz} 1 \mathrm{H}), 3.97(\mathrm{~d}, J=5.8 \mathrm{~Hz}, 1 \mathrm{H}), 3.83$ (hept, $J=6.1 \mathrm{~Hz}, 1 \mathrm{H}), 3.70(\mathrm{dd}, J=6.5,1.1 \mathrm{~Hz}, 1 \mathrm{H}), 2.90(\mathrm{t}, J=6.4 \mathrm{~Hz}$, $1 \mathrm{H}), 1.12(\mathrm{~d}, J=6.1 \mathrm{~Hz}, 3 \mathrm{H}), 1.06(\mathrm{~d}, J=6.1 \mathrm{~Hz}, 3 \mathrm{H}) .{ }^{13} \mathrm{C} \mathrm{NMR}(126 \mathrm{MHz}$, $\left.\mathrm{CD}_{3} \mathrm{OD}\right) \delta 179.67(\mathrm{~s}), 174.78(\mathrm{~s}), 168.25(\mathrm{~s}), 159.04(\mathrm{~d}, J=242.3 \mathrm{~Hz})$, $132.23(\mathrm{~d}, J=2.6 \mathrm{~Hz}), 122.99$ (d, $J=7.6 \mathrm{~Hz}), 117.15$ (d, $J=8.2 \mathrm{~Hz}), 115.33$ (d, $J=23.2 \mathrm{~Hz}), 114.37$ (d, $J=23.6 \mathrm{~Hz}), 72.34$ (s), 69.32 (s), 49.32 (s), 48.15 (d, $J=0.9 \mathrm{~Hz}), 40.63$ (s), 22.44 (s), 22.05 (s). ${ }^{19} \mathrm{~F}$ NMR (377 MHz, $\mathrm{CD}_{3} \mathrm{OD}$ ) $\delta-120.52$ (s, 1F). IR (thin film): 3231, 2977, 1718, 1670, 1502, 1236, 1017, $658 \mathrm{~cm}^{-1}$; HRMS (ESI): $\left[\mathrm{M}+\mathrm{NH}_{4}\right]^{+}$calculated for $\mathrm{C}_{16} \mathrm{H}_{19} \mathrm{FN}_{3} \mathrm{O}_{4}{ }^{+} \mathrm{m} / \mathrm{z} 336.1354$, found $\mathrm{m} / \mathrm{z}$ $336.1347 ; \mathrm{mp}=154-156^{\circ} \mathrm{C} ;[\alpha]_{22}^{\mathrm{D}}+12^{\circ}\left(c 0.39, \mathrm{CH}_{3} \mathrm{OH}\right)$.

(6aR,6bS,9aS,9bR)-6a-Isopropoxy-2-methoxy-9a,9b-dihydro-5H-pyrrolo[3',4':3,4] cyclobuta[1,2-<smiles>[2H][C@@H]1C(=O)NC(=O)[C@@H]2[C@@H]1c1cc(OC)ccc1[C@@H]2C(F)(F)F</smiles>

3i c] quinoline-6,7,9(6a $\boldsymbol{H}, 6 \mathbf{b} \boldsymbol{H}, 8 \boldsymbol{H})$-trione (3i): $60 \%$ yield, white solid, $94 \%$ ee (SFC, Daicel CHIRALPAK ${ }^{\circledR}$ OJ-H, isocratic $20 \% \mathrm{MeOH}, 3.0$ $\left.\mathrm{mL} / \mathrm{min}, 221 \mathrm{~nm} ; \mathrm{t}_{1}=3.19 \mathrm{~min}, \mathrm{t}_{2}=5.07 \mathrm{~min}\right) .{ }^{1} \mathrm{H} \mathrm{NMR}(500 \mathrm{MHz}$, $\left.\mathrm{CD}_{3} \mathrm{OD}\right) \delta 6.79(\mathrm{~d}, J=8.7 \mathrm{~Hz}, 1 \mathrm{H}), 6.74(\mathrm{dd}, J=8.7,2.7 \mathrm{~Hz}, 1 \mathrm{H}), 6.70$ $(\mathrm{d}, J=2.7 \mathrm{~Hz}, 1 \mathrm{H}), 3.80(\mathrm{~d}, J=5.8 \mathrm{~Hz}, 1 \mathrm{H}), 3.71$ (hept, $J=6.1 \mathrm{~Hz}, 1 \mathrm{H})$, 3.67 (s, 3H), 3.57 (dd, $J=6.5,1.2 \mathrm{~Hz}, 1 \mathrm{H}), 2.75$ (t, $J=6.4 \mathrm{~Hz}, 1 \mathrm{H}), 1.00$ $(\mathrm{d}, J=6.1 \mathrm{~Hz}, 3 \mathrm{H}), 0.93(\mathrm{~d}, J=6.1 \mathrm{~Hz}, 3 \mathrm{H}) .{ }^{13} \mathrm{C} \mathrm{NMR}(126 \mathrm{MHz}$, $\left.\mathrm{CD}_{3} \mathrm{OD}\right) \delta 179.98,174.87,168.10,156.50,129.05,122.30,116.81$, $114.45,112.64,72.59,69.21,54.67,49.27,48.66,40.80,22.49,22.05$. IR (thin film): 3233, 2973, 1711, 1669, 1505, 1241, 815, $568 \mathrm{~cm}^{-1}$; HRMS (ESI): $\left[\mathrm{M}+\mathrm{NH}_{4}\right]^{+}$calculated for $\mathrm{C}_{17} \mathrm{H}_{22} \mathrm{~N}_{3} \mathrm{O}_{5}{ }^{+} \mathrm{m} / z$ 348.1554 , found $m / z 348.1549 ; \mathrm{mp}=166-168^{\circ} \mathrm{C} ;[\alpha]_{22}^{\mathrm{D}}+51^{\circ}(c 0.80$, acetone $)$.

(6aR,6bS,9aS,9bR)-3-Bromo-6a-isopropoxy-9a,9b-dihydro-5H-pyrrolo[3',4' :3,4]cyclobuta[1,2-c]<smiles>O=C1Nc2cc(Br)ccc2[C@@H]2[C@H]1[C@@H](C(F)(F)F)[C@H]1C(=O)NC(=O)[C@H]21</smiles>

$3 \mathbf{j}$

quinoline-6,7,9(6aH, $\mathbf{6 b} \mathbf{H}, \mathbf{8 H})$-trione (3j): 92\% yield, white solid, 93\% ee (SFC, Daicel CHIRALPAK ${ }^{\circledR}$ OD-H, isocratic $20 \% \mathrm{MeOH}, 3.0 \mathrm{~mL} / \mathrm{min}$, $\left.221 \mathrm{~nm} ; \mathrm{t}_{1}=8.61 \mathrm{~min}, \mathrm{t}_{2}=9.98 \mathrm{~min}\right) .{ }^{1} \mathrm{H}$ NMR $\left(500 \mathrm{MHz}, \mathrm{CD}_{3} \mathrm{OD}\right) \delta 7.24$ $(\mathrm{dd}, J=8.1,1.9 \mathrm{~Hz}, 1 \mathrm{H}), 7.16(\mathrm{~d}, J=8.1 \mathrm{~Hz}, 1 \mathrm{H}), 7.15(\mathrm{~d}, J=1.8 \mathrm{~Hz}$, $1 \mathrm{H}), 3.93(\mathrm{~d}, J=5.8 \mathrm{~Hz}, 1 \mathrm{H}), 3.84$ (hept, $J=6.1 \mathrm{~Hz}, 1 \mathrm{H}), 3.70$ (dd, $J=6.5$, $1.1 \mathrm{~Hz}, 1 \mathrm{H}), 2.89$ (dd, $J=6.5,5.8 \mathrm{~Hz}, 1 \mathrm{H}), 1.12$ (d, $J=6.1 \mathrm{~Hz}, 3 \mathrm{H}), 1.06$ $(\mathrm{d}, J=6.1 \mathrm{~Hz}, 3 \mathrm{H}) .{ }^{13} \mathrm{C}$ NMR $\left(126 \mathrm{MHz}, \mathrm{CD}_{3} \mathrm{OD}\right) \delta 179.65,174.75$, $168.49,137.39,129.60,126.46,121.84,120.36,118.40,72.58,69.36,49.31,47.90,40.62,22.46$, 22.07. IR (thin film): $3205,2974,1775,1714,1680,1328,1124,1094,719 \mathrm{~cm}^{-1}$; HRMS (ESI): 
$\left[\mathrm{M}+\mathrm{NH}_{4}\right]^{+}$calculated for $\mathrm{C}_{16} \mathrm{H}_{19} \mathrm{BrN}_{3} \mathrm{O}_{4}{ }^{+} m / z$ 396.0554, found $m / z 396.0548 ; \mathrm{mp}>260{ }^{\circ} \mathrm{C} ;[\alpha]_{22}^{\mathrm{D}}+29^{\circ}$ $\left(c 0.55, \mathrm{CH}_{3} \mathrm{OH}\right)$.

(6aR,6bS,9aS,9b $R)$-3-Chloro-6a-isopropoxy-9a,9b-dihydro-5H-pyrrolo[3',4':3,4]cyclobuta[1,2-c]<smiles>O=C1NC(=O)[C@H]2[C@H]1[C@H](F)[C@H]1C(=O)Nc3cc(Cl)ccc3[C@@H]12</smiles>
quinoline-6,7,9(6a $\mathbf{H}, 6 \mathbf{b} \mathbf{H}, 8 \mathrm{H})$-trione (3k): $85 \%$ yield, white solid, $97 \%$ ee (SFC, Daicel CHIRALPAK ${ }^{\circledR}$ OD-H, isocratic $20 \% \mathrm{MeOH}, 3.0 \mathrm{~mL} / \mathrm{min}$, $\left.221 \mathrm{~nm} ; \mathrm{t}_{1}=7.14 \mathrm{~min}, \mathrm{t}_{2}=7.92 \mathrm{~min}\right) .{ }^{1} \mathrm{H}$ NMR $\left(500 \mathrm{MHz}, \mathrm{CD}_{3} \mathrm{OD}\right) \delta 7.23$ $(\mathrm{d}, J=8.2 \mathrm{~Hz}, 1 \mathrm{H}), 7.08(\mathrm{dd}, J=8.2,2.0 \mathrm{~Hz}, 1 \mathrm{H}), 7.00(\mathrm{~d}, J=2.0 \mathrm{~Hz}, 1 \mathrm{H})$, $3.95(\mathrm{~d}, J=5.9 \mathrm{~Hz}, 1 \mathrm{H}), 3.83$ (hept, $J=6.1 \mathrm{~Hz}, 1 \mathrm{H}), 3.70$ (dd, $J=6.4,1.2$ $\mathrm{Hz}, 1 \mathrm{H}), 2.89$ (dd, $J=6.4,5.9 \mathrm{~Hz}, 1 \mathrm{H}), 1.12$ (d, $J=6.1 \mathrm{~Hz}, 3 \mathrm{H}), 1.05$ (d, $J$ $=6.1 \mathrm{~Hz}, 3 \mathrm{H}) .{ }^{13} \mathrm{C} \mathrm{NMR}\left(126 \mathrm{MHz}, \mathrm{CD}_{3} \mathrm{OD}\right) \delta 179.71,174.81,168.54$, $137.23,134.17,129.40,123.48,119.92,115.50,72.62,69.37,49.30,47.82,40.72,22.48,22.09$. IR (thin film): $3196,2975,1728,1675,1587,1358,1211,1080,763 \mathrm{~cm}^{-1}$; HRMS (ESI): $\left[\mathrm{M}+\mathrm{NH}_{4}\right]^{+}$ calculated for $\mathrm{C}_{16} \mathrm{H}_{19} \mathrm{ClN}_{3} \mathrm{O}_{4}{ }^{+} m / z 352.1059$, found $m / z 352.1052 ; \mathrm{mp}=233-235^{\circ} \mathrm{C} ;[\alpha]_{22}^{\mathrm{D}}-21^{\circ}(c 0.29$, $\left.\mathrm{CH}_{3} \mathrm{OH}\right)$.

(6aR,6bS,9aS,9bR)-2,3-Difluoro-6a-isopropoxy-9a,9b-dihydro-5H-pyrrolo[3',4' :3,4]cyclobuta[1,<smiles>O=C1NC(=O)[C@H]2[C@@H]1[C@H]1c3cc(F)c(F)cc3NC(=O)[C@@]21C(F)(F)F</smiles>

2-c]quinoline-6,7,9(6aH,6bH,8H)-trione (31): 54\% yield, white solid, 93\% ee (SFC, Daicel CHIRALPAK ${ }^{\circledR}$ OD-H, isocratic $20 \% \mathrm{MeOH}, 3.0 \mathrm{~mL} / \mathrm{min}$, $\left.221 \mathrm{~nm} ; \mathrm{t}_{1}=5.29 \mathrm{~min}, \mathrm{t}_{2}=5.78 \mathrm{~min}\right) .{ }^{1} \mathrm{H}$ NMR $\left(500 \mathrm{MHz}, \mathrm{CD}_{3} \mathrm{OD}\right) \delta 7.21$ $(\mathrm{dd}, J=10.3,8.3 \mathrm{~Hz}, 1 \mathrm{H}), 6.89(\mathrm{dd}, J=11.2,7.0 \mathrm{~Hz}, 1 \mathrm{H}), 3.97(\mathrm{~d}, J=5.8$ $\mathrm{Hz}, 1 \mathrm{H}), 3.84$ (hept, $J=6.1 \mathrm{~Hz}, 1 \mathrm{H}), 3.70(\mathrm{dd}, J=6.6,1.1 \mathrm{~Hz}, 1 \mathrm{H}), 2.91$ (dd, $J=6.6,5.8 \mathrm{~Hz}, 1 \mathrm{H}), 1.12(\mathrm{~d}, J=6.1 \mathrm{~Hz}, 3 \mathrm{H}), 1.06(\mathrm{~d}, J=6.1 \mathrm{~Hz}, 3 \mathrm{H}) .{ }^{13} \mathrm{C}$ NMR (126 MHz, CD $\left.{ }_{3} \mathrm{OD}\right) \delta 179.55$ (s), 174.69 (s), 168.25 (s), 150.05 (dd, $J$ $=247.1,13.7 \mathrm{~Hz}), 146.23(\mathrm{dd}, J=244.0,13.0 \mathrm{~Hz}), 132.85(\mathrm{dd}, J=8.8,2.7 \mathrm{~Hz}), 117.71(\mathrm{dd}, J=5.7$, $3.9 \mathrm{~Hz}), 116.62$ (d, $J=19.1 \mathrm{~Hz}), 104.79$ (d, $J=21.8 \mathrm{~Hz}), 72.30$ (s), 69.40 (s), 49.25 (s), 47.58 (s), 40.60 (s), 22.44 (s), 22.08 (s). ${ }^{19} \mathrm{~F}$ NMR (377 MHz, CD $\left.{ }_{3} \mathrm{OD}\right) \delta-139.25$ (d, $\left.J=21.1 \mathrm{~Hz}, 1 \mathrm{~F}\right),-146.17$ (d, $J=21.1 \mathrm{~Hz}, 1 \mathrm{~F}$ ). IR(thin film): 3220, 2974, 2778, 1709, 1678, 1648, 1525, 1496, 1341, 1107, 861, $806 \mathrm{~cm}^{-1}$; HRMS (ESI): $\left[\mathrm{M}+\mathrm{NH}_{4}\right]^{+}$calculated for $\mathrm{C}_{16} \mathrm{H}_{18} \mathrm{~F}_{2} \mathrm{~N}_{3} \mathrm{O}_{4}{ }^{+} \mathrm{m} / z$ 354.1260, found $\mathrm{m} / z$ 354.1258; $\mathrm{mp}=153-156^{\circ} \mathrm{C} ;[\alpha]_{22}^{\mathrm{D}}+58^{\circ}(c 0.32$, acetone $)$.

(6aR,6bS,9aS,9bR)-1-Chloro-6a-isopropoxy-9a,9b-dihydro-5H-pyrrolo[3',4':3,4]cyclobuta[1,2-c]

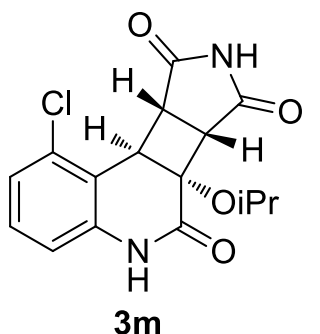
quinoline-6,7,9(6aH,6b $\mathbf{H , 8 H})$-trione (3m): 99\% yield, 6:1 d.r., white solid, 99\% ee (SFC, Daicel CHIRALPAK ${ }^{\circledR}$ OD-H, isocratic 20\% MeOH, $3.0 \mathrm{~mL} / \mathrm{min}, 221$ $\left.\mathrm{nm} ; \mathrm{t}_{1}=7.13 \mathrm{~min}, \mathrm{t}_{2}=10.17 \mathrm{~min}\right) .{ }^{1} \mathrm{H}$ NMR $\left(500 \mathrm{MHz}, \mathrm{CD}_{3} \mathrm{OD}\right) \delta 7.28(\mathrm{t}, J=$ $8.1 \mathrm{~Hz}, 1 \mathrm{H}), 7.17(\mathrm{dd}, J=8.1,0.6 \mathrm{~Hz}, 1 \mathrm{H}), 6.92(\mathrm{dd}, J=8.1,0.6 \mathrm{~Hz}, 1 \mathrm{H}), 4.15$ (dd, $J=5.8,0.9 \mathrm{~Hz}, 1 \mathrm{H}), 3.84$ (hept, $J=6.1 \mathrm{~Hz}, 1 \mathrm{H}), 3.76$ (dd, $J=6.5,0.9 \mathrm{~Hz}$, $1 \mathrm{H}), 2.94(\mathrm{dd}, J=6.5,5.8 \mathrm{~Hz}, 1 \mathrm{H}), 1.14(\mathrm{~d}, J=6.1 \mathrm{~Hz}, 3 \mathrm{H}), 1.06(\mathrm{~d}, J=6.1 \mathrm{~Hz}$, $3 \mathrm{H}) .{ }^{13} \mathrm{C}$ NMR $\left(126 \mathrm{MHz}, \mathrm{CD}_{3} \mathrm{OD}\right) \delta 178.82,174.61,168.01,137.57,133.55$, 129.80, 124.10, 119.09, 114.39, 72.51, 69.48, 49.63, 46.19, 40.55, 22.35, 22.15. IR (thin film): 3211, $3068,2978,1772,1712,1678,1585,1334,1181,785,733 \mathrm{~cm}^{-1}$; HRMS (ESI): [M+NH$]^{+}$calculated for $\mathrm{C}_{16} \mathrm{H}_{19} \mathrm{ClN}_{3} \mathrm{O}_{4}{ }^{+} m / z 352.1059$, found $m / z$ 352.1055; mp $=248-250{ }^{\circ} \mathrm{C} ;[\alpha]_{22}^{\mathrm{D}}+106^{\circ}(c 0.28$, $\left.\mathrm{CH}_{3} \mathrm{OH}\right)$. 
(6a $S, 6 \mathrm{~b} S, 9 \mathrm{a} R, 9 \mathrm{~b} R)$-6a-Isopropoxy-9b-methyl-9a,9b-dihydro-5H-pyrrolo[3',4':3,4]cyclobuta[1,2-

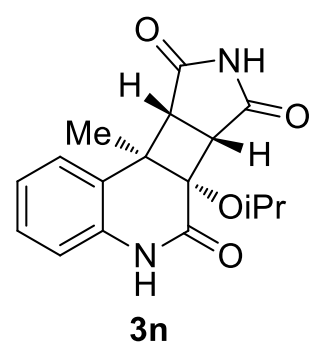
c]quinoline-6,7,9(6aH,6bH,8H)-trione (3n): 78\% yield, 5:1 d.r., white solid, $85 \%$ ee (SFC, Daicel CHIRALPAK ${ }^{\circledR}$ AD-H, isocratic $20 \% \mathrm{MeOH}, 3.0 \mathrm{~mL} / \mathrm{min}$, $\left.221 \mathrm{~nm} ; \mathrm{t}_{1}=5.13 \mathrm{~min}, \mathrm{t}_{2}=6.73 \mathrm{~min}\right) .{ }^{1} \mathrm{H}$ NMR $\left(500 \mathrm{MHz}, \mathrm{CD}_{3} \mathrm{OD}\right) \delta 7.33(\mathrm{dd}$, $J=7.8,1.2 \mathrm{~Hz}, 1 \mathrm{H}), 7.25(\mathrm{td}, J=7.8,1.2 \mathrm{~Hz}, 1 \mathrm{H}), 7.11(\mathrm{td}, J=7.8,1.2 \mathrm{~Hz}$, $1 \mathrm{H}), 6.93$ (dd, $J=7.8,1.2 \mathrm{~Hz}, 1 \mathrm{H}), 4.07$ (hept, $J=6.1 \mathrm{~Hz}, 1 \mathrm{H}), 3.72$ (d, $J=7.0$ $\mathrm{Hz}, 1 \mathrm{H}), 2.94(\mathrm{~d}, J=7.0 \mathrm{~Hz}, 1 \mathrm{H}), 1.65(\mathrm{~s}, 3 \mathrm{H}), 1.19(\mathrm{~d}, J=6.1 \mathrm{~Hz}, 3 \mathrm{H}), 1.03(\mathrm{~d}$, $J=6.1 \mathrm{~Hz}, 3 \mathrm{H}) .{ }^{13} \mathrm{C}$ NMR $\left(126 \mathrm{MHz}, \mathrm{CD}_{3} \mathrm{OD}\right) \delta 178.06,175.69,168.30$, 134.74, 128.39, 127.65, 125.68, 124.00, 115.70, 75.30, 69.54, 49.17, 48.07, 45.29, 22.32, 22.32, 16.86 . IR (thin film): 3121, 3064, 2978, 1771, 1707, 1658, 1589, 1384, 1339, 1162, 851, $772 \mathrm{~cm}^{-1}$; HRMS (ESI): $\left[\mathrm{M}+\mathrm{NH}_{4}\right]^{+}$calculated for $\mathrm{C}_{17} \mathrm{H}_{22} \mathrm{~N}_{3} \mathrm{O}_{4}{ }^{+} \mathrm{m} / z$ 332.1605, found $\mathrm{m} / \mathrm{z} 332.1600 ; \mathrm{mp}=260-261{ }^{\circ} \mathrm{C}$; $[\alpha]_{22}^{\mathrm{D}}+57^{\circ}\left(c 0.46, \mathrm{CH}_{3} \mathrm{OH}\right)$.

(6aR,6bS,9aS,9bR)-4-Chloro-6a-isopropoxy-9a,9b-dihydro-5H-pyrrolo[3',4':3,4]cyclobuta[1,2-c]<smiles>O=C1Nc2c(Cl)cccc2[C@H]2[C@H]1[C@H]1C(=O)NC(=O)[C@H]21</smiles>
quinoline-6,7,9(6aH, $\mathbf{6 b} \mathbf{H}, \mathbf{8 H})$-trione (3o): $74 \%$ yield, white solid, $51 \%$ ee (SFC, Daicel CHIRALPAK ${ }^{\circledR}$ OD-H, isocratic $20 \% \mathrm{MeOH}, 3.0 \mathrm{~mL} / \mathrm{min}, 221$ $\left.\mathrm{nm} ; \mathrm{t}_{1}=5.9 \mathrm{~min}, \mathrm{t}_{2}=6.56 \mathrm{~min}\right) .{ }^{1} \mathrm{H} \mathrm{NMR}\left(500 \mathrm{MHz}, \mathrm{CD}_{3} \mathrm{OD}\right) \delta 7.40(\mathrm{dd}, J=$ $7.8,1.2 \mathrm{~Hz}, 1 \mathrm{H}), 7.22(\mathrm{~d}, J=7.8 \mathrm{~Hz}, 1 \mathrm{H}), 7.09(\mathrm{t}, J=7.8 \mathrm{~Hz}, 1 \mathrm{H}), 4.00(\mathrm{~d}, J=$ $6.5 \mathrm{~Hz}, 1 \mathrm{H}$ ), 3.86 (hept, $J=6.1 \mathrm{~Hz}, 1 \mathrm{H}), 3.74(\mathrm{dd}, J=6.5,1.0 \mathrm{~Hz}, 1 \mathrm{H}), 2.92$ (t, $J=6.5 \mathrm{~Hz}, 1 \mathrm{H}), 1.12(\mathrm{~d}, J=6.1 \mathrm{~Hz}, 3 \mathrm{H}), 1.06(\mathrm{~d}, J=6.1 \mathrm{~Hz}, 3 \mathrm{H}) \cdot{ }^{13} \mathrm{C} \mathrm{NMR}$ $\left(126 \mathrm{MHz}, \mathrm{CD}_{3} \mathrm{OD}\right) \delta 179.51,174.72,168.50,132.51,129.17,126.84,124.31$, 123.20, 119.69, 72.84, 69.51, 49.29, 48.41, 40.55, 22.44, 22.15. IR (thin film): 3168, 3088, 2980, 1773, 1707, 1679, 1479, 1341, 1183, 793, 698, $\mathrm{cm}^{-1}$; HRMS (ESI): $\left[\mathrm{M}+\mathrm{NH}_{4}\right]^{+}$calculated for $\mathrm{C}_{16} \mathrm{H}_{19} \mathrm{ClN}_{3} \mathrm{O}_{4}{ }^{+} m / z 352.1059$, found $m / z 352.1055 ; \mathrm{mp}=199-201^{\circ} \mathrm{C} ;[\alpha]_{22}^{\mathrm{D}}+28^{\circ}\left(c 1.08, \mathrm{CH}_{3} \mathrm{OH}\right)$.

(6a $R, 6 \mathrm{~b} S, 9 \mathrm{a} S, 9 \mathrm{~b} R)$-4-Fluoro-6a-isopropoxy-9a,9b-dihydro-5H-pyrrolo[3',4':3,4]cyclobuta[1,2-c]<smiles>O=C1Nc2c(F)cccc2[C@H]2[C@H]1[C@H]1C(=O)NC(=O)[C@H]21</smiles>
quinoline-6,7,9(6aH, $\mathbf{6 b} \boldsymbol{H}, \mathbf{8 H})$-trione (3p): $79 \%$ yield, white solid, $14 \%$ ee (SFC, Daicel CHIRALPAK ${ }^{\circledR}$ OD-H, isocratic $20 \% \mathrm{MeOH}, 3.0 \mathrm{~mL} / \mathrm{min}, 221$ $\left.\mathrm{nm} ; \mathrm{t}_{1}=4.62 \mathrm{~min}, \mathrm{t}_{2}=5.01 \mathrm{~min}\right) .{ }^{1} \mathrm{H} \mathrm{NMR}\left(500 \mathrm{MHz}, \mathrm{CD}_{3} \mathrm{OD}\right) \delta 7.17-7.05$ (m, 3H), 4.00 (d, $J=5.8 \mathrm{~Hz}, 1 \mathrm{H}), 3.86$ (hept, $J=6.1 \mathrm{~Hz}, 1 \mathrm{H}), 3.73$ (dd, $J=6.5$, $1.2 \mathrm{~Hz}, 1 \mathrm{H}), 2.92(\mathrm{dd}, J=6.5,5.8 \mathrm{~Hz}, 1 \mathrm{H}), 1.13(\mathrm{~d}, J=6.1 \mathrm{~Hz}, 3 \mathrm{H}), 1.06(\mathrm{~d}, J$ $=6.1 \mathrm{~Hz}, 3 \mathrm{H}) \cdot{ }^{13} \mathrm{C}$ NMR $\left(126 \mathrm{MHz}, \mathrm{CD}_{3} \mathrm{OD}\right) \delta 179.60(\mathrm{~s}), 174.75(\mathrm{~s}), 168.20$ (s), $149.82(\mathrm{~d}, J=245.0 \mathrm{~Hz}), 124.25(\mathrm{~d}, J=12.5 \mathrm{~Hz}), 123.90(\mathrm{~d}, J=7.5 \mathrm{~Hz})$, 123.42 (d, $J=2.2 \mathrm{~Hz}), 123.40$ (d, $J=3.6 \mathrm{~Hz}), 114.84$ (d, $J=18.6 \mathrm{~Hz}), 72.70$ (s), 69.42 (s), 49.35 (s), 48.23 (d, $J=2.4 \mathrm{~Hz}$ ), 40.69 (s), 22.45 (s), 22.11 (s). ${ }^{19} \mathrm{~F}$ NMR (377 MHz, CD 3 OD) $\delta-133.88$ (s, 1F).IR (thin film): 3191, 2976, 1778, 1716, 1665, 1499, 1339, 1213, 1120, $787 \mathrm{~cm}^{-1}$; HRMS (ESI): $\left[\mathrm{M}+\mathrm{NH}_{4}\right]^{+}$calculated for $\mathrm{C}_{16} \mathrm{H}_{19} \mathrm{FN}_{3} \mathrm{O}_{4}{ }^{+} m / z 336.1354$, found $m / z 336.1352 ; \mathrm{mp}>260{ }^{\circ} \mathrm{C} ;[\alpha]_{22}^{\mathrm{D}}+11^{\circ}$ (c0.35, acetone).

(6aR,6bS,9aS,9bR)-6a-Isopropoxy-9a,9b-dihydrochromeno $\left[3^{\prime}, 4^{\prime}: 3,4\right]$ cyclobuta[1,2-c]pyrrole-6,7,<smiles>O=C1NC(=O)[C@@H]2[C@H]1[C@H]1C(=O)Oc3ccccc3[C@H]21</smiles>
9(6aH,6bH,8H)-trione (3q): 87\% yield, white solid, 7\% ee (SFC, Daicel CHIRALPAK ${ }^{\circledR} \mathrm{OD}-\mathrm{H}$, isocratic $20 \% \mathrm{MeOH}, 3.0 \mathrm{~mL} / \mathrm{min}, 221 \mathrm{~nm} ; \mathrm{t}_{1}=3.97 \mathrm{~min}$, $\left.\mathrm{t}_{2}=5.28 \mathrm{~min}\right) .{ }^{1} \mathrm{H}$ NMR $\left(500 \mathrm{MHz}, \mathrm{CD}_{3} \mathrm{OD}\right) \delta 7.43-7.38(\mathrm{~m}, 1 \mathrm{H}), 7.35(\mathrm{~d}, J=$ 
$7.6 \mathrm{~Hz}, 1 \mathrm{H}), 7.26(\mathrm{td}, J=7.5,0.9 \mathrm{~Hz}, 1 \mathrm{H}), 7.14(\mathrm{~d}, J=8.2 \mathrm{~Hz}, 1 \mathrm{H}), 4.05(\mathrm{~d}, J=5.1 \mathrm{~Hz}, 1 \mathrm{H}), 3.90$ (hept, $J=6.1 \mathrm{~Hz}, 1 \mathrm{H}), 3.79$ (dd, $J=6.6,1.1 \mathrm{~Hz}, 1 \mathrm{H}), 3.01$ (dd, $J=6.6,5.1 \mathrm{~Hz}, 1 \mathrm{H}), 1.13$ (d, $J=6.1$ $\mathrm{Hz}, 3 \mathrm{H}), 1.10(\mathrm{~d}, J=6.1 \mathrm{~Hz}, 3 \mathrm{H}) .{ }^{13} \mathrm{C} \mathrm{NMR}\left(126 \mathrm{MHz}, \mathrm{CD}_{3} \mathrm{OD}\right) \delta 179.10,174.28,166.58,150.18$, $129.62,128.33,125.47,120.51,116.97,72.84,70.29,49.08,45.72,41.99,22.30,21.90$. IR (thin film): 2974, 2927, 1761, 1703, 1586, 1262, 1228, 1168, $768 \mathrm{~cm}^{-1}$; HRMS (ESI): $\left[\mathrm{M}+\mathrm{NH}_{4}\right]^{+}$calculated for $\mathrm{C}_{16} \mathrm{H}_{19} \mathrm{~N}_{2} \mathrm{O}_{5}^{+} m / z 319.1286$, found $m / z 319.1283 ; \mathrm{mp}>260{ }^{\circ} \mathrm{C} ;[\alpha]_{22}^{\mathrm{D}}+6^{\circ}$ (c0.56, acetone).

(6aR,6bS,9aS,9b $R)$-6a-Isopropoxy-5-methyl-9a,9b-dihydro-5H-pyrrolo[3',4':3,4]cyclobuta[1,2-c]

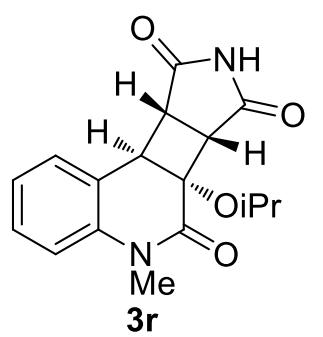
quinoline-6,7,9(6aH,6bH,8H)-trione (3r): 96\% yield, 3:1 d.r., white solid, 72\% ee (SFC, Daicel CHIRALPAK ${ }^{\circledR}$ OD-H, isocratic 20\% MeOH, $3.0 \mathrm{~mL} / \mathrm{min}, 221$ $\left.\mathrm{nm} ; \mathrm{t}_{1}=4.67 \mathrm{~min}, \mathrm{t}_{2}=5.89 \mathrm{~min}\right) .{ }^{1} \mathrm{H}$ NMR $\left(500 \mathrm{MHz}, \mathrm{CDCl}_{3}\right) \delta 8.86(\mathrm{~s}, 1 \mathrm{H})$, $7.36(\mathrm{t}, J=7.9 \mathrm{~Hz}, 1 \mathrm{H}), 7.24(\mathrm{~d}, J=7.1 \mathrm{~Hz}, 1 \mathrm{H}), 7.12(\mathrm{t}, J=7.4 \mathrm{~Hz}, 1 \mathrm{H}), 7.07$ (d, $J=8.3 \mathrm{~Hz}, 1 \mathrm{H}), 3.96-3.83(\mathrm{~m}, 3 \mathrm{H}), 3.49(\mathrm{~s}, 3 \mathrm{H}), 2.88(\mathrm{t}, J=6.2 \mathrm{~Hz}, 1 \mathrm{H})$, $1.12(\mathrm{~d}, J=6.1 \mathrm{~Hz}, 3 \mathrm{H}), 1.00(\mathrm{~d}, J=6.1 \mathrm{~Hz}, 3 \mathrm{H}) .{ }^{13} \mathrm{C} \mathrm{NMR}\left(126 \mathrm{MHz}, \mathrm{CDCl}_{3}\right)$ $\delta 177.91,173.29,167.35,138.17,129.31,128.96,124.22,121.54,115.16$, 77.26, 72.59, 69.79, 49.46, 48.97, 40.07, 30.46, 23.45. IR (thin film): 3135, 3079, 2973, 1772, 1722, $1635,1591,1466,1332,1128,808,763 \mathrm{~cm}^{-1}$; HRMS (ESI): $\left[\mathrm{M}+\mathrm{NH}_{4}\right]^{+}$calculated for $\mathrm{C}_{17} \mathrm{H}_{22} \mathrm{~N}_{3} \mathrm{O}_{4}{ }^{+}$ $\mathrm{m} / \mathrm{z} 332.1605$, found $\mathrm{m} / \mathrm{z} 332.1599 ; \mathrm{mp}=237-239^{\circ} \mathrm{C} ;[\alpha]_{22}^{\mathrm{D}}+43^{\circ}(c 0.63$, acetone $)$.

(6aR,6bS,9aS,9bR)-8-Benzyl-6a-isopropoxy-9a,9b-dihydro-5H-pyrrolo[3',4':3,4]cyclobuta[1,2-c]<smiles>CO[C@@H]1[C@H]2C(=O)NC(=O)[C@@H]1[C@@H]1C(=O)Nc3ccccc3[C@H]21</smiles>
quinoline-6,7,9(6aH,6bH,8H)-trione (3s): $63 \%$ yield, white solid, $88 \%$ ee (SFC, Daicel CHIRALPAK ${ }^{\circledR}$ OD-H, isocratic $20 \% \mathrm{MeOH}, 3.0 \mathrm{~mL} / \mathrm{min}, 221 \mathrm{~nm}$; $\left.\mathrm{t}_{1}=6.35 \mathrm{~min}, \mathrm{t}_{2}=7.51 \mathrm{~min}\right) .{ }^{1} \mathrm{H}$ NMR $\left(500 \mathrm{MHz}\right.$, Acetone- $\left.d_{6}\right) \delta 9.72(\mathrm{~s}, 1 \mathrm{H})$, $7.42(\mathrm{~d}, J=7.4 \mathrm{~Hz}, 2 \mathrm{H}), 7.34$ (t, $J=7.4 \mathrm{~Hz}, 2 \mathrm{H}), 7.31-7.25(\mathrm{~m}, 2 \mathrm{H}), 7.16$ (d, $J=7.4 \mathrm{~Hz}, 1 \mathrm{H}), 7.09-7.02(\mathrm{~m}, 2 \mathrm{H}), 4.75(\mathrm{~d}, J=14.7 \mathrm{~Hz}, 1 \mathrm{H}), 4.68(\mathrm{~d}, J=$ $14.7 \mathrm{~Hz}, 1 \mathrm{H}), 3.88$ (hept, $J=6.1 \mathrm{~Hz}, 1 \mathrm{H}), 3.80(\mathrm{~d}, J=6.6 \mathrm{~Hz}, 1 \mathrm{H}), 3.76(\mathrm{~d}, J=$ $5.7 \mathrm{~Hz}, 1 \mathrm{H}), 3.02(\mathrm{t}, J=6.2 \mathrm{~Hz}, 1 \mathrm{H}), 1.06(\mathrm{~d}, J=6.1 \mathrm{~Hz}, 3 \mathrm{H}), 0.95(\mathrm{~d}, J=6.1$ $\mathrm{Hz}, 3 \mathrm{H}) .{ }^{13} \mathrm{C}$ NMR $\left(126 \mathrm{MHz}\right.$, Acetone- $\left.d_{6}\right) \delta 177.15,172.66,167.11,136.61,136.50,128.89,128.43$, $128.21,127.88,127.47,123.50,121.02,115.78,73.20,69.03,48.48,47.91,41.89,39.38,22.97,22.82$. IR (thin film): 3072, 2977, 2926, 1773, 1699, 1673, 1593, 1380, 1339, 1117, $765 \mathrm{~cm}^{-1}$; HRMS (ESI): $\left[\mathrm{M}+\mathrm{NH}_{4}\right]^{+}$calculated for $\mathrm{C}_{23} \mathrm{H}_{26} \mathrm{~N}_{3} \mathrm{O}_{4}{ }^{+} \mathrm{m} / z$ 408.1918, found $\mathrm{m} / z$ 408.1914; mp $=213-215{ }^{\circ} \mathrm{C}$; $[\alpha]_{22}^{\mathrm{D}}+20^{\circ}\left(c 0.61, \mathrm{CH}_{3} \mathrm{OH}\right)$.

Dimer of NBn-maleimide: Spectral data were consistent with the previous report. ${ }^{10}$

(6a $R, 6 \mathrm{~b} S, 9 \mathrm{a} S, 9 \mathrm{~b} R)-6 \mathrm{a}-I s o p r o p o x y-8-(p r o p-2-y n-1-y \mathrm{l})-9 \mathrm{a}, 9 \mathrm{~b}-\mathrm{dihydro}-5 H$-pyrrolo[3',4':3,4]cyclob

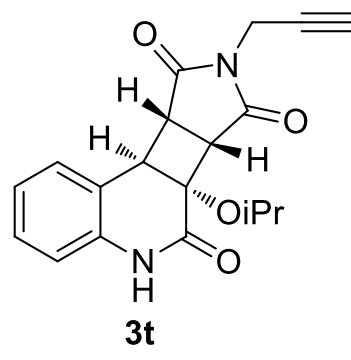
uta[1,2-c]quinoline-6,7,9(6aH,6bH,8H)-trione (3t): 61\% yield, white solid, 97\% ee (HPLC, Daicel CHIRALPAK ${ }^{\circledR}$ OD-H, Gradient 5\% ${ }^{i} \mathrm{PrOH}(95 \%$ Hexane) to $40 \%{ }^{i} \mathrm{PrOH}(60 \%$ Hexane $), 1.0 \mathrm{~mL} / \mathrm{min}, 221 \mathrm{~nm}$; $\mathrm{t}_{1}=22.31 \mathrm{~min}$, $\left.\mathrm{t}_{2}=26.46 \mathrm{~min}\right) .{ }^{1} \mathrm{H}$ NMR (500 MHz, $\left.\mathrm{CD}_{3} \mathrm{OD}\right) \delta 7.29(\mathrm{td}, J=7.8,1.4 \mathrm{~Hz}$, $1 \mathrm{H}), 7.23(\mathrm{~d}, J=7.5 \mathrm{~Hz}, 1 \mathrm{H}), 7.11(\mathrm{td}, J=7.5,1.1 \mathrm{~Hz}, 1 \mathrm{H}), 6.98(\mathrm{~d}, J=8.0$ $\mathrm{Hz}, 1 \mathrm{H}), 4.40-4.29(\mathrm{~m}, 2 \mathrm{H}), 3.84(\mathrm{~d}, J=5.7 \mathrm{~Hz}, 1 \mathrm{H}), 3.81$ (hept, $J=6.3 \mathrm{~Hz}$, $1 \mathrm{H}), 3.76(\mathrm{dd}, J=6.6,1.2 \mathrm{~Hz}, 1 \mathrm{H}), 2.99(\mathrm{dd}, J=6.5,5.8 \mathrm{~Hz}, 1 \mathrm{H}), 2.67$ (t, $J=$ $2.5 \mathrm{~Hz}, 1 \mathrm{H}), 1.11(\mathrm{~d}, J=6.1 \mathrm{~Hz}, 3 \mathrm{H}), 1.04(\mathrm{~d}, J=6.1 \mathrm{~Hz}, 3 \mathrm{H}) .{ }^{13} \mathrm{C}$ NMR $\left(126 \mathrm{MHz}, \mathrm{CD}_{3} \mathrm{OD}\right) \delta$ 
$176.79,172.06,168.32,135.81,128.86,127.92,123.88,120.89,115.76,76.55,72.97,71.14,69.29$, 47.88, 47.73, 39.45, 27.40, 22.58, 21.99. IR (thin film): 3271, 2977, 2925, 1779, 1708, 1674, 1595, 1380, 1325, 1174, $732 \mathrm{~cm}^{-1}$; HRMS (ESI): $\left[\mathrm{M}+\mathrm{NH}_{4}\right]^{+}$calculated for $\mathrm{C}_{19} \mathrm{H}_{22} \mathrm{~N}_{3} \mathrm{O}_{4}{ }^{+} \mathrm{m} / z 356.1605$, found $m / z 356.1599 ; \mathrm{mp}=177-179^{\circ} \mathrm{C} ;[\alpha]_{22}^{\mathrm{D}}+17^{\circ}\left(c 0.63, \mathrm{CH}_{3} \mathrm{OH}\right)$.

(6aR,6b $S, 9 \mathrm{a} S, 9 \mathrm{~b} R)-8$-Allyl-6a-isopropoxy-9a,9b-dihydro-5 $H$-pyrrolo $\left[3^{\prime}, 4^{\prime}: 3,4\right]$ cyclobuta[1,2-c]q

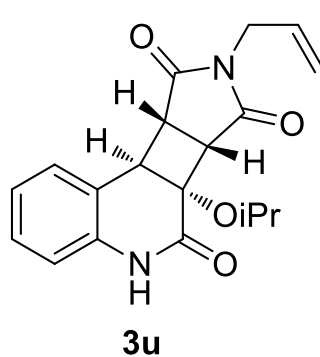
uinoline-6,7,9(6aH, $\mathbf{6 b} \boldsymbol{H}, 8 \mathrm{H})$-trione $(3 \mathrm{u})$ : $44 \%$ yield, white solid, $84 \%$ ee (SFC, Daicel CHIRALPAK ${ }^{\circledR}$ OD-H, isocratic $20 \% \mathrm{MeOH}, 3.0 \mathrm{~mL} / \mathrm{min}, 221$ $\left.\mathrm{nm} ; \mathrm{t}_{1}=3.62 \mathrm{~min}, \mathrm{t}_{2}=4.04 \mathrm{~min}\right) .{ }^{1} \mathrm{H}$ NMR $\left(500 \mathrm{MHz}, \mathrm{CD}_{3} \mathrm{OD}\right) \delta 7.17(\mathrm{td}, J=$ $7.8,1.4 \mathrm{~Hz}, 1 \mathrm{H}), 7.11(\mathrm{~d}, J=7.6 \mathrm{~Hz}, 1 \mathrm{H}), 6.98(\mathrm{td}, J=7.5,1.1 \mathrm{~Hz}, 1 \mathrm{H}), 6.85$ (d, $J=8.0 \mathrm{~Hz}, 1 \mathrm{H}), 5.77$ (ddt, $J=17.1,10.8,5.5 \mathrm{~Hz}, 1 \mathrm{H}), 5.18$ (dq, $J=17.2$, $1.5 \mathrm{~Hz}, 1 \mathrm{H}), 5.09(\mathrm{dq}, J=10.4,1.2 \mathrm{~Hz}, 1 \mathrm{H}), 4.06(\mathrm{dt}, J=5.5,1.4 \mathrm{~Hz}, 2 \mathrm{H})$, $3.75-3.66(\mathrm{~m}, 2 \mathrm{H}), 3.63(\mathrm{dd}, J=6.6,1.2 \mathrm{~Hz}, 1 \mathrm{H}), 2.84(\mathrm{dd}, J=6.6,5.6 \mathrm{~Hz}$, $1 \mathrm{H}), 0.99(\mathrm{~d}, J=6.1 \mathrm{~Hz}, 3 \mathrm{H}), 0.91(\mathrm{~d}, J=6.1 \mathrm{~Hz}, 3 \mathrm{H}) .{ }^{13} \mathrm{C} \mathrm{NMR}\left(126 \mathrm{MHz}, \mathrm{CD}_{3} \mathrm{OD}\right) \delta 177.86$, 173.05, 168.35, 135.75, 130.91, 128.80, 127.94, 123.87, 121.01, 116.53, 115.73, 72.97, 69.30, 48.13, 47.89, 40.45, 39.53, 22.53, 22.02. IR (thin film): 2973, 2920, 1692, 1593, 1373, 1319, 1245, 1189, 1076, $762 \mathrm{~cm}^{-1}$; HRMS (ESI): $\left[\mathrm{M}+\mathrm{NH}_{4}\right]^{+}$calculated for $\mathrm{C}_{19} \mathrm{H}_{24} \mathrm{~N}_{3} \mathrm{O}_{4}{ }^{+} \mathrm{m} / \mathrm{z}$ 358.1761, found $\mathrm{m} / \mathrm{z}$ $358.1757 ; \mathrm{mp}=190-192^{\circ} \mathrm{C} ;[\alpha]_{22}^{\mathrm{D}}+34^{\circ}\left(c 0.50, \mathrm{CH}_{3} \mathrm{OH}\right)$.

(6a $R, 6 \mathrm{~b} S, 9 \mathrm{a} S, 9 \mathrm{~b} R)$-Methyl-6a-isopropoxy-6,7,9-trioxo-6,6a,6b,7,9,9a-hexahydro-5 $H$-pyrrolo[3',4<smiles>COC(=O)N1C(=O)[C@@H]2[C@H](C(=O)N1C(C)(C)C)[C@]1(C)C(=O)Nc3ccccc3[C@H]21</smiles>
::3,4]cyclobuta[1,2-c]quinoline-8(9bH)-carboxylate (3v): 76\% yield, white solid, 99\% ee (SFC, Daicel CHIRALPAK ${ }^{\circledR}$ OD-H, isocratic $20 \% \mathrm{MeOH}, 3.0$ $\left.\mathrm{mL} / \mathrm{min}, 221 \mathrm{~nm} ; \mathrm{t}_{1}=2.17 \mathrm{~min}, \mathrm{t}_{2}=3.77 \mathrm{~min}\right) .{ }^{1} \mathrm{H}$ NMR $\left(500 \mathrm{MHz}, \mathrm{CDCl}_{3}\right) \delta$ $9.60(\mathrm{~s}, 1 \mathrm{H}), 7.23(\mathrm{td}, J=7.8,1.3 \mathrm{~Hz}, 1 \mathrm{H}), 7.15(\mathrm{~d}, J=7.4 \mathrm{~Hz}, 1 \mathrm{H}), 7.03$ (td, $J$ $=7.5,1.0 \mathrm{~Hz}, 1 \mathrm{H}), 6.93(\mathrm{~d}, J=7.8 \mathrm{~Hz}, 1 \mathrm{H}), 3.97(\mathrm{~s}, 3 \mathrm{H}), 3.93-3.84(\mathrm{~m}, 2 \mathrm{H})$, $3.81(\mathrm{dd}, J=6.8,1.2 \mathrm{~Hz}, 1 \mathrm{H}), 2.96(\mathrm{dd}, J=6.7,5.9 \mathrm{~Hz}, 1 \mathrm{H}), 1.10(\mathrm{~d}, J=6.1$ $\mathrm{Hz}, 3 \mathrm{H}), 0.98(\mathrm{~d}, J=6.1 \mathrm{~Hz}, 3 \mathrm{H}) .{ }^{13} \mathrm{C} \mathrm{NMR}\left(126 \mathrm{MHz}, \mathrm{CDCl}_{3}\right) \delta 172.94$, $168.02,167.99,149.01,135.15,129.64,128.49,124.79,119.98,116.51,73.30$, 70.19, 55.17, 49.05, 47.77, 39.46, 23.52, 23.34. IR (thin film): 3063, 2978, 1812, 1768, 1723, 1676, 1595, 1310, 1252, $731 \mathrm{~cm}^{-1}$; HRMS (ESI): $\left[\mathrm{M}+\mathrm{NH}_{4}\right]^{+}$calculated for $\mathrm{C}_{18} \mathrm{H}_{22} \mathrm{~N}_{3} \mathrm{O}_{6}{ }^{+} \mathrm{m} / z$ 376.1503, found $m / z 376.1498 ; \mathrm{mp}=179-181^{\circ} \mathrm{C} ;[\alpha]_{22}^{\mathrm{D}}+17^{\circ}\left(c 0.63, \mathrm{CH}_{3} \mathrm{OH}\right)$.

$(1 R, 2 S, 2 \mathrm{a} R, 8 \mathrm{~b} R)$-Dimethyl-2a-isopropoxy-3-0xo-1,2,2a,3,4,8b-hexahydrocyclobuta $[c]$ quinoline-1 ,2-dicarboxylate (3w): 60\% yield, 7:1 d.r., white solid, 61\% ee (SFC,<smiles>CC(=O)OC1[C@H]2c3ccccc3NC(=O)[C@]2(C(C)=O)[C@H]1C(C)=O</smiles>

$3 w$ Daicel CHIRALPAK ${ }^{\circledR}$ OD-H, isocratic $20 \% \mathrm{MeOH}, 3.0 \mathrm{~mL} / \mathrm{min}, 221 \mathrm{~nm} ; \mathrm{t}_{1}$ $\left.=2.59 \mathrm{~min}, \mathrm{t}_{2}=3.22 \mathrm{~min}\right) .{ }^{1} \mathrm{H} \mathrm{NMR}\left(500 \mathrm{MHz}, \mathrm{CD}_{3} \mathrm{OD}\right) \delta 7.23(\mathrm{td}, J=7.8$, $1.4 \mathrm{~Hz}, 1 \mathrm{H}), 7.17$ (d, $J=7.1 \mathrm{~Hz}, 1 \mathrm{H}), 7.04$ (td, $J=7.5,1.1 \mathrm{~Hz}, 1 \mathrm{H}), 6.89$ (d, $J=7.9 \mathrm{~Hz}, 1 \mathrm{H}), 4.37(\mathrm{~d}, J=11.7 \mathrm{~Hz}, 1 \mathrm{H}), 3.95(\mathrm{dd}, J=4.3,1.1 \mathrm{~Hz}, 1 \mathrm{H})$, $3.84-3.75(\mathrm{~m}, 5 \mathrm{H}), 3.26(\mathrm{~s}, 3 \mathrm{H}), 1.07$ (d, $J=6.1 \mathrm{~Hz}, 3 \mathrm{H}), 1.05(\mathrm{~d}, J=6.1$ $\mathrm{Hz}, 3 \mathrm{H}) .{ }^{13} \mathrm{C}$ NMR $\left(126 \mathrm{MHz}, \mathrm{CD}_{3} \mathrm{OD}\right) \delta 171.41,170.29,169.06,136.89$, 128.66, 128.54, 123.12, 119.49, 115.49, 73.87, 68.69, 51.24, 50.72, 48.66, 44.99, 39.75, 22.71, 22.00 . IR(thin film): 3215, 3063, 2979, 1728, 1680, 1595, 1301, 1168, 1116, $758 \mathrm{~cm}^{-1}$; HRMS (ESI): $\left[\mathrm{M}+\mathrm{NH}_{4}\right]^{+}$calculated for $\mathrm{C}_{18} \mathrm{H}_{25} \mathrm{~N}_{2} \mathrm{O}_{6}{ }^{+} m / z 365.1707$, found $m / z 365.1701 ; \mathrm{mp}=130-132{ }^{\circ} \mathrm{C} ;[\alpha]_{22}^{\mathrm{D}}-3^{\circ}$ 
$\left(c 0.78, \mathrm{CH}_{3} \mathrm{OH}\right)$.

\section{NMR Binding Experiments}

The binding constants were determined through NMR titration studies. ${ }^{2}$

\section{Binding between $4 \mathrm{f}$ and 3-O ${ }^{i}$ Pr quinonlone}

It was found that $3-\mathrm{O}^{i} \mathrm{Pr}$ quinolone $1 \mathbf{c}$ readily dimerizes in solution. This process was analyzed using the method described by Bach, ${ }^{11}$ resulting in a value of $\mathrm{K}_{\mathrm{dim}}=103 \pm 8$.

A stock solution of $3-\mathrm{O}$ Pr quinolone $1 \mathrm{c}\left(2.9 \mathrm{mg}\right.$ in $\left.4 \mathrm{~mL} \mathrm{CD} \mathrm{Cl}_{2}\right)$ was prepared, and $0.45 \mathrm{~mL}$ was transferred to a NMR tube. Next, another stock solution of $1 \mathrm{c}\left(5.6 \mathrm{mg}\right.$ in $\left.1.5 \mathrm{~mL} \mathrm{CD}_{2} \mathrm{Cl}_{2}\right)$ was prepared. Increasing amounts of this solution were titrated into the former solution, acquiring ${ }^{1} \mathrm{H}$ NMR spectra for each titration (Figure S1).

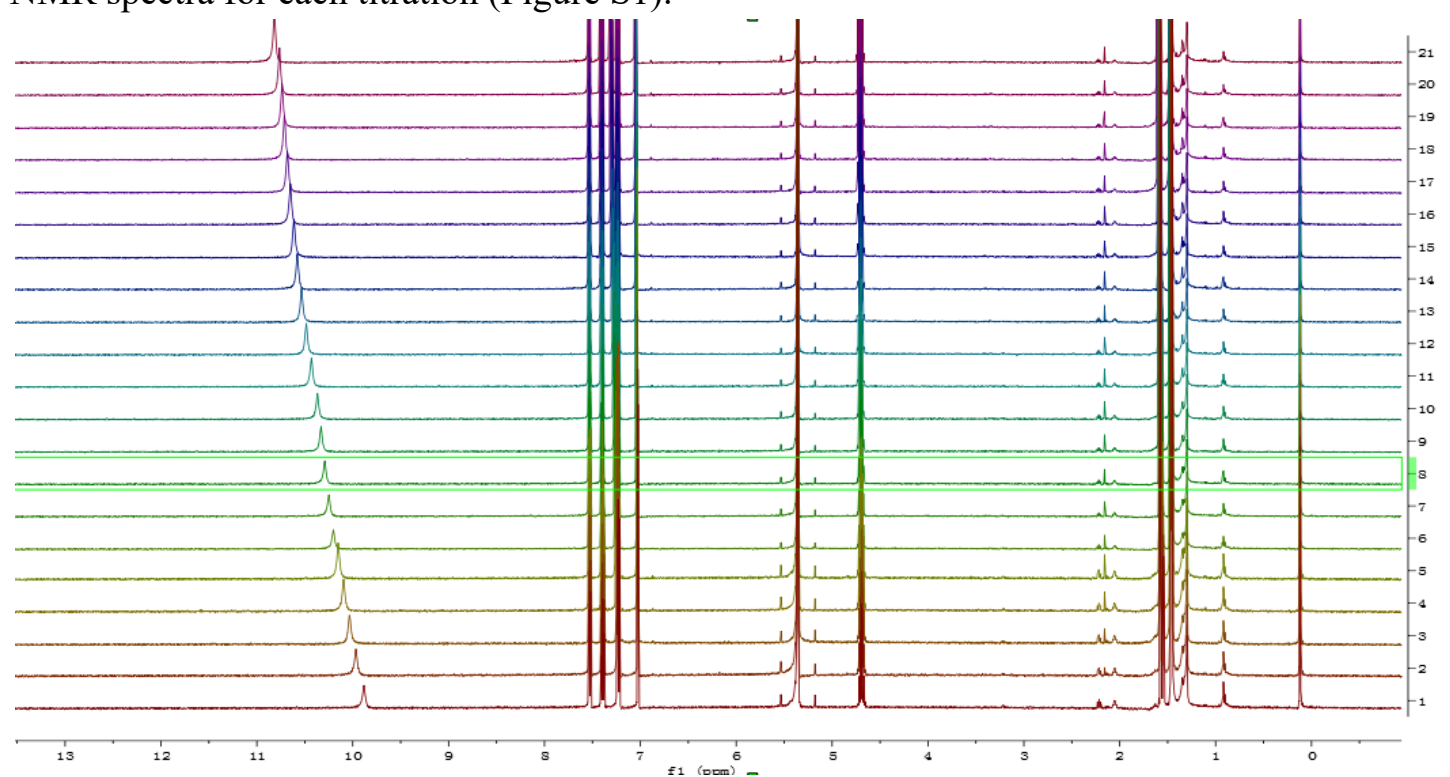

Figure S1. NMR titration of 3-O $\mathrm{O}^{i} \mathrm{Pr}$ quinolone 1c to monitor dimerization.

Similarly, we also performed the titration of $\mathbf{4 f}$ with $3-\mathrm{O}^{i} \operatorname{Pr}$ quinolone $1 \mathbf{c}$. The binding between $\mathbf{4 f}$ and $1 \mathrm{c}$ was analyzed using the method described by Thordarson, ${ }^{12}$ resulting in a value of $K_{a}=603 \pm 30$. A stock solution (A) of $\mathbf{4 f}\left(2.2 \mathrm{mg}\right.$ in $\left.2 \mathrm{~mL} \mathrm{CD}_{2} \mathrm{Cl}_{2}\right)$ was prepared, and $0.45 \mathrm{~mL}$ was transferred to an NMR tube. Next, another stock solution of $1 \mathbf{c}(5.6 \mathrm{mg}$ in $1.0 \mathrm{~mL} \mathrm{~A})$ was prepared, and increasing amounts of this solution were titrated into the former solution, acquiring ${ }^{1} \mathrm{H}$ NMR spectra for each titration (Figure S2). 


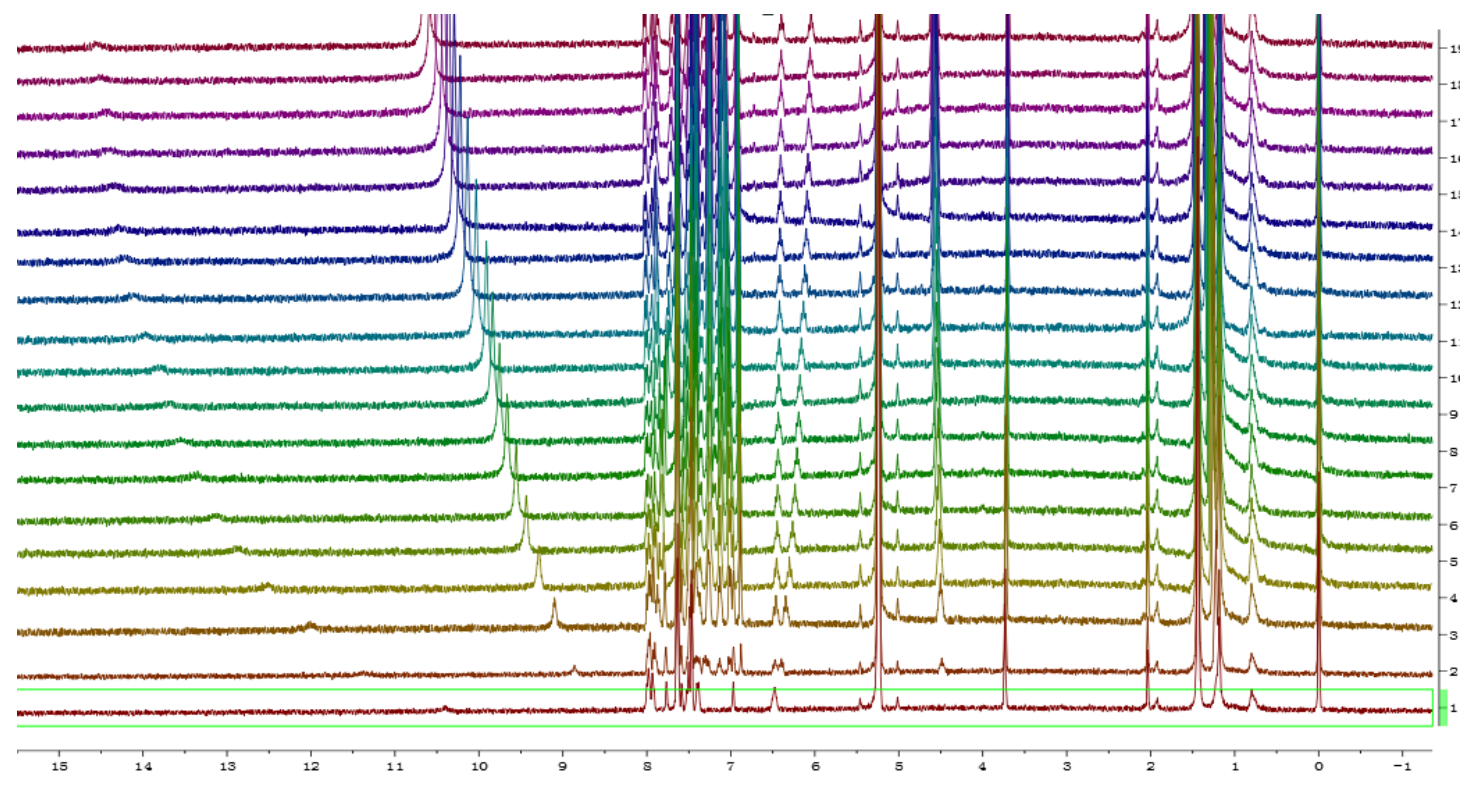

Figure S2. NMR titration of $\mathbf{4 f}$ with 1 c.

We carried out a similar titration experiment for the binding between $\mathbf{4 f}$ and maleimide, resulting in a value of $\mathrm{K}_{\mathrm{a}}=24 \pm 3.8$.

A stock solution (B) of $\mathbf{4 f}\left(1.8 \mathrm{mg}\right.$ in $\left.2 \mathrm{~mL} \mathrm{CD}_{2} \mathrm{Cl}_{2}\right)$ was prepared, and $0.45 \mathrm{~mL}$ was transferred to a NMR tube. Next, another stock solution of 1 c $(10.8 \mathrm{mg}$ in $1.0 \mathrm{~mL} \mathrm{~B})$ was prepared. Increasing amounts of this solution were titrated into the former solution, acquiring ${ }^{1} \mathrm{H}$ NMR spectra for each titration (Figure S3).

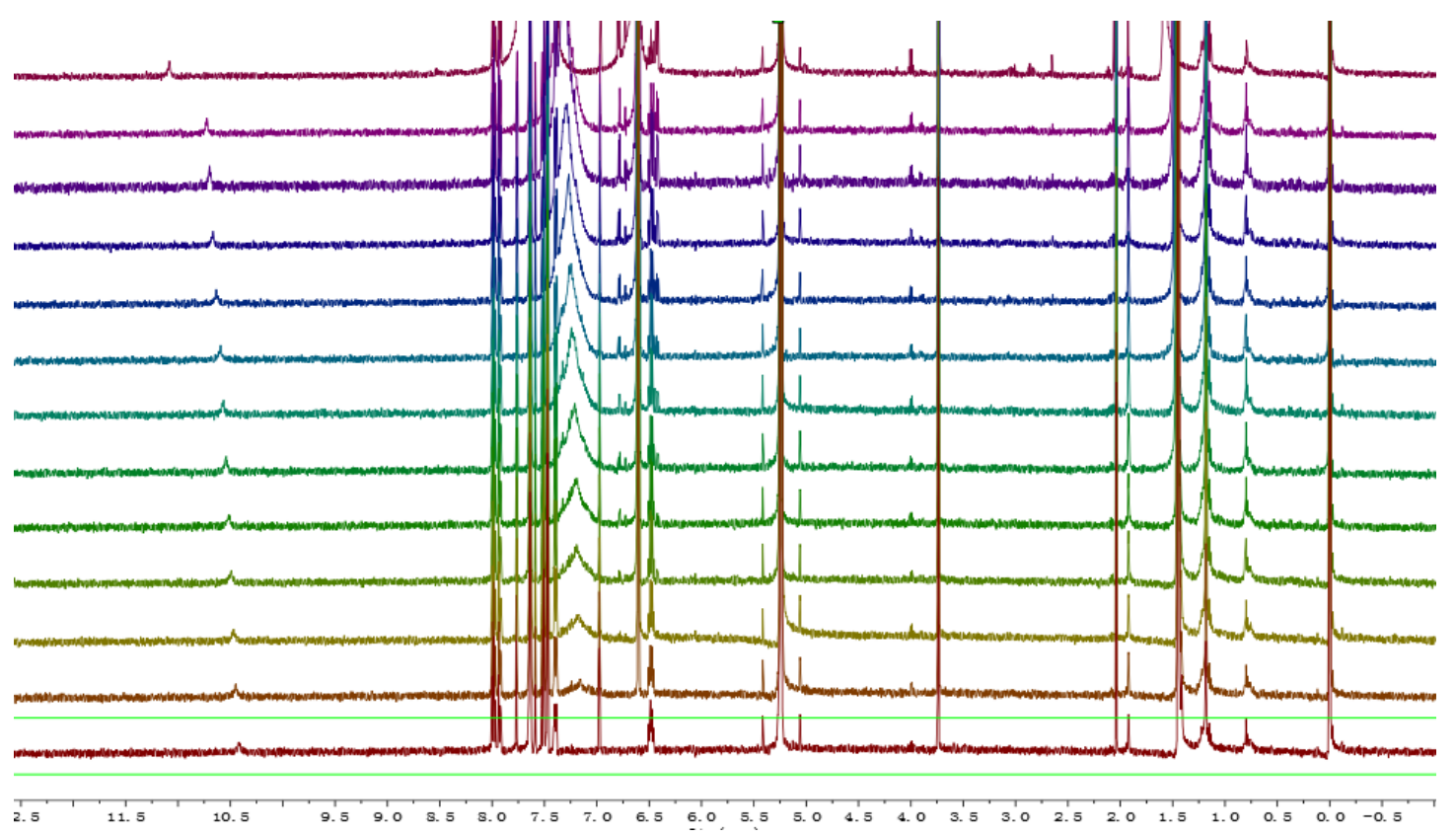

Figure S3. NMR titration of $\mathbf{4 f}$ with maleimide. 


\section{Room Temperature Stern-Volmer Quenching Experiments}

Stern-Volmer quenching experiments were conducted following a known report. ${ }^{13}$

\section{Quenching}

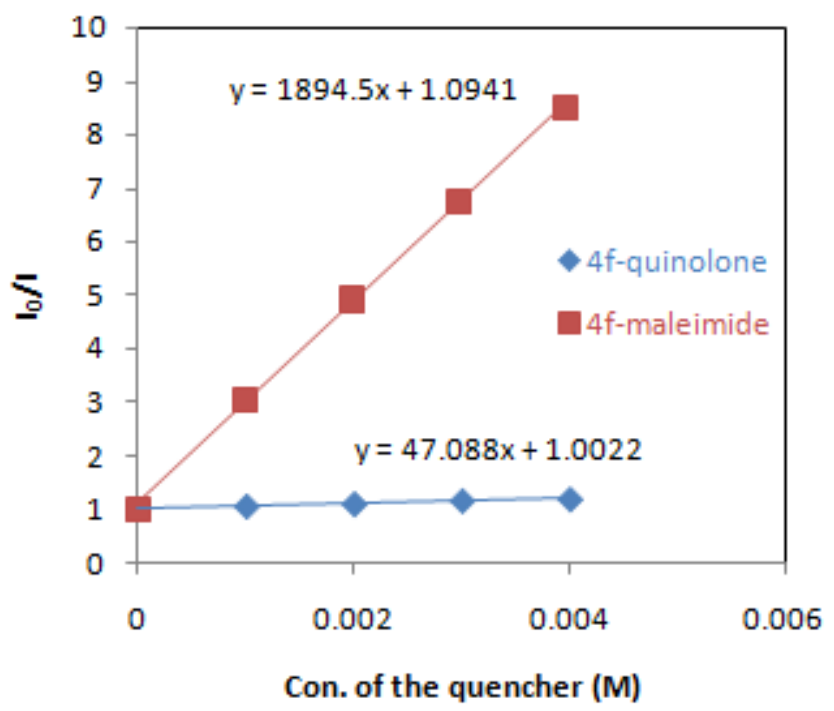

Figure S4. Stern-Volmer Quenching Experiments between the photocalyst $\mathbf{4 f}$ and substrates
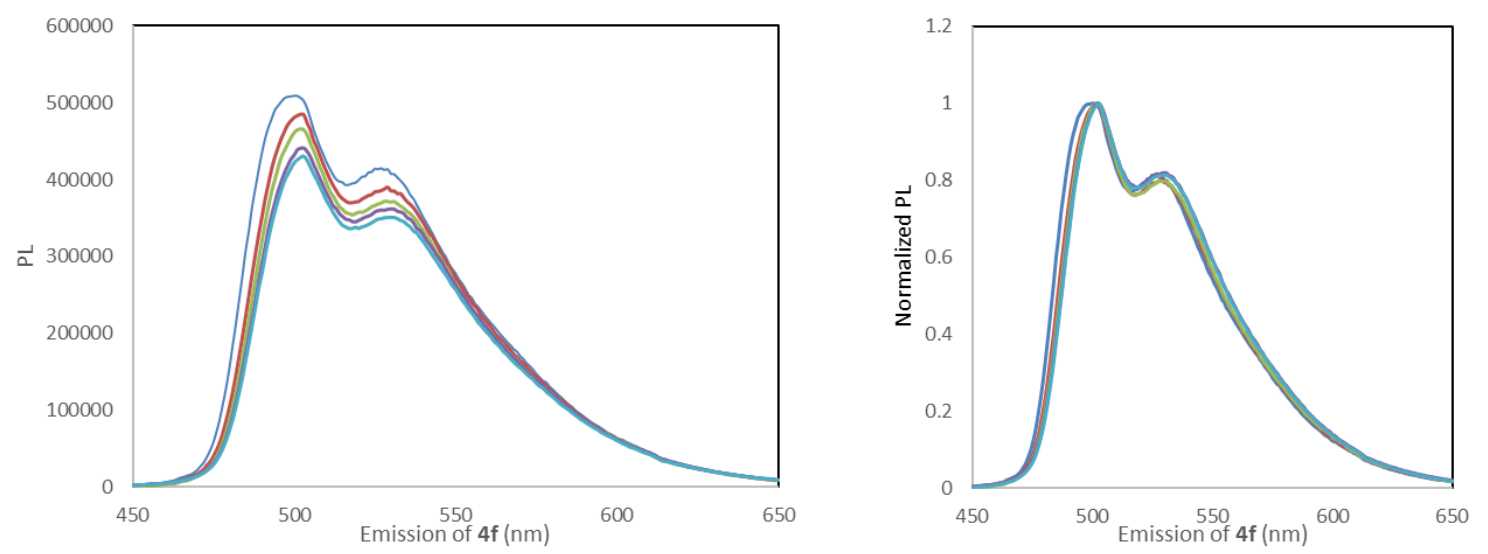

Figure S5. Emission of photocalyst $\mathbf{4 f}$ with different con. of quinolone 1c $(0,1,2,3,4 \mathrm{mM})$. 


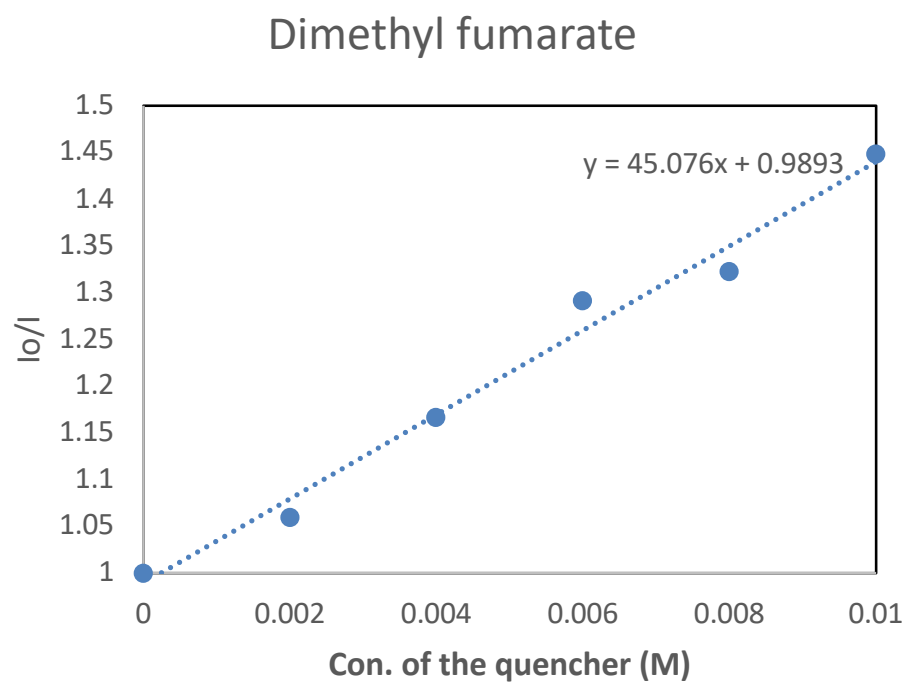

Figure S6. Stern-Volmer Quenching Experiments between the photocalyst $\mathbf{4 f}$ and dimethyl fumarate

\section{Kinetic Studies}

Our kinetic studies focused on the cycloaddition between 1c and maleimide 2. With the optimized reaction conditions, the kinetics of the reaction were monitored by ${ }^{1} \mathrm{H}$ NMR. Each reaction was monitored to roughly $6 \%-11 \%$ conversion, and rate constants were calculated for each reaction using the method of initial rates.

\section{a. Reaction rate dependence on the concentration of quinolone 1c}

The reactions were conducted using the procedure described above (section 4b). The results displayed in Figure S7 show that the reaction rate exhibits a zero-order dependence on the concentration of quinolone 1c.

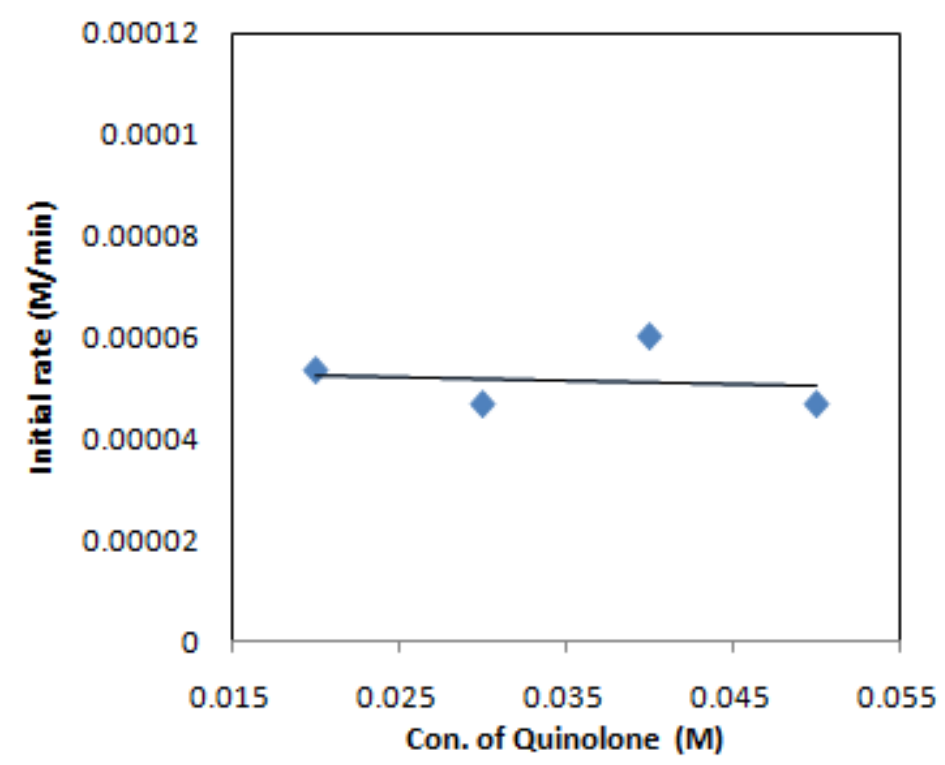

Figure S7. Zero order dependence on the concentration of 1c

\section{b. Reaction rate dependence on the concentration of photocatalyst $4 \mathrm{f}$}


The reactions were conducted using the procedure described above (section $4 \mathrm{~b}$ ). The results displayed in Figure S8 show that the reaction rate exhibits a first-order dependence on the concentration of photocatalyst $\mathbf{4 f}$.

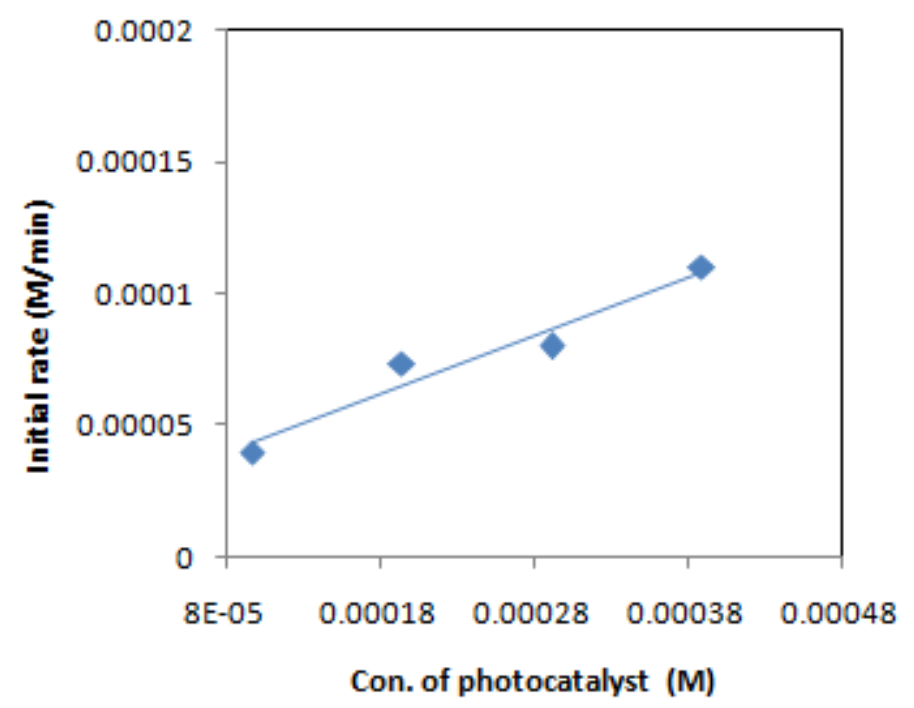

Figure S8. First-order dependence on the concentration of photocatalyst $\mathbf{4 f}$

\section{c. Reaction rate dependence on the concentration of maleimide 2}

The reactions were conducted using the procedure described above (section $4 \mathrm{~b}$ ). The results displayed in Figure S9 show that the reaction rate exhibits a first-order dependence on the concentration of maleimide.

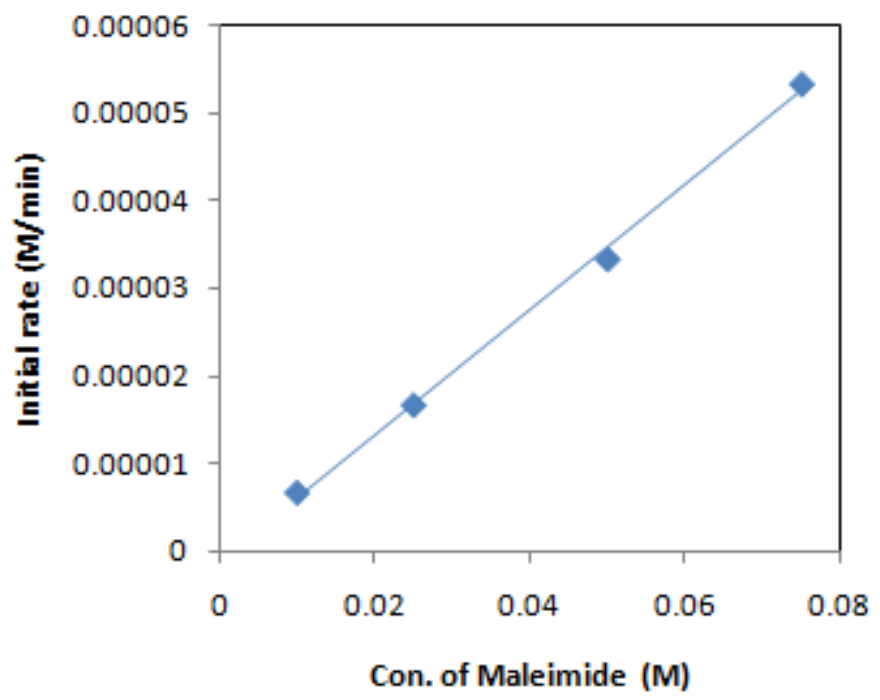

Figure S9. First-order dependence on the concentration of maleimide 


\section{Control Experiments}

These cycloadditions were performed according to the standard procedure described above (section $4 b)$.
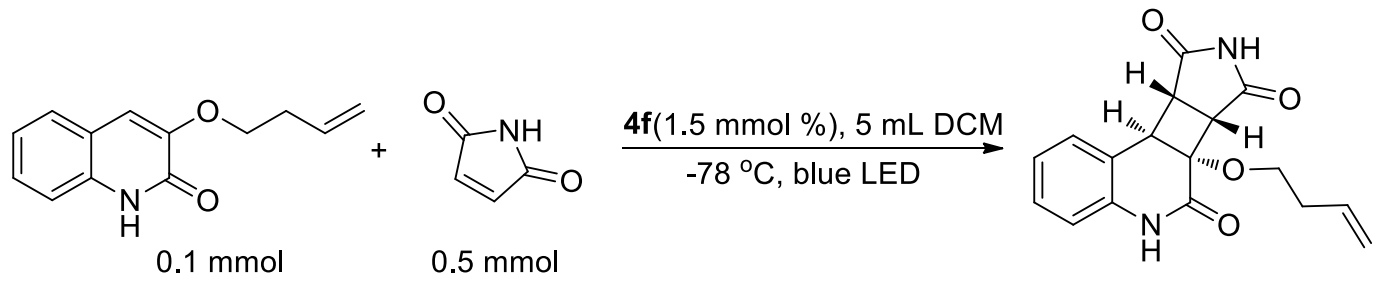

$3 x, 24 \%$ NMR yield, $49 \%$ ee

(6a $R, 6 \mathrm{~b} S, 9 \mathrm{a} S, 9 \mathrm{~b} R)$-6a-(but-3-en-1-yloxy)-9a,9b-dihydro-5H-pyrrolo[3',4':3,4]cyclobuta[1,2-c]qu inoline-6,7,9(6aH, $\mathbf{6 b} \boldsymbol{H}, \mathbf{8 H})$-trione (3x): white solid, 49\% ee (HPLC, Daicel CHIRALPAK ${ }^{\circledR}$ AD-H, Gradient $5 \%$ iPrOH (95\% Hexane) to 50\% ${ }^{i} \mathrm{PrOH}$ (50\% Hexane), $1.0 \mathrm{~mL} / \mathrm{min}, 287 \mathrm{~nm} ; \mathrm{t}_{1}=12.95 \mathrm{~min}$, $\left.\mathrm{t}_{2}=16.69 \mathrm{~min}\right) ;{ }^{1} \mathrm{H}$ NMR $\left(500 \mathrm{MHz}, \mathrm{CD}_{3} \mathrm{OD}\right) \delta 7.15(\mathrm{td}, J=7.7,1.2 \mathrm{~Hz}, 1 \mathrm{H}), 7.12(\mathrm{~d}, J=7.5 \mathrm{~Hz}$, $1 \mathrm{H}), 6.97(\mathrm{td}, J=7.5,0.8 \mathrm{~Hz}, 1 \mathrm{H}), 6.82(\mathrm{~d}, J=7.9 \mathrm{~Hz}, 1 \mathrm{H}), 5.67(\mathrm{ddt}, J=17.1,10.3,6.8 \mathrm{~Hz}, 1 \mathrm{H})$, $4.93(\mathrm{dq}, J=17.2,1.7 \mathrm{~Hz}, 1 \mathrm{H}), 4.90-4.86(\mathrm{~m}, 1 \mathrm{H}), 3.79(\mathrm{~d}, J=5.6 \mathrm{~Hz}, 1 \mathrm{H}), 3.62(\mathrm{dd}, J=6.5,1.1$ $\mathrm{Hz}, 1 \mathrm{H}), 3.47$ (dt, $J=8.7,6.4 \mathrm{~Hz}, 1 \mathrm{H}), 3.30$ (dt, $J=8.6,7.0 \mathrm{~Hz}, 1 \mathrm{H}), 2.77$ (dd, $J=6.4,5.7 \mathrm{~Hz}, 1 \mathrm{H}$ ), $2.19-2.12(\mathrm{~m}, 2 \mathrm{H}) .{ }^{13} \mathrm{C}$ NMR $\left(126 \mathrm{MHz}, \mathrm{CD}_{3} \mathrm{OD}\right) \delta 179.76,174.85,167.16,135.68,134.49,128.68$, $127.88,123.77,121.10,115.79,115.63,73.14,64.81,48.36,47.21,41.03,33.74$. IR (thin film): 3072 , 2922, 2854, 1772, 1714, 1676, 1596, 1494, 1337, 1248, 1183, $759 \mathrm{~cm}^{-1}$; HRMS (ESI): $\left[\mathrm{M}+\mathrm{NH}_{4}\right]^{+}$ calculated for $\mathrm{C}_{17} \mathrm{H}_{20} \mathrm{~N}_{3} \mathrm{O}_{4}{ }^{+} \mathrm{m} / z$ 330.1448, found $\mathrm{m} / z$ 330.1446; $\mathrm{mp}=246-248{ }^{\circ} \mathrm{C}$.

\section{Transient Absorption Spectroscopy}

Transient absorption spectra and single wavelength kinetic data were obtained on a home-built apparatus described previously. ${ }^{14}$ Briefly, samples were excited by a frequency tripled Q-switched, pulsed Nd:YAG laser (Quantel USA (BigSky) Brilliant B) coupled to an optical parametric oscillator (OPO, Opotek, Inc.) to provide an excitation wavelength of $440 \mathrm{~nm}$ (7 ns full width at half-maximum). The laser power was controlled through the Q-switch delay and maintained between $3-4 \mathrm{~mJ} / \mathrm{pulse}$. The beam was directed through a $355 \mathrm{~nm}$ and $532 \mathrm{~nm}$ notch filter and directed to the sample by three silver mirrors. A $150 \mathrm{~W}$ xenon arc lamp (Applied Photophysics), pulsed with $70 \mathrm{~V}$, served as the probe beam and was positioned orthogonal to the excitation laser. Detection was achieved with a monochromator (SPEX 1702/04) optically coupled to an R928 photomultiplier tube (Hamamatsu). Transient data were acquired on a computer-interfaced digital oscilloscope (LeCroy 9450, Dual 350 $\mathrm{MHz}$ ) with $2.5 \mathrm{~ns}$ resolution terminated at $50 \Omega$. Full transient absorption spectra were recorded with a resolution of $10 \mathrm{~nm}$ between 350 and $550 \mathrm{~nm}$. Single wavelength kinetic analysis was performed in Origin2018 (Originlab) with least-squares error minimization accomplished using the Levenberg-Marquardt iteration method 
Samples for measurement were prepared in dichloromethane in a $1 \mathrm{~cm}^{2}$ cuvette affixed with a 24/40 ground glass joint (Quark Glass) and sparged with argon for $10 \mathrm{~min}$. For the equilibrium constant and Stern-Volmer titrations, the titrant was prepared as a more concentrated stock solution in the same mixture of photocatalyst and substrate as required to keep the overall concentrations constant throughout the titration. This stock solution was sparged with argon and titrated into the cuvette with a Hamilton syringe.
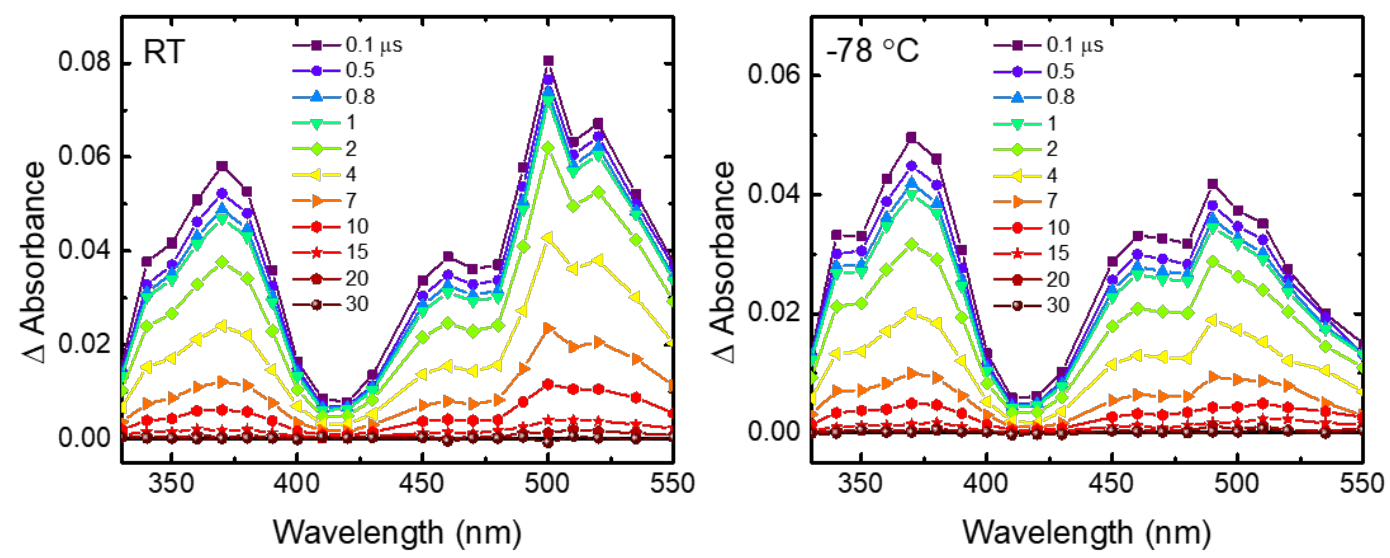

Figure S10. Transient absorption spectra for photocatalyst $4 \mathbf{f}$ in $\mathrm{CH}_{2} \mathrm{Cl}_{2}$ at room temperature (left) and $-78^{\circ} \mathrm{C}$ at the indicated time delays after pulsed laser excitation at $440 \mathrm{~nm}$
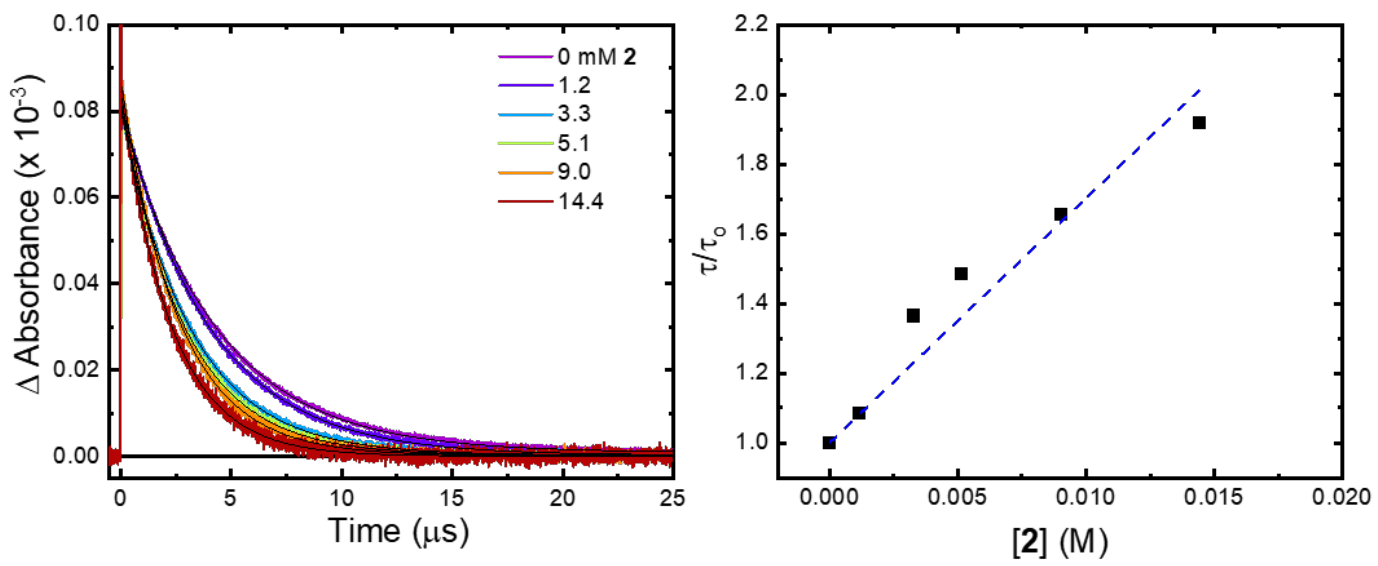

Figure S11. Left, titration of a $150 \mu \mathrm{M} \mathbf{4 f}+10 \mathrm{mM}$ quinolone 1c solution with increasing concentrations of maleimide $2\left(\mathrm{CH}_{2} \mathrm{Cl}_{2}\right)$. The kinetic data were fit with a single exponential, black lines. Right, Stern-Volmer quenching analysis of the photoexcited [Ir-Q] complex lifetime $(\tau)$ with increasing maleimide concentrations. Blue dashed line shows a linear best fit from which a Stern-Volmer constant of $\mathrm{K}_{\mathrm{SV}}=130 \mathrm{M}^{-1}$ and a bimolecular quenching rate constant of $k_{\mathrm{q}}=3.0 \times 10^{7}$ $\mathrm{M}^{-1} \mathrm{~S}^{-1}$ were extracted. 
Table S1. Parameters Extracted from Biexponential Equation and Equilibrium Constant at $-78{ }^{\circ} \mathrm{C}$ in $\mathrm{CH}_{2} \mathrm{Cl}_{2}$.

\begin{tabular}{|l|l|l|l|l|l|}
\hline Quinolone $(\mathrm{mM})$ & $\mathrm{A}_{1}$ & $\tau_{1}\left(\mathrm{x} 10^{-9} \mathrm{~s}\right)$ & $\mathrm{A}_{2}$ & $\tau_{2}\left(\mathrm{x} 10^{-6} \mathrm{~s}\right)$ & $K_{\mathrm{EQ},-78}$ \\
\hline 0 & 0.067 & 570 & $\mathrm{a}$ & $\mathrm{a}$ & $\mathrm{a}$ \\
\hline 0.11 & 0.064 & 620 & 0.010 & 3.09 & 1800 \\
\hline 0.22 & 0.051 & 620 & 0.023 & 3.15 & 2600 \\
\hline 0.32 & 0.039 & 660 & 0.038 & 3.44 & 3900 \\
\hline 0.41 & 0.035 & 620 & 0.038 & 3.33 & 3100 \\
\hline 0.59 & 0.027 & 680 & 0.051 & 3.55 & 3900 \\
\hline 0.82 & 0.021 & 770 & 0.058 & 3.60 & 3900 \\
\hline 1.13 & 0.015 & 930 & 0.066 & 3.76 & 4400 \\
\hline average & - & - & - & - & $3400 \pm 900$ \\
\hline
\end{tabular}

${ }^{a}$ At 0 equivalents of quinoline the quenching of the iridium excited state was first-order and modeled by a single exponential.

To estimate the equilibrium constant between the iridium photocatalyst (Ir) and quinolone 1c (Q) at $-78{ }^{\circ} \mathrm{C}$ in $\mathrm{CH}_{2} \mathrm{Cl}_{2}$ (eqn 1), the kinetic data reported in Figure $1 \mathrm{~B}$ of the manuscript were modeled by a biexponential decay (eqn 2), where $A_{1}$ and $A_{2}$ are the preexponential constants and $\tau_{1}$ and $\tau_{2}$ are the excited-state lifetimes of the free iridium photocatalyst ([Ir $]_{\text {free }}$ ) and quinolone bound photocatalyst ([Ir-Q]) respectively, Table S1. The concentrations of maleimide $2(10 \mathrm{mM})$ and the iridium photocatalyst $(150 \mu \mathrm{M})$ were kept constant throughout the titration. Quinolone 1c was titrated into this solution from a more concentrated stock solution $(3.6 \mathrm{mM})$. It was assumed that the difference in extinction coefficient of photoexcited $[\operatorname{Ir}]_{\text {free }}\left([\operatorname{Ir}]^{*}\right.$ free $)$ and $[\operatorname{Ir}-\mathrm{Q}]\left([\operatorname{Ir}-\mathrm{Q}]^{*}\right)$ was negligible at $370 \mathrm{~nm}$ and therefore the preexponential constants were proportional to the concentrations of the two excited-state species.

$$
\begin{gathered}
{[\mathrm{Ir}]_{\text {free }}+\mathrm{Q} \rightleftharpoons[\mathrm{Ir}-\mathrm{Q}]} \\
\Delta A=A_{1} e^{-t / \tau_{1}}+A_{2} e^{-t / \tau_{2}}
\end{gathered}
$$

To convert $\mathrm{A}_{1}$ and $\mathrm{A}_{2}$ to the relative equilibrium concentrations of the individual compounds ( $[\mathrm{Ir}]_{\text {free, }}[\mathrm{Ir}-\mathrm{Q}]$, and $\mathrm{Q}_{\mathrm{eq}}$ ), the mole fractions were calculated via equations 3 and 4 . The relative concentrations at equilibrium could then be calculated from the known initial concentrations of the iridium photocatalyst ([Ir $\left.]_{0}\right)$ and quinolone $\left([\mathrm{Q}]_{0}\right)$, eqns 5-7.

$$
\begin{array}{cc}
\chi_{[I r]_{\text {free }}}=\frac{A_{1}}{A_{1}+A_{2}} & \text { eqn } 3 \\
\chi_{[I r-Q]}=\frac{A_{2}}{A_{1}+A_{2}} & \text { eqn } 4 \\
{[\text { Ir }]_{\text {free }}=\chi_{[I r]_{\text {free }}} *[\text { Ir }]_{0}} & \text { eqn } 5 \\
{\left[\text { Ir-Q] }=\chi_{[I r-Q]} *[\text { Ir }]_{0}\right.} & \text { eqn } 6 \\
{[Q]_{\text {eq }}=[Q]_{0}-[\text { Ir }-Q]} & \text { eqn } 7
\end{array}
$$

Finally, the equilibrium constant $\left(K_{\mathrm{EQ},-78}\right)$ was estimated from the calculated equilibrium concentrations, eqn 8 . This was performed for each addition of quinolone and the equilibrium 
constants were averaged and standard deviation calculated to provide $K_{\mathrm{EQ}}=3,400 \pm 900$. An outlier test was performed with no outlier found.

$$
K_{E Q,-78}=\frac{[I r-Q]}{[I r]_{\text {free }}^{*[Q]_{e q}}}
$$

As shown in Table $\mathrm{S} 1$, the lifetimes assigned to $[\operatorname{Ir}]_{\text {free }}\left(\tau_{1}\right)$ and $[\operatorname{Ir}-\mathrm{Q}]\left(\tau_{2}\right)$ increase with the quinolone concentration. This result is consistent with the potential for heterodimerization between the quinolone and maleimide ([Q-M]) that reduces the concentration of maleimide available to quench the photoexcited iridium and [Ir-Q] complex. Along with heterodimerization, we have shown that quinolone homodimerizes at room temperature, which would also be expected to affect the calculated $[\mathrm{Q}]_{\mathrm{eq}}$ concentration. Taken together, these competitive association processes prevent accurate determinization of the equilibrium constant. However, the qualitative conclusion can be made that the binding constant between the iridium complex and quinolone is larger at $-78^{\circ} \mathrm{C}$ than at room temperature. 


\section{X-Ray Crystallographic Data}

(6aR,6bS,9a $S, 9 \mathrm{~b} R)$-2-Iodo-6a-isopropoxy-9a,9b-dihydro-5H-pyrrolo[3',4':3,4] cyclobuta[1,2-c]q uinoline-6,7,9(6aH,6b $H, 8 H)$-trione $(3 \mathrm{f})$ :<smiles>O=C1NC(=O)[C@@]2(C(F)(F)F)[C@@H]1[C@@H]1C(=O)Nc3ccc(I)cc3[C@H]12</smiles>

$3 f$

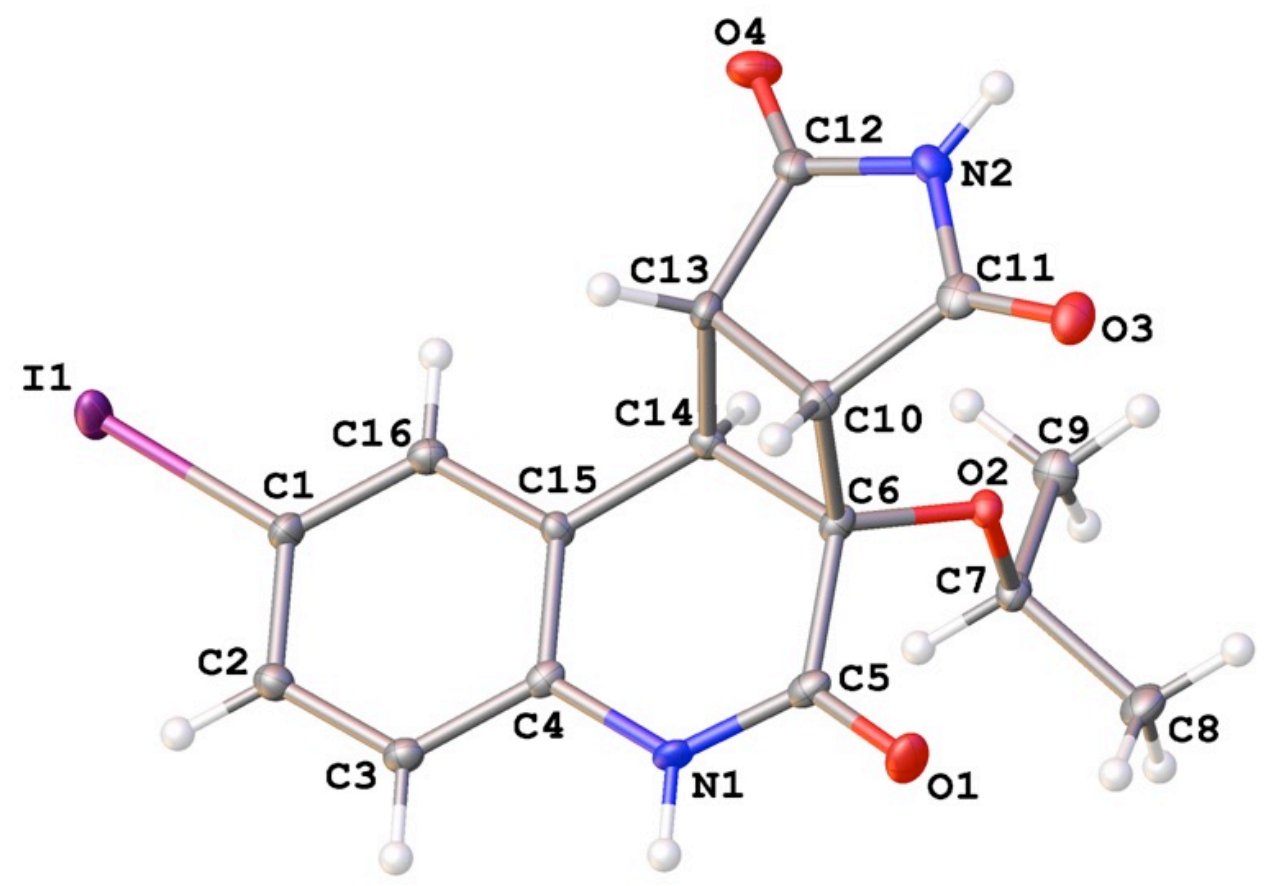

\section{Crystallographic ExperimentalSection}

\section{Data Collection}

A colorless crystal with approximate dimensions $0.035 \times 0.091 \times 0.269 \mathrm{~mm}^{3}$ was selected under oil under ambient conditions and attached to the tip of a MiTeGenMicroMount ${ }^{\mathscr{O}}$. The crystal was mounted in a stream of cold nitrogen at $100(1) \mathrm{K}$ and centered in the X-ray beam by using a video camera.

The crystal evaluation and data collection were performed on a Bruker QuazarSMART APEXII

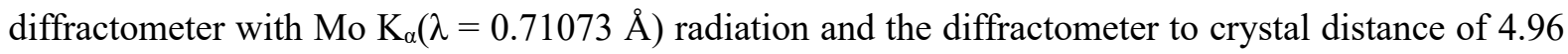
$\mathrm{cm}$.

The initial cell constants were obtained from three series of $\omega$ scans at different starting angles. Each series consisted of 12 frames collected at intervals of $0.5^{\circ}$ in a $6^{\circ}$ range about $\omega$ with the exposure time of 10 seconds per frame. The reflections were successfully indexed by an automated indexing routine built in the APEXII program suite. The final cell constants were calculated from a set of 9245 strong reflections from the actual data collection.

The data were collected by using the full sphere data collection routine to survey the reciprocal space 
to the extent of a full sphere to a resolution of $0.709 \AA$. A total of 18225 data were harvested by collecting 5 sets of frames with $0.7^{\circ}$ scans in $\omega$ and $\varphi$ with exposure times of $26 \mathrm{sec}$ per frame. These highly redundant datasets were corrected for Lorentz and polarization effects. The absorption correction was based on fitting a function to the empirical transmission surface as sampled by multiple equivalent measurements.

\section{Structure Solution and Refinement}

The systematic absences in the diffraction data were consistent for the space groups $P 2_{1}$ and $P 2 / \mathrm{m}$. The $E$-statistics strongly suggested the non-centrosymmetric space group $P 2_{1}$ that yielded chemically reasonable and computationally stable results of refinement.

A successful solution by the direct methods provided most non-hydrogen atoms from the $E$-map. The remaining non-hydrogen atoms were located in an alternating series of least-squares cycles and difference Fourier maps. All non-hydrogen atoms were refined with anisotropic displacement coefficients. All hydrogen atoms were included in the structure factor calculation at idealized positions and were allowed to ride on the neighboring atoms with relative isotropic displacement coefficients.

The absolute configuration was unequivocally assigned based on anomalous dispersion: $\mathrm{C} 6-R, \mathrm{C} 10$ $-S, \mathrm{C} 13-S, \mathrm{C} 14-R$.

The final least-squares refinement of 210 parameters against 4520 data resulted in residuals $R$ (based on $F^{2}$ for $I \geq 2 \sigma$ ) and $w R$ (based on $F^{2}$ for all data) of 0.0196 and 0.0474 , respectively. The final difference Fourier map was featureless.

\section{Summary}

Crystal Data for $\mathrm{C}_{16} \mathrm{H}_{15} \mathrm{IN}_{2} \mathrm{O}_{4} \quad(M=426.20 \mathrm{~g} / \mathrm{mol}):$ monoclinic, space group $P 2_{1}$ (no. 4), $a=9.094(3) \AA, b=7.248(2) \AA, c=11.749(3) \AA, V=769.3(4) \AA^{3}, Z=2, T=100.0 \mathrm{~K}, \mu(\mathrm{MoK} \alpha)=$ $2.104 \mathrm{~mm}^{-1}$, Dcalc $=1.840 \mathrm{~g} / \mathrm{cm}^{3}, 18225$ reflections measured $\left(3.49^{\circ} \leq 2 \Theta \leq 60.264^{\circ}\right), 4520$ unique $\left(R_{\text {int }}=0.0255, \mathrm{R}_{\text {sigma }}=0.0238\right)$ which were used in all calculations. The final $R_{1}$ was $0.0196(\mathrm{I}>2 \sigma(\mathrm{I}))$ and $w R_{2}$ was 0.0474 (all data).

Table S2 Crystal data and structure refinement for Yoon53.

$\begin{array}{ll}\text { Identification code } & \text { Yoon53 } \\ \text { Empirical formula } & \mathrm{C}_{16} \mathrm{H}_{15} \mathrm{IN}_{2} \mathrm{O}_{4} \\ \text { Formula weight } & 426.20 \\ \text { Temperature/K } & 100.02 \\ \text { Crystal system } & \text { monoclinic } \\ \text { Space group } & \mathrm{P} 2_{1} \\ \mathrm{a} / \AA & 9.094(3) \\ \mathrm{b} / \AA & 7.248(2) \\ \mathrm{c} / \AA & 11.749(3) \\ \alpha /{ }^{\circ} & 90\end{array}$




$\begin{array}{ll}\beta /{ }^{\circ} & 96.62(2) \\ \gamma /{ }^{\circ} & 90 \\ \text { Volume } / \AA^{3} & 769.3(4) \\ Z & 2 \\ \rho_{\text {calc }} / \mathrm{cm}^{3} & 1.840 \\ \mu / \mathrm{mm}^{-1} & 2.104 \\ \mathrm{~F}(000) & 420.0 \\ \text { Crystal size } / \mathrm{mm}^{3} & 0.269 \times 0.091 \times 0.035 \\ \text { Radiation } & \mathrm{MoK} \alpha(\lambda=0.71073) \\ 2 \Theta \text { range for data collection } /{ }^{\circ} & 3.49 \text { to } 60.264 \\ \text { Index ranges } & -12 \leq \mathrm{h} \leq 12,-10 \leq \mathrm{k} \leq 10,-16 \leq 1 \leq 16 \\ \text { Reflections collected } & 18225 \\ \text { Independent reflections } & 4520\left[\mathrm{R}_{\text {int }}=0.0255, \mathrm{R}_{\text {sigma }}=0.0238\right] \\ \text { Data/restraints/parameters } & 4520 / 1 / 210 \\ \text { Goodness-of-fit on } \mathrm{F}^{2} & 1.074 \\ \text { Final R indexes }[\mathrm{I}>=2 \sigma(\mathrm{I})] & \mathrm{R}_{1}=0.0196, \mathrm{wR}_{2}=0.0472 \\ \text { Final R indexes [all data }] & \mathrm{R}_{1}=0.0207, \mathrm{wR}_{2}=0.0474 \\ \text { Largest diff. peak/hole } / \mathrm{e} \AA^{-3} & 0.69 /-0.42 \\ \text { Flack parameter } & -0.045(7)\end{array}$

Table S3 Fractional Atomic Coordinates $\left(\times 10^{4}\right)$ and Equivalent Isotropic Displacement Parameters $\left(\AA^{2} \times 10^{3}\right)$ for Yoon53. $U_{\text {eq }}$ is defined as $1 / 3$ of of the trace of the orthogonalised $U_{\text {IJ }}$ tensor.

$\begin{array}{lllll}\text { Atom } & \boldsymbol{x} & \boldsymbol{y} & \boldsymbol{z} & \mathbf{U}(\mathbf{e q}) \\ \mathrm{I} 1 & 7873.3(2) & 375.5(3) & 1909.6(2) & 22.66(6) \\ \mathrm{O} 1 & -212(2) & 5934(3) & 945.8(17) & 19.2(4) \\ \text { O2 } & 961(2) & 6411(2) & 3249.0(16) & 10.9(4) \\ \text { O3 } & 1499(2) & 10501(4) & 2786.7(16) & 19.2(4) \\ \text { O4 } & 5498(2) & 7502(3) & 4644.1(18) & 19.3(4) \\ \text { N1 } & 1481(3) & 3693(3) & 838(2) & 12.5(4) \\ \text { N2 } & 3561(3) & 9332(3) & 3866(2) & 14.4(5) \\ \text { C1 } & 5724(3) & 1428(4) & 1561(2) & 12.5(5) \\ \text { C2 } & 4796(3) & 842(3) & 595(2) & 13.3(5) \\ \text { C3 } & 3385(3) & 1593(4) & 373(2) & 12.1(5) \\ \text { C4 } & 2903(3) & 2932(3) & 1099(2) & 11.0(5)\end{array}$




$\begin{array}{lllll}\text { C5 } & 959(3) & 5205(5) & 1329(2) & 13.0(5) \\ \text { C6 } & 1873(3) & 5987(4) & 2399(2) & 11.0(5) \\ \text { C7 } & 276(3) & 4840(3) & 3756(2) & 12.6(5) \\ \text { C8 } & -1390(3) & 5060(4) & 3536(3) & 18.3(6) \\ \text { C9 } & 816(3) & 4842(4) & 5028(2) & 16.2(5) \\ \text { C10 } & 2759(3) & 7762(4) & 2162(2) & 12.4(5) \\ \text { C11 } & 2472(3) & 9373(4) & 2927(2) & 14.9(5) \\ \text { C12 } & 4535(3) & 7880(4) & 3864(2) & 13.6(5) \\ \text { C13 } & 4187(3) & 6839(4) & 2744(2) & 12.7(5) \\ \text { C14 } & 3347(3) & 4984(3) & 2839(2) & 10.7(5) \\ \text { C15 } & 3823(3) & 3510(3) & 2059(2) & 10.0(5) \\ \text { C16 } & 5236(3) & 2740(4) & 2285(2) & 12.5(5)\end{array}$

Table S4 Anisotropic Displacement Parameters $\left(\AA^{2} \times 10^{3}\right)$ for Yoon53. The Anisotropic displacement factor exponent takes the form: $-2 \pi^{2}\left[h^{2} a^{* 2} U_{11}+2 h k a * b * U_{12}+\ldots\right]$.

$\begin{array}{lllllll}\text { Atom } & \mathbf{U}_{11} & \mathbf{U}_{22} & \mathbf{U}_{33} & \mathbf{U}_{23} & \mathbf{U}_{13} & \mathbf{U}_{12} \\ \text { I1 } & 16.79(9) & 31.07(10) & 18.46(9) & -9.19(9) & -5.07(6) & 12.14(9) \\ \text { O1 } & 13.8(10) & 26.5(11) & 16.3(9) & 0.4(7) & -2.5(7) & 7.3(7) \\ \text { O2 } & 10.8(9) & 9.7(8) & 12.6(9) & 0.2(7) & 3.3(7) & 0.0(7) \\ \text { O3 } & 16.7(8) & 14.0(9) & 27.2(9) & 2.8(11) & 4.3(7) & 2.7(11) \\ \text { O4 } & 19.2(10) & 16.1(9) & 20.8(11) & -1.6(8) & -6.3(8) & -1.0(8) \\ \text { N1 } & 8.9(11) & 17.1(10) & 10.6(11) & -1.5(8) & -2.8(8) & -0.6(8) \\ \text { N2 } & 16.7(12) & 10.5(10) & 16.0(12) & -2.1(8) & 2.1(9) & 0.2(8) \\ \text { C1 } & 9.4(12) & 13.9(12) & 13.9(12) & -0.3(9) & -0.4(9) & 1.6(9) \\ \text { C2 } & 14.0(12) & 13.3(13) & 12.4(12) & -1.4(8) & 0.7(9) & -0.4(9) \\ \text { C3 } & 11.3(12) & 13.9(11) & 10.9(12) & 0.4(9) & -0.1(9) & -1.2(9) \\ \text { C4 } & 9.5(12) & 12.0(11) & 11.2(12) & 3.8(9) & 0.5(9) & -0.8(9) \\ \text { C5 } & 9.9(10) & 17.5(13) & 11.5(10) & 3.2(12) & 0.3(8) & -2.5(12) \\ \text { C6 } & 9.1(11) & 12.3(10) & 11.6(11) & 2.7(8) & 1.1(9) & 0.6(8) \\ \text { C7 } & 11.3(12) & 9.9(11) & 16.8(13) & 0.0(8) & 3.4(10) & 0.0(8) \\ \text { C8 } & 11.9(12) & 19.7(18) & 23.7(13) & -0.4(10) & 3.5(10) & -3.2(10) \\ \text { C9 } & 17.9(13) & 17.3(12) & 13.9(13) & 2.5(9) & 3.3(10) & -0.1(9) \\ \text { C10 } & 12.2(13) & 11.1(11) & 13.7(12) & 2.9(9) & 0.6(9) & 0.3(9) \\ \text { C11 } & 15.0(13) & 12.1(12) & 18.4(14) & 4.3(10) & 4.8(10) & -2.3(10) \\ \text { C12 } & 13.4(12) & 10.9(11) & 16.6(13) & -0.9(9) & 1.7(10) & -2.3(9) \\ \text { C13 } & 8.8(12) & 13.1(11) & 16.7(14) & -1.6(10) & 2.9(10) & 0.7(9)\end{array}$




$\begin{array}{lllllll}\mathrm{C} 14 & 11.1(11) & 11.7(14) & 9.2(11) & -0.9(8) & 0.3(8) & -0.4(8) \\ \mathrm{C} 15 & 11.1(12) & 9.2(10) & 9.6(12) & 0.2(9) & 0.3(9) & 1.8(9) \\ \mathrm{C} 16 & 11.9(12) & 13.5(11) & 11.7(12) & -0.1(9) & -0.5(9) & -0.3(9)\end{array}$

Table S5 Bond Lengths for Yoon53.

\begin{tabular}{llllll}
\multicolumn{2}{c}{ Atom Atom Length/ } & \multicolumn{2}{l}{ Atom Atom Length/Å } \\
$\mathrm{I} 1$ & $\mathrm{C} 1$ & $2.095(3)$ & $\mathrm{C} 3$ & $\mathrm{C} 4$ & $1.396(4)$ \\
$\mathrm{O} 1$ & $\mathrm{C} 5$ & $1.227(3)$ & $\mathrm{C} 4$ & $\mathrm{C} 15$ & $1.389(4)$ \\
$\mathrm{O} 2$ & $\mathrm{C} 6$ & $1.403(3)$ & $\mathrm{C} 5$ & $\mathrm{C} 6$ & $1.533(4)$ \\
$\mathrm{O} 2$ & $\mathrm{C} 7$ & $1.458(3)$ & $\mathrm{C} 6$ & $\mathrm{C} 10$ & $1.560(4)$ \\
$\mathrm{O} 3$ & $\mathrm{C} 11$ & $1.202(4)$ & $\mathrm{C} 6$ & $\mathrm{C} 14$ & $1.560(4)$ \\
$\mathrm{O} 4$ & $\mathrm{C} 12$ & $1.223(3)$ & $\mathrm{C} 7$ & $\mathrm{C} 8$ & $1.516(4)$ \\
$\mathrm{N} 1$ & $\mathrm{C} 4$ & $1.406(3)$ & $\mathrm{C} 7$ & $\mathrm{C} 9$ & $1.518(4)$ \\
$\mathrm{N} 1$ & $\mathrm{C} 5$ & $1.350(4)$ & $\mathrm{C} 10$ & $\mathrm{C} 11$ & $1.514(4)$ \\
$\mathrm{N} 2$ & $\mathrm{C} 11$ & $1.395(4)$ & $\mathrm{C} 10$ & $\mathrm{C} 13$ & $1.548(4)$ \\
$\mathrm{N} 2$ & $\mathrm{C} 12$ & $1.376(3)$ & $\mathrm{C} 12$ & $\mathrm{C} 13$ & $1.518(4)$ \\
$\mathrm{C} 1$ & $\mathrm{C} 2$ & $1.399(4)$ & $\mathrm{C} 13$ & $\mathrm{C} 14$ & $1.557(4)$ \\
$\mathrm{C} 1$ & $\mathrm{C} 16$ & $1.383(4)$ & $\mathrm{C} 14$ & $\mathrm{C} 15$ & $1.504(3)$ \\
$\mathrm{C} 2$ & $\mathrm{C} 3$ & $1.391(4)$ & $\mathrm{C} 15$ & $\mathrm{C} 16$ & $1.398(4)$
\end{tabular}

Table S6 Bond Angles for Yoon53.

\begin{tabular}{llllllll}
\multicolumn{1}{c}{ Atom Atom Atom Angle ${ }^{\circ}$} & \multicolumn{3}{l}{ Atom Atom Atom Angle $/^{\circ}$} \\
$\mathrm{C} 6$ & $\mathrm{O} 2$ & $\mathrm{C} 7$ & $115.81(19)$ & $\mathrm{O} 2$ & $\mathrm{C} 7$ & $\mathrm{C} 9$ & $107.3(2)$ \\
$\mathrm{C} 5$ & $\mathrm{~N} 1$ & $\mathrm{C} 4$ & $125.8(2)$ & $\mathrm{C} 8$ & $\mathrm{C} 7$ & $\mathrm{C} 9$ & $111.8(2)$ \\
$\mathrm{C} 12$ & $\mathrm{~N} 2$ & $\mathrm{C} 11$ & $114.4(2)$ & $\mathrm{C} 11$ & $\mathrm{C} 10$ & $\mathrm{C} 6$ & $113.7(2)$ \\
$\mathrm{C} 2$ & $\mathrm{C} 1$ & $\mathrm{I} 1$ & $120.66(19)$ & $\mathrm{C} 11$ & $\mathrm{C} 10$ & $\mathrm{C} 13$ & $105.3(2)$ \\
$\mathrm{C} 16$ & $\mathrm{C} 1$ & $\mathrm{I} 1$ & $119.24(19)$ & $\mathrm{C} 13$ & $\mathrm{C} 10$ & $\mathrm{C} 6$ & $89.36(19)$ \\
$\mathrm{C} 16$ & $\mathrm{C} 1$ & $\mathrm{C} 2$ & $120.1(2)$ & $\mathrm{O} 3$ & $\mathrm{C} 11$ & $\mathrm{~N} 2$ & $124.5(3)$ \\
$\mathrm{C} 3$ & $\mathrm{C} 2$ & $\mathrm{C} 1$ & $119.4(2)$ & $\mathrm{O} 3$ & $\mathrm{C} 11$ & $\mathrm{C} 10$ & $128.1(3)$ \\
$\mathrm{C} 2$ & $\mathrm{C} 3$ & $\mathrm{C} 4$ & $120.4(2)$ & $\mathrm{N} 2$ & $\mathrm{C} 11$ & $\mathrm{C} 10$ & $107.3(2)$ \\
$\mathrm{C} 3$ & $\mathrm{C} 4$ & $\mathrm{~N} 1$ & $119.0(2)$ & $\mathrm{O} 4$ & $\mathrm{C} 12$ & $\mathrm{~N} 2$ & $125.1(3)$ \\
$\mathrm{C} 15$ & $\mathrm{C} 4$ & $\mathrm{~N} 1$ & $120.7(2)$ & $\mathrm{O} 4$ & $\mathrm{C} 12$ & $\mathrm{C} 13$ & $126.7(3)$ \\
$\mathrm{C} 15$ & $\mathrm{C} 4$ & $\mathrm{C} 3$ & $120.2(2)$ & $\mathrm{N} 2$ & $\mathrm{C} 12$ & $\mathrm{C} 13$ & $108.1(2)$ \\
$\mathrm{O} 1$ & $\mathrm{C} 5$ & $\mathrm{~N} 1$ & $121.7(2)$ & $\mathrm{C} 10$ & $\mathrm{C} 13$ & $\mathrm{C} 14$ & $90.7(2)$ \\
$\mathrm{O} 1$ & $\mathrm{C} 5$ & $\mathrm{C} 6$ & $120.5(3)$ & $\mathrm{C} 12$ & $\mathrm{C} 13$ & $\mathrm{C} 10$ & $104.4(2)$ \\
$\mathrm{N} 1$ & $\mathrm{C} 5$ & $\mathrm{C} 6$ & $117.8(2)$ & $\mathrm{C} 12$ & $\mathrm{C} 13$ & $\mathrm{C} 14$ & $115.0(2)$
\end{tabular}




$\begin{array}{llllllll}\mathrm{O} 2 & \mathrm{C} 6 & \mathrm{C} 5 & 110.8(2) & \mathrm{C} 13 & \mathrm{C} 14 & \mathrm{C} 6 & 89.07(18) \\ \mathrm{O} 2 & \mathrm{C} 6 & \mathrm{C} 10 & 107.8(2) & \mathrm{C} 15 & \mathrm{C} 14 & \mathrm{C} 6 & 115.2(2) \\ \mathrm{O} 2 & \mathrm{C} 6 & \mathrm{C} 14 & 115.0(2) & \mathrm{C} 15 & \mathrm{C} 14 & \mathrm{C} 13 & 113.0(2) \\ \mathrm{C} 5 & \mathrm{C} 6 & \mathrm{C} 10 & 113.6(2) & \mathrm{C} 4 & \mathrm{C} 15 & \mathrm{C} 14 & 121.3(2) \\ \mathrm{C} 5 & \mathrm{C} 6 & \mathrm{C} 14 & 117.6(2) & \mathrm{C} 4 & \mathrm{C} 15 & \mathrm{C} 16 & 119.2(2) \\ \mathrm{C} 14 & \mathrm{C} 6 & \mathrm{C} 10 & 90.09(19) & \mathrm{C} 16 & \mathrm{C} 15 & \mathrm{C} 14 & 119.4(2) \\ \mathrm{O} 2 & \mathrm{C} 7 & \mathrm{C} 8 & 108.3(2) & \mathrm{C} 1 & \mathrm{C} 16 & \mathrm{C} 15 & 120.7(2)\end{array}$

Table S7 Hydrogen Bonds for Yoon53.

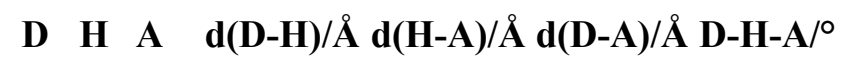

$\begin{array}{lccc}\mathrm{N} 1 \mathrm{H} 1 \mathrm{O}^{1}{ }^{1} 0.88 & 2.21 & 3.027(3) & 154.9 \\ \mathrm{~N} 2 \mathrm{H} 2 \mathrm{O}^{2}{ }^{0} 0.88 & 2.13 & 2.955(3) & 156.1 \\ { }^{1}-\mathrm{X},-1 / 2+\mathrm{Y},-\mathrm{Z} ;{ }^{2} 1-\mathrm{X}, 1 / 2+\mathrm{Y}, 1-\mathrm{Z} & & \end{array}$

Table S8 Torsion Angles for Yoon53.
$\begin{array}{llllllllll}\text { A } & \text { B } & \text { C } & \text { D } & \text { Angle }^{\circ} & \text { A } & \text { B } & \text { C } & \text { D } & \text { Angle }\end{array}{ }^{\circ}$

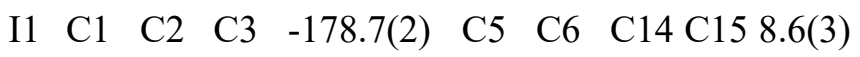

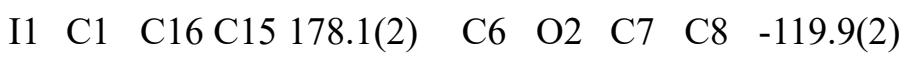

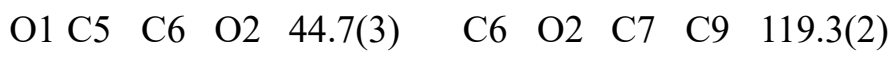
O1 C5 $\quad$ C6 $\quad \mathrm{C} 10-76.9(3) \quad \mathrm{C} 6 \quad \mathrm{C} 10 \mathrm{C} 11 \mathrm{O} 3 \quad-86.0(4)$
$\begin{array}{llllllllll}\text { O1 C5 } & \text { C6 } & \text { C14 } & 179.8(2) & \text { C6 } & \text { C10 } & \text { C11 N2 } & \text { 93.7(3) }\end{array}$
O2 C6 C10 C11 3.0(3) C6 C10 C13 C12 -109.2(2)
O2 C6 C10 C13 109.5(2) C6 C10 C13 C14 6.8(2)
O2 C6 C14 C13 -103.0(2) C6 C14 C15 C4 -8.4(3)
O2 C6 C14 C15 141.8(2) C6 C14 C15 C16 169.9(2)
O4 C12 C13 C10 172.9(3) C7 $\quad$ O2 $\quad$ C6 $\quad$ C5 $\quad 67.3(3)$
O4 C12 C13 C14 75.2(4) C7 $\quad$ O2 $\quad$ C6 $\quad$ C10 $-167.8(2)$

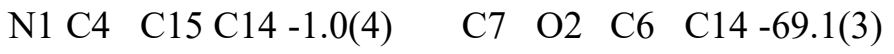
N1 C4 C15 C16 -179.2(2) C10 C6 C14 C13 6.8(2)
N1 C5 $\quad$ C6 $\quad$ O $2 \quad-134.7(2) \quad$ C10 C6 $\quad$ C14 C15 $-108.3(2)$
N1 C5 C6 C10 103.7(3) C10 C13 C14 C6 -6.9(2)
N1 C5 C6 C14 0.3(3) C10 C13 C14 C15 110.3(2)
N2 $\mathrm{C} 12 \mathrm{C} 13 \mathrm{C} 10-6.3(3) \quad \mathrm{C} 11 \mathrm{~N} 2 \quad \mathrm{C} 12 \mathrm{O} 4 \quad-174.0(3)$
N2 C12 C13 C14 -104.0(3) C11 N2 C12 C13 5.2(3)
$\mathrm{C} 1 \mathrm{C} 2 \quad \mathrm{C} 3 \quad \mathrm{C} 4 \quad 0.6(4) \quad \mathrm{C} 11 \mathrm{C} 10 \mathrm{C} 13 \mathrm{C} 12$ 5.2(3)
C2 C1 C16 C15 -0.6(4) C11 C10 C13 C14 121.3(2) 


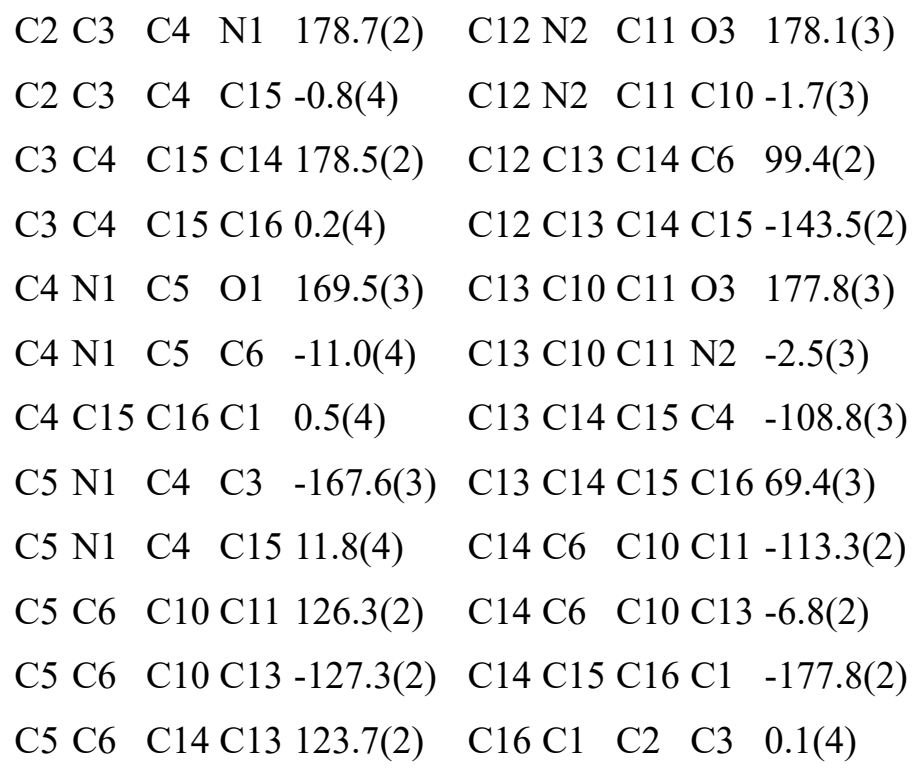

Table S9 Hydrogen Atom Coordinates $\left(\AA \times 10^{4}\right)$ and Isotropic Displacement Parameters $\left(\AA^{2} \times 10^{3}\right)$ for Yoon53.

$\begin{array}{lllll}\text { Atom } & \boldsymbol{x} & \boldsymbol{y} & \boldsymbol{z} & \mathbf{U}(\mathbf{e q )}) \\ \text { H1 } & 878.8 & 3134.79 & 306.84 & 15 \\ \text { H2 } & 3619.88 & 10166.27 & 4413.39 & 17 \\ \text { H2A } & 5127.25 & -60.37 & 96.54 & 16 \\ \text { H3 } & 2745.4 & 1192.26 & -276.93 & 15 \\ \text { H7 } & 581.88 & 3669.59 & 3399.86 & 15 \\ \text { H8A } & -1689.49 & 6175.25 & 3920.95 & 27 \\ \text { H8B } & -1870.67 & 3981.93 & 3834.88 & 27 \\ \text { H8C } & -1687.23 & 5165.16 & 2709.95 & 27 \\ \text { H9A } & 1885.85 & 4616.93 & 5137.67 & 24 \\ \text { H9B } & 307.94 & 3868.03 & 5409.2 & 24 \\ \text { H9C } & 603.82 & 6041.14 & 5357.42 & 24 \\ \text { H10 } & 2771.34 & 8074.62 & 1333.87 & 15 \\ \text { H13 } & 5019.79 & 6784.74 & 2258.93 & 15 \\ \text { H14 } & 3367.88 & 4552.66 & 3649.49 & 13 \\ \text { H16 } & 5868.02 & 3122.57 & 2942.57 & 15\end{array}$


<smiles>COC(=O)C1(OC(C)=O)[C@H](C(C)=O)[C@H]2c3ccccc3NC(=O)[C@@]21C</smiles>

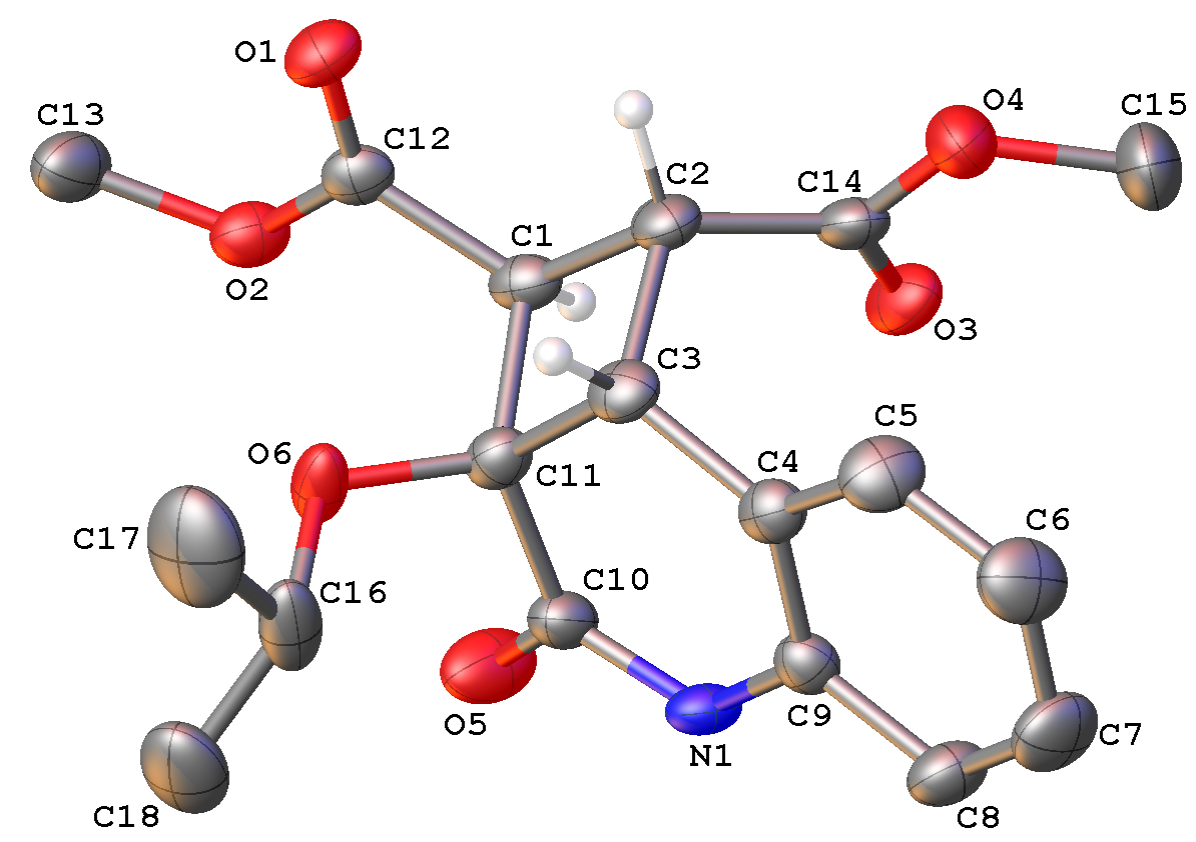

\section{Crystallographic ExperimentalSection}

\section{Data Collection}

A colorless crystal with approximate dimensions $0.161 \times 0.104 \times 0.041 \mathrm{~mm}^{3}$ was selected under oil under ambient conditions and attached to the tip of a MiTeGenMicroMount ${ }^{\mathcal{O}}$. The crystal was mounted in a stream of cold nitrogen at $100(1) \mathrm{K}$ and centered in the X-ray beam by using a video camera.

The crystal evaluation and data collection were performed on a Bruker QuazarSMART APEXII diffractometer with $\mathrm{Mo} \mathrm{K}_{\alpha}(\lambda=0.71073 \AA)$ radiation and the diffractometer to crystal distance of 4.96 $\mathrm{cm}$.

The initial cell constants were obtained from three series of $\omega$ scans at different starting angles. Each series consisted of 12 frames collected at intervals of $0.5^{\circ}$ in a $6^{\circ}$ range about $\omega$ with the exposure time of 10 seconds per frame. The reflections were successfully indexed by an automated indexing routine built in the APEX3 program suite. The final cell constants were calculated from a set of 9920strong reflections from the actual data collection.

The data were collected by using the full sphere data collection routine to survey the reciprocal space to the extent of a full sphere to a resolution of $0.70 \AA$. A total of 55373data were harvested by collecting 3 sets of frames with $0.6^{\circ}$ scans in $\omega$ with exposure times of $80 \mathrm{sec}$ per frame. These highly 
redundant datasets were corrected for Lorentz and polarization effects. The absorption correction was based on fitting a function to the empirical transmission surface as sampled by multiple equivalent measurements.

\section{Structure Solution and Refinement}

The systematic absences in the diffraction data were uniquely consistent for the space group $P 2{ }_{1}{ }_{2}{ }_{1}{ }_{1}$ that yielded chemically reasonable and computationally stable results of refinement.

A successful solution by the direct methods provided most non-hydrogen atoms from the $E$-map. The remaining non-hydrogen atoms were located in an alternating series of least-squares cycles and difference Fourier maps. All non-hydrogen atoms were refined with anisotropic displacement coefficients unless specified otherwise. All hydrogen atoms were included in the structure factor calculation at idealized positions and were allowed to ride on the neighboring atoms with relative isotropic displacement coefficients, except for $\mathrm{H}$ atoms attached to atoms N1, N1a, N1b and C16a. The $\mathrm{H}$ atoms on the latter four were refined without geometrical constraints.

There are three symmetry-independent molecules of interest in the asymmetric unit. The three molecules have the same absolute configuration. The absolute configuration could not be established experimentally and was assigned based on the known configuration of the stereogenic center $\mathrm{C} 11$ (same as $\mathrm{C} 11 \mathrm{a}$ and $\mathrm{C} 11 \mathrm{~b}$ ) as follows: $\mathrm{C} 1-S, \mathrm{C} 2-R, \mathrm{C} 3-R, \mathrm{C} 11-R$. The three molecules are label similarly.

Each molecule exhibits positional disorder. The following groups are disordered over two positions each and only the occupancy of the major component is provided. In molecule N1 the iPrO group is disordered with the major component contribution of 88(4) \%. In molecule N1a two moieties are disordered: the iPrO group (major component at 50.3(8) \%); and the $\mathrm{CO}_{2} \mathrm{Me}$ group at $\mathrm{C} 14$ (55.5(9) \%) with the Me group residing the majority of the time on atom $\mathrm{O} 4 \mathrm{a}$ and the rest of the time on atom $\mathrm{O} 3 \mathrm{a}$. In the $\mathrm{N} 1 \mathrm{~b}$ molecule three moieties are disordered: the $\mathrm{CO}_{2} \mathrm{Me}$ groups at $\mathrm{C} 1 \mathrm{~b}(56.8(13) \%)$ and $\mathrm{C} 1 \mathrm{~b}$ (85.6(5) and the $\mathrm{iPrO}$ group at $\mathrm{C} 11 \mathrm{~b}(87.5(10) \%)$. All disordered moieties were refined with restraints and constraints. Atoms with occupancies less than $15 \%$ were refined isotropically.

The final least-squares refinement of 793 parameters against 10690 data resulted in residuals $R$ (based on $F^{2}$ for $I 2 \sigma$ ) and $w R$ (based on $F^{2}$ for all data) of 0.0467 and 0.1161 , respectively. The final difference Fourier map was featureless.

\section{Summary}

Crystal Data for $\mathrm{C}_{18} \mathrm{H}_{21} \mathrm{NO}_{6}(M=347.36 \mathrm{~g} / \mathrm{mol})$ : orthorhombic, space group $\mathrm{P} 22_{1} 2_{1} 2_{1}$ (no. 19), $a=$ 8.605(4) $\AA, b=19.697(7) \AA, c=30.793(12) \AA, V=5219(4) \AA^{3}, Z=12, T=99.97 \mathrm{~K}, \mu(\mathrm{MoK} \alpha)=$ $0.100 \mathrm{~mm}^{-1}$, Dcalc $=1.326 \mathrm{~g} / \mathrm{cm}^{3}, 55373$ reflections measured $\left(2.454^{\circ} \leq 2 \Theta \leq 52.792^{\circ}\right), 10690$ unique $\left(R_{\text {int }}=0.0459, \mathrm{R}_{\text {sigma }}=0.0359\right)$ which were used in all calculations. The final $R_{1}$ was $0.0467(\mathrm{I}>2 \sigma(\mathrm{I}))$ and $w R_{2}$ was 0.1161 (all data).

Table S10 Crystal data and structure refinement for yoon56.

$\begin{array}{ll}\text { Identification code } & \text { yoon56 } \\ \text { Empirical formula } & \mathrm{C}_{18} \mathrm{H}_{21} \mathrm{NO}_{6} \\ \text { Formula weight } & 347.36\end{array}$


Temperature/K

Crystal system

Space group

$\mathrm{a} / \AA ̊$

$\mathrm{b} / \AA ̊$

$\mathrm{c} / \AA$

$\alpha /{ }^{\circ}$

$\beta /{ }^{\circ}$

$\gamma /{ }^{\circ}$

Volume $/ \AA^{3}$

Z

$\rho_{\text {calc } g / \mathrm{cm}^{3}}$

$\mu / \mathrm{mm}^{-1}$

$\mathrm{F}(000)$

Crystal size $/ \mathrm{mm}^{3}$

Radiation
99.97

orthorhombic

$\mathrm{P} 2{ }_{1} 2_{1} 2_{1}$

8.605(4)

19.697(7)

30.793(12)

90

90

90

5219(4)

12

1.326

0.100

2208.0

$0.161 \times 0.104 \times 0.041$

$\operatorname{MoK} \alpha(\lambda=0.71073)$

$2 \Theta$ range for data collection $/{ }^{\circ} 2.454$ to 52.792

Index ranges $-10 \leq \mathrm{h} \leq 10,-24 \leq \mathrm{k} \leq 24,-36 \leq 1 \leq 38$

Reflections collected 55373

Independent reflections $10690\left[\mathrm{R}_{\text {int }}=0.0459, \mathrm{R}_{\text {sigma }}=0.0359\right]$

Data/restraints/parameters

$10690 / 221 / 793$

Goodness-of-fit on $\mathrm{F}^{2}$

1.024

Final $R$ indexes $[\mathrm{I}>=2 \sigma(\mathrm{I})] \quad \mathrm{R}_{1}=0.0467, \mathrm{wR}_{2}=0.1095$

Final $\mathrm{R}$ indexes [all data] $\quad \mathrm{R}_{1}=0.0588, \mathrm{wR}_{2}=0.1161$

Largest diff. peak/hole / e $\AA^{-3} 0.51 /-0.53$

Flack parameter $-0.1(3)$

Table S11 Fractional Atomic Coordinates $\left(\times 10^{4}\right)$ and Equivalent Isotropic Displacement Parameters $\left(\AA^{2} \times 10^{3}\right)$ for yoon 56 . $U_{\text {eq }}$ is defined as $1 / 3$ of of the trace of the orthogonalised $U_{\mathrm{IJ}}$ tensor.

\begin{tabular}{lrrrr} 
Atom & \multicolumn{1}{c}{$\boldsymbol{x}$} & \multicolumn{2}{c}{$\boldsymbol{U}(\mathbf{z q})$} \\
O1 & $11481(3)$ & $7039.2(12)$ & $7931.1(8)$ & $32.1(6)$ \\
O2 & $13118(3)$ & $6156.8(12)$ & $7858.6(8)$ & $32.3(6)$ \\
O3 & $10378(3)$ & $5768.9(13)$ & $9264.6(8)$ & $38.7(6)$ \\
O4 & $8581(4)$ & $6592.8(13)$ & $9275.2(9)$ & $42.6(7)$ \\
O5 & $11540(3)$ & $4484.8(14)$ & $8213.1(10)$ & $47.5(7)$ \\
C11 & $10018(4)$ & $5506.1(17)$ & $8142.1(10)$ & $27.0(7)$ \\
O6 & $10162(8)$ & $5527(4)$ & $7687.8(9)$ & $33.4(17)$
\end{tabular}




\begin{tabular}{|c|c|c|c|c|}
\hline $\mathrm{C} 16$ & $8968(9)$ & $5154(6)$ & $7459.3(17)$ & $42(2)$ \\
\hline $\mathrm{C} 17$ & $8173(13)$ & $5645(9)$ & $7158(2)$ & $68(3)$ \\
\hline $\mathrm{C} 18$ & $9711(13)$ & $4573(7)$ & $7224(3)$ & $56(3)$ \\
\hline $\mathrm{O} 7$ & $9900(50)$ & $5640(20)$ & 7694(5) & $34(8)$ \\
\hline C19 & $8710(50)$ & $5300(20)$ & 7446(9) & $34(8)$ \\
\hline $\mathrm{C} 20$ & $7910(70)$ & $5830(20)$ & $7173(16)$ & $34(8)$ \\
\hline $\mathrm{C} 21$ & $9370(60)$ & $4770(20)$ & $7161(14)$ & $34(8)$ \\
\hline N1 & 9197(3) & $4469.0(14)$ & $8529.0(10)$ & $27.5(6)$ \\
\hline $\mathrm{C} 1$ & 11121(4) & $6026.8(17)$ & $8362.8(11)$ & $26.8(7)$ \\
\hline $\mathrm{C} 2$ & $9730(4)$ & $6345.3(17)$ & $8604.8(11)$ & $28.9(8)$ \\
\hline $\mathrm{C} 3$ & $8588(4)$ & $5869.9(17)$ & $8341.9(11)$ & $28.6(8)$ \\
\hline $\mathrm{C} 4$ & $7449(4)$ & $5437.0(17)$ & $8585.0(11)$ & $27.4(7)$ \\
\hline $\mathrm{C} 5$ & $6043(4)$ & $5692(2)$ & $8738.5(13)$ & $37.0(9)$ \\
\hline $\mathrm{C} 6$ & $5022(5)$ & $5302(2)$ & $8974.7(15)$ & $44.6(10)$ \\
\hline $\mathrm{C} 7$ & $5390(5)$ & $4631(2)$ & $9057.2(15)$ & $45.1(10)$ \\
\hline $\mathrm{C} 8$ & $6774(4)$ & $4359.0(19)$ & $8906.1(13)$ & $35.2(8)$ \\
\hline C9 & $7801(4)$ & $4761.2(17)$ & $8671.4(11)$ & $25.8(7)$ \\
\hline $\mathrm{C} 10$ & $10327(4)$ & $4775.5(17)$ & $8298.3(12)$ & $29.4(8)$ \\
\hline $\mathrm{C} 12$ & $11896(4)$ & $6475.6(17)$ & $8032.4(11)$ & $25.9(7)$ \\
\hline $\mathrm{C} 13$ & $13754(5)$ & $6476(2)$ & $7474.8(14)$ & $41.8(10)$ \\
\hline $\mathrm{C} 14$ & $9653(5)$ & $6207.2(17)$ & $9078.9(12)$ & $31.7(8)$ \\
\hline $\mathrm{C} 15$ & $8219(7)$ & $6399(2)$ & $9717.9(13)$ & $54.8(12)$ \\
\hline O1A & $7202(3)$ & 1953.3(13) & $5968.2(8)$ & $36.7(6)$ \\
\hline $\mathrm{O} 2 \mathrm{~A}$ & $5966(3)$ & $2226.2(12)$ & $5352.9(7)$ & $30.3(6)$ \\
\hline $\mathrm{O} 3 \mathrm{~A}$ & $3208(4)$ & $2362.0(15)$ & 7086.2(9) & $51.1(8)$ \\
\hline $\mathrm{O} 4 \mathrm{~A}$ & $2357(3)$ & 2974.3(13) & $6520.4(9)$ & $38.6(6)$ \\
\hline $\mathrm{O} 5 \mathrm{~A}$ & $3857(3)$ & $4088.0(12)$ & $5710.5(9)$ & $35.8(6)$ \\
\hline O6A & 6932(3) & $3539.2(13)$ & $5834.5(8)$ & $37.1(5)$ \\
\hline N1A & $4046(4)$ & $4299.4(14)$ & $6427.7(10)$ & $29.6(7)$ \\
\hline C1A & $4966(4)$ & $2678.9(15)$ & $6002.6(10)$ & $22.4(7)$ \\
\hline $\mathrm{C} 2 \mathrm{~A}$ & 4913(4) & $2528.7(16)$ & $6496.5(10)$ & $23.3(7)$ \\
\hline $\mathrm{C} 3 \mathrm{~A}$ & $5996(4)$ & $3162.5(15)$ & $6575.7(11)$ & $21.5(7)$ \\
\hline C4A & $5595(4)$ & $3641.7(16)$ & $6936.5(11)$ & $22.0(7)$ \\
\hline C5A & $6118(5)$ & $3541.0(18)$ & $7359.2(12)$ & $32.0(8)$ \\
\hline C6A & $5628(5)$ & $3965.8(19)$ & $7693.3(12)$ & $38.2(9)$ \\
\hline C7A & $4629(5)$ & $4498.0(19)$ & $7604.4(13)$ & $37.2(9)$ \\
\hline C8A & $4122(4)$ & $4613.5(18)$ & $7186.6(13)$ & $32.9(8)$ \\
\hline C9A & $4600(4)$ & $4182.5(16)$ & $6854.2(11)$ & $24.4(7)$ \\
\hline C10A & $4423(4)$ & $3954.4(15)$ & $6064.6(12)$ & $26.1(7)$ \\
\hline
\end{tabular}




\begin{tabular}{|c|c|c|c|c|}
\hline C11A & $5664(4)$ & $3394.8(15)$ & $6105.6(11)$ & $21.8(7)$ \\
\hline $\mathrm{C} 12 \mathrm{~A}$ & $6178(4)$ & $2243.3(16)$ & $5781.9(11)$ & $25.8(7)$ \\
\hline C13A & $7168(5)$ & $1857(2)$ & $5116.0(13)$ & $40.6(9)$ \\
\hline C14A & $3366(4)$ & $2610.4(15)$ & $6711.6(10)$ & $27.8(8)$ \\
\hline $\mathrm{C} 15 \mathrm{~A}$ & $1110(8)$ & $3245(4)$ & $6756(3)$ & $43(2)$ \\
\hline C19A & 2089(9) & $2615(5)$ & $7372(3)$ & $41(3)$ \\
\hline C16A & $8222(8)$ & $3885(4)$ & $6033(3)$ & $37.1(5)$ \\
\hline C17A & $9623(9)$ & $3743(5)$ & $5760(3)$ & $37.1(5)$ \\
\hline C18A & $7937(10)$ & $4605(4)$ & $6107(3)$ & $37.1(5)$ \\
\hline $\mathrm{C} 20 \mathrm{~A}$ & 7991(9) & $4067(4)$ & $5980(3)$ & $37.1(5)$ \\
\hline $\mathrm{C} 21 \mathrm{~A}$ & $9356(9)$ & $4008(5)$ & $5665(3)$ & $37.1(5)$ \\
\hline $\mathrm{C} 22 \mathrm{~A}$ & $7430(10)$ & $4768(4)$ & $5977(3)$ & $37.1(5)$ \\
\hline $\mathrm{C} 1 \mathrm{~B}$ & $428(4)$ & $6785.0(16)$ & $6092.6(12)$ & $27.5(7)$ \\
\hline O1B & $600(20)$ & $7965(4)$ & $6282(4)$ & $31(2)$ \\
\hline $\mathrm{O} 2 \mathrm{~B}$ & $-203(12)$ & $7239(4)$ & 6789.7(19) & $41.0(18)$ \\
\hline C12B & $320(30)$ & 7393(4) & $6398(3)$ & $25(3)$ \\
\hline C13B & $-400(13)$ & $7800(4)$ & $7095(2)$ & $55(3)$ \\
\hline O7B & $760(30)$ & $8003(5)$ & $6151(4)$ & $24(3)$ \\
\hline O8B & $534(12)$ & $7341(3)$ & $6743(3)$ & $23.9(18)$ \\
\hline C19B & $560(40)$ & $7463(5)$ & $6319(4)$ & 24(3) \\
\hline $\mathrm{C} 20 \mathrm{~B}$ & $830(11)$ & $7941(4)$ & $7006(2)$ & $27(3)$ \\
\hline O3B & $-683(4)$ & $5771.3(14)$ & $5472.2(12)$ & $41.9(10)$ \\
\hline O4B & $-1963(4)$ & $6613.9(14)$ & $5153.0(11)$ & $37.3(9)$ \\
\hline C14B & $-869(5)$ & $6366.8(19)$ & $5411.7(13)$ & $26.7(9)$ \\
\hline C15B & $-3009(8)$ & $6111(4)$ & 4971(3) & $50.1(16)$ \\
\hline O9B & $-795(19)$ & $6880(8)$ & $4861(4)$ & $31(5)$ \\
\hline O10B & $-1961(19)$ & $6352(8)$ & $5421(5)$ & $32(5)$ \\
\hline $\mathrm{C} 21 \mathrm{~B}$ & $-880(20)$ & $6726(12)$ & $5232(4)$ & $29(6)$ \\
\hline $\mathrm{C} 22 \mathrm{~B}$ & $-3100(40)$ & $6070(30)$ & $5125(10)$ & $42(13)$ \\
\hline O5B & 2088(3) & $5474.6(13)$ & $6336.9(9)$ & $38.8(6)$ \\
\hline O6B & $3198(4)$ & $6869(3)$ & $6255.9(11)$ & $28.8(10)$ \\
\hline C16B & $4787(4)$ & $6864(3)$ & $6109.2(17)$ & $40.5(13)$ \\
\hline C17B & $5664(5)$ & $6256(3)$ & $6267(2)$ & $58.0(19)$ \\
\hline C18B & $5490(6)$ & $7503(3)$ & $6285(3)$ & $79(3)$ \\
\hline C23B & $4510(30)$ & $7140(15)$ & $6217(9)$ & $53(8)$ \\
\hline C24B & $5570(30)$ & $6647(18)$ & $5993(11)$ & $53(8)$ \\
\hline $\mathrm{C} 25 \mathrm{~B}$ & $5280(40)$ & $7410(20)$ & $6613(11)$ & $53(8)$ \\
\hline O11B & $3060(30)$ & $6820(30)$ & $6310(8)$ & $53(8)$ \\
\hline N1B & $2742(3)$ & $5469.0(14)$ & $5630.9(10)$ & $28.0(6)$ \\
\hline
\end{tabular}




$\begin{array}{lrrrr}\text { C2B } & 115(4) & 6929.2(17) & 5608.6(12) & 31.7(8) \\ \text { C3B } & 1908(4) & 6855.8(16) & 5517.0(12) & 27.1(7) \\ \text { C4B } & 2441(4) & 6436.4(17) & 5144.7(12) & 26.4(7) \\ \text { C5B } & 2512(4) & 6695.5(18) & 4723.9(13) & 32.0(8) \\ \text { C6B } & 3001(4) & 6297.9(19) & 4379.1(13) & 34.7(8) \\ \text { C7B } & 3426(4) & 5627(2) & 4452.6(13) & 35.7(9) \\ \text { C8B } & 3350(4) & 5358.0(18) & 4866.6(12) & 31.5(8) \\ \text { C9B } & 2846(4) & 5758.7(16) & 5209.8(12) & 25.9(7) \\ \text { C10B } & 2286(4) & 5782.6(17) & 5993.9(12) & 28.6(8) \\ \text { C11B } & 2100(4) & 6561.2(16) & 5978.3(12) & 26.4(7)\end{array}$

Table S12 Anisotropic Displacement Parameters $\left(\AA^{2} \times 10^{3}\right)$ for yoon56. The Anisotropic displacement factor exponent takes the form: $-2 \pi^{2}\left[h^{2} a^{* 2} U_{11}+2 h k a * b * U_{12}+\ldots\right]$.

\begin{tabular}{|c|c|c|c|c|c|c|}
\hline Atom & $\mathrm{U}_{11}$ & $\mathbf{U}_{22}$ & $\mathbf{U}_{33}$ & $\mathbf{U}_{23}$ & $\mathbf{U}_{13}$ & $\mathbf{U}_{12}$ \\
\hline $\mathrm{O} 1$ & $34.2(14)$ & $25.3(12)$ & $36.8(14)$ & 7.6(11) & $-1.5(12)$ & $-0.9(10)$ \\
\hline $\mathrm{O} 2$ & $28.4(13)$ & $27.0(12)$ & $41.5(15)$ & $-0.4(11)$ & $0.3(11)$ & $-1.3(11)$ \\
\hline $\mathrm{O} 3$ & $54.4(17)$ & $31.4(14)$ & $30.2(14)$ & $8.5(11)$ & $-7.0(13)$ & $-7.4(13)$ \\
\hline $\mathrm{O} 4$ & $60.0(18)$ & $33.5(14)$ & $34.4(15)$ & $-3.3(12)$ & $10.1(14)$ & $-3.3(13)$ \\
\hline O5 & $37.8(16)$ & $30.8(14)$ & $74(2)$ & $-2.1(14)$ & $19.8(15)$ & $3.6(12)$ \\
\hline C11 & $27.9(17)$ & $26.1(17)$ & $27.1(17)$ & $2.3(14)$ & $-2.6(14)$ & $-5.2(15)$ \\
\hline O6 & $31(3)$ & $49(3)$ & $19.6(17)$ & $0.6(13)$ & $-0.6(12)$ & $-18(2)$ \\
\hline $\mathrm{C} 16$ & $31(3)$ & $69(5)$ & $27(2)$ & $-8(2)$ & $3.5(19)$ & $-24(3)$ \\
\hline $\mathrm{C} 17$ & $55(5)$ & $104(8)$ & $45(3)$ & $-6(4)$ & $-17(3)$ & $-9(5)$ \\
\hline $\mathrm{C} 18$ & $54(5)$ & $71(6)$ & $43(3)$ & $-19(4)$ & $-2(3)$ & $-24(4)$ \\
\hline N1 & $28.4(15)$ & $16.4(13)$ & $37.5(17)$ & $-1.0(12)$ & $0.1(13)$ & $-2.2(12)$ \\
\hline $\mathrm{C} 1$ & $31.0(18)$ & $20.5(16)$ & $28.9(18)$ & $2.1(14)$ & $-7.5(15)$ & $-2.4(14)$ \\
\hline $\mathrm{C} 2$ & $37.0(19)$ & $18.7(15)$ & $30.9(18)$ & $3.6(14)$ & $0.4(15)$ & $-1.9(15)$ \\
\hline $\mathrm{C} 3$ & $34.5(19)$ & $22.0(16)$ & $29.2(18)$ & $5.5(14)$ & $-4.8(15)$ & $2.1(14)$ \\
\hline $\mathrm{C} 4$ & $29.9(18)$ & $26.8(17)$ & $25.5(18)$ & $1.4(14)$ & $-5.0(14)$ & $-1.6(15)$ \\
\hline $\mathrm{C} 5$ & $31.0(19)$ & $32(2)$ & $48(2)$ & $3.6(17)$ & $-1.5(17)$ & 7.1(17) \\
\hline C6 & $30(2)$ & $46(2)$ & $58(3)$ & $-6(2)$ & 7.8(19) & $3.7(18)$ \\
\hline $\mathrm{C} 7$ & $34(2)$ & $43(2)$ & $58(3)$ & $2(2)$ & 11.3(19) & $-12.4(19)$ \\
\hline $\mathrm{C} 8$ & $34.6(19)$ & $26.8(18)$ & $44(2)$ & $3.8(16)$ & $2.3(17)$ & $-6.3(15)$ \\
\hline C9 & $25.3(17)$ & $26.5(17)$ & $25.5(17)$ & $-3.5(14)$ & $-1.9(14)$ & $-1.9(14)$ \\
\hline $\mathrm{C} 10$ & $32.5(19)$ & $22.9(17)$ & $32.7(19)$ & $-3.8(14)$ & $3.5(16)$ & $-3.6(15)$ \\
\hline $\mathrm{C} 12$ & $22.7(16)$ & $25.1(17)$ & $30.0(18)$ & $-0.4(14)$ & $-6.2(14)$ & $-3.9(14)$ \\
\hline $\mathrm{C} 13$ & $40(2)$ & $34(2)$ & $51(3)$ & $-6.9(18)$ & $15.9(19)$ & $-7.0(18)$ \\
\hline C14 & $42(2)$ & $19.6(16)$ & $33.3(19)$ & $-1.1(15)$ & $-1.6(17)$ & $-8.6(16)$ \\
\hline $\mathrm{C} 15$ & $80(3)$ & $52(3)$ & $32(2)$ & $-6(2)$ & $17(2)$ & $-10(3)$ \\
\hline
\end{tabular}




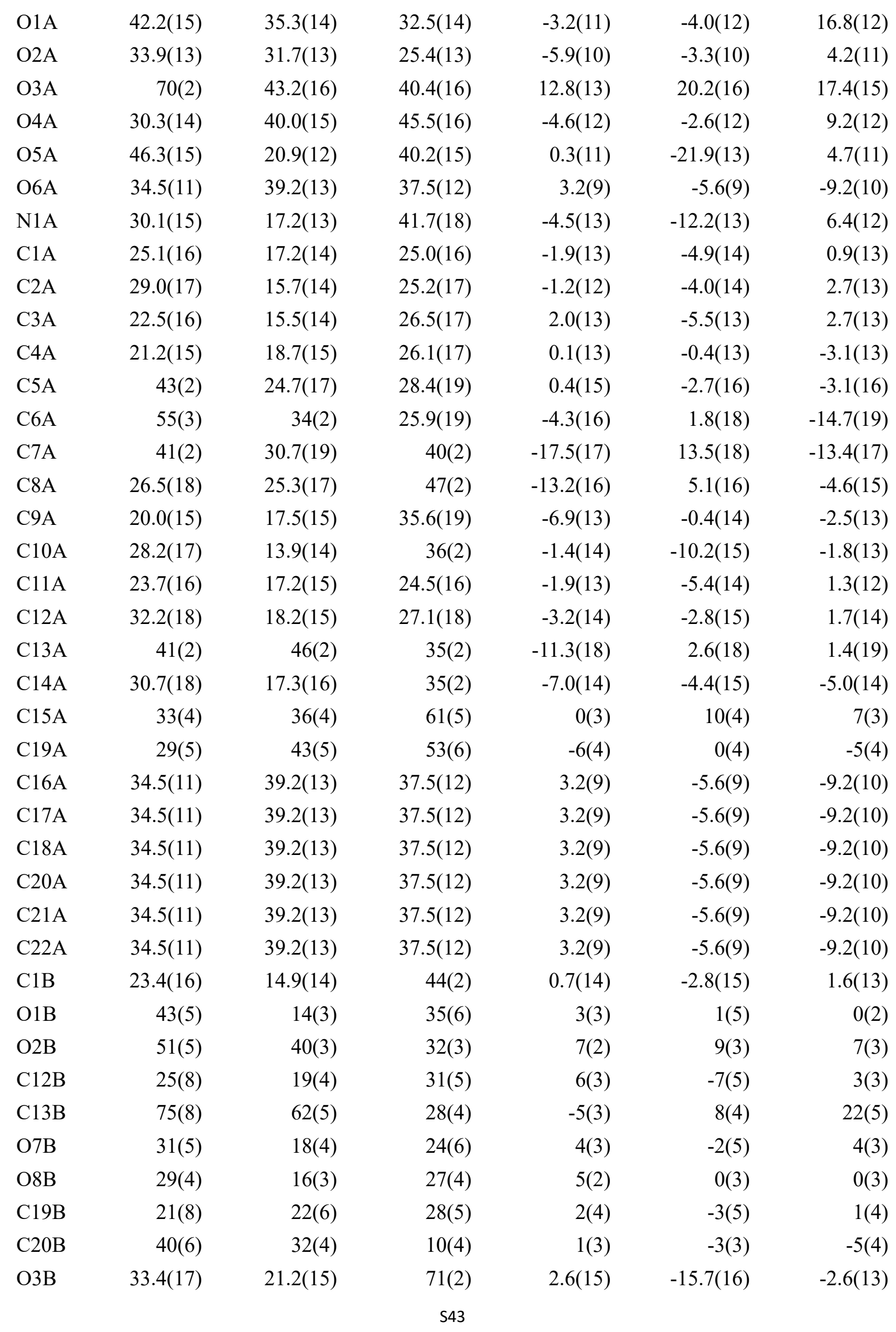




$\begin{array}{lrrrrrr}\text { O4B } & 36.8(18) & 25.2(15) & 50(2) & -6.5(14) & -19.3(15) & 0.9(13) \\ \text { C14B } & 23(2) & 20(2) & 37(2) & -2.6(17) & 0.2(17) & 2.6(16) \\ \text { C15B } & 45(3) & 45(4) & 60(5) & -8(4) & -24(3) & -8(3) \\ \text { O5B } & 40.9(15) & 28.8(13) & 46.5(16) & 17.0(12) & 13.2(13) & 15.4(12) \\ \text { O6B } & 17.2(15) & 29.8(18) & 39.4(19) & 9.5(15) & -7.6(12) & -4.0(12) \\ \text { C16B } & 20(2) & 53(3) & 48(3) & 3(2) & -5(2) & -1(2) \\ \text { C17B } & 25(2) & 58(4) & 90(5) & -7(3) & -2(3) & 6(2) \\ \text { C18B } & 31(3) & 46(3) & 159(8) & 29(4) & -27(4) & -15(2) \\ \text { N1B } & 31.1(15) & 14.7(13) & 38.1(17) & 6.2(12) & -0.6(13) & 3.6(12) \\ \text { C2B } & 25.6(17) & 23.6(17) & 46(2) & -4.1(16) & -8.8(17) & 7.8(14) \\ \text { C3B } & 25.7(16) & 15.3(15) & 40(2) & 7.5(14) & -5.3(15) & 0.1(13) \\ \text { C4B } & 20.7(16) & 19.5(16) & 39(2) & 5.5(14) & -0.9(14) & -6.1(13) \\ \text { C5B } & 29.3(18) & 22.1(17) & 45(2) & 11.0(16) & -3.6(16) & -6.2(14) \\ \text { C6B } & 32.3(19) & 36(2) & 36(2) & 7.4(16) & 0.1(17) & -10.9(16) \\ \text { C7B } & 29.4(19) & 37(2) & 41(2) & -2.3(17) & 0.5(17) & -3.5(16) \\ \text { C8B } & 26.5(18) & 26.0(18) & 42(2) & 0.2(16) & -4.3(16) & -2.3(15) \\ \text { C9B } & 19.2(15) & 19.9(15) & 39(2) & 3.5(14) & -3.1(14) & -2.2(13) \\ \text { C10B } & 22.0(16) & 21.2(16) & 43(2) & 9.3(15) & 3.3(15) & 3.9(14) \\ \text { C11B } & 20.3(16) & 21.5(16) & 37.3(19) & 5.1(14) & -4.1(15) & 1.7(13) \\ & & & & & \end{array}$

Table S13 Bond Lengths for yoon56.

\begin{tabular}{|c|c|c|c|c|}
\hline \multicolumn{2}{|c|}{ Atom Atom } & Length/Å & Atom Atom & Length/Å \\
\hline $\mathrm{O} 1$ & $\mathrm{C} 12$ & $1.207(4)$ & $\mathrm{C} 4 \mathrm{~A} \quad \mathrm{C} 5 \mathrm{~A}$ & $1.391(5)$ \\
\hline $\mathrm{O} 2$ & $\mathrm{C} 12$ & $1.336(4)$ & $\mathrm{C} 4 \mathrm{~A} \quad \mathrm{C} 9 \mathrm{~A}$ & $1.390(5)$ \\
\hline $\mathrm{O} 2$ & $\mathrm{C} 13$ & $1.446(5)$ & C5A C6A & $1.392(5)$ \\
\hline $\mathrm{O} 3$ & $\mathrm{C} 14$ & $1.209(4)$ & C6A C7A & $1.383(6)$ \\
\hline $\mathrm{O} 4$ & $\mathrm{C} 14$ & $1.339(5)$ & C7A C8A & $1.377(6)$ \\
\hline $\mathrm{O} 4$ & $\mathrm{C} 15$ & $1.449(5)$ & C8A C9A & $1.392(5)$ \\
\hline O5 & $\mathrm{C} 10$ & $1.219(5)$ & C10AC11A & $1.540(4)$ \\
\hline C11 & O6 & $1.405(4)$ & C16AC17A & $1.496(8)$ \\
\hline C11 & $\mathrm{O} 7$ & $1.409(11)$ & C16A C18A & $1.457(8)$ \\
\hline C11 & $\mathrm{C} 1$ & $1.554(5)$ & $\mathrm{C} 20 \mathrm{AC} 21 \mathrm{~A}$ & $1.529(8)$ \\
\hline C11 & $\mathrm{C} 3$ & $1.551(5)$ & $\mathrm{C} 20 \mathrm{AC} 22 \mathrm{~A}$ & $1.462(8)$ \\
\hline C11 & $\mathrm{C} 10$ & $1.540(5)$ & $\mathrm{C} 1 \mathrm{~B} \quad \mathrm{C} 12 \mathrm{~B}$ & $1.525(7)$ \\
\hline O6 & $\mathrm{C} 16$ & $1.446(5)$ & C1B C19B & $1.511(9)$ \\
\hline $\mathrm{C} 16$ & $\mathrm{C} 17$ & $1.506(7)$ & $\mathrm{C} 1 \mathrm{~B}$ C2B & $1.541(5)$ \\
\hline C16 & $\mathrm{C} 18$ & $1.499(6)$ & C1B C11B & $1.546(5)$ \\
\hline $\mathrm{O} 7$ & C19 & $1.439(11)$ & O1B C12B & $1.207(8)$ \\
\hline C19 & $\mathrm{C} 20$ & $1.508(12)$ & $\mathrm{O} 2 \mathrm{~B} \quad \mathrm{C} 12 \mathrm{~B}$ & $1.322(9)$ \\
\hline
\end{tabular}




\begin{tabular}{|c|c|c|c|c|}
\hline C19 & $\mathrm{C} 21$ & $1.485(12)$ & O2B C13B & $1.461(8)$ \\
\hline N1 & C9 & $1.402(5)$ & O7B C19B & $1.194(9)$ \\
\hline N1 & $\mathrm{C} 10$ & $1.348(5)$ & O8B C19B & $1.328(10)$ \\
\hline $\mathrm{C} 1$ & $\mathrm{C} 2$ & $1.543(5)$ & O8B C20B & $1.455(8)$ \\
\hline $\mathrm{C} 1$ & $\mathrm{C} 12$ & $1.504(5)$ & O3B C14B & $1.198(5)$ \\
\hline $\mathrm{C} 2$ & $\mathrm{C} 3$ & $1.580(5)$ & O4B C14B & $1.326(5)$ \\
\hline $\mathrm{C} 2$ & $\mathrm{C} 14$ & $1.486(5)$ & O4B C15B & $1.451(5)$ \\
\hline $\mathrm{C} 3$ & $\mathrm{C} 4$ & $1.499(5)$ & C14B C2B & $1.521(5)$ \\
\hline $\mathrm{C} 4$ & $\mathrm{C} 5$ & $1.393(5)$ & O9B C21B & $1.185(11)$ \\
\hline $\mathrm{C} 4$ & $\mathrm{C} 9$ & $1.391(5)$ & O10B C21B & $1.322(11)$ \\
\hline $\mathrm{C} 5$ & C6 & $1.375(6)$ & $\mathrm{O} 10 \mathrm{BC} 22 \mathrm{~B}$ & $1.452(12)$ \\
\hline C6 & $\mathrm{C} 7$ & $1.382(6)$ & $\mathrm{C} 21 \mathrm{~B} C 2 \mathrm{~B}$ & $1.496(10)$ \\
\hline $\mathrm{C} 7$ & $\mathrm{C} 8$ & $1.386(6)$ & O5B C10B & $1.230(4)$ \\
\hline $\mathrm{C} 8$ & $\mathrm{C} 9$ & $1.390(5)$ & O6B C16B & $1.439(5)$ \\
\hline $\mathrm{O} 1 \mathrm{~A}$ & $\mathrm{C} 12 \mathrm{~A}$ & $1.197(4)$ & O6B C11B & $1.411(4)$ \\
\hline $\mathrm{O} 2 \mathrm{~A}$ & $\mathrm{C} 12 \mathrm{~A}$ & $1.334(4)$ & C16B C17B & $1.498(6)$ \\
\hline $\mathrm{O} 2 \mathrm{~A}$ & $\mathrm{C} 13 \mathrm{~A}$ & $1.460(5)$ & C16B C18B & $1.497(7)$ \\
\hline O3A & $\mathrm{C} 14 \mathrm{~A}$ & $1.260(4)$ & C23B C24B & $1.498(12)$ \\
\hline $\mathrm{O} 3 \mathrm{~A}$ & C19A & $1.396(7)$ & C23B C25B & $1.488(12)$ \\
\hline $\mathrm{O} 4 \mathrm{~A}$ & $\mathrm{C} 14 \mathrm{~A}$ & $1.271(4)$ & C23B O11B & $1.432(11)$ \\
\hline $\mathrm{O} 4 \mathrm{~A}$ & $\mathrm{C} 15 \mathrm{~A}$ & $1.400(6)$ & O11B C11B & $1.407(11)$ \\
\hline O5A & $\mathrm{C} 10 \mathrm{~A}$ & $1.223(4)$ & N1B C9B & $1.419(5)$ \\
\hline O6A & $\mathrm{C} 11 \mathrm{~A}$ & $1.403(4)$ & N1B C10B & $1.336(5)$ \\
\hline O6A & $\mathrm{C} 16 \mathrm{~A}$ & $1.438(7)$ & $\mathrm{C} 2 \mathrm{~B} \quad \mathrm{C} 3 \mathrm{~B}$ & $1.575(5)$ \\
\hline O6A & $\mathrm{C} 20 \mathrm{~A}$ & $1.453(7)$ & $\mathrm{C} 3 \mathrm{~B} \quad \mathrm{C} 4 \mathrm{~B}$ & $1.485(5)$ \\
\hline N1A & C9A & $1.416(5)$ & C3B C11B & $1.544(5)$ \\
\hline N1A & $\mathrm{C} 10 \mathrm{~A}$ & $1.348(5)$ & C4B C5B & $1.394(5)$ \\
\hline $\mathrm{C} 1 \mathrm{~A}$ & $\mathrm{C} 2 \mathrm{~A}$ & $1.550(5)$ & C4B C9B & $1.394(5)$ \\
\hline $\mathrm{C} 1 \mathrm{~A}$ & $\mathrm{C} 11 \mathrm{~A}$ & $1.565(4)$ & C5B C6B & $1.385(6)$ \\
\hline $\mathrm{C} 1 \mathrm{~A}$ & $\mathrm{C} 12 \mathrm{~A}$ & $1.512(5)$ & C6B C7B & $1.391(5)$ \\
\hline $\mathrm{C} 2 \mathrm{~A}$ & $\mathrm{C} 3 \mathrm{~A}$ & $1.577(4)$ & $\mathrm{C} 7 \mathrm{~B} \quad \mathrm{C} 8 \mathrm{~B}$ & $1.382(5)$ \\
\hline $\mathrm{C} 2 \mathrm{~A}$ & $\mathrm{C} 14 \mathrm{~A}$ & $1.495(5)$ & C8B C9B & $1.389(5)$ \\
\hline $\mathrm{C} 3 \mathrm{~A}$ & $\mathrm{C} 4 \mathrm{~A}$ & $1.498(5)$ & C10B C11B & $1.543(4)$ \\
\hline $\mathrm{C} 3 \mathrm{~A}$ & $\mathrm{C} 11 \mathrm{~A}$ & $1.545(5)$ & & \\
\hline
\end{tabular}

Table S14 Bond Angles for yoon56.

\begin{tabular}{lllrlr}
\multicolumn{2}{c}{ Atom Atom Atom } & \multicolumn{1}{c}{ Angle $^{\circ}$} & Atom Atom Atom & \multicolumn{1}{c}{ Angle $/^{\circ}$} \\
C12 & O2 & C13 & $114.9(3)$ & O6A C11A C3A & $118.3(3)$ \\
C14 & O4 & C15 & $115.0(3)$ & O6A C11A C10A & $110.2(3)$
\end{tabular}




\begin{tabular}{|c|c|c|}
\hline O6 & C11 & $\mathrm{C} 1$ \\
\hline O6 & $\mathrm{C} 11$ & $\mathrm{C} 3$ \\
\hline O6 & $\mathrm{C} 11$ & $\mathrm{C} 10$ \\
\hline O7 & $\mathrm{C} 11$ & $\mathrm{C} 1$ \\
\hline O7 & $\mathrm{C} 11$ & $\mathrm{C} 3$ \\
\hline O7 & $\mathrm{C} 11$ & $\mathrm{C} 10$ \\
\hline C3 & $\mathrm{C} 11$ & $\mathrm{C} 1$ \\
\hline $\mathrm{C} 10$ & C11 & $\mathrm{C} 1$ \\
\hline C10 & C11 & $\mathrm{C} 3$ \\
\hline C11 & O6 & $\mathrm{C} 16$ \\
\hline O6 & C16 & $\mathrm{C} 17$ \\
\hline O6 & C16 & $\mathrm{C} 18$ \\
\hline C18 & $\mathrm{C} 16$ & $\mathrm{C} 17$ \\
\hline C11 & $\mathrm{O} 7$ & C19 \\
\hline O7 & C19 & $\mathrm{C} 20$ \\
\hline O7 & C19 & C21 \\
\hline $\mathrm{C} 21$ & C19 & C20 \\
\hline $\mathrm{C} 10$ & N1 & C9 \\
\hline $\mathrm{C} 2$ & $\mathrm{C} 1$ & $\mathrm{C} 11$ \\
\hline $\mathrm{C} 12$ & $\mathrm{C} 1$ & C11 \\
\hline $\mathrm{C} 12$ & $\mathrm{C} 1$ & $\mathrm{C} 2$ \\
\hline $\mathrm{C} 1$ & $\mathrm{C} 2$ & $\mathrm{C} 3$ \\
\hline C14 & $\mathrm{C} 2$ & $\mathrm{C} 1$ \\
\hline $\mathrm{C} 14$ & $\mathrm{C} 2$ & $\mathrm{C} 3$ \\
\hline C11 & $\mathrm{C} 3$ & $\mathrm{C} 2$ \\
\hline $\mathrm{C} 4$ & $\mathrm{C} 3$ & $\mathrm{C} 11$ \\
\hline $\mathrm{C} 4$ & $\mathrm{C} 3$ & $\mathrm{C} 2$ \\
\hline $\mathrm{C} 5$ & $\mathrm{C} 4$ & $\mathrm{C} 3$ \\
\hline C9 & $\mathrm{C} 4$ & $\mathrm{C} 3$ \\
\hline C9 & $\mathrm{C} 4$ & $\mathrm{C} 5$ \\
\hline C6 & $\mathrm{C} 5$ & $\mathrm{C} 4$ \\
\hline $\mathrm{C} 5$ & C6 & $\mathrm{C} 7$ \\
\hline C6 & C7 & C8 \\
\hline $\mathrm{C} 7$ & C8 & C9 \\
\hline $\mathrm{C} 4$ & C9 & N1 \\
\hline $\mathrm{C} 8$ & C9 & N1 \\
\hline $\mathrm{C} 8$ & C9 & $\mathrm{C} 4$ \\
\hline O5 & $\mathrm{C} 10$ & C11 \\
\hline O5 & $\mathrm{C} 10$ & N1 \\
\hline
\end{tabular}

\begin{tabular}{|c|c|c|}
\hline 111.3(3) & C3A C11AC1A & $89.7(2)$ \\
\hline $116.8(5)$ & C10A C11A C1A & $111.2(3)$ \\
\hline $108.8(5)$ & C10A C11A C3A & $114.7(3)$ \\
\hline $110.4(14)$ & O1A C12A O2A & $124.3(3)$ \\
\hline $104(2)$ & O1A C12AC1A & $124.3(3)$ \\
\hline $119(2)$ & $\mathrm{O} 2 \mathrm{~A} \quad \mathrm{C} 12 \mathrm{AC} 1 \mathrm{~A}$ & $111.4(3)$ \\
\hline $90.3(3)$ & O3A C14A O4A & $124.7(3)$ \\
\hline $112.0(3)$ & O3A C14AC2A & $117.4(3)$ \\
\hline $116.4(3)$ & $\mathrm{O} 4 \mathrm{~A} \quad \mathrm{C} 14 \mathrm{AC} 2 \mathrm{~A}$ & $117.6(3)$ \\
\hline $114.0(4)$ & O6A C16AC17A & $107.2(6)$ \\
\hline 107.3(4) & O6A C16AC18A & $113.5(6)$ \\
\hline $108.7(4)$ & C18A C16A C17A & $114.0(6)$ \\
\hline $112.7(4)$ & O6A C20AC21A & $103.3(5)$ \\
\hline $118.9(19)$ & O6A C20AC22A & $117.8(6)$ \\
\hline $107.6(16)$ & C22A C20A C21A & $108.8(6)$ \\
\hline $111.6(17)$ & $\mathrm{C} 12 \mathrm{~B}$ C1B $\mathrm{C} 2 \mathrm{~B}$ & $116.1(6)$ \\
\hline 109.7(16) & C12B C1B C11B & $115.0(9)$ \\
\hline $126.8(3)$ & C19B C1B C2B & $107.2(6)$ \\
\hline $90.3(3)$ & C19B C1B C11B & $106.6(12)$ \\
\hline 111.3(3) & C2B C1B C11B & $89.7(3)$ \\
\hline $115.6(3)$ & $\mathrm{C} 12 \mathrm{~B} \mathrm{O} 2 \mathrm{~B}$ C13B & $117.0(6)$ \\
\hline $89.6(3)$ & O1B C12B C1B & $122.5(8)$ \\
\hline $115.7(3)$ & O1B C12B O2B & $123.5(8)$ \\
\hline 111.5(3) & O2B C12B C1B & $113.8(6)$ \\
\hline $89.1(3)$ & C19B O8B C20B & $113.3(7)$ \\
\hline $117.0(3)$ & O7B C19B C1B & $126.8(10)$ \\
\hline 119.2(3) & O7B C19B O8B & $126.1(10)$ \\
\hline 122.1(3) & O8B C19B C1B & $106.9(8)$ \\
\hline $119.8(3)$ & C14B O4B C15B & $114.9(4)$ \\
\hline $118.0(3)$ & O3B C14B O4B & $123.2(4)$ \\
\hline $122.1(4)$ & O3B C14B C2B & $125.2(4)$ \\
\hline 119.1(4) & O4B C14B C2B & $111.6(3)$ \\
\hline $120.3(4)$ & C21B O10B C22B & $114.6(15)$ \\
\hline 120.1(3) & O9B C21B O10B & $127.6(13)$ \\
\hline 121.3(3) & O9B C21B C2B & $130.0(13)$ \\
\hline $118.3(3)$ & O10B C21B C2B & $102.2(10)$ \\
\hline $120.4(3)$ & C11B O6B C16B & $116.3(4)$ \\
\hline 121.3(3) & O6B C16B C17B & $112.4(4)$ \\
\hline 121.4(3) & O6B C16B C18B & $105.4(5)$ \\
\hline
\end{tabular}




\begin{tabular}{|c|c|c|c|c|}
\hline N1 & $\mathrm{C} 10 \quad \mathrm{C} 11$ & $117.3(3)$ & C18B C16B C17B & $110.5(4)$ \\
\hline $\mathrm{O} 1$ & $\mathrm{C} 12 \mathrm{O} 2$ & $124.1(3)$ & C25B C23B C24B & $110.0(15)$ \\
\hline $\mathrm{O} 1$ & $\mathrm{C} 12 \mathrm{C} 1$ & $125.8(3)$ & O11B C23B C24B & $109.7(16)$ \\
\hline $\mathrm{O} 2$ & $\mathrm{C} 12 \mathrm{C} 1$ & $110.1(3)$ & O11B C23B C25B & $112.6(16)$ \\
\hline $\mathrm{O} 3$ & $\mathrm{C} 14 \quad \mathrm{O} 4$ & $123.2(3)$ & C11B O11B C23B & $121.7(17)$ \\
\hline $\mathrm{O} 3$ & $\mathrm{C} 14 \quad \mathrm{C} 2$ & $124.9(3)$ & C10B N1B C9B & $126.6(3)$ \\
\hline $\mathrm{O} 4$ & $\mathrm{C} 14 \quad \mathrm{C} 2$ & $111.7(3)$ & $\mathrm{C} 1 \mathrm{~B} \quad \mathrm{C} 2 \mathrm{~B} \quad \mathrm{C} 3 \mathrm{~B}$ & $89.2(3)$ \\
\hline $\mathrm{C} 12 \mathrm{~A}$ & $\mathrm{AO} 2 \mathrm{~A} \mathrm{C} 13 \mathrm{~A}$ & $114.3(3)$ & C14B C2B C1B & $110.4(3)$ \\
\hline $\mathrm{C} 14 \mathrm{~A}$ & $\mathrm{AO} 3 \mathrm{~A}$ C19A & $120.9(5)$ & C14B C2B C3B & $114.0(3)$ \\
\hline $\mathrm{C} 14 \mathrm{~A}$ & A O4A C15A & $119.9(4)$ & $\mathrm{C} 21 \mathrm{~B} C 2 \mathrm{~B}$ C1B & $143.2(6)$ \\
\hline C11A & O O6A C16A & $116.3(4)$ & C21B C2B C3B & $113.4(9)$ \\
\hline C11A & O O6A C20A & $116.7(4)$ & $\mathrm{C} 4 \mathrm{~B} \quad \mathrm{C} 3 \mathrm{~B} \quad \mathrm{C} 2 \mathrm{~B}$ & $119.4(3)$ \\
\hline $\mathrm{C} 10 \mathrm{~A}$ & AN1A C9A & $127.3(3)$ & C4B C3B C11B & $117.9(3)$ \\
\hline $\mathrm{C} 2 \mathrm{~A}$ & C1A C11A & $89.1(2)$ & C11B C3B C2B & $88.5(3)$ \\
\hline $\mathrm{C} 12 \mathrm{~A}$ & $\mathrm{AC} 1 \mathrm{~A} \quad \mathrm{C} 2 \mathrm{~A}$ & $110.7(3)$ & $\mathrm{C} 5 \mathrm{~B} \quad \mathrm{C} 4 \mathrm{~B} \quad \mathrm{C} 3 \mathrm{~B}$ & $121.8(3)$ \\
\hline $\mathrm{C} 12 \mathrm{~A}$ & $\mathrm{AC} 1 \mathrm{~A} \quad \mathrm{C} 11 \mathrm{~A}$ & $109.8(3)$ & C5B C4B C9B & $118.2(3)$ \\
\hline $\mathrm{C} 1 \mathrm{~A}$ & $\mathrm{C} 2 \mathrm{~A} \quad \mathrm{C} 3 \mathrm{~A}$ & $89.0(2)$ & C9B C4B C3B & $119.9(3)$ \\
\hline $\mathrm{C} 14 \mathrm{~A}$ & $\mathrm{AC} 2 \mathrm{~A} \quad \mathrm{C} 1 \mathrm{~A}$ & $116.1(3)$ & C6B C5B C4B & $121.3(3)$ \\
\hline C14A & $\mathrm{AC} 2 \mathrm{~A} \quad \mathrm{C} 3 \mathrm{~A}$ & $111.9(3)$ & $\mathrm{C} 5 \mathrm{~B}$ C6B C7B & $119.5(4)$ \\
\hline $\mathrm{C} 4 \mathrm{~A}$ & $\mathrm{C} 3 \mathrm{~A} \quad \mathrm{C} 2 \mathrm{~A}$ & $118.5(3)$ & C8B C7B C6B & $120.1(4)$ \\
\hline $\mathrm{C} 4 \mathrm{~A}$ & $\mathrm{C} 3 \mathrm{~A} \quad \mathrm{C} 11 \mathrm{~A}$ & $117.7(3)$ & C7B C8B C9B & $120.0(3)$ \\
\hline C11A & $\mathrm{AC} 3 \mathrm{~A} \quad \mathrm{C} 2 \mathrm{~A}$ & $88.9(2)$ & C4B C9B N1B & $120.0(3)$ \\
\hline C5A & $\mathrm{C} 4 \mathrm{~A} \quad \mathrm{C} 3 \mathrm{~A}$ & $122.0(3)$ & C8B C9B N1B & $119.1(3)$ \\
\hline C9A & $\mathrm{C} 4 \mathrm{~A} \quad \mathrm{C} 3 \mathrm{~A}$ & $119.3(3)$ & C8B C9B C4B & $120.8(3)$ \\
\hline C9A & $\mathrm{C} 4 \mathrm{~A} \quad \mathrm{C} 5 \mathrm{~A}$ & $118.6(3)$ & O5B C10B N1B & $122.1(3)$ \\
\hline $\mathrm{C} 4 \mathrm{~A}$ & $\mathrm{C} 5 \mathrm{~A} \quad \mathrm{C} 6 \mathrm{~A}$ & $120.5(4)$ & O5B C10B C11B & $120.2(3)$ \\
\hline C7A & C6A C5A & $119.9(4)$ & N1B C10B C11B & $117.6(3)$ \\
\hline $\mathrm{C} 8 \mathrm{~A}$ & C7A C6A & $120.5(3)$ & O6B C11B C1B & $111.3(3)$ \\
\hline C7A & C8A C9A & $119.5(3)$ & O6B C11B C3B & $117.9(3)$ \\
\hline $\mathrm{C} 4 \mathrm{~A}$ & C9A N1A & $120.1(3)$ & O6B C11B C10B & $109.8(3)$ \\
\hline $\mathrm{C} 4 \mathrm{~A}$ & C9A C8A & $121.0(3)$ & O11B C11B C1B & $106.0(15)$ \\
\hline C8A & C9A N1A & $118.9(3)$ & O11B C11B C3B & $126.5(19)$ \\
\hline O5A & C10AN1A & $122.4(3)$ & O11B C11B C10B & $106(2)$ \\
\hline O5A & C10AC11A & $120.2(3)$ & C3B C11B C1B & $90.1(2)$ \\
\hline N1A & C10AC11A & $117.4(3)$ & C10B C11B C1B & $111.9(3)$ \\
\hline O6A & $\mathrm{C} 11 \mathrm{AC} 1 \mathrm{~A}$ & $111.1(3)$ & C10B C11B C3B & $114.4(3)$ \\
\hline
\end{tabular}

Table S15 Hydrogen Bonds for yoon56.

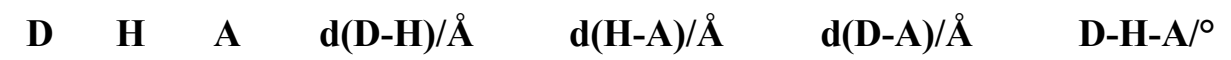




$\begin{array}{lllllrl}\mathrm{N} 1 & \mathrm{H} 1 & \mathrm{O}_{1}{ }^{1} & 0.87(2) & 2.17(3) & 3.024(9) & 170(4) \\ \mathrm{N} 1 & \mathrm{H} 1 & \mathrm{O}^{1} & 0.87(2) & 2.21(3) & 3.051(12) & 164(4) \\ \mathrm{N} 1 \mathrm{AH} 1 \mathrm{~A} & \mathrm{O} 5 \mathrm{~B} & 0.87(2) & 2.02(3) & 2.877(4) & 171(4) \\ \mathrm{N} 1 \mathrm{~B} & \mathrm{H} 1 \mathrm{BB} & 5 \mathrm{~A} & 0.87(2) & 2.04(3) & 2.895(4) & 168(4) \\ { }^{1} 1-\mathrm{X},-1 / 2+\mathrm{Y}, 3 / 2-\mathrm{Z} & & & & \end{array}$

Table S16 Torsion Angles for yoon56.

\begin{tabular}{|c|c|c|c|c|c|c|c|}
\hline $\mathbf{A}$ & B & $\mathbf{C}$ & D & Angle $/^{\circ}$ & $\mathbf{A}$ & $\mathbf{C}$ & Angle $/^{\circ}$ \\
\hline C11 & O6 & $\mathrm{C} 16$ & $\mathrm{C} 17$ & $122.8(5)$ & C11A C1A & $\mathrm{C} 2 \mathrm{~A} \quad \mathrm{C} 3 \mathrm{~A}$ & $-13.6(2)$ \\
\hline C11 & O6 & $\mathrm{C} 16$ & $\mathrm{C} 18$ & $-115.1(6)$ & C11A C1A & $\mathrm{C} 2 \mathrm{~A} \quad \mathrm{C} 14 \mathrm{~A}$ & $100.4(3)$ \\
\hline C11 & $\mathrm{O} 7$ & C19 & $\mathrm{C} 20$ & $132(4)$ & C11A C1A & $\mathrm{C} 12 \mathrm{AO} 1 \mathrm{~A}$ & $79.6(4)$ \\
\hline C11 & $\mathrm{O} 7$ & C19 & $\mathrm{C} 21$ & $-107(4)$ & C11A C1A & $\mathrm{C} 12 \mathrm{AO} 2 \mathrm{~A}$ & $-100.0(3)$ \\
\hline C11 & $\mathrm{C} 1$ & $\mathrm{C} 2$ & $\mathrm{C} 3$ & $-5.8(2)$ & C11A C3A & $\mathrm{C} 4 \mathrm{~A} \quad \mathrm{C} 5 \mathrm{~A}$ & $-167.2(3)$ \\
\hline C11 & $\mathrm{C} 1$ & $\mathrm{C} 2$ & $\mathrm{C} 14$ & $108.0(3)$ & C11A C3A & C4A C9A & $16.5(4)$ \\
\hline C11 & $\mathrm{C} 1$ & $\mathrm{C} 12$ & O1 & $97.7(4)$ & $\mathrm{C} 12 \mathrm{AC} 1 \mathrm{~A}$ & $\mathrm{C} 2 \mathrm{~A} \quad \mathrm{C} 3 \mathrm{~A}$ & $97.2(3)$ \\
\hline $\mathrm{C} 11$ & $\mathrm{C} 1$ & $\mathrm{C} 12$ & $\mathrm{O} 2$ & $-81.5(3)$ & $\mathrm{C} 12 \mathrm{AC} 1 \mathrm{~A}$ & $\mathrm{C} 2 \mathrm{~A} \quad \mathrm{C} 14 \mathrm{~A}$ & $-148.8(3)$ \\
\hline C11 & $\mathrm{C} 3$ & $\mathrm{C} 4$ & $\mathrm{C} 5$ & $-173.0(3)$ & $\mathrm{C} 12 \mathrm{AC} 1 \mathrm{~A}$ & C11A O6A & $22.6(4)$ \\
\hline C11 & $\mathrm{C} 3$ & $\mathrm{C} 4$ & C9 & $8.4(5)$ & $\mathrm{C} 12 \mathrm{AC} 1 \mathrm{~A}$ & C11AC3A & $-97.8(3)$ \\
\hline O6 & $\mathrm{C} 11$ & $\mathrm{C} 1$ & $\mathrm{C} 2$ & $125.1(5)$ & $\mathrm{C} 12 \mathrm{AC} 1 \mathrm{~A}$ & $\mathrm{C} 11 \mathrm{~A} \mathrm{C10A}$ & $145.8(3)$ \\
\hline O6 & $\mathrm{C} 11$ & $\mathrm{C} 1$ & $\mathrm{C} 12$ & $7.3(6)$ & $\mathrm{C} 13 \mathrm{~A} \mathrm{O} 2 \mathrm{~A}$ & $\mathrm{C} 12 \mathrm{AO} 1 \mathrm{~A}$ & $-4.2(5)$ \\
\hline O6 & $\mathrm{C} 11$ & $\mathrm{C} 3$ & $\mathrm{C} 2$ & $-119.9(4)$ & $\mathrm{C} 13 \mathrm{AO} 2 \mathrm{~A}$ & $\mathrm{C} 12 \mathrm{AC} 1 \mathrm{~A}$ & $175.5(3)$ \\
\hline O6 & $\mathrm{C} 11$ & $\mathrm{C} 3$ & $\mathrm{C} 4$ & $117.4(4)$ & $\mathrm{C} 14 \mathrm{~A} C 2 \mathrm{~A}$ & $\mathrm{C} 3 \mathrm{~A} \quad \mathrm{C} 4 \mathrm{~A}$ & $17.2(4)$ \\
\hline O6 & $\mathrm{C} 11$ & $\mathrm{C} 10$ & O5 & $56.7(5)$ & $\mathrm{C} 14 \mathrm{~A} C 2 \mathrm{~A}$ & $\mathrm{C} 3 \mathrm{~A} \quad \mathrm{C} 11 \mathrm{~A}$ & $-104.1(3)$ \\
\hline O6 & C11 & $\mathrm{C} 10$ & N1 & $-122.6(4)$ & $\mathrm{C} 15 \mathrm{~A} \mathrm{O} 4 \mathrm{~A}$ & C14A O3A & $13.7(6)$ \\
\hline $\mathrm{O} 7$ & $\mathrm{C} 11$ & $\mathrm{C} 1$ & $\mathrm{C} 2$ & 111(2) & $\mathrm{C} 15 \mathrm{~A} \mathrm{O} 4 \mathrm{~A}$ & $\mathrm{C} 14 \mathrm{AC} 2 \mathrm{~A}$ & $-159.9(4)$ \\
\hline $\mathrm{O} 7$ & C11 & $\mathrm{C} 1$ & $\mathrm{C} 12$ & $-6(2)$ & C19A O3A & $\mathrm{C} 14 \mathrm{~A} \mathrm{O} 4 \mathrm{~A}$ & $-17.7(7)$ \\
\hline $\mathrm{O} 7$ & $\mathrm{C} 11$ & $\mathrm{C} 3$ & $\mathrm{C} 2$ & $-117.0(15)$ & C19A O3A & $\mathrm{C} 14 \mathrm{AC} 2 \mathrm{~A}$ & $155.8(5)$ \\
\hline $\mathrm{O} 7$ & C11 & $\mathrm{C} 3$ & $\mathrm{C} 4$ & $120.4(15)$ & C16A O6A & $\mathrm{C} 11 \mathrm{AC} 1 \mathrm{~A}$ & $-141.0(4)$ \\
\hline $\mathrm{O} 7$ & $\mathrm{C} 11$ & $\mathrm{C} 10$ & $\mathrm{O} 5$ & $65(2)$ & C16A O6A & C11A C3A & $-39.4(5)$ \\
\hline $\mathrm{O} 7$ & $\mathrm{C} 11$ & $\mathrm{C} 10$ & N1 & $-115(2)$ & C16A O6A & $\mathrm{C} 11 \mathrm{~A} \mathrm{C} 10 \mathrm{~A}$ & $95.3(5)$ \\
\hline $\mathrm{C} 1$ & $\mathrm{C} 11$ & O6 & $\mathrm{C} 16$ & $-168.7(4)$ & C20A O6A & $\mathrm{C} 11 \mathrm{AC} 1 \mathrm{~A}$ & $-160.5(4)$ \\
\hline $\mathrm{C} 1$ & $\mathrm{C} 11$ & $\mathrm{O} 7$ & $\mathrm{C} 19$ & $-168(2)$ & C20A O6A & C11AC3A & $-58.9(5)$ \\
\hline $\mathrm{C} 1$ & C11 & $\mathrm{C} 3$ & $\mathrm{C} 2$ & $-5.8(2)$ & C20A O6A & $\mathrm{C} 11 \mathrm{~A} \mathrm{C10A}$ & $75.7(5)$ \\
\hline $\mathrm{C} 1$ & $\mathrm{C} 11$ & $\mathrm{C} 3$ & $\mathrm{C} 4$ & $-128.4(3)$ & $\mathrm{C} 1 \mathrm{~B} \quad \mathrm{C} 2 \mathrm{~B}$ & $\mathrm{C} 3 \mathrm{~B} \quad \mathrm{C} 4 \mathrm{~B}$ & $133.3(3)$ \\
\hline $\mathrm{C} 1$ & C11 & $\mathrm{C} 10$ & O5 & $-66.7(4)$ & $\mathrm{C} 1 \mathrm{~B} \quad \mathrm{C} 2 \mathrm{~B}$ & $\mathrm{C} 3 \mathrm{~B} \quad \mathrm{C} 11 \mathrm{~B}$ & $11.7(2)$ \\
\hline $\mathrm{C} 1$ & C11 & $\mathrm{C} 10$ & N1 & $114.0(3)$ & $\mathrm{C} 12 \mathrm{~B}$ C1B & $\mathrm{C} 2 \mathrm{~B} \quad \mathrm{C} 14 \mathrm{~B}$ & $-138.4(10)$ \\
\hline $\mathrm{C} 1$ & $\mathrm{C} 2$ & $\mathrm{C} 3$ & $\mathrm{C} 11$ & $5.8(2)$ & $\mathrm{C} 12 \mathrm{~B}$ C1B & $\mathrm{C} 2 \mathrm{~B} \quad \mathrm{C} 3 \mathrm{~B}$ & $106.2(9)$ \\
\hline $\mathrm{C} 1$ & $\mathrm{C} 2$ & $\mathrm{C} 3$ & $\mathrm{C} 4$ & $126.5(3)$ & $\mathrm{C} 12 \mathrm{~B}$ C1B & C11B O6B & $13.2(6)$ \\
\hline $\mathrm{C} 1$ & $\mathrm{C} 2$ & $\mathrm{C} 14$ & $\mathrm{O} 3$ & $-15.8(5)$ & $\mathrm{C} 12 \mathrm{~B}$ C1B & C11B C3B & $-107.0(5)$ \\
\hline
\end{tabular}




\begin{tabular}{|c|c|c|c|c|c|c|}
\hline $\mathrm{C} 1$ & $\mathrm{C} 2$ & $\mathrm{C} 14$ & $\mathrm{O} 4$ & $169.3(3)$ & C12B C1B C11B C10B & $136.5(5)$ \\
\hline $\mathrm{C} 2$ & $\mathrm{C} 1$ & $\mathrm{C} 12$ & $\mathrm{O} 1$ & $-3.5(5)$ & $\mathrm{C} 13 \mathrm{~B}$ O2B C12B C1B & $-177.6(11)$ \\
\hline $\mathrm{C} 2$ & $\mathrm{C} 1$ & $\mathrm{C} 12$ & $\mathrm{O} 2$ & $177.4(3)$ & C13B O2B C12B O1B & $-2(3)$ \\
\hline $\mathrm{C} 2$ & $\mathrm{C} 3$ & $\mathrm{C} 4$ & $\mathrm{C} 5$ & $81.7(4)$ & C19B C1B C2B C21B & $-134(2)$ \\
\hline $\mathrm{C} 2$ & $\mathrm{C} 3$ & $\mathrm{C} 4$ & $\mathrm{C} 9$ & $-96.9(4)$ & C19B C1B C2B C3B & $95.6(12)$ \\
\hline $\mathrm{C} 3$ & $\mathrm{C} 11$ & O6 & $\mathrm{C} 16$ & $-66.9(5)$ & C19B C1B C11B O11B & $32(2)$ \\
\hline $\mathrm{C} 3$ & $\mathrm{C} 11$ & O7 & C19 & $-72(3)$ & C19B C1B C11B C3B & $-95.9(6)$ \\
\hline C3 & $\mathrm{C} 11$ & $\mathrm{C} 1$ & $\mathrm{C} 2$ & $5.9(2)$ & C19B C1B C11B C10B & $147.5(6)$ \\
\hline $\mathrm{C} 3$ & $\mathrm{C} 11$ & $\mathrm{C} 1$ & $\mathrm{C} 12$ & $-111.8(3)$ & C20B O8B C19B C1B & $173.5(12)$ \\
\hline $\mathrm{C} 3$ & $\mathrm{C} 11$ & $\mathrm{C} 10$ & O5 & $-168.7(3)$ & C20B O8B C19B O7B & $-2(4)$ \\
\hline $\mathrm{C} 3$ & $\mathrm{C} 11$ & $\mathrm{C} 10$ & N1 & $12.0(4)$ & O3B C14B C2B C1B & $-43.5(5)$ \\
\hline $\mathrm{C} 3$ & $\mathrm{C} 2$ & $\mathrm{C} 14$ & $\mathrm{O} 3$ & $84.7(5)$ & O3B C14B C2B C3B & $55.0(6)$ \\
\hline $\mathrm{C} 3$ & $\mathrm{C} 2$ & $\mathrm{C} 14$ & $\mathrm{O} 4$ & $-90.2(3)$ & O4B C14B C2B C1B & $137.7(3)$ \\
\hline $\mathrm{C} 3$ & $\mathrm{C} 4$ & $\mathrm{C} 5$ & C6 & $-177.6(4)$ & O4B C14B C2B C3B & $-123.8(4)$ \\
\hline $\mathrm{C} 3$ & $\mathrm{C} 4$ & C9 & N1 & $-1.1(5)$ & C14B C2B C3B C4B & $21.3(5)$ \\
\hline $\mathrm{C} 3$ & $\mathrm{C} 4$ & C9 & $\mathrm{C} 8$ & $178.3(3)$ & C14B C2B C3B C11B & $-100.3(3)$ \\
\hline $\mathrm{C} 4$ & $\mathrm{C} 5$ & C6 & $\mathrm{C} 7$ & $-1.0(6)$ & C15B O4B $\mathrm{C} 14 \mathrm{~B}$ O3B & $4.4(7)$ \\
\hline $\mathrm{C} 5$ & $\mathrm{C} 4$ & C9 & N1 & $-179.8(3)$ & C15B O4B C14B C2B & $-176.7(5)$ \\
\hline C5 & $\mathrm{C} 4$ & C9 & $\mathrm{C} 8$ & $-0.3(5)$ & O9B C21B C2B C1B & $-171.9(17)$ \\
\hline $\mathrm{C} 5$ & C6 & $\mathrm{C} 7$ & $\mathrm{C} 8$ & $0.5(7)$ & O9B C21B C2B C3B & $-48(3)$ \\
\hline C6 & $\mathrm{C} 7$ & $\mathrm{C} 8$ & C9 & $0.2(6)$ & O10B C21B C2B C1B & $11(3)$ \\
\hline $\mathrm{C} 7$ & $\mathrm{C} 8$ & C9 & N1 & $179.2(4)$ & O10B C21B C2B $\mathrm{C} 3 \mathrm{~B}$ & $134.8(14)$ \\
\hline $\mathrm{C} 7$ & $\mathrm{C} 8$ & C9 & $\mathrm{C} 4$ & $-0.3(6)$ & $\mathrm{C} 21 \mathrm{~B} \mathrm{C} 2 \mathrm{~B} \quad \mathrm{C} 3 \mathrm{~B} \quad \mathrm{C} 4 \mathrm{~B}$ & $-16.7(10)$ \\
\hline C9 & N1 & $\mathrm{C} 10$ & O5 & $175.6(3)$ & $\mathrm{C} 21 \mathrm{~B}$ C2B C3B C11B & $-138.3(9)$ \\
\hline C9 & N1 & $\mathrm{C} 10$ & $\mathrm{C} 11$ & $-5.1(5)$ & C22B O10B C21B O9B & $4(5)$ \\
\hline C9 & $\mathrm{C} 4$ & $\mathrm{C} 5$ & C6 & $0.9(6)$ & C22B O10B C21B C2B & $-179(3)$ \\
\hline $\mathrm{C} 10$ & $\mathrm{C} 11$ & O6 & $\mathrm{C} 16$ & $67.5(5)$ & O5B C10B C11B C1B & $-62.4(4)$ \\
\hline $\mathrm{C} 10$ & $\mathrm{C} 11$ & O7 & C19 & $60(4)$ & O5B C10B C11B O6B & $61.7(4)$ \\
\hline $\mathrm{C} 10$ & $\mathrm{C} 11$ & $\mathrm{C} 1$ & $\mathrm{C} 2$ & $-112.9(3)$ & O5B C10B C11B O11B & $52.7(12)$ \\
\hline $\mathrm{C} 10$ & $\mathrm{C} 11$ & $\mathrm{C} 1$ & $\mathrm{C} 12$ & $129.4(3)$ & O5B C10B C11B C3B & $-163.1(3)$ \\
\hline $\mathrm{C} 10$ & $\mathrm{C} 11$ & $\mathrm{C} 3$ & $\mathrm{C} 2$ & 109.1(3) & C16B O6B C11B C1B & $-162.4(4)$ \\
\hline $\mathrm{C} 10$ & $\mathrm{C} 11$ & $\mathrm{C} 3$ & $\mathrm{C} 4$ & $-13.5(4)$ & C16B O6B C11B C3B & $-60.3(6)$ \\
\hline $\mathrm{C} 10$ & N1 & C9 & $\mathrm{C} 4$ & $-0.7(5)$ & C16B O6B C11B C10B & $73.1(5)$ \\
\hline $\mathrm{C} 10$ & N1 & C9 & $\mathrm{C} 8$ & $179.9(3)$ & C23B O11B C11B C1B & $-136(3)$ \\
\hline $\mathrm{C} 12$ & $\mathrm{C} 1$ & $\mathrm{C} 2$ & $\mathrm{C} 3$ & $108.1(3)$ & C23B O11B C11B C3B & $-34(5)$ \\
\hline $\mathrm{C} 12$ & $\mathrm{C} 1$ & $\mathrm{C} 2$ & $\mathrm{C} 14$ & $-138.0(3)$ & C23B O11B C11B C10B & $105(4)$ \\
\hline $\mathrm{C} 13$ & $\mathrm{O} 2$ & $\mathrm{C} 12$ & $\mathrm{O} 1$ & $-11.7(5)$ & C24B C23B O11B C11B & $-61(4)$ \\
\hline $\mathrm{C} 13$ & $\mathrm{O} 2$ & $\mathrm{C} 12$ & $\mathrm{C} 1$ & $167.5(3)$ & C25B C23B O11B C11B & $176(4)$ \\
\hline $\mathrm{C} 14$ & $\mathrm{C} 2$ & $\mathrm{C} 3$ & $\mathrm{C} 11$ & $-111.9(3)$ & N1B C10B C11B C1B & $120.9(3)$ \\
\hline
\end{tabular}


C14 $\mathrm{C} 2 \quad \mathrm{C} 3 \quad \mathrm{C} 4$

$\begin{array}{llll}\mathrm{C} 15 & \mathrm{O} 4 & \mathrm{C} 14 & \mathrm{O} 3\end{array}$

C15 O4 C14 C2

O5A C10AC11A O6A

O5A C10AC11AC1A

O5A C10AC11AC3A

N1A C10AC11A O6A

N1A C10AC11AC1A

N1A C10AC11AC3A

C1A C2A C3A C4A

C1A C2A C3A C11A

C1A C2A C14AO3A

C1A C2A C14AO4A

C2A C1A C11AO6A

C2A C1A C11AC3A

C2A C1A C11AC10A

C2A C1A C12AO1A

C2A C1A C12AO2A

C2A C3A C4A C5A

C2A C3A C4A C9A

C2A C3A C11AO6A

C2A C3A C11AC1A

C2A C3A C11AC10A

C3A C2A C14AO3A

C3A C2A C14AO4A

C3A C4A C5A C6A

C3A C4A C9A N1A

C3A C4A C9A C8A

C4A C3A C11A O6A

C4A C3A C11AC1A

C4A C3A C11AC10A

C4A C5A C6A C7A

C5A C4A C9A N1A

C5A C4A C9A C8A

C5A C6A C7A C8A

C6A C7A C8A C9A

C7A C8A C9A N1A

C7A C8A C9A C4A

C9A N1A C10AO5A

$$
\text { 8.9(4) N1B C10B C11B O6B }
$$

$-6.5(5)$ N1B C10B C11B O11B

168.5(3) N1B C10B C11B C3B

57.3(4) $\mathrm{C} 2 \mathrm{~B}$ C1B C12B O1B

$-66.5(4)$ C2B C1B C12B O2B

-166.3(3) C2B C1B C19B O7B

$-120.2(3)$ C2B C1B C19B O8B

116.1(3) C2B C1B C11B O6B

16.2(4) C2B C1B C11B O11B

135.1(3) C2B C1B C11B C3B

13.8(2) C2B C1B C11B C10B

165.8(3) C2B C3B C4B C5B

-20.2(4) C2B C3B C4B C9B

134.3(3) C2B C3B C11B C1B

13.9(2) C2B C3B C11B O6B

$-102.5(3) \mathrm{C} 2 \mathrm{~B}$ C3B C11B O11B

-17.2(5) C2B C3B C11B C10B

163.2(3) $\mathrm{C} 3 \mathrm{~B}$ C4B C5B C6B

87.7(4) C3B C4B C9B N1B

-88.6(4) C3B C4B C9B C8B

$-127.6(3) \mathrm{C} 4 \mathrm{~B}$ C3B C11B C1B

-13.7(2) C4B C3B C11B O6B

99.6(3) C4B C3B C11B O11B

-94.1(4) C4B C3B C11B C10B

79.9(4) C4B C5B C6B C7B

$-175.0(3) \mathrm{C} 5 \mathrm{~B}$ C4B C9B N1B

-3.6(5) C5B C4B C9B C8B

175.9(3) C5B C6B C7B C8B

110.5(3) C6B C7B C8B C9B

-135.6(3) C7B C8B C9B N1B

-22.3(4) C7B C8B C9B C4B

$-0.9(6)$ C9B N1B C10B O5B

180.0(3) C9B N1B C10B C11B

$-0.5(5)$ C9B C4B C5B C6B

-0.4(6) C10B N1B C9B C4B

1.1(5) $\mathrm{C} 10 \mathrm{~B}$ N1B $\mathrm{C} 9 \mathrm{~B} \quad \mathrm{C} 8 \mathrm{~B}$

178.8(3) C11B C1B C12B O1B

$-0.7(5)$ C11B C1B C12B O2B

178.7(3) C11B C1B C19B O7B
$-115.0(4)$

$-123.9(11)$

20.2(4)

$-29(2)$

$146.9(12)$

$-16(3)$

$168.3(15)$

132.2(3)

140(2)

$12.0(2)$

$-104.6(3)$

$83.5(4)$

$-94.7(4)$

$-11.7(2)$

$-126.0(4)$

$-122(2)$

102.5(3)

$-179.5(3)$

$0.3(5)$

$-179.8(3)$

$-134.6(3)$

111.1(4)

115(2)

$-20.3(4)$

$0.2(5)$

$-178.0(3)$

1.9(5)

$0.4(5)$

$0.1(5)$

178.6(3)

$-1.3(5)$

172.8(3)

$-10.6(5)$

$-1.3(5)$

$-0.4(5)$

179.8(3)

74(2)

$-110.2(14)$

79(3) 


$\begin{array}{llrllr}\text { C9A N1A C10AC11A } & -3.9(5) & \text { C11B C1B C19B O8B } & -96.7(17) \\ \text { C9A C4A C5A C6A } & 1.3(5) & \text { C11B C1B C2B C14B } & 103.7(3) \\ \text { C10A N1A C9A C4A } & -3.1(5) & \text { C11B C1B C2B C21B } & 118.4(17) \\ \text { C10A N1A C9A C8A } & 177.4(3) & \text { C11B C1B C2B C3B } & -11.7(2) \\ \text { C11A 06A C16AC17A } & 157.6(5) & \text { C11B O6B C16B C17B } & -91.5(5) \\ \text { C11A O6A C16AC18A } & -75.7(7) & \text { C11B O6B C16B C18B } & 148.0(5) \\ \text { C11A O6A C20AC21A } & 170.1(5) & \text { C11B C3B C4B C5B } & -171.0(3) \\ \text { C11A O6A C20AC22A } & -69.9(8) & \text { C11B C3B C4B C9B } & 10.7(5)\end{array}$

Table S17 Hydrogen Atom Coordinates $\left(\AA \times 10^{4}\right)$ and Isotropic Displacement Parameters $\left(\AA^{2} \times 10^{3}\right)$ for yoon 56 .

\begin{tabular}{|c|c|c|c|c|}
\hline Atom & $x$ & $y$ & $z$ & $\mathbf{U}(\mathbf{e q})$ \\
\hline H16 & 8195.48 & 4974.51 & 7673.09 & 51 \\
\hline $\mathrm{H} 17 \mathrm{G}$ & 7720.02 & 6017.51 & 7326.65 & 102 \\
\hline $\mathrm{H} 17 \mathrm{H}$ & 7348.93 & 5409.44 & 6997.93 & 102 \\
\hline H17I & 8933.09 & 5828.93 & 6951.62 & 102 \\
\hline $\mathrm{H} 18 \mathrm{G}$ & 10437.72 & 4748.44 & 7006.17 & 84 \\
\hline $\mathrm{H} 18 \mathrm{H}$ & 8906.41 & 4301.95 & 7079.87 & 84 \\
\hline H18I & 10274.8 & 4287.52 & 7431.73 & 84 \\
\hline H19 & 7943.29 & 5091.81 & 7649.38 & 41 \\
\hline $\mathrm{H} 20 \mathrm{E}$ & 7372.19 & 6155.21 & 7363.29 & 51 \\
\hline $\mathrm{H} 20 \mathrm{~F}$ & 7145.04 & 5610.17 & 6983.13 & 51 \\
\hline $\mathrm{H} 20 \mathrm{G}$ & 8675.07 & 6071.65 & 6996.29 & 51 \\
\hline H21D & 9908.56 & 4980.25 & 6915.23 & 51 \\
\hline $\mathrm{H} 21 \mathrm{E}$ & 8532.48 & 4477.25 & 7052.05 & 51 \\
\hline $\mathrm{H} 21 \mathrm{~F}$ & 10111.63 & 4491.43 & 7326.46 & 51 \\
\hline H1 & $9380(50)$ & $4047(13)$ & $8590(12)$ & 33 \\
\hline $\mathrm{H} 1 \mathrm{C}$ & 11880.91 & 5806.53 & 8564.03 & 32 \\
\hline $\mathrm{H} 2$ & 9585.73 & 6836.85 & 8534.24 & 35 \\
\hline H3 & 8036.62 & 6138.6 & 8113.51 & 34 \\
\hline H5 & 5779.75 & 6151.13 & 8678.07 & 44 \\
\hline H6 & 4078.45 & 5490.62 & 9079.79 & 54 \\
\hline H7 & 4691.07 & 4355.15 & 9218.12 & 54 \\
\hline H8 & 7019.64 & 3897.35 & 8962.99 & 42 \\
\hline $\mathrm{H} 13 \mathrm{G}$ & 12932.79 & 6529.23 & 7256.97 & 63 \\
\hline $\mathrm{H} 13 \mathrm{H}$ & 14586.33 & 6191.07 & 7356.05 & 63 \\
\hline H13I & 14174.22 & 6922.28 & 7551.17 & 63 \\
\hline $\mathrm{H} 15 \mathrm{G}$ & 7867.68 & 5926.15 & 9723.67 & 82 \\
\hline $\mathrm{H} 15 \mathrm{H}$ & 7395.56 & 6693.93 & 9830.9 & 82 \\
\hline
\end{tabular}




\begin{tabular}{|c|c|c|c|c|}
\hline H15I & 9150.79 & 6446.92 & 9898.55 & 82 \\
\hline $\mathrm{H} 1 \mathrm{~A}$ & $3370(40)$ & $4624(17)$ & $6402(12)$ & 36 \\
\hline H1AA & 3929.67 & 2686.59 & 5855.8 & 27 \\
\hline $\mathrm{H} 2 \mathrm{~A}$ & 5429.93 & 2090.22 & 6573.37 & 28 \\
\hline $\mathrm{H} 3 \mathrm{~A}$ & $6990(40)$ & 2961(16) & $6606(11)$ & 26 \\
\hline $\mathrm{H} 5 \mathrm{~A}$ & 6815.8 & 3179.53 & 7420.2 & 38 \\
\hline H6A & 5978.27 & 3890.36 & 7981.8 & 46 \\
\hline H7A & 4290.68 & 4786.06 & 7833.05 & 45 \\
\hline H8A & 3451.09 & 4984.47 & 7125.7 & 39 \\
\hline H13D & 6979.67 & 1897.75 & 4803.18 & 61 \\
\hline H13E & 7142.94 & 1377.24 & 5199.62 & 61 \\
\hline H13F & 8188.86 & 2048.75 & 5186.07 & 61 \\
\hline H15D & 594.24 & 3595.96 & 6581.85 & 65 \\
\hline $\mathrm{H} 15 \mathrm{E}$ & 1496.08 & 3445.77 & 7026.11 & 65 \\
\hline $\mathrm{H} 15 \mathrm{~F}$ & 366.58 & 2883.44 & 6823.37 & 65 \\
\hline H19A & 2114.4 & 3112.11 & 7368.19 & 62 \\
\hline H19B & 2310.07 & 2452.38 & 7666.56 & 62 \\
\hline H19C & 1057.8 & 2457.09 & 7282.32 & 62 \\
\hline H16A & 8400.28 & 3670.83 & 6322.7 & 44 \\
\hline H17D & 9824.77 & 3252.98 & 5757.08 & 56 \\
\hline H17E & 10524.33 & 3979.85 & 5882.43 & 56 \\
\hline H17F & 9439.82 & 3901.64 & 5463.2 & 56 \\
\hline H18D & 7842.36 & 4839.1 & 5827.04 & 56 \\
\hline $\mathrm{H} 18 \mathrm{E}$ & 8805.22 & 4799.98 & 6270.98 & 56 \\
\hline $\mathrm{H} 18 \mathrm{~F}$ & 6972.55 & 4661.53 & 6272.14 & 56 \\
\hline H20D & 8361.82 & 3952.64 & 6279.16 & 44 \\
\hline $\mathrm{H} 21 \mathrm{~A}$ & 9500.48 & 3531.11 & 5583.11 & 56 \\
\hline $\mathrm{H} 21 \mathrm{~B}$ & 10302.59 & 4177.33 & 5804.46 & 56 \\
\hline $\mathrm{H} 21 \mathrm{C}$ & 9139.4 & 4277.33 & 5404.01 & 56 \\
\hline $\mathrm{H} 22 \mathrm{D}$ & 7126.06 & 4894.12 & 5681.63 & 56 \\
\hline $\mathrm{H} 22 \mathrm{E}$ & 8258.49 & 5070.86 & 6078.09 & 56 \\
\hline $\mathrm{H} 22 \mathrm{~F}$ & 6530.41 & 4807.78 & 6170.85 & 56 \\
\hline H1B & -208.18 & 6394.66 & 6199.86 & 33 \\
\hline H1BA & -250.02 & 6437.85 & 6234.88 & 33 \\
\hline H13A & -1056.48 & 8150.21 & 6963.73 & 83 \\
\hline H13B & 618.22 & 7992.89 & 7166.17 & 83 \\
\hline $\mathrm{H} 13 \mathrm{C}$ & -894.33 & 7631.49 & 7361.16 & 83 \\
\hline $\mathrm{H} 20 \mathrm{~A}$ & 1839.41 & 8134.96 & 6926.34 & 41 \\
\hline H20B & 838.06 & 7814.56 & 7313.38 & 41 \\
\hline
\end{tabular}




\begin{tabular}{|c|c|c|c|c|}
\hline $\mathrm{H} 20 \mathrm{C}$ & 10.94 & 8277.18 & 6954.06 & 41 \\
\hline $\mathrm{H} 15 \mathrm{~A}$ & -3764.09 & 6335.7 & 4781.05 & 75 \\
\hline H15B & -3558.07 & 5877.66 & 5206.42 & 75 \\
\hline $\mathrm{H} 15 \mathrm{C}$ & -2410.02 & 5779.72 & 4802.37 & 75 \\
\hline $\mathrm{H} 22 \mathrm{~A}$ & -2597.21 & 5954.35 & 4848.59 & 62 \\
\hline $\mathrm{H} 22 \mathrm{~B}$ & -3931.53 & 6396.23 & 5074.84 & 62 \\
\hline $\mathrm{H} 22 \mathrm{C}$ & -3541.4 & 5651.89 & 5252.39 & 62 \\
\hline H16B & 4811.1 & 6876.47 & 5784.61 & 49 \\
\hline H17A & 5650.49 & 6247.53 & 6585.64 & 87 \\
\hline H17B & 6741.19 & 6280.44 & 6165.21 & 87 \\
\hline $\mathrm{H} 17 \mathrm{C}$ & 5174.68 & 5842.75 & 6155.08 & 87 \\
\hline H18A & 4982.83 & 7896.79 & 6151.98 & 118 \\
\hline H18B & 6603.16 & 7511.58 & 6218.17 & 118 \\
\hline $\mathrm{H} 18 \mathrm{C}$ & 5344.25 & 7518.49 & 6600.7 & 118 \\
\hline $\mathrm{H} 23 \mathrm{~B}$ & 4314.46 & 7527.28 & 6014.83 & 64 \\
\hline $\mathrm{H} 24 \mathrm{~A}$ & 5299.58 & 6624.23 & 5684.34 & 80 \\
\hline H24B & 5447.57 & 6196.34 & 6124.2 & 80 \\
\hline $\mathrm{H} 24 \mathrm{C}$ & 6648.08 & 6797.11 & 6024.75 & 80 \\
\hline $\mathrm{H} 25 \mathrm{~A}$ & 6207.29 & 7141.54 & 6678.89 & 80 \\
\hline $\mathrm{H} 25 \mathrm{~B}$ & 4558.09 & 7390.74 & 6858.66 & 80 \\
\hline $\mathrm{H} 25 \mathrm{C}$ & 5586.31 & 7885.75 & 6563.22 & 80 \\
\hline H1BB & $2930(50)$ & $5038(13)$ & $5652(12)$ & 34 \\
\hline $\mathrm{H} 2 \mathrm{~B}$ & -297.65 & 7395.34 & 5551.67 & 38 \\
\hline $\mathrm{H} 2 \mathrm{BA}$ & -15.43 & 7433.69 & 5609.02 & 38 \\
\hline $\mathrm{H} 3 \mathrm{~B}$ & 2398.13 & 7315.62 & 5501.95 & 33 \\
\hline $\mathrm{H} 5 \mathrm{~B}$ & 2220.22 & 7153.7 & 4672.67 & 38 \\
\hline H6B & 3045.44 & 6482.42 & 4094.38 & 42 \\
\hline H7B & 3769.36 & 5352 & 4217.76 & 43 \\
\hline H8B & 3643.48 & 4899.6 & 4916.44 & 38 \\
\hline
\end{tabular}

Table S18 Atomic Occupancy for yoon56.

$\begin{array}{lrlrlr}\text { Atom } & \text { Occupancy } & \text { Atom } & \text { Occupancy } & \text { Atom } & \text { Occupancy } \\ \text { O6 } & 0.88(4) & \mathrm{C} 16 & 0.88(4) & \mathrm{H} 16 & 0.88(4) \\ \mathrm{C} 17 & 0.88(4) & \mathrm{H} 17 \mathrm{G} & 0.88(4) & \mathrm{H} 17 \mathrm{H} & 0.88(4) \\ \mathrm{H} 17 \mathrm{I} & 0.88(4) \mathrm{C} 18 & 0.88(4) \mathrm{H} 18 \mathrm{G} & 0.88(4) \\ \mathrm{H} 18 \mathrm{H} & 0.88(4) \mathrm{H} 18 \mathrm{I} & 0.88(4) \mathrm{O} 7 & 0.12(4) \\ \mathrm{C} 19 & 0.12(4) \mathrm{H} 19 & 0.12(4) \mathrm{C} 20 & 0.12(4) \\ \text { H20E } & 0.12(4) \mathrm{H} 20 \mathrm{~F} & 0.12(4) \mathrm{H} 20 \mathrm{G} & 0.12(4) \\ \mathrm{C} 21 & 0.12(4) \mathrm{H} 21 \mathrm{D} & 0.12(4) \mathrm{H} 21 \mathrm{E} & 0.12(4)\end{array}$




\begin{tabular}{|c|c|c|c|}
\hline $\mathrm{H} 21 \mathrm{~F}$ & $0.12(4) \mathrm{C} 15 \mathrm{~A}$ & $0.555(9) \mathrm{H} 15 \mathrm{D}$ & $0.555(9)$ \\
\hline H15E & $0.555(9) \mathrm{H} 15 \mathrm{~F}$ & $0.555(9) \mathrm{C} 19 \mathrm{~A}$ & $0.445(9)$ \\
\hline H19A & $0.445(9) \mathrm{H} 19 \mathrm{~B}$ & $0.445(9) \mathrm{H} 19 \mathrm{C}$ & $0.445(9)$ \\
\hline $\mathrm{C} 16 \mathrm{~A}$ & $0.503(8) \mathrm{H} 16 \mathrm{~A}$ & $0.503(8) \mathrm{C} 17 \mathrm{~A}$ & $0.503(8)$ \\
\hline H17D & $0.503(8)$ H17E & $0.503(8) \mathrm{H} 17 \mathrm{~F}$ & $0.503(8)$ \\
\hline C18A & $0.503(8) \mathrm{H} 18 \mathrm{D}$ & $0.503(8) \mathrm{H} 18 \mathrm{E}$ & $0.503(8)$ \\
\hline $\mathrm{H} 18 \mathrm{~F}$ & $0.503(8) \mathrm{C} 20 \mathrm{~A}$ & $0.497(8) \mathrm{H} 20 \mathrm{D}$ & $0.497(8)$ \\
\hline $\mathrm{C} 21 \mathrm{~A}$ & $0.497(8) \mathrm{H} 21 \mathrm{~A}$ & $0.497(8) \mathrm{H} 21 \mathrm{~B}$ & $0.497(8)$ \\
\hline $\mathrm{H} 21 \mathrm{C}$ & $0.497(8) \mathrm{C} 22 \mathrm{~A}$ & $0.497(8) \mathrm{H} 22 \mathrm{D}$ & $0.497(8)$ \\
\hline $\mathrm{H} 22 \mathrm{E}$ & $0.497(8) \mathrm{H} 22 \mathrm{~F}$ & $0.497(8) \mathrm{H} 1 \mathrm{~B}$ & $0.568(13)$ \\
\hline H1BA & $0.432(13) \mathrm{O} 1 \mathrm{~B}$ & $0.568(13) \mathrm{O} 2 \mathrm{~B}$ & $0.568(13)$ \\
\hline $\mathrm{C} 12 \mathrm{~B}$ & $0.568(13) \mathrm{C} 13 \mathrm{~B}$ & $0.568(13) \mathrm{H} 13 \mathrm{~A}$ & $0.568(13)$ \\
\hline H13B & $0.568(13) \mathrm{H} 13 \mathrm{C}$ & $0.568(13)$ O7B & $0.432(13)$ \\
\hline O8B & $0.432(13) \mathrm{C} 19 \mathrm{~B}$ & $0.432(13) \mathrm{C} 20 \mathrm{~B}$ & $0.432(13)$ \\
\hline $\mathrm{H} 20 \mathrm{~A}$ & $0.432(13) \mathrm{H} 20 \mathrm{~B}$ & $0.432(13) \mathrm{H} 20 \mathrm{C}$ & $0.432(13)$ \\
\hline O3B & $0.856(6) \mathrm{O} 4 \mathrm{~B}$ & $0.856(6) \mathrm{C} 14 \mathrm{~B}$ & $0.856(6)$ \\
\hline C15B & $0.856(6) \mathrm{H} 15 \mathrm{~A}$ & $0.856(6) \mathrm{H} 15 \mathrm{~B}$ & $0.856(6)$ \\
\hline $\mathrm{H} 15 \mathrm{C}$ & $0.856(6)$ O9B & $0.144(6) \mathrm{O} 10 \mathrm{~B}$ & $0.144(6)$ \\
\hline $\mathrm{C} 21 \mathrm{~B}$ & $0.144(6) \mathrm{C} 22 \mathrm{~B}$ & $0.144(6) \mathrm{H} 22 \mathrm{~A}$ & $0.144(6)$ \\
\hline $\mathrm{H} 22 \mathrm{~B}$ & $0.144(6) \mathrm{H} 22 \mathrm{C}$ & $0.144(6)$ O6B & $0.875(10)$ \\
\hline $\mathrm{C} 16 \mathrm{~B}$ & $0.875(10)$ H16B & $0.875(10) \mathrm{C} 17 \mathrm{~B}$ & $0.875(10)$ \\
\hline H17A & $0.875(10)$ H17B & $0.875(10) \mathrm{H} 17 \mathrm{C}$ & $0.875(10)$ \\
\hline C18B & $0.875(10) \mathrm{H} 18 \mathrm{~A}$ & $0.875(10) \mathrm{H} 18 \mathrm{~B}$ & $0.875(10)$ \\
\hline $\mathrm{H} 18 \mathrm{C}$ & $0.875(10) \mathrm{C} 23 \mathrm{~B}$ & $0.125(10) \mathrm{H} 23 \mathrm{~B}$ & $0.125(10)$ \\
\hline $\mathrm{C} 24 \mathrm{~B}$ & $0.125(10) \mathrm{H} 24 \mathrm{~A}$ & $0.125(10)$ H24B & $0.125(10)$ \\
\hline $\mathrm{H} 24 \mathrm{C}$ & $0.125(10) \mathrm{C} 25 \mathrm{~B}$ & $0.125(10) \mathrm{H} 25 \mathrm{~A}$ & $0.125(10)$ \\
\hline $\mathrm{H} 25 \mathrm{~B}$ & $0.125(10) \mathrm{H} 25 \mathrm{C}$ & $0.125(10)$ O11B & $0.125(10)$ \\
\hline H2B & $0.856(6) \mathrm{H} 2 \mathrm{BA}$ & $0.144(6)$ & \\
\hline
\end{tabular}




\section{Computational Studies}

\section{a. General Information}

All calculations were done using density functional theory ${ }^{15}$ (DFT) implemented in Jaguar 9.1 suite of program. ${ }^{16}$ Geometry of all structures were optimized with B3LYP-D3 ${ }^{17,18,19}$ levels of theory and 6-31G** basis set. ${ }^{20}$ For the Ir metal center, Los Alamos LACVP ${ }^{21,22,23}$ basis set was applied. The single point energies of all optimized structures were re-evaluated using triple- $\zeta$ quality of basis set, cc-pVTZ(-f), ${ }^{24}$ where Ir metal was evaluated with LACV3P** basis set. Vibrational frequencies were calculated using the optimized structures at the same level as the geometry optimizations. Solvation correction energies were derived from the self-consistent reaction field (SCRF) approximations, ${ }^{25,26,27}$ which is the solution for the linearized Poisson-Boltzmann equations with the proper dielectric constants $(\varepsilon=9.08$ for dichloromethane). Finally, the solution phase Gibbs free energies were computed as follows:

$$
\begin{aligned}
& \mathrm{G}(\text { Sol })=\mathrm{G}(\text { gas })+\mathrm{G}^{\text {solv }} \\
& \mathrm{G}(\text { gas })=\mathrm{H}(\text { gas })-\mathrm{TS}(\text { gas }) \\
& \mathrm{H}(\mathrm{Gas})=\mathrm{E}(\mathrm{SCF})+\mathrm{ZPE} \\
& \Delta \mathrm{G}(\mathrm{Sol})=\Sigma \mathrm{G}(\text { Sol }) \text { for products }-\Sigma \mathrm{G}(\text { Sol }) \text { for reactants }
\end{aligned}
$$

$\mathrm{G}(\mathrm{Sol})$ is the solvation corrected Gibbs free energy; $\mathrm{G}(\mathrm{gas})$ is the gas phase free energy; H(gas) is the enthalpy in the gas phase; $\mathrm{T}$ is the temperature $(191.15 \mathrm{~K})$; $\mathrm{S}$ (gas) is the entropy in the gas phase; $\mathrm{E}(\mathrm{SCF})$ is the electronic energy converged from the self-consistent field method; ZPE is the vibrational zero-point energy; and $\mathrm{S}$ for the vibrational entropy correction. Note that here entropy refers specifically to the vibrational/rotational/translational entropy of the solute(s). The solvent entropies are implicitly included in the continuum model.

\section{b. Benchmark calculations to evaluate triplet energies}

Before we evaluate the triplet energies of molecules using DFT, we tested popular functionals to mimic the current system. Hence, B3LYP-D3, M06 and BH and HLYP levels were adopted for evaluation of triplet energies of photocatalyst (4f), maleimide (2), and quinolone substrate (1c). As summarized in Table S19, both B3LYP-D3 and BHandHLYP are closer to the experimental estimates, whereas the M06 functional severely underestimates the singlet-triplet gap.

Table S19 Comparison of different functionals to evaluate triplet energies.

\begin{tabular}{|c|c|c|c|}
\hline $\begin{array}{c}\text { Triplet energy } \\
(\mathrm{kcal} / \mathrm{mol})\end{array}$ & $\begin{array}{c}\Delta \mathrm{G}\left({ }^{\mathbf{3}} \mathbf{4 f}\right)-\Delta \mathrm{G}\left({ }^{\mathbf{4}} \mathbf{4 f}\right) \\
\text { (Photocatalyst) }\end{array}$ & $\begin{array}{c}\Delta \mathrm{G}\left({ }^{\mathbf{3}} \mathbf{2}\right)-\Delta \mathrm{G}\left({ }^{\mathbf{1}} \mathbf{2}\right) \\
(\text { maleimide) }\end{array}$ & $\begin{array}{c}\Delta \mathrm{G}\left({ }^{\mathbf{3}} \mathbf{1} \mathbf{c}\right)-\Delta \mathrm{G}\left({ }^{\mathbf{1} 1 \mathbf{c})}\right. \\
\text { (quinolone) }\end{array}$ \\
\hline B3LYP-D3 & 53.14 & 64.42 & 55.44 \\
\hline M06 & 47.73 & 67.96 & 56.37 \\
\hline BH and HLYP & 66.33 & 77.94 & 54.88 \\
\hline Experimental & $\sim 60 \mathrm{kcal} / \mathrm{mol}$ & & \\
\hline
\end{tabular}


Additionally, to be sure that our computational level is high enough to mimic triplet state molecules, we further evaluated the triplet energies of known photocatalysts. ${ }^{28}$ As shown in Table S20, the experimental values and computational estimations match in both qualitative and quantitative manners.

Table S20 Comparison of experimental triplet energies with computed values for known $\operatorname{Ir}(\mathrm{III})$ photocatalysts
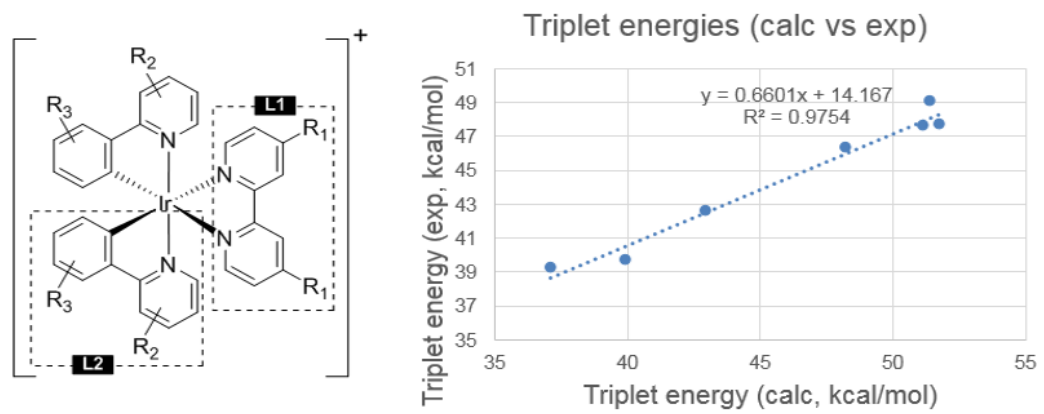

\begin{tabular}{|c|c|c|c|}
\hline L1 & L2 & $\begin{array}{c}\mathbf{E}_{\mathbf{T}} \text { (exp, } \\
\mathbf{k c a l} / \mathbf{m o l})\end{array}$ & $\begin{array}{c}\mathbf{E}_{\mathbf{T}} \text { (calc, } \\
\mathbf{k a l} / \mathbf{m o l})\end{array}$ \\
\hline $5,5^{\prime}-\mathrm{Me}_{2}$ bpy & ppy & 49.0 & 51.39 \\
\hline $4,4^{\prime}-(\mathrm{MeO})_{2}$ bpy & ppy & 47.7 & 51.78 \\
\hline $4,4^{\prime}-\mathrm{Me}_{2}$ bpy & ppy & 47.6 & 51.16 \\
\hline bpy & ppy & 46.3 & 48.25 \\
\hline $4,4^{\prime}-\mathrm{Cl}_{2}$ bpy & ppy & 42.6 & 43.00 \\
\hline $4,4^{\prime}-\left(\mathrm{CO}_{2} \mathrm{Me}\right)_{2}$ bpy & ppy & 39.7 & 39.92 \\
\hline $4,4^{\prime}-\left(\mathrm{F}_{3} \mathrm{C}\right)_{2}$ bpy & ppy & 39.2 & 37.09 \\
\hline
\end{tabular}

\section{c. Conformers for encounter complexes}

To evaluate the energies of possible encounter complexes for donor-acceptor pairs ([Ir-Q] and [Ir-2]), various of conformations were optimized and compared. For [Ir-Q] complexes, since $\mathrm{N}-\mathrm{H}$ bond in the pyrazole ring participates in the hydrogen bonds, we can consider two major hydrogen bond donor-acceptor pairs, where quinolone substrate possess two hydrogen bond acceptors and one hydrogen bond donor. Additionally, the two possible facial approaches give four major encounter complexes $\left[\mathbf{I r}-\mathbf{Q}_{A}\right],\left[\mathbf{I r}-\mathbf{Q}_{B}\right],\left[\mathbf{I r}-\mathbf{Q}_{C}\right]$, and $\left[\mathbf{I r}-\mathbf{Q}_{\mathbf{D}}\right]$. For $\left[\mathbf{I r}-\mathbf{Q}_{A}\right]$ and $\left[\mathbf{I r}-\mathbf{Q}_{B}\right]$, encounter complex containing a non-classical hydrogen bond was observed, where two $\mathrm{N}_{\text {pyrazole }}-\mathrm{H}$ to $\mathrm{O}=\mathrm{C}_{\mathrm{Q}}$ an $\mathrm{O}-\mathrm{C}_{\mathrm{Q}}$ hydrogen bonds were found for $\left[\mathbf{I r}-\mathbf{Q}_{\mathbf{C}}\right]$, and $\left[\mathbf{I r}-\mathbf{Q}_{\mathbf{D}}\right]$. For $[\operatorname{Ir}-2]$, since there is only one hydrogen bond donor in maleimide, two facial approaches were considered. ([Ir-2 $\mathbf{A}]$ and $\left[\mathbf{I r}-\mathbf{2}_{\mathrm{B}}\right]$ )

For [Ir-Q] complexes, the conformation of isopropyl group tethered on the quinolone was also considered. Three major conformations showing the staggered relationship were optimized, and the geometry showing the lowest energy was chosen for the energy comparison. 


\section{d. Mulliken spin densities for triplet state intermediates}

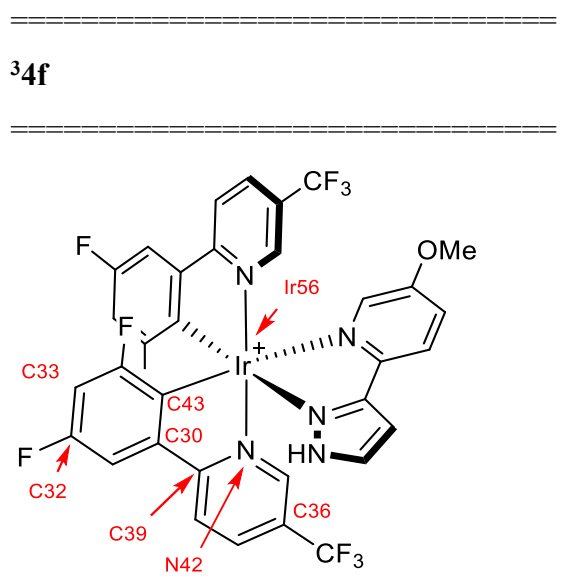

$\begin{array}{lllll}\mathrm{H} 1 & \mathrm{H} 2 & \mathrm{H} 3 & \mathrm{H} 4 & \mathrm{H} 5\end{array}$

$\begin{array}{lllll}0.00018 & 0.00067 & 0.00083 & 0.00018 & 0.00002\end{array}$

$\begin{array}{lllll}\text { H6 } & \mathrm{H} 7 & \mathrm{H} 8 & \mathrm{H} 9 & \mathrm{H} 10\end{array}$

$\begin{array}{lllll}0.00003 & 0.00346 & -0.00681 & -0.00602 & -0.0002\end{array}$

$\begin{array}{lllll}\text { H11 } & \text { H12 } & \text { F13 } & \text { F14 } & \text { F15 }\end{array}$

$\begin{array}{lllll}-0.00004 & 0.00011 & 0.00002 & 0.00011 & 0.00004\end{array}$

$\begin{array}{lllll}\text { C16 } & \text { F17 } & \text { F18 } & \text { F19 } & \text { C20 }\end{array}$

$\begin{array}{lllll}-0.00016 & 0.00293 & 0.00202 & 0.00733 & -0.00789\end{array}$

$\begin{array}{lllll}\mathrm{C} 21 & \mathrm{C} 22 & \mathrm{C} 23 & \mathrm{~N} 24 & \mathrm{C} 25\end{array}$

$\begin{array}{lllll}-0.00177 & 0.0008 & -0.00203 & 0.00308 & -0.00192\end{array}$

$\begin{array}{lllll}\mathrm{C} 26 & \mathrm{C} 27 & \mathrm{C} 28 & \mathrm{C} 29 & \mathbf{C 3 0}\end{array}$

$\begin{array}{lllll}0.00004 & -0.00009 & -0.00037 & -0.00011 & \mathbf{0 . 1 9 6 6 4}\end{array}$

$\begin{array}{lllll}\text { C31 } & \text { C32 } & \text { C33 } & \text { C34 } & \text { C35 } \\ 0.05453 & \mathbf{0 . 1 5 0 6 4} & \mathbf{0 . 2 7 8 0 5} & -0.07205 & -0.0883\end{array}$

$\begin{array}{lllll}\mathbf{C} 36 & \mathrm{C} 37 & \mathrm{C} 38 & \mathbf{C 3 9} & \mathrm{N} 40\end{array}$

$\begin{array}{lllll}\mathbf{0 . 2 4 1 6 1} & 0.0652 & 0.0703 & \mathbf{0 . 1 6 3 5 2} & -0.00149\end{array}$

$\begin{array}{lllll}\mathrm{N} 41 & \mathbf{N} 42 & \mathbf{C 4 3} & \mathrm{C} 44 & \mathrm{C} 45\end{array}$

$\begin{array}{lllll}0.01058 & \mathbf{0 . 1 9 7 8 8} & \mathbf{0 . 3 9 2 8 7} & -0.01548 & 0.02327\end{array}$

$\begin{array}{lllll}\mathrm{C} 46 & \mathrm{C} 47 & \mathrm{C} 48 & \mathrm{C} 49 & \mathrm{C} 50\end{array}$

$\begin{array}{lllll}-0.01371 & 0.02937 & -0.00606 & 0.02031 & -0.00242\end{array}$
C51 C5

0.00267

0.00014

$\mathrm{C} 53 \quad \mathrm{C} 54 \quad \mathrm{~N} 55$

$\begin{array}{lllll}\text { Ir56 } & \text { H57 } & \text { H58 } & \text { H59 } & \text { H60 }\end{array}$

0.29218

$\begin{array}{lll}0.00014 & 0.00041 & -0.02143\end{array}$

$-0.00553$

$\begin{array}{lllll}\text { F61 } & \text { F62 } & \text { F63 } & \text { F64 } & \text { O65 }\end{array}$

$\begin{array}{lllll}0.00248 & 0.03011 & 0.0032 & 0.00423 & -0.00006\end{array}$

$\begin{array}{llll}\text { C66 } & \text { H67 } & \text { H68 } & \text { H69 }\end{array}$

$\begin{array}{llll}0.00005 & -0.00001 & -0.00001 & 0.00001\end{array}$

32

O9

$\begin{array}{lllll}\text { N1 } & \text { C2 } & \text { C3 } & \text { C4 } & \text { C5 } \\ 0.05125 & \mathbf{0 . 1 5 3 0 5} & \mathbf{0 . 2 1 6 3 2} & \mathbf{0 . 2 1 6 2 7} & \mathbf{0 . 1 5 3 0 7}\end{array}$

$\begin{array}{lllll}\text { H6 } & \text { H7 } & \text { H8 } & \text { O9 } & 010\end{array}$

$\begin{array}{lllll}-0.00887 & -0.0064 & -0.0064 & \mathbf{0 . 6 1 5 8 3} & \mathbf{0 . 6 1 5 8 7}\end{array}$

${ }^{3} 1 \mathrm{c}$<smiles>CCCOc1cc2ccccc2[nH]c1=O</smiles>

$\begin{array}{lllll}\text { C1 } & \text { C2 } & \text { C3 } & \text { H4 } & \text { C5 }\end{array}$

$\begin{array}{lllll}\mathbf{0 . 3 6 0 5 3} & \mathbf{- 0 . 1 1 1 0 5} & \mathbf{0 . 2 5 6 0 1} & 0.00475 & -0.09182\end{array}$

$\begin{array}{lllll}\text { H6 } & \text { C7 } & \text { H8 } & \text { C9 } & \text { N10 }\end{array}$

$\begin{array}{lllll}-0.02605 & \mathbf{0 . 2 0 2 2 1} & 0.00264 & -0.04077 & 0.09992\end{array}$

$\begin{array}{lllll}\text { C11 } & \text { H12 } & \text { C13 } & \text { H14 } & \text { C15 }\end{array}$

$\begin{array}{llllll}\mathbf{0 . 4 5 2 1 9} & -0.01753 & -0.02216 & -0.00616 & \mathbf{0 . 5 2 8 3 2}\end{array}$

$\begin{array}{lllll}016 & 017 & \text { H18 } & \text { C19 } & \text { H20 }\end{array}$ 


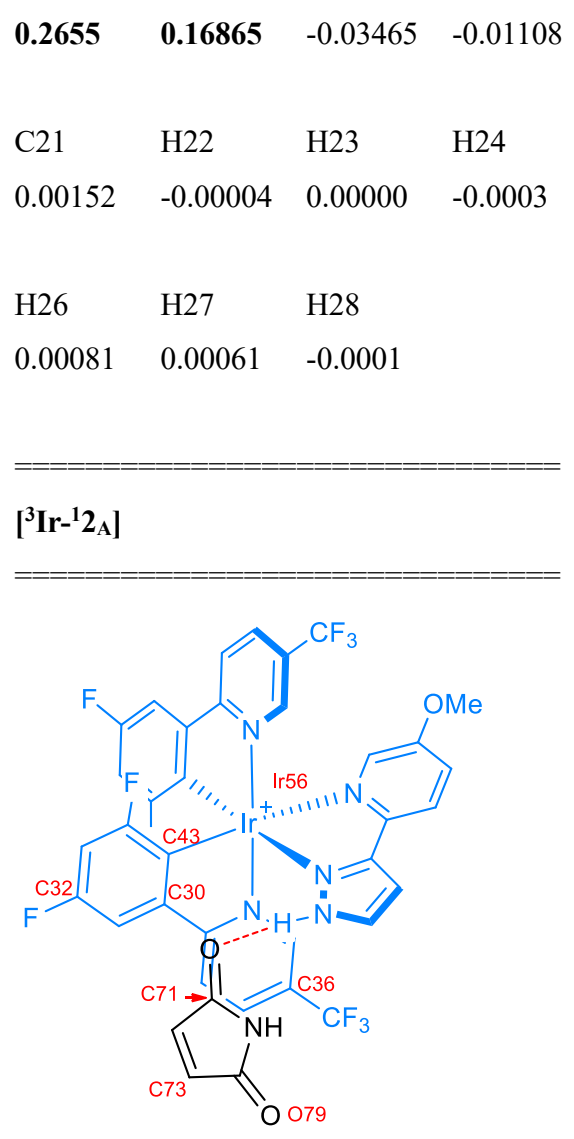

$\begin{array}{lllll}\text { H1 } & \text { H2 } & \text { H3 } & \text { H4 } & \text { H5 } \\ 0.00008 & 0.00057 & -0.00116 & 0.00014 & 0.00000\end{array}$

$\begin{array}{lllll}\text { H6 } & \mathrm{H} 7 & \mathrm{H} 8 & \mathrm{H} 9 & \mathrm{H} 10\end{array}$

$\begin{array}{lllll}-0.00004 & 0.00159 & -0.00004 & -0.00542 & -0.00021\end{array}$

$\begin{array}{lllll}\text { H11 } & \text { H12 } & \text { F13 } & \text { F14 } & \text { F15 }\end{array}$

$\begin{array}{llllll}0.00003 & 0.00008 & 0.00002 & 0.0001 & 0.00004\end{array}$

$\begin{array}{lllll}\text { C16 } & \text { F17 } & \text { F18 } & \text { F19 } & \text { C20 }\end{array}$

$\begin{array}{lllll}-0.00015 & 0.00071 & 0.00218 & 0.00385 & -0.00421\end{array}$

$\begin{array}{lllll}\mathrm{C} 21 & \mathrm{C} 22 & \mathrm{C} 23 & \mathrm{~N} 24 & \mathrm{C} 25\end{array}$

$\begin{array}{lllll}-0.00106 & -0.00032 & -0.00007 & 0.00229 & -0.00109\end{array}$

$\begin{array}{lllll}\mathrm{C} 26 & \mathrm{C} 27 & \mathrm{C} 28 & \mathrm{C} 29 & \mathbf{C} 30\end{array}$

$\begin{array}{lllll}0.00052 & -0.00014 & 0.00027 & 0.0001 & \mathbf{0 . 1 2 1 8 6}\end{array}$

$\begin{array}{lllll}\text { C31 } & \text { C32 } & \text { C33 } & \text { C34 } & \text { C35 }\end{array}$

$\begin{array}{lllll}0.01246 & \mathbf{0 . 1 9 9 3 1} & 0.08099 & 0.04542 & -0.03801\end{array}$ $\begin{array}{lllll}\text { C36 } & \text { C37 } & \text { C38 } & \text { C39 } & \text { N40 } \\ \mathbf{0 . 1 3 1 4 1} & -0.01337 & 0.06997 & 0.07889 & -0.00116\end{array}$

N41 N42 $\quad$ C43 $\quad$ C44

$\begin{array}{lllll}0.01303 & 0.07001 & \mathbf{0 . 2 7 3 9 3} & -0.00501 & 0.02321\end{array}$

$\begin{array}{lllll}\mathrm{C} 46 & \mathrm{C} 47 & \mathrm{C} 48 & \mathrm{C} 49 & \mathrm{C} 50\end{array}$

$\begin{array}{lllll}-0.01323 & 0.02735 & -0.00687 & 0.02015 & -0.00325\end{array}$

$\begin{array}{lllll}\mathrm{C} 51 & \mathrm{C} 52 & \mathrm{C} 53 & \mathrm{C} 54 & \mathrm{~N} 55\end{array}$

$\begin{array}{lllll}0.00313 & -0.00092 & 0.00301 & -0.00148 & 0.00016\end{array}$

$\begin{array}{lllll}\text { Ir56 } & \text { H57 } & \text { H58 } & \text { H59 } & \text { H60 }\end{array}$

$\begin{array}{lllll}\mathbf{0 . 3 0 4 5 1} & 0.00024 & 0.00062 & -0.00829 & -0.00226\end{array}$

$\begin{array}{lllll}\text { F61 } & \text { F62 } & \text { F63 } & \text { F64 } & \text { O65 }\end{array}$

$\begin{array}{llllll}0.01184 & 0.03433 & 0.00334 & 0.00398 & 0.00033\end{array}$

$\begin{array}{lllll}\text { C66 } & \text { H67 } & \text { H68 } & \text { H69 } & \text { N70 }\end{array}$

$\begin{array}{lllll}-0.00007 & 0.00000 & 0.00006 & 0.00000 & -0.02152\end{array}$

$\begin{array}{llllll}\mathbf{C} 71 & \mathrm{C} 72 & \mathbf{C 7 3} & \mathrm{C} 74 & \mathrm{H} 75\end{array}$

$\begin{array}{lllll}\mathbf{0 . 1 3 2 0 3} & 0.06882 & \mathbf{0 . 1 4 2 6 8} & 0.04127 & -0.00032\end{array}$

H76 $\mathrm{H77} \quad \mathrm{O} 78 \quad \mathbf{O 7 9}$

$\begin{array}{llll}-0.00706 & -0.01078 & 0.08727 & \mathbf{0 . 1 2 9 3 3}\end{array}$

$\left[{ }^{1} \mathbf{I r}^{3}{ }^{3} \mathbf{2}_{\mathrm{A}}\right]$

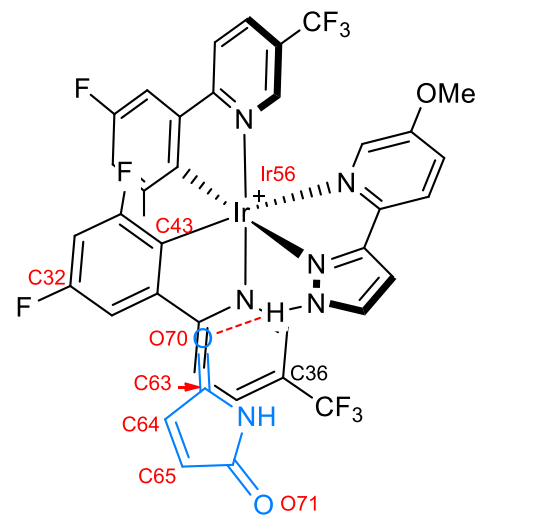

$\begin{array}{lllll}\text { H1 } & \text { H2 } & \text { H3 } & \text { H4 } & \text { H5 } \\ -0.00002 & 0.00026 & -0.00167 & -0.00005 & 0.00002\end{array}$ 


\begin{tabular}{|c|c|c|c|c|c|c|c|c|c|}
\hline & & & & & F76 & F77 & H78 & H79 & \\
\hline H6 & $\mathrm{H} 7$ & H8 & H9 & H10 & 0.00094 & 0.00111 & 0.00008 & 0.00017 & \\
\hline-0.00003 & 0.00006 & -0.00004 & 0.00125 & -0.00005 & & & & & \\
\hline H11 & H12 & F13 & F14 & F15 & {$\left[{ }^{3} \mathbf{I r}-{ }^{1} 2_{B}\right]$} & & & & \\
\hline 0.00004 & 0.00001 & 0.00000 & 0.00002 & 0.00000 & $===$ & & & $=====$ & \\
\hline $\mathrm{C} 16$ & F17 & F18 & F19 & $\mathrm{C} 20$ & & & & & \\
\hline-0.00002 & 0.00000 & 0.00000 & -0.00001 & 0.00003 & & & & & \\
\hline $\mathrm{C} 21$ & $\mathrm{C} 22$ & $\mathrm{C} 23$ & N24 & $\mathrm{C} 25$ & & & & & \\
\hline-0.00047 & -0.00023 & 0.00072 & 0.00005 & -0.00059 & & & & & \\
\hline $\mathrm{C} 26$ & $\mathrm{C} 27$ & $\mathrm{C} 28$ & $\mathrm{C} 29$ & C30 & & & & & \\
\hline 0.00017 & -0.00025 & 0.00017 & 0.00002 & -0.00552 & & & & & \\
\hline $\mathrm{C} 31$ & C32 & $\mathrm{C} 33$ & C34 & C35 & H1 & $\mathrm{H} 2$ & $\mathrm{H} 3$ & $\mathrm{H} 4$ & H5 \\
\hline 0.06796 & 0.15769 & -0.03014 & 0.06958 & -0.00046 & 0.00000 & 0.00056 & -0.00028 & -0.0001 & -0.00006 \\
\hline C36 & C37 & C38 & C39 & N40 & H6 & $\mathrm{H} 7$ & H8 & H9 & $\mathrm{H} 10$ \\
\hline-0.00053 & 0.00076 & -0.00104 & 0.00025 & -0.00114 & -0.00001 & 0.00262 & -0.00375 & -0.00471 & -0.00036 \\
\hline N41 & N42 & $\mathrm{C43}$ & $\mathrm{C} 44$ & $\mathrm{C} 45$ & H11 & H12 & F13 & F14 & F15 \\
\hline 0.00453 & -0.00108 & 0.14133 & 0.0006 & 0.00587 & 0.00022 & 0.00013 & 0.00002 & 0.00004 & 0.00014 \\
\hline $\mathrm{C} 46$ & $\mathrm{C} 47$ & $\mathrm{C} 48$ & C49 & $\mathrm{C} 50$ & $\mathrm{C} 16$ & F17 & F18 & F19 & $\mathrm{C} 20$ \\
\hline-0.00305 & 0.00701 & -0.00172 & 0.00512 & -0.00136 & -0.00045 & 0.00236 & 0.00113 & 0.00463 & -0.00504 \\
\hline $\mathrm{C} 51$ & C52 & C53 & C54 & N55 & C21 & $\mathrm{C} 22$ & $\mathrm{C} 23$ & N24 & $\mathrm{C} 25$ \\
\hline 0.00068 & -0.00069 & 0.00054 & -0.00058 & -0.00028 & -0.00229 & 0.00088 & -0.00147 & 0.00305 & -0.00056 \\
\hline Ir56 & O57 & C58 & H59 & H60 & $\mathrm{C} 26$ & $\mathrm{C} 27$ & $\mathrm{C} 28$ & $\mathrm{C} 29$ & C30 \\
\hline 0.1235 & 0.0001 & -0.00003 & 0.00000 & 0.00002 & -0.00019 & 0.00082 & -0.00044 & 0.00083 & 0.20248 \\
\hline H61 & N62 & $\mathrm{C63}$ & C64 & C65 & $\mathrm{C} 31$ & C32 & C33 & C34 & $\mathrm{C} 35$ \\
\hline 0.00000 & 0.0212 & 0.10187 & 0.42612 & 0.54495 & -0.01322 & 0.20446 & 0.1263 & 0.01414 & -0.06146 \\
\hline C66 & H67 & H68 & H69 & O70 & C36 & $\mathrm{C} 37$ & C38 & C39 & N40 \\
\hline 0.02336 & -0.00307 & -0.02869 & -0.03432 & 0.16057 & 0.16439 & 0.03299 & 0.06144 & 0.07894 & -0.0035 \\
\hline O71 & $\mathrm{H} 72$ & H73 & F74 & F75 & N41 & N42 & $\mathrm{C} 43$ & $\mathrm{C} 44$ & $\mathrm{C} 45$ \\
\hline 0.22519 & 0.00017 & -0.0057 & 0.01112 & 0.01761 & 0.00989 & 0.1399 & 0.29344 & -0.00587 & 0.02402 \\
\hline
\end{tabular}




\begin{tabular}{|c|c|c|c|c|}
\hline $\mathrm{C} 46$ & $\mathrm{C} 47$ & $\mathrm{C} 48$ & $\mathrm{C} 49$ & C50 \\
\hline-0.01401 & 0.03327 & -0.00932 & 0.02366 & -0.00528 \\
\hline C51 & C52 & $\mathrm{C} 53$ & C54 & N55 \\
\hline 0.00539 & -0.00313 & 0.00455 & -0.00266 & 0.00064 \\
\hline Ir56 & H57 & H58 & H59 & H60 \\
\hline 0.31857 & 0.00032 & 0.00047 & -0.01251 & -0.00152 \\
\hline F61 & F62 & F63 & F64 & O65 \\
\hline 0.01044 & 0.03339 & 0.00324 & 0.00495 & 0.00007 \\
\hline C66 & H67 & H68 & H69 & N70 \\
\hline-0.00004 & 0.00000 & 0.00001 & 0.00000 & -0.01694 \\
\hline C71 & C72 & C73 & C74 & H75 \\
\hline 0.02547 & 0.16304 & 0.00376 & 0.0704 & -0.01112 \\
\hline H76 & O77 & $\mathrm{O} 78$ & H79 & \\
\hline-0.00261 & 0.07678 & 0.03459 & 0.00005 & \\
\hline
\end{tabular}

$\left[{ }^{1} \mathrm{Ir}^{3}{ }^{3} \mathbf{B}_{\mathrm{B}}\right]$

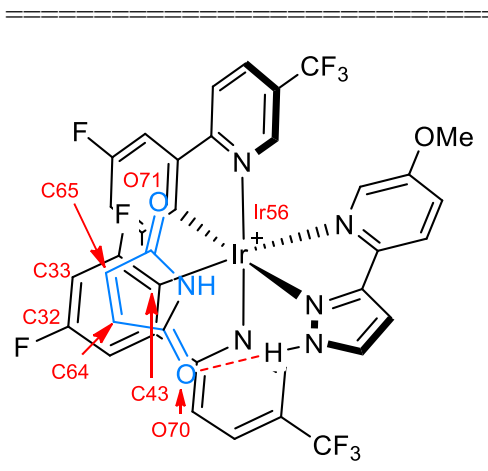

$\begin{array}{lllll}\mathrm{H} 1 & \mathrm{H} 2 & \mathrm{H} 3 & \mathrm{H} 4 & \mathrm{H} 5\end{array}$

$\begin{array}{lllll}-0.00003 & 0.00031 & -0.00066 & -0.0001 & 0.00000\end{array}$

$\begin{array}{lllll}\text { H6 } & \text { H7 } & \text { H8 } & \text { H9 } & \text { H10 }\end{array}$

$\begin{array}{lllll}-0.00001 & 0.00043 & 0.00039 & -0.0013 & -0.00005\end{array}$

$\begin{array}{lllll}\text { H11 } & \text { H12 } & \text { F13 } & \text { F14 } & \text { F15 } \\ 0.00004 & 0.00011 & 0.00000 & 0.00000 & 0.00001\end{array}$

$\begin{array}{lllll}\text { C16 } & \text { F17 } & \text { F18 } & \text { F19 } & \text { C20 }\end{array}$ $\begin{array}{lllll}-0.00009 & 0.00017 & 0.00024 & 0.0006 & -0.00087\end{array}$

$\begin{array}{lllll}\mathrm{C} 21 & \mathrm{C} 22 & \mathrm{C} 23 & \mathrm{~N} 24 & \mathrm{C} 25\end{array}$

$\begin{array}{lllll}-0.00090 & 0.00011 & 0.00018 & -0.00023 & -0.00025\end{array}$

$\begin{array}{lllll}\mathrm{C} 26 & \mathrm{C} 27 & \mathrm{C} 28 & \mathrm{C} 29 & \mathrm{C} 30\end{array}$

$\begin{array}{lllll}-0.00011 & 0.00002 & 0.00002 & 0.00041 & 0.07114\end{array}$

$\begin{array}{lllll}\text { C31 } & \mathbf{C 3 2} & \mathrm{C} 33 & \mathrm{C} 34 & \mathrm{C} 35\end{array}$

$\begin{array}{lllll}0.00425 & \mathbf{0 . 1 4 6 6 5} & 0.04457 & -0.01411 & -0.00937\end{array}$

$\begin{array}{lllll}\text { C36 } & \text { C37 } & \text { C38 } & \text { C39 } & \text { N40 }\end{array}$

$\begin{array}{lllll}0.02133 & -0.00841 & 0.02153 & -0.01821 & -0.00142\end{array}$

N41 N42 C43 $\quad$ C44

$\begin{array}{lllll}0.00343 & 0.01764 & \mathbf{0 . 1 1 5 8 1} & -0.00019 & 0.0045\end{array}$

$\begin{array}{lllll}\mathrm{C} 46 & \mathrm{C} 47 & \mathrm{C} 48 & \mathrm{C} 49 & \mathrm{C} 50\end{array}$

$\begin{array}{lllll}-0.00257 & 0.00604 & -0.00194 & 0.0045 & -0.00117\end{array}$

$\begin{array}{lllll}\mathrm{C} 51 & \mathrm{C} 52 & \mathrm{C} 53 & \mathrm{C} 54 & \mathrm{~N} 55\end{array}$

$\begin{array}{lllll}0.00055 & -0.00066 & 0.00053 & -0.00075 & 0.00033\end{array}$

$\begin{array}{lllll}\text { Ir56 } & \text { O57 } & \text { C58 } & \text { H59 } & \text { H60 }\end{array}$

$\begin{array}{llllll}0.09739 & 0.00000 & -0.00001 & 0.00000 & 0.00001\end{array}$

$\begin{array}{lllll}\text { H61 } & \text { N62 } & \text { C63 } & \text { C64 } & \text { C65 } \\ 0.00000 & 0.04397 & 0.08216 & \mathbf{0 . 4 6 7 2 6} & \mathbf{0 . 5 9 5 2 9}\end{array}$

$\begin{array}{lllll}\text { C66 } & \text { H67 } & \text { H68 } & \text { H69 } & \text { O70 }\end{array}$

$\begin{array}{lllll}-0.0076 & -0.0038 & -0.03047 & -0.03628 & \mathbf{0 . 1 4 4 9 9}\end{array}$

$\begin{array}{lllll}\text { O71 } & \text { H72 } & \text { H73 } & \text { F74 } & \text { F75 }\end{array}$

$\begin{array}{lllll}\mathbf{0 . 2 3 4 1 4} & -0.00495 & -0.00149 & -0.0003 & 0.01532\end{array}$

$\begin{array}{llll}\text { F76 } & \text { F77 } & \text { H78 } & \text { H79 }\end{array}$

$\begin{array}{llll}0.00080 & 0.00093 & 0.00009 & 0.0001\end{array}$

$\left[{ }^{3} \mathbf{I r}-{ }^{-1} \mathbf{Q}_{A}\right]$ 


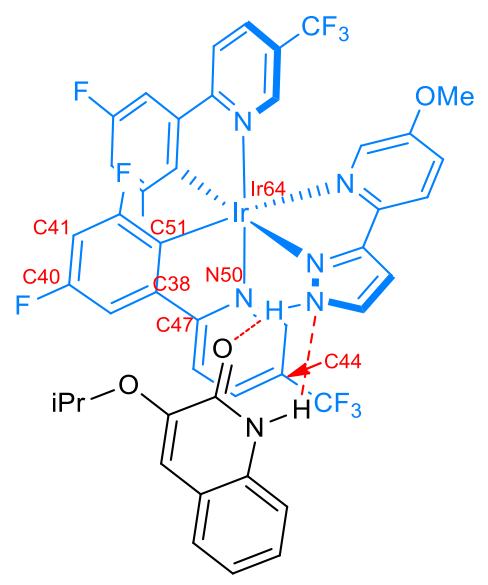

$\begin{array}{lllll}\mathrm{H} 1 & \mathrm{H} 2 & \mathrm{H} 3 & \mathrm{H} 4 & \mathrm{H} 5\end{array}$

$\begin{array}{llllll}0.00014 & 0.00066 & -0.00016 & 0.00036 & 0.00005\end{array}$

$\begin{array}{lllll}\text { H6 } & \text { H7 } & \text { H8 } & \text { H9 } & \text { H10 }\end{array}$

$\begin{array}{lllll}0.00002 & -0.00571 & 0.00321 & -0.00799 & -0.00538\end{array}$

$\begin{array}{lllll}\text { H11 } & \text { H12 } & \text { H13 } & \text { H14 } & \text { F15 }\end{array}$

$\begin{array}{lllll}0.00001 & -0.00002 & -0.00044 & -0.00006 & 0.00000\end{array}$

$\begin{array}{lllll}\text { F16 } & \text { F17 } & \text { C18 } & \text { F19 } & \text { F20 }\end{array}$

$\begin{array}{llllll}0.00012 & 0.00004 & -0.00009 & 0.00765 & 0.00216\end{array}$

$\begin{array}{lllll}\text { F21 } & \text { C22 } & \text { F23 } & \text { H24 } & \text { F25 }\end{array}$

$\begin{array}{llllll}0.00228 & -0.0053 & 0.00203 & -0.00004 & 0.00156\end{array}$

$\begin{array}{lllll}\text { F26 } & \text { H27 } & \text { F28 } & \text { C29 } & \text { C30 }\end{array}$

$\begin{array}{lllll}0.02902 & -0.01912 & 0.00476 & -0.00125 & 0.00003\end{array}$

$\begin{array}{lllll}\mathrm{C} 31 & \mathrm{~N} 32 & \mathrm{C} 33 & \mathrm{C} 34 & \mathrm{C} 35\end{array}$

$\begin{array}{lllll}-0.0006 & 0.0027 & -0.00218 & 0.00059 & -0.00051\end{array}$

$\begin{array}{lllll}\text { C36 } & \text { C37 } & \mathbf{C 3 8} & \mathrm{C} 39 & \mathbf{C 4 0}\end{array}$

$\begin{array}{lllll}0.00054 & -0.00004 & \mathbf{0 . 1 9 8 9 2} & 0.04238 & \mathbf{0 . 1 7 4 2 9}\end{array}$

$\begin{array}{lllll}\mathbf{C} 41 & \mathrm{C} 42 & \mathrm{C} 43 & \mathbf{C 4 4} & \mathrm{C} 45\end{array}$

$\begin{array}{lllll}\mathbf{0 . 2 4 3 4 5} & -0.04406 & -0.0822 & \mathbf{0 . 2 2 6 9} & 0.0807\end{array}$

$\begin{array}{lllll}\mathrm{C} 46 & \mathbf{C 4 7} & \mathrm{N} 48 & \mathrm{~N} 49 & \mathbf{N 5 0}\end{array}$

$\begin{array}{lllll}0.06398 & \mathbf{0 . 1 7 2 7} & -0.00112 & 0.01414 & \mathbf{0 . 1 8 4 9 6}\end{array}$

$\begin{array}{lllll}\mathbf{C} 51 & \mathrm{C} 52 & \mathrm{C} 53 & \mathrm{C} 54 & \mathrm{C} 55\end{array}$

$\begin{array}{lllll}\mathbf{0 . 4 0 2 0 6} & -0.0087 & 0.0104 & -0.0036 & 0.01427\end{array}$

$\begin{array}{lllll}\text { C56 } & \text { C57 } & \text { C58 } & \text { C59 } & \text { C60 } \\ -0.00191 & 0.01112 & 0.00490 & -0.00115 & 0.00585 \\ & & & & \\ \text { C61 } & \text { C62 } & \text { N63 } & \text { Ir64 } & \text { O65 } \\ 0.00284 & -0.00074 & 0.00139 & \mathbf{0 . 2 6 6 1 1} & 0.00021 \\ & & & & \\ \text { C66 } & \text { H67 } & \text { H68 } & \text { H69 } & \text { C70 } \\ -0.00004 & 0.00002 & 0.00003 & 0.00000 & 0.00018 \\ & & & & \\ \text { C71 } & \text { C72 } & \text { H73 } & \text { C74 } & \text { H75 } \\ 0.00001 & 0.00068 & 0.00009 & -0.0003 & 0.00001\end{array}$

$\begin{array}{lllll}\text { C76 } & \mathrm{H} 77 & \mathrm{C} 78 & \mathrm{~N} 79 & \mathrm{C} 80\end{array}$

$\begin{array}{lllll}-0.00017 & 0.00023 & 0.0009 & 0.00126 & -0.00125\end{array}$

$\begin{array}{lllll}\mathrm{H} 81 & \mathrm{C} 82 & \mathrm{H} 83 & \mathrm{C} 84 & \mathrm{O} 85\end{array}$

$\begin{array}{lllll}-0.00002 & 0.0022 & 0.00016 & 0.00075 & 0.00112\end{array}$

$\begin{array}{lllll}086 & \mathrm{H} 87 & \mathrm{C} 88 & \mathrm{H} 89 & \mathrm{C} 90\end{array}$

$\begin{array}{lllll}0.0059 & 0.00023 & -0.0005 & 0.00014 & 0.00000\end{array}$

$\begin{array}{lllll}\text { H91 } & \text { H92 } & \text { H93 } & \text { C94 } & \text { H95 }\end{array}$

$\begin{array}{lllll}0.00012 & 0.00048 & 0.00013 & 0.00049 & 0.00005\end{array}$

H96 H97

$-0.00003-0.00003$

$\left[{ }^{1} \mathbf{I r}-{ }^{3} \mathbf{Q}_{\mathrm{A}}\right]$

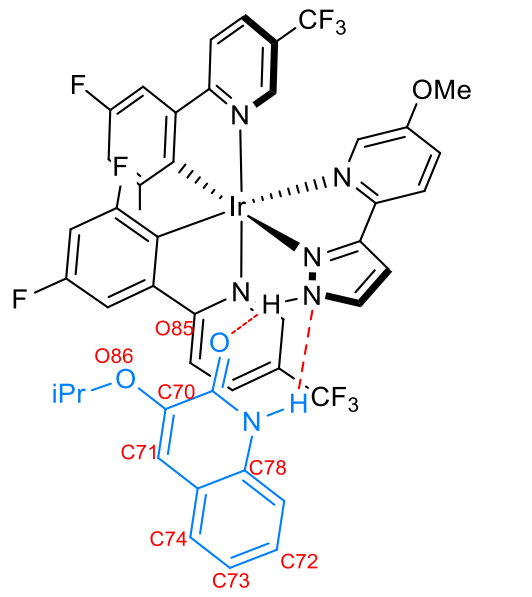




\begin{tabular}{|c|c|c|c|c|c|c|c|c|c|}
\hline & & & & & C71 & C72 & C73 & C74 H75 & \\
\hline H1 & $\mathrm{H} 2$ & H3 & $\mathrm{H} 4$ & H5 & 0.52655 & 0.35091 & -0.12998 & 0.2714 & 0.00594 \\
\hline \multirow[t]{2}{*}{-0.00003} & 0.00014 & -0.00186 & 0.00003 & 0.00000 & & & & & \\
\hline & & & & & C76 & $\mathrm{H} 77$ & C78 & H79 & $\mathrm{C} 80$ \\
\hline H6 & H7 & H8 & H9 & H10 & -0.08433 & -0.02497 & 0.18400 & 0.00283 & -0.08675 \\
\hline \multirow[t]{2}{*}{0.00000} & -0.00001 & 0.0006 & -0.00004 & 0.00000 & & & & & \\
\hline & & & & & N81 & H8 & $\mathrm{C} 83$ & H84 & 085 \\
\hline H11 & H12 & F13 & F14 & F15 & 0.08386 & -0.01757 & 0.07907 & -0.00489 & 0.19396 \\
\hline \multirow[t]{2}{*}{0.00000} & -0.00003 & 0.00000 & 0.00000 & 0.00000 & & & & & \\
\hline & & & & & 086 & H87 & C88 & H89 & $\mathrm{C} 90$ \\
\hline $\mathrm{C} 16$ & F17 & F18 & F19 & $\mathrm{C} 20$ & 0.19683 & -0.03843 & -0.01186 & 0.00602 & 0.00232 \\
\hline \multirow[t]{2}{*}{0.00000} & 0.00003 & 0.00002 & 0.00008 & -0.00011 & & & & & \\
\hline & & & & & H91 & H92 H93 & C94 H95 & & \\
\hline $\mathrm{C} 21$ & $\mathrm{C} 22$ & $\mathrm{C} 23$ & $\mathrm{~N} 24$ & $\mathrm{C} 25$ & -0.00015 & -0.00019 & -0.00022 & 0.01377 & 0.00063 \\
\hline \multirow[t]{2}{*}{0.00004} & 0.00015 & 0.0006 & -0.00094 & -0.00003 & & & & & \\
\hline & & & & & H96 & H97 & & & \\
\hline $\mathrm{C} 26$ & $\mathrm{C} 27$ & $\mathrm{C} 28$ & $\mathrm{C} 29$ & $\mathrm{C} 30$ & -0.00015 & -0.00014 & & & \\
\hline 0.00003 & -0.00001 & 0.00002 & -0.00001 & 0.00093 & & & & & \\
\hline $\mathrm{C} 31$ & $\mathrm{C} 32$ & C33 & $\mathrm{C} 34$ & $\mathrm{C} 35$ & {$\left[{ }^{3} \mathbf{I r}-{ }^{1} \mathbf{Q}_{B}\right]$} & & & & \\
\hline 0.00027 & 0.0058 & -0.0017 & 0.0162 & -0.00043 & & & & & \\
\hline C36 & $\mathrm{C} 37$ & C38 & C39 & N40 & & & & & \\
\hline 0.00298 & -0.00131 & 0.00309 & 0.00257 & -0.00004 & & & & & \\
\hline N41 & N42 & $\mathrm{C} 43$ & $\mathrm{C} 44$ & $\mathrm{C} 45$ & & & & & \\
\hline-0.00009 & 0.00063 & 0.00861 & 0.00016 & -0.00001 & & & & & \\
\hline C46 & $\mathrm{C} 47$ & $\mathrm{C} 48$ & $\mathrm{C} 49$ & C50 & & & & & \\
\hline \multirow[t]{2}{*}{0.00001} & -0.00002 & 0.00002 & -0.00009 & -0.00008 & & & $\mathrm{CF}_{3}$ & & \\
\hline & & & & & $\mathrm{H} 1$ & $\mathrm{H} 2$ & $\mathrm{H} 3$ & $\mathrm{H} 4$ & H5 \\
\hline C51 & C52 & $\mathrm{C} 53$ & $\mathrm{C} 54$ & N55 & 0.00000 & 0.00018 & 0.00008 & 0.00139 & 0.00018 \\
\hline \multirow[t]{2}{*}{0.00001} & -0.00003 & 0.00004 & -0.00002 & 0.00002 & & & & & \\
\hline & & & & & H6 & $\mathrm{H} 7$ & H8 & H9 & H10 \\
\hline Ir56 & H57 & H58 & H59 & H60 & 0.00001 & -0.00014 & 0.00016 & -0.00038 & -0.00404 \\
\hline \multirow[t]{2}{*}{0.00334} & 0.00000 & 0.00001 & -0.00006 & 0.00017 & & & & & \\
\hline & & & & & H11 & H12 & F13 & F14 & F15 \\
\hline F61 & F62 & F63 & F64 & O65 & -0.00906 & 0.00355 & 0.00671 & 0.00342 & 0.00171 \\
\hline \multirow[t]{2}{*}{0.00183} & 0.00066 & 0.00000 & 0.00000 & 0.00001 & & & & & \\
\hline & & & & & $\mathrm{C} 16$ & F17 & F18 & F19 & $\mathrm{C} 20$ \\
\hline C66 & H67 & H68 & H69 & C70 & -0.00454 & 0.00004 & 0.00005 & 0.00014 & -0.00019 \\
\hline \multirow[t]{3}{*}{0.00000} & 0.00000 & 0.00000 & 0.00000 & 0.43942 & & & & & \\
\hline & & & & & $\mathrm{H} 21$ & $\mathrm{H} 22$ & $\mathrm{C} 23$ & $\mathrm{C} 24$ & $\mathrm{C} 25$ \\
\hline & & & & & & & & & \\
\hline
\end{tabular}




\begin{tabular}{|c|c|c|c|c|c|c|c|c|c|}
\hline-0.01941 & 0.00071 & -0.00134 & -0.00013 & -0.00026 & & & & & \\
\hline & & & & & F96 & F97 & & & \\
\hline N26 & $\mathrm{C} 27$ & $\mathrm{C} 28$ & $\mathrm{C} 29$ & $\mathrm{C} 30$ & 0.00458 & 0.00519 & & & \\
\hline 0.00141 & 0.00056 & 0.00184 & -0.00184 & 0.00207 & & & & & \\
\hline $\mathrm{C} 31$ & C32 & C33 & C34 & $\mathrm{C} 35$ & {$\left[{ }^{1} \mathbf{I r}-{ }^{3} \mathbf{Q}_{\mathrm{B}}\right]$} & & & & \\
\hline-0.00060 & 0.02973 & -0.01226 & 0.04247 & -0.02030 & & & & & \\
\hline $\mathrm{C} 36$ & C37 & $\mathrm{C} 38$ & C39 & $\mathrm{C} 40$ & & & & $\mathrm{Me}$ & \\
\hline 0.03193 & -0.00363 & 0.00491 & -0.00282 & 0.00543 & & & & & \\
\hline $\mathrm{C} 41$ & N42 & N43 & N44 & $\mathrm{C} 45$ & & & & & \\
\hline-0.00624 & 0.00992 & -0.00241 & 0.00110 & -0.01643 & & & & & \\
\hline C46 & $\mathrm{C} 47$ & C48 & C49 & $\mathrm{C} 50$ & & & $\mathrm{CF}_{3}$ & & \\
\hline 0.34418 & -0.04254 & 0.24469 & 0.14119 & 0.06206 & & & & & \\
\hline C51 & C52 & $\mathrm{C} 53$ & C54 & C55 & $\mathrm{H} 1$ & $\mathrm{H} 2$ & $\mathrm{H} 3$ & $\mathrm{H} 4$ & H5 \\
\hline 0.16716 & 0.19828 & 0.03602 & 0.10091 & 0.22049 & 0.00004 & 0.00012 & -0.00117 & -0.00002 & 0.00000 \\
\hline $\mathrm{C} 56$ & N57 & Ir58 & C59 & $\mathrm{C} 60$ & H6 & $\mathrm{H} 7$ & $\mathrm{H} 8$ & H9 & $\mathrm{H} 10$ \\
\hline-0.08404 & 0.19305 & 0.33673 & -0.00021 & 0.00057 & 0.00000 & 0.00006 & 0.00003 & -0.00020 & 0.00001 \\
\hline C61 & C62 & C63 & C64 & N65 & H11 & H12 & F13 & F14 & F15 \\
\hline 0.00000 & -0.00001 & -0.00008 & 0.00064 & -0.00013 & 0.00001 & 0.00009 & 0.00002 & 0.00001 & 0.00000 \\
\hline C66 & C67 & C68 & O69 & $\mathrm{O} 70$ & $\mathrm{C} 16$ & F17 & F18 & F19 & $\mathrm{C} 20$ \\
\hline-0.00007 & 0.00011 & 0.00038 & 0.00008 & -0.00009 & -0.00004 & 0.00003 & 0.00003 & 0.00009 & -0.00012 \\
\hline C71 & $\mathrm{C} 72$ & $\mathrm{H} 73$ & $\mathrm{H} 74$ & $\mathrm{H} 75$ & $\mathrm{C} 21$ & $\mathrm{C} 22$ & $\mathrm{C} 23$ & $\mathrm{~N} 24$ & $\mathrm{C} 25$ \\
\hline 0.00006 & 0.00000 & 0.00000 & -0.00001 & 0.00018 & -0.00005 & 0.00003 & 0.00050 & -0.00039 & 0.00002 \\
\hline H76 & H77 & $\mathrm{H} 78$ & H79 & H80 & $\mathrm{C} 26$ & $\mathrm{C} 27$ & $\mathrm{C} 28$ & $\mathrm{C} 29$ & $\mathrm{C} 30$ \\
\hline-0.00004 & 0.00013 & -0.00003 & 0.00001 & 0.00000 & 0.00002 & 0.00000 & -0.00001 & -0.00005 & 0.01232 \\
\hline H81 & H82 & $\mathrm{C} 83$ & H84 & H85 & $\mathrm{C} 31$ & C32 & $\mathrm{C} 33$ & $\mathrm{C} 34$ & $\mathrm{C} 35$ \\
\hline-0.00002 & 0.00000 & 0.00001 & 0.00000 & 0.00000 & -0.00406 & 0.00445 & -0.00087 & 0.00663 & -0.00152 \\
\hline H86 & O87 & $\mathrm{C} 88$ & H89 & H90 & $\mathrm{C} 36$ & C37 & C38 & C39 & N40 \\
\hline 0.00000 & 0.00077 & -0.00005 & 0.00011 & -0.00011 & 0.00344 & -0.00084 & 0.00281 & -0.00183 & 0.00004 \\
\hline H91 & H92 & F93 & F94 & H95 & N41 & N42 & $\mathrm{C} 43$ & $\mathrm{C} 44$ & $\mathrm{C} 45$ \\
\hline-0.00001 & -0.00629 & 0.02793 & 0.00414 & 0.00039 & -0.00056 & 0.00287 & -0.00519 & 0.00030 & -0.00014 \\
\hline
\end{tabular}




\begin{tabular}{|c|c|c|c|c|c|c|c|c|c|}
\hline $\mathrm{C} 46$ & $\mathrm{C} 47$ & $\mathrm{C} 48$ & C49 & C50 & & & & & \\
\hline 0.00027 & -0.00009 & 0.00024 & -0.00014 & 0.00068 & & & & & \\
\hline C51 & $\mathrm{C} 52$ & C53 & C54 & N55 & & & & & \\
\hline-0.00017 & 0.00044 & 0.00065 & -0.00027 & -0.00001 & & & & & \\
\hline $\operatorname{Ir} 56$ & H57 & H58 & H59 & H60 & & & & & \\
\hline \multirow[t]{2}{*}{0.00198} & -0.00002 & -0.00002 & 0.00111 & 0.00032 & & $\mathrm{H}$ & & & \\
\hline & & & & & $\mathrm{H} 1$ & $\mathrm{H} 2$ & $\mathrm{H} 3$ & $\mathrm{H} 4$ & H5 \\
\hline F61 & F62 & F63 & F64 & O65 & 0.00009 & 0.00062 & -0.00092 & 0.00019 & 0.00003 \\
\hline \multirow[t]{2}{*}{0.00136} & 0.00086 & 0.00001 & 0.00000 & 0.00001 & & & & & \\
\hline & & & & & H6 & H7 & $\mathrm{H} 8$ & H9 & H10 \\
\hline C66 & H67 & H68 & H69 & C70 & 0.00001 & 0.00344 & -0.00609 & -0.00659 & -0.00006 \\
\hline \multirow[t]{2}{*}{0.00000} & 0.00000 & 0.00000 & 0.00000 & 0.34999 & & & & & \\
\hline & & & & & H11 & H12 & F13 & F14 & F15 \\
\hline C71 & C72 & $\mathrm{H} 73$ & $\mathrm{C} 74$ & $\mathrm{H} 75$ & -0.00016 & 0.00005 & 0.00001 & 0.00008 & 0.00003 \\
\hline \multirow[t]{2}{*}{-0.12796} & 0.26702 & 0.00583 & -0.08625 & -0.02448 & & & & & \\
\hline & & & & & $\mathrm{C} 16$ & F17 & F18 & F19 & $\mathrm{C} 20$ \\
\hline C76 & $\mathrm{H} 77$ & $\mathrm{C} 78$ & N79 & C80 & -0.00011 & 0.00399 & 0.00165 & 0.00692 & -0.00676 \\
\hline \multirow[t]{2}{*}{0.18678} & 0.00277 & -0.08432 & 0.08791 & 0.53743 & & & & & \\
\hline & & & & & $\mathrm{C} 21$ & $\mathrm{C} 22$ & $\mathrm{C} 23$ & $\mathrm{~N} 24$ & $\mathrm{C} 25$ \\
\hline H81 & $\mathrm{C} 82$ & $\mathrm{H} 83$ & C84 & 085 & -0.00186 & 0.00025 & -0.00122 & 0.00245 & -0.0019 \\
\hline \multirow[t]{2}{*}{-0.01763} & 0.07934 & -0.00489 & 0.45776 & 0.17983 & & & & & \\
\hline & & & & & $\mathrm{C} 26$ & $\mathrm{C} 27$ & $\mathrm{C} 28$ & $\mathrm{C} 29$ & $\mathrm{C} 30$ \\
\hline 086 & H87 & $\mathrm{C} 88$ & H89 & $\mathrm{C} 90$ & 0.00008 & -0.0003 & 0.00004 & 0.00000 & 0.23447 \\
\hline \multirow[t]{2}{*}{0.19664} & -0.04014 & -0.01179 & 0.00693 & 0.01430 & & & & & \\
\hline & & & & & C31 & C32 & C33 & $\mathrm{C} 34$ & $\mathrm{C} 35$ \\
\hline H91 & H92 & H93 & C94 & H95 & 0.03411 & 0.15255 & 0.28577 & -0.05146 & -0.08872 \\
\hline \multirow[t]{2}{*}{-0.00028} & 0.00013 & 0.00031 & 0.00132 & -0.00049 & & & & & \\
\hline & & & & & C36 & $\mathrm{C} 37$ & $\mathrm{C} 38$ & C39 & N40 \\
\hline H96 & H97 & & & & 0.24585 & 0.05279 & 0.08202 & 0.15235 & -0.00183 \\
\hline \multirow[t]{3}{*}{-0.00013} & -0.00008 & & & & & & & & \\
\hline & & & & & N41 & N42 & $\mathrm{C43}$ & $\mathrm{C} 44$ & $\mathrm{C} 45$ \\
\hline & & & & & 0.01155 & 0.19768 & 0.38215 & -0.01015 & 0.01317 \\
\hline \multicolumn{10}{|l|}{$\left[{ }^{3} \mathbf{I r}-{ }^{1} \mathbf{Q}_{\mathbf{C}}\right]$} \\
\hline \multirow{5}{*}{$====$} & & & 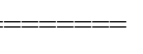 & & $\mathrm{C} 46$ & $\mathrm{C} 47$ & $\mathrm{C} 48$ & $\mathrm{C} 49$ & $\mathrm{C} 50$ \\
\hline & & & & & -0.0075 & 0.01599 & -0.00442 & 0.01245 & 0.00044 \\
\hline & & & & & $\mathrm{C} 51$ & C52 & $\mathrm{C} 53$ & C54 & N55 \\
\hline & & & & & 0.00041 & 0.00211 & 0.00206 & -0.0015 & 0.00043 \\
\hline & & & & & Ir56 & H57 & H58 & H59 & H60 \\
\hline & & & & & & & & & \\
\hline
\end{tabular}




\begin{tabular}{|c|c|c|c|c|c|c|c|c|c|}
\hline \multirow[t]{2}{*}{0.24492} & 0.00014 & 0.00023 & -0.02253 & -0.00544 & H11 & H12 & H13 & H14 & F15 \\
\hline & & & & & 0.00000 & 0.00000 & 0.00000 & -0.00001 & 0.00000 \\
\hline F61 & F62 & F63 & F64 & O65 & & & & & \\
\hline \multirow[t]{2}{*}{0.0028} & 0.0271 & 0.00225 & 0.00215 & 0.00006 & F16 & F17 & $\mathrm{C} 18$ & F19 & F20 \\
\hline & & & & & 0.00000 & 0.00000 & -0.00001 & 0.00003 & 0.00009 \\
\hline C66 & H67 & H68 & H69 & $\mathrm{C} 70$ & & & & & \\
\hline \multirow[t]{2}{*}{-0.00002} & 0.00000 & 0.00000 & 0.00000 & -0.00165 & F21 & $\mathrm{C} 22$ & F23 & $\mathrm{H} 24$ & F25 \\
\hline & & & & & 0.00005 & -0.00009 & 0.00000 & 0.00000 & 0.00001 \\
\hline C71 & $\mathrm{C} 72$ & $\mathrm{H} 73$ & C74 & H75 & & & & & \\
\hline \multirow[t]{2}{*}{0.00513} & -0.00232 & -0.00034 & 0.00287 & 0.00007 & F26 & $\mathrm{H} 27$ & F28 & $\mathrm{C} 29$ & $\mathrm{C} 30$ \\
\hline & & & & & 0.00286 & 0.00034 & -0.00004 & -0.00009 & 0.00008 \\
\hline $\mathrm{C} 76$ & H77 & $\mathrm{C} 78$ & N79 & $\mathrm{C} 80$ & & & & & \\
\hline \multirow[t]{2}{*}{0.0013} & -0.00015 & 0.0034 & 0.01402 & 0.00398 & C31 & N32 & C33 & C34 & C35 \\
\hline & & & & & 0.00021 & -0.00084 & -0.00002 & -0.00001 & 0.00000 \\
\hline H81 & $\mathrm{C} 82$ & H83 & C84 & O85 & & & & & \\
\hline \multirow[t]{2}{*}{0.00011} & -0.00441 & 0.00023 & 0.00388 & 0.01329 & $\mathrm{C} 36$ & $\mathrm{C} 37$ & $\mathrm{C} 38$ & C39 & $\mathrm{C} 40$ \\
\hline & & & & & -0.00001 & 0.00003 & 0.00308 & 0.00904 & 0.00933 \\
\hline O86 & H87 & C88 & H89 & C90 & & & & & \\
\hline \multirow[t]{2}{*}{0.00219} & -0.00023 & -0.00018 & 0.00009 & 0.00018 & $\mathrm{C} 41$ & $\mathrm{C} 42$ & $\mathrm{C} 43$ & $\mathrm{C} 44$ & $\mathrm{C} 45$ \\
\hline & & & & & -0.00114 & 0.00134 & -0.00164 & 0.00353 & 0.00286 \\
\hline H91 & H92 & H93 & C94 & H95 & & & & & \\
\hline \multirow[t]{2}{*}{-0.00002} & 0.00002 & 0.00008 & 0.00004 & -0.00001 & $\mathrm{C} 46$ & $\mathrm{C} 47$ & N48 & N49 & N50 \\
\hline & & & & & 0.00080 & 0.00188 & -0.00002 & -0.00010 & 0.00388 \\
\hline H96 & H97 & & & & & & & & \\
\hline \multirow{10}{*}{$\begin{array}{l}0.00000 \\
====== \\
{\left[{ }^{1} \mathbf{I r}-{ }^{3} \mathbf{Q}_{\mathbf{C}}\right]}\end{array}$} & 0.00001 & & & & C51 & C52 & C53 & C54 & C55 \\
\hline & & & & & 0.00329 & 0.00018 & -0.00004 & 0.00001 & -0.00002 \\
\hline & & & & & & & & & \\
\hline & & & $====$ & & C56 & C57 & C58 & C59 & C60 \\
\hline & & & & & 0.00001 & -0.00007 & 0.00000 & -0.00001 & -0.00001 \\
\hline & & & & & C61 & C62 & N63 & Ir64 & O65 \\
\hline & & & & & -0.00001 & -0.00005 & -0.00003 & 0.00255 & 0.00000 \\
\hline & & & & & C66 & H67 & H68 & H69 & C70 \\
\hline & & & & & 0.00000 & 0.00000 & 0.00000 & 0.00000 & 0.35802 \\
\hline & $\mathrm{H}$ & & & & C71 & C72 & $\mathrm{H} 73$ & C74 & H75 \\
\hline H1 & $\mathrm{H} 2$ & $\mathrm{H} 3$ & $\mathrm{H} 4$ & H5 & -0.13380 & 0.27231 & 0.00614 & -0.08431 & -0.02499 \\
\hline \multirow[t]{2}{*}{0.00000} & 0.00005 & -0.00150 & -0.00001 & 0.00000 & & & & & \\
\hline & & & & & C76 & $\mathrm{H} 77$ & C78 & N79 & $\mathrm{C} 80$ \\
\hline H6 & $\mathrm{H} 7$ & H8 & H9 & $\mathrm{H} 10$ & 0.18173 & 0.00281 & -0.07563 & 0.09039 & 0.51567 \\
\hline \multirow[t]{3}{*}{0.00000} & -0.00050 & 0.00005 & -0.00020 & -0.00001 & & & & & \\
\hline & & & & & H81 & $\mathrm{C} 82$ & H83 & C84 & 085 \\
\hline & & & & & & & & & \\
\hline
\end{tabular}




\begin{tabular}{|c|c|c|c|c|c|c|c|c|c|}
\hline \multirow[t]{2}{*}{-0.01777} & 0.06130 & -0.00550 & 0.48680 & 0.17920 & \multirow[b]{2}{*}{ C36 } & \multirow[b]{2}{*}{$\mathrm{C} 37$} & \multirow[b]{2}{*}{ C38 } & \multirow[b]{2}{*}{ C39 } & \multirow[b]{2}{*}{$\mathrm{C} 40$} \\
\hline & & & & & & & & & \\
\hline 086 & H87 & C88 & H89 & C90 & -0.00281 & 0.00457 & -0.00249 & 0.00515 & -0.00549 \\
\hline \multirow[t]{2}{*}{0.17771} & -0.04013 & -0.00990 & 0.00653 & 0.00037 & & & & & \\
\hline & & & & & N41 & N42 & N43 & $\mathrm{C} 44$ & $\mathrm{C45}$ \\
\hline H91 & H92 & H93 & C94 & H95 & 0.01132 & -0.00282 & 0.00054 & -0.02178 & 0.35339 \\
\hline \multirow[t]{2}{*}{0.00002} & 0.00022 & -0.00061 & 0.01404 & 0.00018 & & & & & \\
\hline & & & & & $\mathrm{C} 46$ & $\mathrm{C} 47$ & $\mathrm{C} 48$ & C49 & C50 \\
\hline H96 & H97 & & & & -0.04670 & 0.26047 & 0.14613 & 0.05367 & 0.19264 \\
\hline \multirow[t]{2}{*}{0.00036} & -0.00027 & & & & & & & & \\
\hline & & & & & C51 & C52 & C53 & C54 & C55 \\
\hline & & & $===$ & & 0.17797 & 0.06585 & 0.07328 & 0.24134 & -0.08737 \\
\hline \multicolumn{10}{|l|}{$\left[{ }^{3} \mathbf{I r}-{ }^{1} \mathbf{Q}_{\mathrm{D}}\right]$} \\
\hline & & & $=====$ & & N56 & Ir57 & C58 & C59 & C60 \\
\hline & & & & & 0.18737 & 0.31192 & 0.00005 & -0.00006 & 0.00011 \\
\hline & & & & & C61 & C62 & C63 & N64 & C65 \\
\hline & & & & & -0.00005 & 0.00001 & -0.00005 & 0.00015 & 0.00004 \\
\hline & & & & & C66 & C67 & O68 & O69 & $\mathrm{C} 70$ \\
\hline & & & & & 0.00007 & -0.00033 & 0.00015 & 0.00001 & 0.00012 \\
\hline & $\mathrm{iPr}$ & & & & C71 & $\mathrm{H} 72$ & $\mathrm{H} 73$ & $\mathrm{H} 74$ & $\mathrm{H} 75$ \\
\hline H1 & $\mathrm{H} 2$ & $\mathrm{H} 3$ & $\mathrm{H} 4$ & H5 & -0.00005 & 0.00000 & 0.00000 & 0.00008 & 0.00000 \\
\hline \multirow[t]{2}{*}{-0.00002} & 0.00013 & 0.00002 & 0.00071 & 0.00012 & & & & & \\
\hline & & & & & H76 & $\mathrm{H} 77$ & $\mathrm{H} 78$ & H79 & H80 \\
\hline H6 & H7 & H8 & H9 & H10 & 0.00001 & 0.00003 & -0.00012 & 0.00000 & -0.00004 \\
\hline \multirow[t]{2}{*}{0.00005} & -0.00022 & 0.00014 & -0.00037 & -0.00585 & & & & & \\
\hline & & & & & F81 & F82 & H83 & O84 & $\mathrm{C} 85$ \\
\hline H11 & H12 & F13 & F14 & F15 & 0.00356 & 0.02826 & -0.00611 & 0.00074 & -0.00010 \\
\hline \multirow[t]{2}{*}{-0.00723} & 0.00369 & 0.00293 & 0.00841 & 0.00229 & & & & & \\
\hline & & & & & H86 & H87 & H88 & F89 & F90 \\
\hline C16 & F17 & F18 & F19 & $\mathrm{C} 20$ & 0.00024 & -0.00004 & -0.00002 & 0.00430 & 0.00340 \\
\hline \multirow[t]{2}{*}{-0.00347} & 0.00004 & 0.00005 & 0.00012 & -0.00017 & & & & & \\
\hline & & & & & H91 & H92 & H93 & C94 & H95 \\
\hline $\mathrm{H} 21$ & $\mathrm{C} 22$ & $\mathrm{C} 23$ & $\mathrm{C} 24$ & N25 & 0.00070 & 0.00039 & 0.00000 & 0.00002 & 0.00000 \\
\hline \multirow[t]{2}{*}{-0.02046} & -0.00122 & -0.00004 & -0.00038 & 0.00126 & & & & & \\
\hline & & & & & H96 & H97 & & & \\
\hline $\mathrm{C} 26$ & $\mathrm{C} 27$ & $\mathrm{C} 28$ & $\mathrm{C} 29$ & $\mathrm{C} 30$ & 0.00000 & 0.00000 & & & \\
\hline 0.00116 & 0.00180 & -0.00117 & 0.00148 & -0.00041 & & & & & \\
\hline $\mathrm{C} 31$ & $\mathrm{C} 32$ & $\mathrm{C} 33$ & C34 & $\mathrm{C} 35$ & {$\left[{ }^{1} \mathbf{I r}-{ }^{3} Q_{D}\right]$} & & & & \\
\hline 0.02840 & -0.01170 & 0.03649 & -0.01898 & 0.03078 & $=====$ & & & & \\
\hline
\end{tabular}




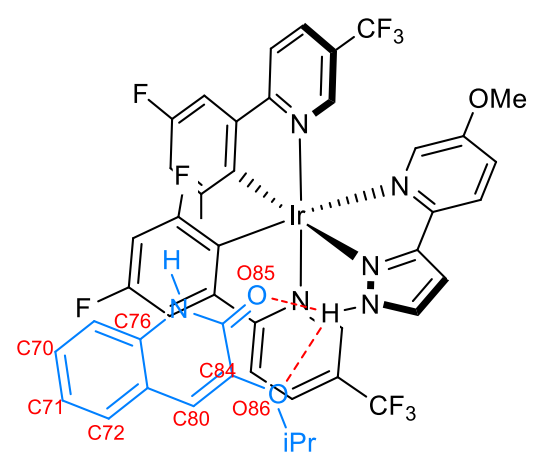

$\begin{array}{lllll}\text { H1 } & \text { H2 } & \text { H3 } & \text { H4 } & \text { H5 } \\ 0.00003 & 0.00003 & -0.00100 & 0.00000 & 0.00000\end{array}$

$\begin{array}{lllll}\text { H6 } & \mathrm{H} 7 & \mathrm{H} 8 & \mathrm{H} 9 & \mathrm{H} 10\end{array}$

$\begin{array}{lllll}0.00000 & 0.00014 & 0.00000 & -0.00027 & -0.00006\end{array}$

H11 H12 F13 F14 F15

$\begin{array}{lllll}-0.00003 & -0.00052 & 0.00000 & -0.00002 & 0.00001\end{array}$

$\begin{array}{lllll}\text { C16 } & \text { F17 } & \text { F18 } & \text { F19 } & \text { C20 }\end{array}$

$\begin{array}{lllll}0.00003 & 0.00006 & 0.00007 & 0.00018 & -0.00020\end{array}$

$\begin{array}{lllll}\mathrm{C} 21 & \mathrm{C} 22 & \mathrm{C} 23 & \mathrm{~N} 24 & \mathrm{C} 25\end{array}$

$\begin{array}{lllll}-0.00015 & 0.00029 & 0.00024 & 0.00022 & 0.00001\end{array}$

$\begin{array}{lllll}\mathrm{C} 26 & \mathrm{C} 27 & \mathrm{C} 28 & \mathrm{C} 29 & \mathrm{C} 30\end{array}$

$\begin{array}{lllll}0.00000 & 0.00000 & -0.00002 & 0.00002 & 0.01292\end{array}$

$\begin{array}{lllll}\mathrm{C} 31 & \mathrm{C} 32 & \mathrm{C} 33 & \mathrm{C} 34 & \mathrm{C} 35\end{array}$

$\begin{array}{lllll}0.00831 & 0.00198 & 0.01428 & 0.01105 & -0.00267\end{array}$

$\begin{array}{lllll}\text { C36 } & \text { C37 } & \text { C38 } & \text { C39 } & \text { N40 }\end{array}$

$\begin{array}{llllll}0.00645 & -0.00078 & 0.00403 & 0.00054 & 0.00004\end{array}$

$\begin{array}{lllll}\text { N41 } & \text { N42 } & \text { C43 } & \text { C44 } & \text { C45 } \\ -0.00003 & 0.00479 & -0.00816 & 0.00002 & -0.00001\end{array}$

$\begin{array}{lllll}\mathrm{C} 46 & \mathrm{C} 47 & \mathrm{C} 48 & \mathrm{C} 49 & \mathrm{C} 50\end{array}$

$\begin{array}{lllll}-0.00001 & 0.00001 & -0.00003 & 0.00003 & -0.00013\end{array}$

$\begin{array}{lllll}\mathrm{C} 51 & \mathrm{C} 52 & \mathrm{C} 53 & \mathrm{C} 54 & \mathrm{~N} 55\end{array}$

$\begin{array}{llllll}0.00082 & 0.00037 & 0.00038 & 0.00210 & 0.00082\end{array}$

$\begin{array}{lllll}\text { Ir56 } & \text { H57 } & \text { H58 } & \text { H59 } & \text { H60 } \\ 0.00000 & 0.00000 & 0.00000 & 0.00032 & -0.00062 \\ & & & & \\ \text { F61 } & \text { F62 } & \text { F63 } & \text { F64 } & \text { O65 } \\ 0.00079 & 0.00009 & 0.00001 & 0.00000 & 0.00000 \\ & & & & \\ \text { C66 } & \text { H67 } & \text { H68 } & \text { H69 } & \text { C70 } \\ 0.00000 & 0.00000 & 0.00000 & 0.00000 & \mathbf{0 . 3 4 0 9 8} \\ & & & & \\ \text { C71 } & \mathbf{C 7 2} & \text { H73 } & \text { C74 } & \text { H75 } \\ \mathbf{- 0 . 1 2 0 6 7} & \mathbf{0 . 2 5 6 6 9} & 0.00539 & -0.08049 & -0.02384 \\ & & & & \\ \text { C76 } & \text { H77 } & \text { C78 } & \text { N79 } & \mathbf{C 8 0} \\ \mathbf{0 . 1 7 8 2 3} & 0.00272 & -0.07871 & 0.09027 & \mathbf{0 . 5 1 9 6 8}\end{array}$

$\begin{array}{lllll}\mathrm{H} 81 & \mathrm{C} 82 & \mathrm{H} 83 & \mathbf{C 8 4} & \mathbf{0 8 5}\end{array}$

$\begin{array}{lllll}-0.01691 & 0.06030 & -0.00508 & \mathbf{0 . 4 9 3 2 3} & \mathbf{0 . 1 7 0 7 0}\end{array}$

$\begin{array}{lllll}086 & \mathrm{H} 87 & \mathrm{C} 88 & \mathrm{H} 89 & \mathrm{C} 90\end{array}$

$\begin{array}{llllll}\mathbf{0 . 1 7 9 3 7} & -0.03970 & -0.01187 & 0.00881 & 0.01381\end{array}$

$\begin{array}{lllll}\text { H91 } & \text { H92 } & \text { H93 } & \text { C94 } & \text { H95 }\end{array}$

$\begin{array}{lllll}-0.00042 & 0.00069 & 0.00004 & 0.00060 & -0.00066\end{array}$

H96 H97

$-0.00004 \quad 0.00008$

[ $\left.{ }^{3}{ }^{1}-{ }^{1} Q-{ }^{12}\right]$

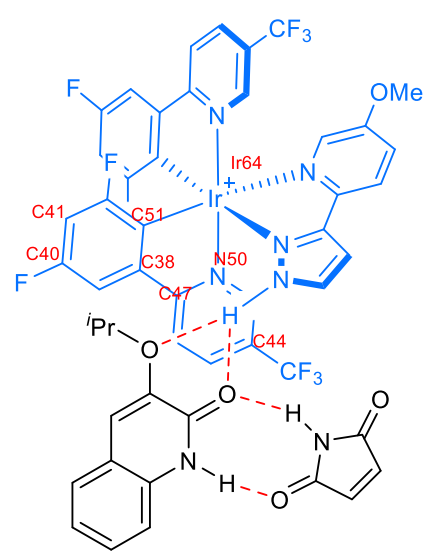

$\begin{array}{lllll}\mathrm{H} 1 & \mathrm{H} 2 & \mathrm{H} 3 & \mathrm{H} 4 & \mathrm{H} 5\end{array}$ 
$\begin{array}{lllll}0.00007 & 0.00067 & -0.00063 & 0.00011 & 0.00002\end{array}$

$\begin{array}{lllll}\text { H6 } & \text { H7 } & \text { H8 } & \text { H9 } & \text { H10 } \\ 0.00000 & -0.00482 & 0.00295 & -0.00776 & -0.00396\end{array}$

$\begin{array}{lllll}\text { H11 } & \text { H12 } & \text { H13 } & \text { H14 } & \text { F15 } \\ 0.00024 & -0.00017 & 0.00007 & 0.00018 & 0.00004\end{array}$

$\begin{array}{lllll}\text { F16 } & \text { F17 } & \text { C18 } & \text { F19 } & \text { F20 } \\ 0.00007 & 0.00002 & -0.00010 & 0.00175 & 0.00697\end{array}$

$\begin{array}{lllll}\text { F21 } & \text { C22 } & \text { F23 } & \text { H24 } & \text { F25 }\end{array}$

$\begin{array}{llllll}0.00320 & -0.00573 & 0.00249 & 0.00031 & 0.00216\end{array}$

$\begin{array}{lllll}\text { F26 } & \text { H27 } & \text { F28 } & \text { C29 } & \text { C30 }\end{array}$

$\begin{array}{llllll}0.02951 & -0.01627 & 0.00525 & -0.00097 & 0.00029\end{array}$

$\begin{array}{lllll}\text { C31 } & \text { N32 } & \text { C33 } & \text { C34 } & \text { C35 } \\ -0.00062 & 0.00275 & -0.00092 & 0.00058 & -0.00040\end{array}$

$\begin{array}{lllll}\text { C36 } & \text { C37 } & \text { C38 } & \text { C39 } & \text { C40 } \\ 0.00049 & 0.00039 & \mathbf{0 . 1 5 6 9 3} & 0.02880 & \mathbf{0 . 1 6 2 2 1}\end{array}$

$\begin{array}{llllll}\mathbf{C 4 1} & \mathrm{C} 42 & \mathrm{C} 43 & \mathbf{C} 44 & \mathrm{C} 45\end{array}$

$\begin{array}{llllll}\mathbf{0 . 1 9 3 9 3} & -0.01098 & -0.06932 & \mathbf{0 . 2 1 2 3 9} & 0.07619\end{array}$

$\begin{array}{llllll}\mathrm{C} 46 & \mathbf{C 4 7} & \mathrm{N} 48 & \mathrm{~N} 49 & \mathbf{N 5 0}\end{array}$

$\begin{array}{llllll}0.04030 & \mathbf{0 . 1 6 2 4 0} & -0.00336 & 0.01358 & \mathbf{0 . 1 3 9 7 4}\end{array}$

$\begin{array}{llllll}\mathbf{C 5 1} & \mathrm{C} 52 & \mathrm{C} 53 & \mathrm{C} 54 & \mathrm{C} 55\end{array}$

$\begin{array}{llllll}\mathbf{0 . 3 0 2 9 5} & -0.00576 & 0.01401 & -0.00820 & 0.01772\end{array}$

$\begin{array}{llllll}\mathrm{C} 56 & \mathrm{C} 57 & \mathrm{C} 58 & \mathrm{C} 59 & \mathrm{C} 60\end{array}$

$\begin{array}{llllll}-0.00581 & 0.01340 & -0.00236 & 0.00210 & -0.00136\end{array}$

$\begin{array}{lllll}\text { C61 } & \text { C62 } & \text { N63 } & \text { Ir64 } & \text { O65 }\end{array}$

$\begin{array}{llllll}0.00274 & -0.00249 & -0.00059 & \mathbf{0 . 2 7 0 0 6} & 0.00017\end{array}$

$\begin{array}{lllll}\text { C66 } & \text { H67 } & \text { H68 } & \text { H69 } & \text { C70 }\end{array}$

$\begin{array}{llllll}-0.00006 & 0.00002 & 0.00005 & -0.00001 & 0.01342\end{array}$

$\begin{array}{lllll}\text { C71 } & \text { C72 } & \text { H73 } & \text { C74 } & \text { H75 } \\ -0.00219 & 0.00485 & 0.00008 & -0.00665 & -0.00087\end{array}$ $\begin{array}{lllll}\text { C76 } & \mathrm{H} 77 & \mathrm{C} 78 & \mathrm{~N} 79 & \mathrm{C} 80\end{array}$

$\begin{array}{lllll}0.01195 & 0.00026 & 0.00438 & 0.00110 & 0.01133\end{array}$

$\begin{array}{lllll}\mathrm{H} 81 & \mathrm{C} 82 & \mathrm{H} 83 & \mathrm{C} 84 & \mathrm{O} 85\end{array}$

$\begin{array}{lllll}-0.00037 & -0.00201 & 0.00002 & 0.01388 & 0.02306\end{array}$

$\begin{array}{lllll}086 & \mathrm{H} 87 & \mathrm{C} 88 & \mathrm{H} 89 & \mathrm{C} 90\end{array}$

$\begin{array}{llllll}0.01102 & -0.00070 & -0.00015 & 0.00091 & -0.00015\end{array}$

$\begin{array}{lllll}\text { H91 } & \text { H92 } & \text { H93 } & \text { C94 } & \text { H95 }\end{array}$

$\begin{array}{llllll}0.00000 & 0.00055 & 0.00003 & 0.00114 & -0.00004\end{array}$

$\begin{array}{lllll}\text { H96 } & \text { H97 } & \text { N98 } & \text { C99 } & \text { C100 }\end{array}$

$\begin{array}{lllll}-0.00001 & 0.00000 & -0.01103 & 0.01773 & 0.05335\end{array}$

$\begin{array}{lllll}\mathrm{C} 101 & \mathrm{C} 102 & \mathrm{H} 103 & \mathrm{H} 104 & \mathrm{H} 105\end{array}$

$\begin{array}{llllll}0.03515 & 0.03074 & -0.00011 & -0.00439 & -0.00302\end{array}$

$0106 \quad 0107$

$0.03933 \quad 0.03982$

$\left[{ }^{1} \mathbf{I r}-{ }^{3} \mathbf{Q}-{ }^{1} \mathbf{2}\right]$

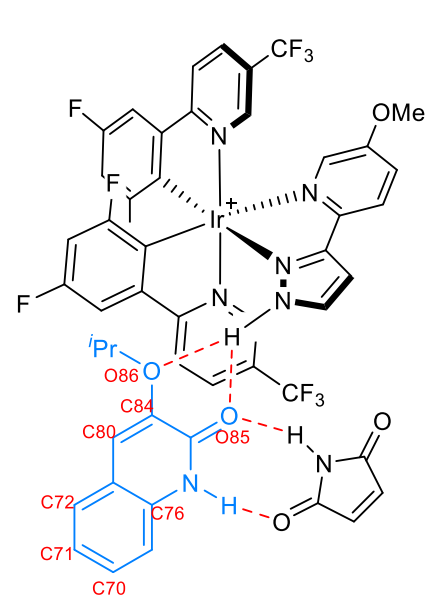

$\begin{array}{lllll}\mathrm{H} 1 & \mathrm{H} 2 & \mathrm{H} 3 & \mathrm{H} 4 & \mathrm{H} 5\end{array}$

$\begin{array}{llllll}0.00003 & 0.00008 & -0.00087 & -0.00001 & 0.00000\end{array}$

$\begin{array}{lllll}\mathrm{H} 6 & \mathrm{H} 7 & \mathrm{H} 8 & \mathrm{H} 9 & \mathrm{H} 10\end{array}$

$\begin{array}{lllll}0.00000 & 0.00010 & -0.00007 & -0.00015 & 0.00000\end{array}$ 


\begin{tabular}{|c|c|c|c|c|c|c|c|c|c|}
\hline H11 & H12 & F13 & F14 & F15 & -0.01771 & 0.09666 & -0.00471 & 0.45038 & 0.14404 \\
\hline \multirow[t]{2}{*}{0.00000} & 0.00000 & 0.00000 & 0.00000 & 0.00000 & & & & & \\
\hline & & & & & 086 & H87 & C88 & H89 & C90 \\
\hline $\mathrm{C} 16$ & F17 & F18 & F19 & $\mathrm{C} 20$ & 0.17823 & -0.03742 & -0.01133 & 0.00670 & 0.00085 \\
\hline \multirow[t]{2}{*}{0.00000} & 0.00005 & 0.00005 & 0.00016 & -0.00015 & & & & & \\
\hline & & & & & H91 & H92 & H93 & C94 & H95 \\
\hline $\mathrm{C} 21$ & $\mathrm{C} 22$ & $\mathrm{C} 23$ & N24 & $\mathrm{C} 25$ & 0.00007 & 0.00002 & -0.00053 & 0.01352 & 0.00034 \\
\hline \multirow[t]{2}{*}{-0.00004} & -0.00002 & 0.00057 & -0.00081 & -0.00001 & & & & & \\
\hline & & & & & H96 & H97 & N98 & C99 & $\mathrm{C} 100$ \\
\hline $\mathrm{C} 26$ & $\mathrm{C} 27$ & $\mathrm{C} 28$ & $\mathrm{C} 29$ & $\mathrm{C} 30$ & 0.00079 & -0.00016 & -0.00215 & 0.00685 & 0.00273 \\
\hline \multirow[t]{2}{*}{0.00000} & 0.00000 & 0.00000 & -0.00002 & 0.01421 & & & & & \\
\hline & & & & & C101 & $\mathrm{C} 102$ & H103 & H104 & H105 \\
\hline $\mathrm{C} 31$ & C32 & C33 & $\mathrm{C} 34$ & C35 & 0.00713 & 0.00237 & -0.00066 & -0.0003 & -0.00057 \\
\hline \multirow[t]{2}{*}{-0.00236} & 0.00869 & -0.00057 & 0.00195 & -0.00220 & & & & & \\
\hline & & & & & O106 & O107 & & & \\
\hline $\mathrm{C} 36$ & $\mathrm{C} 37$ & $\mathrm{C} 38$ & C39 & N40 & 0.00265 & 0.0037 & & & \\
\hline 0.00527 & 0.00055 & 0.00248 & -0.00095 & 0.00002 & & & & & \\
\hline N41 & N42 & $\mathrm{C} 43$ & $\mathrm{C} 44$ & $\mathrm{C} 45$ & {$\left[{ }^{1} \mathbf{I r}-{ }^{1} \mathbf{Q}-{ }^{3} 2\right.$} & & & & \\
\hline-0.00014 & 0.00611 & -0.00009 & 0.00002 & -0.00003 & & & & $======$ & \\
\hline $\mathrm{C} 46$ & $\mathrm{C} 47$ & $\mathrm{C} 48$ & C49 & C50 & & & & & \\
\hline 0.00000 & 0.00002 & 0.00000 & 0.00003 & 0.00000 & & & & & \\
\hline C51 & C52 & C53 & C54 & N55 & & & & & \\
\hline 0.00000 & 0.00002 & -0.00001 & -0.00012 & -0.00003 & & & & & \\
\hline Ir56 & H57 & $\mathrm{H} 58$ & H59 & H60 & & & & & \\
\hline 0.00194 & 0.00000 & 0.00000 & 0.00172 & 0.00006 & & & & & \\
\hline F61 & F62 & F63 & F64 & O65 & & & & & \\
\hline \multirow[t]{2}{*}{0.00058} & 0.00260 & 0.00002 & 0.00000 & 0.00000 & H1 & $\mathrm{H} 2$ & H3 & $\mathrm{H} 4$ & H5 \\
\hline & & & & & 0.00004 & 0.00000 & -0.00021 & 0.00002 & 0.00001 \\
\hline C66 & H67 & H68 & H69 & C70 & & & & & \\
\hline \multirow[t]{2}{*}{0.00000} & 0.00000 & 0.00000 & 0.00000 & 0.35621 & H6 & $\mathrm{H} 7$ & H8 & H9 & $\mathrm{H} 10$ \\
\hline & & & & & -0.00001 & 0.00024 & -0.00012 & 0.00019 & 0.00044 \\
\hline C71 & C72 & $\mathrm{H} 73$ & $\mathrm{C} 74$ & H75 & & & & & \\
\hline \multirow[t]{2}{*}{-0.12368} & 0.26762 & 0.00544 & -0.07122 & -0.02471 & H11 & H12 & H13 & H14 & F15 \\
\hline & & & & & 0.00004 & -0.00003 & 0.00002 & 0.00003 & 0.00001 \\
\hline C76 & $\mathrm{H} 77$ & $\mathrm{C} 78$ & N79 & C80 & & & & & \\
\hline \multirow[t]{2}{*}{0.17259} & 0.00212 & -0.05694 & 0.09880 & 0.49362 & F16 & F17 & $\mathrm{C} 18$ & F19 & $\mathrm{F} 20$ \\
\hline & & & & & 0.00000 & 0.00000 & 0.00000 & 0.00103 & 0.00099 \\
\hline \multirow[t]{2}{*}{ H81 } & $\mathrm{C} 82$ & H83 & C84 & 085 & & & & & \\
\hline & & & & & & & & & \\
\hline
\end{tabular}




\begin{tabular}{|c|c|c|c|c|c|c|c|c|c|}
\hline F21 & $\mathrm{C} 22$ & F23 & $\mathrm{H} 24$ & F25 & C66 & H67 & H68 & H69 & $\mathrm{C} 70$ \\
\hline 0.00083 & 0.00089 & 0.00049 & 0.00002 & 0.00030 & -0.00001 & 0.00000 & 0.00000 & 0.00000 & 0.01733 \\
\hline F26 & $\mathrm{H} 27$ & F28 & $\mathrm{C} 29$ & $\mathrm{C} 30$ & C71 & $\mathrm{C} 72$ & $\mathrm{H} 73$ & C74 & H75 \\
\hline 0.00454 & -0.00108 & 0.00040 & -0.00015 & 0.00041 & 0.00121 & 0.00193 & -0.00015 & -0.00795 & -0.00108 \\
\hline C31 & N32 & $\mathrm{C} 33$ & $\mathrm{C} 34$ & $\mathrm{C} 35$ & C76 & H77 & C78 & N79 & $\mathrm{C} 80$ \\
\hline 0.00113 & -0.00013 & -0.00016 & 0.00000 & -0.00008 & 0.01483 & 0.00004 & 0.01047 & -0.00183 & 0.00718 \\
\hline $\mathrm{C} 36$ & $\mathrm{C} 37$ & C38 & C39 & $\mathrm{C} 40$ & H81 & $\mathrm{C} 82$ & H83 & $\mathrm{C} 84$ & O85 \\
\hline 0.00001 & -0.00024 & 0.01264 & -0.00800 & 0.02023 & -0.00027 & -0.00312 & -0.00051 & 0.02011 & 0.02300 \\
\hline C41 & $\mathrm{C} 42$ & $\mathrm{C} 43$ & $\mathrm{C} 44$ & $\mathrm{C} 45$ & O86 & H87 & C88 & H89 & C90 \\
\hline 0.01453 & -0.00187 & -0.00308 & 0.03253 & 0.00729 & 0.01419 & -0.00058 & -0.00083 & 0.00042 & 0.00008 \\
\hline C46 & $\mathrm{C} 47$ & N48 & N49 & N50 & H91 & H92 & H93 & C94 & H95 \\
\hline-0.00453 & 0.00819 & -0.00035 & 0.00175 & 0.02913 & -0.00002 & 0.00014 & 0.00000 & 0.00114 & -0.00002 \\
\hline C51 & C52 & $\mathrm{C} 53$ & C54 C55 & & H96 & H97 & N98 & C99 & C100 \\
\hline 0.03422 & 0.00001 & 0.00164 & -0.00101 & 0.00287 & -0.00005 & -0.00005 & 0.14597 & 0.02466 & 0.63692 \\
\hline C56 & C57 & C58 & C59 & C60 & C101 & C102 & H103 & H104 & H105 \\
\hline-0.00063 & 0.00182 & -0.00049 & 0.00033 & -0.00033 & 0.65976 & -0.00623 & -0.00933 & -0.03881 & -0.04054 \\
\hline C61 & C62 & N63 & Ir64 & O65 & 0106 & 0107 & & & \\
\hline 0.00028 & -0.00041 & -0.00001 & 0.04588 & 0.00001 & 0.14482 & 0.18465 & & & \\
\hline
\end{tabular}




\section{e. Energies, Coordinates, and Vibrational Frequencies of Optimized Structures}

Table S21 Computed energies of the optimized geometries

\begin{tabular}{|c|c|c|c|c|}
\hline & $\begin{array}{c}\mathbf{E}(\mathbf{S C F}) /(\mathbf{e V}) \\
\text { cc-pVTZ(-f)/LACV3P** }\end{array}$ & $\begin{array}{c}\text { ZPE/(kcal/mol) } \\
\text { 6-31G**/LACVP** }\end{array}$ & $\begin{array}{l}\mathbf{S}(\mathbf{g a s}) /(\mathbf{c a l} / \mathbf{m o l} \cdot \mathbf{K}) \\
6-31 \mathrm{G}^{* *} / \mathrm{LACVP}^{* *}\end{array}$ & $\begin{array}{c}\text { G(solv) } /(\mathbf{k c a l} / \mathbf{m o l}) \\
6-31 \mathrm{G}^{* *} / \mathrm{LACVP} * * \\
\end{array}$ \\
\hline $14 f$ & -74067.844 & 297.89 & 255.76 & -44.05 \\
\hline${ }^{3} 4 f$ & -74065.398 & 295.77 & 261.44 & -44.09 \\
\hline${ }^{1} 2$ & -9784.128 & 42.92 & 75.07 & -9.99 \\
\hline${ }^{3} 2$ & -9781.297 & 40.27 & 78.26 & -7.59 \\
\hline${ }^{1} 1 \mathrm{c}$ & -18247.131 & 144.33 & 112.73 & -11.71 \\
\hline${ }^{3} 1 \mathrm{c}$ & -18244.570 & 141.61 & 119.74 & -11.24 \\
\hline${ }^{1}\left[\mid r-2_{A}\right]$ & -83852.781 & 341.73 & 292.01 & -43.71 \\
\hline$\left[{ }^{3} \mid r-{ }^{1} 2_{A}\right]$ & -83850.375 & 339.31 & 285.63 & -47.34 \\
\hline$\left[{ }^{1} \mid r r^{3} 2_{A}\right]$ & -83850.508 & 340.02 & 279.81 & -46.42 \\
\hline${ }^{1}\left[\mid r-2_{B}\right]$ & -83852.797 & 341.95 & 284.06 & -43.09 \\
\hline$\left[{ }^{3} \mid r-{ }^{1}{ }^{2} B\right]$ & -83850.383 & 339.75 & 284.63 & -44.36 \\
\hline$\left[{ }^{1} \mid r_{-}{ }^{3} 2_{B}\right]$ & -83850.320 & 339.34 & 284.96 & -46.36 \\
\hline${ }^{1}\left[I r-Q_{A}\right]$ & -92316.211 & 443.40 & 315.15 & -41.42 \\
\hline$\left[{ }^{3} \mid r-{ }^{1} Q_{A}\right]$ & -92313.805 & 440.99 & 320.22 & -41.60 \\
\hline$\left[{ }^{1} \mid r-{ }^{3} Q_{A}\right]$ & -92313.734 & 440.30 & 319.74 & -41.20 \\
\hline${ }^{1}\left[\mid r-Q_{B}\right]$ & -92316.266 & 443.24 & 311.77 & -41.14 \\
\hline$\left[{ }^{3} \mid r-{ }^{1} Q_{B}\right]$ & -92313.914 & 441.05 & 316.42 & -39.84 \\
\hline$\left[{ }^{1} \mid r-{ }^{3} Q_{B}\right]$ & -92313.766 & 440.52 & 317.81 & -40.84 \\
\hline${ }^{1}\left[\mid r-Q_{c}\right]$ & -92316.391 & 443.27 & 309.96 & -41.70 \\
\hline$\left[{ }^{3} \mid r-{ }^{1} Q_{c}\right]$ & -92314.000 & 441.23 & 319.50 & -41.64 \\
\hline$\left[{ }^{1} \mid r-{ }^{3} Q_{c}\right]$ & -92313.867 & 440.78 & 323.56 & -41.40 \\
\hline${ }^{1}\left[\mid r-Q_{D}\right]$ & -92316.469 & 444.69 & 306.34 & -38.57 \\
\hline$\left[{ }^{3} \mid r-{ }^{1} Q_{D}\right]$ & -92314.016 & 441.36 & 322.01 & -37.91 \\
\hline$\left[{ }^{1} \mid r-{ }^{3} Q_{D}\right]$ & -92313.836 & 440.84 & 323.50 & -38.97 \\
\hline${ }^{1}[I r-Q-2]$ & -102101.555 & 487.79 & 347.59 & -41.29 \\
\hline$\left[^{3} \mid r-{ }^{1} Q-{ }^{1} 2\right]$ & -102099.234 & 486.05 & 345.49 & -42.18 \\
\hline$\left[{ }^{1} \mid r-{ }^{3} Q-{ }^{1} 2\right]$ & -102098.969 & 484.27 & 352.61 & -41.46 \\
\hline$\left[{ }^{1} \mid r-{ }^{1} Q-{ }^{3} 2\right]$ & -102099.008 & 485.28 & 344.56 & -42.17 \\
\hline
\end{tabular}


Table S22 Cartesian coordinates of the optimized geometries

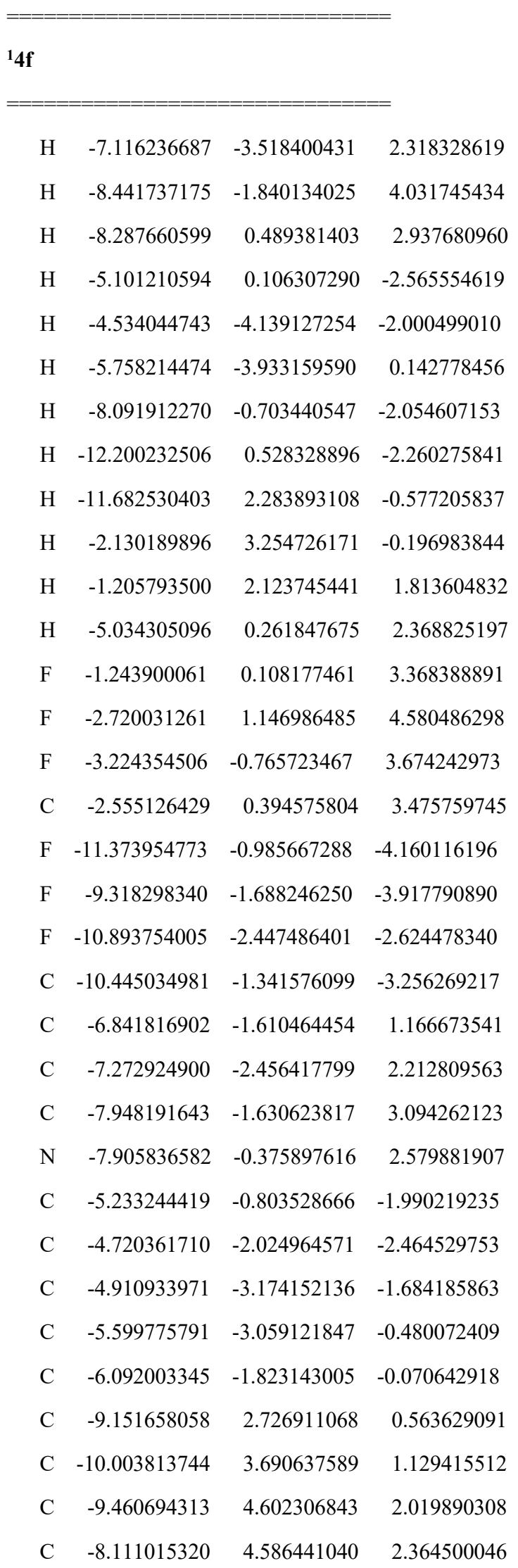

C $\quad-7.304566383 \quad 3.614867210 \quad 1.784804821$

$\begin{array}{llll}\text { C } & -8.912469864 & -0.074096225 & -1.740086436\end{array}$

C $\quad-10.180202484 \quad-0.229293004 \quad-2.275862694$

$\begin{array}{llll}\text { C } & -11.200547218 & 0.625814974 & -1.850150347\end{array}$

C $\quad-10.908425331 \quad 1.604297638 \quad-0.912045062$

$\begin{array}{llll}\text { C } & -9.608821869 & 1.728083491 & -0.404301196\end{array}$

N $\quad-5.900036812 \quad-0.711138070 \quad-0.839357138$

$\begin{array}{llll}\mathrm{N} & -7.236082077 & -0.353174955 & 1.418304205\end{array}$

$\begin{array}{llll}\mathrm{N} & -8.635836601 & 0.871744335 & -0.830873251\end{array}$

$\begin{array}{llll}\text { C } & -7.769884586 & 2.660511971 & 0.881155372\end{array}$

$\begin{array}{llll}\text { C } & -6.056531429 & 2.448809385 & -1.413197398\end{array}$

$\begin{array}{llll}\text { C } & -6.688385487 & 2.958450317 & -2.547501087\end{array}$

C $\quad-6.092801571 \quad 3.844428301 \quad-3.435737133$

$\begin{array}{llll}\text { C } & -4.787086964 & 4.250243187 & -3.173589945\end{array}$

C $\quad-4.086929321 \quad 3.797917128 \quad-2.067922115$

$\begin{array}{llll}\text { C } & -4.728242874 & 2.901546001 & -1.196176052\end{array}$

C $\quad-4.076897621 \quad 2.354183674 \quad-0.005912429$

$\begin{array}{llll}\text { C } & -2.753830194 & 2.592013121 & 0.390052408\end{array}$

$\begin{array}{llll}\text { C } & -2.235694408 & 1.964716673 & 1.512212634\end{array}$

$\begin{array}{llll}\text { C } & -3.056495190 & 1.101132512 & 2.243085623\end{array}$

$\begin{array}{llll}\text { C } & -4.365548611 & 0.917589009 & 1.828842521\end{array}$

$\begin{array}{llll}\mathrm{N} & -4.860301971 & 1.518072605 & 0.736283004\end{array}$

Ir $\quad-6.756163597 \quad 1.154689789 \quad-0.016781118$

H $\quad-3.075404167 \quad 4.153194427 \quad-1.912709951$

H $\quad-6.628821373 \quad 4.211077690 \quad-4.303159237$

$\begin{array}{llll}\mathrm{H} & -7.704516888 & 5.312085152 & 3.059226274\end{array}$

H $\quad-11.059863091 \quad 3.755826712 \quad 0.897487700$

F $\quad-5.995019436 \quad 3.611964464 \quad 2.134866714$

F $\quad \begin{array}{llll}-10.256696701 & 5.533036709 & 2.573063374\end{array}$

F $\quad-7.962292194 \quad 2.584748268 \quad-2.828151464$

F $\quad-4.193397522 \quad 5.106340408 \quad-4.022323132$

O $\quad-4.084672928 \quad-1.967413902 \quad-3.645471334$

C $\quad-3.596229792 \quad-3.185268641 \quad-4.222447395$

H $\quad-2.822300673 \quad-3.636461735 \quad-3.590576887$

H $\quad-4.412341595 \quad-3.899490356 \quad-4.382086754$

H $\quad-3.165674686 \quad-2.902578592 \quad-5.182186604$ 


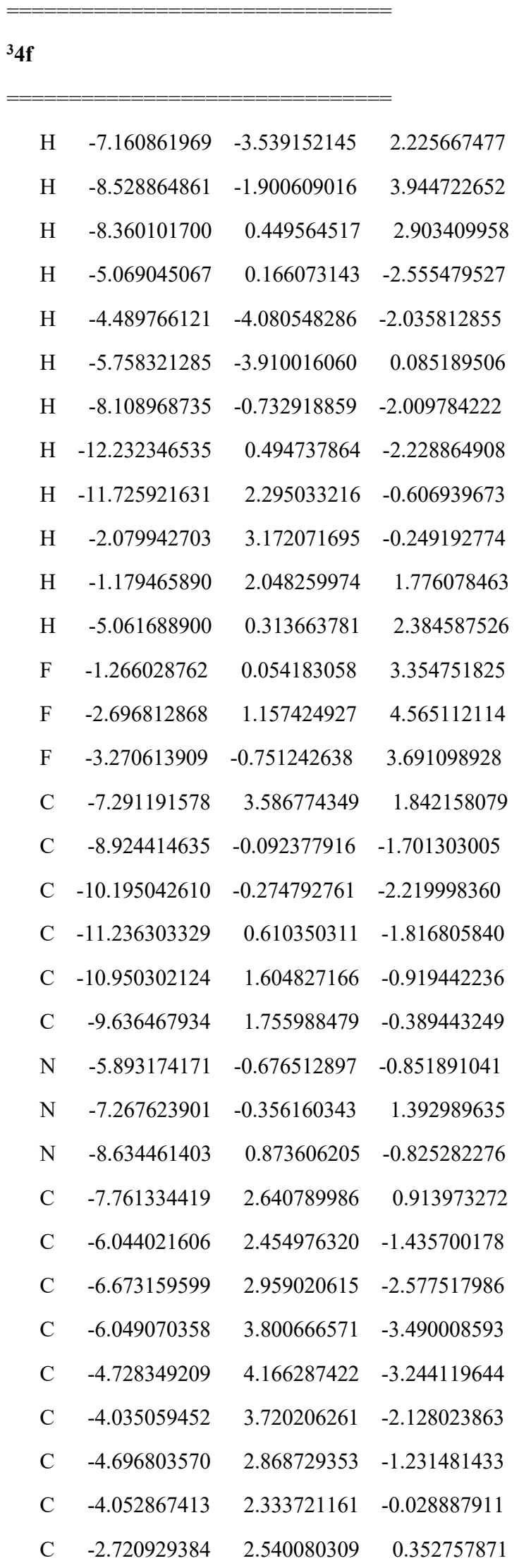

C $\quad-2.566064119 \quad 0.383193433 \quad 3.471566200$

F $\quad-11.343870163 \quad-1.094178081 \quad-4.103425980$

F $\quad-9.333895683 \quad-1.874335885 \quad-3.729723454$

F $\quad-11.000040054 \quad-2.482056141 \quad-2.470364332$

C $\quad-10.468039513 \quad-1.426566839 \quad-3.134799957$

$\begin{array}{llll}\text { C } & -6.866022587 & -1.606439114 & 1.123049498\end{array}$

$\begin{array}{llll}\text { C } & -7.316864491 & -2.475258112 & 2.140740871\end{array}$

$\begin{array}{llll}\text { C } & -8.013570786 & -1.669858932 & 3.024142027\end{array}$

N $\quad \begin{array}{llll}-7.962123394 & -0.405153394 & 2.538057327\end{array}$

$\begin{array}{llll}\text { C } & -5.205070019 & -0.749820471 & -1.991388798\end{array}$

$\begin{array}{llll}\text { C } & -4.674459934 & -1.960709929 & -2.471104622\end{array}$

C $\quad-4.876209259-3.121810198 \quad-1.712173104$

C $\quad-5.589794159 \quad-3.026542902 \quad-0.521199524$

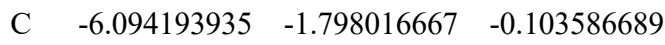

$\begin{array}{llll}\text { C } & -9.200945854 & 2.716948032 & 0.541388094\end{array}$

C $\quad \begin{array}{llll}-10.012727737 & 3.726056337 & 1.114782691\end{array}$

$\begin{array}{llll}\text { C } & -9.444615364 & 4.597327709 & 2.013762474\end{array}$

$\begin{array}{llll}\text { C } & -8.075594902 & 4.563727379 & 2.416338444\end{array}$

$\begin{array}{llll}\text { C } & -2.215556383 & 1.915570617 & 1.483358622\end{array}$

$\begin{array}{llll}\text { C } & -3.056674480 & 1.088188767 & 2.232811689\end{array}$

$\begin{array}{llll}\text { C } & -4.374514103 & 0.939589620 & 1.831455469\end{array}$

$\begin{array}{llll}\mathrm{N} & -4.856053352 & 1.539668441 & 0.734208584\end{array}$

Ir $\quad-6.771409512 \quad 1.210240841 \quad-0.023898991$

$\mathrm{H} \quad-3.012374163 \quad 4.048592567 \quad-1.988258362$

H $\quad-6.577938557 \quad 4.165374756 \quad-4.362481117$

H $\quad-7.687249660 \quad 5.278684139 \quad 3.128880024$

H $\quad-11.060444832 \quad 3.843076944 \quad 0.868544996$

$\begin{array}{llll}\text { F } & -5.987686157 & 3.547530174 & 2.197051287\end{array}$

F $\quad \begin{array}{llll}-10.203649521 & 5.547518730 & 2.561438560\end{array}$

F $\quad-7.956250668 \quad 2.632831573 \quad-2.831124067$

F $\quad-4.109597683 \quad 4.977768898 \quad-4.113626957$

O $\quad-4.012152195 \quad-1.881037712 \quad-3.635588884$

C $\quad-3.512935877 \quad-3.088438034 \quad-4.225383282$

H $\quad-2.750503778 \quad-3.550245523 \quad-3.587250710$

H $\quad-4.325500965 \quad-3.799595833 \quad-4.413065434$

H $\quad-3.064435482 \quad-2.788168669 \quad-5.171390533$ 


\begin{tabular}{|c|c|c|c|}
\hline \multicolumn{4}{|l|}{12} \\
\hline $\mathrm{N}$ & -5.401314735 & -5.177960873 & -1.448237896 \\
\hline $\mathrm{C}$ & -4.244321346 & -4.988488674 & -0.688426197 \\
\hline $\mathrm{C}$ & -4.732588291 & -4.642112255 & 0.692367196 \\
\hline $\mathrm{C}$ & -6.069819450 & -4.642560482 & 0.692854762 \\
\hline $\mathrm{C}$ & -6.558351517 & -4.988847733 & -0.687616289 \\
\hline $\mathrm{H}$ & -5.400140285 & -5.426800728 & -2.426285982 \\
\hline $\mathrm{H}$ & -4.045629978 & -4.436492443 & 1.502683878 \\
\hline $\mathrm{H}$ & -6.755875111 & -4.436982155 & 1.503975153 \\
\hline $\mathrm{O}$ & -3.102493286 & -5.087026119 & -1.080698609 \\
\hline $\mathrm{O}$ & -7.699919701 & -5.087652206 & -1.080486178 \\
\hline
\end{tabular}

32

\begin{tabular}{|c|c|c|c|}
\hline $\mathrm{N}$ & -5.401309013 & -5.169267654 & -1.411703467 \\
\hline $\mathrm{C}$ & -4.252712250 & -4.973077774 & -0.627950370 \\
\hline $\mathrm{C}$ & -4.709108829 & -4.641987801 & 0.691583216 \\
\hline $\mathrm{C}$ & -6.093164921 & -4.642360210 & 0.691760540 \\
\hline $\mathrm{C}$ & -6.549767971 & -4.973627567 & -0.627633989 \\
\hline $\mathrm{H}$ & -5.401390553 & -5.418319702 & -2.390043259 \\
\hline $\mathrm{H}$ & -4.029210567 & -4.437185764 & 1.504037976 \\
\hline $\mathrm{H}$ & -6.772819996 & -4.437557697 & 1.504404306 \\
\hline $\mathrm{O}$ & -3.116608620 & -5.102596283 & -1.142523050 \\
\hline $\mathrm{O}$ & -7.685942650 & -5.103370667 & -1.142005801 \\
\hline
\end{tabular}

$11 \mathrm{c}$

\begin{tabular}{|c|c|c|c|}
\hline $\mathrm{C}$ & -4.543751717 & -5.725379944 & -7.707424641 \\
\hline $\mathrm{C}$ & -4.205198288 & -6.751370907 & -6.812257290 \\
\hline $\mathrm{C}$ & -2.879380226 & -6.940680027 & -6.448442936 \\
\hline $\mathrm{H}$ & -4.978406429 & -7.395055294 & -6.404709339 \\
\hline $\mathrm{C}$ & -3.560262918 & -4.896360397 & -8.231888771 \\
\hline
\end{tabular}

H $\quad-5.580173969 \quad-5.572836399 \quad-7.992820740$

C $\quad-2.221414566 \quad-5.086802959 \quad-7.863057137$

H $\quad-3.818204165 \quad-4.097725868 \quad-8.922571182$

C $\quad-1.861637950 \quad-6.115831375 \quad-6.961922169$

N $\quad-1.210260272 \quad-4.282943726 \quad-8.361979485$

C $\quad-0.473127455-6.276317596 \quad-6.613619328$

H $\quad-2.607845306 \quad-7.733871937 \quad-5.756604195$

C $\quad 0.146296144 \quad-4.367679596 \quad-8.071142197$

$\mathrm{H} \quad-1.438167810 \quad-3.541676521 \quad-9.013060570$

C $\quad 0.488690823 \quad-5.450310230 \quad-7.112569809$

O $\quad 0.956194699 \quad-3.602336407 \quad-8.576104164$

$\begin{array}{llll}\text { O } & 1.809076905 & -5.469650745 & -6.843156815\end{array}$

H $\quad-0.221271932 \quad-7.072253227 \quad-5.922000408$

$\begin{array}{llll}\text { C } & 2.343781710 & -6.423734188 & -5.905042648\end{array}$

H $\quad 1.647080779 \quad-6.524668694 \quad-5.061117172$

C $\quad 3.647845745 \quad-5.817024708 \quad-5.407205105$

H $\quad 4.333292007 \quad-5.663054943 \quad-6.245986462$

H $\quad 3.462913990 \quad-4.850524426 \quad-4.931600094$

H $\quad 4.123596191 \quad-6.483860970 \quad-4.682004929$

$\begin{array}{lllll}\text { C } & 2.541244030 & -7.775304317 & -6.591827869\end{array}$

H $\quad 2.952757359 \quad-8.505214691 \quad-5.887086391$

H $\quad \begin{array}{llll}1.598896980 & -8.166687965 & -6.983465672\end{array}$

H $\quad 3.239263535 \quad-7.666171551 \quad-7.427474022$

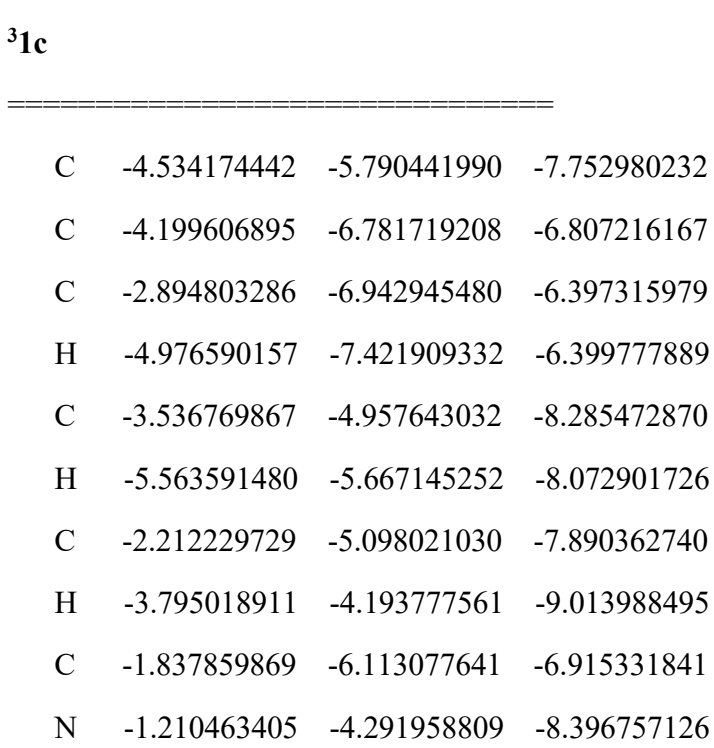



C $\quad-0.519122779 \quad-6.245542526 \quad-6.526681423$
H $\quad-2.632166624 \quad-7.704838753 \quad-5.669260979$
C $\quad 0.164643466 \quad-4.354452610 \quad-8.063117027$
H $\quad-1.432172537 \quad-3.575185061 \quad-9.073771477$
C $\quad 0.511834443 \quad-5.360540390 \quad-7.101419926$
O $\quad 0.948677957 \quad-3.565221310 \quad-8.605455399$
O $\quad 1.814853549 \quad-5.415703297 \quad-6.782294273$
H $\quad-0.249540314 \quad-6.997785091 \quad-5.797329426$
$\begin{array}{llll}\text { C } & 2.339714289 & -6.434602261 & -5.899791241\end{array}$

${ }^{1}\left[\mathbf{I r}-2_{\mathrm{A}}\right]$

\begin{tabular}{lrrr}
$\mathrm{H}$ & -6.475228310 & -3.729193687 & 2.057127953 \\
$\mathrm{H}$ & -7.955019474 & -2.310611725 & 3.885442972 \\
$\mathrm{H}$ & -8.269604683 & 0.018864932 & 2.897753477 \\
$\mathrm{H}$ & -5.046683788 & 0.327891082 & -2.638877869 \\
$\mathrm{H}$ & -4.092015266 & -3.876075029 & -2.414601803 \\
$\mathrm{H}$ & -5.338447094 & -3.950219870 & -0.242916465 \\
$\mathrm{H}$ & -7.891872883 & -0.924475551 & -2.202095985 \\
$\mathrm{H}$ & -12.058433533 & 0.034350656 & -2.613791943 \\
$\mathrm{H}$ & -11.733354568 & 1.848663926 & -0.950766027 \\
$\mathrm{H}$ & -2.323287249 & 3.434365511 & 0.190065607 \\
$\mathrm{H}$ & -1.534036279 & 2.362557650 & 2.289825439 \\
$\mathrm{H}$ & -5.254977226 & 0.228957549 & 2.450295925 \\
$\mathrm{~F}$ & -1.596005440 & 0.336827219 & 3.843206406 \\
$\mathrm{~F}$ & -3.247082233 & 1.296431780 & 4.882373810 \\
$\mathrm{~F}$ & -3.541763067 & -0.653161049 & 3.961313725 \\
$\mathrm{C}$ & -2.926481485 & 0.542936981 & 3.813219309 \\
$\mathrm{~F}$ & -11.082397461 & -1.478348851 & -4.422053814 \\
$\mathrm{~F}$ & -8.976689339 & -1.988828182 & -4.135712147 \\
$\mathrm{~F}$ & -10.497200966 & -2.857074738 & -2.845576286 \\
$\mathrm{C}$ & -10.139968872 & -1.730216861 & -3.497720242 \\
$\mathrm{C}$ & -6.602149963 & -1.790007234 & 0.939147174 \\
$\mathrm{C}$ & -6.819513798 & -2.709636927 & 1.984916806 \\
$\mathrm{C}$ & -7.563922405 & -2.013947725 & 2.923286676 \\
\hline & -7.766405106 & -0.765197694 & 2.444190264
\end{tabular}

$$
\begin{array}{llll}
\mathrm{H} & 1.657524347 & -6.558432579 & -5.049778938 \\
\mathrm{C} & 3.668677807 & -5.883683205 & -5.401753426 \\
\mathrm{H} & 4.343403339 & -5.711336613 & -6.245219707 \\
\mathrm{H} & 3.519534111 & -4.935246468 & -4.879617214 \\
\mathrm{H} & 4.137442112 & -6.594909191 & -4.715234280 \\
\mathrm{C} & 2.485708237 & -7.748656273 & -6.664750099 \\
\mathrm{H} & 2.911288023 & -8.519833565 & -6.014791965 \\
\mathrm{H} & 1.518987298 & -8.100571632 & -7.034865379 \\
\mathrm{H} & 3.151170015 & -7.606130123 & -7.521453381
\end{array}
$$$$
\text { C } \quad-5.078671932 \quad-0.642106295 \quad-2.160519600
$$$$
\text { C } \quad-4.468959808 \quad-1.778967500 \quad-2.706979513
$$$$
\begin{array}{llll}
\text { C } & -4.561181545 & -2.990365028 & -2.000094414
\end{array}
$$$$
\text { C } \quad-5.250715733 \quad-3.024859190 \quad-0.801142097
$$$$
\text { C } \quad-5.846978664 \quad-1.857531309 \quad-0.310020864
$$$$
\begin{array}{llll}
\text { C } & -9.280737877 & 2.506640196 & 0.258859336
\end{array}
$$$$
\begin{array}{llll}
\text { C } & -10.207478523 & 3.464728832 & 0.706276596
\end{array}
$$$$
\begin{array}{llll}
\text { C } & -9.799005508 & 4.364062309 & 1.675064683
\end{array}
$$$$
\begin{array}{llll}
\text { C } & -8.504609108 & 4.361792564 & 2.190268755
\end{array}
$$$$
\begin{array}{llll}
\text { C } & -7.614507198 & 3.415267467 & 1.700318694
\end{array}
$$$$
\text { C } \quad-8.760049820 \quad-0.332143307 \quad-1.949695110
$$$$
\begin{array}{llll}
\text { C } & -9.990002632 & -0.576487303 & -2.540451288
\end{array}
$$$$
\text { C } \quad-11.081721306 \quad 0.215997353 \quad-2.178002357
$$$$
\text { C } \quad-10.893819809 \quad 1.239657760 \quad-1.259977460
$$$$
\begin{array}{llll}
\text { C } & -9.624376297 & 1.466419339 & -0.711131155
\end{array}
$$$$
\begin{array}{llll}
\mathrm{N} & -5.748414993 & -0.692674577 & -0.995788872
\end{array}
$$$$
\text { N } \quad \begin{array}{llll}
-7.192091942 & -0.617422402 & 1.238983512
\end{array}
$$$$
\begin{array}{llll}
\mathrm{N} & -8.582983017 & 0.651358783 & -1.055506945
\end{array}
$$$$
\begin{array}{llll}
\text { C } & -7.947879791 & 2.459573746 & 0.740374327
\end{array}
$$$$
\text { C } \quad-6.060895443 \quad 2.400818110 \quad-1.395272613
$$$$
\text { C } \quad-6.616093636 \quad 2.878146172 \quad-2.582110405
$$$$
\begin{array}{llll}
\text { C } & -5.984477043 & 3.785584927 & -3.423874855
\end{array}
$$$$
\text { C } \quad \begin{array}{llll}
-4.724827766 & 4.247876167 & -3.052563667
\end{array}
$$$$
\text { C } \quad-4.101925850 \quad 3.828406334 \quad-1.888516903
$$$$
\text { C } \quad-4.776552200 \quad 2.909029722 \quad-1.067396998
$$$$
\begin{array}{llll}
\text { C } & -4.205708027 & 2.388903379 & 0.177082241
\end{array}
$$$$
\begin{array}{llll}
\text { C } & -2.952637911 & 2.720928907 & 0.707539439
\end{array}
$$ 


$\begin{array}{cccc}\text { C } & -2.511044025 & 2.128229618 & 1.880729079 \\ \text { C } & -3.340445042 & 1.206662416 & 2.524969578 \\ \text { C } & -4.580811501 & 0.927267611 & 1.975340128 \\ \text { N } & -4.997391224 & 1.490682483 & 0.831682265 \\ \text { Ir } & -6.787919998 & 1.024507999 & -0.097623341 \\ \text { H } & -3.122634649 & 4.226133823 & -1.650673151 \\ \text { H } & -6.459802151 & 4.128595352 & -4.335437775 \\ \text { H } & -8.201988220 & 5.078717709 & 2.944539070 \\ \text { H } & -11.224877357 & 3.515724421 & 0.338735044 \\ \text { F } & -6.359756947 & 3.434567928 & 2.208449841 \\ \text { F } & -10.684433937 & 5.259761810 & 2.151557446 \\ \text { F } & -7.840592861 & 2.442415237 & -2.969202042 \\ \text { F } & -4.095927238 & 5.124047279 & -3.854288578 \\ \text { O } & -3.796719074 & -1.801065683 & -3.869344473 \\ \text { C } & -3.722483158 & -0.597258985 & -4.642057896 \\ \text { H } & -4.723265171 & -0.248869717 & -4.924767494 \\ \text { H } & -3.196417093 & 0.193204463 & -4.093376160 \\ \text { H } & -3.159486532 & -0.857030571 & -5.537763119 \\ \text { N } & -11.083022118 & 0.881024063 & 2.419051886 \\ \text { C } & -10.367640495 & 1.427957058 & 3.468280077 \\ \text { C } & -11.262471199 & 2.443449259 & 4.106326580 \\ \text { C } & -12.385523796 & 2.535135746 & 3.384420395 \\ \text { C } & -12.284923553 & 1.589972973 & 2.222002983 \\ \text { H } & -10.667908669 & 0.315718234 & 1.691600442 \\ \text { H } & -10.965968132 & 2.991307259 & 4.990859985 \\ \text { H } & -13.243194580 & 3.178310871 & 3.529656410 \\ \text { O } & -9.221057892 & 1.140047431 & 3.789697170 \\ \text { O } & -13.022194862 & 1.464184999 & 1.271288514\end{array}$

\section{$\left[{ }^{3} \mid r-{ }^{-1}{ }_{A}\right]$}

$\begin{array}{lrrc}\mathrm{H} & -6.118937016 & -3.337928057 & 2.440487146 \\ \mathrm{H} & -7.500397682 & -1.836147070 & 4.277724266 \\ \mathrm{H} & -8.322208405 & 0.340177238 & 2.979394913 \\ \mathrm{H} & -5.478712082 & 0.366921604 & -2.701752424 \\ \mathrm{H} & -4.486200809 & -3.818557262 & -2.390184641\end{array}$

\begin{tabular}{|c|c|c|c|}
\hline $\mathrm{H}$ & -5.417649269 & -3.774968624 & -0.062260594 \\
\hline $\mathrm{H}$ & -8.242876053 & -0.815472722 & -1.759911776 \\
\hline $\mathrm{H}$ & -12.254722595 & 0.634010732 & -2.260346651 \\
\hline $\mathrm{H}$ & -11.659366608 & 2.585536718 & -0.842608035 \\
\hline $\mathrm{H}$ & -2.018703222 & 2.863351583 & -0.133152947 \\
\hline $\mathrm{H}$ & -1.259942770 & 1.802092195 & 1.982371569 \\
\hline $\mathrm{H}$ & -5.298635960 & 0.510144174 & 2.640583038 \\
\hline $\mathrm{F}$ & -1.591539264 & -0.075128272 & 3.712002039 \\
\hline $\mathrm{F}$ & -2.870751143 & 1.317171812 & 4.786177635 \\
\hline $\mathrm{F}$ & -3.684899569 & -0.579050064 & 4.093091965 \\
\hline $\mathrm{C}$ & -2.838479519 & 0.427500278 & 3.777997971 \\
\hline $\mathrm{F}$ & -11.706736565 & -1.364165664 & -3.619027615 \\
\hline $\mathrm{F}$ & -9.558623314 & -1.761931658 & -3.740932226 \\
\hline $\mathrm{F}$ & -10.719803810 & -2.572555780 & -2.099267960 \\
\hline $\mathrm{C}$ & -10.586052895 & -1.494270444 & -2.889858007 \\
\hline C & -6.536083698 & -1.552167058 & 1.154407859 \\
\hline C & -6.549966335 & -2.358053684 & 2.306980133 \\
\hline $\mathrm{C}$ & -7.235684872 & -1.608051419 & 3.255144358 \\
\hline $\mathrm{N}$ & -7.581837177 & -0.427997231 & 2.696767569 \\
\hline C & -5.438885212 & -0.572277665 & -2.167956829 \\
\hline $\mathrm{C}$ & -4.911931038 & -1.738508224 & -2.735479593 \\
\hline $\mathrm{C}$ & -4.892117977 & -2.910108328 & -1.958145857 \\
\hline C & -5.404798508 & -2.880012989 & -0.673953533 \\
\hline $\mathrm{C}$ & -5.932920933 & -1.686650634 & -0.166834667 \\
\hline C & -9.164245605 & 2.924820423 & 0.415841281 \\
\hline C & -9.894536972 & 4.047239780 & 0.812092364 \\
\hline $\mathrm{C}$ & -9.340638161 & 4.897893429 & 1.762159586 \\
\hline C & -8.071599960 & 4.705037594 & 2.352011919 \\
\hline C & -7.359588623 & 3.610694885 & 1.926280618 \\
\hline C & -9.018486023 & -0.087623887 & -1.565166712 \\
\hline $\mathrm{C}$ & -10.293479919 & -0.248251289 & -2.099254847 \\
\hline $\mathrm{C}$ & -11.256929398 & 0.738755345 & -1.850936770 \\
\hline $\mathrm{C}$ & -10.923434258 & 1.821358919 & -1.056529760 \\
\hline $\mathrm{C}$ & -9.634723663 & 1.919986606 & -0.498752415 \\
\hline $\mathrm{N}$ & -5.931303024 & -0.558858216 & -0.915942669 \\
\hline $\mathrm{N}$ & -7.187472343 & -0.400203288 & 1.409828782 \\
\hline $\mathrm{N}$ & -8.694923401 & 0.953244627 & -0.797728240 \\
\hline
\end{tabular}




\begin{tabular}{|c|c|c|c|}
\hline $\mathrm{C}$ & -7.817971230 & 2.699875355 & 0.939227462 \\
\hline $\mathrm{C}$ & -6.022465706 & 2.465634823 & -1.345556021 \\
\hline $\mathrm{C}$ & -6.597005367 & 2.977971554 & -2.509855747 \\
\hline $\mathrm{C}$ & -5.895211697 & 3.728149891 & -3.446726799 \\
\hline $\mathrm{C}$ & -4.547875404 & 3.979002714 & -3.200423241 \\
\hline $\mathrm{C}$ & -3.903362989 & 3.508575439 & -2.063681364 \\
\hline $\mathrm{C}$ & -4.644745827 & 2.755724907 & -1.142446280 \\
\hline $\mathrm{C}$ & -4.061502457 & 2.214166164 & 0.091423318 \\
\hline $\mathrm{C}$ & -2.724866629 & 2.330032110 & 0.491385609 \\
\hline $\mathrm{C}$ & -2.299144268 & 1.744062543 & 1.675854564 \\
\hline $\mathrm{C}$ & -3.228568554 & 1.065560937 & 2.467535019 \\
\hline $\mathrm{C}$ & -4.545874596 & 1.002012610 & 2.042835474 \\
\hline $\mathrm{N}$ & -4.942398071 & 1.536310673 & 0.878176212 \\
\hline Ir & -6.833709717 & 1.245287657 & 0.051791344 \\
\hline $\mathrm{H}$ & -2.855305672 & 3.745797873 & -1.925850391 \\
\hline $\mathrm{H}$ & -6.382393837 & 4.114120007 & -4.334616661 \\
\hline $\mathrm{H}$ & -7.692336559 & 5.388704300 & 3.100384474 \\
\hline $\mathrm{H}$ & -10.880518913 & 4.272655964 & 0.425974488 \\
\hline $\mathrm{F}$ & -6.143680096 & 3.405361176 & 2.459528208 \\
\hline $\mathrm{F}$ & -10.041156769 & 5.959774017 & 2.147571325 \\
\hline $\mathrm{F}$ & -7.900969028 & 2.744056940 & -2.764968157 \\
\hline $\mathrm{F}$ & -3.853623867 & 4.699646950 & -4.090529919 \\
\hline $\mathrm{O}$ & -4.421744347 & -1.824553490 & -3.981663704 \\
\hline $\mathrm{C}$ & -4.497101784 & -0.673659444 & -4.831182957 \\
\hline $\mathrm{H}$ & -5.537896633 & -0.358200222 & -4.972809792 \\
\hline $\mathrm{H}$ & -3.908603907 & 0.157671764 & -4.424113750 \\
\hline $\mathrm{H}$ & -4.076556683 & -0.985445023 & -5.786517620 \\
\hline $\mathrm{N}$ & -10.508604050 & -0.306313813 & 1.657298326 \\
\hline $\mathrm{C}$ & -10.433274269 & 0.876600206 & 2.371155262 \\
\hline $\mathrm{C}$ & -11.718180656 & 1.533267498 & 2.213740349 \\
\hline $\mathrm{C}$ & -12.514376640 & 0.759925723 & 1.415683508 \\
\hline $\mathrm{C}$ & -11.767211914 & -0.446620256 & 1.022660971 \\
\hline $\mathrm{H}$ & -9.771013260 & -0.982582688 & 1.541102529 \\
\hline $\mathrm{H}$ & -11.944724083 & 2.478745699 & 2.686515331 \\
\hline $\mathrm{H}$ & -13.534418106 & 0.939580321 & 1.105780721 \\
\hline $\mathrm{O}$ & -9.429770470 & 1.271997333 & 3.031055212 \\
\hline $\mathrm{O}$ & -12.062644958 & -1.384564996 & 0.295402139 \\
\hline
\end{tabular}

$\left[{ }^{1} \mathbf{I r}-{ }^{3} \mathbf{2}_{\mathrm{A}}\right]$

H $\quad-2.851521015 \quad-2.673525572 \quad 6.110894203$

H $\quad-2.137258291 \quad-3.878309727 \quad 3.747082233$

H $\quad-0.515332699 \quad-2.271195889 \quad 2.526218891$

$\begin{array}{llll}\mathrm{H} & -0.009758662 & 3.028645277 & 6.102147579\end{array}$

$\mathrm{H} \quad-2.403014421 \quad 1.302121162 \quad 9.248602867$

H $\quad-2.596321106 \quad-0.765854359 \quad 7.843238831$

H $\quad \begin{array}{lll}1.411056876 & 0.245253533 & 6.688470840\end{array}$

H $\quad 5.181571007 \quad-1.723754525 \quad 6.088684082$

H $\quad 4.845962048 \quad-1.470100999 \quad 3.640497446$

H $\quad-2.388773441 \quad 4.766954899 \quad 2.120796680$

H $\quad-4.405004025 \quad 3.470822096 \quad 1.452338457$

H $\quad-2.374303341 \quad-0.100485288 \quad 2.698348999$

$\begin{array}{llll}\text { F } & -5.870357513 & 1.397444248 & 1.788815618\end{array}$

F $\quad \begin{array}{llll}-4.632222652 & 0.437138677 & 0.282641590\end{array}$

F $\quad-4.759380817 \quad-0.418612361 \quad 2.279985905$

$\begin{array}{llll}\text { C } & -4.711343288 & 0.742336750 & 1.590902209\end{array}$

F $\quad 4.710159779 \quad-1.014374733 \quad 8.456863403$

F $\quad 2.849260569 \quad 0.100175716 \quad 8.722590446$

F $\quad 2.799967766 \quad-2.056561232 \quad 8.453880310$

$\begin{array}{llll}\text { C } & 3.429137945 & -0.928666174 & 8.064098358\end{array}$

$\begin{array}{llll}\text { C } & -1.446317315 & -1.102640629 & 5.350574970\end{array}$

C $\quad-2.167171240 \quad-2.310197353 \quad 5.359920025$

$\begin{array}{llll}\text { C } & -1.821459770 & -2.935541868 & 4.170032501\end{array}$

N $\quad-0.946522951 \quad-2.143740654 \quad 3.513861656$

$\begin{array}{llll}\text { C } & -0.591924608 & 2.227980375 & 6.536587715\end{array}$

$\begin{array}{llll}\text { C } & -1.194755793 & 2.329554081 & 7.795914650\end{array}$

$\begin{array}{llll}\text { C } & -1.934082866 & 1.233209133 & 8.272879601\end{array}$

$\begin{array}{llll}\text { C } & -2.037569046 & 0.095303141 & 7.493548870\end{array}$

$\begin{array}{llll}\text { C } & -1.405763745 & 0.049316388 & 6.244518280\end{array}$

C $\quad 2.669011116 \quad-0.153854504 \quad 2.449305534$

$\begin{array}{llll}\text { C } & 3.571414709 & -0.394574940 & 1.415061355\end{array}$

$\begin{array}{llll}\text { C } & 3.150818586 & -0.143074304 & 0.100847803\end{array}$

$\begin{array}{llll}\text { C } & 1.886556625 & 0.376120955 & -0.198970392\end{array}$ 


\begin{tabular}{|c|c|c|c|}
\hline $\mathrm{C}$ & 1.034654856 & 0.622694731 & 0.856091440 \\
\hline $\mathrm{C}$ & 2.191648960 & -0.135116160 & 6.045370102 \\
\hline $\mathrm{C}$ & 3.323358536 & -0.740233421 & 6.571759701 \\
\hline $\mathrm{C}$ & 4.300928116 & -1.223698735 & 5.700846195 \\
\hline $\mathrm{C}$ & 4.125571728 & -1.056926131 & 4.334055901 \\
\hline $\mathrm{C}$ & 2.981328726 & -0.412583828 & 3.856052399 \\
\hline $\mathrm{N}$ & -0.699374199 & 1.113082767 & 5.790933132 \\
\hline $\mathrm{N}$ & -0.705513358 & -1.030843735 & 4.225414753 \\
\hline $\mathrm{N}$ & 2.020845652 & 0.015519170 & 4.724000454 \\
\hline $\mathrm{C}$ & 1.368505001 & 0.392039448 & 2.210989475 \\
\hline $\mathrm{C}$ & 0.920751750 & 2.766177893 & 3.741943121 \\
\hline $\mathrm{C}$ & 2.118908167 & 3.344497442 & 4.157888412 \\
\hline $\mathrm{C}$ & 2.378028393 & 4.709216118 & 4.105967999 \\
\hline $\mathrm{C}$ & 1.380393267 & 5.539774895 & 3.601968765 \\
\hline $\mathrm{C}$ & 0.162499547 & 5.044315338 & 3.160322189 \\
\hline $\mathrm{C}$ & -0.057312194 & 3.659378052 & 3.231469154 \\
\hline $\mathrm{C}$ & -1.305427074 & 3.028666019 & 2.790027618 \\
\hline $\mathrm{C}$ & -2.411423922 & 3.691231012 & 2.243366480 \\
\hline $\mathrm{C}$ & -3.535018682 & 2.970264435 & 1.864306569 \\
\hline $\mathrm{C}$ & -3.537060022 & 1.582800388 & 2.028049946 \\
\hline $\mathrm{C}$ & -2.415762424 & 0.970877767 & 2.564414978 \\
\hline $\mathrm{N}$ & -1.340129733 & 1.674397469 & 2.948221684 \\
\hline $\mathrm{Ir}$ & 0.321238905 & 0.825845361 & 3.852094889 \\
\hline $\mathrm{O}$ & -1.115111351 & 3.406388521 & 8.594458580 \\
\hline $\mathrm{C}$ & -0.329947233 & 4.523920536 & 8.163620949 \\
\hline $\mathrm{H}$ & 0.716594994 & 4.233602047 & 8.010182381 \\
\hline $\mathrm{H}$ & -0.732992113 & 4.958228588 & 7.240673065 \\
\hline $\mathrm{H}$ & -0.391956508 & 5.255009174 & 8.968790054 \\
\hline $\mathrm{N}$ & 2.041230202 & -3.173531294 & 2.191149712 \\
\hline $\mathrm{C}$ & 1.266828418 & -2.796586275 & 1.096734047 \\
\hline $\mathrm{C}$ & 2.129219294 & -2.843324661 & -0.039031137 \\
\hline $\mathrm{C}$ & 3.447368145 & -3.210317373 & 0.416779399 \\
\hline $\mathrm{C}$ & 3.398914099 & -3.405370712 & 1.847469687 \\
\hline $\mathrm{H}$ & 1.707526326 & -3.226408482 & 3.142440557 \\
\hline $\mathrm{H}$ & 1.803647399 & -2.665342569 & -1.053051710 \\
\hline $\mathrm{H}$ & 4.342018127 & -3.318464756 & -0.179016218 \\
\hline $\mathrm{O}$ & 0.059389222 & -2.429704666 & 1.161760807 \\
\hline
\end{tabular}

$$
\begin{array}{lrrr}
\mathrm{O} & 4.289082527 & -3.638777018 & 2.668347359 \\
\mathrm{H} & 1.593986511 & 0.575366020 & -1.222814202 \\
\mathrm{H} & 4.577337265 & -0.758280814 & 1.577277064 \\
\mathrm{~F} & -0.172508463 & 1.132169843 & 0.563130319 \\
\mathrm{~F} & 4.008647919 & -0.346636713 & -0.896454036 \\
\mathrm{~F} & 3.100271225 & 2.554658890 & 4.653512955 \\
\mathrm{~F} & 1.607773185 & 6.860859871 & 3.545743227 \\
\mathrm{H} & -0.572214663 & 5.740647316 & 2.773905516 \\
\mathrm{H} & 3.326818228 & 5.113109112 & 4.439771175
\end{array}
$$$$
{ }^{1}\left[\operatorname{Ir}-2_{B}\right]
$$$$
\text { H } \quad \begin{array}{llll}
-7.196782112 & -3.638515711 & 2.094232559
\end{array}
$$$$
\mathrm{H} \quad-8.508371353 \quad-2.061096907 \quad 3.919263363
$$$$
\mathrm{H} \quad-8.344578743 \quad 0.330950916 \quad 3.042173147
$$$$
\text { H } \quad-5.100132942 \quad 0.277131557 \quad-2.482889652
$$$$
\text { H } \quad-4.605488777 \quad-4.005450249 \quad-2.251375437
$$$$
\text { H } \quad-5.891592979 \quad-3.946677208 \quad-0.102031723
$$$$
\text { H } \quad-8.086749077 \quad-0.782005847 \quad-2.027866364
$$$$
\text { Н } \quad-12.210862160 \quad 0.365195543 \quad-2.341888428
$$$$
\text { H } \quad-11.749270439 \quad 2.196161985 \quad-0.725279868
$$$$
\mathrm{H} \quad-2.438805580 \quad 3.677864790 \quad 0.413133413
$$$$
\text { H } \quad \begin{array}{llll}
-1.700420856 & 2.628102541 & 2.545874119
\end{array}
$$$$
\text { H } \quad-5.203759193 \quad 0.152040407 \quad 2.378512621
$$$$
\text { F } \quad-2.139811277 \quad 1.255941510 \quad 4.618574142
$$$$
\text { F } \quad-4.050828457 \quad 0.185080439 \quad 4.596945286
$$$$
\text { F } \quad-2.341056108 \quad-0.607054591 \quad 3.515258074
$$$$
\text { C } \quad-2.965834856 \quad 0.542885661 \quad 3.849365473
$$$$
\text { F } \quad-11.368773460 \quad-1.228567243 \quad-4.133421421
$$$$
\text { F } \quad-9.278595924 \quad-1.826846838 \quad-3.908733368
$$$$
\text { F } \quad-10.791561127 \quad-2.612298489 \quad-2.558574677
$$$$
\text { C } \quad-10.407607079 \quad-1.509768248 \quad-3.236446142
$$$$
\begin{array}{llll}
\text { C } & -6.939293861 & -1.664391875 & 1.061969876
\end{array}
$$$$
\begin{array}{llll}
\text { C } & -7.357849598 & -2.572729111 & 2.055402040
\end{array}
$$$$
\begin{array}{llll}
\text { C } & -8.024262428 & -1.797640800 & 2.990357876
\end{array}
$$$$
\text { N } \quad-7.996426582 \quad-0.516183615 \quad 2.558083773
$$ 


\begin{tabular}{|c|c|c|c|}
\hline $\mathrm{C}$ & -5.241009235 & -0.683865130 & -2.005993366 \\
\hline $\mathrm{C}$ & -4.746819496 & -1.879886985 & -2.542811394 \\
\hline $\mathrm{C}$ & -4.984206200 & -3.075091600 & -1.842114449 \\
\hline $\mathrm{C}$ & -5.695511341 & -3.036047220 & -0.656523287 \\
\hline $\mathrm{C}$ & -6.171275139 & -1.811317921 & -0.173099980 \\
\hline $\mathrm{C}$ & -9.245398521 & 2.743278027 & 0.420923322 \\
\hline $\mathrm{C}$ & -10.116237640 & 3.755500078 & 0.858860612 \\
\hline $\mathrm{C}$ & -9.605420113 & 4.756866455 & 1.665224910 \\
\hline $\mathrm{C}$ & -8.268306732 & 4.778038979 & 2.050949335 \\
\hline $\mathrm{C}$ & -7.452306271 & 3.740016699 & 1.619462013 \\
\hline $\mathrm{C}$ & -8.921152115 & -0.153255880 & -1.751448750 \\
\hline $\mathrm{C}$ & -10.177119255 & -0.352449924 & -2.299999952 \\
\hline $\mathrm{C}$ & -11.218949318 & 0.499218971 & -1.923739910 \\
\hline $\mathrm{C}$ & -10.957431793 & 1.520231128 & -1.023257375 \\
\hline $\mathrm{C}$ & -9.668010712 & 1.688499212 & -0.501886070 \\
\hline $\mathrm{N}$ & -5.935907841 & -0.663424790 & -0.854734898 \\
\hline $\mathrm{N}$ & -7.341452122 & -0.421928108 & 1.389604688 \\
\hline $\mathrm{N}$ & -8.673889160 & 0.833032668 & -0.876362801 \\
\hline $\mathrm{C}$ & -7.879193306 & 2.694291353 & 0.804679334 \\
\hline $\mathrm{C}$ & -6.063262939 & 2.472230673 & -1.328946233 \\
\hline $\mathrm{C}$ & -6.606442451 & 2.925942421 & -2.528942347 \\
\hline $\mathrm{C}$ & -5.981825352 & 3.844863892 & -3.363320827 \\
\hline $\mathrm{C}$ & -4.743207932 & 4.343555450 & -2.968096733 \\
\hline $\mathrm{C}$ & -4.132445812 & 3.947983265 & -1.788912296 \\
\hline $\mathrm{C}$ & -4.798730850 & 3.014888525 & -0.977613568 \\
\hline $\mathrm{C}$ & -4.237924576 & 2.503738403 & 0.273983926 \\
\hline $\mathrm{C}$ & -3.034570932 & 2.898915052 & 0.872769117 \\
\hline $\mathrm{C}$ & -2.615226984 & 2.311575413 & 2.057524920 \\
\hline $\mathrm{C}$ & -3.394413710 & 1.297536135 & 2.620033979 \\
\hline $\mathrm{C}$ & -4.572780609 & 0.940608740 & 1.990256190 \\
\hline $\mathrm{N}$ & -5.001525402 & 1.547526598 & 0.876135111 \\
\hline$r$ & -6.827001095 & 1.147731423 & 0.003308177 \\
\hline $\mathrm{H}$ & -3.166776657 & 4.368567467 & -1.534755945 \\
\hline $\mathrm{H}$ & -6.446209908 & 4.170392036 & -4.286805630 \\
\hline $\mathrm{H}$ & -7.877082348 & 5.577811241 & 2.666887283 \\
\hline $\mathrm{H}$ & -11.161242485 & 3.797172785 & 0.577517390 \\
\hline $\mathrm{F}$ & -6.159106255 & 3.776679516 & 2.048608303 \\
\hline
\end{tabular}

$$
\begin{array}{lrrr}
\text { F } & -10.414246559 & 5.743514061 & 2.089239597 \\
\text { F } & -7.813747406 & 2.455460787 & -2.929798841 \\
\text { F } & -4.122567177 & 5.232865810 & -3.761549950 \\
\text { O } & -4.058771610 & -1.976384282 & -3.691686630 \\
\text { C } & -3.833622694 & -0.788328886 & -4.459418297 \\
\text { H } & -4.782948971 & -0.330943704 & -4.763295650 \\
\text { H } & -3.234939575 & -0.062132847 & -3.896806002 \\
\text { H } & -3.283132315 & -1.109335184 & -5.342935085 \\
\text { N } & -6.406517029 & 2.045437336 & 4.545182705 \\
\text { C } & -5.614670753 & 3.145071507 & 4.929874420 \\
\text { C } & -6.594388485 & 4.223141193 & 5.291922092 \\
\text { C } & -7.833150387 & 3.774190664 & 5.056975842 \\
\text { C } & -7.741906643 & 2.380937099 & 4.510426521 \\
\text { H } & -6.006986618 & 1.174211144 & 4.234811306 \\
\text { H } & -6.273094177 & 5.187215805 & 5.663395882 \\
\text { H } & -8.783496857 & 4.275450230 & 5.181673050 \\
\text { O } & -4.406589985 & 3.171858788 & 4.935277462 \\
\text { O } & -8.660143852 & 1.684348941 & 4.090713978
\end{array}
$$$$
\left[{ }^{3} I r-{ }^{1} 2_{B}\right]
$$$$
\begin{array}{lrrr}
\mathrm{H} & -7.311179161 & -3.442727327 & 2.213648319 \\
\mathrm{H} & -8.633615494 & -1.793228507 & 3.968427181 \\
\mathrm{H} & -8.410120010 & 0.589654326 & 3.054162502 \\
\mathrm{H} & -5.009476185 & 0.316892684 & -2.397759199 \\
\mathrm{H} & -4.647300720 & -3.969849348 & -2.066931009 \\
\mathrm{H} & -5.991279125 & -3.830860138 & 0.042283840 \\
\mathrm{H} & -7.988668442 & -0.591167271 & -2.162447214 \\
\mathrm{H} & -12.233819008 & 0.111036159 & -2.140063047 \\
\mathrm{H} & -11.868640900 & 1.904637456 & -0.466906041 \\
\mathrm{H} & -2.335046291 & 3.643111706 & 0.380100399 \\
\mathrm{H} & -1.680819631 & 2.680666447 & 2.581082821 \\
\mathrm{H} & -5.311403751 & 0.392475665 & 2.503591061 \\
\mathrm{~F} & -2.252442360 & 1.451729655 & 4.748515606 \\
\mathrm{~F} & -4.145262241 & 0.346701950 & 4.678145409 \\
\mathrm{~F} & -2.389917612 & -0.418806404 & 3.653815031
\end{array}
$$ 


\begin{tabular}{|c|c|c|c|}
\hline $\mathrm{C}$ & -3.047888756 & 0.721224964 & 3.966680527 \\
\hline $\mathrm{F}$ & -11.176205635 & -1.181471109 & -4.216454029 \\
\hline $\mathrm{F}$ & -9.136885643 & -1.863916636 & -3.820931196 \\
\hline $\mathrm{F}$ & -10.798548698 & -2.609275341 & -2.625917673 \\
\hline $\mathrm{C}$ & -10.307510376 & -1.508844376 & -3.240478754 \\
\hline $\mathrm{C}$ & -7.002609730 & -1.503010154 & 1.134863734 \\
\hline $\mathrm{C}$ & -7.455175877 & -2.376138926 & 2.142121553 \\
\hline $\mathrm{C}$ & -8.128334999 & -1.562451482 & 3.041886806 \\
\hline $\mathrm{N}$ & -8.075287819 & -0.293887109 & 2.580469370 \\
\hline $\mathrm{C}$ & -5.191845417 & -0.627717316 & -1.903386474 \\
\hline $\mathrm{C}$ & -4.717522144 & -1.847141862 & -2.403856993 \\
\hline $\mathrm{C}$ & -5.009001732 & -3.020516157 & -1.686550498 \\
\hline $\mathrm{C}$ & -5.751837730 & -2.937358379 & -0.523033977 \\
\hline $\mathrm{C}$ & -6.206929207 & -1.690604210 & -0.076424211 \\
\hline $\mathrm{C}$ & -9.359424591 & 2.626566410 & 0.550812662 \\
\hline $\mathrm{C}$ & -10.260860443 & 3.488255739 & 1.196058512 \\
\hline $\mathrm{C}$ & -9.750113487 & 4.501790524 & 1.984897017 \\
\hline $\mathrm{C}$ & -8.365146637 & 4.736097813 & 2.185451508 \\
\hline $\mathrm{C}$ & -7.498043060 & 3.842461824 & 1.594453692 \\
\hline $\mathrm{C}$ & -8.860586166 & -0.067689784 & -1.796731114 \\
\hline $\mathrm{C}$ & -10.122859001 & -0.380515456 & -2.270390272 \\
\hline $\mathrm{C}$ & -11.237703323 & 0.337353170 & -1.775751591 \\
\hline $\mathrm{C}$ & -11.032047272 & 1.330269814 & -0.846227288 \\
\hline $\mathrm{C}$ & -9.724822998 & 1.617908835 & -0.384429783 \\
\hline $\mathrm{N}$ & -5.917758942 & -0.564907372 & -0.772446632 \\
\hline $\mathrm{N}$ & -7.393506050 & -0.248406291 & 1.425143957 \\
\hline $\mathrm{N}$ & -8.657041550 & 0.885681570 & -0.881202459 \\
\hline $\mathrm{C}$ & -7.910709858 & 2.742926836 & 0.821555257 \\
\hline $\mathrm{C}$ & -5.997665405 & 2.517148495 & -1.357076049 \\
\hline $\mathrm{C}$ & -6.501742840 & 2.914740801 & -2.596381664 \\
\hline $\mathrm{C}$ & -5.807548523 & 3.731467009 & -3.480348110 \\
\hline $\mathrm{C}$ & -4.547415733 & 4.181072235 & -3.093463182 \\
\hline $\mathrm{C}$ & -3.978342056 & 3.839254141 & -1.873435855 \\
\hline $\mathrm{C}$ & -4.707180977 & 3.009425163 & -1.012179255 \\
\hline $\mathrm{C}$ & -4.193930626 & 2.559916496 & 0.285017848 \\
\hline $\mathrm{C}$ & -2.979848146 & 2.927550793 & 0.875644863 \\
\hline $\mathrm{C}$ & -2.607720375 & 2.387472391 & 2.100060463 \\
\hline
\end{tabular}

$\begin{array}{llll}\text { C } & -3.445390701 & 1.451376677 & 2.708424807\end{array}$

$\begin{array}{llll}\text { C } & -4.637086391 & 1.124972701 & 2.084218979\end{array}$

$\begin{array}{llll}\mathrm{N} & -5.016977310 & 1.689516425 & 0.931278050\end{array}$

Ir $\quad-6.839319229 \quad 1.300661087 \quad 0.016455309$

$\mathrm{H} \quad-2.994152784 \quad 4.222826004 \quad-1.632062912$

H $\quad-6.235407352 \quad 4.019967079 \quad-4.433280468$

$\mathrm{H} \quad \begin{array}{llll}8.016703606 & 5.594079971 & 2.741298437\end{array}$

H $\quad-11.335622787 \quad 3.406441927 \quad 1.090121984$

F $\quad-6.182002068 \quad 4.030022144 \quad 1.814702988$

$\begin{array}{llll}\text { F } & -10.593291283 & 5.322362900 & 2.609642029\end{array}$

$\begin{array}{llll}\text { F } & -7.725577354 & 2.494683743 & -2.976885080\end{array}$

F $\quad-3.860183477 \quad 4.968509197 \quad-3.931680441$

$\begin{array}{llll}\text { O } & -4.000653267 & -1.985866189 & -3.530210733\end{array}$

$\begin{array}{llll}\text { C } & -3.729494572 & -0.824861407 & -4.323580265\end{array}$

H $\quad-4.660386562 \quad-0.352531701 \quad-4.660048008$

H $\quad-3.123514414 \quad-0.099340111 \quad-3.767644405$

H $\quad-3.169085979 \quad-1.183004022 \quad-5.186414242$

$\begin{array}{llll}\mathrm{N} & -6.541077137 & 2.242883444 & 4.426558018\end{array}$

$\begin{array}{llll}\text { C } & -5.732511044 & 3.292165041 & 4.932363033\end{array}$

C $\quad-6.683653355 \quad 4.362415314 \quad 5.292850018$

$\begin{array}{llll}\text { C } & -7.940429211 & 3.960514069 & 4.965554237\end{array}$

$\begin{array}{llll}\text { C } & -7.872843742 & 2.601895571 & 4.395249367\end{array}$

$\mathrm{H} \quad-6.362584114 \quad 5.304003239 \quad 5.716310501$

$\mathrm{H} \quad-8.877143860 \quad 4.487055302 \quad 5.085294724$

$\begin{array}{llll}\text { O } & -4.516366482 & 3.249615431 & 4.994721413\end{array}$

$\begin{array}{llll}\text { O } & -8.791933060 & 1.910189271 & 3.921333313\end{array}$

$\begin{array}{llll}\mathrm{H} & -6.175652981 & 1.322285891 & 4.248908043\end{array}$

$$
\left[{ }^{1} \mathbf{I r}-{ }^{3} 2_{B}\right]
$$

H $\quad-2.738180161-2.627372026$

6.580168724

H $\quad-2.156804323 \quad-3.945400953 \quad 4.239408970$

H $\quad-0.642779469 \quad-2.398889303 \quad 2.846325874$

H $\quad 0.084930249 \quad 3.067533970 \quad 6.189333439$

$\mathrm{H} \quad-2.065521240 \quad 1.418328166 \quad 9.542337418$

H $\quad-2.446770191 \quad-0.643274486 \quad 8.167936325$ 


\begin{tabular}{|c|c|c|c|}
\hline $\mathrm{H}$ & 1.598430991 & 0.349152565 & 6.585376263 \\
\hline $\mathrm{H}$ & 4.976823330 & -2.106800556 & 5.576139450 \\
\hline $\mathrm{H}$ & 4.323028088 & -1.895260215 & 3.184397697 \\
\hline $\mathrm{H}$ & -2.579723358 & 4.799164295 & 2.182112455 \\
\hline $\mathrm{H}$ & -4.473173141 & 3.455115318 & 1.282606840 \\
\hline $\mathrm{H}$ & -2.534621477 & -0.060130361 & 2.814296246 \\
\hline $\mathrm{F}$ & -5.481534958 & 1.313505650 & 0.519454181 \\
\hline $\mathrm{F}$ & -4.164868355 & -0.408158213 & 0.841790974 \\
\hline $\mathrm{F}$ & -5.522878647 & 0.235188290 & 2.416835785 \\
\hline $\mathrm{C}$ & -4.720243454 & 0.688025296 & 1.428440690 \\
\hline $\mathrm{F}$ & 4.982906342 & -1.182303548 & 7.919905186 \\
\hline $\mathrm{F}$ & 3.284884453 & 0.119428493 & 8.389454842 \\
\hline $\mathrm{F}$ & 3.013196945 & -2.039509058 & 8.290359497 \\
\hline $\mathrm{C}$ & 3.663432360 & -0.997878075 & 7.727314472 \\
\hline $\mathrm{C}$ & -1.500671387 & -1.036909103 & 5.602032185 \\
\hline $\mathrm{C}$ & -2.151611328 & -2.274707794 & 5.746073723 \\
\hline $\mathrm{C}$ & -1.873408318 & -2.956683397 & 4.569972992 \\
\hline $\mathrm{N}$ & -1.114046812 & -2.160632133 & 3.789535046 \\
\hline $\mathrm{C}$ & -0.475899935 & 2.282825947 & 6.676601410 \\
\hline $\mathrm{C}$ & -0.957280993 & 2.404122353 & 7.984565735 \\
\hline $\mathrm{C}$ & -1.687063217 & 1.333455086 & 8.529392242 \\
\hline $\mathrm{C}$ & -1.894760609 & 0.199187264 & 7.766091347 \\
\hline $\mathrm{C}$ & -1.375800252 & 0.130560622 & 6.466588974 \\
\hline $\mathrm{C}$ & 2.177594662 & -0.352251053 & 2.239306211 \\
\hline $\mathrm{C}$ & 2.900052309 & -0.727043629 & 1.110543847 \\
\hline $\mathrm{C}$ & 2.487189770 & -0.239754155 & -0.132271051 \\
\hline $\mathrm{C}$ & 1.404168367 & 0.642325997 & -0.269476891 \\
\hline $\mathrm{C}$ & 0.674136937 & 0.932678401 & 0.867994964 \\
\hline $\mathrm{C}$ & 2.217663765 & -0.166775897 & 5.864421844 \\
\hline $\mathrm{C}$ & 3.335995436 & -0.886427462 & 6.259560108 \\
\hline $\mathrm{C}$ & 4.102367401 & -1.533182025 & 5.288486958 \\
\hline $\mathrm{C}$ & 3.737021685 & -1.410289407 & 3.955418348 \\
\hline $\mathrm{C}$ & 2.616668224 & -0.650307119 & 3.603790998 \\
\hline $\mathrm{N}$ & -0.682749033 & 1.170937181 & 5.946341515 \\
\hline $\mathrm{N}$ & -0.875123024 & -0.992990375 & 4.406628609 \\
\hline $\mathrm{N}$ & 1.864795923 & -0.057987783 & 4.576771259 \\
\hline $\mathrm{C}$ & 0.992787778 & 0.458266199 & 2.150323868 \\
\hline
\end{tabular}

$\begin{array}{llll}\text { C } & 0.736704648 & 2.794336557 & 3.783357620\end{array}$

$\begin{array}{llll}\text { C } & 1.962768674 & 3.348743916 & 4.141452312\end{array}$

$\begin{array}{llll}\text { C } & 2.214271307 & 4.715885162 & 4.156032562\end{array}$

$\begin{array}{llll}\text { C } & 1.180415869 & 5.568788528 & 3.775178909\end{array}$

C $\quad-0.063363470 \quad 5.095073700 \quad 3.383425236$

C $\quad-0.274219126 \quad 3.707863331 \quad 3.383979797$

$\begin{array}{llll}\text { C } & -1.527374983 & 3.084242582 & 2.948237419\end{array}$

$\begin{array}{llll}\text { C } & -2.600443125 & 3.725345850 & 2.320257187\end{array}$

$\begin{array}{llll}\text { C } & -3.657977819 & 2.977792740 & 1.815023541\end{array}$

$\begin{array}{llll}\text { C } & -3.643118382 & 1.591081738 & 1.972677588\end{array}$

$\begin{array}{llll}\text { C } & -2.585901499 & 1.008898497 & 2.653168917\end{array}$

$\mathrm{N} \quad-1.560594440 \quad 1.730975986 \quad 3.119326830$

Ir $\quad 0.132448792 \quad 0.865278840 \quad 3.911217213$

$\begin{array}{llll}\text { O } & -0.768967569 & 3.479121208 & 8.768765450\end{array}$

$\begin{array}{llll}\text { C } & 0.013181668 & 4.567228794 & 8.263439178\end{array}$

H $\quad \begin{array}{lll}1.031058788 & 4.239977837 & 8.018358231\end{array}$

$\begin{array}{llll}\mathrm{H} & -0.454296827 & 5.013689518 & 7.377480030\end{array}$

$\mathrm{H} \quad 0.049393076 \quad 5.302016735 \quad 9.066786766$

N $\quad-1.180980682-1.493893981 \quad 0.150306612$

$\begin{array}{llll}\text { C } & -0.102317475 & -2.300748825 & 0.493673563\end{array}$

$\begin{array}{llll}\text { C } & 0.727280974 & -2.380327940 & -0.667571425\end{array}$

C $\quad 0.123968802 \quad-1.566900492 \quad-1.704803228$

C $\quad-1.099843144 \quad-0.996756554 \quad-1.184051514$

H $\quad-1.978346586 \quad-1.293474078 \quad 0.737658858$

H $\quad \begin{array}{llll}1.611930370 & -2.994625568 & -0.738396108\end{array}$

H $\quad 0.488272339 \quad-1.402738094 \quad-2.708420277$

$\begin{array}{lllll}\text { O } & 0.103251033 & -2.805099964 & 1.629440904\end{array}$

O $\quad-1.914655805 \quad-0.230117440 \quad-1.686259031$

$\mathrm{H} \quad 1.139972210 \quad 1.064510345 \quad-1.230192184$

$\mathrm{H} \quad 3.786184072 \quad-1.348210812 \quad 1.149169803$

F $\quad-0.392526597 \quad 1.741952538 \quad 0.699468434$

F $\quad 3.207260132 \quad-0.544992983 \quad-1.213129401$

F $\quad 2.971562624 \quad 2.527127504 \quad 4.519024372$

F $\quad 1.400305748 \quad 6.892560482 \quad 3.788664103$

$\mathrm{H} \quad-0.825470626 \quad 5.807582855 \quad 3.090763807$

H $\quad 3.182752848 \quad 5.107227325 \quad 4.444752216$ 


\begin{tabular}{|c|c|c|c|}
\hline \multicolumn{4}{|c|}{${ }^{1}\left[\mathbf{I r}-\mathbf{Q}_{\mathrm{A}}\right]$} \\
\hline $\mathrm{H}$ & -1.749826908 & 0.818890810 & -10.842705727 \\
\hline $\mathrm{H}$ & 0.318346292 & -0.988075554 & -10.769630432 \\
\hline $\mathrm{H}$ & 0.432449102 & -1.854939461 & -8.321844101 \\
\hline $\mathrm{H}$ & -3.522577286 & 1.688202739 & -4.790636063 \\
\hline $\mathrm{H}$ & -5.414916992 & 3.317163706 & -8.310636520 \\
\hline $\mathrm{H}$ & -3.786702156 & 1.993459225 & -9.687515259 \\
\hline $\mathrm{H}$ & 0.055133291 & -4.853688240 & -4.049341202 \\
\hline $\mathrm{H}$ & -4.107126236 & -1.034284830 & -6.338964939 \\
\hline $\mathrm{H}$ & -4.431562901 & -5.138910294 & -5.116033554 \\
\hline $\mathrm{H}$ & -2.040984392 & -5.047150612 & -4.442137241 \\
\hline $\mathrm{H}$ & -0.007181963 & 3.025339603 & -1.979666352 \\
\hline $\mathrm{H}$ & 1.134277344 & 3.783391953 & -3.636134863 \\
\hline $\mathrm{H}$ & 2.340095520 & 4.544425011 & -5.675672531 \\
\hline $\mathrm{H}$ & 0.590732157 & 1.251009226 & -7.792864323 \\
\hline $\mathrm{F}$ & 2.346485853 & 4.686758041 & -8.266481400 \\
\hline $\mathrm{F}$ & 3.531092167 & 2.867831945 & -8.158097267 \\
\hline $\mathrm{F}$ & 1.647791743 & 2.863538980 & -9.249799728 \\
\hline $\mathrm{C}$ & 2.272226095 & 3.346432924 & -8.150250435 \\
\hline $\mathrm{F}$ & -5.959186554 & -2.546298504 & -7.503108501 \\
\hline $\mathrm{F}$ & -6.399742126 & -4.311709881 & -6.296379089 \\
\hline $\mathrm{F}$ & -6.663369179 & -2.316172361 & -5.461579800 \\
\hline $\mathrm{C}$ & -5.877270222 & -3.073900938 & -6.253575325 \\
\hline $\mathrm{F}$ & -1.401682019 & 2.112275839 & -0.023204627 \\
\hline $\mathrm{H}$ & -2.794518232 & 0.022403322 & -0.732974529 \\
\hline $\mathrm{F}$ & -2.942685366 & -1.157641649 & -3.008590937 \\
\hline $\mathrm{F}$ & 2.612575531 & -4.650358200 & -3.767576218 \\
\hline $\mathrm{H}$ & 3.452822685 & -2.276467085 & -4.432164192 \\
\hline $\mathrm{F}$ & 2.131905317 & -0.234966606 & -5.274087429 \\
\hline $\mathrm{C}$ & -1.811558008 & 0.341632843 & -8.651891708 \\
\hline $\mathrm{C}$ & -1.370633602 & 0.277390450 & -9.989776611 \\
\hline $\mathrm{C}$ & -0.313917011 & -0.621947646 & -9.973715782 \\
\hline $\mathrm{N}$ & -0.141745269 & -1.044153929 & -8.699089050 \\
\hline $\mathrm{C}$ & -3.636215210 & 1.791447520 & -5.861876488 \\
\hline $\mathrm{C}$ & -4.610281467 & 2.610691786 & -6.447295189 \\
\hline
\end{tabular}

\begin{tabular}{|c|c|c|c|}
\hline $\mathrm{C}$ & -4.663270950 & 2.685322523 & -7.849644661 \\
\hline $\mathrm{C}$ & -3.761715174 & 1.953714252 & -8.604121208 \\
\hline $\mathrm{C}$ & -2.812514544 & 1.151250601 & -7.958835602 \\
\hline $\mathrm{C}$ & -0.360725522 & -2.831175804 & -4.772910595 \\
\hline $\mathrm{C}$ & 0.448069006 & -3.877796888 & -4.302150726 \\
\hline $\mathrm{C}$ & 1.806475639 & -3.652098656 & -4.193944454 \\
\hline $\mathrm{C}$ & 2.383839846 & -2.430267811 & -4.518711090 \\
\hline $\mathrm{C}$ & 1.544184923 & -1.417316198 & -4.961315632 \\
\hline $\mathrm{C}$ & -3.687128782 & -1.939430237 & -5.919582844 \\
\hline $\mathrm{C}$ & -4.451208591 & -3.087641716 & -5.775153160 \\
\hline $\mathrm{C}$ & -3.860589981 & -4.223426819 & -5.219647408 \\
\hline $\mathrm{C}$ & -2.525790215 & -4.169494152 & -4.850614071 \\
\hline $\mathrm{C}$ & -1.786061406 & -2.995860338 & -5.037107468 \\
\hline $\mathrm{N}$ & -2.767987728 & 1.086133480 & -6.605632305 \\
\hline $\mathrm{N}$ & -1.062434912 & -0.483195066 & -7.891315937 \\
\hline $\mathrm{N}$ & -2.396493435 & -1.891187310 & -5.563961983 \\
\hline $\mathrm{C}$ & 0.166811600 & -1.560029626 & -5.109274387 \\
\hline $\mathrm{C}$ & -1.377293825 & 0.432626605 & -3.835077524 \\
\hline $\mathrm{C}$ & -2.152298927 & -0.076123789 & -2.791998863 \\
\hline $\mathrm{C}$ & -2.181615353 & 0.462110341 & -1.511178493 \\
\hline $\mathrm{C}$ & -1.390322447 & 1.578284621 & -1.256772876 \\
\hline $\mathrm{C}$ & -0.600403011 & 2.155982018 & -2.236773014 \\
\hline $\mathrm{C}$ & -0.600288033 & 1.578808308 & -3.518106461 \\
\hline $\mathrm{C}$ & 0.189320982 & 2.118545294 & -4.627743244 \\
\hline $\mathrm{C}$ & 1.024139166 & 3.244069815 & -4.568570137 \\
\hline $\mathrm{C}$ & 1.700824022 & 3.668207645 & -5.702964306 \\
\hline $\mathrm{C}$ & 1.539564133 & 2.953305960 & -6.893312931 \\
\hline $\mathrm{C}$ & 0.719497621 & 1.838309169 & -6.896692753 \\
\hline $\mathrm{N}$ & 0.055634852 & 1.436606050 & -5.802188873 \\
\hline Ir & -1.202706456 & -0.210475922 & -5.751942158 \\
\hline $\mathrm{O}$ & -5.507606030 & 3.339250565 & -5.761473179 \\
\hline $\mathrm{C}$ & -5.488477707 & 3.283624411 & -4.329926014 \\
\hline $\mathrm{H}$ & -5.674385071 & 2.263747931 & -3.971888065 \\
\hline $\mathrm{H}$ & -4.532952785 & 3.647005081 & -3.932619333 \\
\hline $\mathrm{H}$ & -6.293726444 & 3.938845158 & -3.999306679 \\
\hline $\mathrm{C}$ & -4.646603584 & -5.295475483 & -8.742573738 \\
\hline $\mathrm{C}$ & -4.443714142 & -6.615521908 & -8.304064751 \\
\hline
\end{tabular}

$\begin{array}{llll}\text { C } & -4.663270950 & 2.685322523 & -7.849644661\end{array}$

C $\quad \begin{array}{llll}-3.761715174 & 1.953714252 & -8.604121208\end{array}$

C $\quad \begin{array}{llll}-2.812514544 & 1.151250601 & -7.958835602\end{array}$

C $\quad-0.360725522 \quad-2.831175804 \quad-4.772910595$

C $\quad 1.806475639 \quad-3.652098656 \quad-4.193944454$

C $\quad 2.383839846 \quad-2.430267811 \quad-4.518711090$

$\begin{array}{lll}-1.417316198 & -4.961315632\end{array}$

$\begin{array}{llll}\text { C } & -4.451208591 & -3.087641716 & -5.775153160\end{array}$

$\begin{array}{llll}\text { C } & -3.860589981 & -4.223426819 & -5.219647408\end{array}$

$\begin{array}{llll}\text { C } & -2.525790215 & -4.169494152 & -4.850614071\end{array}$

N $\quad-2.767987728 \quad 1.086133480 \quad-6.605632305$

N $\quad-1.062434912 \quad-0.483195066 \quad-7.891315937$

$\begin{array}{llll}C & -2.152298927 & -0.076123789 & -2.791998863\end{array}$

$\begin{array}{llll}\text { C } & -0.600403011 & 2.155982018 & -2.236773014\end{array}$

$\begin{array}{lll}-0.600288033 & 1.578808308 & -3.518106461\end{array}$

C $\quad 1.024139166 \quad 3.244069815 \quad-4.568570137$

C $\quad \begin{array}{llll}1.700824022 & 3.668207645 & -5.702964306\end{array}$

N $\quad 0.055634852 \quad 1.436606050 \quad-5.802188873$

Ir $\quad-1.202706456 \quad-0.210475922 \quad-5.751942158$

$-4.443714142-6.615521908-8.304064751$ 


$$
\begin{array}{lrrr}
\mathrm{C} & -3.194104671 & -7.018523693 & -7.859673023 \\
\mathrm{H} & -5.269487858 & -7.319895267 & -8.311388016 \\
\mathrm{C} & -3.598395109 & -4.387733459 & -8.739655495 \\
\mathrm{H} & -5.629655838 & -4.974947453 & -9.070366859 \\
\mathrm{C} & -2.338794708 & -4.795601368 & -8.282999039 \\
\mathrm{H} & -3.754554510 & -3.360585451 & -9.057742119 \\
\mathrm{C} & -2.112525940 & -6.113882065 & -7.834538460 \\
\mathrm{~N} & -1.275633335 & -3.905504942 & -8.228810310 \\
\mathrm{C} & -0.809090972 & -6.458271503 & -7.340346813 \\
\mathrm{H} & -3.032942057 & -8.037788391 & -7.518901825 \\
\mathrm{C} & -0.009815557 & -4.167518139 & -7.760834217 \\
\mathrm{H} & -1.446365237 & -2.957967758 & -8.536708832 \\
\mathrm{C} & 0.202209592 & -5.540534496 & -7.266159058 \\
\mathrm{O} & 0.878145039 & -3.292417288 & -7.763250351 \\
\mathrm{O} & 1.441267490 & -5.726644993 & -6.786264896 \\
\mathrm{H} & -0.651962042 & -7.476272106 & -7.004116535 \\
\mathrm{C} & 1.874204993 & -7.048661232 & -6.363115311 \\
\mathrm{H} & 1.545284033 & -7.768661976 & -7.126018524 \\
\mathrm{C} & 1.298766136 & -7.414438725 & -4.993212223 \\
\mathrm{H} & 1.701798081 & -6.743922710 & -4.232245445 \\
\mathrm{H} & 0.206922904 & -7.359361172 & -4.967739582 \\
\mathrm{H} & 1.586655736 & -8.439116478 & -4.738341331 \\
\mathrm{C} & 3.394000053 & -6.972550392 & -6.337801933 \\
\mathrm{H} & 3.814877510 & -7.939008713 & -6.045039177 \\
\mathrm{H} & 3.784889936 & -6.701341152 & -7.321410179 \\
\mathrm{H} & 3.709191799 & -6.217543125 & -5.611722469
\end{array}
$$

$$
\left[{ }^{3} \mathbf{I r}-{ }^{1} \mathbf{Q}_{\mathrm{A}}\right]
$$$$
\begin{array}{lrrr}
\mathrm{H} & -1.865385890 & 1.133404493 & -10.826369286 \\
\mathrm{H} & 0.079749800 & -0.804257333 & -10.937374115 \\
\mathrm{H} & 0.284688205 & -1.788926363 & -8.544072151 \\
\mathrm{H} & -3.323840380 & 1.805692434 & -4.680446625 \\
\mathrm{H} & -5.237122536 & 3.734686136 & -8.029606819 \\
\mathrm{H} & -3.788087845 & 2.350586891 & -9.538247108 \\
\mathrm{H} & -0.718297601 & -5.005943775 & -3.936392546
\end{array}
$$

$$
\begin{aligned}
& \text { H } \quad-4.228680611 \quad-0.737996042 \quad-6.640041351 \\
& \text { H } \quad-5.240662098 \quad-4.660435200 \quad-5.169378757 \\
& \text { H } \quad-2.892035246 \quad-4.929477215 \quad-4.434250832 \\
& \text { H } \quad 0.240657195 \quad 2.646041870 \quad-1.921018958 \\
& \text { H } \quad 1.447539330 \quad 3.364547253 \quad-3.547095776 \\
& \mathrm{H} \quad 2.700679064 \quad 4.109308720 \quad-5.559663296 \\
& \mathrm{H} \quad 0.699394763 \quad 1.041235685 \quad-7.793212414 \\
& \text { F } \quad 2.717897415 \quad 4.359053612 \quad-8.126503944 \\
& \text { F } \quad 3.730015278 \quad 2.435625315 \quad-8.147203445 \\
& \text { F } \quad 1.835217118 \quad 2.666777849 \quad-9.193073273 \\
& \begin{array}{llll}
\text { C } & 2.522142172 & 3.027040243 & -8.084463120
\end{array} \\
& \text { F } \quad-6.232636452 \quad-3.067047596 \quad-7.913351059 \\
& \text { F } \quad-7.188823223 \quad-3.194693565 \quad-5.965697289 \\
& \text { F } \quad-6.605341911 \quad-1.249346495 \quad-6.778984070 \\
& \text { C } \quad-6.229208469-2.544259071 \quad-6.650109768 \\
& \begin{array}{lllll}
\text { F } & -1.314178348 & 1.872005582 & -0.029538397
\end{array} \\
& \text { H } \quad-2.950734138 \quad 0.003488176 \quad-0.829724491 \\
& \text { F } \quad-3.181366682-1.090119243 \quad-3.140078068 \\
& \text { F } \quad \begin{array}{llll}
1.801916957 & -5.074798584 & -3.580461025
\end{array} \\
& \text { H } \quad 3.030470610 \quad-2.937335253 \quad-4.410484314 \\
& \text { F } \quad 2.011912823 \quad-0.788555741 \quad-5.453423500 \\
& \begin{array}{llll}
\text { C } & -1.898124695 & 0.512476563 & -8.672574997
\end{array} \\
& \text { C } \quad-1.502591252 \quad 0.508449435-10.025013924 \\
& \text { C } \quad-0.513617635-0.460497379-10.102405548 \\
& \text { N } \quad-0.344482273 \quad-0.986113369 \quad-8.867145538 \\
& \begin{array}{llll}
\text { C } & -3.469560146 & 1.969399452 & -5.739388943
\end{array} \\
& \begin{array}{llll}
\text { C } & -4.401739597 & 2.886974812 & -6.239243507
\end{array} \\
& \begin{array}{llll}
\text { C } & -4.515228748 & 3.028865099 & -7.632763386
\end{array} \\
& \begin{array}{llll}
\text { C } & -3.711717606 & 2.263408899 & -8.460527420
\end{array} \\
& \begin{array}{llll}
\text { C } & -2.799776077 & 1.361418724 & -7.897685051
\end{array} \\
& \text { C } \quad-0.880098581 \quad-3.033815622 \quad-4.887587547 \\
& \text { C } \quad-0.206830949 \quad-4.113709927 \quad-4.272999763 \\
& \text { C } \quad \begin{array}{llll}
1.158792496 & -4.035764694 & -4.128482342
\end{array} \\
& \text { C } \quad \begin{array}{llll}
1.955765247 & -2.926818132 & -4.529217720
\end{array} \\
& \begin{array}{lllll}
\text { C } & 1.284452796 & -1.862261057 & -5.084653378
\end{array} \\
& \text { C } \quad-3.951873541 \quad-1.672476411 \quad-6.169711590 \\
& \text { C } \quad-4.890360832 \quad-2.678910732 \quad-6.005049229
\end{aligned}
$$




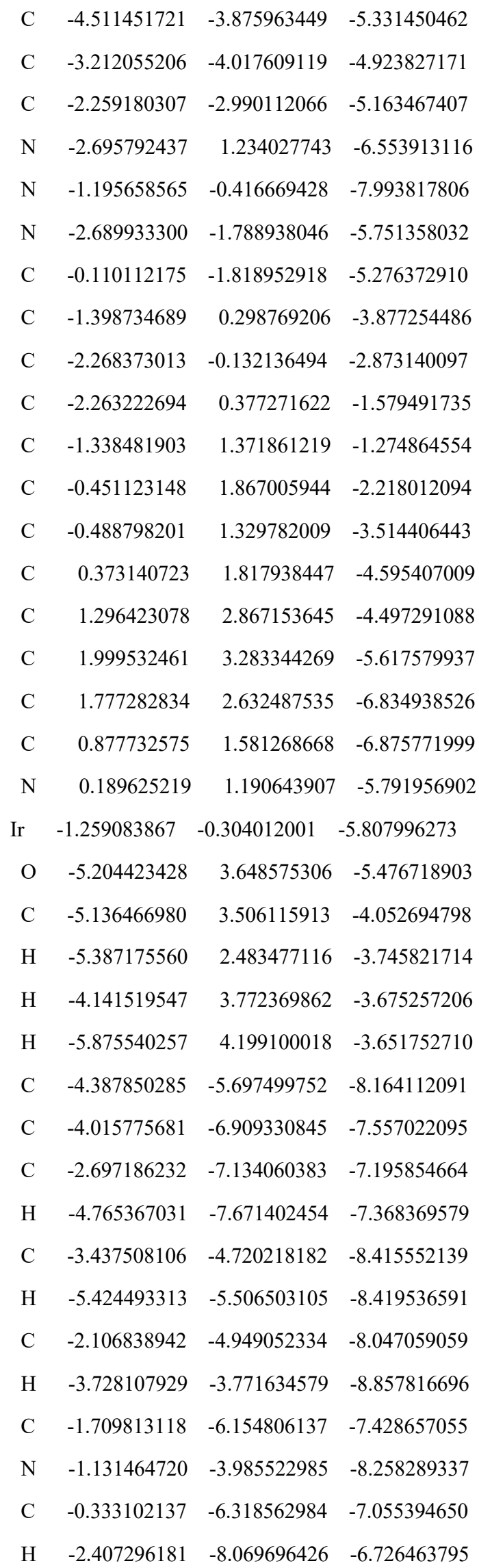

C $\quad 0.185322046 \quad-4.066206932 \quad-7.887835503$

$\begin{array}{lllll}\mathrm{H} & -1.425338984 & -3.106830597 & -8.664731979\end{array}$

$\begin{array}{lllll}\text { C } & 0.591206610 & -5.333792210 & -7.260833263\end{array}$

$\begin{array}{llll}\text { O } & 0.969102859 & -3.109539270 & -8.073263168\end{array}$

$\begin{array}{llll}\text { O } & 1.890959859 & -5.337420464 & -6.918677330\end{array}$

H $\quad-0.044621773 \quad-7.249989986 \quad-6.581654072$

$\begin{array}{llll}\text { C } & 2.511130810 & -6.550416470 & -6.411393166\end{array}$

$\begin{array}{llll}\mathrm{H} & 1.873595715 & -6.950079441 & -5.610468388\end{array}$

C $\quad 3.841720343 \quad-6.103405952 \quad-5.822802544$

H $\quad 4.447212696 \quad-5.614336491 \quad-6.591420650$

H $\quad 3.686561823 \quad-5.407997131 \quad-4.997179031$

H $\quad 4.391059875 \quad-6.970820427 \quad-5.445115566$

$\begin{array}{llll}\text { C } & 2.693799019 & -7.569167614 & -7.536123753\end{array}$

H $\quad 3.156825781 \quad-8.478909492 \quad-7.141875267$

H $\quad \begin{array}{llll}1.748260498 & -7.846978664 & -8.007627487\end{array}$

H $\quad 3.350111723 \quad-7.153118134 \quad-8.306647301$

\section{$\left[{ }^{1} \mathbf{I} \mathbf{r}^{-3} \mathbf{Q}_{\mathrm{A}}\right]$}

$\begin{array}{crrr}\text { H } & -6.936486721 & -3.626012325 & 2.060577869 \\ \text { H } & -8.197248459 & -2.082767725 & 3.952319145 \\ \text { H } & -8.257007599 & 0.326365769 & 3.035560369 \\ \text { H } & -5.108707428 & 0.349429905 & -2.590814114 \\ \text { H } & -4.582865715 & -3.932624578 & -2.393854856 \\ \text { H } & -5.817648888 & -3.901932001 & -0.249776214 \\ \text { H } & -8.074191093 & -0.665173292 & -2.138704538 \\ \text { H } & -12.194012642 & 0.510223866 & -2.437165737 \\ \text { H } & -11.746255875 & 2.255678654 & -0.728491783 \\ \text { H } & -2.220318317 & 3.302629471 & 0.187669158 \\ \text { H } & -1.444358826 & 2.135205269 & 2.241024256 \\ \text { H } & -5.293332100 & 0.250634253 & 2.470673561 \\ \text { F } & -1.601302147 & 0.074514821 & 3.755112886 \\ \text { F } & -3.145875692 & 1.127022505 & 4.865581989 \\ \text { F } & -3.609357119 & -0.774334788 & 3.914486885 \\ \text { C } & -2.914281607 & 0.377902895 & 3.770483971 \\ \text { F } & -11.313806534 & -0.988440633 & -4.318118095\end{array}$




\begin{tabular}{|c|c|c|c|}
\hline $\mathrm{F}$ & -9.247836113 & -1.650208950 & -4.051779270 \\
\hline $\mathrm{F}$ & -10.822853088 & -2.442173958 & -2.778307915 \\
\hline $\mathrm{C}$ & -10.387367249 & -1.324305415 & -3.402741432 \\
\hline $\mathrm{C}$ & -6.847799301 & -1.657923460 & 0.990501046 \\
\hline $\mathrm{C}$ & -7.157259464 & -2.570984125 & 2.018376827 \\
\hline $\mathrm{C}$ & -7.795578957 & -1.811724186 & 2.986701488 \\
\hline $\mathrm{N}$ & -7.858933449 & -0.534751296 & 2.543510914 \\
\hline $\mathrm{C}$ & -5.248616695 & -0.605204940 & -2.096049786 \\
\hline $\mathrm{C}$ & -4.740019321 & -1.783175111 & -2.669070482 \\
\hline $\mathrm{C}$ & -4.949567795 & -2.994500160 & -1.995705009 \\
\hline $\mathrm{C}$ & -5.644604206 & -2.977931499 & -0.790222943 \\
\hline $\mathrm{C}$ & -6.123456955 & -1.776892304 & -0.272397429 \\
\hline $\mathrm{C}$ & -9.245417595 & 2.749279261 & 0.450081319 \\
\hline $\mathrm{C}$ & -10.118077278 & 3.730385542 & 0.954367638 \\
\hline $\mathrm{C}$ & -9.609614372 & 4.646172047 & 1.856760740 \\
\hline $\mathrm{C}$ & -8.286387444 & 4.621911526 & 2.280432701 \\
\hline $\mathrm{C}$ & -7.463209152 & 3.629980803 & 1.765548706 \\
\hline $\mathrm{C}$ & -8.908720970 & -0.044591300 & -1.844682932 \\
\hline $\mathrm{C}$ & -10.159732819 & -0.210409030 & -2.417050600 \\
\hline $\mathrm{C}$ & -11.203642845 & 0.624917626 & -2.009568691 \\
\hline $\mathrm{C}$ & -10.949695587 & 1.601705194 & -1.059992671 \\
\hline $\mathrm{C}$ & -9.663188934 & 1.744652748 & -0.522972345 \\
\hline $\mathrm{N}$ & -5.918921471 & -0.606712639 & -0.941860914 \\
\hline $\mathrm{N}$ & -7.289926529 & -0.433303446 & 1.331815600 \\
\hline $\mathrm{N}$ & -8.667866707 & 0.897941053 & -0.921907008 \\
\hline $\mathrm{C}$ & -7.888074875 & 2.667233467 & 0.850310862 \\
\hline $\mathrm{C}$ & -6.059412956 & 2.530986309 & -1.314415693 \\
\hline $\mathrm{C}$ & -6.622883797 & 3.078237057 & -2.466056108 \\
\hline $\mathrm{C}$ & -5.973675728 & 3.990465879 & -3.288743258 \\
\hline $\mathrm{C}$ & -4.685152054 & 4.382262707 & -2.935372353 \\
\hline $\mathrm{C}$ & -4.051484585 & 3.891272545 & -1.806121826 \\
\hline $\mathrm{C}$ & -4.744770527 & 2.969792366 & -1.002861381 \\
\hline $\mathrm{C}$ & -4.168760300 & 2.385717154 & 0.210268512 \\
\hline $\mathrm{C}$ & -2.881130457 & 2.621829510 & 0.709951222 \\
\hline $\mathrm{C}$ & -2.446613073 & 1.975678563 & 1.857618093 \\
\hline $\mathrm{C}$ & -3.316814661 & 1.098020673 & 2.509735346 \\
\hline $\mathrm{C}$ & -4.587515831 & 0.913413763 & 1.991548061 \\
\hline
\end{tabular}

\begin{tabular}{|c|c|c|c|}
\hline $\mathrm{N}$ & -4.996346474 & 1.525574684 & 0.870677948 \\
\hline Ir & -6.829223633 & 1.175456882 & -0.015725311 \\
\hline $\mathrm{H}$ & -3.049809933 & 4.236634254 & -1.579765201 \\
\hline $\mathrm{H}$ & -6.455149651 & 4.387974262 & -4.174479961 \\
\hline $\mathrm{H}$ & -7.909650326 & 5.340261936 & 2.997550249 \\
\hline $\mathrm{H}$ & -11.162199974 & 3.793635130 & 0.674993753 \\
\hline $\mathrm{F}$ & -6.179853916 & 3.619174957 & 2.193800449 \\
\hline $\mathrm{F}$ & -10.437934875 & 5.584421635 & 2.374068022 \\
\hline $\mathrm{F}$ & -7.877087116 & 2.713224411 & -2.832094193 \\
\hline $\mathrm{F}$ & -4.040172100 & 5.264145374 & -3.718833685 \\
\hline $\mathrm{O}$ & -4.090537071 & -1.627168179 & -3.835922241 \\
\hline $\mathrm{C}$ & -3.560777187 & -2.788790703 & -4.483848572 \\
\hline $\mathrm{H}$ & -2.808811426 & -3.282169580 & -3.856538296 \\
\hline $\mathrm{H}$ & -4.358207226 & -3.498052835 & -4.735685349 \\
\hline $\mathrm{H}$ & -3.092064857 & -2.427960873 & -5.398749828 \\
\hline $\mathrm{C}$ & -11.005683899 & 2.757628679 & 3.916326761 \\
\hline $\mathrm{C}$ & -12.408967972 & 2.897045851 & 3.480479956 \\
\hline $\mathrm{C}$ & -14.039504051 & -0.004465527 & 0.881940067 \\
\hline $\mathrm{C}$ & -14.833505630 & 1.076228857 & 1.299992919 \\
\hline $\mathrm{C}$ & -14.313496590 & 2.035458326 & 2.147867680 \\
\hline $\mathrm{H}$ & -15.861265182 & 1.156036139 & 0.959709942 \\
\hline $\mathrm{C}$ & -12.705888748 & -0.114381492 & 1.321159005 \\
\hline $\mathrm{H}$ & -14.449514389 & -0.762443900 & 0.222485736 \\
\hline $\mathrm{C}$ & -12.168308258 & 0.834597111 & 2.173837900 \\
\hline $\mathrm{H}$ & -12.092715263 & -0.950623333 & 0.994839311 \\
\hline $\mathrm{C}$ & -12.962458611 & 1.956148624 & 2.624115229 \\
\hline $\mathrm{N}$ & -10.849730492 & 0.745964468 & 2.615592718 \\
\hline $\mathrm{H}$ & -14.922612190 & 2.872130156 & 2.477288485 \\
\hline $\mathrm{C}$ & -10.207529068 & 1.667898297 & 3.454453468 \\
\hline $\mathrm{H}$ & -10.282511711 & -0.015482791 & 2.273427486 \\
\hline $\mathrm{O}$ & -8.991813660 & 1.503833890 & 3.739334345 \\
\hline $\mathrm{O}$ & -10.381545067 & 3.633515596 & 4.699195862 \\
\hline $\mathrm{H}$ & -13.003470421 & 3.732801676 & 3.821661711 \\
\hline $\mathrm{C}$ & -11.071319580 & 4.794372082 & 5.264418125 \\
\hline $\mathrm{H}$ & -11.720199585 & 5.213767529 & 4.491233349 \\
\hline $\mathrm{C}$ & -9.973060608 & 5.793341160 & 5.592990875 \\
\hline $\mathrm{H}$ & -9.242350578 & 5.349282742 & 6.275019169 \\
\hline
\end{tabular}




$\begin{array}{rrrr}\mathrm{H} & -9.466251373 & 6.113885880 & 4.681092739 \\ \mathrm{H} & -10.409666061 & 6.674226284 & 6.072923660 \\ \mathrm{C} & -11.870711327 & 4.346828461 & 6.482309341 \\ \mathrm{H} & -12.378602028 & 5.206865311 & 6.928805828 \\ \mathrm{H} & -12.625113487 & 3.601248503 & 6.214726448 \\ \mathrm{H} & -11.202269554 & 3.910458803 & 7.230175972\end{array}$

\section{${ }^{1}\left[\mathbf{I r}-\mathbf{Q}_{\mathbf{B}}\right]$}

\begin{tabular}{rrrr} 
H & -6.952991486 & -3.785371780 & 1.778955817 \\
H & -8.361519814 & -2.313198566 & 3.623346806 \\
H & -8.329577446 & 0.113561511 & 2.822575569 \\
H & -5.011402607 & 0.331802309 & -2.685812950 \\
H & -4.375203133 & -3.935209990 & -2.590304136 \\
H & -5.618253231 & -3.980826855 & -0.415528178 \\
H & -7.957523823 & -0.691897273 & -2.247767687 \\
H & -12.101089478 & 0.426482767 & -2.359850645 \\
H & -11.605179787 & 2.156621456 & -0.646385670 \\
H & -2.098351479 & 3.281794071 & 0.226469874 \\
H & -1.391970396 & 2.171668768 & 2.333734274 \\
H & -5.162037373 & 0.108094901 & 2.352780581 \\
F & -1.971317649 & -0.702590585 & 3.484916925 \\
F & -2.225877047 & 1.126300454 & 4.629201412 \\
F & -3.912834167 & -0.237445012 & 4.361779213 \\
C & -2.826434612 & 0.300845742 & 3.745420933 \\
F & -11.288866997 & -1.035576820 & -4.288397312 \\
F & -9.187984467 & -1.624462128 & -4.150935173 \\
F & -10.662335396 & -2.512233734 & -2.821032763 \\
C & -10.304126740 & -1.363438964 & -3.433248758 \\
H & -6.224997044 & 4.262441635 & -4.275938511 \\
H & -7.621141434 & 5.307828426 & 2.876950264 \\
C & -6.740546703 & -1.770314813 & 0.821735740 \\
C & -7.146509647 & -2.724220514 & 1.774802923 \\
C & -7.860132217 & -2.001511335 & 2.718724489 \\
\hline
\end{tabular}

\section{$=============================$}

\begin{tabular}{|c|c|c|c|}
\hline $\mathrm{C}$ & -4.596744537 & -1.808928967 & -2.820018053 \\
\hline $\mathrm{C}$ & -4.776014328 & -3.029848337 & -2.147185564 \\
\hline $\mathrm{C}$ & -5.463280201 & -3.048563480 & -0.946738720 \\
\hline $\mathrm{C}$ & -5.971844196 & -1.855218530 & -0.418495774 \\
\hline $\mathrm{C}$ & -9.065381050 & 2.660416603 & 0.450510412 \\
\hline $\mathrm{C}$ & -9.918668747 & 3.638604164 & 0.987292588 \\
\hline $\mathrm{C}$ & -9.375111580 & 4.572570324 & 1.849486947 \\
\hline $\mathrm{C}$ & -8.028559685 & 4.570482731 & 2.196677685 \\
\hline $\mathrm{C}$ & -7.230029583 & 3.566107035 & 1.667565465 \\
\hline $\mathrm{C}$ & -8.788927078 & -0.089313947 & -1.910055637 \\
\hline $\mathrm{C}$ & -10.059454918 & -0.266440243 & -2.431764126 \\
\hline $\mathrm{C}$ & -11.096640587 & 0.547531486 & -1.968035936 \\
\hline $\mathrm{C}$ & -10.815876961 & 1.513581991 & -1.014482141 \\
\hline $\mathrm{C}$ & -9.511761665 & 1.665516019 & -0.524373412 \\
\hline $\mathrm{N}$ & -5.783936024 & -0.681873858 & -1.069796920 \\
\hline $\mathrm{N}$ & -7.191339016 & -0.553308189 & 1.186041951 \\
\hline $\mathrm{N}$ & -8.524196625 & 0.842938542 & -0.983432114 \\
\hline $\mathrm{C}$ & -7.691159248 & 2.584839344 & 0.794689357 \\
\hline $\mathrm{C}$ & -5.876945019 & 2.439659119 & -1.388084412 \\
\hline $\mathrm{C}$ & -6.425823689 & 2.980143785 & -2.549735069 \\
\hline $\mathrm{C}$ & -5.755490303 & 3.867640018 & -3.382388353 \\
\hline $\mathrm{C}$ & -4.461975574 & 4.243422985 & -3.026151896 \\
\hline $\mathrm{C}$ & -3.843866825 & 3.761452198 & -1.884225249 \\
\hline $\mathrm{C}$ & -4.559053421 & 2.864316940 & -1.071792126 \\
\hline $\mathrm{C}$ & -4.008369446 & 2.288504124 & 0.154950082 \\
\hline $\mathrm{C}$ & -2.753256083 & 2.569525957 & 0.713118315 \\
\hline $\mathrm{C}$ & -2.356628656 & 1.951542616 & 1.888080597 \\
\hline $\mathrm{C}$ & -3.224533796 & 1.039680600 & 2.497938156 \\
\hline $\mathrm{C}$ & -4.456304550 & 0.799843550 & 1.914343357 \\
\hline $\mathrm{N}$ & -4.837606430 & 1.405594826 & 0.781494200 \\
\hline $\mathrm{r}$ & -6.669382095 & 1.084699512 & -0.109247506 \\
\hline $\mathrm{C}$ & -4.853622913 & 5.020448208 & 6.447037220 \\
\hline $\mathrm{C}$ & -4.221938133 & 3.858218193 & 5.972847462 \\
\hline $\mathrm{C}$ & -4.962725163 & 2.854823351 & 5.367642403 \\
\hline $\mathrm{C}$ & -6.344460487 & 3.012024403 & 5.223963737 \\
\hline $\mathrm{C}$ & -7.005179882 & 4.158751965 & 5.713535786 \\
\hline $\mathrm{C}$ & -6.226599693 & 5.163391590 & 6.325170517 \\
\hline
\end{tabular}

$\begin{array}{llll}\text { C } & -4.596744537 & -1.808928967 & -2.820018053\end{array}$

$\begin{array}{llll}\text { C } & -4.776014328 & -3.029848337 & -2.147185564\end{array}$

$\begin{array}{llll}\text { C } & -5.463280201 & -3.048563480 & -0.946738720\end{array}$

$\begin{array}{llll}\text { C } & -5.971844196 & -1.855218530 & -0.418495774\end{array}$

$\begin{array}{llll}\text { C } & -9.065381050 & 2.660416603 & 0.450510412\end{array}$

$\begin{array}{llll}\text { C } & -9.918668747 & 3.638604164 & 0.987292588\end{array}$

$\begin{array}{llll}\text { C } & -9.375111580 & 4.572570324 & 1.849486947\end{array}$

$\begin{array}{llll}\text { C } & -8.028559685 & 4.570482731 & 2.196677685\end{array}$

C $-7.230029583-3.566107035-1.667565465$

(1)

(1) 


\begin{tabular}{lrrr} 
N & -7.115968704 & 2.041623831 & 4.594741821 \\
C & -8.465471268 & 2.106021643 & 4.359377384 \\
C & -9.148859024 & 3.281939268 & 4.922761440 \\
C & -8.429483414 & 4.250906944 & 5.563863277 \\
O & -9.053249359 & 1.225908756 & 3.700076103 \\
O & -10.468376160 & 3.276730537 & 4.669239044 \\
C & -11.281893730 & 4.405169487 & 5.090122700 \\
C & -12.535072327 & 4.336041451 & 4.230325699 \\
H & -4.266468048 & 5.803454876 & 6.916701317 \\
H & -3.148435593 & 3.735644341 & 6.079490662 \\
H & -4.489800930 & 1.945292354 & 5.024208069 \\
H & -6.722559452 & 6.054251194 & 6.701148987 \\
H & -6.641733170 & 1.234745502 & 4.208725929 \\
H & -8.921604156 & 5.132172108 & 5.958161354 \\
H & -10.735034943 & 5.326508045 & 4.852323532 \\
H & -13.222463608 & 5.141190052 & 4.506025314 \\
H & -12.276693344 & 4.443820953 & 3.175526142 \\
H & -13.042845726 & 3.378458977 & 4.379611969 \\
C & -11.581886292 & 4.311133862 & 6.587310791 \\
H & -12.166749954 & 3.410074711 & 6.794487000 \\
H & -10.669479370 & 4.264310360 & 7.187402248 \\
H & -12.157687187 & 5.182675362 & 6.914249420 \\
O & -3.934927225 & -1.847520232 & -3.989037991 \\
C & -3.785251617 & -0.632577479 & -4.731898308 \\
H & -4.762320995 & -0.208721802 & -4.994163036 \\
H & -3.200441837 & 0.106033564 & -4.170435429 \\
H & -3.250524759 & -0.906996667 & -5.640642643 \\
H & -2.837367058 & 4.092627048 & -1.657300353 \\
F & -3.797171593 & 5.099282742 & -3.821375608 \\
F & -7.682388306 & 2.627395391 & -2.914476871 \\
H & -10.972663879 & 3.697927713 & 0.746391714 \\
\hline & -5.927300453 & 3.560639381 & 2.058630943 \\
\hline
\end{tabular}

$\left[{ }^{3} \mathbf{I r}-{ }^{1} \mathbf{Q}_{\mathbf{B}}\right]$

\begin{tabular}{|c|c|c|c|}
\hline & -7.036416054 & -3.782127142 & 096 \\
\hline & -8.364546776 & -2.289368868 & 3.592141628 \\
\hline & -8.273761749 & 0.139148280 & 2.826657057 \\
\hline & -5.010686874 & 0.289475620 & -2.74 \\
\hline & -4.557030201 & -4.000929832 & -2.687180281 \\
\hline & -5.783264637 & -4.008553982 & -0.502945125 \\
\hline & -7.989049435 & -0.545047462 & -2.36502 \\
\hline & -12.160825729 & 0.462120712 & -2.184351444 \\
\hline & -11.607104301 & & -0.425315887 \\
\hline & -1.890667915 & 2.967161655 & 0.155744970 \\
\hline & -1.3 & 1.945 & \\
\hline & -5.243496418 & 0.168893054 & \\
\hline & -2.161266565 & -0.770586252 & 3.733398914 \\
\hline & -2.313492298 & 1.171 & \\
\hline & -4.091557980 & -0.086649984 & 4.483 \\
\hline & -2.949723959 & 0.317692131 & \\
\hline & -11.439547539 & -0.899067998 & -4.213681221 \\
\hline & -9.316921234 & -1.421 & -4.242163658 \\
\hline & -10.668607712 & -2.411557674 & -2.855537415 \\
\hline & -10.389114380 & -1.228832841 & -3.442437887 \\
\hline & -6.051496983 & 4.1340 & \\
\hline & -7.489555836 & & \\
\hline & -6.792943001 & -1.765165091 & 0.760635674 \\
\hline & -7.205219746 & -2.716881037 & 1.711762786 \\
\hline & -7.878098965 & -1.984 & \\
\hline & -7.856887817 & $-0.68 c$ & \\
\hline & -5.146996021 & -0.686253846 & 8330 \\
\hline & -4.685508251 & & -2.8981504 \\
\hline & -4.912449360 & -3.082039118 & -2.233330488 \\
\hline & -5.589711666 & -3.079852343 & -1.02 \\
\hline & -6.039660454 & -1.868662715 & -0.486103058 \\
\hline & -9.01569 & 2.652440071 & \\
\hline & -9.852049828 & 3.595663548 & 1.149532080 \\
\hline & -9.279249191 & 4.517520428 & 2.010070324 \\
\hline & -7.915217876 & 4.540983200 & 2.280944586 \\
\hline & -7.122471809 & 3.570764780 & 1.683856010 \\
\hline & -8.814494133 & 0.021756468 & -1.956507444 \\
\hline
\end{tabular}




\begin{tabular}{|c|c|c|c|}
\hline $\mathrm{C}$ & -10.110429764 & -0.166660562 & -2.410804033 \\
\hline $\mathrm{C}$ & -11.137620926 & 0.595119953 & -1.849057913 \\
\hline $\mathrm{C}$ & -10.824758530 & 1.526478171 & -0.869819462 \\
\hline $\mathrm{C}$ & -9.497740746 & 1.688560724 & -0.452654839 \\
\hline $\mathrm{N}$ & -5.805005074 & -0.702012181 & -1.128197908 \\
\hline $\mathrm{N}$ & -7.206341267 & -0.540951550 & 1.141747475 \\
\hline $\mathrm{N}$ & -8.520535469 & 0.915014565 & -1.003157496 \\
\hline $\mathrm{C}$ & -7.620565414 & 2.596560717 & 0.815014005 \\
\hline $\mathrm{C}$ & -5.789095402 & 2.351443291 & -1.439544678 \\
\hline $\mathrm{C}$ & -6.321786880 & 2.909297705 & -2.615044594 \\
\hline $\mathrm{C}$ & -5.614848614 & 3.719282866 & -3.479041576 \\
\hline $\mathrm{C}$ & -4.270337582 & 4.005184174 & -3.115379810 \\
\hline $\mathrm{C}$ & -3.647733927 & 3.524904251 & -1.984166265 \\
\hline $\mathrm{C}$ & -4.380774021 & 2.686265707 & -1.111167431 \\
\hline $\mathrm{C}$ & -3.889335394 & 2.132971287 & 0.089265041 \\
\hline $\mathrm{C}$ & -2.607227087 & 2.338705778 & 0.672798634 \\
\hline $\mathrm{C}$ & -2.281767607 & 1.775300980 & 1.877923250 \\
\hline $\mathrm{C}$ & -3.253578901 & 0.968691170 & 2.545114040 \\
\hline $\mathrm{C}$ & -4.485736847 & 0.778424561 & 1.946859956 \\
\hline $\mathrm{N}$ & -4.811976910 & 1.325637221 & 0.773671031 \\
\hline Ir & -6.634621143 & 1.128435373 & -0.144103989 \\
\hline $\mathrm{C}$ & -5.010346413 & 5.307405949 & 6.458684921 \\
\hline $\mathrm{C}$ & -4.292156219 & 4.218052387 & 5.935965538 \\
\hline $\mathrm{C}$ & -4.955861092 & 3.162007809 & 5.330348492 \\
\hline $\mathrm{C}$ & -6.351837635 & 3.194084406 & 5.238040447 \\
\hline $\mathrm{C}$ & -7.096040249 & 4.271965981 & 5.765343666 \\
\hline $\mathrm{C}$ & -6.392904282 & 5.330009937 & 6.378500938 \\
\hline $\mathrm{N}$ & -7.055308342 & 2.168262959 & 4.612731934 \\
\hline $\mathrm{C}$ & -8.407559395 & 2.135086775 & 4.397343636 \\
\hline $\mathrm{C}$ & -9.172470093 & 3.248358011 & 4.978741169 \\
\hline $\mathrm{C}$ & -8.524781227 & 4.254182816 & 5.638660908 \\
\hline $\mathrm{O}$ & -8.947242737 & 1.226402760 & 3.733025551 \\
\hline $\mathrm{O}$ & -10.487905502 & 3.153325081 & 4.710003853 \\
\hline $\mathrm{C}$ & -11.394066811 & 4.198083401 & 5.153170109 \\
\hline $\mathrm{C}$ & -12.615612030 & 4.077354908 & 4.254335880 \\
\hline $\mathrm{H}$ & -4.480939865 & 6.130716324 & 6.927847385 \\
\hline $\mathrm{H}$ & -3.208875895 & 4.195548534 & 6.002463818 \\
\hline
\end{tabular}

$$
\begin{array}{lrrr}
\text { H } & -4.406744957 & 2.317316771 & 4.937102318 \\
\text { H } & -6.955371857 & 6.166416168 & 6.784474373 \\
\text { H } & -6.522247791 & 1.402106524 & 4.218900681 \\
\text { H } & -9.078277588 & 5.086850643 & 6.055892467 \\
\text { H } & -10.914752960 & 5.168148994 & 4.971505165 \\
\text { H } & -13.367922783 & 4.818322182 & 4.540774822 \\
\text { H } & -12.334856033 & 4.250988483 & 3.212974787 \\
\text { H } & -13.056389809 & 3.079918861 & 4.344463348 \\
\text { C } & -11.724292755 & 4.016918182 & 6.633019924 \\
\text { H } & -12.221492767 & 3.054974079 & 6.789094448 \\
\text { H } & -10.828585625 & 4.042869091 & 7.259050369 \\
\text { H } & -12.396655083 & 4.812995911 & 6.967786312 \\
\text { O } & -4.039797783 & -1.922685862 & -4.075546741 \\
\mathrm{C} & -3.859460592 & -0.711083353 & -4.816998005 \\
\mathrm{H} & -4.825788021 & -0.253984272 & -5.063814640 \\
\mathrm{H} & -3.242163897 & 0.005534098 & -4.262125492 \\
\mathrm{H} & -3.348028660 & -1.000636220 & -5.734308243 \\
\mathrm{H} & -2.621308565 & 3.813369036 & -1.794615507 \\
\mathrm{~F} & -3.582353592 & 4.794795513 & -3.942387819 \\
\mathrm{~F} & -7.606019020 & 2.635341167 & -2.933265209 \\
\mathrm{~F} & -10.919894218 & 3.639483690 & 0.973428249 \\
\hline & -10.068629265 & 5.425910473 & 2.615224361
\end{array}
$$

\section{$\left[{ }^{1} \mathbf{I r}-{ }^{3} \mathbf{Q}_{B}\right]$}

$\begin{array}{lrrr}\text { H } & -6.941859245 & -3.691555500 & 2.112322569 \\ \text { H } & -8.365129471 & -2.206360340 & 3.934562445 \\ \text { H } & -8.431901932 & 0.197882086 & 3.060852051 \\ \text { H } & -5.199610710 & 0.336841226 & -2.508823633 \\ \text { H } & -4.451673508 & -3.907839060 & -2.300001621 \\ \text { H } & -5.643086433 & -3.917875528 & -0.094962575 \\ \text { H } & -8.139372826 & -0.714200556 & -1.929017544 \\ \text { H } & -12.275310516 & 0.438304603 & -1.988651872 \\ \mathrm{H} & -11.726794243 & 2.220432043 & -0.343017787 \\ \mathrm{H} & -2.199570417 & 3.328961611 & 0.184905156\end{array}$




\begin{tabular}{|c|c|c|c|}
\hline $\mathrm{H}$ & -1.386849165 & 2.236226797 & 2.262966394 \\
\hline $\mathrm{H}$ & -5.164209843 & 0.200617567 & 2.511354208 \\
\hline $\mathrm{F}$ & -1.809238911 & -0.523939490 & 3.478069067 \\
\hline $\mathrm{F}$ & -2.197112322 & 1.255483508 & 4.661256790 \\
\hline $\mathrm{F}$ & -3.766580820 & -0.241412267 & 4.393312454 \\
\hline $\mathrm{C}$ & -2.743109703 & 0.397560298 & 3.771610737 \\
\hline $\mathrm{F}$ & -11.530004501 & -1.098875642 & -3.875954628 \\
\hline $\mathrm{F}$ & -9.422276497 & -1.673109889 & -3.795063972 \\
\hline $\mathrm{F}$ & -10.844473839 & -2.533644676 & -2.393247128 \\
\hline $\mathrm{C}$ & -10.514842033 & -1.399188519 & -3.046539783 \\
\hline $\mathrm{C}$ & -6.793528557 & -1.697938681 & 1.099915147 \\
\hline $\mathrm{C}$ & -7.161293030 & -2.635781288 & 2.083667040 \\
\hline $\mathrm{C}$ & -7.880481243 & -1.905386806 & 3.017205238 \\
\hline $\mathrm{N}$ & -7.920833588 & -0.619036496 & 2.603793144 \\
\hline $\mathrm{C}$ & -5.268039227 & -0.629722178 & -2.027768850 \\
\hline $\mathrm{C}$ & -4.733144283 & -1.795771718 & -2.587986469 \\
\hline $\mathrm{C}$ & -4.865943909 & -2.999755383 & -1.874980569 \\
\hline $\mathrm{C}$ & -5.524512291 & -2.998789549 & -0.658195615 \\
\hline $\mathrm{C}$ & -6.050419331 & -1.802257299 & -0.154041797 \\
\hline $\mathrm{C}$ & -9.156042099 & 2.743361235 & 0.668857396 \\
\hline $\mathrm{C}$ & -9.985673904 & 3.755218267 & 1.181677103 \\
\hline $\mathrm{C}$ & -9.411422729 & 4.720276356 & 1.985297322 \\
\hline $\mathrm{C}$ & -8.056932449 & 4.717638016 & 2.299829006 \\
\hline $\mathrm{C}$ & -7.281461716 & 3.681033611 & 1.797899008 \\
\hline $\mathrm{C}$ & -8.958403587 & -0.092643641 & -1.594910979 \\
\hline $\mathrm{C}$ & -10.241778374 & -0.274770886 & -2.083014011 \\
\hline $\mathrm{C}$ & -11.261582375 & 0.563011169 & -1.622901678 \\
\hline $\mathrm{C}$ & -10.951735497 & 1.557718039 & -0.707846284 \\
\hline $\mathrm{C}$ & -9.635126114 & 1.715644717 & -0.255103856 \\
\hline $\mathrm{N}$ & -5.908695698 & -0.645741582 & -0.845168471 \\
\hline $\mathrm{N}$ & -7.265594959 & -0.481699675 & 1.439967513 \\
\hline $\mathrm{N}$ & -8.665236473 & 0.868299603 & -0.707430601 \\
\hline $\mathrm{C}$ & -7.774704933 & 2.667180300 & 0.981354475 \\
\hline $\mathrm{C}$ & -6.046770573 & 2.462955713 & -1.241346478 \\
\hline $\mathrm{C}$ & -6.643835545 & 2.978087902 & -2.391131163 \\
\hline $\mathrm{C}$ & -6.013843536 & 3.856880903 & -3.263397217 \\
\hline $\mathrm{C}$ & -4.712039471 & 4.250291348 & -2.962112427 \\
\hline
\end{tabular}

\begin{tabular}{|c|c|c|c|}
\hline $\mathrm{C}$ & -4.046326160 & 3.792541981 & -1.837009788 \\
\hline $\mathrm{C}$ & -4.721419811 & 2.902654648 & -0.983234465 \\
\hline $\mathrm{C}$ & -4.116680145 & 2.347096443 & 0.228113055 \\
\hline $\mathrm{C}$ & -2.832772017 & 2.628967524 & 0.716161549 \\
\hline $\mathrm{C}$ & -2.377003193 & 2.020428419 & 1.874567270 \\
\hline $\mathrm{C}$ & -3.216057539 & 1.120897412 & 2.540835619 \\
\hline $\mathrm{C}$ & -4.479150772 & 0.882790029 & 2.027683258 \\
\hline $\mathrm{N}$ & -4.916984558 & 1.477656245 & 0.908984423 \\
\hline Ir & -6.784947872 & 1.135032177 & 0.097685285 \\
\hline $\mathrm{H}$ & -3.035296440 & 4.136856556 & -1.654166818 \\
\hline $\mathrm{H}$ & -6.520423412 & 4.231810570 & -4.145218849 \\
\hline $\mathrm{H}$ & -7.625623226 & 5.480884552 & 2.934697151 \\
\hline $\mathrm{H}$ & -11.046377182 & 3.811654568 & 0.969098210 \\
\hline $\mathrm{F}$ & -5.970184803 & 3.682430983 & 2.150860786 \\
\hline $\mathrm{F}$ & -10.188131332 & 5.697909832 & 2.507496595 \\
\hline $\mathrm{F}$ & -7.910056591 & 2.609512806 & -2.704403877 \\
\hline $\mathrm{F}$ & -4.085643291 & 5.099265099 & -3.795501709 \\
\hline $\mathrm{O}$ & -4.095141888 & -1.853605390 & -3.770137072 \\
\hline $\mathrm{C}$ & -3.987650633 & -0.657773316 & -4.550171852 \\
\hline $\mathrm{H}$ & -4.978868008 & -0.264320850 & -4.806774139 \\
\hline $\mathrm{H}$ & -3.410784245 & 0.110522911 & -4.021182537 \\
\hline $\mathrm{H}$ & -3.462600231 & -0.945941210 & -5.460368633 \\
\hline $\mathrm{C}$ & -4.167139530 & 3.866518021 & 5.855829716 \\
\hline $\mathrm{C}$ & -4.712010860 & 5.096849918 & 6.257894993 \\
\hline $\mathrm{C}$ & -6.073354721 & 5.317742348 & 6.165586948 \\
\hline $\mathrm{H}$ & -4.063760757 & 5.875375748 & 6.648034096 \\
\hline $\mathrm{C}$ & -5.004269123 & 2.857661486 & 5.345660210 \\
\hline $\mathrm{H}$ & -3.101145983 & 3.679850817 & 5.933488846 \\
\hline $\mathrm{C}$ & -6.363129139 & 3.069373131 & 5.223248482 \\
\hline $\mathrm{H}$ & -4.589871407 & 1.900203586 & 5.059232712 \\
\hline $\mathrm{C}$ & -6.959054947 & 4.312613487 & 5.653871059 \\
\hline $\mathrm{N}$ & -7.197671413 & 2.085402012 & 4.687805653 \\
\hline $\mathrm{C}$ & -8.333963394 & 4.483930111 & 5.568022728 \\
\hline $\mathrm{H}$ & -6.503290653 & 6.262392044 & 6.486002922 \\
\hline $\mathrm{C}$ & -8.570089340 & 2.222710133 & 4.463701725 \\
\hline $\mathrm{H}$ & -6.774847984 & 1.238743901 & 4.337688923 \\
\hline $\mathrm{C}$ & -9.166525841 & 3.421083689 & 4.963110447 \\
\hline
\end{tabular}




$\begin{array}{rrrr}\mathrm{O} & -9.194944382 & 1.321320057 & 3.845878124 \\ \mathrm{O} & -10.472032547 & 3.543587208 & 4.732538223 \\ \mathrm{H} & -8.787433624 & 5.407758236 & 5.898589611 \\ \mathrm{C} & -11.2444486662 & 4.672903061 & 5.248342991 \\ \mathrm{H} & -10.697174072 & 5.591836929 & 5.016061783 \\ \mathrm{C} & -11.448291779 & 4.505735397 & 6.750852108 \\ \mathrm{H} & -11.974191666 & 3.568513393 & 6.956135750 \\ \mathrm{H} & -10.496442795 & 4.493200779 & 7.289083004 \\ \mathrm{H} & -12.050518990 & 5.333871365 & 7.136632919 \\ \mathrm{C} & -12.542428970 & 4.649889946 & 4.459175110 \\ \mathrm{H} & -13.192800522 & 5.461991787 & 4.796522141 \\ \mathrm{H} & -12.338293076 & 4.785136223 & 3.395486593 \\ \mathrm{H} & -13.062307358 & 3.699131012 & 4.609587669\end{array}$

\section{${ }^{1}$ [Ir-QC]}

\begin{tabular}{|c|c|c|c|}
\hline $\mathrm{H}$ & -2.118559837 & \multicolumn{2}{|c|}{$0.836123168-10.844901085$} \\
\hline $\mathrm{H}$ & -0.683409393 & \multicolumn{2}{|c|}{$-1.507934451-10.936098099$} \\
\hline $\mathrm{H}$ & -0.553363085 & -2.348882198 & -8.520690918 \\
\hline $\mathrm{H}$ & -3.340011358 & 1.893538475 & -4.679376125 \\
\hline $\mathrm{H}$ & -4.752205372 & 4.178472996 & -8.052974701 \\
\hline $\mathrm{H}$ & -3.482542038 & 2.604607105 & -9.533401489 \\
\hline $\mathrm{H}$ & -0.898324430 & -5.183304310 & -4.220157146 \\
\hline $\mathrm{H}$ & -4.349461555 & -0.721054494 & -6.563162327 \\
\hline $\mathrm{H}$ & -5.208057880 & -4.885630608 & -5.951587677 \\
\hline $\mathrm{H}$ & -2.900548220 & -5.149572372 & -5.053893089 \\
\hline $\mathrm{H}$ & 0.005274235 & 2.904047251 & -2.117754698 \\
\hline $\mathrm{H}$ & 1.358186960 & 3.403248072 & -3.771955013 \\
\hline $\mathrm{H}$ & 2.790104628 & 3.803714514 & -5.768895149 \\
\hline $\mathrm{H}$ & 0.664722800 & 0.647650123 & -7.766866684 \\
\hline $\mathrm{F}$ & 3.204710722 & 3.617329836 & -8.270012856 \\
\hline $\mathrm{F}$ & 3.782153368 & 1.529227972 & -8.047316551 \\
\hline $\mathrm{F}$ & 2.053713560 & 2.042980433 & -9.260865211 \\
\hline $\mathrm{C}$ & 2.724425077 & 2.369711399 & -8.130917549 \\
\hline $\mathrm{F}$ & -6.830972195 & -3.666081190 & -7.437645435 \\
\hline $\mathrm{F}$ & -7.194347382 & -2.169877052 & -5.901680470 \\
\hline
\end{tabular}

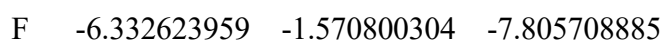

$$
\begin{aligned}
& \text { C } \quad-6.337471485 \quad-2.548686266 \quad-6.871866226 \\
& \text { F } \quad-1.518743634 \quad 2.195305109 \quad-0.169379145 \\
& \text { H } \quad-3.007581949 \quad 0.152153030 \quad-0.806875885 \\
& \text { F } \quad-3.153043985 \quad-1.144853115 \quad-3.038686991 \\
& \text { F } \quad \begin{array}{llll}
1.571251392 & -5.260356903 & -3.509988785
\end{array} \\
& \mathrm{H} \quad 2.726716518 \quad-2.940582275 \quad-3.845263720 \\
& \text { F } \quad 1.778337836 \quad-0.752926469 \quad-4.793587208 \\
& \begin{array}{llll}
\text { C } & -2.020365000 & 0.420609325 & -8.645051003
\end{array} \\
& \text { C } \quad-1.793006778 \quad 0.222291037-10.019524574 \\
& \text { C } \quad-1.061728358-0.954769790-10.089425087
\end{aligned}
$$

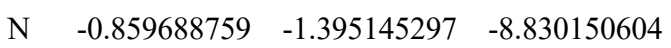

$$
\begin{aligned}
& \begin{array}{llll}
\text { C } & -3.415591240 & 2.095530748 & -5.738942146
\end{array} \\
& \begin{array}{llll}
\text { C } & -4.161500454 & 3.162612200 & -6.252039433
\end{array} \\
& \begin{array}{llll}
\text { C } & -4.179934025 & 3.352667093 & -7.644102573
\end{array} \\
& \begin{array}{llll}
\text { C } & -3.475974083 & 2.481120110 & -8.456370354
\end{array} \\
& \begin{array}{llll}
\text { C } & -2.754613400 & 1.426270127 & -7.883693218
\end{array} \\
& \text { C } \quad-0.987377644 \quad-3.105695248 \quad-4.913913250
\end{aligned}
$$

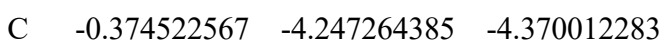

$$
\begin{aligned}
& \text { C } \quad 0.958735466 \quad-4.170779228 \quad-4.010279179 \\
& \text { C } \quad 1.689275980 \quad-2.995090246 \quad-4.152963161
\end{aligned}
$$

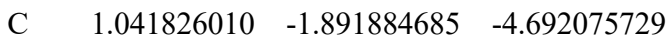

$$
\begin{aligned}
& \text { C } \quad-4.071336746 \quad-1.712463856 \quad-6.230402470 \\
& \begin{array}{llll}
\text { C } & -4.952582836 & -2.778052330 & -6.326447487
\end{array} \\
& \begin{array}{llll}
\text { C } & -4.536382198 & -4.036471844 & -5.884647369
\end{array} \\
& \begin{array}{llll}
\text { C } & -3.250271797 & -4.180115223 & -5.387724400
\end{array} \\
& \begin{array}{llll}
\text { C } & -2.385634899 & -3.080221653 & -5.336838722
\end{array} \\
& \begin{array}{llll}
\mathrm{N} & -2.735259056 & 1.257581949 & -6.540243149
\end{array} \\
& \mathrm{~N} \quad-1.441508651 \quad-0.577900290 \quad-7.943958282 \\
& \begin{array}{llll}
\mathrm{N} & -2.829146862 & -1.857056856 & -5.748775005
\end{array} \\
& \begin{array}{llll}
\text { C } & -0.288615555 & -1.887561321 & -5.106513977
\end{array} \\
& \begin{array}{llll}
\text { C } & -1.490994692 & 0.318558455 & -3.881247520
\end{array} \\
& \text { C } \quad-2.315259457 \quad-0.099196754 \quad-2.838474274 \\
& \begin{array}{llll}
\text { C } & -2.348213434 & 0.509548366 & -1.589328051
\end{array} \\
& \begin{array}{llll}
\text { C } & -1.498089075 & 1.591788292 & -1.370688677
\end{array} \\
& \begin{array}{llll}
\text { C } & -0.637576163 & 2.062907696 & -2.349195004
\end{array} \\
& \text { C } \quad-0.637293220 \quad 1.417557240 \quad-3.597735643
\end{aligned}
$$




$$
\begin{aligned}
& \begin{array}{llll}
\text { C } & 0.243509874 & 1.809551001 & -4.700661182
\end{array} \\
& \begin{array}{llll}
\text { C } & 1.222091794 & 2.812147141 & -4.669217587
\end{array} \\
& \begin{array}{lllll}
\text { C } & 2.026023388 & 3.033624887 & -5.779564381
\end{array} \\
& \begin{array}{llll}
\text { C } & 1.838524938 & 2.247715235 & -6.920277119
\end{array} \\
& \text { C } \quad 0.846639931 \quad 1.280639887 \quad-6.909821510 \\
& \text { N } \quad 0.068975307 \quad 1.077256083 \quad-5.838578224 \\
& \text { Ir } \quad-1.420718193 \quad-0.346642852 \quad-5.790858746 \\
& \text { O } \quad-4.866880894 \quad 4.029953480 \quad-5.504207134 \\
& \text { C } \quad \begin{array}{llll}
-4.883626938 & 3.851924658 & -4.083669186
\end{array} \\
& \mathrm{H} \quad-5.318936825 \quad 2.882326841 \quad-3.812477350 \\
& \text { H } \quad \begin{array}{llll}
-3.874605656 & 3.933446169 & -3.661089182
\end{array} \\
& \mathrm{H} \quad-5.509049416 \quad 4.654232025 \quad-3.693390846 \\
& \begin{array}{llll}
\text { C } & 1.532276869 & -8.658623695 & -5.330741405
\end{array} \\
& \begin{array}{llll}
\text { C } & 2.859611034 & -8.204258919 & -5.253196716
\end{array} \\
& \begin{array}{llll}
\text { C } & 3.211856365 & -6.990107059 & -5.817484856
\end{array} \\
& \text { H } \quad 3.608250856 \quad-8.805612564 \quad-4.747474670 \\
& \text { C } \quad 0.563066125 \quad-7.897396564 \quad-5.967605114 \\
& \text { H } \quad \begin{array}{llll}
1.259355903 & -9.610790253 & -4.886691093
\end{array} \\
& \text { C } \quad 0.919386446 \quad-6.665502548 \quad-6.530775070 \\
& \mathrm{H} \quad-0.465171546 \quad-8.244481087 \quad-6.028576374 \\
& \begin{array}{llll}
\text { C } & 2.249531507 & -6.192737103 & -6.468363762
\end{array} \\
& \text { N } \quad-0.023348197 \quad-5.853208065 \quad-7.147343159 \\
& \text { C } \quad 2.557305098 \quad-4.913893700 \quad-7.044054985 \\
& \text { H } \quad 4.235787392 \quad-6.631694794 \quad-5.757292747 \\
& \begin{array}{llll}
\text { C } & 0.196604624 & -4.597836971 & -7.642539024
\end{array} \\
& \text { H } \quad-0.986016035 \quad-6.163229465 \quad-7.207210064 \\
& \text { C } \quad 1.588546276 \quad-4.133445740 \quad-7.601022720 \\
& \text { O } \quad-0.752506256 \quad-3.928197861 \quad-8.094596863 \\
& \text { O } \quad 1.727369428 \quad-2.911056995 \quad-8.163326263 \\
& \text { H } \quad 3.584542274 \quad-4.572399616 \quad-6.994642735 \\
& \begin{array}{llll}
\text { C } & 3.052625418 & -2.364853621 & -8.404055595
\end{array} \\
& \text { H } \quad 3.707204819 \quad-3.192259312 \quad-8.710485458 \\
& \text { C } \quad 3.594385147 \quad-1.691894293 \quad-7.147214890 \\
& \text { H } \quad 2.953532934 \quad-0.859438121 \quad-6.854197502 \\
& \text { H } \quad 3.644610882 \quad-2.385081768 \quad-6.304430485 \\
& \text { H } \quad 4.599584103 \quad-1.303143859 \quad-7.336788654 \\
& \begin{array}{llll}
\text { C } & 2.890358448 & -1.396647573 & -9.567189217
\end{array}
\end{aligned}
$$

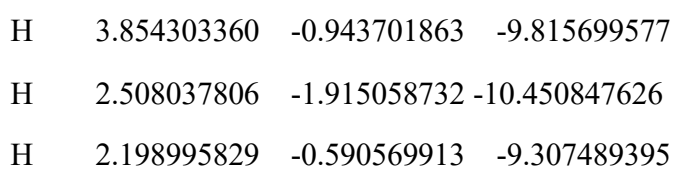

[ $\left.^{3} \mathbf{r}{ }^{1} \mathbf{Q}_{\mathrm{C}}\right]$

$\begin{array}{crrc}\mathrm{H} & -6.588360310 & -3.376048326 & 2.358070135 \\ \mathrm{H} & -8.305275917 & -1.951704741 & 3.965594769 \\ \mathrm{H} & -8.729705811 & 0.287729919 & 2.776106596 \\ \mathrm{H} & -5.096166134 & 0.499556065 & -2.482746124 \\ \mathrm{H} & -3.868224859 & -3.600022793 & -1.939172268 \\ \mathrm{H} & -5.244513988 & -3.640318155 & 0.156133085 \\ \mathrm{H} & -7.967574596 & -0.866964757 & -1.989048958 \\ \mathrm{H} & -12.122750282 & 0.042335588 & -2.680523396 \\ \mathrm{H} & -11.887038231 & 1.993730783 & -1.176815152 \\ \mathrm{H} & -2.360171556 & 3.438483953 & 0.169405460 \\ \mathrm{H} & -1.564201474 & 2.262360096 & 2.208096266 \\ \mathrm{H} & -5.445878506 & 0.444515526 & 2.512764454 \\ \mathrm{~F} & -1.700342655 & 0.186148062 & 3.677757263 \\ \mathrm{~F} & -3.210826874 & 1.199335337 & 4.868049622 \\ \mathrm{~F} & -3.705212593 & -0.665541530 & 3.861168861 \\ \mathrm{C} & -3.009695053 & 0.488535047 & 3.734637260 \\ \mathrm{~F} & -10.829917908 & -1.560613275 & -4.417757511 \\ \mathrm{~F} & -8.952072144 & -2.313497543 & -3.587350607 \\ \mathrm{~F} & -10.850379944 & -2.786340952 & -2.628607988 \\ \mathrm{C} & -10.167892456 & -1.805988908 & -3.266947031 \\ \mathrm{C} & -6.726760864 & -1.511013627 & 1.122931004 \\ \mathrm{C} & -6.976797104 & -2.382031679 & 2.199812412 \\ \mathrm{C} & --7.841405392 & -1.679801822 & 3.029500246 \\ \mathrm{~N} & -8.067944527 & -0.473813474 & 2.470808506 \\ \mathrm{C} & -5.089874744 & -0.439501286 & -1.945850492 \\ \mathrm{C} & -4.369253159 & -1.557204127 & -2.382315636 \\ \mathrm{C} & -4.422924042 & -2.728724718 & -1.607886553 \\ \mathrm{C} & -5.183474064 & -2.745002747 & -0.452653021 \\ \mathrm{C} & -5.887831688 & -1.596547723 & -0.069545478 \\ \mathrm{C} & -9.525177956 & 2.653790951 & 0.184070244\end{array}$




$$
\begin{aligned}
& \begin{array}{llll}
\text { C } & -10.441409111 & 3.664603233 & 0.576307833
\end{array} \\
& \begin{array}{llll}
\text { C } & -10.034731865 & 4.590094566 & 1.503515601
\end{array} \\
& \begin{array}{llll}
\text { C } & -8.745298386 & 4.613397598 & 2.115088701
\end{array} \\
& \begin{array}{llll}
\text { C } & -7.852037430 & 3.648422003 & 1.694648623
\end{array} \\
& \begin{array}{lllll}
\text { C } & -8.845527649 & -0.258996844 & -1.818476081
\end{array} \\
& \text { C } \quad-10.048954010-0.574710190 \quad-2.427697182 \\
& \text { C } \quad-11.176934242 \quad 0.263782233 \quad-2.198730230 \\
& \text { C } \quad-11.040566444 \quad 1.344716430 \quad-1.368742108 \\
& \begin{array}{llll}
\text { C } & -9.796984673 & 1.626142859 & -0.733675241
\end{array} \\
& \text { N } \quad-5.824785233 \quad-0.471478045 \quad-0.820258915 \\
& \text { N } \quad \begin{array}{llll}
-7.405680180 & -0.362445414 & 1.314061642
\end{array} \\
& \begin{array}{llll}
\mathrm{N} & -8.701988220 & 0.794459939 & -1.012192607
\end{array} \\
& \begin{array}{llll}
\text { C } & -8.146458626 & 2.652714968 & 0.753529906
\end{array} \\
& \begin{array}{llll}
\text { C } & -6.177814960 & 2.591949463 & -1.351703048
\end{array} \\
& \text { C } \quad-6.711716175 \quad 3.055517197 \quad-2.555816412 \\
& \text { C } \quad-6.039761543 \quad 3.916883469 \quad-3.414815426 \\
& \begin{array}{llll}
\text { C } & -4.768373966 & 4.346374512 & -3.043608189
\end{array} \\
& \text { C } \quad-4.167635918 \quad 3.940015554 \quad-1.862664223 \\
& \text { C } \quad-4.877450466 \quad 3.065290689 \quad-1.024126410 \\
& \begin{array}{llll}
\text { C } & -4.319415092 & 2.542431593 & 0.225362569
\end{array} \\
& \begin{array}{llll}
\text { C } & -3.024361849 & 2.774042130 & 0.708103836
\end{array} \\
& \begin{array}{llll}
\text { C } & -2.577648401 & 2.121768236 & 1.847476244
\end{array} \\
& \begin{array}{llll}
\text { C } & -3.445978880 & 1.250488043 & 2.510989904
\end{array} \\
& \begin{array}{llll}
\text { C } & -4.734636784 & 1.094364285 & 2.023822308
\end{array} \\
& \begin{array}{llll}
\mathrm{N} & -5.155797482 & 1.714019537 & 0.912951827
\end{array} \\
& \text { Ir } \quad-6.964049816 \quad 1.274935484 \quad-0.030504070 \\
& \text { H } \quad-3.177929163 \quad 4.313024521 \quad-1.627856374 \\
& \text { H } \quad-6.494674683 \quad 4.252834797 \quad-4.339238644 \\
& \mathrm{H} \quad-8.490884781 \quad 5.366882801 \quad 2.847562075 \\
& \begin{array}{llll}
\mathrm{H} & -11.437734604 & 3.741987228 & 0.158340931
\end{array} \\
& \text { F } \quad \begin{array}{llll}
-6.608041286 & 3.687140226 & 2.229682207
\end{array} \\
& \begin{array}{llll}
\text { F } & -10.893842697 & 5.540622711 & 1.892400622
\end{array} \\
& \text { F } \quad \begin{array}{llll}
-7.944832802 & 2.659487009 & -2.931562901
\end{array} \\
& \text { F } \quad-4.104150772 \quad 5.179318428 \quad-3.861034155 \\
& \text { O } \quad-3.618880272 \quad-1.596612692 \quad-3.497789383 \\
& \text { C } \quad-3.576444149 \quad-0.437278360 \quad-4.336496830 \\
& \text { H } \quad-4.576529503 \quad-0.182421997 \quad-4.707716465
\end{aligned}
$$
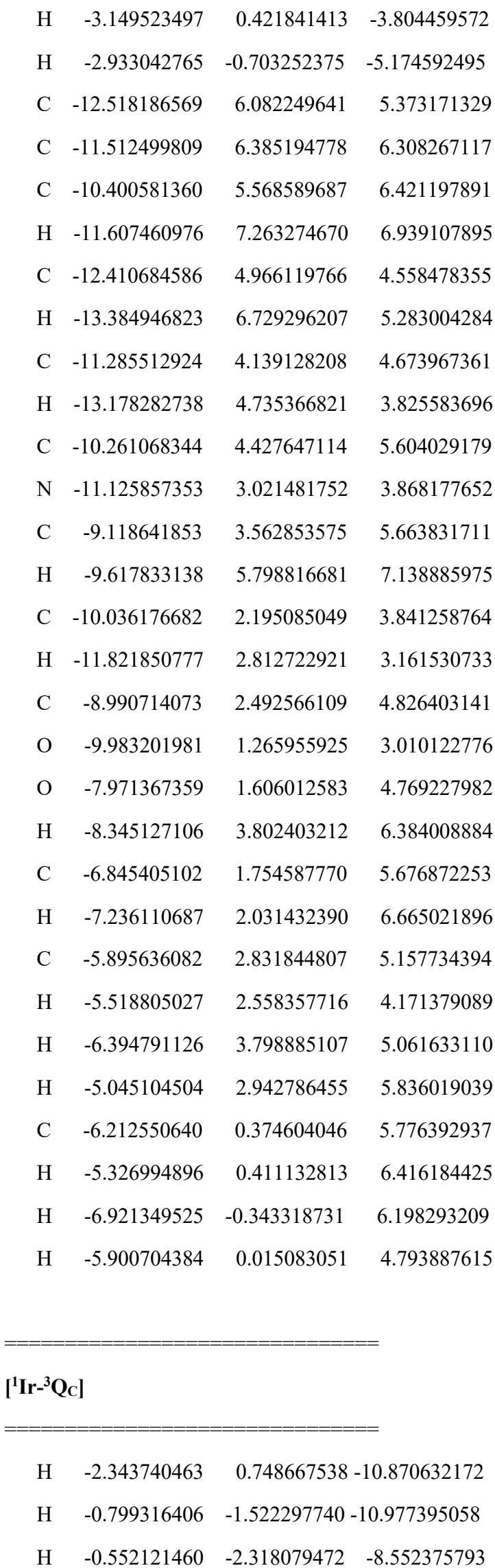


\begin{tabular}{|c|c|c|c|}
\hline & 3 & 1.86 & \\
\hline & -4.974240303 & 4.055295467 & -8.0269107 \\
\hline & 3.711353540 & .499744177 & -9.536037445 \\
\hline & -0.584115088 & -5.120618343 & -4.286975861 \\
\hline & 42507204 & -0.811 & -6.428066254 \\
\hline & -4.995538235 & -5.009808540 & -5.77 \\
\hline & -2.623399734 & -5.180647850 & \\
\hline & 0.084260069 & 2.951206684 & \\
\hline & & & \\
\hline & & & \\
\hline & & & \\
\hline & 2.55 & & \\
\hline & & & \\
\hline & & & \\
\hline & & & \\
\hline & -6.7612 & -3.8 & -7.1 \\
\hline & -7.085680962 & -2.411556482 & \\
\hline & -6.39 & -1.71 & \\
\hline & -6.28 & -2.7 & \\
\hline & & & \\
\hline & -2.740096807 & 0.062132 & -0.7 \\
\hline & & & \\
\hline & & & \\
\hline & & -2.699473619 & \\
\hline & & -0.56 & \\
\hline & & & \\
\hline & & & \\
\hline $\mathrm{C}$ & -1.165194869 & $-0.969176650-$ & \\
\hline & & & \\
\hline & -3.483660698 & & \\
\hline & -4.280 & 3.086 & -6.2 \\
\hline $\mathrm{C}$ & -4.360539913 & & -7.630610466 \\
\hline $\mathrm{C}$ & & & -8.458582878 \\
\hline $\mathrm{C}$ & -2.884022713 & 1.370974422 & -7.896542072 \\
\hline $\mathrm{C}$ & -0.803054035 & -3.049057484 & -4.971544743 \\
\hline $\mathrm{C}$ & -0.114729129 & -4.158438683 & -4.453039646 \\
\hline & 1.226005912 & -4.013918400 & -4.13595914 \\
\hline
\end{tabular}

C $\quad 1.898175478 \quad-2.807135344 \quad-4.315210819$

C $\quad \begin{array}{llll}1.174540043 & -1.739615321 & -4.829174042\end{array}$

C $\quad-4.011356831 \quad-1.791260839 \quad-6.117429733$

C $\quad-4.853280067-2.891414165 \quad-6.157541275$

C $\quad-4.356438637 \quad-4.133635521 \quad-5.751463413$

C $\quad-3.036390066 \quad-4.225319386 \quad-5.338278770$

C $\quad-2.218993902 \quad-3.088300705 \quad-5.331017494$

$\mathrm{N} \quad-2.809111834 \quad 1.222464085 \quad-6.552112103$

N $\quad-1.479135036 \quad-0.567712784 \quad-7.974258900$

N $\quad-2.736358643 \quad-1.885144830 \quad-5.715586662$

C $\quad-0.171078622-1.797984600 \quad-5.189010143$

C $\quad-1.420305729 \quad 0.345273495 \quad-3.915175915$

C $\quad-2.165878057 \quad-0.121385358-2.834412575$

C $\quad \begin{array}{llll}-2.143915176 & 0.460248381 & -1.572210550\end{array}$

$\begin{array}{lllll}\text { C } & -1.320846438 & 1.568302274 & -1.381357431\end{array}$

$\begin{array}{llll}\text { C } & -0.541669965 & 2.092172384 & -2.400410891\end{array}$

$\begin{array}{llll}\text { C } & -0.594782650 & 1.472335219 & -3.660895109\end{array}$

$\begin{array}{llll}\text { C } & 0.194639191 & 1.925435662 & -4.808604717\end{array}$

C $\quad 1.101891041 \quad 2.993190765 \quad-4.824888706$

C $\quad 1.806985974 \quad 3.292013645 \quad-5.981759548$

$\begin{array}{llll}\text { C } & 1.593997002 & 2.515056849 & -7.124382496\end{array}$

$\begin{array}{llll}\text { C } & 0.678920329 & 1.478177071 & -7.064412594\end{array}$

$\begin{array}{llll}\mathrm{N} & -0.001140893 & 1.196326375 & -5.944374084\end{array}$

Ir $\quad-1.400964737 \quad-0.313959926 \quad-5.829716206$

$\begin{array}{llll}\text { O } & -4.982047558 & 3.944953442 & -5.475174427\end{array}$

C $\quad-4.933224201 \quad 3.794207573 \quad-4.052076340$

H $\quad \begin{array}{llll}-5.326648712 & 2.817589283 & -3.744438410\end{array}$

H $\quad-3.910249472 \quad 3.914459229 \quad-3.674558401$

$\mathrm{H} \quad-5.565880775 \quad 4.584833145 \quad-3.649605513$

C $\quad \begin{array}{llll}1.125128746 & -8.937490463 & -5.597948551\end{array}$

C $\quad 2.450180531 \quad-8.554242134 \quad-5.336945534$

$\begin{array}{llll}\text { C } & 2.903538227 & -7.310433388 & -5.730060101\end{array}$

H $\quad 3.119254351 \quad-9.236226082 \quad-4.821796894$

C $\quad 0.248249263 \quad-8.052727699 \quad-6.257877350$

H $\quad 0.767103314 \quad-9.915199280 \quad-5.292523861$

C $\quad 0.685584247 \quad-6.805249691 \quad-6.661510468$

H $\quad-0.777331769-8.353437424 \quad-6.457136154$ 


$\begin{array}{lrll}\mathrm{C} & 2.048448086 & -6.385353088 & -6.414206505 \\ \mathrm{~N} & -0.171276823 & -5.910597324 & -7.308907032 \\ \mathrm{C} & 2.473557234 & -5.127421379 & -6.819065571 \\ \mathrm{H} & 3.924268484 & -7.004100323 & -5.523898602 \\ \mathrm{C} & 0.159069315 & -4.611641884 & -7.681374550 \\ \mathrm{H} & -1.123656392 & -6.180473328 & -7.511303425 \\ \mathrm{C} & 1.512143612 & -4.212048531 & -7.461110592 \\ \mathrm{O} & -0.741094708 & -3.885401487 & -8.173153877 \\ \mathrm{O} & 1.791342854 & -2.954506874 & -7.825534344 \\ \mathrm{H} & 3.486341238 & -4.806953430 & -6.619733810 \\ \mathrm{C} & 3.166002274 & -2.456343412 & -7.854365826 \\ \mathrm{H} & 3.632737160 & -2.702219486 & -6.893795013 \\ \mathrm{C} & 3.046712875 & -0.947283566 & -7.985346317 \\ \mathrm{H} & 2.500430346 & -0.682050407 & -8.895917892 \\ \mathrm{H} & 2.532228708 & -0.531926274 & -7.120040417 \\ \mathrm{H} & 4.040494919 & -0.496013224 & -8.042229652 \\ \mathrm{C} & 3.919394732 & -3.102115393 & -9.012734413 \\ \mathrm{H} & 4.944004059 & -2.719720364 & -9.045104980 \\ \mathrm{H} & 3.960669518 & -4.189772129 & -8.911870956 \\ \mathrm{H} & 3.429391623 & -2.859271526 & -9.960523605\end{array}$

\section{${ }^{1}[\mathbf{I r}-\mathbf{Q D}]$}

$\begin{array}{crrr}\text { H } & -7.434926987 & -3.789075375 & 1.641417027 \\ \text { H } & -8.444661140 & -2.260595798 & 3.689471245 \\ \text { H } & -7.779453754 & 0.147396624 & 3.126267195 \\ \text { H } & -5.003381729 & 0.307902426 & -2.598613024 \\ \text { H } & -4.885756493 & -4.002468586 & -2.746135950 \\ \text { H } & -6.123375893 & -4.019751072 & -0.567131221 \\ \text { H } & -8.029067993 & -0.654126167 & -2.202550173 \\ \text { H } & -12.194568634 & 0.373577505 & -2.109282494 \\ \text { H } & -11.666553497 & 2.053623438 & -0.354700118 \\ \text { H } & -2.111431837 & 3.291546345 & -0.047274612 \\ \text { H } & -1.381890059 & 2.458455086 & 2.179772377 \\ \text { H } & -5.175437927 & 0.471464157 & 2.541320562 \\ \text { F } & -1.565780520 & 0.793954849 & 4.084653378\end{array}$

\begin{tabular}{|c|c|c|c|}
\hline $\mathrm{F}$ & -3.264642239 & 1.927424788 & 4.826305866 \\
\hline $\mathrm{F}$ & -3.517386436 & -0.166637674 & 4.275283337 \\
\hline $\mathrm{C}$ & -2.888072968 & 0.965267718 & 3.922633171 \\
\hline $\mathrm{F}$ & -11.432519913 & -0.985183477 & -4.122331142 \\
\hline $\mathrm{F}$ & -9.317603111 & -1.535256743 & -4.097848892 \\
\hline $\mathrm{F}$ & -10.716095924 & -2.509314775 & -2.747287512 \\
\hline $\mathrm{C}$ & -10.405993462 & -1.327945352 & -3.323763371 \\
\hline $\mathrm{H}$ & -6.430853844 & 4.028544903 & -4.402197361 \\
\hline $\mathrm{C}$ & -6.927282333 & -1.764356971 & 0.823238015 \\
\hline $\mathrm{C}$ & -7.447692871 & -2.713737726 & 1.724989414 \\
\hline $\mathrm{C}$ & -7.976772308 & -1.962563992 & 2.762880564 \\
\hline $\mathrm{N}$ & -7.803116798 & -0.656160533 & 2.463702440 \\
\hline $\mathrm{C}$ & -5.215227604 & -0.673786700 & -2.197336674 \\
\hline $\mathrm{C}$ & -4.838741302 & -1.854828477 & -2.847643375 \\
\hline $\mathrm{C}$ & -5.171338558 & -3.081807613 & -2.248645782 \\
\hline $\mathrm{C}$ & -5.855915070 & -3.084841251 & -1.046409607 \\
\hline $\mathrm{C}$ & -6.209651470 & -1.870943785 & -0.444527745 \\
\hline $\mathrm{C}$ & -9.101188660 & 2.580700874 & 0.653341889 \\
\hline $\mathrm{C}$ & -9.974688530 & 3.482513905 & 1.288123608 \\
\hline $\mathrm{C}$ & -9.433998108 & 4.411045551 & 2.156033754 \\
\hline $\mathrm{C}$ & -8.065372467 & 4.498955250 & 2.389264584 \\
\hline $\mathrm{C}$ & -7.243493080 & 3.573842525 & 1.761948109 \\
\hline $\mathrm{C}$ & -8.857321739 & -0.086634167 & -1.803517580 \\
\hline $\mathrm{C}$ & -10.143396378 & -0.270537734 & -2.284315586 \\
\hline $\mathrm{C}$ & -11.179157257 & 0.499057084 & -1.748484969 \\
\hline $\mathrm{C}$ & -10.881046295 & 1.433920741 & -0.769289255 \\
\hline $\mathrm{C}$ & -9.561373711 & 1.596186876 & -0.325533926 \\
\hline $\mathrm{N}$ & -5.884122849 & -0.692522764 & -1.030516624 \\
\hline $\mathrm{N}$ & -7.149775505 & -0.525521338 & 1.299273849 \\
\hline $\mathrm{N}$ & -8.574477196 & 0.810391665 & -0.847214997 \\
\hline $\mathrm{C}$ & -7.703563690 & 2.576836109 & 0.901498437 \\
\hline $\mathrm{C}$ & -5.968730450 & 2.394134998 & -1.418563843 \\
\hline $\mathrm{C}$ & -6.569457531 & 2.875728607 & -2.581442833 \\
\hline $\mathrm{C}$ & -5.922027588 & 3.679927349 & -3.511016607 \\
\hline $\mathrm{C}$ & -4.598381042 & 4.031742573 & -3.256632328 \\
\hline $\mathrm{C}$ & -3.929949522 & 3.609610081 & -2.119519472 \\
\hline $\mathrm{C}$ & -4.623131752 & 2.796997070 & -1.205444813 \\
\hline
\end{tabular}

$\begin{array}{llll}\text { F } & -3.264642239 & 1.927424788 & 4.826305866\end{array}$

$\begin{array}{llll}\text { F } & -3.517386436 & -0.166637674 & 4.275283337\end{array}$

$\begin{array}{llll}\text { C } & -2.888072968 & 0.965267718 & 3.922633171\end{array}$

F $\quad-11.432519913 \quad-0.985183477 \quad-4.122331142$

F $\quad-9.317603111 \quad-1.535256743 \quad-4.097848892$

F $\quad-10.716095924 \quad-2.509314775 \quad-2.747287512$

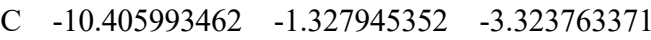

H $\quad-6.430853844 \quad 4.028544903 \quad-4.402197361$

$-6.927282333-1.764356971$

$\begin{array}{llll}\text { C } & -7.976772308 & -1.962563992 & 2.762880564\end{array}$

$\begin{array}{llll}\text { N } & -7.803116798 & -0.656160533 & 2.463702440\end{array}$

$\begin{array}{llll}\text { C } & -5.215227604 & -0.673786700 & -2.197336674\end{array}$

$\begin{array}{llll}\text { C } & -4.838741302 & -1.854828477 & -2.847643375\end{array}$

C $\quad-5.171338558 \quad-3.081807613 \quad-2.248645782$

$\begin{array}{llll}C & -5.855915070 & -3.084841251 & -1.046409607\end{array}$

C $\quad-6.209651470 \quad-1.870943785 \quad-0.444527745$

$\begin{array}{llll}\text { C } & -9.101188660 & 2.580700874 & 0.653341889\end{array}$

$\begin{array}{llll}\text { C } & -9.974688530 & 3.482513905 & 1.288123608\end{array}$

$\begin{array}{lll}-9.433998108 & 4.411045551 & 2.156033754\end{array}$

$\begin{array}{llll}\text { C } & -7.243493080 & 3.573842525 & 1.761948109\end{array}$

$\begin{array}{llll}\text { C } & -8.857321739 & -0.086634167 & -1.803517580\end{array}$

C $\quad-11.179157257 \quad 0.499057084 \quad-1.748484969$

$\begin{array}{llll}\text { C } & -10.881046295 & 1.433920741 & -0.769289255\end{array}$

$\begin{array}{llll}\text { C } & -9.561373711 & 1.596186876 & -0.325533926\end{array}$

N $\quad-5.884122849 \quad-0.692522764 \quad-1.030516624$

$\begin{array}{llll}\mathrm{N} & -7.149775505 & -0.525521338 & 1.299273849\end{array}$

$\begin{array}{llll}\mathrm{N} & -8.574477196 & 0.810391665 & -0.847214997\end{array}$

$\begin{array}{llll}\text { C } & -7.703563690 & 2.576836109 & 0.901498437\end{array}$

$\begin{array}{llll}\text { C } & -5.968730450 & 2.394134998 & -1.418563843\end{array}$

$\begin{array}{llll}\text { C } & -6.569457531 & 2.875728607 & -2.581442833\end{array}$

C $\quad-5.922027588 \quad 3.679927349 \quad-3.511016607$

$\begin{array}{llll}\text { C } & -4.598381042 & 4.031742573 & -3.256632328\end{array}$

$\begin{array}{llll}\text { C } & -4.623131752 & 2.796997070 & -1.205444813\end{array}$ 


\begin{tabular}{|c|c|c|c|}
\hline $\mathrm{C}$ & -4.030230045 & 2.318201065 & 0.045070969 \\
\hline $\mathrm{C}$ & -2.763364077 & 2.654470205 & 0.537579894 \\
\hline $\mathrm{C}$ & -2.356791019 & 2.197164536 & 1.781904459 \\
\hline $\mathrm{C}$ & -3.227801085 & 1.394901037 & 2.523813963 \\
\hline $\mathrm{C}$ & -4.461924553 & 1.062402129 & 1.986273050 \\
\hline $\mathrm{N}$ & -4.843473434 & 1.504026413 & 0.779258549 \\
\hline Ir & -6.688253403 & 1.098889112 & -0.040715560 \\
\hline $\mathrm{C}$ & -6.891095161 & 6.746356010 & 6.829528332 \\
\hline $\mathrm{C}$ & -5.561442852 & 6.347519398 & 6.607327461 \\
\hline $\mathrm{C}$ & -5.281461716 & 5.112773418 & 6.041651249 \\
\hline $\mathrm{C}$ & -6.339803696 & 4.263472557 & 5.690631390 \\
\hline $\mathrm{C}$ & -7.684100151 & 4.642477512 & 5.910516262 \\
\hline $\mathrm{C}$ & -7.935852051 & 5.904395580 & 6.487350941 \\
\hline $\mathrm{N}$ & -6.102169037 & 3.031268597 & 5.100784302 \\
\hline $\mathrm{C}$ & -7.055131435 & 2.156711340 & 4.669885635 \\
\hline $\mathrm{C}$ & -8.446818352 & 2.551509142 & 4.915005684 \\
\hline $\mathrm{C}$ & -8.730630875 & 3.741928101 & 5.516755104 \\
\hline $\mathrm{O}$ & -6.729500294 & 1.102228403 & 4.088852882 \\
\hline $\mathrm{O}$ & -9.313898087 & 1.636851072 & 4.424936771 \\
\hline $\mathrm{C}$ & -10.740013123 & 1.899723053 & 4.460197449 \\
\hline $\mathrm{C}$ & -11.341716766 & 0.956993818 & 3.426878452 \\
\hline $\mathrm{H}$ & -7.098118305 & 7.716846943 & 7.269147396 \\
\hline $\mathrm{H}$ & -4.745560646 & 7.010965824 & 6.876739025 \\
\hline $\mathrm{H}$ & -4.257130623 & 4.797037125 & 5.862660885 \\
\hline $\mathrm{H}$ & -8.965545654 & 6.208038330 & 6.654424191 \\
\hline $\mathrm{H}$ & -5.147111416 & 2.743493557 & 4.916143417 \\
\hline $\mathrm{H}$ & -9.754641533 & 4.059784412 & 5.672331810 \\
\hline $\mathrm{H}$ & -10.900941849 & 2.940160513 & 4.146726608 \\
\hline $\mathrm{H}$ & -12.422734261 & 1.111736178 & 3.358711958 \\
\hline $\mathrm{H}$ & -10.895391464 & 1.129669189 & 2.445049047 \\
\hline $\mathrm{F}$ & -7.855540752 & 2.542628050 & -2.853395700 \\
\hline $\mathrm{F}$ & -3.953418732 & 4.804904938 & -4.148155689 \\
\hline $\mathrm{H}$ & -2.902888536 & 3.921980858 & -1.972274661 \\
\hline $\mathrm{O}$ & -4.180099964 & -1.904728174 & -4.019189835 \\
\hline $\mathrm{C}$ & -3.869728804 & -0.674362063 & -4.681777000 \\
\hline $\mathrm{H}$ & -4.781919479 & -0.112798557 & -4.917955399 \\
\hline $\mathrm{H}$ & -3.201936960 & -0.053223666 & -4.072613239 \\
\hline
\end{tabular}

$\begin{array}{llll}\mathrm{H} & -3.364619970 & -0.956181467 & -5.605096817\end{array}$

F $\quad \begin{array}{llll}-10.259399414 & 5.238719463 & 2.832722425\end{array}$

F $\quad \begin{array}{llll}-5.918444633 & 3.659112692 & 2.052219152\end{array}$

$\mathrm{H} \quad-7.661297321 \quad 5.235605717 \quad 3.073045015$

H $\quad-11.046534538 \quad 3.476858854 \quad 1.130209446$

H $\quad-11.160387993 \quad-0.083595708 \quad 3.714053631$

C $\quad-11.296161652 \quad 1.660521626 \quad 5.863664150$

$\mathrm{H} \quad-12.370843887 \quad 1.867041945 \quad 5.880943775$

$\begin{array}{llll}\mathrm{H} & -11.139990807 & 0.617148161 & 6.154081345\end{array}$

H $\quad-10.816576004 \quad 2.295050859 \quad 6.612903595$

$\left[{ }^{3} \mathbf{I r}-{ }^{1} \mathbf{Q}_{\mathrm{D}}\right]$

$\begin{array}{lrrr}\mathrm{H} & -7.491701126 & -3.699460745 & 1.632797480 \\ \mathrm{H} & -8.483799934 & -2.121760845 & 3.653893948 \\ \mathrm{H} & -7.764183521 & 0.252101749 & 3.082494974 \\ \mathrm{H} & -4.958108902 & 0.254111707 & -2.668331146 \\ \mathrm{H} & -5.076239109 & -4.055659771 & -2.792368650 \\ \mathrm{H} & -6.260439396 & -3.994445801 & -0.585579872 \\ \mathrm{H} & -7.971971035 & -0.531026304 & -2.334578037 \\ \mathrm{H} & -12.173970222 & 0.284371614 & -2.003019810 \\ \mathrm{H} & -11.638483047 & 1.947168946 & -0.234226629 \\ \mathrm{H} & -1.938207626 & 3.199039698 & -0.073452428 \\ \mathrm{H} & -1.328401208 & 2.494634867 & 2.219738960 \\ \mathrm{H} & -5.196726322 & 0.617501318 & 2.569938898 \\ \mathrm{~F} & -1.687959433 & 0.952198923 & 4.261850834 \\ \mathrm{~F} & -3.321555614 & 2.281482697 & 4.794610500 \\ \mathrm{~F} & -3.716940641 & 0.163295791 & 4.459005356 \\ \mathrm{C} & -2.984122515 & 1.193767309 & 3.999545097 \\ \mathrm{~F} & -11.453684807 & -1.003078580 & -4.079286098 \\ \mathrm{~F} & -9.316520691 & -1.454156041 & -4.168396473 \\ \mathrm{~F} & -10.598476410 & -2.506773233 & -2.761642456 \\ \mathrm{C} & -10.373014450 & -1.307521582 & -3.339784861 \\ \mathrm{H} & -6.237095356 & 3.895448208 & -4.552958965 \\ \mathrm{C} & -6.936538696 & -1.696685553 & 0.793594599 \\ \mathrm{C} & -7.484594345 & -2.623044252 & 1.702006578\end{array}$




\begin{tabular}{|c|c|c|c|}
\hline $\mathrm{C}$ & -8.005285263 & -1.847182274 & 2.725452185 \\
\hline $\mathrm{N}$ & -7.802025795 & -0.549113214 & 2.412219048 \\
\hline $\mathrm{C}$ & -5.210206509 & -0.712398887 & -2.253324032 \\
\hline $\mathrm{C}$ & -4.915991783 & -1.913856506 & -2.908623219 \\
\hline $\mathrm{C}$ & -5.296719551 & -3.118394613 & -2.292733669 \\
\hline $\mathrm{C}$ & -5.950777054 & -3.077867985 & -1.074772716 \\
\hline $\mathrm{C}$ & -6.227826595 & -1.842931151 & -0.474104583 \\
\hline $\mathrm{C}$ & -9.051990509 & 2.555271149 & 0.677296996 \\
\hline $\mathrm{C}$ & -9.935220718 & 3.388583899 & 1.380964994 \\
\hline $\mathrm{C}$ & -9.401402473 & 4.295478821 & 2.280124664 \\
\hline $\mathrm{C}$ & -8.031193733 & 4.431235790 & 2.476494312 \\
\hline $\mathrm{C}$ & -7.189918995 & 3.578439951 & 1.775286317 \\
\hline $\mathrm{C}$ & -8.805936813 & -0.014559226 & -1.881146550 \\
\hline $\mathrm{C}$ & -10.105836868 & -0.252080113 & -2.298657179 \\
\hline $\mathrm{C}$ & -11.148094177 & 0.453460634 & -1.692720413 \\
\hline $\mathrm{C}$ & -10.845535278 & 1.379827142 & -0.705672264 \\
\hline $\mathrm{C}$ & -9.513934135 & 1.590021729 & -0.323793352 \\
\hline $\mathrm{N}$ & -5.850032330 & -0.688707530 & -1.071559668 \\
\hline $\mathrm{N}$ & -7.135785103 & -0.447872490 & 1.251491070 \\
\hline $\mathrm{N}$ & -8.520035744 & 0.871289968 & -0.917063355 \\
\hline $\mathrm{C}$ & -7.644516468 & 2.604548216 & 0.880861521 \\
\hline $\mathrm{C}$ & -5.857478619 & 2.355238676 & -1.493511438 \\
\hline $\mathrm{C}$ & -6.434657097 & 2.817925692 & -2.688272953 \\
\hline $\mathrm{C}$ & -5.762585640 & 3.551960945 & -3.643458366 \\
\hline $\mathrm{C}$ & -4.401773930 & 3.863999367 & -3.359390736 \\
\hline $\mathrm{C}$ & -3.736333370 & 3.481566429 & -2.217463732 \\
\hline $\mathrm{C}$ & -4.437775135 & 2.724014997 & -1.246535182 \\
\hline $\mathrm{C}$ & -3.905395031 & 2.287824392 & -0.019296227 \\
\hline $\mathrm{C}$ & -2.630017281 & 2.615076303 & 0.522997618 \\
\hline $\mathrm{C}$ & -2.291099548 & 2.234850645 & 1.794077396 \\
\hline $\mathrm{C}$ & -3.232158422 & 1.488759995 & 2.562277317 \\
\hline $\mathrm{C}$ & -4.452934265 & 1.151040196 & 1.995508671 \\
\hline $\mathrm{N}$ & -4.784196854 & 1.509779215 & 0.754093587 \\
\hline Ir & -6.617988586 & 1.177801609 & -0.106644660 \\
\hline $\mathrm{C}$ & -6.974573612 & 6.721209049 & 6.986698151 \\
\hline $\mathrm{C}$ & -5.644359589 & 6.350630283 & 6.720356941 \\
\hline $\mathrm{C}$ & -5.357820988 & 5.135204315 & 6.118439198 \\
\hline
\end{tabular}

\begin{tabular}{|c|c|c|c|}
\hline & -6.411 & .2766962 & 5.77 \\
\hline & -7.756338120 & 4.627964020 & 6.0355129 \\
\hline & -8.014345169 & 5.870931625 & 6.650347233 \\
\hline & -6.165793896 & 3.063992500 & 5.149859428 \\
\hline & -7.113518715 & 2.184452057 & 4.722526550 \\
\hline & -8.504930496 & 2.546815634 & 5.009600639 \\
\hline & -8.796420097 & 3.718494654 & \\
\hline & -6.787468910 & 1.149042606 & 5009 \\
\hline & -9.366230011 & 1.625731945 & 4.517195702 \\
\hline & -10.795196533 & & \\
\hline & -11.409596443 & 0.915996969 & 3.573201180 \\
\hline & -7.1857 & 6871 & 396175 \\
\hline & -4.833078384 & & \\
\hline & -4.334355354 & 4.839755535 & \\
\hline & -9.044287682 & & 79600 \\
\hline & -5.205667 & & \\
\hline & -9.821726799 & & 175445 \\
\hline & -10.992018700 & & \\
\hline & -12.495510 & & \\
\hline & -10.999242783 & & \\
\hline & -7.729995251 & 2.521496296 & -2.935432911 \\
\hline & -3.747169495 & & \\
\hline & -2.702792645 & & \\
\hline & -4.298693180 & -2.003072739 & -4.099438667 \\
\hline & -3.971573591 & -0.793516755 & \\
\hline & -4.871894360 & -0.199959874 & -4.994874001 \\
\hline & -3.252772331 & -0.192003116 & \\
\hline & -3.521417618 & -1.106395960 & -5.734171867 \\
\hline & -10.237533569 & & \\
\hline & -5.869386673 & 3.713668823 & 2.010546207 \\
\hline & -7.635922432 & 5.151746750 & 3.182489634 \\
\hline & -11.010693550 & 3.349059820 & 1.258268356 \\
\hline & -11.195138931 & -0.125611067 & 3.832949638 \\
\hline & -11.297719955 & 1.564289808 & 6.019527435 \\
\hline & -12.376647949 & 1.738307357 & 6.076481819 \\
\hline & -11.101701736 & 0.519240379 & 6.278281689 \\
\hline & -10.811704636 & 2.194022894 & 6.76824808 \\
\hline
\end{tabular}




\begin{tabular}{|c|c|c|c|}
\hline \multicolumn{3}{|c|}{$\left[{ }^{1} \mathbf{I r}-{ }^{3} \mathbf{Q}_{\mathrm{D}}\right]$} & \\
\hline $\mathrm{H}$ & -7.221777439 & -3.857878447 & 1.755020618 \\
\hline $\mathrm{H}$ & -8.406969070 & -2.442592859 & 3.788477421 \\
\hline $\mathrm{H}$ & -7.957308292 & 0.018009208 & 3.250687122 \\
\hline $\mathrm{H}$ & -5.004109383 & 0.467888147 & -2.365717888 \\
\hline $\mathrm{H}$ & -4.635128975 & -3.823411226 & -2.608054161 \\
\hline $\mathrm{H}$ & -5.922379971 & -3.964220285 & -0.462672710 \\
\hline $\mathrm{H}$ & -7.964441776 & -0.794590592 & -2.026764631 \\
\hline $\mathrm{H}$ & -12.160180092 & 0.071948931 & -2.244158030 \\
\hline $\mathrm{H}$ & -11.807045937 & 1.849366426 & -0.542776346 \\
\hline $\mathrm{H}$ & -2.362382412 & 3.505453110 & 0.391006023 \\
\hline $\mathrm{H}$ & -1.655754447 & 2.596055269 & 2.592751741 \\
\hline $\mathrm{H}$ & -5.391825676 & 0.478690833 & 2.776461601 \\
\hline $\mathrm{F}$ & -1.799187303 & 0.752593160 & 4.345528603 \\
\hline $\mathrm{F}$ & -3.483454466 & 1.844492912 & 5.179275990 \\
\hline $\mathrm{F}$ & -3.754225492 & -0.208172724 & 4.496615887 \\
\hline $\mathrm{C}$ & -3.122588158 & 0.941532612 & 4.211786270 \\
\hline $\mathrm{F}$ & -11.228178024 & -1.354677558 & -4.128824711 \\
\hline $\mathrm{F}$ & -9.088604927 & -1.775455594 & -3.974891901 \\
\hline $\mathrm{F}$ & -10.497963905 & -2.782566786 & -2.660707474 \\
\hline $\mathrm{C}$ & -10.227576256 & -1.606050014 & -3.266141415 \\
\hline $\mathrm{C}$ & -6.898452759 & -1.790881991 & 0.950390637 \\
\hline $\mathrm{C}$ & -7.340943813 & -2.789231300 & 1.840198517 \\
\hline $\mathrm{C}$ & -7.955288410 & -2.096297741 & 2.870684862 \\
\hline $\mathrm{N}$ & -7.903690815 & -0.777975678 & 2.577244520 \\
\hline $\mathrm{C}$ & -5.167920589 & -0.533368826 & -1.991448164 \\
\hline $\mathrm{C}$ & -4.709988117 & -1.674950480 & -2.659564495 \\
\hline $\mathrm{C}$ & -4.983666420 & -2.932893991 & -2.096137047 \\
\hline $\mathrm{C}$ & -5.695451260 & -3.004119158 & -0.912066519 \\
\hline $\mathrm{C}$ & -6.135286331 & -1.827185273 & -0.293456048 \\
\hline $\mathrm{C}$ & -9.331510544 & 2.533888578 & 0.582964420 \\
\hline $\mathrm{C}$ & -10.258901596 & 3.495346546 & 1.028193951 \\
\hline $\mathrm{C}$ & -9.796743393 & 4.525949478 & 1.821702242 \\
\hline $\mathrm{C}$ & -8.456091881 & 4.646286011 & 2.175593615 \\
\hline
\end{tabular}

\begin{tabular}{|c|c|c|c|}
\hline $\mathrm{C}$ & -7.588727474 & 3.642190456 & 1.762715697 \\
\hline $\mathrm{C}$ & -8.834836960 & -0.241144672 & -1.706409216 \\
\hline $\mathrm{C}$ & -10.079072952 & -0.496657521 & -2.259345293 \\
\hline $\mathrm{C}$ & -11.175265312 & 0.254317462 & -1.827513456 \\
\hline $\mathrm{C}$ & -10.973981857 & 1.244314075 & -0.878671706 \\
\hline $\mathrm{C}$ & -9.692123413 & 1.480241895 & -0.362163454 \\
\hline $\mathrm{N}$ & -5.863985538 & -0.618543744 & -0.843163252 \\
\hline $\mathrm{N}$ & -7.248721600 & -0.579630315 & 1.422791004 \\
\hline $\mathrm{N}$ & -8.645241737 & 0.708825946 & -0.778941393 \\
\hline $\mathrm{C}$ & -7.964478493 & 2.562659264 & 0.963790476 \\
\hline $\mathrm{C}$ & -6.103263855 & 2.457824230 & -1.180648685 \\
\hline $\mathrm{C}$ & -6.666139126 & 2.924271345 & -2.367876053 \\
\hline $\mathrm{C}$ & -6.020221710 & 3.787459373 & -3.244128704 \\
\hline $\mathrm{C}$ & -4.738805294 & 4.216117859 & -2.906285286 \\
\hline $\mathrm{C}$ & -4.109861851 & 3.811052561 & -1.740505457 \\
\hline $\mathrm{C}$ & -4.800407410 & 2.937362909 & -0.882510185 \\
\hline $\mathrm{C}$ & -4.242074966 & 2.454981327 & 0.382976890 \\
\hline $\mathrm{C}$ & -3.006639481 & 2.822375298 & 0.929940343 \\
\hline $\mathrm{C}$ & -2.613654613 & 2.323602915 & 2.162281036 \\
\hline $\mathrm{C}$ & -3.472153187 & 1.457902074 & 2.844811678 \\
\hline $\mathrm{C}$ & -4.684897423 & 1.115505815 & 2.264499664 \\
\hline $\mathrm{N}$ & -5.047760963 & 1.587205052 & 1.061972499 \\
\hline$[\mathrm{r}$ & -6.825707436 & 1.100001693 & 0.135404155 \\
\hline $\mathrm{H}$ & -3.113318443 & 4.181225777 & -1.531002283 \\
\hline $\mathrm{H}$ & -6.498507023 & 4.121927738 & -4.157392025 \\
\hline $\mathrm{H}$ & -8.105696678 & 5.476891994 & 2.773152590 \\
\hline $\mathrm{H}$ & -11.308509827 & 3.472601175 & 0.760752976 \\
\hline $\mathrm{F}$ & -6.298672199 & 3.759210587 & 2.173263788 \\
\hline $\mathrm{F}$ & -10.675735474 & 5.434528351 & 2.299832344 \\
\hline $\mathrm{F}$ & -7.909609795 & 2.514340878 & -2.719555140 \\
\hline $\mathrm{F}$ & -4.094727039 & 5.047167301 & -3.744640350 \\
\hline $\mathrm{O}$ & -4.026403904 & -1.659760118 & -3.817567587 \\
\hline $\mathrm{C}$ & -3.790290356 & -0.400411934 & -4.456360340 \\
\hline $\mathrm{H}$ & -4.734792709 & 0.103649966 & -4.695416927 \\
\hline $\mathrm{H}$ & -3.172218084 & 0.253129929 & -3.828982353 \\
\hline $\mathrm{H}$ & -3.256855011 & -0.632898450 & -5.377483845 \\
\hline $\mathrm{C}$ & -5.467906475 & 6.427463055 & 6.080979347 \\
\hline
\end{tabular}




$\begin{array}{llll}\mathrm{C} & -6.735816002 & 7.031647205 & 6.068229675 \\ \mathrm{C} & -7.858055592 & 6.284579754 & 5.763839722 \\ \mathrm{H} & -6.834081173 & 8.088718414 & 6.294747353 \\ \mathrm{C} & -5.329788685 & 5.058443069 & 5.784080982 \\ \mathrm{H} & -4.586415768 & 7.014161587 & 6.318371773 \\ \mathrm{C} & -6.442745209 & 4.293475628 & 5.484914303 \\ \mathrm{H} & -4.348500252 & 4.591593742 & 5.785895824 \\ \mathrm{C} & -7.761081696 & 4.887375355 & 5.460712433 \\ \mathrm{~N} & -6.323472500 & 2.933719873 & 5.198031425 \\ \mathrm{C} & -8.861910820 & 4.116641521 & 5.105369568 \\ \mathrm{H} & -8.841559410 & 6.745428562 & 5.744812012 \\ \mathrm{C} & -7.345671654 & 2.135783195 & 4.707787037 \\ \mathrm{H} & -5.406123638 & 2.508720636 & 5.192085743 \\ \mathrm{C} & -8.653790474 & 2.712066889 & 4.699198246 \\ \mathrm{O} & -7.073661804 & 0.971463442 & 4.318357944 \\ \mathrm{O} & -9.614819527 & 1.915393591 & 4.220454216 \\ \mathrm{H} & -9.841172218 & 4.568897247 & 5.031208038 \\ \mathrm{C} & -11.026275635 & 2.287842035 & 4.283987999 \\ \mathrm{H} & -11.133965492 & 3.292344809 & 3.860397816 \\ \mathrm{C} & -11.509414673 & 2.245406151 & 5.730858326 \\ \mathrm{H} & -11.362592697 & 1.244194269 & 6.146887302 \\ \mathrm{H} & -10.973258972 & 2.962033987 & 6.358259678 \\ \mathrm{H} & -12.576131821 & 2.485785484 & 5.771292210 \\ \mathrm{C} & -11.732879639 & 1.278180480 & 3.392307043 \\ \mathrm{H} & -12.800929070 & 1.509272456 & 3.342133045 \\ \mathrm{H} & -11.317384720 & 1.302893996 & 2.382596254 \\ \mathrm{H} & -11.615180016 & 0.267931014 & 3.795826435\end{array}$

\begin{tabular}{llll}
${ }^{1}[$ Ir-Q-2] \\
$==========================$ \\
\hline H & -2.316545486 & 0.830758035 & -10.882968903 \\
$\mathrm{H}$ & -0.545235395 & -1.265898228 & -11.025624275 \\
$\mathrm{H}$ & -0.312387675 & -2.161561251 & -8.638537407 \\
$\mathrm{H}$ & -3.436925411 & 1.780997515 & -4.699950695 \\
$\mathrm{H}$ & -5.392278194 & 3.710461855 & -8.024693489 \\
$\mathrm{H}$ & -4.068212032 & 2.222603559 & -9.549769402
\end{tabular}

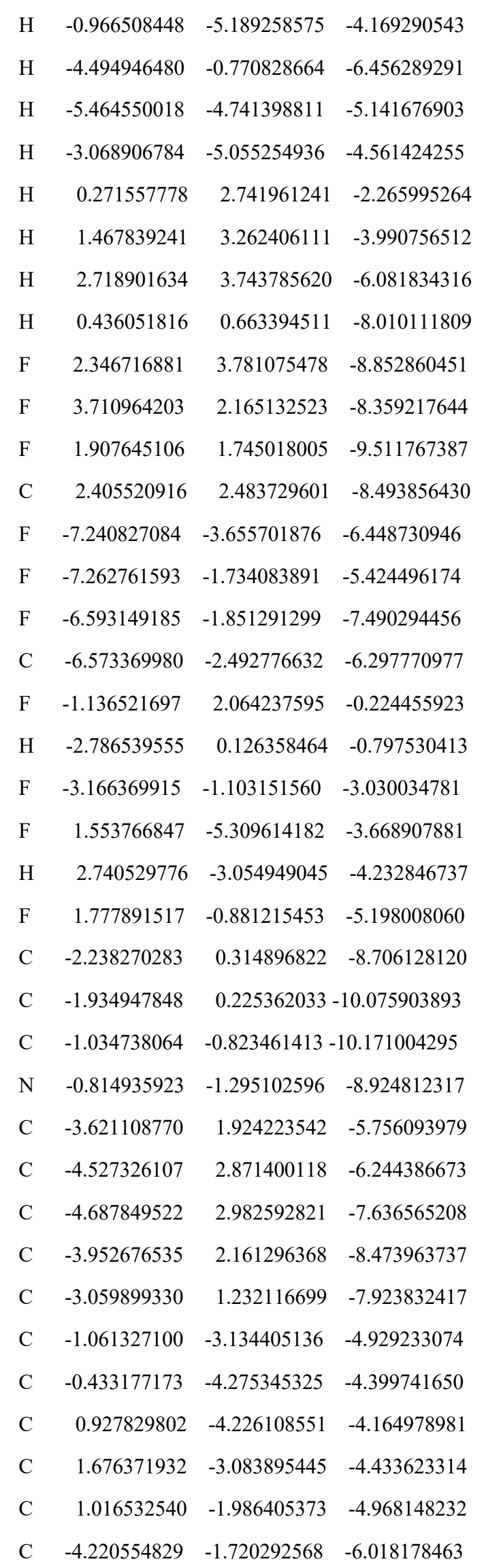




$$
\begin{aligned}
& \begin{array}{llll}
\text { C } & -5.157011509 & -2.728223324 & -5.848159313
\end{array} \\
& \text { C } \quad-4.749063969-3.939671755 \quad-5.286318302 \\
& \text { C } \quad-3.413103104 \quad-4.110134125 \quad-4.961696148 \\
& \text { C } \quad-2.497707367 \quad-3.072941780 \quad-5.178490162 \\
& \text { N } \quad-2.913321972 \quad 1.132189512 \quad-6.581068039 \\
& \text { N } \quad-1.555444360 \quad-0.628742576 \quad-8.027226448
\end{aligned}
$$

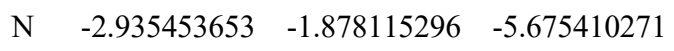

$$
\begin{aligned}
& \text { C } \quad-0.348204523 \quad-1.949046135 \quad-5.245729923 \\
& \text { C } \quad-1.472216249 \quad 0.271421969 \quad-3.967230320 \\
& \text { C } \quad-2.250328302 \quad-0.114342168 \quad-2.876648664 \\
& \text { C } \quad-2.162554741 \quad 0.465377599 \quad-1.616318345 \\
& \text { C } \quad-1.238386750 \quad 1.491081595 \quad-1.436619163 \\
& \begin{array}{llll}
\text { C } & -0.428289413 & 1.939541578 & -2.466788530
\end{array} \\
& \text { C } \quad-0.550498486 \quad 1.324730396 \quad-3.724548340 \\
& \text { C } \quad 0.256880015 \quad 1.719247103 \quad-4.881725311 \\
& \begin{array}{llll}
\text { C } & 1.248787403 & 2.709740162 & -4.896029472
\end{array} \\
& \begin{array}{llll}
\text { C } & 1.950676441 & 2.977413893 & -6.060602188
\end{array} \\
& \text { C } \quad 1.655145049 \quad 2.240843296 \quad-7.211930752 \\
& \begin{array}{lllll}
\text { C } & 0.673318803 & 1.268364310 & -7.149106979
\end{array} \\
& \mathrm{~N} \quad-0.013277883 \quad 1.022552371 \quad-6.021677971 \\
& \text { Ir } \quad-1.504059792 \quad-0.402469456 \quad-5.881799221 \\
& \begin{array}{llll}
\text { O } & -5.265981197 & 3.690454721 & -5.473047733
\end{array} \\
& \text { C } \quad-5.140245438 \quad 3.587647676 \quad-4.050609112 \\
& \text { H } \quad-5.427397728 \quad 2.589007854 \quad-3.698899984 \\
& \mathrm{H} \quad-4.117019653 \quad 3.812179565 \quad-3.725020647 \\
& \text { H } \quad-5.824366093 \quad 4.329302788 \quad-3.639174938 \\
& \text { C } \quad \begin{array}{llll}
1.261522055 & -8.793376923 & -5.534723759
\end{array} \\
& \text { C } \quad 2.624080420 \quad-8.495985985 \quad-5.346675873 \\
& \begin{array}{lllll}
\text { C } & 3.150130510 & -7.308325768 & -5.822280407
\end{array} \\
& \text { H } \quad 3.262666702 \quad-9.200788498 \quad-4.823617458 \\
& \text { C } \quad 0.431490511 \quad-7.903452396 \quad-6.196558952 \\
& \text { H } \quad 0.855419934 \quad-9.727090836 \quad-5.157914162 \\
& \text { C } \quad 0.966906250 \quad-6.698170185 \quad-6.674846649 \\
& \text { H } \quad-0.621971965 \quad-8.119849205 \quad-6.350368500 \\
& \text { C } \quad 2.331493139-6.378359795 \quad-6.496644020 \\
& \text { N } \quad \begin{array}{llll}
0.160696149 & -5.778667450 & -7.326160431
\end{array} \\
& \begin{array}{llll}
\text { C } & 2.810871124 & -5.118651867 & -6.983103752
\end{array}
\end{aligned}
$$

$$
\begin{array}{lrrr}
\mathrm{H} & 4.199913025 & -7.073068142 & -5.673660755 \\
\mathrm{C} & 0.563560486 & -4.563804150 & -7.768891335 \\
\mathrm{H} & -0.840112746 & -5.993457317 & -7.433207512 \\
\mathrm{C} & 1.969810367 & -4.223030567 & -7.582131386 \\
\mathrm{O} & -0.256987929 & -3.778294563 & -8.314076424 \\
\mathrm{O} & 2.249624014 & -2.975326300 & -8.020676613 \\
\mathrm{H} & 3.857640505 & -4.883191109 & -6.832800865 \\
\mathrm{C} & 3.601284027 & -2.457880497 & -7.916616917 \\
\mathrm{H} & 3.990206718 & -2.713334799 & -6.920629978 \\
\mathrm{C} & 3.463011980 & -0.947264194 & -8.024820328 \\
\mathrm{H} & 3.005415440 & -0.669916093 & -8.979197502 \\
\mathrm{H} & 2.843211889 & -0.570102811 & -7.210617065 \\
\mathrm{H} & 4.444194794 & -0.468858004 & -7.966551304 \\
\mathrm{C} & 4.481771469 & -3.057991028 & -9.009765625 \\
\mathrm{H} & 5.505111694 & -2.681863546 & -8.915549278 \\
\mathrm{H} & -3.950509787 & -2.300231934 & -9.276141167 \\
\mathrm{H} & 4.513997555 & -4.149317741 & -8.959301949 \\
\mathrm{H} & 4.097374916 & -2.773054600 & -9.993677139 \\
\mathrm{~N} & -2.942891836 & -4.209035397 & -8.411781311 \\
\mathrm{C} & -4.007133007 & -3.452738523 & -8.899759293 \\
\mathrm{C} & -5.212672234 & -4.358451843 & -8.828199387 \\
\mathrm{C} & -4.829502583 & -5.538366795 & -8.326971054 \\
\mathrm{H} & -3.356651545 & -5.468033314 & -8.040672302 \\
\mathrm{H} & -1.947793841 & -3.932226896 & -8.383039474 \\
\mathrm{H} & -6.198347092 & -4.024341583 & -9.121798515 \\
\mathrm{H} & -6.37898695 & -6.423354626 & -8.122384071 \\
\mathrm{H} & -6.338743687 & -7.550594330
\end{array}
$$

\section{$\left[{ }^{3} \mathbf{I} \mathbf{r}^{-1} \mathbf{Q}-{ }^{-12}\right]$}
H $\quad-2.370742559$
$0.756500542-10.877194405$
H $\quad-0.570645928 \quad-1.315468907-10.984118462$
H $\quad-0.300298929-2.135344982 \quad-8.558104515$
H $\quad-3.520613670 \quad 1.778672934 \quad-4.704863548$
H $\quad-5.520676136 \quad 3.600541592 \quad-8.059839249$
H $\quad-4.130940914 \quad 2.148214340 \quad-9.561596870$ 


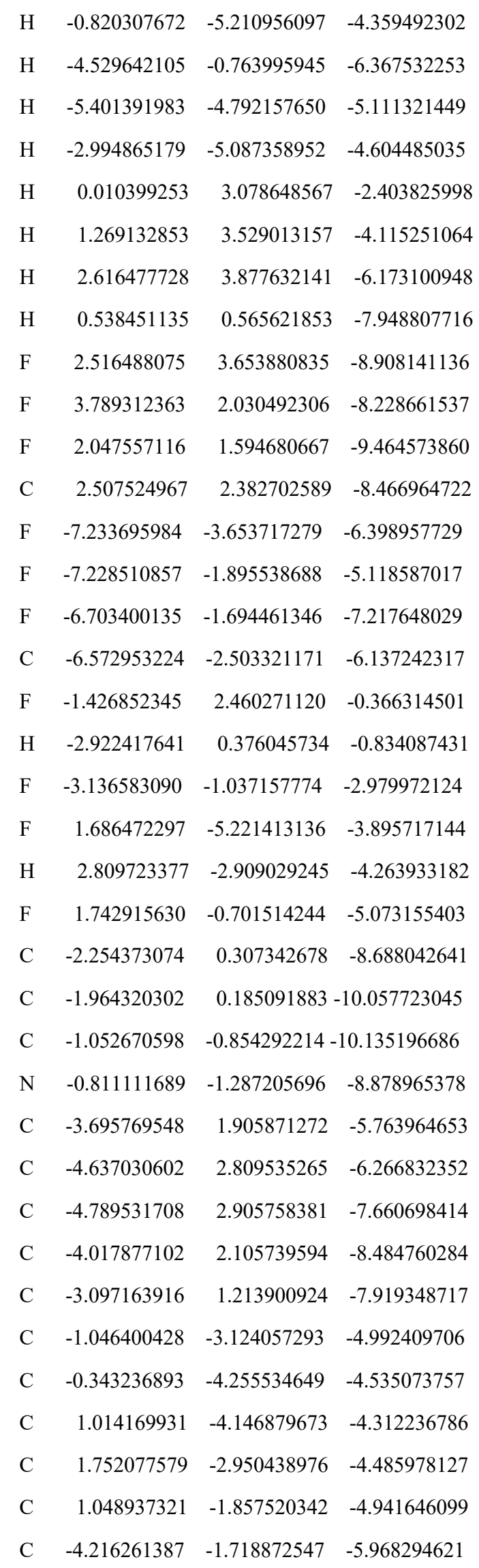

\begin{tabular}{|c|c|c|c|}
\hline & -5.134 & -2.753442049 & -5.8 \\
\hline & -4.695182800 & -3.986240864 & -5.268751144 \\
\hline & -3.360449314 & -4.141474724 & -4.984743118 \\
\hline & -2.447864294 & -3.080922365 & -5.201303482 \\
\hline & -2.950940132 & 1.135770202 & -6.576260567 \\
\hline & -1.553706288 & -0.607973456 & -7.994311333 \\
\hline & -2.929225683 & & -5.657787323 \\
\hline & -0.323873818 & -1.863782287 & -5.254959106 \\
\hline & -1.523917198 & 0.391292125 & -3.968177080 \\
\hline & -2.310179234 & 0.023614410 & -2.875387669 \\
\hline & -2.299636126 & 0.699450493 & -1.660107493 \\
\hline & -1.451372266 & 1.796518564 & -1.53 \\
\hline & -0.632753193 & 2.222384214 & -2.568126678 \\
\hline & -0.672193944 & 1.515054822 & -3.779 \\
\hline & 0.166424140 & 1.866008997 & -4.931309223 \\
\hline & 1.118448853 & & \\
\hline & 1.874785066 & 3.086760998 & \\
\hline & 1.672270417 & 2862940 & \\
\hline & 0.715 & 1.2 & \\
\hline & -0.021710442 & 1.075479746 & -6.023060799 \\
\hline & -1.489556551 & -0.379059076 & -5.841306686 \\
\hline & -5.418220997 & 3.598346233 & \\
\hline & -5.323565006 & 3.484588623 & -4.082027912 \\
\hline & -5.576889038 & 2.470519066 & -3.748896360 \\
\hline & -4.319354057 & 3.74883 & \\
\hline & -6.048774242 & 4.191622734 & \\
\hline & 1.191849828 & -9.018050194 & \\
\hline & 2.561560869 & -8.769458771 & -5.702251434 \\
\hline & 3.108244896 & -7.545778275 & -6.040822506 \\
\hline & 3.188183069 & -9.541950226 & -5.267956734 \\
\hline & 0.374707699 & -8.045160294 & -6.469442844 \\
\hline & 0.770510674 & -9.981656075 & \\
\hline & 0.929974079 & -6.802521229 & -6.812307835 \\
\hline & -0.683154643 & -8.221861839 & -6.641263485 \\
\hline & 2.304116011 & -6.530833721 & -6.603522778 \\
\hline & 0.136060566 & -5.804720879 & -7.348590374 \\
\hline & 2.810849428 & -5.240931511 & -6.958464146 \\
\hline
\end{tabular}




$$
\begin{array}{lrll}
\mathrm{H} & 4.163100243 & -7.348237038 & -5.874317169 \\
\mathrm{C} & 0.569903851 & -4.564731121 & -7.675950527 \\
\mathrm{H} & -0.881643057 & -5.978812218 & -7.454898834 \\
\mathrm{C} & 1.989378810 & -4.277503967 & -7.479373932 \\
\mathrm{O} & -0.234702662 & -3.701920986 & -8.107069016 \\
\mathrm{O} & 2.301352501 & -3.012387037 & -7.830738544 \\
\mathrm{H} & 3.863243341 & -5.043962479 & -6.791707516 \\
\mathrm{C} & 3.675654888 & -2.550583363 & -7.748324871 \\
\mathrm{H} & 4.081537247 & -2.857605696 & -6.773987770 \\
\mathrm{C} & 3.595602036 & -1.032454371 & -7.808136940 \\
\mathrm{H} & 3.131905794 & -0.712017119 & -8.745923042 \\
\mathrm{H} & 3.009639263 & -0.646264017 & -6.973340511 \\
\mathrm{H} & 4.597581387 & -0.597683430 & -7.760397911 \\
\mathrm{C} & 4.501502991 & -3.142332792 & -8.887796402 \\
\mathrm{H} & 5.538458347 & -2.801095724 & -8.813323021 \\
\mathrm{H} & 4.500768661 & -4.234952927 & -8.874918938 \\
\mathrm{H} & 4.096751690 & -2.811353207 & -9.848901749 \\
\mathrm{~N} & -2.962045908 & -4.120354176 & -8.337709427 \\
\mathrm{C} & -4.033564091 & -3.334183693 & -8.769106865 \\
\mathrm{C} & -5.236623287 & -4.212670803 & -8.651937485 \\
\mathrm{C} & -4.849643707 & -5.413381577 & -8.175256729 \\
\mathrm{C} & -3.378639221 & -5.379077911 & -7.959911346 \\
\mathrm{H} & -1.980495811 & -3.836188555 & -8.288924217 \\
\mathrm{H} & -6.230033398 & -3.866194487 & -8.897892952 \\
\mathrm{H} & -5.449358940 & -6.286672115 & -7.956844330 \\
\mathrm{O} & -3.952688456 & -2.176554918 & -9.146916389 \\
\mathrm{O} & -2.639017344 & -6.274369240 & -7.534823895
\end{array}
$$

\begin{tabular}{|c|c|c|c|}
\hline & -7.532674313 & -1.050506234 & -2.467486143 \\
\hline & -11.518402100 & -0.283671200 & -3.854382515 \\
\hline & -11.703345299 & 1.488176942 & -2.120552778 \\
\hline & -2.881982088 & 3.670123577 & 0.977042615 \\
\hline & -2.476744413 & 2.639381886 & 3.199660301 \\
\hline & -6.017752171 & 0.283550084 & 2.614042759 \\
\hline & -2.818170786 & 0.067562886 & 4.445051193 \\
\hline & -3.996996880 & 1.618838549 & 5.400903702 \\
\hline & -4.970957756 & -0.195881054 & 4.677742958 \\
\hline & -4.001655579 & 0.708524644 & 4.403253555 \\
\hline & -10.083025932 & -1.781770825 & -5.343979359 \\
\hline & -8.108585358 & -2.241651058 & -4.524374485 \\
\hline & -9.895611763 & -3.102754116 & -3.626822233 \\
\hline & -9.394169807 & -1.983094454 & -4.205033302 \\
\hline & -6.821937084 & -1.819552898 & 0.857497811 \\
\hline & -7.141485214 & -2.755300999 & 1.858271003 \\
\hline & -8.067127228 & -2.110794544 & 2.662150383 \\
\hline & -8.257778168 & -0.871020496 & 2.161430359 \\
\hline & -4.906720638 & -0.546449423 & -1.961939216 \\
\hline & -4.207726002 & -1.654273510 & -2.453834295 \\
\hline & -4.365250587 & -2.887944937 & -1.798970938 \\
\hline & -5.204871655 & -2.971936941 & -0.703010619 \\
\hline & -5.889408588 & -1.831789613 & -0.264220625 \\
\hline & -9.696921349 & 2.165175200 & -0.290162474 \\
\hline & -10.810827255 & 2.959466934 & 0.026617697 \\
\hline & -10.678075790 & 3.901732922 & 1.029537678 \\
\hline & -9.481245995 & 4.083264828 & 1.717640281 \\
\hline & -8.411872864 & 3.264859200 & 1.380213618 \\
\hline & -8.464189529 & -0.507301390 & -2.416950226 \\
\hline & -9.507664680 & -0.808539391 & -3.275681734 \\
\hline & -10.692155838 & -0.074361712 & -3.183651686 \\
\hline & -10.790979385 & 0.913417816 & -2.217778683 \\
\hline & -9.716281891 & 1.168622255 & -1.357311845 \\
\hline & -5.727102757 & -0.646129668 & -0.899579048 \\
\hline & -7.524120808 & -0.689897180 & 1.056540608 \\
\hline & -8.557544708 & 0.460086435 & -1.493981481 \\
\hline & -8.461706161 & 2.277842283 & 0.401619911 \\
\hline
\end{tabular}

\section{$\left[{ }^{1} \mid r^{-3}{ }^{3}{ }^{-1}{ }^{-2}\right]$}

$\begin{array}{lrrr}\mathrm{H} & -6.757909775 & -3.755635262 & 1.980958462 \\ \mathrm{H} & -8.599616051 & -2.440130234 & 3.541865826 \\ \mathrm{H} & -8.943930626 & -0.154328436 & 2.476874590 \\ \mathrm{H} & -4.839481354 & 0.436560541 & -2.408666611 \\ \mathrm{H} & -3.830311298 & -3.752594948 & -2.176984787 \\ \mathrm{H} & -5.353492737 & -3.916174412 & -0.190783098\end{array}$




\begin{tabular}{|c|c|c|c|}
\hline $\mathrm{C}$ & -6.164102554 & 2.423044920 & -1.323975444 \\
\hline $\mathrm{C}$ & -6.503703594 & 2.885836840 & -2.595102549 \\
\hline $\mathrm{C}$ & -5.779132366 & 3.844771147 & -3.292374611 \\
\hline $\mathrm{C}$ & -4.649239540 & 4.379540920 & -2.678623676 \\
\hline $\mathrm{C}$ & -4.242095947 & 3.982255220 & -1.416208625 \\
\hline $\mathrm{C}$ & -5.003774643 & 3.008181095 & -0.747853041 \\
\hline $\mathrm{C}$ & -4.656692505 & 2.510744810 & 0.584883153 \\
\hline $\mathrm{C}$ & -3.557275295 & 2.914610386 & 1.357481837 \\
\hline $\mathrm{C}$ & -3.329833508 & 2.341171503 & 2.598603725 \\
\hline $\mathrm{C}$ & -4.218371868 & 1.367659450 & 3.067483425 \\
\hline $\mathrm{C}$ & -5.298162460 & 1.014456630 & 2.278249741 \\
\hline $\mathrm{N}$ & -5.505758286 & 1.560451269 & 1.069912791 \\
\hline Ir & -7.029766560 & 0.974305272 & -0.197473347 \\
\hline $\mathrm{H}$ & -3.355954885 & 4.435882092 & -0.988639235 \\
\hline $\mathrm{H}$ & -6.086519241 & 4.172117710 & -4.278789520 \\
\hline $\mathrm{H}$ & -9.396587372 & 4.833945274 & 2.493721724 \\
\hline $\mathrm{H}$ & -11.768645287 & 2.858815193 & -0.468800008 \\
\hline $\mathrm{F}$ & -7.251627922 & 3.469257355 & 2.062061310 \\
\hline $\mathrm{F}$ & -11.738122940 & 4.659376144 & 1.366538405 \\
\hline $\mathrm{F}$ & -7.596214294 & 2.377674580 & -3.218427896 \\
\hline $\mathrm{F}$ & -3.933964491 & 5.307540894 & -3.338309526 \\
\hline $\mathrm{O}$ & -3.392952442 & -1.631649256 & -3.524613857 \\
\hline $\mathrm{C}$ & -3.273044109 & -0.412837684 & -4.265083313 \\
\hline $\mathrm{H}$ & -4.246979237 & -0.090702832 & -4.654113770 \\
\hline $\mathrm{H}$ & -2.839751005 & 0.385077566 & -3.649841309 \\
\hline $\mathrm{H}$ & -2.604176998 & -0.636692107 & -5.095561504 \\
\hline $\mathrm{C}$ & -15.127209663 & 4.109539509 & 3.444025040 \\
\hline $\mathrm{C}$ & -14.540888786 & 5.032289028 & 4.325845242 \\
\hline $\mathrm{C}$ & -13.235915184 & 4.859848022 & 4.742754459 \\
\hline $\mathrm{H}$ & -15.114573479 & 5.882968426 & 4.680154324 \\
\hline $\mathrm{C}$ & -14.390074730 & 3.002717257 & 2.975395441 \\
\hline $\mathrm{H}$ & -16.153257370 & 4.243876934 & 3.117189169 \\
\hline $\mathrm{C}$ & -13.083037376 & 2.817406416 & 3.381686449 \\
\hline $\mathrm{H}$ & -14.840676308 & 2.290912151 & 2.289843321 \\
\hline $\mathrm{C}$ & -12.451685905 & 3.746571064 & 4.291769028 \\
\hline $\mathrm{N}$ & -12.339381218 & 1.730392337 & 2.915753603 \\
\hline $\mathrm{C}$ & -11.139452934 & 3.540493488 & 4.693719387 \\
\hline
\end{tabular}

$\begin{array}{llll}\mathrm{H} & -12.774486542 & 5.568461418 & 5.423860550\end{array}$

$\begin{array}{llll}\text { C } & -11.019379616 & 1.493366957 & 3.235448837\end{array}$

$\begin{array}{llll}\mathrm{H} & -12.757693291 & 1.128477335 & 2.201398373\end{array}$

$\begin{array}{llll}\text { C } & -10.400409698 & 2.381617785 & 4.165041447\end{array}$

$\begin{array}{llll}\text { O } & -10.423524857 & 0.515419841 & 2.691045761\end{array}$

$\begin{array}{llll}\text { O } & -9.127374649 & 2.100640059 & 4.453889370\end{array}$

$\begin{array}{llll}\mathrm{H} & -10.665095329 & 4.230652332 & 5.376481533\end{array}$

$\begin{array}{llll}\text { C } & -8.390460014 & 2.863993883 & 5.459178448\end{array}$

$\mathrm{H} \quad-8.564044952 \quad 3.929867983 \quad 5.271586895$

$\begin{array}{llll}\text { C } & -6.928968430 & 2.535215855 & 5.212619305\end{array}$

$\mathrm{H} \quad-6.751303196 \quad 1.465342522 \quad 5.348685741$

$\mathrm{H} \quad-6.645772457 \quad 2.817819834 \quad 4.198076725$

$\mathrm{H} \quad-6.296852112 \quad 3.077914715 \quad 5.920099735$

$\begin{array}{llll}\text { C } & -8.869572639 & 2.466975689 & 6.851801395\end{array}$

$\begin{array}{llll}\mathrm{H} & -8.307591438 & 3.022379398 & 7.608621597\end{array}$

$\begin{array}{llll}\mathrm{H} & -9.933535576 & 2.676504612 & 6.989146233\end{array}$

$\begin{array}{llll}\mathrm{H} & -8.703358650 & 1.397531152 & 7.012483120\end{array}$

$\begin{array}{llll}\mathrm{N} & -11.495888710 & -0.932615995 & 0.619186819\end{array}$

$\begin{array}{llll}\text { C } & -12.817072868 & -0.704786897 & 0.294287026\end{array}$

$\begin{array}{lllll}\text { C } & -13.146484375 & -1.637328267 & -0.834956110\end{array}$

$\begin{array}{lllll}\text { C } & -12.039081573 & -2.323322773 & -1.146374702\end{array}$

$\begin{array}{llll}\text { C } & -10.931859016 & -1.888623118 & -0.221498579\end{array}$

$\begin{array}{llll}\mathrm{H} & -11.020123482 & -0.421199977 & 1.380836129\end{array}$

H $\quad-14.131833076 \quad-1.677138925 \quad-1.280846834$

H $\quad-11.872990608 \quad-3.057913780 \quad-1.923275590$

$\begin{array}{llll}\text { O } & -13.548068047 & 0.124811575 & 0.824110329\end{array}$

$\begin{array}{lllll}\text { O } & -9.775622368 & -2.264621496 & -0.207721144\end{array}$

\section{$\left[{ }^{1} \mid{ }^{-1}{ }^{1} \mathbf{Q}^{-3}{ }^{3}\right]$}

$\begin{array}{lrrr}\text { H } & -2.240832090 & 0.867076457 & -10.903055191 \\ \text { H } & -0.450267494 & -1.212116599 & -11.019316673 \\ \text { H } & -0.242801756 & -2.095714092 & -8.623231888 \\ \text { H } & -3.558171511 & 1.745657921 & -4.744448662 \\ \text { H } & -5.451944828 & 3.648408413 & -8.119336128 \\ \text { H } & -4.013734818 & 2.231719255 & -9.608615875\end{array}$




\begin{tabular}{|c|c|c|c|}
\hline & 0.69656 & & \\
\hline & -4.499821663 & -0.820529699 & -6.366803169 \\
\hline & -5.231753826 & -4.884921551 & -5.171788692 \\
\hline & -2.800339937 & -5.119106293 & -4.738010883 \\
\hline & -0.070698649 & 3.018567801 & -2.319 \\
\hline & 1.219 & 3.522998333 & -4.00005960 \\
\hline & & & \\
\hline & 0.549874842 & & \\
\hline & 2.679 & & \\
\hline & & & \\
\hline & & & \\
\hline & & & \\
\hline & -7.134027481 & & \\
\hline & -7.131378174 & -2.140181065 & \\
\hline & -6.731830597 & -1.73 & -7.04 \\
\hline & -6.51907 & -2.61 & \\
\hline & -1.501338959 & & \\
\hline & & & \\
\hline & -3.12 & -1.1 & \\
\hline & & & \\
\hline & 2.880676985 & -2.795195103 & \\
\hline & & & \\
\hline & & & \\
\hline & 1.850636601 & & \\
\hline & -0.944106 & & \\
\hline & -0.72 & & \\
\hline & -5.10 & & \\
\hline & -4.626814842 & & \\
\hline & & & \\
\hline & -3.938471794 & & \\
\hline & -3.035156727 & $1.25 t$ & \\
\hline & -0.912796557 & -3.069243670 & -5.010674953 \\
\hline & -0.219407111 & -4.192610264 & \\
\hline & 1.137327552 & -4.074305058 & -4.2 \\
\hline & 1.821650743 & -2.876613140 & \\
\hline & 1.100616336 & -1.796154499 & -4.978405476 \\
\hline & -4.162169456 & -1.772866368 & -5.982671261 \\
\hline
\end{tabular}

C $\quad-5.047906876 \quad-2.831463575 \quad-5.806212425$

C $\quad-4.559923172 \quad-4.047512054 \quad-5.321406841$

C $\quad-3.203182459 \quad-4.170033455 \quad-5.066576958$

$\begin{array}{llll}\text { C } & -2.349928379 & -3.079084635 & -5.258358002\end{array}$

N $\quad-2.937175512 \quad 1.141232491 \quad-6.612430573$

N $\quad-1.480040073 \quad-0.558952451 \quad-8.031953812$

N $\quad-2.858830929 \quad-1.885241628 \quad-5.702745914$

C $\quad-0.264613658-1.829600692 \quad-5.271326065$

$\begin{array}{llll}\text { C } & -1.534654021 & 0.351484179 & -3.985310078\end{array}$

C $\quad-2.311035633 \quad-0.070001327 \quad-2.906925201$

$\begin{array}{llll}\text { C } & -2.323417902 & 0.560296118 & -1.668308616\end{array}$

C $\quad-1.502756238 \quad 1.672296047 \quad-1.498121262$

C $\quad-0.692159653 \quad 2.152649164 \quad-2.514258623$

$\begin{array}{llll}\text { C } & -0.711026728 & 1.485540986 & -3.750445127\end{array}$

$\begin{array}{llll}\text { C } & 0.128408700 & 1.886641979 & -4.883903503\end{array}$

C $\quad \begin{array}{llll}\text { C } & 1.083874345 & 2.913180828 & -4.884725094\end{array}$

C $\quad 1.870393634 \quad 3.128322363 \quad-6.007017136$

$\begin{array}{lllll}\text { C } & 1.690322161 & 2.312062740 & -7.128882885\end{array}$

C $\quad 0.714614153 \quad 1.332611084 \quad-7.091982841$

N $\quad-0.049542107 \quad 1.134437680 \quad-6.006589890$

Ir $\quad-1.486409307 \quad-0.343524903 \quad-5.891213417$

$\begin{array}{lllll}\text { O } & -5.426430702 & 3.594856977 & -5.566541195\end{array}$

C $\quad-5.355962276 \quad 3.475868702 \quad-4.142331123$

H $\quad-5.622255802 \quad 2.462962389 \quad-3.815881729$

H $\quad-4.355268002 \quad 3.730629206 \quad-3.771725416$

H $\quad-6.082032681 \quad 4.187529564 \quad-3.750185013$

C $\quad 1.004634738 \quad-8.911451340 \quad-5.745452404$

C $\quad 2.385882854 \quad-8.696852684 \quad-5.562773705$

$\begin{array}{llll}\text { C } & 2.968717098 & -7.515687943 & -5.981824398\end{array}$

H $\quad 2.991948843 \quad-9.462665558 \quad-5.089218616$

$\begin{array}{llll}\text { C } & 0.212475836 & -7.946957111 & -6.345963478\end{array}$

H $\quad 0.555643022 \quad-9.842438698 \quad-5.413196564$

C $\quad 0.804840684 \quad-6.747763634 \quad-6.771287441$

H $\quad-0.852411985 \quad-8.097202301 \quad-6.498525143$

$\begin{array}{llll}\text { C } & 2.190110207 & -6.509743214 & -6.595637321\end{array}$

N $\quad 0.035292208 \quad-5.761154175 \quad-7.359342098$

$\begin{array}{llll}\text { C } & 2.731002331 & -5.258450985 & -7.028179646\end{array}$ 

$\mathrm{H} \quad 4.031766891 \quad-7.343235016 \quad-5.838726044$
C $\quad 0.503885925 \quad-4.561618328 \quad-7.769181252$
$\mathrm{H} \quad-0.991978645 \quad-5.921424866 \quad-7.456146717$
C $\quad \begin{array}{llll}1.930279732 & -4.298104763 & -7.587157249\end{array}$
O $\quad-0.279124111 \quad-3.709447622 \quad-8.264277458$
O $\quad 2.268743753 \quad-3.055023432 \quad-7.985995770$
H $\quad 3.789148808 \quad-5.082052708 \quad-6.878156662$
C $\quad 3.646475554 \quad-2.602339506 \quad-7.895081520$
$\mathrm{H} \quad 4.043951511 \quad-2.915103912 \quad-6.919131756$
$\begin{array}{lllll}\text { C } & 3.574759483 & -1.083549857 & -7.945039749\end{array}$
H $\quad 3.107252598 \quad-0.751584291 \quad-8.877133369$
H $\quad 2.997074127 \quad-0.701771796 \quad-7.102423668$
$\mathrm{H} \quad 4.578783989 \quad-0.653888762 \quad-7.898545265$
C $\quad 4.474092484 \quad-3.198626280 \quad-9.031413078$
$\mathrm{H} \quad 5.513922691 \quad-2.868037462 \quad-8.949978828$
H $\quad 4.462386131 \quad-4.291408062 \quad-9.020606995$
$\mathrm{H} \quad 4.078350067 \quad-2.862483978 \quad-9.994303703$
N $\quad-2.971920013 \quad-4.090448856 \quad-8.466091156$
C $\quad-3.424090862-5.328105450 \quad-8.014547348$
C $\quad-4.851023674 \quad-5.306580544 \quad-8.191294670$
$\begin{array}{llll}\text { C } & -5.212961674 & -3.993055820 & -8.748676300\end{array}$
C $\quad-4.001392841 \quad-3.223484516 \quad-8.903775215$
H $\quad-1.968461275 \quad-3.854877949 \quad-8.466996193$
H $\quad-5.511296272 \quad-6.134027481 \quad-7.974907875$
$\mathrm{H} \quad-6.204300404 \quad-3.624113083 \quad-8.963182449$
O $\quad-2.690855265 \quad-6.215506077 \quad-7.527282238$
O $\quad-3.825152636 \quad-2.060525417 \quad-9.268111229$ 
Table S23Vibrational frequencies $\left(\right.$ in $\mathrm{cm}^{-1}$ ) of the optimized geometries

\begin{tabular}{|c|c|c|c|c|c|}
\hline \multicolumn{6}{|l|}{${ }^{14} \mathrm{f}$} \\
\hline 16.81 & 17.24 & 19.96 & 22.46 & 24.70 & 28.19 \\
\hline 30.87 & 36.28 & 39.15 & 43.90 & 66.03 & 70.90 \\
\hline 75.92 & 78.47 & 92.02 & 104.49 & 111.85 & 136.63 \\
\hline 138.87 & 150.89 & 152.42 & 160.04 & 164.40 & 178.58 \\
\hline 191.07 & 196.70 & 205.10 & 209.37 & 221.03 & 224.37 \\
\hline 231.29 & 232.25 & 234.27 & 249.34 & 253.16 & 266.46 \\
\hline 267.05 & 272.60 & 286.65 & 288.59 & 322.78 & 348.81 \\
\hline 349.19 & 353.65 & 368.28 & 375.99 & 379.98 & 394.07 \\
\hline 394.76 & 403.82 & 422.53 & 433.59 & 451.34 & 454.07 \\
\hline 459.32 & 465.71 & 469.20 & 470.85 & 503.99 & 504.14 \\
\hline 530.26 & 531.63 & 536.40 & 537.97 & 569.49 & 577.66 \\
\hline 578.25 & 581.59 & 586.30 & 587.65 & 588.42 & 608.71 \\
\hline 610.08 & 627.40 & 630.02 & 630.98 & 639.50 & 639.67 \\
\hline 653.28 & 670.17 & 682.95 & 688.32 & 692.68 & 695.43 \\
\hline 709.81 & 724.46 & 737.67 & 763.79 & 791.30 & 792.28 \\
\hline 793.42 & 799.85 & 802.48 & 841.62 & 855.93 & 856.28 \\
\hline 860.76 & 868.43 & 868.73 & 877.49 & 878.13 & 886.76 \\
\hline 896.56 & 905.56 & 935.47 & 940.13 & 944.45 & 945.77 \\
\hline 979.50 & 985.36 & 1000.49 & 1001.18 & 1018.10 & 1028.02 \\
\hline 1043.24 & 1054.32 & 1065.44 & 1070.79 & 1071.10 & 1082.43 \\
\hline 1090.74 & 1110.93 & 1114.23 & 1121.44 & 1126.52 & 1129.23 \\
\hline 1164.28 & 1170.03 & 1170.63 & 1176.85 & 1183.47 & 1187.75 \\
\hline 1190.28 & 1199.12 & 1205.42 & 1214.94 & 1224.40 & 1228.73 \\
\hline 1234.51 & 1257.34 & 1261.43 & 1304.89 & 1314.77 & 1315.89 \\
\hline 1320.75 & 1327.06 & 1328.44 & 1341.83 & 1347.40 & 1348.92 \\
\hline 1349.81 & 1350.48 & 1366.90 & 1369.66 & 1374.75 & 1417.80 \\
\hline 1433.66 & 1437.41 & 1454.23 & 1465.24 & 1466.67 & 1478.21 \\
\hline 1478.53 & 1481.94 & 1498.17 & 1506.55 & 1510.02 & 1512.30 \\
\hline 1543.39 & 1546.52 & 1556.66 & 1563.52 & 1610.46 & 1611.45 \\
\hline 1624.80 & 1625.83 & 1626.20 & 1657.80 & 1660.76 & 1662.83 \\
\hline 1676.51 & 1676.64 & 3033.14 & 3107.19 & 3179.08 & 3214.26 \\
\hline 3219.23 & 3220.23 & 3224.84 & 3227.45 & 3228.41 & 3235.73 \\
\hline 3236.48 & 3238.39 & 3238.56 & 3240.23 & 3261.17 & 3265.41 \\
\hline 3283.27 & 3298.68 & 3646.54 & & & \\
\hline \multicolumn{6}{|l|}{${ }^{3} 4 \mathrm{f}$} \\
\hline 14.27 & 19.03 & 20.39 & 21.49 & 24.65 & 26.78 \\
\hline 29.70 & 34.37 & 36.65 & 42.44 & 63.26 & 65.07 \\
\hline 71.75 & 79.23 & 90.52 & 102.71 & 109.11 & 130.34 \\
\hline 138.56 & 147.92 & 150.73 & 150.85 & 161.61 & 175.11 \\
\hline 184.30 & 194.05 & 197.04 & 199.21 & 201.68 & 218.90 \\
\hline 222.39 & 229.53 & 230.17 & 244.35 & 250.54 & 255.77 \\
\hline 266.23 & 270.09 & 278.84 & 286.77 & 312.47 & 326.07 \\
\hline 348.86 & 351.33 & 356.34 & 365.98 & 377.72 & 379.35 \\
\hline 391.73 & 396.43 & 421.19 & 426.94 & 432.93 & 447.66 \\
\hline 456.43 & 460.21 & 465.09 & 465.72 & 480.80 & 503.15 \\
\hline 512.59 & 526.68 & 529.34 & 536.11 & 546.96 & 565.81 \\
\hline 575.59 & 577.14 & 583.52 & 589.48 & 591.20 & 594.75 \\
\hline 614.82 & 617.70 & 627.30 & 628.29 & 630.46 & 639.87 \\
\hline 648.92 & 663.58 & 673.21 & 676.77 & 684.83 & 689.09 \\
\hline 693.35 & 707.37 & 730.31 & 736.44 & 741.25 & 762.55 \\
\hline 786.46 & 792.34 & 792.89 & 801.20 & 810.40 & 823.88 \\
\hline 836.47 & 858.19 & 861.64 & 866.02 & 869.46 & 878.67 \\
\hline 888.06 & 906.53 & 932.88 & 941.09 & 941.99 & 945.89 \\
\hline 969.24 & 979.40 & 980.99 & 983.60 & 1002.28 & 1026.09 \\
\hline 1032.57 & 1043.11 & 1045.96 & 1052.99 & 1070.36 & 1074.18 \\
\hline 1088.33 & 1089.68 & 1094.18 & 1113.06 & 1120.50 & 1131.42 \\
\hline 1163.04 & 1163.64 & 1173.11 & 1174.01 & 1176.83 & 1180.61 \\
\hline 1182.15 & 1190.80 & 1207.50 & 1214.48 & 1215.01 & 1225.62 \\
\hline 1234.30 & 1241.16 & 1259.13 & 1269.72 & 1305.59 & 1314.78 \\
\hline 1321.30 & 1327.78 & 1336.22 & 1342.25 & 1344.46 & 1348.31 \\
\hline 1350.24 & 1352.93 & 1365.92 & 1376.21 & 1387.75 & 1417.22 \\
\hline 1425.47 & 1435.14 & 1445.86 & 1452.78 & 1468.33 & 1476.61 \\
\hline 1478.64 & 1482.28 & 1498.46 & 1503.61 & 1507.75 & 1510.00 \\
\hline 1512.21 & 1517.92 & 1543.66 & 1557.69 & 1560.68 & 1566.67 \\
\hline 1587.62 & 1605.32 & 1616.35 & 1623.77 & 1626.19 & 1649.64 \\
\hline 1663.33 & 1675.33 & 3034.19 & 3108.73 & 3179.95 & 3212.86 \\
\hline 3216.49 & 3221.19 & 3227.90 & 3229.21 & 3230.13 & 3236.81 \\
\hline 3238.96 & 3239.20 & 3241.11 & 3252.46 & 3256.30 & 3257.55 \\
\hline 3284.02 & 3299.33 & 3645.34 & & & \\
\hline
\end{tabular}

$\begin{array}{llllll}136.28 & 298.32 & 387.20 & 518.70 & 531.23 & 635.05\end{array}$ $\begin{array}{rrrrrr}639.13 & 669.32 & 772.19 & 845.23 & 905.53 & 917.11\end{array}$ $\begin{array}{llllll}977.33 & 1079.96 & 1137.20 & 1320.64 & 1350.16 & 1350.93\end{array}$ $\begin{array}{llllll}1654.32 & 1835.64 & 1871.50 & 3249.28 & 3269.75 & 3669.36\end{array}$

${ }^{3} 2$

$\begin{array}{llllll}172.47 & 261.57 & 356.57 & 401.23 & 498.07 & 504.73\end{array}$ $\begin{array}{llllll}638.31 & 641.95 & 690.67 & 726.83 & 754.20 & 893.68\end{array}$ $\begin{array}{llllll}979.45 & 1068.99 & 1105.32 & 1212.26 & 1227.00 & 1307.07\end{array}$ $\begin{array}{llllll}1362.70 & 1483.55 & 1643.12 & 3277.52 & 3291.80 & 3671.49\end{array}$

${ }^{1} 1 \mathrm{c}$

$\begin{array}{llllll}49.14 & 72.27 & 103.24 & 117.14 & 136.28 & 221.71\end{array}$ $\begin{array}{llllll}248.89 & 253.34 & 261.77 & 308.31 & 332.88 & 352.11\end{array}$ $\begin{array}{llllll}406.11 & 431.02 & 451.00 & 469.27 & 482.74 & 535.45\end{array}$ $\begin{array}{llllll}567.03 & 607.72 & 632.89 & 657.69 & 696.12 & 746.02\end{array}$

$\begin{array}{llllll}746.58 & 761.19 & 766.82 & 850.27 & 857.41 & 888.35\end{array}$ $\begin{array}{llllll}894.40 & 931.96 & 940.91 & 949.82 & 964.59 & 969.02\end{array}$ $\begin{array}{lllllll}977.64 & 1065.07 & 1150.57 & 1156.33 & 1164.90 & 1195.97\end{array}$ $\begin{array}{llllll}1209.28 & 1236.13 & 1254.39 & 1279.07 & 1303.85 & 1326.32\end{array}$ $\begin{array}{llllll}1374.37 & 1383.90 & 1395.81 & 1408.95 & 1423.59 & 1436.50\end{array}$ $\begin{array}{llllll}1456.98 & 1493.63 & 1503.01 & 1508.03 & 1521.13 & 1527.71\end{array}$ $\begin{array}{lllllll}1542.51 & 1624.62 & 1666.51 & 1673.00 & 1789.24 & 3023.19\end{array}$ $\begin{array}{lllllll}3051.50 & 3056.85 & 3125.68 & 3135.16 & 3137.33 & 3141.15\end{array}$ $\begin{array}{lllllll}3176.14 & 3179.88 & 3195.32 & 3209.32 & 3210.64 & 3606.79\end{array}$

${ }^{3} 1 \mathrm{c}$

$\begin{array}{llllll}29.51 & 49.20 & 98.54 & 107.66 & 119.85 & 193.34\end{array}$ $\begin{array}{lllllll}224.93 & 252.37 & 254.67 & 263.30 & 302.91 & 342.27\end{array}$ $\begin{array}{llllll}365.92 & 405.28 & 428.25 & 432.40 & 456.90 & 494.09\end{array}$ $\begin{array}{llllll}524.30 & 526.39 & 595.59 & 615.12 & 644.44 & 672.42\end{array}$ $\begin{array}{lllllll}682.57 & 721.34 & 722.72 & 741.94 & 819.78 & 822.27\end{array}$ $\begin{array}{llllll}864.83 & 885.70 & 913.20 & 949.00 & 953.87 & 958.22\end{array}$ $\begin{array}{llllll}963.90 & 1038.43 & 1100.66 & 1145.41 & 1156.88 & 1169.11\end{array}$ $\begin{array}{llllll}1180.84 & 1202.43 & 1212.28 & 1276.21 & 1301.45 & 1310.38\end{array}$ $\begin{array}{llllll}1361.47 & 1371.40 & 1393.50 & 1412.23 & 1422.43 & 1435.37\end{array}$ $\begin{array}{lllllll}1443.69 & 1483.63 & 1493.48 & 1502.17 & 1506.70 & 1510.91\end{array}$ $\begin{array}{lllllll}1526.26 & 1535.08 & 1568.17 & 1590.71 & 1668.58 & 3048.65\end{array}$ $\begin{array}{lllllll}3051.13 & 3058.32 & 3128.74 & 3135.79 & 3138.20 & 3143.02\end{array}$ $\begin{array}{llllll}3178.34 & 3182.21 & 3197.05 & 3212.32 & 3232.29 & 3631.47\end{array}$

${ }^{1}\left[\mathrm{Ir}-\mathbf{2}_{\mathrm{A}}\right]$

$\begin{array}{llllll}12.99 & 15.00 & 17.66 & 20.38 & 21.77 & 24.29\end{array}$ $\begin{array}{rrrrrr}26.38 & 29.24 & 30.58 & 35.00 & 40.35 & 44.45 \\ 57.44 & 58.80 & 71.91 & 74.82 & 78.01 & 81.87\end{array}$ $\begin{array}{llllll}97.14 & 98.30 & 102.91 & 123.79 & 132.70 & 138.53\end{array}$ $\begin{array}{rrrrrr}142.55 & 148.83 & 155.30 & 165.05 & 172.46 & 182.11\end{array}$ $\begin{array}{llllll}187.87 & 192.33 & 196.74 & 211.65 & 216.38 & 218.63\end{array}$ $\begin{array}{llllll}222.74 & 228.69 & 231.22 & 238.09 & 248.79 & 254.72\end{array}$ $\begin{array}{llllll}268.64 & 270.58 & 282.54 & 286.78 & 288.70 & 297.06\end{array}$ $\begin{array}{llllll}341.85 & 346.19 & 350.37 & 358.72 & 372.47 & 376.18\end{array}$ $\begin{array}{llllll}379.81 & 388.90 & 395.11 & 405.57 & 408.58 & 424.67\end{array}$ $\begin{array}{llllll}433.17 & 451.13 & 454.79 & 462.47 & 465.44 & 469.38\end{array}$ $\begin{array}{llllll}472.38 & 503.68 & 503.73 & 525.38 & 532.83 & 537.33\end{array}$ $\begin{array}{llllll}541.79 & 547.26 & 568.22 & 577.06 & 577.95 & 583.01\end{array}$ $\begin{array}{llllll}587.12 & 588.07 & 589.56 & 608.66 & 613.23 & 618.19\end{array}$ $\begin{array}{llllll}618.47 & 627.39 & 629.54 & 630.53 & 639.38 & 639.60\end{array}$ $\begin{array}{llllll}661.18 & 674.85 & 676.31 & 680.95 & 688.11 & 691.45\end{array}$ $\begin{array}{llllll}694.47 & 707.96 & 726.88 & 739.53 & 765.69 & 766.76\end{array}$ $\begin{array}{llllll}791.30 & 792.52 & 795.88 & 796.98 & 802.42 & 846.29\end{array}$ $\begin{array}{llllll}848.66 & 856.20 & 864.13 & 865.87 & 866.17 & 867.72\end{array}$ $\begin{array}{llllll}876.34 & 877.76 & 880.57 & 898.16 & 904.54 & 905.11\end{array}$ $\begin{array}{llllll}914.05 & 930.90 & 938.54 & 940.79 & 942.36 & 951.44\end{array}$ $\begin{array}{llllll}977.02 & 986.18 & 991.82 & 997.98 & 1000.36 & 1019.42\end{array}$ $\begin{array}{lllllll}1027.64 & 1049.13 & 1055.58 & 1065.71 & 1068.64 & 1072.11\end{array}$ $\begin{array}{llllll}1083.31 & 1087.89 & 1094.81 & 1106.58 & 1114.57 & 1128.95\end{array}$ $\begin{array}{llllll}1129.85 & 1132.47 & 1144.73 & 1165.38 & 1170.13 & 1171.01\end{array}$ $\begin{array}{lllllll}1176.02 & 1177.52 & 1188.15 & 1190.07 & 1199.78 & 1206.71\end{array}$ $\begin{array}{lllllll}1215.52 & 1223.84 & 1229.96 & 1258.29 & 1260.60 & 1262.79\end{array}$ $\begin{array}{lllllll}1314.47 & 1316.57 & 1321.36 & 1324.37 & 1326.27 & 1328.33\end{array}$ $\begin{array}{llllll}1329.31 & 1345.78 & 1347.28 & 1348.03 & 1349.51 & 1350.89\end{array}$ $\begin{array}{llllll}1356.09 & 1356.79 & 1369.72 & 1373.68 & 1375.66 & 1402.88\end{array}$ $\begin{array}{llllll}1432.22 & 1437.35 & 1444.06 & 1465.45 & 1466.71 & 1477.32\end{array}$ 
$\begin{array}{llllll}1481.62 & 1490.26 & 1510.21 & 1511.19 & 1515.02 & 1521.32\end{array}$ $\begin{array}{llllll}1542.66 & 1546.04 & 1573.45 & 1587.06 & 1610.48 & 1612.29\end{array}$ $\begin{array}{lllllll}1623.90 & 1624.85 & 1626.73 & 1650.19 & 1657.81 & 1658.49\end{array}$ $\begin{array}{lllllll}1660.04 & 1673.26 & 1676.70 & 1791.48 & 1860.79 & 3028.08\end{array}$ $\begin{array}{lllllll}3099.62 & 3174.32 & 3176.18 & 3218.44 & 3219.89 & 3222.81\end{array}$ $\begin{array}{llllll}3226.43 & 3231.14 & 3231.92 & 3234.56 & 3234.66 & 3239.30\end{array}$ $\begin{array}{lllllll}3244.62 & 3249.98 & 3258.17 & 3266.14 & 3270.63 & 3276.68\end{array}$ $\begin{array}{lll}3281.10 & 3297.59 & 3650.27\end{array}$

\section{$\left[{ }^{3} \mathrm{Ir}-{ }^{1} \mathbf{2}_{\mathrm{A}}\right]$}

$\begin{array}{llllll}12.17 & 21.08 & 23.91 & 26.52 & 28.31 & 29.23 \\ 35.22 & 37.88 & 41.88 & 46.84 & 49.22 & 57.60\end{array}$

$\begin{array}{lrrrrr}67.38 & 74.29 & 76.20 & 78.61 & 92.38 & 93.59\end{array}$

$\begin{array}{lrrrrr}101.01 & 105.06 & 126.34 & 128.79 & 139.65 & 146.02\end{array}$

$\begin{array}{llllll}148.60 & 156.17 & 168.17 & 170.32 & 182.36 & 185.17\end{array}$

$\begin{array}{llllll}188.96 & 195.46 & 202.01 & 210.83 & 212.38 & 218.32\end{array}$

$\begin{array}{llllll}223.22 & 227.10 & 230.66 & 238.66 & 248.95 & 254.13\end{array}$

$\begin{array}{llllll}262.13 & 272.84 & 284.91 & 286.74 & 291.20 & 332.03\end{array}$

$\begin{array}{llllll}345.02 & 350.56 & 353.53 & 357.46 & 365.14 & 378.23\end{array}$

$\begin{array}{llllll}381.90 & 387.18 & 389.00 & 400.78 & 407.71 & 425.21\end{array}$

$\begin{array}{llllll}428.99 & 432.40 & 446.83 & 451.69 & 460.84 & 463.20\end{array}$

$\begin{array}{llllll}468.87 & 472.56 & 491.82 & 503.45 & 520.25 & 526.36\end{array}$

$\begin{array}{llllll}535.48 & 557.57 & 566.08 & 568.79 & 578.29 & 578.92\end{array}$

$\begin{array}{llllll}588.93 & 592.49 & 594.49 & 608.60 & 611.35 & 616.10\end{array}$

$\begin{array}{llllll}622.69 & 626.18 & 629.32 & 633.76 & 635.34 & 641.17\end{array}$

$\begin{array}{llllll}654.30 & 678.05 & 683.72 & 688.81 & 693.13 & 695.41\end{array}$

$\begin{array}{llllll}705.47 & 706.95 & 710.23 & 720.26 & 735.44 & 760.83\end{array}$

$\begin{array}{llllll}766.49 & 786.30 & 793.63 & 796.05 & 800.29 & 803.61\end{array}$

$\begin{array}{llllll}814.79 & 834.00 & 840.76 & 847.30 & 851.00 & 862.34\end{array}$

$\begin{array}{llllll}868.62 & 869.65 & 879.34 & 890.68 & 892.53 & 894.12\end{array}$

$\begin{array}{llllll}906.43 & 909.14 & 924.25 & 927.20 & 952.89 & 965.02\end{array}$

$\begin{array}{llllll}981.94 & 982.18 & 995.62 & 1003.62 & 1005.64 & 1026.32\end{array}$

$\begin{array}{rrrrrr}1050.17 & 1051.12 & 1057.73 & 1059.45 & 1065.92 & 1074.20\end{array}$

$\begin{array}{lllllll}1078.01 & 1082.86 & 1104.00 & 1110.67 & 1116.76 & 1128.89\end{array}$

$\begin{array}{llllll}1133.28 & 1140.98 & 1149.79 & 1168.60 & 1171.49 & 1174.10\end{array}$

$\begin{array}{llllll}1177.64 & 1184.20 & 1186.10 & 1190.73 & 1211.53 & 1213.89\end{array}$

$\begin{array}{llllll}1216.04 & 1225.56 & 1234.76 & 1258.69 & 1263.69 & 1273.26\end{array}$

$\begin{array}{llllll}1293.90 & 1310.27 & 1317.14 & 1318.53 & 1326.32 & 1328.36\end{array}$

$\begin{array}{lllllll}1332.78 & 1336.77 & 1345.03 & 1346.23 & 1351.31 & 1351.52\end{array}$

$\begin{array}{lllllll}1353.12 & 1360.10 & 1368.30 & 1377.71 & 1391.73 & 1405.54\end{array}$

$\begin{array}{llllll}1436.30 & 1442.90 & 1444.10 & 1452.84 & 1460.95 & 1468.19\end{array}$

$\begin{array}{lllllll}1479.11 & 1490.08 & 1495.89 & 1509.81 & 1511.56 & 1514.63\end{array}$

$\begin{array}{llllll}1521.03 & 1524.56 & 1540.30 & 1545.71 & 1568.52 & 1570.57\end{array}$

$\begin{array}{lllllll}1580.30 & 1607.30 & 1611.74 & 1620.54 & 1626.76 & 1640.41\end{array}$

$\begin{array}{llllll}1651.02 & 1658.11 & 1660.97 & 1675.90 & 1766.33 & 2155.30\end{array}$

$\begin{array}{llllll}3026.59 & 3098.34 & 3176.74 & 3219.62 & 3219.63 & 3227.95\end{array}$

$\begin{array}{lllllll}3231.26 & 3231.93 & 3235.69 & 3237.35 & 3239.02 & 3246.74\end{array}$

$\begin{array}{llllll}3252.02 & 3254.83 & 3255.58 & 3258.82 & 3272.22 & 3275.77\end{array}$

$\begin{array}{lll}3280.52 & 3293.88 & 3688.55\end{array}$

$\left[{ }^{1} \mathbf{I r}-{ }^{3} \mathbf{2}_{\mathrm{A}}\right]$

$\begin{array}{llllll}7.13 & 10.76 & 19.31 & 24.33 & 25.65 & 29.16\end{array}$

$\begin{array}{llllll}31.49 & 36.03 & 37.94 & 42.66 & 48.82 & 55.13\end{array}$

$\begin{array}{llllll}63.26 & 73.72 & 78.80 & 79.61 & 92.53 & 99.30\end{array}$

$\begin{array}{rrrrrr}105.94 & 113.42 & 120.00 & 124.63 & 139.90 & 145.91 \\ 151.59 & 156.41 & 164.38 & 176.98 & 184.32 & 190.42\end{array}$

$\begin{array}{lllllll}151.59 & 156.41 & 164.38 & 176.98 & 184.32 & 190.42\end{array}$

$\begin{array}{llllll}196.57 & 199.78 & 202.89 & 216.58 & 220.30 & 223.98\end{array}$

$\begin{array}{llllll}228.58 & 231.72 & 234.77 & 236.49 & 251.13 & 256.92\end{array}$

$\begin{array}{llllll}269.28 & 275.22 & 284.00 & 288.63 & 291.09 & 339.80\end{array}$

$\begin{array}{llllll}347.76 & 350.95 & 353.29 & 363.23 & 370.70 & 379.47\end{array}$

$\begin{array}{llllll}381.49 & 388.70 & 395.54 & 403.32 & 410.14 & 433.41\end{array}$

$\begin{array}{llllll}433.79 & 452.30 & 455.54 & 462.21 & 466.12 & 471.28\end{array}$

$\begin{array}{llllll}473.62 & 502.35 & 504.53 & 506.98 & 530.63 & 532.92\end{array}$

$\begin{array}{lllllll}536.82 & 561.50 & 567.57 & 579.06 & 579.41 & 582.99\end{array}$

$\begin{array}{llllll}586.67 & 587.23 & 590.58 & 592.96 & 602.62 & 612.39\end{array}$

$\begin{array}{llllll}625.58 & 628.07 & 630.32 & 632.33 & 634.44 & 636.71\end{array}$

$\begin{array}{llllll}640.51 & 641.06 & 682.59 & 688.70 & 691.98 & 694.90\end{array}$

$\begin{array}{llllll}697.12 & 707.50 & 713.13 & 719.13 & 728.59 & 740.89\end{array}$

$\begin{array}{lllllll}743.63 & 766.98 & 790.29 & 793.91 & 800.16 & 803.65\end{array}$

$\begin{array}{llllll}806.08 & 850.64 & 856.22 & 859.64 & 868.24 & 868.78\end{array}$

$\begin{array}{llllll}869.17 & 875.38 & 878.10 & 891.26 & 904.99 & 906.81\end{array}$

$\begin{array}{llllll}907.73 & 911.84 & 935.10 & 941.58 & 948.03 & 979.95\end{array}$

$\begin{array}{llllll}988.22 & 995.63 & 1001.55 & 1003.16 & 1008.80 & 1022.04\end{array}$

$\begin{array}{lllllll}1027.29 & 1051.15 & 1057.97 & 1065.56 & 1073.82 & 1074.94\end{array}$

$\begin{array}{lllllll}1086.02 & 1091.96 & 1114.71 & 1117.57 & 1131.91 & 1134.20\end{array}$

$\begin{array}{llllll}1138.90 & 1140.20 & 1165.76 & 1172.16 & 1177.56 & 1179.06\end{array}$

$\begin{array}{llllll}1182.80 & 1190.82 & 1194.04 & 1201.17 & 1205.74 & 1211.93\end{array}$ $\begin{array}{llllll}1216.24 & 1226.55 & 1235.54 & 1251.64 & 1261.18 & 1276.27\end{array}$ $\begin{array}{llllll}283.10 & 1315.26 & 1316.95 & 1324.27 & 1326.10 & 1326.83\end{array}$ $\begin{array}{llllll}1331.21 & 1332.10 & 1346.78 & 1348.06 & 1349.35 & 1350.25\end{array}$ $\begin{array}{lllllll}1354.22 & 1361.48 & 1363.60 & 1364.67 & 1377.76 & 1380.56\end{array}$ $\begin{array}{lllllll}1400.26 & 1434.33 & 1437.70 & 1444.16 & 1463.12 & 1466.43\end{array}$ $\begin{array}{llllll}1482.20 & 1491.29 & 1492.96 & 1510.32 & 1511.74 & 1518.56\end{array}$ $\begin{array}{lllllll}1522.16 & 1537.89 & 1544.88 & 1549.12 & 1579.45 & 1608.25\end{array}$ $\begin{array}{llllll}1612.19 & 1623.80 & 1626.79 & 1627.24 & 1633.83 & 1646.81\end{array}$ $\begin{array}{lllllll}1656.37 & 1661.67 & 1676.14 & 1676.40 & 1718.21 & 2328.99\end{array}$ $\begin{array}{llllll}3026.87 & 3098.02 & 3176.84 & 3217.50 & 3220.10 & 3227.15\end{array}$ $\begin{array}{lllllll}3228.86 & 3230.62 & 3234.97 & 3240.35 & 3240.38 & 3246.55\end{array}$ $\begin{array}{lllllll}3254.52 & 3255.41 & 3269.97 & 3272.73 & 3272.82 & 3277.23\end{array}$ $\begin{array}{lll}3282.54 & 3293.82 & 3658.94\end{array}$

${ }^{1}\left[\mathbf{I r}-2_{B}\right]$

$\begin{array}{llllll}15.20 & 20.50 & 23.23 & 24.23 & 29.45 & 32.22\end{array}$

$\begin{array}{llllll}34.07 & 39.06 & 42.97 & 47.63 & 51.44 & 59.78\end{array}$

$\begin{array}{llllll}60.48 & 70.80 & 73.83 & 78.60 & 83.45 & 89.66\end{array}$

$\begin{array}{lrrrrr}96.84 & 106.84 & 112.20 & 123.66 & 134.94 & 140.09\end{array}$

$\begin{array}{llllll}147.73 & 153.55 & 154.56 & 165.73 & 170.76 & 173.27\end{array}$

$\begin{array}{llllll}183.59 & 191.18 & 197.08 & 204.50 & 217.79 & 219.80\end{array}$

$\begin{array}{llllll}225.96 & 232.39 & 234.53 & 246.89 & 248.99 & 254.82\end{array}$

$\begin{array}{llllll}265.13 & 270.49 & 279.89 & 286.51 & 288.28 & 310.64\end{array}$

$\begin{array}{llllll}337.69 & 347.41 & 350.23 & 355.23 & 371.95 & 376.36\end{array}$

$\begin{array}{lllllll}382.61 & 388.26 & 396.92 & 407.74 & 408.61 & 425.94\end{array}$

$\begin{array}{llllll}434.09 & 451.56 & 456.53 & 463.36 & 465.37 & 467.73\end{array}$

$\begin{array}{llllll}473.68 & 500.83 & 504.55 & 522.58 & 526.69 & 532.69\end{array}$

$\begin{array}{llllll}536.85 & 547.74 & 568.26 & 577.36 & 578.64 & 580.72\end{array}$

$\begin{array}{llllll}587.74 & 588.12 & 591.20 & 606.43 & 610.50 & 614.64\end{array}$

$\begin{array}{lllllll}619.53 & 626.54 & 630.87 & 632.08 & 640.20 & 641.29\end{array}$

$\begin{array}{llllll}654.57 & 673.16 & 675.24 & 683.74 & 690.20 & 693.53\end{array}$

$\begin{array}{llllll}696.24 & 707.69 & 726.56 & 739.40 & 765.50 & 770.57\end{array}$

$\begin{array}{llllll}792.06 & 793.10 & 795.97 & 798.73 & 804.13 & 843.34\end{array}$

$\begin{array}{lllllll}845.99 & 857.12 & 859.71 & 865.53 & 867.35 & 867.95\end{array}$

$\begin{array}{llllll}873.47 & 876.52 & 876.75 & 896.69 & 906.15 & 908.43\end{array}$

$\begin{array}{llllll}911.41 & 914.76 & 937.40 & 944.35 & 948.81 & 959.63\end{array}$

$\begin{array}{llllll}976.35 & 982.33 & 991.41 & 1001.18 & 1001.44 & 1018.25\end{array}$ $\begin{array}{llllll}1026.66 & 1046.39 & 1053.24 & 1061.45 & 1070.27 & 1071.22\end{array}$ $\begin{array}{llllll}1082.61 & 1087.22 & 1094.05 & 1106.96 & 1112.65 & 1124.70\end{array}$ $\begin{array}{llllll}1128.49 & 1128.63 & 1144.93 & 1164.42 & 1164.99 & 1168.75\end{array}$ $\begin{array}{llllll}1174.31 & 1176.02 & 1177.35 & 1188.44 & 1190.37 & 1199.63\end{array}$ $\begin{array}{llllll}1215.06 & 1228.96 & 1238.05 & 1257.63 & 1261.34 & 1262.98\end{array}$ $\begin{array}{llllll}1315.12 & 1315.76 & 1321.49 & 1323.42 & 1326.93 & 1327.94\end{array}$ $\begin{array}{lllllll}1331.70 & 1345.66 & 1347.96 & 1348.75 & 1349.64 & 1353.28\end{array}$ $\begin{array}{lllllll}1354.19 & 1357.27 & 1366.47 & 1374.52 & 1379.26 & 1402.14\end{array}$ $\begin{array}{lllllll}1434.17 & 1438.21 & 1442.55 & 1462.38 & 1466.86 & 1479.64\end{array}$ $\begin{array}{lllllll}1482.60 & 1488.95 & 1509.27 & 1510.39 & 1513.91 & 1521.66\end{array}$ $\begin{array}{lllllll}1544.80 & 1547.84 & 1572.21 & 1587.33 & 1611.34 & 1612.41\end{array}$ $\begin{array}{lllllll}1623.08 & 1625.50 & 1626.75 & 1647.79 & 1657.79 & 1660.31\end{array}$ $\begin{array}{lllllll}1661.62 & 1676.82 & 1679.05 & 1787.54 & 1865.81 & 3028.83\end{array}$ $\begin{array}{llllll}3100.80 & 3176.74 & 3189.20 & 3219.84 & 3220.99 & 3223.24\end{array}$ $\begin{array}{llllll}3228.19 & 3229.89 & 3232.60 & 3234.96 & 3240.21 & 3240.73\end{array}$ $\begin{array}{llllll}3244.27 & 3244.34 & 3245.28 & 3256.70 & 3268.05 & 3275.55\end{array}$ $\begin{array}{lll}3281.35 & 3297.32 & 3694.40\end{array}$

$\left[{ }^{3} \mathrm{Ir}-{ }^{1} \mathbf{2}_{\mathrm{B}}\right]$

$\begin{array}{llllll}16.19 & 23.44 & 26.51 & 27.81 & 29.82 & 34.12\end{array}$

$\begin{array}{llllll}36.91 & 42.83 & 44.43 & 53.01 & 58.58 & 60.23\end{array}$

$\begin{array}{rrrrrr}64.98 & 68.37 & 75.39 & 81.67 & 91.56 & 95.84 \\ 103.32 & 108.45 & 115.40 & 124.55 & 132.92 & 139.69\end{array}$

$\begin{array}{llllll}103.32 & 108.45 & 115.40 & 124.55 & 132.92 & 139.69 \\ 148.37 & 152.23 & 158.42 & 165.68 & 168.89 & 172.59\end{array}$

$\begin{array}{llllll}148.37 & 152.23 & 158.42 & 165.68 & 168.89 & 172.59 \\ 185.04 & 190.70 & 197.23 & 199.38 & 214.00 & 217.35\end{array}$

$\begin{array}{llllll}221.85 & 230.02 & 230.90 & 236.89 & 245.16 & 253.13\end{array}$

$\begin{array}{lllllll}259.16 & 269.25 & 277.62 & 284.32 & 288.44 & 330.40\end{array}$

$\begin{array}{llllll}338.00 & 347.05 & 350.55 & 351.33 & 360.42 & 373.65\end{array}$

$\begin{array}{llllll}381.12 & 383.92 & 386.45 & 400.13 & 402.49 & 421.00\end{array}$

$\begin{array}{llllll}432.70 & 440.31 & 447.64 & 457.08 & 463.20 & 464.94\end{array}$

$\begin{array}{llllll}466.47 & 490.79 & 495.69 & 500.94 & 513.35 & 520.74\end{array}$

$\begin{array}{lllllll}535.25 & 552.89 & 563.74 & 565.49 & 576.09 & 579.15\end{array}$

$\begin{array}{llllll}587.28 & 591.39 & 592.96 & 605.88 & 613.57 & 614.41\end{array}$

$\begin{array}{llllll}617.64 & 623.33 & 624.88 & 629.99 & 631.77 & 641.31\end{array}$

$\begin{array}{llllll}659.04 & 671.76 & 674.84 & 684.75 & 691.95 & 693.28\end{array}$

$\begin{array}{llllll}696.61 & 701.99 & 705.39 & 735.60 & 738.37 & 755.14\end{array}$

$\begin{array}{llllll}765.33 & 782.52 & 786.86 & 793.07 & 799.11 & 805.55\end{array}$

$\begin{array}{llllll}806.80 & 830.54 & 842.88 & 848.01 & 862.47 & 868.49\end{array}$

$\begin{array}{llllll}869.90 & 872.47 & 878.27 & 888.63 & 904.36 & 907.76\end{array}$

$\begin{array}{llllll}910.44 & 912.27 & 923.66 & 946.65 & 948.60 & 963.20\end{array}$ 
$\begin{array}{lllllll}976.49 & 977.87 & 986.77 & 994.77 & 1005.39 & 1024.87\end{array}$ $\begin{array}{llllll}1040.36 & 1046.33 & 1046.90 & 1053.56 & 1054.68 & 1071.50\end{array}$ $\begin{array}{llllll}1074.76 & 1078.22 & 1089.15 & 1093.29 & 1100.35 & 1109.88\end{array}$ $\begin{array}{llllll}1132.19 & 1132.52 & 1137.94 & 1164.71 & 1166.33 & 1170.12\end{array}$ $\begin{array}{llllll}1177.30 & 1177.35 & 1177.65 & 1182.74 & 1188.47 & 1190.45\end{array}$ $\begin{array}{lllllll}1214.86 & 1216.45 & 1242.48 & 1245.45 & 1261.48 & 1269.61\end{array}$ $\begin{array}{llllll}1282.17 & 1306.65 & 1311.69 & 1322.39 & 1324.03 & 1328.19\end{array}$ $\begin{array}{llllll}1333.01 & 1339.81 & 1346.60 & 1347.36 & 1349.38 & 1351.22\end{array}$ $\begin{array}{lllllll}1354.44 & 1357.76 & 1362.01 & 1379.72 & 1399.91 & 1403.46\end{array}$ $\begin{array}{llllll}1435.08 & 1438.83 & 1441.53 & 1446.69 & 1470.87 & 1473.55\end{array}$ $\begin{array}{lllllll}1479.06 & 1485.67 & 1489.34 & 1509.02 & 1510.44 & 1515.42\end{array}$ $\begin{array}{lllllll}1521.26 & 1529.23 & 1546.71 & 1552.61 & 1561.11 & 1573.88\end{array}$ $\begin{array}{lllllll}1591.31 & 1600.40 & 1607.83 & 1624.53 & 1627.24 & 1634.43\end{array}$ $\begin{array}{llllll}1649.74 & 1658.62 & 1677.20 & 1696.24 & 1793.70 & 2794.92\end{array}$ $\begin{array}{llllll}3027.70 & 3099.61 & 3176.60 & 3218.60 & 3219.60 & 3222.47\end{array}$ $\begin{array}{lllllll}3228.01 & 3230.81 & 3231.76 & 3236.92 & 3237.35 & 3238.70\end{array}$ $\begin{array}{lllllll}3247.08 & 3257.95 & 3264.31 & 3266.07 & 3273.65 & 3274.46\end{array}$ $3279.64 \quad 3296.20 \quad 3706.11$

\section{$\left[{ }^{1} \mathbf{I r}-{ }^{3} 2_{B}\right]$}

$\begin{array}{llllll}20.73 & 21.87 & 25.45 & 27.20 & 31.14 & 33.34\end{array}$

$\begin{array}{llllll}34.61 & 35.96 & 40.12 & 45.08 & 48.18 & 51.73\end{array}$

$\begin{array}{llllll}60.33 & 64.70 & 76.73 & 80.00 & 84.77 & 95.76\end{array}$

$\begin{array}{llllll}100.03 & 109.28 & 121.97 & 124.02 & 137.34 & 141.78\end{array}$

$\begin{array}{llllll}146.71 & 156.05 & 162.45 & 166.22 & 179.92 & 183.32\end{array}$

$\begin{array}{llllll}190.26 & 197.51 & 203.58 & 210.24 & 215.97 & 221.10\end{array}$

$\begin{array}{llllll}231.42 & 233.98 & 234.77 & 246.34 & 253.81 & 258.35\end{array}$

$\begin{array}{llllll}265.98 & 274.65 & 281.97 & 287.51 & 291.17 & 334.14\end{array}$

$\begin{array}{llllll}346.49 & 347.49 & 352.55 & 356.03 & 370.52 & 371.55\end{array}$

$\begin{array}{lllllll}381.74 & 388.19 & 398.57 & 399.66 & 410.28 & 422.14\end{array}$

$\begin{array}{llllll}432.53 & 450.47 & 455.25 & 461.88 & 465.11 & 471.37\end{array}$

$\begin{array}{llllll}473.56 & 500.62 & 503.38 & 508.38 & 531.91 & 535.53\end{array}$

$\begin{array}{llllll}542.78 & 556.98 & 567.40 & 573.44 & 576.19 & 577.58\end{array}$

$\begin{array}{llllll}580.20 & 583.07 & 587.74 & 589.48 & 591.06 & 613.05\end{array}$

$\begin{array}{llllll}622.13 & 623.20 & 625.64 & 627.85 & 632.57 & 633.84\end{array}$

$\begin{array}{llllll}639.00 & 641.76 & 680.55 & 684.29 & 690.30 & 692.26\end{array}$

$\begin{array}{llllll}695.46 & 704.87 & 706.71 & 717.32 & 725.51 & 733.91\end{array}$

$\begin{array}{llllll}738.90 & 766.83 & 787.90 & 792.16 & 798.87 & 799.90\end{array}$

$\begin{array}{llllll}803.59 & 847.55 & 848.55 & 860.60 & 864.48 & 867.95\end{array}$

$\begin{array}{llllll}868.55 & 873.97 & 877.58 & 888.57 & 889.88 & 907.44\end{array}$

$\begin{array}{llllll}908.84 & 913.35 & 932.89 & 947.99 & 954.25 & 965.92\end{array}$

$\begin{array}{lllllr}981.82 & 988.32 & 995.09 & 1003.16 & 1004.18 & 1012.06\end{array}$

$\begin{array}{llllll}1025.26 & 1050.60 & 1057.94 & 1059.43 & 1071.19 & 1071.57\end{array}$

$\begin{array}{llllll}1082.92 & 1090.52 & 1106.56 & 1110.24 & 1121.35 & 1125.39\end{array}$

$\begin{array}{llllll}1129.70 & 1138.12 & 1155.84 & 1165.83 & 1169.67 & 1174.64\end{array}$

$\begin{array}{llllll}1175.75 & 1176.97 & 1180.68 & 1187.14 & 1189.05 & 1196.25\end{array}$

$\begin{array}{lllllll}1216.02 & 1225.79 & 1230.93 & 1239.53 & 1259.36 & 1267.63\end{array}$

$\begin{array}{lllllll}1279.54 & 1303.54 & 1315.31 & 1317.81 & 1325.73 & 1327.49\end{array}$

$\begin{array}{lllllll}1330.61 & 1331.53 & 1345.70 & 1347.97 & 1348.47 & 1349.39\end{array}$

$\begin{array}{lllllll}1354.35 & 1357.00 & 1359.08 & 1364.98 & 1374.68 & 1378.28\end{array}$

$\begin{array}{llllll}1399.53 & 1433.93 & 1437.86 & 1444.26 & 1459.35 & 1467.15\end{array}$

$\begin{array}{llllll}1482.16 & 1482.78 & 1491.24 & 1509.49 & 1511.58 & 1519.56\end{array}$

$\begin{array}{lllllll}1522.76 & 1536.73 & 1542.08 & 1544.40 & 1581.35 & 1609.37\end{array}$

$\begin{array}{llllll}1613.10 & 1622.87 & 1627.61 & 1627.90 & 1629.84 & 1654.20\end{array}$

$\begin{array}{llllll}1657.66 & 1662.43 & 1673.21 & 1676.24 & 1722.83 & 2398.10\end{array}$

$\begin{array}{llllll}3026.74 & 3097.42 & 3176.80 & 3218.03 & 3223.30 & 3224.88\end{array}$

$\begin{array}{lllllll}3228.85 & 3231.24 & 3233.02 & 3235.81 & 3240.86 & 3241.73\end{array}$

$\begin{array}{lllllll}3247.08 & 3253.49 & 3259.33 & 3260.94 & 3275.00 & 3277.96\end{array}$ $3286.12 \quad 3294.46 \quad 3654.01$

\section{${ }^{1}\left[\mathrm{Ir}-\mathbf{Q}_{\mathrm{A}}\right]$}

$\begin{array}{llllll}16.04 & 20.40 & 22.04 & 25.83 & 26.88 & 30.08\end{array}$

$\begin{array}{llllll}30.91 & 36.08 & 40.07 & 41.46 & 45.69 & 47.62\end{array}$

$\begin{array}{llllll}49.84 & 58.66 & 70.32 & 71.86 & 75.47 & 76.25\end{array}$

$\begin{array}{llllll}82.12 & 85.72 & 95.84 & 103.71 & 111.81 & 121.51\end{array}$

$\begin{array}{llllll}129.34 & 133.56 & 139.67 & 145.19 & 148.64 & 153.78\end{array}$

$\begin{array}{llllll}157.91 & 167.32 & 169.99 & 180.37 & 184.11 & 196.84\end{array}$

$\begin{array}{llllll}203.97 & 211.03 & 218.42 & 221.24 & 229.29 & 231.19\end{array}$

$\begin{array}{lllllll}233.93 & 239.65 & 250.28 & 251.31 & 253.89 & 262.56\end{array}$

$\begin{array}{llllll}267.88 & 270.26 & 272.65 & 283.03 & 285.58 & 286.44\end{array}$

$\begin{array}{llllll}289.51 & 320.44 & 340.69 & 346.93 & 351.08 & 352.42\end{array}$

$\begin{array}{llllll}358.58 & 370.44 & 373.74 & 379.30 & 382.15 & 393.04\end{array}$

$\begin{array}{lllllll}396.69 & 408.64 & 418.53 & 430.65 & 437.72 & 445.31\end{array}$

$\begin{array}{llllll}449.89 & 454.88 & 455.62 & 464.08 & 467.74 & 468.00\end{array}$

$\begin{array}{llllll}472.03 & 473.79 & 487.76 & 503.12 & 504.37 & 530.43\end{array}$

$\begin{array}{llllll}537.52 & 538.66 & 539.42 & 562.96 & 569.26 & 578.12\end{array}$ $\begin{array}{llllll}609.19 & 610.67 & 617.89 & 620.63 & 628.87 & 632.72\end{array}$

$\begin{array}{llllll}635.66 & 637.42 & 639.28 & 642.54 & 680.97 & 684.54\end{array}$

$\begin{array}{llllll}689.86 & 692.43 & 694.73 & 698.27 & 709.37 & 725.25\end{array}$

$\begin{array}{llllll}738.07 & 744.36 & 752.36 & 755.81 & 764.18 & 767.97\end{array}$

$\begin{array}{llllll}787.51 & 792.38 & 798.25 & 798.85 & 802.76 & 844.87\end{array}$

$\begin{array}{llllll}847.06 & 853.21 & 856.93 & 858.01 & 860.85 & 865.78\end{array}$

$\begin{array}{llllll}866.02 & 873.72 & 875.42 & 885.29 & 895.55 & 900.55\end{array}$

$\begin{array}{llllll}901.15 & 907.49 & 911.52 & 915.01 & 925.80 & 934.97\end{array}$

$\begin{array}{llllll}938.05 & 944.92 & 954.21 & 960.99 & 969.17 & 982.12\end{array}$

$\begin{array}{llllll}986.60 & 989.42 & 991.60 & 998.23 & 1026.91 & 1027.74\end{array}$

$\begin{array}{rrrrrr}1047.66 & 1053.72 & 1064.18 & 1066.64 & 1068.67 & 1069.88\end{array}$ $\begin{array}{llllll}1073.00 & 1083.56 & 1095.02 & 1109.87 & 1113.03 & 1128.63\end{array}$ $\begin{array}{llllll}1133.37 & 1135.24 & 1145.60 & 1158.88 & 1162.52 & 1163.45\end{array}$ $\begin{array}{llllll}1166.61 & 1169.06 & 1173.14 & 1177.01 & 1183.67 & 1188.29\end{array}$ $\begin{array}{llllll}1194.87 & 1201.79 & 1206.95 & 1214.81 & 1216.97 & 1220.40\end{array}$ $\begin{array}{lllllll}1228.23 & 1239.45 & 1259.42 & 1263.72 & 1266.01 & 1269.47\end{array}$ $\begin{array}{llllll}1297.57 & 1313.96 & 1316.12 & 1317.20 & 1325.16 & 1326.62\end{array}$ $\begin{array}{llllll}1329.14 & 1329.97 & 1336.76 & 1345.58 & 1347.64 & 1349.24\end{array}$ $\begin{array}{llllll}1352.91 & 1356.15 & 1356.88 & 1372.40 & 1374.81 & 1375.99\end{array}$ $\begin{array}{lllllll}1390.07 & 1397.72 & 1398.78 & 1415.44 & 1426.38 & 1435.20\end{array}$ $\begin{array}{lllllll}1438.50 & 1442.14 & 1443.48 & 1460.08 & 1464.42 & 1470.47\end{array}$ $\begin{array}{lllllll}1478.97 & 1482.39 & 1490.24 & 1495.33 & 1508.69 & 1510.59\end{array}$ $\begin{array}{lllllll}1511.02 & 1514.02 & 1515.32 & 1517.18 & 1524.53 & 1540.05\end{array}$ $\begin{array}{llllll}1542.25 & 1544.26 & 1545.94 & 1571.96 & 1599.96 & 1611.30\end{array}$ $\begin{array}{lllllll}1617.37 & 1623.24 & 1624.97 & 1625.48 & 1630.14 & 1656.39\end{array}$ $\begin{array}{lllllll}1659.71 & 1662.14 & 1663.81 & 1671.23 & 1674.84 & 1676.21\end{array}$ $\begin{array}{lllllll}1708.67 & 2742.91 & 3024.33 & 3027.57 & 3059.12 & 3064.16\end{array}$ $\begin{array}{llllll}3099.07 & 3128.32 & 3139.99 & 3147.42 & 3166.02 & 3174.68\end{array}$ $\begin{array}{lllllll}3185.53 & 3188.26 & 3206.44 & 3216.76 & 3219.01 & 3221.45\end{array}$ $\begin{array}{llllll}3222.54 & 3226.53 & 3227.40 & 3230.53 & 3234.12 & 3236.92\end{array}$ $\begin{array}{llllll}3238.25 & 3240.89 & 3242.55 & 3245.23 & 3246.21 & 3277.16\end{array}$ $\begin{array}{lll}3289.56 & 3292.87 & 3628.77\end{array}$

${ }^{3}\left[{ }^{3}{ }^{1} \mathbf{Q}_{A}\right]$

$\begin{array}{llllll}19.54 & 20.08 & 22.11 & 25.76 & 27.01 & 28.85\end{array}$

$\begin{array}{llllll}31.28 & 33.99 & 37.73 & 39.54 & 44.01 & 48.40\end{array}$

$\begin{array}{llllll}52.76 & 56.66 & 67.18 & 70.60 & 73.65 & 79.48\end{array}$

$\begin{array}{llllll}80.79 & 83.56 & 94.99 & 109.02 & 111.72 & 116.19\end{array}$

$\begin{array}{llllll}127.13 & 131.79 & 139.85 & 141.90 & 143.43 & 150.59\end{array}$

$\begin{array}{llllll}155.74 & 160.97 & 166.02 & 179.59 & 182.17 & 191.17\end{array}$

$\begin{array}{llllll}196.73 & 204.34 & 206.11 & 210.75 & 216.67 & 219.34\end{array}$

$\begin{array}{llllll}225.41 & 229.53 & 232.52 & 243.69 & 249.40 & 256.35\end{array}$

$\begin{array}{lllllll}257.80 & 267.02 & 268.74 & 277.97 & 285.01 & 287.56\end{array}$

$\begin{array}{llllll}290.78 & 315.70 & 335.55 & 341.06 & 344.63 & 349.82\end{array}$

$\begin{array}{llllll}354.01 & 359.79 & 371.34 & 376.50 & 378.29 & 381.84\end{array}$

$\begin{array}{llllll}391.78 & 396.44 & 419.46 & 423.95 & 429.04 & 429.83\end{array}$

$\begin{array}{llllll}439.08 & 447.98 & 454.75 & 460.13 & 461.10 & 465.05\end{array}$

$\begin{array}{llllll}470.71 & 471.59 & 481.49 & 490.88 & 503.18 & 511.36\end{array}$

$\begin{array}{llllll}529.02 & 532.07 & 534.54 & 548.02 & 562.29 & 566.08\end{array}$

$\begin{array}{llllll}575.49 & 577.76 & 582.99 & 586.07 & 591.05 & 594.93\end{array}$

$\begin{array}{llllll}609.88 & 612.50 & 619.27 & 621.28 & 623.80 & 628.56\end{array}$

$\begin{array}{lllllll}629.92 & 638.86 & 641.74 & 648.26 & 675.48 & 677.03\end{array}$

$\begin{array}{llllll}678.95 & 682.98 & 684.90 & 691.23 & 697.30 & 708.69\end{array}$

$\begin{array}{llllll}727.31 & 735.78 & 745.42 & 746.00 & 751.14 & 758.91\end{array}$

$\begin{array}{llllll}766.75 & 769.22 & 785.04 & 791.68 & 796.08 & 798.53\end{array}$

$\begin{array}{lllllll}800.11 & 816.76 & 843.70 & 847.21 & 858.14 & 862.27\end{array}$

$\begin{array}{llllll}865.95 & 866.58 & 870.37 & 875.15 & 886.98 & 887.76\end{array}$

$\begin{array}{llllll}900.20 & 904.93 & 914.60 & 918.71 & 934.44 & 936.93\end{array}$

$\begin{array}{llllll}940.89 & 951.52 & 954.20 & 955.99 & 961.89 & 966.68\end{array}$

$\begin{array}{lllllr}983.08 & 991.52 & 992.55 & 993.76 & 1000.03 & 1025.10\end{array}$ $\begin{array}{lllllll}1031.27 & 1045.03 & 1047.46 & 1053.79 & 1063.47 & 1064.57\end{array}$ $\begin{array}{llllll}1068.45 & 1080.23 & 1087.09 & 1095.19 & 1110.85 & 1128.73\end{array}$ $\begin{array}{llllll}1132.55 & 1137.70 & 1140.67 & 1144.01 & 1160.85 & 1162.57\end{array}$ $\begin{array}{llllll}1162.80 & 1166.16 & 1171.37 & 1173.04 & 1177.02 & 1179.98\end{array}$ $\begin{array}{llllll}1188.39 & 1201.93 & 1208.17 & 1209.87 & 1215.08 & 1220.52\end{array}$ $\begin{array}{lllllll}1222.44 & 1232.00 & 1239.85 & 1257.55 & 1265.00 & 1269.23\end{array}$ $\begin{array}{lllllll}1269.78 & 1298.97 & 1314.70 & 1319.18 & 1326.02 & 1326.87\end{array}$ $\begin{array}{llllll}1330.24 & 1334.22 & 1336.42 & 1343.85 & 1345.72 & 1348.37\end{array}$ $\begin{array}{lllllll}1349.86 & 1351.13 & 1355.27 & 1369.87 & 1373.22 & 1386.08\end{array}$ $\begin{array}{llllll}1392.35 & 1396.94 & 1400.27 & 1416.89 & 1421.52 & 1427.60\end{array}$ $\begin{array}{lllllll}1434.43 & 1440.50 & 1442.93 & 1451.88 & 1459.87 & 1463.33\end{array}$ $\begin{array}{lllllll}1477.27 & 1485.64 & 1490.73 & 1495.43 & 1498.20 & 1503.62\end{array}$ $\begin{array}{lllllll}1510.74 & 1511.43 & 1511.74 & 1513.33 & 1516.00 & 1517.81\end{array}$ $\begin{array}{lllllll}1526.36 & 1530.51 & 1540.20 & 1544.70 & 1555.98 & 1575.01\end{array}$ $\begin{array}{lllllll}1582.76 & 1606.87 & 1609.03 & 1613.08 & 1623.86 & 1624.54\end{array}$ $\begin{array}{lllllll}1626.82 & 1651.46 & 1657.58 & 1663.01 & 1670.59 & 1672.56\end{array}$ $\begin{array}{lllllll}1697.01 & 2601.54 & 3026.92 & 3030.54 & 3057.87 & 3066.66\end{array}$ $\begin{array}{llllll}3098.33 & 3131.54 & 3139.65 & 3142.02 & 3171.62 & 3174.04\end{array}$ 
$\begin{array}{llllll}3189.53 & 3192.87 & 3207.06 & 3215.90 & 3218.98 & 3219.73\end{array}$ $\begin{array}{llllll}3220.41 & 3223.75 & 3227.82 & 3232.72 & 3234.83 & 3236.57\end{array}$ $\begin{array}{llllll}3238.77 & 3240.92 & 3247.85 & 3251.12 & 3257.90 & 3276.84\end{array}$ $3287.01 \quad 3293.21 \quad 3613.71$

\section{$\left[{ }^{1} \mathbf{I r}-{ }^{3} \mathbf{Q}_{\mathrm{A}}\right]$}

$\begin{array}{rrrrrr}8.79 & 15.15 & 18.45 & 19.74 & 22.32 & 26.8\end{array}$

$\begin{array}{llllll}27.91 & 29.24 & 32.78 & 37.06 & 38.20 & 42.60\end{array}$

$\begin{array}{llllll}47.84 & 58.38 & 63.12 & 70.64 & 74.20 & 76.10\end{array}$

$\begin{array}{rrrrrr}79.69 & 81.16 & 87.74 & 94.11 & 103.16 & 106.35\end{array}$

$\begin{array}{llllll}117.02 & 121.76 & 129.40 & 137.68 & 141.63 & 143.80\end{array}$

$\begin{array}{llllll}155.85 & 157.15 & 173.72 & 180.06 & 182.57 & 198.23\end{array}$

$\begin{array}{llllll}201.20 & 208.48 & 210.66 & 212.99 & 221.90 & 226.51\end{array}$

$\begin{array}{llllll}228.79 & 232.81 & 234.54 & 240.10 & 248.95 & 255.75\end{array}$

$\begin{array}{llllll}257.26 & 259.92 & 267.67 & 269.52 & 273.34 & 282.89\end{array}$

$\begin{array}{llllll}287.82 & 292.27 & 314.99 & 323.44 & 349.42 & 349.69\end{array}$

$\begin{array}{llllll}357.54 & 369.18 & 372.84 & 378.16 & 380.92 & 386.80\end{array}$

$\begin{array}{llllll}395.21 & 395.28 & 405.89 & 407.19 & 412.65 & 428.01\end{array}$

$\begin{array}{llllll}433.16 & 434.79 & 435.12 & 452.41 & 455.13 & 460.53\end{array}$

$\begin{array}{llllll}461.16 & 465.50 & 471.65 & 473.35 & 504.18 & 504.89\end{array}$

$\begin{array}{llllll}524.30 & 528.32 & 532.27 & 535.76 & 536.88 & 568.54\end{array}$

$\begin{array}{llllll}577.87 & 578.16 & 584.45 & 585.09 & 588.34 & 590.66\end{array}$

$\begin{array}{llllll}598.62 & 608.18 & 609.90 & 622.18 & 626.90 & 627.84\end{array}$

$\begin{array}{llllll}628.45 & 628.70 & 631.78 & 638.55 & 639.91 & 666.05\end{array}$

$\begin{array}{llllll}669.52 & 683.37 & 686.10 & 689.06 & 693.20 & 696.27\end{array}$

$\begin{array}{llllll}709.37 & 722.76 & 726.83 & 738.21 & 739.71 & 751.33\end{array}$

$\begin{array}{llllll}764.76 & 788.41 & 792.97 & 794.96 & 800.00 & 803.36\end{array}$

$\begin{array}{llllll}827.56 & 837.22 & 840.37 & 849.61 & 855.79 & 858.48\end{array}$

$\begin{array}{llllll}860.98 & 867.08 & 868.58 & 873.40 & 875.72 & 882.61\end{array}$

$\begin{array}{llllll}895.51 & 898.30 & 905.75 & 913.83 & 925.70 & 935.09\end{array}$

$\begin{array}{llllll}941.00 & 942.34 & 942.68 & 953.92 & 968.09 & 968.17\end{array}$

$\begin{array}{llllll}977.28 & 981.88 & 991.45 & 998.71 & 1018.78 & 1027.18\end{array}$

$\begin{array}{rrrrrr}1044.56 & 1048.39 & 1057.21 & 1065.27 & 1069.89 & 1072.58\end{array}$

$\begin{array}{llllll}1083.10 & 1090.33 & 1112.36 & 1112.77 & 1116.03 & 1129.21\end{array}$

$\begin{array}{lllllll}1129.36 & 1131.04 & 1131.97 & 1151.69 & 1164.90 & 1169.14\end{array}$

$\begin{array}{llllll}1169.91 & 1171.26 & 1177.38 & 1178.24 & 1183.24 & 1185.57\end{array}$

$\begin{array}{lllllll}1188.52 & 1189.07 & 1194.27 & 1205.97 & 1206.66 & 1214.79\end{array}$

$\begin{array}{lllllll}1221.79 & 1226.49 & 1227.53 & 1257.11 & 1260.59 & 1265.50\end{array}$

$\begin{array}{lllllll}1283.93 & 1301.40 & 1305.86 & 1316.65 & 1317.31 & 1322.45\end{array}$

$\begin{array}{lllllll}1325.08 & 1328.80 & 1330.51 & 1346.71 & 1348.94 & 1349.69\end{array}$

$\begin{array}{llllll}1350.66 & 1352.08 & 1353.66 & 1361.89 & 1368.44 & 1371.23\end{array}$

$\begin{array}{lllllll}1374.57 & 1399.00 & 1408.05 & 1416.05 & 1425.70 & 1433.05\end{array}$

$\begin{array}{llllll}1436.93 & 1437.76 & 1450.36 & 1457.19 & 1465.85 & 1468.21\end{array}$

$\begin{array}{llllll}1475.53 & 1480.75 & 1482.42 & 1485.68 & 1494.35 & 1499.72\end{array}$

$\begin{array}{lllllll}1506.10 & 1507.70 & 1509.02 & 1510.18 & 1512.56 & 1522.19\end{array}$

$\begin{array}{lllllll}1525.48 & 1537.06 & 1543.39 & 1546.77 & 1564.80 & 1578.24\end{array}$

$\begin{array}{lllllll}1588.67 & 1600.02 & 1611.30 & 1612.42 & 1624.95 & 1626.33\end{array}$

$\begin{array}{lllllll}1626.63 & 1651.89 & 1660.15 & 1664.90 & 1666.91 & 1674.65\end{array}$

$\begin{array}{lllllll}1677.14 & 2604.93 & 3029.94 & 3054.59 & 3064.68 & 3098.35\end{array}$

$\begin{array}{lllllll}3102.34 & 3133.73 & 3135.81 & 3143.52 & 3162.10 & 3175.37\end{array}$

$\begin{array}{llllll}3177.36 & 3190.52 & 3203.56 & 3216.86 & 3218.50 & 3218.75\end{array}$

$\begin{array}{llllll}3219.62 & 3225.86 & 3226.90 & 3231.98 & 3234.65 & 3238.60\end{array}$

$\begin{array}{lllllll}3239.03 & 3242.73 & 3243.47 & 3248.84 & 3267.41 & 3274.62\end{array}$ $\begin{array}{lll}3277.22 & 3294.81 & 3642.12\end{array}$

\section{${ }^{1}\left[\mathbf{I r}-Q_{\mathbf{B}}\right]$}

$\begin{array}{llllll}2.95 & 12.29 & 17.95 & 22.04 & 25.92 & 28.19\end{array}$

$\begin{array}{llllll}28.60 & 30.61 & 34.73 & 40.75 & 48.33 & 52.48\end{array}$

$\begin{array}{llllll}60.11 & 61.02 & 66.40 & 71.55 & 74.05 & 78.39\end{array}$

$\begin{array}{llllll}82.71 & 87.79 & 95.33 & 100.13 & 103.65 & 112.34\end{array}$

$\begin{array}{llllll}124.69 & 127.80 & 138.87 & 141.34 & 143.39 & 151.51\end{array}$

$\begin{array}{llllll}153.73 & 158.17 & 167.97 & 173.20 & 183.33 & 191.71\end{array}$

$\begin{array}{llllll}197.96 & 207.62 & 213.59 & 218.62 & 219.25 & 226.36\end{array}$

$\begin{array}{llllll}230.97 & 232.69 & 248.42 & 249.17 & 252.93 & 255.18\end{array}$

$\begin{array}{llllll}265.68 & 269.51 & 271.94 & 280.63 & 283.10 & 287.68\end{array}$

$\begin{array}{llllll}289.31 & 315.04 & 337.84 & 342.04 & 349.59 & 351.04\end{array}$

$\begin{array}{llllll}356.30 & 368.60 & 374.03 & 376.11 & 382.73 & 389.90\end{array}$

$\begin{array}{llllll}395.56 & 406.87 & 416.81 & 421.52 & 432.77 & 442.15\end{array}$

$\begin{array}{lllllll}452.27 & 457.77 & 458.41 & 463.38 & 465.76 & 467.36\end{array}$

$\begin{array}{llllll}470.98 & 474.35 & 486.30 & 504.53 & 506.37 & 525.74\end{array}$

$\begin{array}{llllll}532.36 & 536.65 & 538.09 & 564.72 & 568.88 & 576.33\end{array}$

$\begin{array}{llllll}537.70 & 582.30 & 586.94 & 588.62 & 590.45 & 605.34\end{array}$

$\begin{array}{llllll}609.80 & 612.98 & 613.97 & 626.20 & 626.97 & 631.70\end{array}$

$\begin{array}{llllll}638.35 & 639.69 & 640.28 & 652.30 & 675.81 & 681.10\end{array}$

$\begin{array}{llllll}688.28 & 691.91 & 695.49 & 699.83 & 708.02 & 727.36\end{array}$

$\begin{array}{llllll}740.26 & 751.54 & 760.23 & 761.93 & 766.55 & 772.55\end{array}$

$\begin{array}{llllll}791.17 & 793.19 & 797.10 & 798.16 & 802.50 & 847.90\end{array}$ $\begin{array}{llllll}848.56 & 855.33 & 858.67 & 865.61 & 866.04 & 867.02\end{array}$

$\begin{array}{llllll}868.51 & 876.67 & 877.04 & 884.86 & 889.70 & 901.29\end{array}$

$\begin{array}{llllll}904.37 & 906.12 & 914.09 & 930.48 & 935.68 & 945.06\end{array}$

$\begin{array}{llllll}952.17 & 953.23 & 954.59 & 963.74 & 968.17 & 983.70\end{array}$

$\begin{array}{llllll}991.12 & 994.72 & 995.24 & 999.37 & 1013.93 & 1026.91\end{array}$ $\begin{array}{lllllll}1049.74 & 1056.00 & 1063.88 & 1064.68 & 1070.17 & 1070.52\end{array}$ $\begin{array}{llllll}1071.96 & 1083.27 & 1093.32 & 1108.83 & 1111.61 & 1124.94\end{array}$ $\begin{array}{lllllll}1128.61 & 1133.91 & 1144.23 & 1154.49 & 1164.65 & 1165.50\end{array}$ $\begin{array}{llllll}1167.69 & 1168.71 & 1174.35 & 1177.45 & 1184.29 & 1186.86\end{array}$ $\begin{array}{llllll}1194.54 & 1198.89 & 1204.70 & 1209.95 & 1213.96 & 1215.43\end{array}$ $\begin{array}{lllllll}1227.46 & 1238.91 & 1254.83 & 1260.99 & 1267.76 & 1269.25\end{array}$ $\begin{array}{llllll}1301.82 & 1309.70 & 1314.42 & 1316.67 & 1324.89 & 1328.40\end{array}$ $\begin{array}{lllllll}1329.66 & 1331.73 & 1337.69 & 1345.80 & 1349.07 & 1350.08\end{array}$ $\begin{array}{lllllll}1350.75 & 1352.11 & 1356.01 & 1364.08 & 1373.11 & 1375.06\end{array}$ $\begin{array}{llllll}1392.58 & 1400.01 & 1401.95 & 1415.42 & 1426.16 & 1433.52\end{array}$ $\begin{array}{lllllll}1437.49 & 1438.03 & 1442.78 & 1455.70 & 1465.71 & 1467.20\end{array}$ $\begin{array}{llllll}1477.31 & 1480.24 & 1490.39 & 1495.55 & 1502.55 & 1509.70\end{array}$ $\begin{array}{lllllll}1510.02 & 1511.01 & 1512.88 & 1516.40 & 1521.04 & 1528.10\end{array}$ $\begin{array}{lllllll}1544.24 & 1545.07 & 1547.63 & 1573.68 & 1597.09 & 1609.64\end{array}$ $\begin{array}{lllllll}1613.34 & 1623.70 & 1624.51 & 1626.49 & 1627.13 & 1658.38\end{array}$ $\begin{array}{lllllll}1659.04 & 1660.51 & 1664.23 & 1674.08 & 1677.00 & 1677.79\end{array}$ $\begin{array}{lllllll}1707.30 & 2772.67 & 3027.36 & 3048.31 & 3056.26 & 3062.41\end{array}$ $\begin{array}{lllllll}3098.47 & 3130.78 & 3136.75 & 3138.22 & 3159.08 & 3174.52\end{array}$ $\begin{array}{llllll}3186.38 & 3198.84 & 3213.65 & 3215.86 & 3218.53 & 3219.67\end{array}$ $\begin{array}{lllllll}3221.44 & 3224.41 & 3231.00 & 3231.52 & 3233.67 & 3238.11\end{array}$ $\begin{array}{llllll}3242.47 & 3243.16 & 3245.94 & 3260.12 & 3265.24 & 3275.40\end{array}$ $\begin{array}{lll}3279.43 & 3296.13 & 3609.58\end{array}$

${ }^{3}\left[{ }^{3} \mathbf{r}^{-1} \mathbf{Q}_{\mathbf{B}}\right]$

$\begin{array}{llllll}8.47 & 18.01 & 19.77 & 23.34 & 25.26 & 27.54\end{array}$ $\begin{array}{llllll}28.34 & 30.29 & 34.67 & 41.16 & 46.73 & 56.42\end{array}$ $\begin{array}{llllll}58.14 & 59.57 & 63.07 & 65.01 & 67.04 & 75.89\end{array}$ $\begin{array}{llllll}78.05 & 83.45 & 86.81 & 97.06 & 102.34 & 108.10\end{array}$ $\begin{array}{llllll}123.13 & 124.73 & 134.59 & 139.67 & 141.01 & 148.16\end{array}$ $\begin{array}{llllll}151.52 & 156.94 & 165.99 & 167.03 & 173.80 & 189.42\end{array}$ $\begin{array}{lllllll}195.54 & 201.17 & 203.00 & 207.22 & 216.56 & 220.76\end{array}$ $\begin{array}{llllll}222.79 & 230.06 & 241.71 & 250.32 & 253.34 & 254.87\end{array}$ $\begin{array}{lllllll}256.87 & 268.17 & 271.12 & 276.13 & 280.58 & 285.83\end{array}$ $\begin{array}{llllll}289.26 & 315.36 & 329.14 & 337.90 & 340.96 & 349.82\end{array}$ $\begin{array}{llllll}350.93 & 357.42 & 370.98 & 372.75 & 376.53 & 383.82\end{array}$ $\begin{array}{llllll}386.78 & 397.79 & 416.31 & 419.63 & 425.87 & 429.54\end{array}$ $\begin{array}{llllll}441.28 & 449.02 & 458.54 & 459.64 & 460.50 & 466.36\end{array}$ $\begin{array}{llllll}466.48 & 470.04 & 480.59 & 484.98 & 503.62 & 516.72\end{array}$ $\begin{array}{llllll}520.95 & 530.30 & 538.01 & 552.59 & 563.50 & 565.19\end{array}$ $\begin{array}{llllll}572.25 & 577.17 & 586.79 & 588.11 & 593.29 & 596.30\end{array}$ $\begin{array}{llllll}611.60 & 613.00 & 614.90 & 620.04 & 625.37 & 627.29\end{array}$ $\begin{array}{lllllll}631.77 & 636.62 & 640.75 & 664.82 & 669.69 & 676.88\end{array}$ $\begin{array}{llllll}679.73 & 682.41 & 687.26 & 693.44 & 699.56 & 705.81\end{array}$ $\begin{array}{llllll}731.36 & 739.52 & 746.38 & 749.71 & 759.94 & 762.32\end{array}$ $\begin{array}{llllll}764.79 & 770.14 & 786.38 & 792.03 & 797.79 & 798.37\end{array}$ $\begin{array}{llllll}801.28 & 824.51 & 843.05 & 849.57 & 862.86 & 863.79\end{array}$ $\begin{array}{llllll}866.42 & 870.02 & 877.16 & 880.65 & 887.16 & 889.57\end{array}$ $\begin{array}{llllll}901.38 & 902.84 & 909.98 & 929.37 & 934.81 & 951.04\end{array}$ $\begin{array}{llllll}951.42 & 952.40 & 953.55 & 962.60 & 964.81 & 968.00\end{array}$ $\begin{array}{llllll}976.32 & 983.97 & 992.23 & 995.65 & 1002.04 & 1014.62\end{array}$ $\begin{array}{lllllll}1035.65 & 1043.85 & 1047.30 & 1054.06 & 1054.92 & 1062.96\end{array}$ $\begin{array}{llllll}1069.17 & 1072.68 & 1083.95 & 1093.84 & 1097.75 & 1110.05\end{array}$ $\begin{array}{llllll}1127.80 & 1131.95 & 1144.72 & 1147.95 & 1162.14 & 1166.00\end{array}$ $\begin{array}{lllllll}1168.01 & 1171.86 & 1173.19 & 1175.80 & 1177.74 & 1181.56\end{array}$ $\begin{array}{llllll}1187.02 & 1192.04 & 1201.26 & 1202.78 & 1210.14 & 1215.46\end{array}$ $\begin{array}{lllllll}1229.56 & 1236.30 & 1242.55 & 1255.38 & 1264.92 & 1267.33\end{array}$ $\begin{array}{lllllll}1272.70 & 1300.18 & 1307.07 & 1313.19 & 1323.24 & 1325.64\end{array}$ $\begin{array}{llllll}1329.71 & 1333.77 & 1336.80 & 1346.57 & 1347.21 & 1349.42\end{array}$ $\begin{array}{lllllll}1352.58 & 1357.79 & 1359.77 & 1367.64 & 1375.31 & 1392.15\end{array}$ $\begin{array}{llllll}1394.33 & 1399.42 & 1400.76 & 1414.99 & 1426.72 & 1433.12\end{array}$ $\begin{array}{lllllll}1437.04 & 1440.36 & 1441.50 & 1449.21 & 1455.44 & 1470.01\end{array}$ $\begin{array}{lllllll}1473.44 & 1485.04 & 1489.66 & 1495.32 & 1496.72 & 1501.12\end{array}$ $\begin{array}{llllll}1509.48 & 1509.69 & 1511.00 & 1512.12 & 1516.18 & 1517.57\end{array}$ $\begin{array}{lllllll}1523.82 & 1526.84 & 1544.44 & 1544.63 & 1560.49 & 1576.32\end{array}$ $\begin{array}{llllll}1595.49 & 1599.93 & 1607.04 & 1614.20 & 1625.24 & 1625.56\end{array}$ $\begin{array}{lllllll}1626.11 & 1645.72 & 1659.66 & 1663.35 & 1672.71 & 1676.23\end{array}$ $\begin{array}{llllll}1703.89 & 2751.40 & 3026.89 & 3051.07 & 3055.35 & 3059.57\end{array}$ $\begin{array}{lllllll}3098.09 & 3130.57 & 3137.22 & 3138.35 & 3146.97 & 3175.45\end{array}$ $\begin{array}{lllllll}3187.23 & 3200.99 & 3210.88 & 3215.22 & 3219.51 & 3220.55\end{array}$ $\begin{array}{llllll}3223.55 & 3227.74 & 3228.49 & 3232.22 & 3234.18 & 3241.73\end{array}$ $\begin{array}{lllllll}3242.79 & 3244.96 & 3245.18 & 3254.04 & 3260.10 & 3270.95\end{array}$ $3279.78 \quad 3297.25 \quad 3604.61$ 
$\left[{ }^{1} \mathbf{I} \mathbf{r}^{3} \mathbf{Q}_{\mathrm{B}}\right]$

\begin{tabular}{|c|c|c|c|c|c|}
\hline 4.56 & 11.81 & 18.40 & 21.13 & 26.14 & 28.26 \\
\hline 30.22 & 31.82 & 35.37 & 40.85 & 47.87 & 49.53 \\
\hline 55.75 & 60.36 & 61.67 & 66.24 & 72.28 & 73.89 \\
\hline 78.12 & 82.60 & 94.30 & 97.98 & 101.25 & 113.55 \\
\hline 121.11 & 124.78 & 126.77 & 138.57 & 140.47 & 142.95 \\
\hline 153.04 & 157.13 & 168.01 & 173.88 & 183.33 & 191.29 \\
\hline 197.76 & 207.14 & 211.16 & 219.00 & 219.44 & 225.30 \\
\hline 228.04 & 229.25 & 232.17 & 248.64 & 252.95 & 256.20 \\
\hline 260.11 & 262.30 & 266.49 & 271.18 & 283.37 & 284.43 \\
\hline 287.85 & 290.11 & 310.19 & 340.85 & 349.12 & 351.20 \\
\hline 357.16 & 365.84 & 373.28 & 376.57 & 379.22 & 382.13 \\
\hline 389.82 & 395.22 & 405.14 & 408.44 & 414.14 & 421.51 \\
\hline 432.32 & 435.23 & 436.28 & 451.98 & 458.00 & 462.60 \\
\hline 464.45 & 467.25 & 469.94 & 474.04 & 504.57 & 506.52 \\
\hline 526.63 & 527.06 & 528.85 & 532.16 & 536.82 & 568.81 \\
\hline 577.26 & 577.68 & 581.75 & 587.04 & 589.07 & 590.75 \\
\hline 601.39 & 605.36 & 609.52 & 618.58 & 622.67 & 623.88 \\
\hline 626.98 & 628.27 & 632.19 & 639.82 & 640.45 & 659.06 \\
\hline 677.31 & 681.72 & 687.45 & 688.31 & 691.80 & 695.74 \\
\hline 708.20 & 725.43 & 727.81 & 740.02 & 740.75 & 759.17 \\
\hline 766.42 & 791.42 & 793.27 & 797.03 & 798.81 & 802.49 \\
\hline 827.59 & 848.91 & 849.40 & 854.97 & 858.31 & 864.63 \\
\hline 866.13 & 866.54 & 874.04 & 875.19 & 876.34 & 885.36 \\
\hline 890.28 & 905.73 & 909.43 & 914.03 & 919.62 & 927.47 \\
\hline 944.55 & 944.83 & 952.70 & 955.99 & 968.25 & 968.81 \\
\hline 984.49 & 991.60 & 992.90 & 999.35 & 1015.18 & 1026.97 \\
\hline 1050.11 & 1050.23 & 1056.78 & 1064.55 & 1070.18 & 1071.41 \\
\hline 1082.98 & 1092.32 & 1106.72 & 1109.08 & 1111.98 & 1124.43 \\
\hline 1126.15 & 1128.80 & 1134.64 & 1137.63 & 1161.44 & 1165.88 \\
\hline 1167.43 & 1168.52 & 1174.52 & 1176.89 & 1177.32 & 1179.99 \\
\hline 1186.52 & 1188.33 & 1198.95 & 1205.49 & 1206.67 & 1210.23 \\
\hline 1215.33 & 1227.47 & 1227.85 & 1254.63 & 1260.32 & 1268.93 \\
\hline 1275.80 & 1304.85 & 1314.27 & 1315.91 & 1319.72 & 1324.65 \\
\hline 1327.88 & 1329.61 & 1331.34 & 1345.42 & 1348.65 & 1348.84 \\
\hline 1350.41 & 1351.76 & 1353.47 & 1359.11 & 1365.27 & 1369.89 \\
\hline 1374.63 & 1397.23 & 1399.68 & 1415.28 & 1425.72 & 1433.17 \\
\hline 1436.96 & 1438.04 & 1443.59 & 1457.60 & 1464.78 & 1466.77 \\
\hline 1477.08 & 1479.99 & 1485.79 & 1490.40 & 1495.16 & 1501.15 \\
\hline 1508.02 & 1509.05 & 1509.77 & 1511.33 & 1516.42 & 1519.83 \\
\hline 1526.67 & 1538.02 & 1543.64 & 1546.98 & 1574.69 & 1579.88 \\
\hline 1594.45 & 1599.81 & 1609.46 & 1613.13 & 1623.54 & 1624.28 \\
\hline 1626.64 & 1657.93 & 1658.87 & 1660.29 & 1670.24 & 1676.16 \\
\hline 1676.87 & 2648.84 & 3026.86 & 3054.48 & 3063.20 & 3083.19 \\
\hline 3097.79 & 3133.59 & 3136.05 & 3142.07 & 3159.26 & 3173.71 \\
\hline 3188.20 & 3202.71 & 3214.45 & 3216.26 & 3216.37 & 3219.14 \\
\hline 3224.28 & 3226.64 & 3229.80 & 3232.85 & 3237.44 & 3238.44 \\
\hline 3246.00 & 3246.74 & 3247.72 & 3257.82 & 3261.78 & 3263.07 \\
\hline 3278.45 & 3295.29 & 3647.90 & & & \\
\hline
\end{tabular}

${ }^{1}\left[\mathrm{Ir}-\mathbf{Q C}_{\mathrm{C}}\right]$

$\begin{array}{llllll}9.99 & 20.24 & 22.42 & 26.41 & 27.79 & 29.49\end{array}$

$\begin{array}{llllll}30.07 & 33.63 & 38.32 & 41.62 & 44.99 & 45.47\end{array}$

$\begin{array}{rrrrrr}48.42 & 60.00 & 65.15 & 70.16 & 75.91 & 78.33 \\ 81.08 & 85.55 & 93.63 & 98.32 & 117.06 & 121.79\end{array}$

$\begin{array}{llllll}123.16 & 129.59 & 131.74 & 138.42 & 142.88 & 150.54\end{array}$

$\begin{array}{llllll}152.67 & 153.99 & 162.75 & 179.39 & 185.04 & 191.44\end{array}$

$\begin{array}{llllll}197.85 & 206.10 & 218.42 & 219.81 & 222.97 & 227.16\end{array}$

$\begin{array}{llllll}232.66 & 233.93 & 245.34 & 248.40 & 253.69 & 259.55\end{array}$

$\begin{array}{llllll}265.87 & 270.27 & 272.30 & 278.03 & 285.18 & 286.68\end{array}$

$\begin{array}{llllll}289.19 & 317.48 & 340.31 & 346.95 & 348.52 & 350.55\end{array}$

$\begin{array}{llllll}357.42 & 361.99 & 373.57 & 379.50 & 380.04 & 389.46\end{array}$

$\begin{array}{llllll}396.69 & 409.08 & 419.39 & 430.68 & 432.51 & 433.86\end{array}$

$\begin{array}{llllll}452.54 & 453.79 & 462.28 & 463.69 & 465.78 & 467.64\end{array}$

$\begin{array}{llllll}470.61 & 472.37 & 479.66 & 504.06 & 504.84 & 532.22\end{array}$

$\begin{array}{llllll}535.21 & 536.93 & 538.64 & 560.69 & 567.47 & 576.23\end{array}$

$\begin{array}{llllll}577.39 & 582.80 & 587.24 & 588.58 & 590.29 & 608.91\end{array}$

$\begin{array}{llllll}610.00 & 613.46 & 622.67 & 628.35 & 629.42 & 632.66\end{array}$

$\begin{array}{llllll}637.40 & 638.78 & 639.45 & 675.27 & 681.52 & 683.33\end{array}$

$\begin{array}{llllll}687.73 & 692.52 & 694.09 & 699.68 & 708.20 & 726.49\end{array}$

$\begin{array}{llllll}738.83 & 746.96 & 758.18 & 763.96 & 764.74 & 767.68\end{array}$

$\begin{array}{llllll}787.13 & 791.42 & 793.90 & 795.95 & 801.32 & 848.69\end{array}$

$\begin{array}{llllll}850.42 & 853.19 & 855.61 & 856.77 & 863.41 & 864.86\end{array}$

$\begin{array}{llllll}865.30 & 867.21 & 874.32 & 876.23 & 885.79 & 894.22\end{array}$

$\begin{array}{llllll}901.21 & 905.33 & 906.78 & 914.61 & 934.93 & 936.58\end{array}$

$\begin{array}{llllll}941.25 & 950.41 & 952.17 & 961.57 & 962.80 & 966.64\end{array}$

$\begin{array}{lllrrr}990.68 & 993.91 & 995.72 & 1000.52 & 1001.66 & 1025.46\end{array}$ $\begin{array}{llllll}1026.41 & 1051.20 & 1058.09 & 1059.60 & 1064.65 & 1065.67\end{array}$ $\begin{array}{lllllll}1071.38 & 1081.80 & 1092.74 & 1107.48 & 1111.71 & 1126.48\end{array}$ $\begin{array}{llllll}1128.22 & 1137.55 & 1143.87 & 1159.98 & 1165.35 & 1165.94\end{array}$ $\begin{array}{llllll}1166.38 & 1168.97 & 1177.46 & 1180.83 & 1182.99 & 1187.07\end{array}$ $\begin{array}{llllll}1188.54 & 1201.01 & 1202.22 & 1208.81 & 1216.13 & 1224.93\end{array}$ $\begin{array}{lllllll}1225.35 & 1234.51 & 1255.90 & 1259.14 & 1263.83 & 1278.61\end{array}$ $\begin{array}{lllllll}1301.00 & 1305.13 & 1313.61 & 1317.09 & 1325.81 & 1327.41\end{array}$ $\begin{array}{llllll}1329.37 & 1333.29 & 1338.46 & 1347.17 & 1347.64 & 1350.10\end{array}$ $\begin{array}{llllll}1350.83 & 1351.79 & 1358.78 & 1372.01 & 1374.27 & 1378.84\end{array}$ $\begin{array}{lllllll}1389.80 & 1397.61 & 1402.81 & 1419.49 & 1428.83 & 1433.63\end{array}$ $\begin{array}{lllllll}1437.81 & 1439.62 & 1445.86 & 1456.49 & 1464.05 & 1465.21\end{array}$ $\begin{array}{lllllll}1477.94 & 1481.03 & 1489.26 & 1491.71 & 1499.86 & 1504.66\end{array}$ $\begin{array}{llllll}1510.49 & 1512.25 & 1516.34 & 1519.40 & 1522.99 & 1524.97\end{array}$ $\begin{array}{lllllll}1542.47 & 1545.56 & 1545.77 & 1578.24 & 1596.30 & 1610.85\end{array}$ $\begin{array}{llllll}1613.55 & 1624.34 & 1625.23 & 1625.96 & 1627.82 & 1658.62\end{array}$ $\begin{array}{lllllll}1661.50 & 1662.87 & 1667.15 & 1673.37 & 1674.99 & 1676.96\end{array}$ $\begin{array}{lllllll}1715.61 & 2985.76 & 3025.76 & 3032.79 & 3061.41 & 3070.01\end{array}$ $\begin{array}{lllllll}3096.05 & 3138.65 & 3141.42 & 3145.66 & 3169.84 & 3173.34\end{array}$ $\begin{array}{llllll}3181.03 & 3188.31 & 3204.04 & 3217.27 & 3217.67 & 3218.11\end{array}$ $\begin{array}{lllllll}3219.29 & 3220.86 & 3224.51 & 3226.39 & 3231.35 & 3233.62\end{array}$ $\begin{array}{lllllll}3233.91 & 3234.45 & 3238.38 & 3251.17 & 3252.13 & 3267.45\end{array}$ $\begin{array}{lll}3280.34 & 3295.50 & 3602.09\end{array}$

$\left[{ }^{3} \mid \mathbf{r}-{ }^{-1} \mathbf{Q}_{\mathrm{C}}\right]$

$\begin{array}{llllll}12.82 & 18.85 & 19.55 & 22.74 & 28.86 & 31.82\end{array}$

$\begin{array}{llllll}34.11 & 37.37 & 40.73 & 46.95 & 51.64 & 56.71\end{array}$

$\begin{array}{llllll}57.60 & 60.48 & 65.35 & 72.20 & 76.27 & 82.50\end{array}$

$\begin{array}{rrrrrr}84.20 & 92.07 & 97.75 & 103.45 & 118.02 & 119.10\end{array}$

$\begin{array}{llllll}124.98 & 128.88 & 133.85 & 138.84 & 146.24 & 148.06\end{array}$

$\begin{array}{lllllll}153.49 & 157.19 & 163.68 & 175.55 & 184.59 & 192.28\end{array}$

$\begin{array}{llllll}195.41 & 198.61 & 212.32 & 215.37 & 217.05 & 220.39\end{array}$

$\begin{array}{llllll}226.14 & 229.76 & 231.38 & 243.30 & 249.44 & 257.32\end{array}$

$\begin{array}{llllll}263.44 & 272.76 & 273.49 & 279.99 & 285.85 & 287.51\end{array}$

$\begin{array}{lllllll}287.74 & 318.65 & 333.70 & 340.38 & 342.86 & 348.44\end{array}$

$\begin{array}{llllll}349.60 & 358.73 & 363.12 & 370.18 & 374.86 & 379.95\end{array}$

$\begin{array}{llllll}389.42 & 398.29 & 416.23 & 417.40 & 427.58 & 432.28\end{array}$

$\begin{array}{llllll}433.82 & 449.35 & 460.63 & 462.12 & 465.92 & 468.74\end{array}$

$\begin{array}{llllll}469.77 & 470.29 & 482.93 & 484.53 & 504.99 & 508.91\end{array}$

$\begin{array}{llllll}523.10 & 530.40 & 536.47 & 546.91 & 561.48 & 566.64\end{array}$

$\begin{array}{llllll}575.34 & 576.91 & 579.07 & 587.65 & 589.97 & 595.38\end{array}$

$\begin{array}{llllll}611.99 & 612.89 & 618.12 & 618.74 & 624.78 & 627.97\end{array}$

$\begin{array}{llllll}631.56 & 634.52 & 639.44 & 670.47 & 672.17 & 673.39\end{array}$

$\begin{array}{lllllll}677.84 & 681.38 & 686.60 & 692.19 & 698.52 & 706.39\end{array}$

$\begin{array}{llllll}727.94 & 731.22 & 739.98 & 746.83 & 747.35 & 758.75\end{array}$

$\begin{array}{llllll}764.93 & 768.02 & 783.64 & 791.34 & 793.26 & 799.15\end{array}$

$\begin{array}{llllll}806.54 & 821.00 & 845.50 & 846.92 & 850.30 & 855.68\end{array}$

$\begin{array}{lllllll}861.69 & 866.74 & 867.24 & 876.50 & 877.04 & 895.03\end{array}$

$\begin{array}{llllll}904.71 & 906.92 & 912.40 & 927.34 & 934.70 & 936.58\end{array}$

$\begin{array}{llllll}941.10 & 950.20 & 954.29 & 961.87 & 963.48 & 965.71\end{array}$

$\begin{array}{llllll}978.96 & 981.31 & 992.67 & 996.47 & 998.55 & 1012.93\end{array}$

$\begin{array}{lrrrrr}023.28 & 1029.16 & 1044.30 & 1049.90 & 1056.86 & 1063.71\end{array}$ $\begin{array}{llllll}1068.28 & 1071.22 & 1080.54 & 1087.91 & 1092.31 & 1112.88\end{array}$

$\begin{array}{llllll}1131.36 & 1135.34 & 1141.95 & 1156.61 & 1160.23 & 1165.80\end{array}$ $\begin{array}{llllll}1170.04 & 1172.09 & 1173.51 & 1177.62 & 1180.62 & 1181.62\end{array}$ $\begin{array}{llllll}1185.49 & 1189.99 & 1200.97 & 1207.37 & 1208.03 & 1215.59\end{array}$ $\begin{array}{llllll}1222.96 & 1232.84 & 1233.60 & 1258.61 & 1265.12 & 1265.48\end{array}$ $\begin{array}{llllll}1278.58 & 1300.06 & 1307.72 & 1316.00 & 1323.74 & 1330.43\end{array}$ $\begin{array}{llllll}1330.73 & 1335.12 & 1338.89 & 1343.98 & 1346.59 & 1347.42\end{array}$ $\begin{array}{lllllll}1350.33 & 1352.13 & 1358.62 & 1375.68 & 1376.73 & 1389.35\end{array}$ $\begin{array}{lllllll}1394.02 & 1401.59 & 1402.51 & 1417.79 & 1425.70 & 1431.25\end{array}$ $\begin{array}{lllllll}1436.05 & 1439.57 & 1442.50 & 1446.56 & 1456.17 & 1467.38\end{array}$ $\begin{array}{lllllll}1475.87 & 1477.86 & 1489.59 & 1490.72 & 1497.07 & 1504.49\end{array}$ $\begin{array}{llllll}1508.12 & 1510.04 & 1510.90 & 1514.23 & 1516.11 & 1516.44\end{array}$ $\begin{array}{lllllll}1520.04 & 1524.09 & 1543.09 & 1544.29 & 1559.51 & 1576.02\end{array}$ $\begin{array}{lllllll}1585.37 & 1602.19 & 1607.96 & 1616.34 & 1621.16 & 1624.46\end{array}$ $\begin{array}{lllllll}1625.22 & 1652.89 & 1660.45 & 1661.00 & 1672.69 & 1673.13\end{array}$ $\begin{array}{lllllll}1701.28 & 2869.07 & 3026.35 & 3037.44 & 3067.69 & 3074.10\end{array}$ $\begin{array}{lllllll}3097.20 & 3142.08 & 3145.65 & 3159.71 & 3170.82 & 3174.02\end{array}$ $\begin{array}{llllll}3187.24 & 3192.01 & 3205.77 & 3213.26 & 3217.27 & 3218.50\end{array}$ $\begin{array}{llllll}3219.44 & 3220.69 & 3225.83 & 3226.62 & 3230.92 & 3231.59\end{array}$ $\begin{array}{lllllll}3235.45 & 3239.38 & 3247.37 & 3257.65 & 3258.23 & 3273.21\end{array}$ $\begin{array}{lll}3281.96 & 3297.13 & 3600.23\end{array}$

$\left[{ }^{1} \mathbf{I r}-{ }^{3} \mathbf{Q}_{\mathbf{C}}\right]$

$\begin{array}{llllll}13.36 & 14.41 & 22.05 & 24.16 & 25.13 & 26.85\end{array}$ $\begin{array}{llllll}30.15 & 31.77 & 35.17 & 40.67 & 45.06 & 47.89\end{array}$ $\begin{array}{llllll}50.98 & 57.38 & 61.64 & 67.24 & 72.27 & 76.95\end{array}$ 


\begin{tabular}{|c|c|c|c|c|c|}
\hline & & & & & \\
\hline 80.40 & 86.11 & 96.12 & 97.53 & 116.58 & 118.31 \\
\hline 120.39 & 125.55 & 130.68 & 138.71 & 145.49 & 147.72 \\
\hline 153.44 & 155.92 & 163.83 & 181.00 & 186.52 & 193.44 \\
\hline 198.30 & 208.98 & 217.27 & 219.16 & 221.47 & 224.42 \\
\hline 231.38 & 232.63 & 234.34 & 247.10 & 249.09 & 255.94 \\
\hline 260.60 & 269.26 & 270.42 & 277.48 & 284.67 & 285.82 \\
\hline 288.24 & 289.88 & 311.54 & 347.26 & 349.37 & 349.87 \\
\hline 355.25 & 361.48 & 373.71 & 379.02 & 380.33 & 388.50 \\
\hline 390.60 & 396.24 & 408.54 & 416.10 & 424.85 & 429.78 \\
\hline 434.23 & 447.33 & 450.94 & 455.29 & 458.47 & 464.56 \\
\hline 466.72 & 468.14 & 469.17 & 474.74 & 504.84 & 505.37 \\
\hline 524.36 & 527.22 & 532.25 & 532.51 & 538.05 & 567.99 \\
\hline 577.98 & 578.21 & 583.30 & 587.38 & 589.40 & 590.76 \\
\hline 600.05 & 608.64 & 610.40 & 619.29 & 621.26 & 623.30 \\
\hline 627.88 & 630.75 & 634.34 & 639.61 & 641.06 & 656.68 \\
\hline 681.96 & 685.28 & 687.24 & 689.97 & 692.80 & 696.71 \\
\hline 708.42 & 718.89 & 727.56 & 738.19 & 739.87 & 753.06 \\
\hline 765.38 & 790.39 & 792.29 & 794.48 & 799.29 & 803.27 \\
\hline 826.40 & 841.07 & 848.68 & 855.72 & 856.06 & 859.36 \\
\hline 861.77 & 864.95 & 867.02 & 870.91 & 875.99 & 876.55 \\
\hline 886.67 & 887.74 & 902.03 & 906.82 & 914.28 & 914.78 \\
\hline 941.84 & 946.75 & 949.14 & 957.37 & 967.30 & 969.24 \\
\hline 970.60 & 990.63 & 994.35 & 998.24 & 1005.64 & 1023.81 \\
\hline 1024.39 & 1048.61 & 1049.33 & 1056.82 & 1062.90 & 1067.54 \\
\hline 1070.68 & 1081.23 & 1093.13 & 1109.23 & 1113.87 & 1123.42 \\
\hline 1126.79 & 1127.36 & 1133.33 & 1138.11 & 1163.29 & 1165.40 \\
\hline 1165.57 & 1169.37 & 1177.00 & 1177.38 & 1182.36 & 1185.67 \\
\hline 1186.72 & 1188.32 & 1190.45 & 1202.12 & 1206.97 & 1215.50 \\
\hline 1222.32 & 1225.52 & 1229.29 & 1257.28 & 1259.56 & 1263.51 \\
\hline 1278.55 & 1300.05 & 1315.61 & 1316.35 & 1318.42 & 1325.82 \\
\hline 1328.16 & 1330.66 & 1332.44 & 1346.21 & 1347.36 & 1349.10 \\
\hline 1349.76 & 1350.76 & 1351.29 & 1359.55 & 1369.36 & 1370.39 \\
\hline 1373.31 & 1394.88 & 1404.47 & 1415.74 & 1425.40 & 1434.15 \\
\hline 1437.56 & 1438.03 & 1446.89 & 1458.10 & 1464.06 & 1465.57 \\
\hline 1477.72 & 1480.54 & 1483.18 & 1491.35 & 1496.57 & 1504.60 \\
\hline 1508.25 & 1510.34 & 1510.96 & 1511.92 & 1519.58 & 1522.98 \\
\hline 1529.18 & 1532.36 & 1543.12 & 1546.42 & 1575.69 & 1578.07 \\
\hline 1596.39 & 1598.21 & 1608.54 & 1610.31 & 1623.39 & 1624.63 \\
\hline 1625.30 & 1654.59 & 1657.83 & 1660.33 & 1670.44 & 1675.57 \\
\hline 1676.29 & 2920.33 & 3025.50 & 3055.55 & 3063.46 & 3070.21 \\
\hline 3095.81 & 3133.55 & 3139.73 & 3142.83 & 3172.70 & 3178.54 \\
\hline 3191.57 & 3194.14 & 3206.63 & 3216.10 & 3216.96 & 3218.78 \\
\hline 3218.94 & 3224.02 & 3225.61 & 3231.91 & 3232.93 & 3233.52 \\
\hline 3234.72 & 3236.18 & 3247.41 & 3249.96 & 3252.46 & 3279.00 \\
\hline 3281.57 & 3294.36 & 3635.70 & & & \\
\hline \multicolumn{6}{|l|}{${ }^{1}\left[\mathbf{I r}-\mathbf{Q}_{\mathrm{D}}\right]$} \\
\hline 26.62 & 29.12 & 30.32 & 34.77 & 37.10 & 39.02 \\
\hline 42.07 & 42.69 & 45.67 & 49.59 & 52.91 & 60.04 \\
\hline 66.98 & 71.32 & 73.17 & 79.53 & 81.31 & 86.38 \\
\hline 87.34 & 91.10 & 98.92 & 101.53 & 113.58 & 119.28 \\
\hline 133.09 & 135.21 & 140.94 & 145.01 & 149.43 & 156.08 \\
\hline 159.81 & 163.97 & 171.72 & 179.91 & 190.39 & 196.94 \\
\hline 199.35 & 207.90 & 219.80 & 223.79 & 224.33 & 227.48 \\
\hline 234.70 & 235.14 & 250.07 & 251.52 & 254.99 & 265.90 \\
\hline 270.21 & 276.96 & 279.78 & 280.40 & 284.99 & 291.50 \\
\hline 298.13 & 322.35 & 338.78 & 347.02 & 349.78 & 352.43 \\
\hline 360.51 & 363.96 & 375.53 & 376.84 & 383.26 & 389.74 \\
\hline 399.41 & 410.99 & 420.93 & 422.12 & 433.74 & 447.44 \\
\hline 454.19 & 459.67 & 465.03 & 467.15 & 469.40 & 473.49 \\
\hline 476.47 & 479.36 & 491.84 & 505.47 & 507.83 & 523.53 \\
\hline 534.34 & 538.37 & 540.85 & 567.40 & 570.22 & 577.30 \\
\hline 577.94 & 582.30 & 587.85 & 589.37 & 592.51 & 611.01 \\
\hline 612.87 & 617.25 & 618.09 & 627.14 & 630.67 & 634.29 \\
\hline 639.50 & 641.98 & 642.83 & 672.89 & 681.44 & 688.85 \\
\hline 692.30 & 696.26 & 702.95 & 708.92 & 729.03 & 730.93 \\
\hline 741.51 & 754.94 & 765.56 & 766.39 & 770.15 & 781.49 \\
\hline 785.34 & 792.69 & 795.86 & 796.66 & 798.83 & 802.96 \\
\hline 846.46 & 853.47 & 858.06 & 865.71 & 868.65 & 871.53 \\
\hline 872.76 & 875.31 & 879.07 & 883.70 & 889.87 & 894.53 \\
\hline 902.63 & 905.78 & 906.93 & 916.38 & 940.33 & 946.96 \\
\hline 950.96 & 953.87 & 954.05 & 962.34 & 966.75 & 974.01 \\
\hline 989.02 & 992.99 & 1002.58 & 1002.84 & 1006.23 & 1017.41 \\
\hline 1027.15 & 1048.15 & 1056.62 & 1064.02 & 1066.89 & 1070.43 \\
\hline 1070.65 & 1082.82 & 1093.13 & 1104.79 & 1111.12 & 1125.73 \\
\hline 1128.75 & 1131.99 & 1149.63 & 1154.20 & 1162.99 & 1166.12 \\
\hline 1167.01 & 1170.58 & 1172.57 & 1179.26 & 1180.11 & 1188.61 \\
\hline 1191.79 & 1199.45 & 1204.11 & 1210.12 & 1216.85 & 1228.41 \\
\hline
\end{tabular}

$\begin{array}{llllll}1239.03 & 1240.74 & 1256.41 & 1260.63 & 1271.25 & 1275.20\end{array}$ $\begin{array}{lllllll}1306.15 & 1314.71 & 1319.16 & 1322.98 & 1324.97 & 1328.45\end{array}$ $\begin{array}{llllll}1331.08 & 1331.78 & 1344.59 & 1345.70 & 1347.68 & 1350.73\end{array}$ $\begin{array}{llllll}1352.64 & 1354.89 & 1357.18 & 1366.71 & 1374.64 & 1386.09\end{array}$ $\begin{array}{lllllll}1394.02 & 1405.93 & 1408.23 & 1426.28 & 1430.66 & 1435.08\end{array}$ $\begin{array}{lllllll}1440.52 & 1442.10 & 1446.13 & 1465.10 & 1466.85 & 1474.89\end{array}$ $\begin{array}{lllllll}1475.84 & 1479.85 & 1491.65 & 1496.31 & 1503.92 & 1509.66\end{array}$ $\begin{array}{lllllll}1511.61 & 1512.06 & 1519.40 & 1522.47 & 1525.17 & 1530.92\end{array}$ $\begin{array}{lllllll}1544.44 & 1547.37 & 1549.17 & 1574.21 & 1584.22 & 1610.88\end{array}$ $\begin{array}{lllllll}1612.21 & 1623.24 & 1625.68 & 1626.86 & 1627.58 & 1658.82\end{array}$ $\begin{array}{lllllll}1659.75 & 1661.17 & 1667.04 & 1675.44 & 1675.78 & 1676.42\end{array}$ $\begin{array}{llllll}1724.88 & 3030.99 & 3044.62 & 3058.58 & 3063.68 & 3101.26\end{array}$ $\begin{array}{llllll}3127.73 & 3130.95 & 3138.67 & 3143.58 & 3153.94 & 3175.65\end{array}$ $\begin{array}{lllllll}3189.06 & 3194.02 & 3208.79 & 3221.30 & 3221.55 & 3221.99\end{array}$ $\begin{array}{lllllll}3222.16 & 3226.54 & 3229.47 & 3234.19 & 3234.81 & 3240.54\end{array}$ $\begin{array}{lllllll}3240.76 & 3241.27 & 3241.93 & 3253.93 & 3274.66 & 3281.71\end{array}$ $\begin{array}{lll}3288.88 & 3297.57 & 3592.31\end{array}$

$\left[{ }^{3} \mathbf{I r}-{ }^{1} \mathbf{Q}_{\mathbf{D}}\right]$

$\begin{array}{llllll}16.49 & 18.01 & 22.08 & 24.93 & 26.37 & 28.37\end{array}$

$\begin{array}{llllll}29.44 & 34.25 & 37.89 & 40.29 & 45.94 & 51.04\end{array}$

$\begin{array}{rrrrrr}53.20 & 57.94 & 65.36 & 67.35 & 73.59 & 77.31 \\ 79.35 & 89.84 & 90.94 & 100.95 & 102.44 & 110.52\end{array}$

$\begin{array}{rrrrrr}122.73 & 126.18 & 131.47 & 139.53 & 142.85 & 150.23\end{array}$

$\begin{array}{llllll}152.25 & 162.58 & 165.16 & 171.15 & 176.01 & 192.16\end{array}$

$\begin{array}{llllll}195.36 & 200.24 & 202.58 & 206.80 & 211.86 & 216.77\end{array}$

$\begin{array}{llllll}223.00 & 231.90 & 242.11 & 248.29 & 252.69 & 254.24\end{array}$

$\begin{array}{llllll}259.05 & 271.74 & 272.59 & 274.48 & 280.69 & 285.45\end{array}$

$\begin{array}{llllll}289.75 & 317.71 & 329.11 & 339.16 & 342.99 & 349.16\end{array}$

$\begin{array}{llllll}352.89 & 356.81 & 361.27 & 372.28 & 375.46 & 383.26\end{array}$

$\begin{array}{llllll}385.98 & 398.95 & 417.45 & 420.35 & 425.88 & 431.07\end{array}$

$\begin{array}{llllll}442.02 & 450.58 & 459.04 & 461.27 & 464.65 & 466.46\end{array}$

$\begin{array}{llllll}472.94 & 474.26 & 480.54 & 486.87 & 504.54 & 515.84\end{array}$

$\begin{array}{llllll}518.38 & 533.20 & 537.48 & 550.46 & 562.36 & 563.41\end{array}$

$\begin{array}{llllll}575.05 & 576.92 & 584.44 & 587.07 & 592.19 & 594.62\end{array}$

$\begin{array}{lllllll}614.80 & 615.82 & 617.67 & 618.94 & 626.18 & 628.92\end{array}$

$\begin{array}{llllll}632.57 & 636.80 & 641.08 & 668.18 & 674.60 & 679.11\end{array}$

$\begin{array}{llllll}679.40 & 687.00 & 692.68 & 700.61 & 705.62 & 728.24\end{array}$

$\begin{array}{llllll}732.64 & 738.13 & 739.91 & 750.70 & 758.23 & 763.17\end{array}$

$\begin{array}{llllll}766.10 & 778.60 & 783.68 & 789.89 & 793.32 & 797.83\end{array}$

$\begin{array}{llllll}800.77 & 805.63 & 822.78 & 843.28 & 851.83 & 864.75\end{array}$

$\begin{array}{llllll}866.44 & 869.92 & 872.04 & 876.05 & 885.13 & 889.93\end{array}$

$\begin{array}{llllll}892.29 & 896.16 & 903.27 & 905.83 & 938.52 & 947.34\end{array}$

$\begin{array}{llllll}951.13 & 952.87 & 956.25 & 958.90 & 963.02 & 967.66\end{array}$ $\begin{array}{llllll}972.70 & 986.13 & 988.71 & 991.89 & 998.57 & 1001.29\end{array}$ $\begin{array}{rrrrrr}1020.63 & 1027.82 & 1039.01 & 1045.30 & 1054.64 & 1055.88\end{array}$ $\begin{array}{lllllll}1065.22 & 1067.22 & 1071.16 & 1093.25 & 1097.11 & 1110.58\end{array}$ $\begin{array}{llllll}1125.28 & 1127.61 & 1132.12 & 1148.51 & 1159.85 & 1163.30\end{array}$ $\begin{array}{lllllll}1165.72 & 1166.54 & 1174.58 & 1176.11 & 1177.25 & 1182.02\end{array}$ $\begin{array}{lllllll}1187.47 & 1200.20 & 1200.63 & 1207.33 & 1215.49 & 1229.48\end{array}$ $\begin{array}{llllll}1231.69 & 1233.89 & 1243.83 & 1254.84 & 1270.21 & 1270.95\end{array}$ $\begin{array}{lllllll}1273.54 & 1303.74 & 1313.59 & 1321.42 & 1322.58 & 1328.10\end{array}$ $\begin{array}{lllllll}1328.47 & 1339.81 & 1342.91 & 1346.10 & 1347.56 & 1350.30\end{array}$ $\begin{array}{lllllll}1351.54 & 1355.27 & 1356.42 & 1368.71 & 1383.86 & 1393.47\end{array}$ $\begin{array}{lllllll}1394.46 & 1401.47 & 1406.26 & 1424.76 & 1429.74 & 1432.56\end{array}$ $\begin{array}{lllllll}1437.20 & 1438.38 & 1445.08 & 1452.19 & 1467.49 & 1472.89\end{array}$ $\begin{array}{lllllll}1473.67 & 1489.18 & 1489.73 & 1494.48 & 1499.93 & 1502.12\end{array}$ $\begin{array}{llllll}1508.89 & 1508.97 & 1510.36 & 1514.53 & 1515.24 & 1522.66\end{array}$ $\begin{array}{lllllll}1524.71 & 1529.30 & 1544.19 & 1547.87 & 1561.69 & 1574.52\end{array}$ $\begin{array}{llllll}1585.62 & 1595.45 & 1607.17 & 1619.13 & 1622.17 & 1624.78\end{array}$ $\begin{array}{lllllll}1625.58 & 1647.37 & 1660.27 & 1664.50 & 1674.47 & 1674.94\end{array}$ $\begin{array}{llllll}1721.11 & 3026.82 & 3037.36 & 3054.11 & 3056.19 & 3059.66\end{array}$ $\begin{array}{lllllll}3098.11 & 3127.46 & 3134.89 & 3143.76 & 3145.58 & 3175.09\end{array}$ $\begin{array}{lllllll}3187.34 & 3191.46 & 3204.78 & 3211.75 & 3217.53 & 3218.57\end{array}$ $\begin{array}{lllllll}3218.93 & 3225.82 & 3227.32 & 3228.18 & 3231.72 & 3234.13\end{array}$ $\begin{array}{lllllll}3237.66 & 3238.63 & 3246.40 & 3253.49 & 3268.67 & 3274.85\end{array}$ $3280.77 \quad 3297.00 \quad 3556.11$

$\left[{ }^{1} \mathbf{I} \mathbf{r}^{-3} \mathbf{Q} \mathbf{D}\right]$

$\begin{array}{llllll}13.56 & 17.78 & 23.57 & 24.87 & 25.69 & 29.66\end{array}$

$\begin{array}{llllll}30.07 & 32.13 & 34.85 & 36.50 & 40.70 & 44.32\end{array}$

$\begin{array}{llllll}52.02 & 61.27 & 65.25 & 70.53 & 75.59 & 76.17\end{array}$

$\begin{array}{llllll}80.15 & 82.54 & 88.88 & 97.73 & 107.39 & 114.77\end{array}$

$\begin{array}{llllll}122.60 & 124.25 & 130.16 & 137.11 & 140.24 & 147.01\end{array}$

$\begin{array}{llllll}152.50 & 159.40 & 169.01 & 177.63 & 185.49 & 196.65\end{array}$

$\begin{array}{llllll}199.97 & 206.08 & 211.66 & 216.44 & 219.59 & 222.12\end{array}$ 


\begin{tabular}{rrrrrr}
259.64 & 263.32 & 270.98 & 273.33 & 277.06 & 280.78 \\
286.53 & 289.62 & 311.46 & 335.03 & 346.52 & 350.28 \\
352.82 & 359.51 & 373.69 & 375.81 & 381.47 & 387.51 \\
390.62 & 397.69 & 409.53 & 415.61 & 424.05 & 433.29 \\
433.84 & 447.53 & 452.66 & 457.51 & 461.48 & 463.41 \\
467.45 & 468.85 & 475.43 & 504.10 & 505.94 & 520.51 \\
525.27 & 529.78 & 532.79 & 536.93 & 559.15 & 566.98 \\
576.74 & 577.07 & 579.77 & 587.55 & 588.83 & 591.89 \\
601.96 & 607.78 & 609.31 & 610.87 & 617.79 & 622.61 \\
626.02 & 629.70 & 634.31 & 637.83 & 639.35 & 661.15 \\
669.89 & 680.23 & 687.29 & 688.97 & 690.72 & 694.33 \\
707.73 & 725.92 & 727.41 & 739.37 & 740.52 & 758.95 \\
764.31 & 789.68 & 789.94 & 792.02 & 794.02 & 800.25 \\
801.37 & 828.58 & 841.27 & 851.24 & 855.23 & 858.69 \\
864.58 & 866.04 & 867.05 & 875.03 & 875.57 & 882.63 \\
885.99 & 895.55 & 905.64 & 913.98 & 916.77 & 918.17 \\
941.76 & 944.00 & 945.38 & 953.78 & 955.24 & 967.08 \\
970.02 & 989.70 & 991.04 & 996.90 & 998.35 & 1016.81 \\
1026.83 & 1046.12 & 1049.88 & 1054.24 & 1060.19 & 1067.45 \\
1068.89 & 1081.39 & 1092.82 & 1104.09 & 1107.06 & 1122.88 \\
1124.93 & 1128.45 & 1130.36 & 1135.27 & 1154.91 & 1160.92 \\
1165.47 & 1169.24 & 1171.42 & 1177.04 & 1177.29 & 1179.11 \\
1186.90 & 1189.95 & 1191.19 & 1197.75 & 1204.96 & 1215.38 \\
1227.25 & 1233.34 & 1238.97 & 1254.47 & 1259.81 & 1271.96 \\
1279.27 & 1303.25 & 1314.75 & 1318.17 & 1321.51 & 1321.97 \\
1327.94 & 1329.61 & 1330.05 & 1345.37 & 1345.71 & 1349.36 \\
1351.36 & 1352.71 & 1355.98 & 1356.25 & 1367.02 & 1374.38 \\
1378.45 & 1397.62 & 1405.27 & 1424.58 & 1425.75 & 1433.09 \\
1437.09 & 1440.17 & 1444.24 & 1458.56 & 1461.25 & 1466.46 \\
1473.97 & 1479.41 & 1489.73 & 1493.37 & 1494.45 & 1501.25 \\
1507.25 & 1509.10 & 1510.27 & 1512.56 & 1514.70 & 1523.91 \\
1526.69 & 1537.59 & 1542.42 & 1545.93 & 1573.68 & 1578.83 \\
1588.86 & 1598.41 & 1607.46 & 1612.03 & 1623.05 & 1623.78 \\
1626.56 & 1649.58 & 1658.62 & 1660.32 & 1671.06 & 1674.19 \\
1676.68 & 3026.99 & 3055.82 & 3060.82 & 3069.70 & 3077.98 \\
3097.75 & 3132.21 & 3139.55 & 3140.97 & 3152.31 & 3174.14 \\
3185.75 & 3189.14 & 3203.86 & 3217.55 & 3218.08 & 3218.70 \\
3218.98 & 3224.62 & 3225.21 & 3231.82 & 3233.32 & 3237.70 \\
3240.93 & 3245.38 & 3250.91 & 3254.05 & 3275.68 & 3278.46 \\
3280.70 & 3296.80 & 3627.65 & & & \\
\hline & & & & &
\end{tabular}

\section{${ }^{1}[\mathbf{I r}-\mathbf{Q}-2]$}

$\begin{array}{llllll}10.38 & 18.79 & 23.21 & 26.32 & 28.01 & 29.21\end{array}$

$\begin{array}{llllll}32.12 & 33.19 & 36.52 & 39.92 & 43.99 & 45.48\end{array}$

$\begin{array}{llllll}51.77 & 52.74 & 59.32 & 63.28 & 66.51 & 69.32\end{array}$

$\begin{array}{rrrrrr}74.36 & 76.30 & 80.07 & 82.24 & 86.39 & 96.07 \\ 96.64 & 98.49 & 107.24 & 114.60 & 118.14 & 122.74\end{array}$

$\begin{array}{llllll}127.54 & 130.38 & 143.32 & 144.75 & 151.83 & 153.52\end{array}$

$\begin{array}{llllll}158.97 & 160.95 & 165.47 & 167.42 & 179.85 & 183.09\end{array}$

$\begin{array}{llllll}197.56 & 202.64 & 211.81 & 218.40 & 219.97 & 224.44\end{array}$

$\begin{array}{llllll}229.96 & 232.48 & 236.88 & 242.74 & 249.28 & 253.22\end{array}$

$\begin{array}{llllll}266.52 & 268.86 & 271.15 & 277.76 & 284.48 & 287.78\end{array}$

$\begin{array}{llllll}289.35 & 291.41 & 304.38 & 320.13 & 342.61 & 346.64\end{array}$

$\begin{array}{llllll}348.13 & 350.83 & 360.71 & 372.26 & 375.96 & 378.63\end{array}$

$\begin{array}{llllll}381.45 & 393.03 & 396.33 & 407.97 & 410.99 & 424.30\end{array}$

$\begin{array}{llllll}427.61 & 436.14 & 445.65 & 449.77 & 456.22 & 464.82\end{array}$

$\begin{array}{llllll}467.52 & 470.03 & 474.00 & 476.71 & 478.36 & 488.10\end{array}$

$\begin{array}{lllllll}505.18 & 505.87 & 530.19 & 531.96 & 539.07 & 539.62\end{array}$

$\begin{array}{llllll}549.17 & 561.82 & 568.45 & 577.29 & 578.38 & 582.07\end{array}$

$\begin{array}{llllll}586.24 & 587.80 & 588.91 & 607.14 & 608.45 & 619.17\end{array}$

$\begin{array}{llllll}620.58 & 623.95 & 628.48 & 630.13 & 631.76 & 639.03\end{array}$

$\begin{array}{llllll}640.37 & 644.09 & 651.02 & 681.67 & 685.06 & 685.88\end{array}$

$\begin{array}{llllll}688.97 & 692.33 & 695.18 & 702.94 & 707.92 & 724.76\end{array}$

$\begin{array}{llllll}737.43 & 745.47 & 752.76 & 763.98 & 770.07 & 770.45\end{array}$

$\begin{array}{lllllll}771.50 & 787.01 & 791.58 & 793.16 & 798.14 & 802.53\end{array}$

$\begin{array}{llllll}822.64 & 844.47 & 845.84 & 853.11 & 855.29 & 855.85\end{array}$

$\begin{array}{llllll}858.05 & 863.05 & 864.42 & 869.64 & 871.14 & 871.83\end{array}$

$\begin{array}{llllll}874.44 & 888.58 & 901.24 & 905.20 & 907.50 & 908.62\end{array}$

$\begin{array}{llllll}912.58 & 913.33 & 914.90 & 925.22 & 940.27 & 943.82\end{array}$

$\begin{array}{lllllll}955.70 & 959.62 & 961.35 & 965.86 & 969.04 & 971.15\end{array}$

$\begin{array}{llllll}976.11 & 989.16 & 991.63 & 991.78 & 997.61 & 997.70\end{array}$

$\begin{array}{llllll}1024.51 & 1027.21 & 1049.74 & 1055.72 & 1061.46 & 1064.16\end{array}$

$\begin{array}{lllllll}1067.66 & 1069.76 & 1080.40 & 1082.73 & 1093.56 & 1110.33\end{array}$

$\begin{array}{llllll}1114.75 & 1126.72 & 1127.14 & 1135.56 & 1145.54 & 1160.27\end{array}$

$\begin{array}{llllll}1163.87 & 1166.04 & 1168.21 & 1169.80 & 1175.70 & 1177.08\end{array}$

$\begin{array}{llllll}1178.77 & 1186.64 & 1189.09 & 1197.38 & 1200.53 & 1200.84\end{array}$

$\begin{array}{llllll}1209.23 & 1215.42 & 1216.47 & 1220.51 & 1234.33 & 1257.92\end{array}$

$\begin{array}{llllll}1261.92 & 1271.85 & 1274.54 & 1305.50 & 1316.80 & 1318.07\end{array}$ $\begin{array}{llllll}1323.93 & 1326.11 & 1328.15 & 1329.92 & 1330.63 & 1337.54\end{array}$ $\begin{array}{lllllll}1345.82 & 1348.49 & 1349.13 & 1350.34 & 1352.64 & 1354.37\end{array}$ $\begin{array}{lllllll}1357.52 & 1371.09 & 1374.30 & 1375.11 & 1384.49 & 1393.10\end{array}$ $\begin{array}{lllllll}1402.91 & 1403.78 & 1425.97 & 1433.79 & 1435.73 & 1439.35\end{array}$ $\begin{array}{lllllll}1440.73 & 1445.48 & 1459.95 & 1463.07 & 1465.89 & 1478.08\end{array}$ $\begin{array}{lllllll}1481.17 & 1488.17 & 1491.27 & 1497.23 & 1506.09 & 1510.31\end{array}$ $\begin{array}{llllll}1511.35 & 1511.87 & 1519.42 & 1525.63 & 1530.27 & 1533.76\end{array}$ $\begin{array}{lllllll}1544.80 & 1548.63 & 1552.67 & 1579.15 & 1592.52 & 1611.00\end{array}$ $\begin{array}{lllllll}1613.00 & 1623.38 & 1623.97 & 1625.81 & 1628.21 & 1647.57\end{array}$ $\begin{array}{llllll}1658.66 & 1660.51 & 1663.94 & 1664.67 & 1672.39 & 1676.20\end{array}$ $\begin{array}{lllllll}1677.69 & 1717.07 & 1787.32 & 1848.21 & 3023.51 & 3025.30\end{array}$ $\begin{array}{llllll}3056.97 & 3068.63 & 3076.58 & 3095.36 & 3132.04 & 3139.21\end{array}$ $\begin{array}{lllllll}3141.75 & 3172.42 & 3176.72 & 3189.06 & 3193.70 & 3204.73\end{array}$ $\begin{array}{lllllll}3212.37 & 3214.21 & 3216.65 & 3220.32 & 3222.74 & 3223.38\end{array}$ $\begin{array}{lllllll}3225.36 & 3229.56 & 3233.47 & 3233.76 & 3235.11 & 3238.08\end{array}$ $\begin{array}{lllllll}3242.93 & 3248.52 & 3254.34 & 3263.49 & 3277.43 & 3281.52\end{array}$ $3294.39 \quad 3297.09 \quad 3311.10$

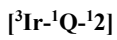

$\begin{array}{llllll}17.11 & 21.36 & 26.21 & 27.56 & 28.69 & 33.15\end{array}$

$\begin{array}{llllll}35.47 & 39.17 & 43.26 & 43.84 & 45.90 & 48.20\end{array}$

$\begin{array}{llllll}53.81 & 57.93 & 62.19 & 70.04 & 71.98 & 75.94\end{array}$

$\begin{array}{rrrrrr}79.22 & 81.21 & 83.18 & 87.90 & 94.36 & 97.04\end{array}$

$\begin{array}{lrrrrr}101.09 & 107.57 & 113.57 & 117.32 & 121.12 & 124.28\end{array}$

$\begin{array}{llllll}127.35 & 129.58 & 143.11 & 146.15 & 154.01 & 157.24\end{array}$

$\begin{array}{llllll}159.02 & 160.51 & 168.77 & 178.65 & 179.82 & 184.05\end{array}$

$\begin{array}{llllll}197.02 & 200.41 & 209.27 & 211.62 & 219.01 & 223.88\end{array}$

$\begin{array}{llllll}226.19 & 227.61 & 231.90 & 239.57 & 247.80 & 252.18\end{array}$

$\begin{array}{llllll}263.54 & 270.31 & 270.97 & 278.64 & 283.39 & 288.73\end{array}$

$\begin{array}{llllll}290.77 & 292.03 & 321.54 & 329.14 & 334.96 & 342.92\end{array}$

$\begin{array}{llllll}348.78 & 350.04 & 353.29 & 363.97 & 374.50 & 375.66\end{array}$

$\begin{array}{llllll}378.01 & 383.07 & 391.43 & 398.74 & 408.04 & 423.39\end{array}$

$\begin{array}{llllll}424.95 & 426.85 & 436.16 & 449.50 & 452.45 & 462.15\end{array}$

$\begin{array}{llllll}464.22 & 467.81 & 471.46 & 477.24 & 479.35 & 487.26\end{array}$

$\begin{array}{llllll}490.76 & 505.85 & 520.68 & 528.39 & 531.34 & 538.18\end{array}$

$\begin{array}{llllll}553.63 & 558.76 & 560.10 & 565.70 & 576.17 & 577.80\end{array}$

$\begin{array}{llllll}584.98 & 588.83 & 592.97 & 601.50 & 614.25 & 616.76\end{array}$

$\begin{array}{llllll}617.48 & 621.04 & 621.54 & 623.97 & 627.50 & 632.81\end{array}$

$\begin{array}{llllll}637.13 & 639.99 & 644.58 & 680.64 & 681.60 & 684.78\end{array}$

$\begin{array}{llllll}688.75 & 692.37 & 692.89 & 695.32 & 701.32 & 704.31\end{array}$

$\begin{array}{llllll}731.95 & 744.11 & 745.47 & 747.56 & 753.35 & 760.76\end{array}$

$\begin{array}{llllll}762.45 & 763.23 & 768.41 & 774.53 & 788.66 & 792.41\end{array}$

$\begin{array}{llllll}792.98 & 798.66 & 807.33 & 818.94 & 832.91 & 845.17\end{array}$

$\begin{array}{llllll}851.86 & 858.73 & 865.16 & 865.64 & 872.15 & 876.34\end{array}$

$\begin{array}{llllll}878.37 & 883.08 & 890.31 & 901.69 & 908.27 & 909.07\end{array}$

$\begin{array}{llllll}910.78 & 915.63 & 928.27 & 930.83 & 942.23 & 949.02\end{array}$

$\begin{array}{llllll}952.72 & 955.92 & 956.60 & 963.67 & 968.13 & 968.82\end{array}$

$\begin{array}{llllll}973.25 & 975.97 & 993.24 & 995.36 & 997.50 & 999.72\end{array}$ $\begin{array}{llllll}1002.72 & 1012.77 & 1045.01 & 1049.37 & 1050.36 & 1056.72\end{array}$ $\begin{array}{lllllll}1062.68 & 1066.50 & 1071.45 & 1075.67 & 1092.61 & 1093.87\end{array}$ $\begin{array}{llllll}1098.13 & 1110.82 & 1127.51 & 1137.00 & 1142.98 & 1160.18\end{array}$ $\begin{array}{llllll}1162.33 & 1164.04 & 1166.03 & 1166.77 & 1170.16 & 1172.64\end{array}$ $\begin{array}{llllll}1176.58 & 1177.25 & 1180.18 & 1187.15 & 1197.06 & 1200.04\end{array}$ $\begin{array}{lllllll}1208.41 & 1210.11 & 1215.71 & 1220.75 & 1229.67 & 1250.95\end{array}$ $\begin{array}{lllllll}1257.80 & 1274.30 & 1276.74 & 1279.49 & 1305.82 & 1317.18\end{array}$ $\begin{array}{llllll}1322.08 & 1325.04 & 1330.58 & 1332.16 & 1333.40 & 1338.42\end{array}$ $\begin{array}{lllllll}1341.82 & 1346.83 & 1347.73 & 1350.62 & 1351.92 & 1353.29\end{array}$ $\begin{array}{llllll}1358.81 & 1364.26 & 1372.34 & 1374.22 & 1388.21 & 1393.96\end{array}$ $\begin{array}{lllllll}1405.28 & 1407.36 & 1426.89 & 1429.07 & 1431.78 & 1435.37\end{array}$ $\begin{array}{lllllll}1439.48 & 1442.90 & 1447.51 & 1457.11 & 1466.03 & 1475.89\end{array}$ $\begin{array}{lllllll}1479.18 & 1488.84 & 1491.03 & 1491.69 & 1497.40 & 1506.25\end{array}$ $\begin{array}{lllllll}1510.16 & 1511.78 & 1512.14 & 1518.07 & 1521.00 & 1529.56\end{array}$ $\begin{array}{llllll}1532.69 & 1539.31 & 1543.13 & 1547.32 & 1553.16 & 1581.64\end{array}$ $\begin{array}{lllllll}1587.00 & 1594.63 & 1604.57 & 1606.49 & 1609.11 & 1623.78\end{array}$ $\begin{array}{llllll}1625.36 & 1625.74 & 1651.23 & 1655.21 & 1661.94 & 1669.63\end{array}$ $\begin{array}{lllllll}1677.07 & 1722.11 & 1741.73 & 1793.46 & 3023.97 & 3024.47\end{array}$ $\begin{array}{lllllll}3058.30 & 3069.42 & 3080.11 & 3094.56 & 3132.95 & 3136.13\end{array}$ $\begin{array}{lllllll}3141.73 & 3142.27 & 3173.06 & 3176.86 & 3190.81 & 3196.50\end{array}$ $\begin{array}{lllllll}3206.88 & 3215.60 & 3217.97 & 3220.67 & 3221.96 & 3223.94\end{array}$ $\begin{array}{llllll}3227.38 & 3234.50 & 3235.63 & 3238.05 & 3238.56 & 3243.08\end{array}$ $\begin{array}{lllllll}3254.04 & 3255.18 & 3257.60 & 3258.73 & 3281.79 & 3282.14\end{array}$ $\begin{array}{lll}3283.23 & 3297.23 & 3385.33\end{array}$

$\left[{ }^{1} \mathbf{I r}-{ }^{3} \mathbf{Q}-{ }^{-12}\right]$

$\begin{array}{llllll}17.53 & 23.50 & 25.00 & 26.52 & 28.11 & 30.59 \\ 33.38 & 34.02 & 36.87 & 41.51 & 42.12 & 50.07\end{array}$

$\begin{array}{llllll}33.38 & 34.02 & 36.87 & 41.51 & 42.12 & 50.07 \\ 50.55 & 54.05 & 56.62 & 62.15 & 66.05 & 67.32\end{array}$ 


\begin{tabular}{|c|c|c|c|c|c|}
\hline 71.98 & 73.74 & 76.97 & 81.68 & 83.27 & 92.93 \\
\hline 95.48 & 96.32 & 103.82 & 106.34 & 114.07 & 117.71 \\
\hline 118.40 & 126.69 & 133.04 & 141.80 & 142.35 & 148.78 \\
\hline 152.92 & 157.99 & 167.99 & 169.38 & 176.98 & 180.67 \\
\hline 195.90 & 203.97 & 206.19 & 210.44 & 219.67 & 221.29 \\
\hline 221.95 & 231.16 & 231.47 & 232.70 & 244.25 & 248.13 \\
\hline 252.26 & 263.65 & 265.26 & 267.04 & 270.45 & 278.52 \\
\hline 288.43 & 290.20 & 291.05 & 305.94 & 314.33 & 332.95 \\
\hline 344.72 & 350.82 & 354.48 & 365.90 & 369.96 & 374.32 \\
\hline 380.94 & 390.04 & 393.25 & 394.82 & 403.71 & 405.78 \\
\hline 407.37 & 417.44 & 432.47 & 435.34 & 443.46 & 446.29 \\
\hline 452.21 & 459.42 & 459.68 & 468.63 & 470.39 & 472.11 \\
\hline 504.58 & 506.08 & 517.72 & 522.30 & 528.26 & 530.73 \\
\hline 535.28 & 550.63 & 566.97 & 575.91 & 576.61 & 580.45 \\
\hline 584.72 & 587.52 & 588.51 & 601.31 & 603.81 & 606.01 \\
\hline 608.60 & 616.38 & 619.67 & 621.87 & 622.56 & 628.59 \\
\hline 629.93 & 633.49 & 637.75 & 638.84 & 649.30 & 667.10 \\
\hline 668.70 & 684.34 & 685.79 & 686.95 & 688.33 & 693.04 \\
\hline 706.10 & 706.80 & 723.20 & 725.11 & 738.20 & 741.02 \\
\hline 760.52 & 763.00 & 770.88 & 786.60 & 790.05 & 791.96 \\
\hline 792.87 & 799.42 & 821.47 & 829.12 & 839.61 & 847.25 \\
\hline 849.31 & 852.30 & 854.70 & 861.50 & 861.52 & 866.70 \\
\hline 866.95 & 869.86 & 872.66 & 874.75 & 878.50 & 889.14 \\
\hline 904.68 & 912.79 & 918.13 & 920.12 & 931.76 & 941.64 \\
\hline 944.53 & 950.51 & 959.67 & 961.80 & 968.44 & 968.52 \\
\hline 971.06 & 971.49 & 988.60 & 991.52 & 991.96 & 994.80 \\
\hline 1008.43 & 1026.24 & 1045.98 & 1047.55 & 1049.47 & 1053.32 \\
\hline 1064.18 & 1068.93 & 1079.19 & 1080.42 & 1093.27 & 1094.07 \\
\hline 1112.59 & 1113.87 & 1121.88 & 1125.91 & 1128.36 & 1134.74 \\
\hline 1162.14 & 1163.40 & 1164.08 & 1168.84 & 1173.03 & 1175.68 \\
\hline 1177.38 & 1177.65 & 1181.73 & 1182.91 & 1187.91 & 1189.87 \\
\hline 1200.31 & 1207.84 & 1215.17 & 1215.40 & 1225.20 & 1234.01 \\
\hline 1245.73 & 1259.67 & 1268.26 & 1286.24 & 1305.47 & 1310.74 \\
\hline 1316.47 & 1319.40 & 1324.78 & 1326.55 & 1327.54 & 1328.78 \\
\hline 1330.74 & 1343.24 & 1345.19 & 1346.74 & 1346.96 & 1348.46 \\
\hline 1351.62 & 1353.11 & 1357.71 & 1361.54 & 1372.68 & 1373.47 \\
\hline 1393.67 & 1404.37 & 1426.35 & 1431.50 & 1432.88 & 1438.03 \\
\hline 1439.85 & 1442.38 & 1458.68 & 1464.96 & 1465.47 & 1474.21 \\
\hline 1477.06 & 1478.99 & 1489.47 & 1495.77 & 1503.97 & 1506.04 \\
\hline 1508.95 & 1509.90 & 1510.21 & 1513.93 & 1515.64 & 1523.33 \\
\hline 1533.31 & 1537.20 & 1540.95 & 1546.62 & 1571.55 & 1575.27 \\
\hline 1589.56 & 1596.04 & 1608.26 & 1609.63 & 1623.45 & 1623.52 \\
\hline 1626.24 & 1632.37 & 1657.40 & 1659.51 & 1661.14 & 1671.06 \\
\hline 1675.53 & 1676.58 & 1775.65 & 1819.66 & 3025.55 & 3056.21 \\
\hline 3061.96 & 3074.89 & 3076.26 & 3095.84 & 3135.15 & 3140.26 \\
\hline 3150.10 & 3173.13 & 3178.41 & 3190.31 & 3193.74 & 3195.60 \\
\hline 3205.70 & 3213.98 & 3216.88 & 3218.14 & 3219.82 & 3225.15 \\
\hline 3228.47 & 3230.15 & 3233.45 & 3238.29 & 3239.95 & 3243.20 \\
\hline 3245.81 & 3250.75 & 3251.71 & 3271.55 & 3281.16 & 3281.27 \\
\hline 3286.71 & 3297.64 & 3372.84 & & & \\
\hline
\end{tabular}

[ ${ }^{1} \mathbf{r}-{ }^{-1} \mathbf{Q}^{-3}$ 2]

\begin{tabular}{|c|c|c|c|c|c|}
\hline 18.70 & 23.49 & 27.81 & 29.41 & 31.81 & \\
\hline 35.15 & 36.19 & 40.24 & 45.72 & & \\
\hline 55.39 & 58.70 & 60.41 & 66.17 & & \\
\hline 77.48 & 1.59 & 82.90 & & & \\
\hline 104.36 & 106.95 & 113.82 & 116.57 & & \\
\hline 128.14 & 130.16 & 44.61 & & & \\
\hline 159.49 & 163.99 & 172.86 & & & \\
\hline 197.9 & 206. & & & & \\
\hline 229.64 & 232. & 6 & 243 & & \\
\hline 268.15 & 69.70 & 6 & 277 & & \\
\hline 289.86 & 2.23 & 1 & 320 & & 8.2 \\
\hline 350.23 & 1.31 & & & & \\
\hline 380.70 & 2.33 & 57 & 399 & & 8.2 \\
\hline 421.96 & 8.97 & 2 & 4 & & \\
\hline 464.89 & 467.65 & 469. & 472 & & \\
\hline 489.00 & 504.91 & 506 & 5 & & \\
\hline 538.93 & 558.99 & 561 & 565 & & 578.2 \\
\hline 578.38 & 584.42 & 587. & 589 & 590 & \\
\hline 610.42 & 612.39 & 20. & 622 & & \\
\hline 30.75 & 7.99 & 9 & & & \\
\hline 35.49 & & & & & \\
\hline 714.12 & 720.7 & 726 & & & \\
\hline 762.78 & 764.98 & & & & \\
\hline 799.13 & 801.3 & & & & \\
\hline 857.1 & & & & & \\
\hline 875.82 & & & & & \\
\hline 906.9 & & & 92 & & 46.2 \\
\hline 954.8 & & & 96 & & 977.6 \\
\hline 978.3 & & & & & 1002.6 \\
\hline 1005.47 & 25.52 & 1 & 7 & 8 & 060.9 \\
\hline 2.63 & 6.25 & & & & 9.0 \\
\hline 1.85 & 25.15 & 28.38 & & 113 & 146.8 \\
\hline 0.43 & 5.25 & & & 77 & 175.4 \\
\hline 7.61 & 49 & & & & 199.7 \\
\hline 7.56 & & & & & 231.9 \\
\hline 7.43 & 0.54 & & & & \\
\hline 16.33 & 21.02 & & & 0 & \\
\hline 33.81 & 41.80 & & 4 & 65 & \\
\hline & & & & & \\
\hline 1393.06 & 06.08 & & & & 1431. \\
\hline & & & & & 1468.2 \\
\hline & & & & & \\
\hline & & & & & 530.2 \\
\hline & & & & & 590.8 \\
\hline & & & & & \\
\hline & & & & & \\
\hline & & & & 3004 & 3024.5 \\
\hline & & & & & \\
\hline & & & & 3188 & \\
\hline & & & & 3219. & \\
\hline & & & 3233 & 3233 & \\
\hline & & 3251.52 & 3266.30 & 3273.61 & 3280. \\
\hline 288.14 & 3290.51 & & & & \\
\hline
\end{tabular}




\section{Cyclic Voltammetry Data}

Cyclic voltammetry experiments were performed in a standard three electrode setup in $\mathrm{CH}_{2} \mathrm{Cl}_{2}$ with the indicated analyte $(1 \mathrm{mM})$ and $\operatorname{TBAPF}_{6}(0.1 \mathrm{M})$ using a glassy carbon working electrode, platinum wire counter electrode, and a $\mathrm{Ag} / \mathrm{AgNO}_{3}(\mathrm{MeCN})$ reference electrode at a scan rate of $50 \mathrm{mV} / \mathrm{s}$. The potentials were referenced to SCE through an external ferrocene standard $\left(\mathrm{Fc}^{+/ 0}=460 \mathrm{mV}\right.$ vs. SCE $){ }^{29}$ All the analytes showed irreversible reduction and oxidation. Half-peak potentials were estimated from the voltammograms.

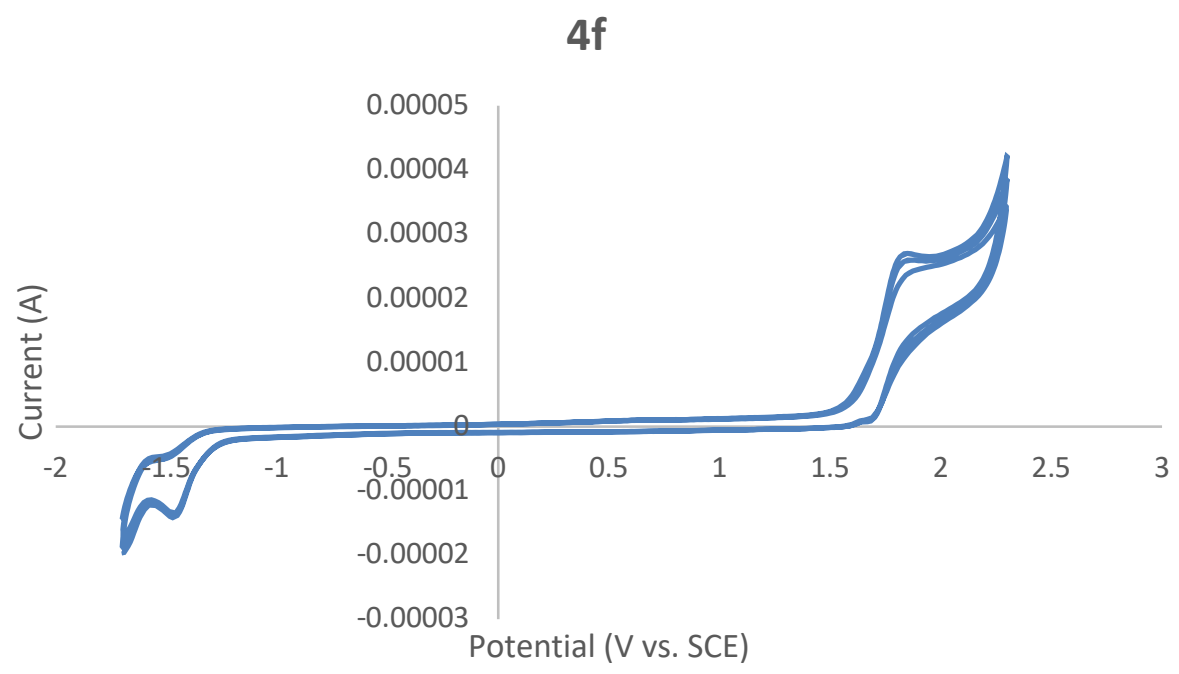

4f:

$$
\begin{aligned}
& \mathrm{E}_{\mathrm{p} / 2}\left(\mathrm{Ir}^{\mathrm{III}} / \mathrm{Ir}^{\mathrm{II}}\right)=-1.36 \mathrm{~V} \text { vs. SCE in } \mathrm{CH}_{2} \mathrm{Cl}_{2} \\
& \mathrm{E}_{\mathrm{p} / 2}\left(\mathrm{Ir}^{\mathrm{IV}} / \mathrm{Ir}^{\mathrm{III}}\right)=+1.71 \mathrm{~V} \text { vs. SCE in } \mathrm{CH}_{2} \mathrm{Cl}_{2}
\end{aligned}
$$

\section{Quinolone 1c}

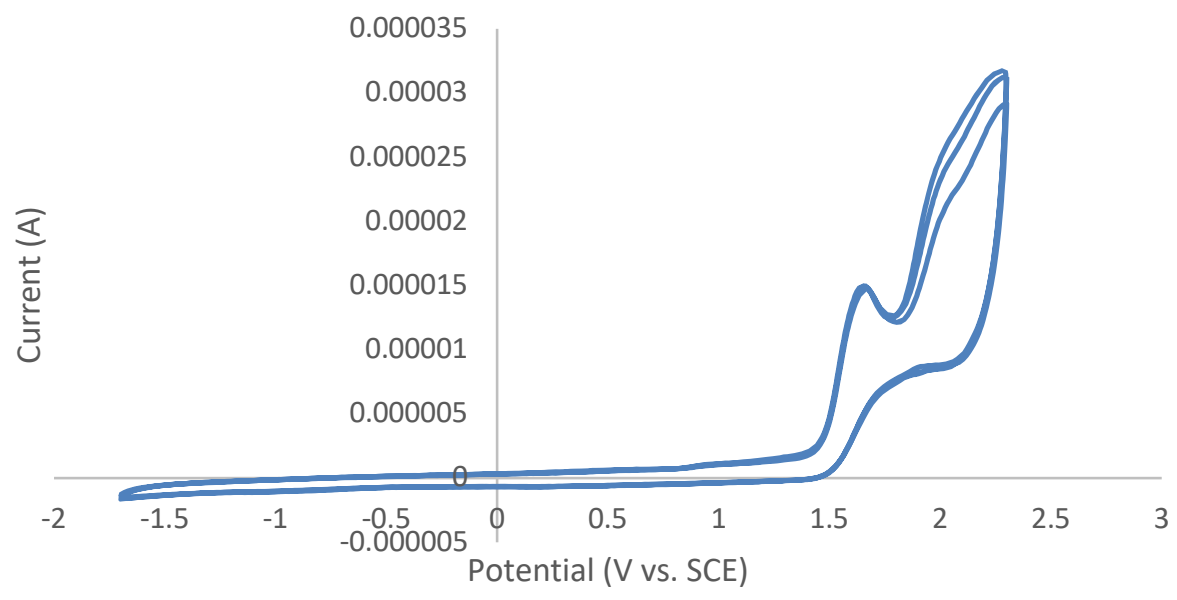

Quinolone 1c:

$$
\begin{gathered}
\mathrm{E}_{\mathrm{p} / 2}\left(\mathbf{1} \mathbf{c} / \mathbf{1} \mathbf{c}^{\bullet-}\right)<-1.69 \mathrm{~V} \text { vs. SCE in } \mathrm{CH}_{2} \mathrm{Cl}_{2} \\
\mathrm{E}_{\mathrm{p} / 2}\left(\mathbf{1} \mathbf{c}^{\bullet+} / \mathbf{1} \mathbf{c}\right)=+1.54 \mathrm{~V} \text { vs. SCE in } \mathrm{CH}_{2} \mathrm{Cl}_{2} \\
\text { s113 }
\end{gathered}
$$




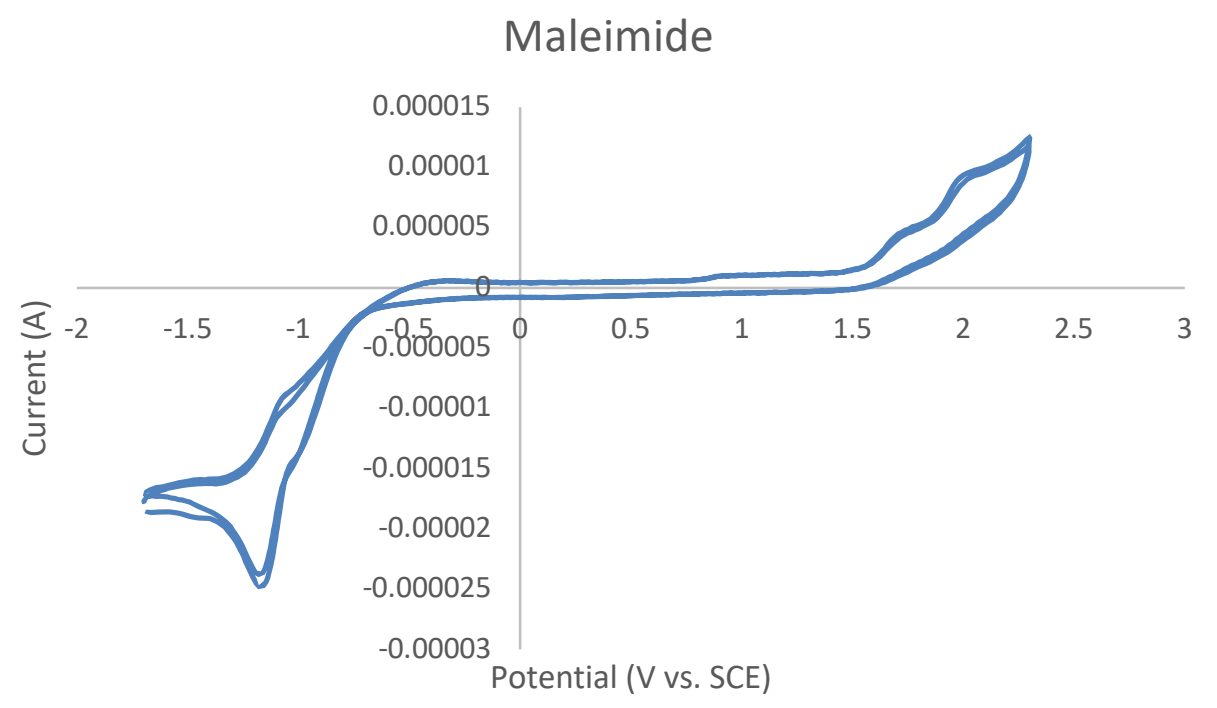

Maleimide 2:

$$
\begin{aligned}
& \mathrm{E}_{\mathrm{p} / 2}\left(\mathbf{2}^{-\mathbf{2}^{--}}\right)=-1.09 \mathrm{~V} \text { vs. SCE in } \mathrm{CH}_{2} \mathrm{Cl}_{2} \\
& \mathrm{E}_{\mathrm{p} / 2}\left(\mathbf{2}^{\mathbf{+}} \mathbf{2}\right)=+1.64 \mathrm{~V} \text { vs. SCE in } \mathrm{CH}_{2} \mathrm{Cl}_{2}
\end{aligned}
$$

The excited-state redox potentials of photocatalyst $\mathbf{4 f}$ were calculated through equations below ${ }^{30,31}$ using the ground state potentials above and the Gibbs free energy change for the $S_{0}$ to $T_{1}$ transition $\left(\Delta \mathrm{G}_{\mathrm{ES}}\right)$ estimated as the energy at $10 \%$ of the PL maxima. ${ }^{32,33,34}$ This provides a generous estimate for the excited-state redox potentials, and demonstrates that photoinduced electron transfer is endergonic in our reactions.

$$
\begin{aligned}
& \mathrm{E}_{\mathrm{p} / 2}\left(\mathrm{Ir}^{\mathrm{III}} / \mathrm{Ir}^{\mathrm{II}}\right)=\mathrm{E}_{\mathrm{p} / 2}\left(\mathrm{Ir}^{\mathrm{III}} / \mathrm{Ir}^{\mathrm{II}}\right)+\Delta \mathrm{G}_{\mathrm{ES}}=-1.36 \mathrm{~V}+2.61 \mathrm{~V}=+1.25 \mathrm{~V} \text { vs. SCE in } \mathrm{CH}_{2} \mathrm{Cl}_{2} \\
& \mathrm{E}_{\mathrm{p} / 2}\left(\mathrm{Ir}^{\mathrm{IV}} / \mathrm{Ir}^{\mathrm{III}}\right)=\mathrm{E}_{\mathrm{p} / 2}\left(\mathrm{Ir}^{\mathrm{IV}} / \mathrm{Ir}^{\mathrm{III}}\right)-\Delta \mathrm{G}_{\mathrm{ES}}=1.71 \mathrm{~V}-2.61 \mathrm{~V}=-0.90 \mathrm{~V} \text { vs. SCE in } \mathrm{CH}_{2} \mathrm{Cl}_{2}
\end{aligned}
$$

\section{Quantum yield of the parent reaction}

Determination of the light intensity at $436 \mathrm{~nm}$ : The intensity of the $436 \mathrm{~nm}$ blue LED light source used in these studies was determined as previously reported. ${ }^{35,36,37,38} \mathrm{~A} 0.15 \mathrm{M}$ solution of potassium ferrioxalate was prepared by dissolving $2.21 \mathrm{~g}$ of potassium ferrioxalate hydrate in $30 \mathrm{~mL} \mathrm{H}_{2} \mathrm{SO}_{4}(0.05 \mathrm{M})$. A buffered solution of phenanthroline was prepared by dissolving $50 \mathrm{mg}$ phenanthroline and $11.25 \mathrm{~g}$ sodium acetate in $50 \mathrm{~mL} \mathrm{H}_{2} \mathrm{SO}_{4}(0.5 \mathrm{M})$. Both solutions were stored in the dark. Next, a Schlenk tube was completely covered in black tape except for a small hole near the base (see Figure S12). The prepared tube was charged with $5 \mathrm{~mL}$ of the ferrioxalate solution and irradiated for 10 seconds. This solution was treated with $0.875 \mathrm{~mL}$ buffered phenanthroline solution and stirred for $1 \mathrm{~h}$ in dark to allow the ferrous ions to completely coordinate to the phenanthroline. The absorbance of the resulting solution was measured at 510 $\mathrm{nm}$. A non-irradiated sample was also prepared and the absorbance was measured at $510 \mathrm{~nm}$. Finally, the conversion of ferrioxalate was calculated as below:

$$
\mathrm{mol} \mathrm{Fe}{ }^{2+}=\mathrm{V} \bullet \Delta \mathrm{A} /(1 \bullet \varepsilon)=0.005875 \mathrm{~L} \cdot(0.698881-0.097828) /(1 \bullet 11,100)=3.18 \times 10^{-7} \mathrm{~mol}
$$


where $\mathrm{V}$ is the total volume $(0.005875 \mathrm{~L})$ of the solution after addition of phenanthroline, $\Delta \mathrm{A}$ is the difference in absorbance at $510 \mathrm{~nm}$ between the irradiated and non-irradiated solutions, 1 is the path length $(1.000 \mathrm{~cm})$, and $\varepsilon$ is the molar absorptivity at $510 \mathrm{~nm}\left(11,100 \mathrm{~L} \mathrm{~mol}^{-1} \mathrm{~cm}^{-1}\right) \cdot{ }^{36}$

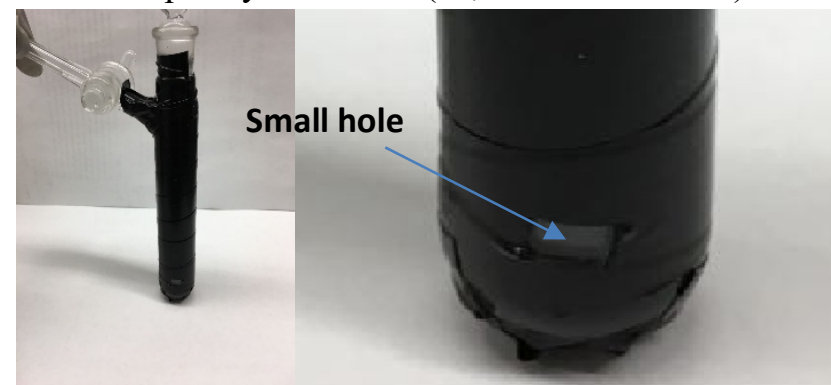

Figure S12. Taped Schlenk tube

The photon flux was calculated as follows:

photon flux $=\operatorname{mol~Fe}{ }^{2+} /(\Phi \bullet \mathrm{t} \bullet \mathrm{f})=3.18 \times 10^{-7} /(1.01 \cdot 10 \bullet 0.99833)=3.15 \times 10^{-8}$ einstein $^{-1}$ where $\Phi$ is the quantum yield for the ferrioxalate actinometer (1.01 for a $0.15 \mathrm{M}$ solution), ${ }^{36} \mathrm{t}$ is the time (10 s), and $\mathrm{f}$ is the fraction of light absorbed at $\lambda=436 \mathrm{~nm}(0.99833)^{2}$.

Determination of quantum yield: The parent reaction was conducted in the same taped Schlenk tube at the optimal conditions in $0.1 \mathrm{mmol}$ scale. After irradiation for $6 \mathrm{~h}$, the yield of product was determined by ${ }^{1} \mathrm{H}$ NMR (6\% yield). The quantum yield was determined as below.

$$
\Phi=\text { mol product } /(\text { flux } \bullet t \cdot f)=\left(6 \% \cdot 0.1 \bullet 10^{-3}\right) /\left(3.15 \times 10^{-8} \bullet 21600 \bullet 0.691\right)=1.3 \times 10^{-2}
$$

Where $\Phi$ is the quantum yield for the reaction, $\mathrm{t}$ is the reaction time (21600 s), and $\mathrm{f}$ is 0.691 (vide infra) for the photocatalyst.

Determination of fraction of light absorbed at $436 \mathbf{~ n m}$ for the photocatalyst $\mathbf{4 f}$ : The absorbance of the photocatalyst solution at $436 \mathrm{~nm}$ was measured to be 0.5094453692 . The fraction of light absorbed (f) by this solution was calculated as below, where $A$ is the measured absorbance at $436 \mathrm{~nm}$.

$$
\mathrm{f}=1-10^{-\mathrm{A}}=1-10^{-0.5094453692}=0.691
$$

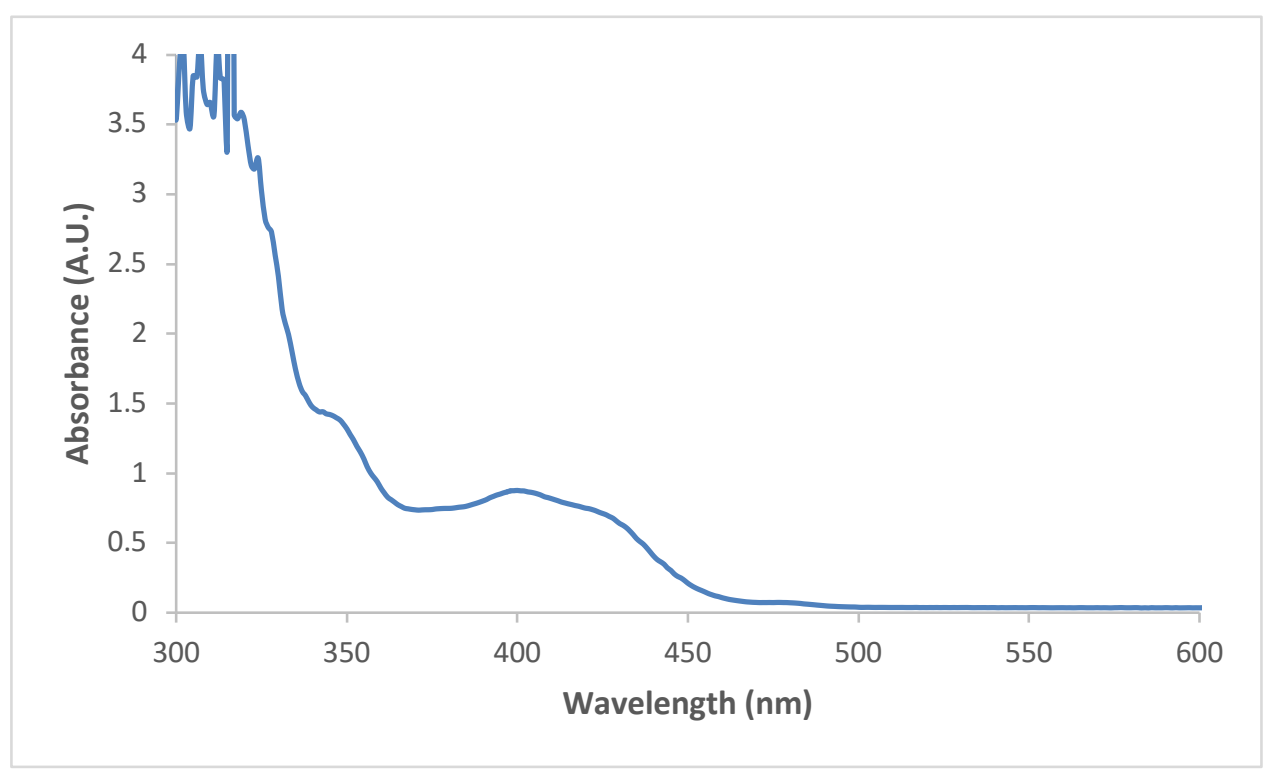

Figure S13. Absorbance of the photocatalyst $\mathbf{4 f}$ solution. 


\section{SFC and HPLC Chromatograms}

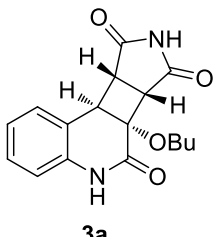

Racemic (HPLC, Daicel CHIRALPAK ${ }^{\circledR}$ AD-H, Gradient 5\% iPrOH (95\% Hexane) to 40\% $\mathrm{iPrOH}(60 \%$ Hexane), $1.0 \mathrm{~mL} / \mathrm{min})$

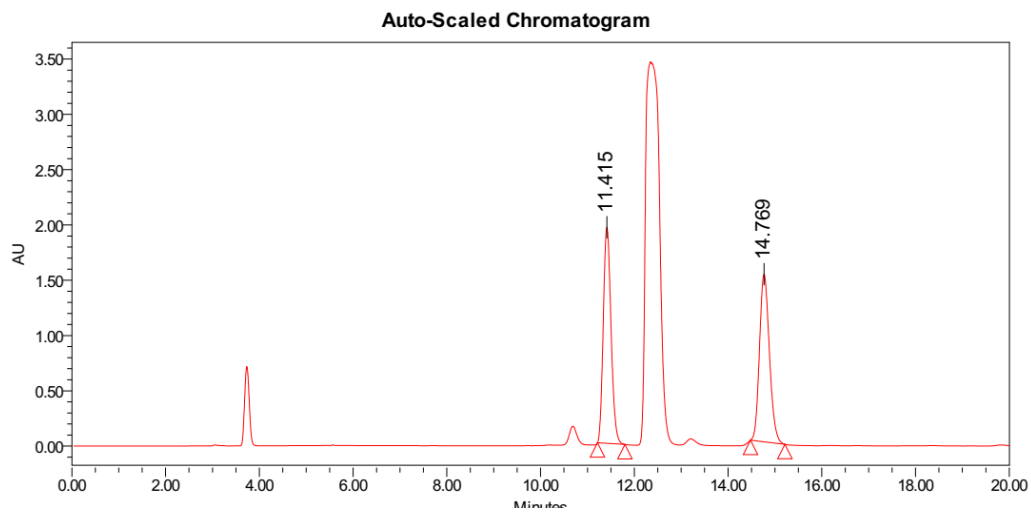

Peak Results

\begin{tabular}{|c|c|c|c|r|}
\hline & RT & Area & Height & $\%$ Area \\
\hline 1 & 11.415 & 22356786 & 1953516 & 50.09 \\
\hline 2 & 14.769 & 22280382 & 1515989 & 49.91 \\
\hline
\end{tabular}

\section{Enantioenriched}

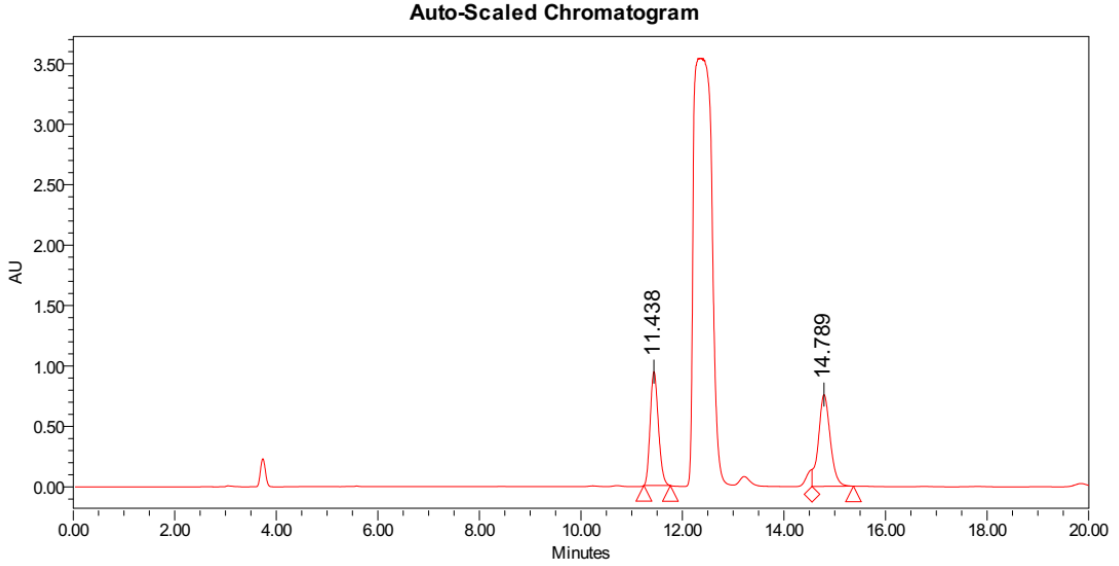

Peak Results

\begin{tabular}{|c|c|c|r|c|c|c|c|}
\hline & Name & RT & $\%$ Area & Area & Height & Amount & Units \\
\hline 1 & & 11.438 & 46.38 & 10562264 & 941755 & & \\
\hline 2 & & 14.789 & 53.62 & 12213120 & 759237 & & \\
\hline
\end{tabular}




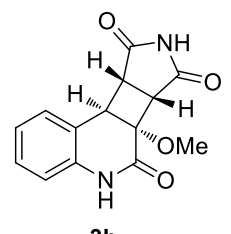

3b

Racemic (HPLC, Daicel CHIRALPAK ${ }^{\circledR} \mathrm{OD}-\mathrm{H}$, Gradient 5\% $\mathrm{iPrOH}(95 \%$ Hexane) to $40 \% \mathrm{PrOH}(60 \%$ Hexane), $1.0 \mathrm{~mL} / \mathrm{min}$ )

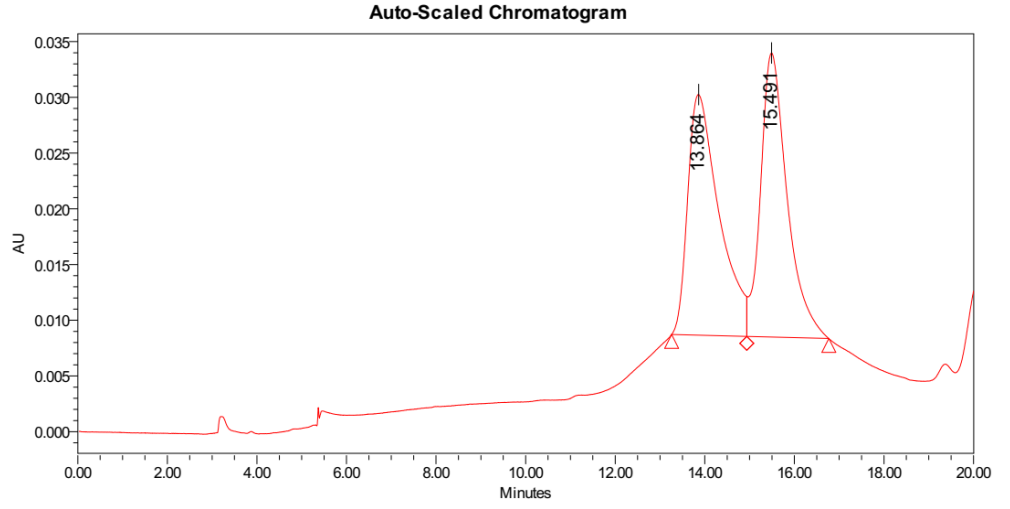

Peak Results

\begin{tabular}{|c|c|c|r|c|c|c|c|}
\hline & Name & RT & \% Area & Area & Height & Amount & Units \\
\hline 1 & & 13.864 & 50.01 & 1051987 & 21593 & & \\
\hline 2 & & 15.491 & 49.99 & 1051664 & 25490 & & \\
\hline
\end{tabular}

Enantioenriched

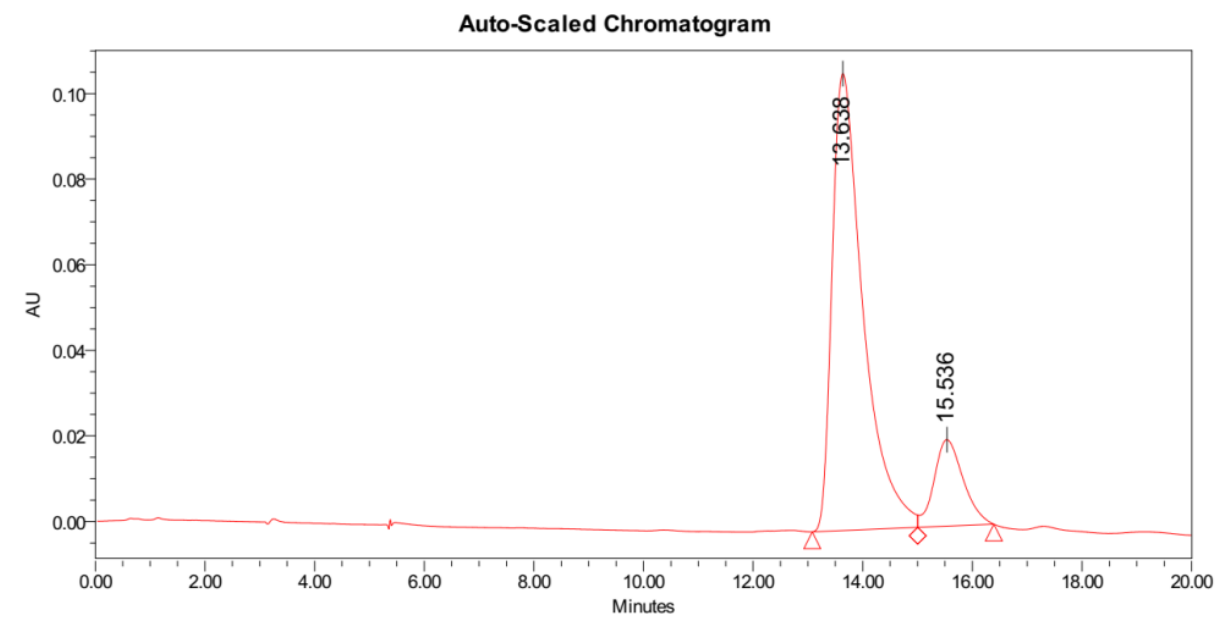

Peak Results

\begin{tabular}{|c|c|c|r|r|r|r|r|}
\hline & Name & RT & \% Area & Area & Height & Amount & Units \\
\hline 1 & & 13.638 & 84.81 & 4189844 & 106765 & & \\
\hline 2 & & 15.536 & 15.19 & 750234 & 20224 & & \\
\hline
\end{tabular}




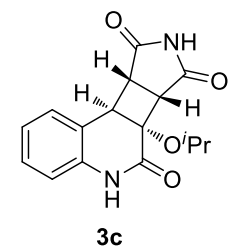

Racemic (Daicel CHIRALPAK ${ }^{\circledR}$ AD-H, isocratic 20\% MeOH, $3.0 \mathrm{~mL} / \mathrm{min}$ )

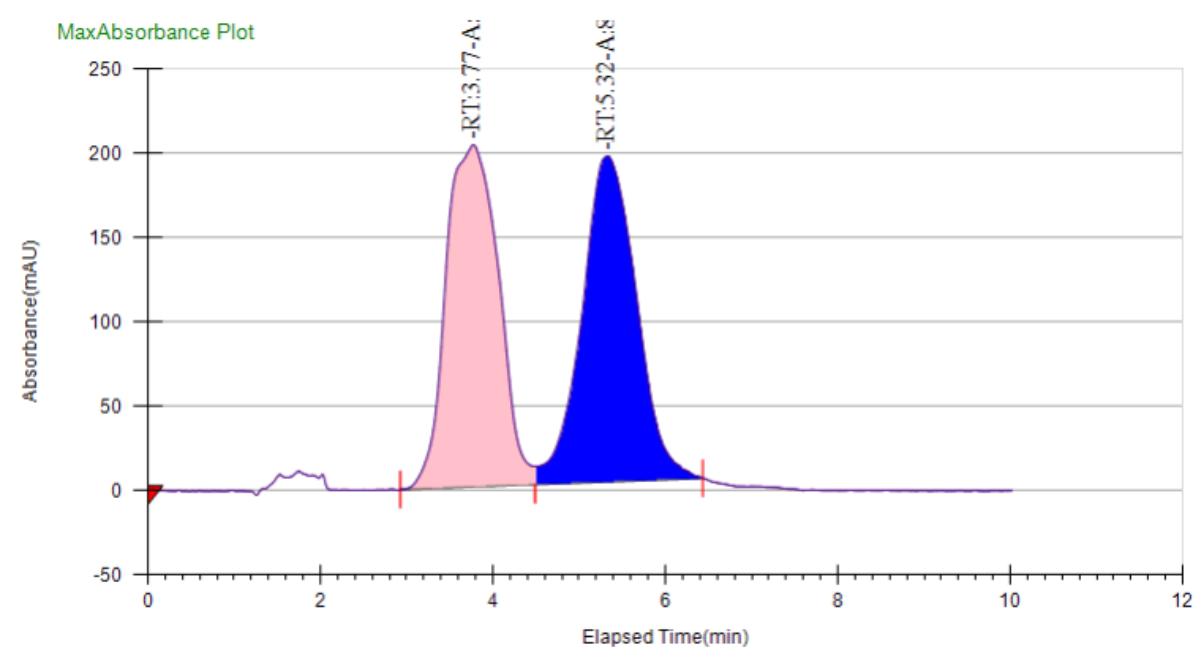

\section{Peak Information}

\begin{tabular}{|l|l|l|l|l|l|}
\hline Peak No & $\%$ Area & Area & Ret. Time & Height & Cap. Factor \\
\hline 1 & 50.2078 & 8546.8427 & $3.77 \mathrm{~min}$ & 202.8821 & 3773.95 \\
\hline 2 & 49.7922 & 8476.0871 & $5.32 \mathrm{~min}$ & 193.2605 & 5323.9333 \\
\hline
\end{tabular}

\section{Enantioenriched}

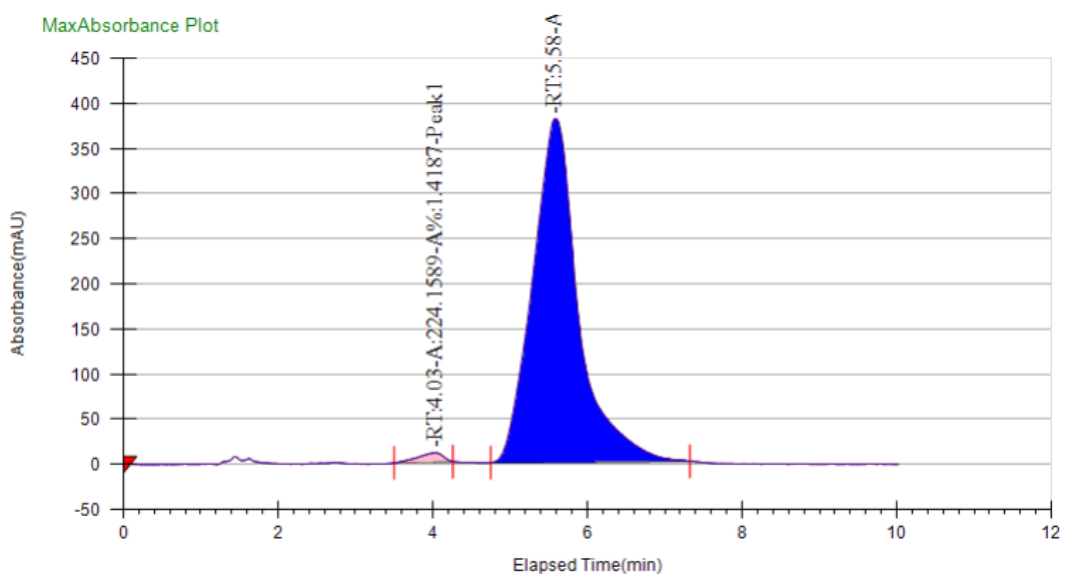

Peak Information

\begin{tabular}{|l|l|l|l|l|l|}
\hline Peak No & \% Area & Area & Ret. Time & Height & Cap. Factor \\
\hline 1 & 1.4187 & 224.1589 & $4.03 \mathrm{~min}$ & 10.0525 & 4032.2833 \\
\hline 2 & 98.5813 & $\begin{array}{l}15575.888 \\
7\end{array}$ & $5.58 \mathrm{~min}$ & 380.7977 & 5582.25 \\
\hline
\end{tabular}




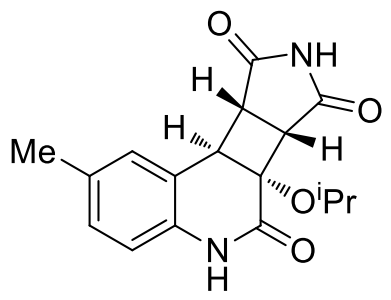

$3 e$

Racemic (Daicel CHIRALPAK ${ }^{\circledR}$ OJ-H, isocratic 20\% MeOH, $3.0 \mathrm{~mL} / \mathrm{min}$ )

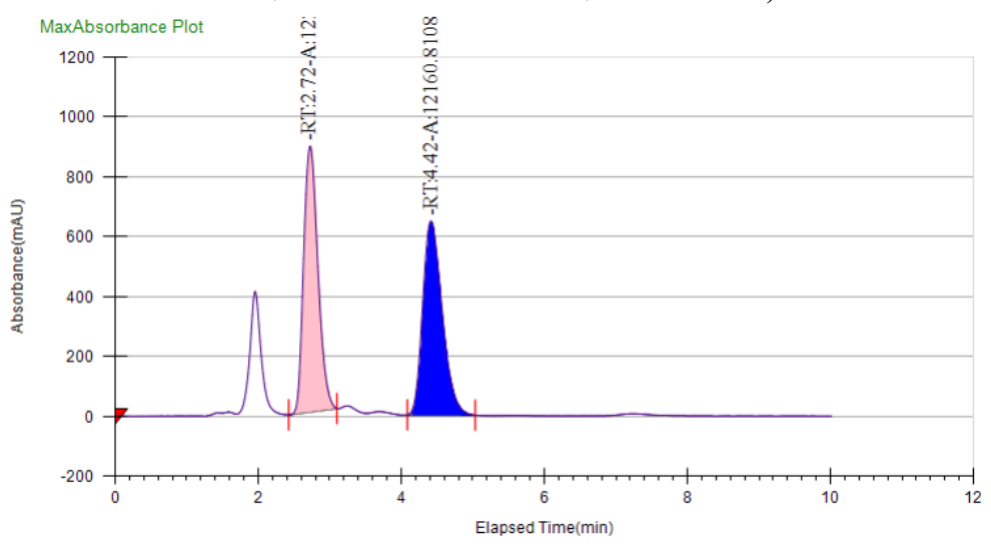

Peak Information

\begin{tabular}{|l|l|l|l|l|l|}
\hline Peak No & $\%$ Area & Area & Ret. Time & Height & Cap. Factor \\
\hline 1 & 50.0965 & $\begin{array}{l}12207.838 \\
1\end{array}$ & $2.72 \mathrm{~min}$ & 889.3199 & 0 \\
\hline 2 & 49.9035 & $\begin{array}{l}12160.810 \\
8\end{array}$ & $4.42 \mathrm{~min}$ & 647.833 & 0 \\
\hline
\end{tabular}

\section{Enantioenriched}

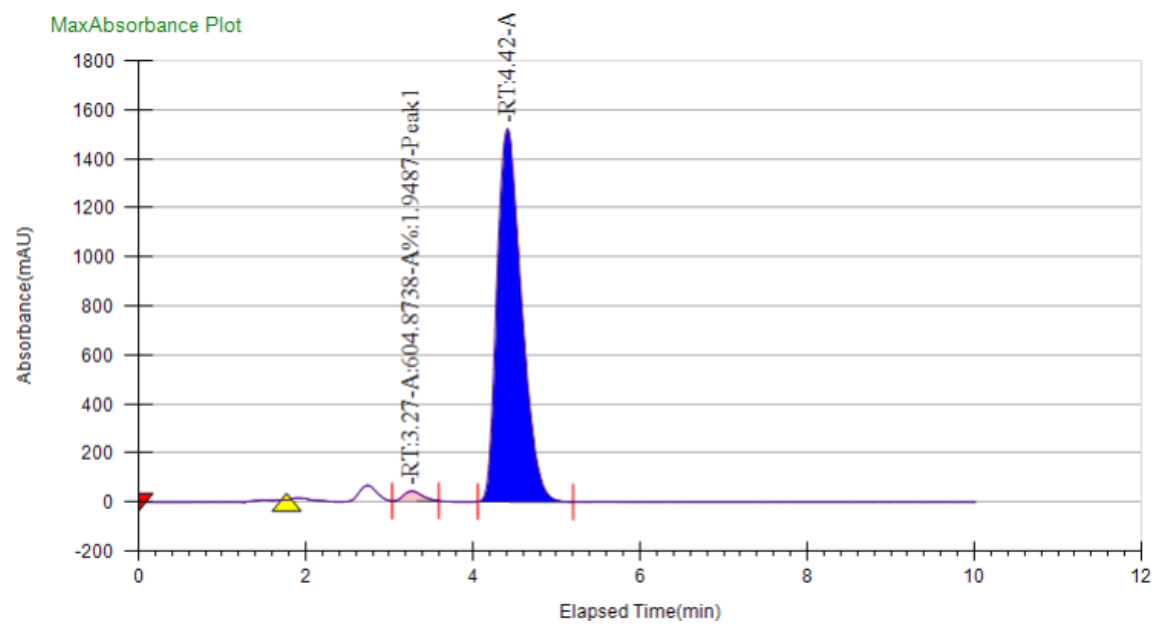

Peak Information

\begin{tabular}{|l|l|l|l|l|l|}
\hline Peak No & \% Area & Area & Ret. Time & Height & Cap. Factor \\
\hline 1 & 1.9487 & 604.8738 & $3.27 \mathrm{~min}$ & 40.7334 & 3273.95 \\
\hline 2 & 98.0513 & $\begin{array}{l}30435.732 \\
4\end{array}$ & $4.42 \mathrm{~min}$ & 1519.2618 & 4415.6 \\
\hline
\end{tabular}




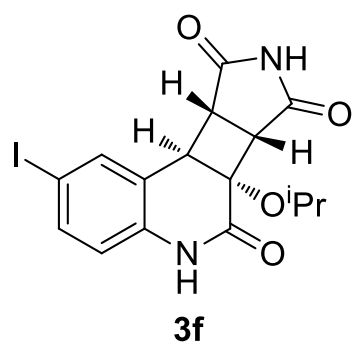

Racemic (Daicel CHIRALPAK ${ }^{\circledR}$ OD-H, isocratic 20\% MeOH, $3.0 \mathrm{~mL} / \mathrm{min}$ )

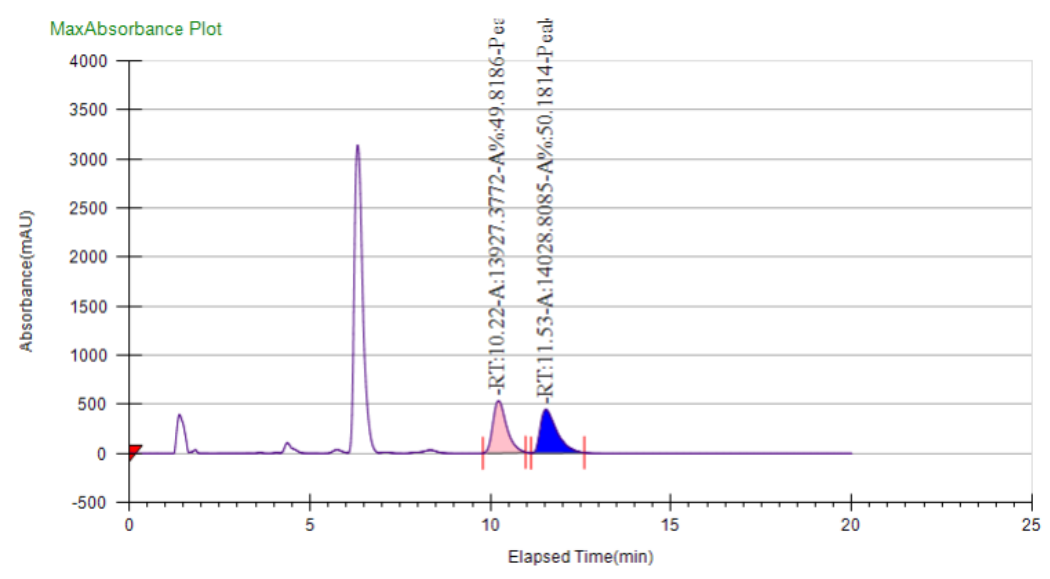

Peak Information

\begin{tabular}{|l|l|l|l|l|l|}
\hline Peak No & \% Area & Area & Ret. Time & Height & Cap. Factor \\
\hline 1 & 49.8186 & $\begin{array}{l}13927.377 \\
2\end{array}$ & $10.22 \mathrm{~min}$ & 532.1485 & 10223.85 \\
\hline 2 & 50.1814 & $\begin{array}{l}14028.808 \\
5\end{array}$ & $11.53 \mathrm{~min}$ & 443.0015 & 11532.1667 \\
\hline
\end{tabular}

\section{Enantioenriched}

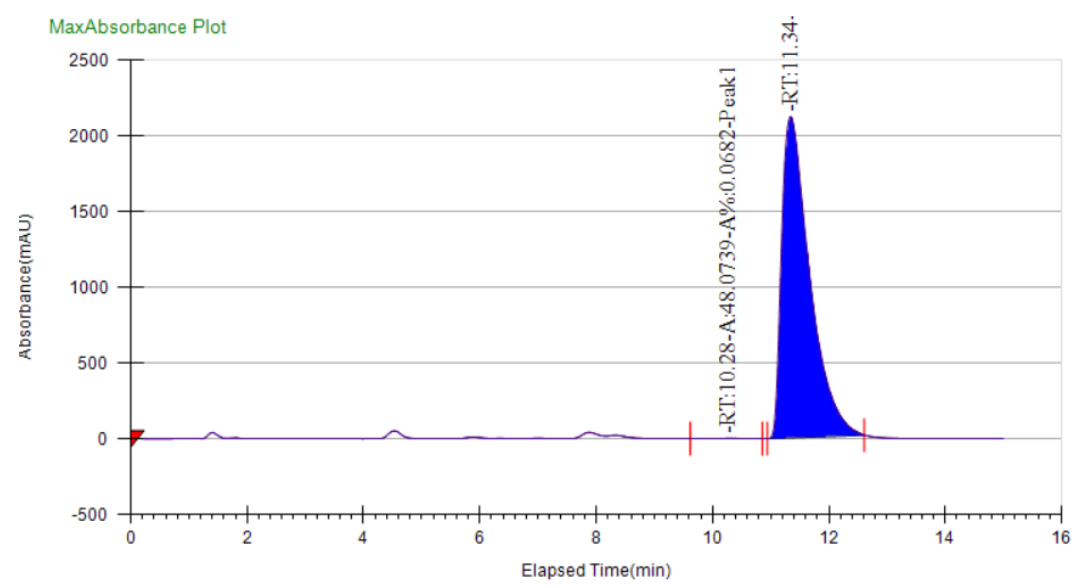

Peak Information

\begin{tabular}{|l|l|l|l|l|l|}
\hline Peak No & $\%$ Area & Area & Ret. Time & Height & Cap. Factor \\
\hline 1 & 0.0682 & 48.0739 & $10.28 \mathrm{~min}$ & 1.9283 & 10282.1833 \\
\hline 2 & 99.9318 & $\begin{array}{l}70486.723 \\
8\end{array}$ & $11.34 \mathrm{~min}$ & 2120.3538 & 11340.5 \\
\hline
\end{tabular}


<smiles>CCC[C@]12C(=O)NC(=O)[C@@H]1[C@@H]1c3cc(Br)ccc3NC(=O)[C@H]12</smiles>

Racemic (Daicel CHIRALPAK ${ }^{\circledR}$ OD-H, isocratic $20 \% \mathrm{MeOH}, 3.0 \mathrm{~mL} / \mathrm{min}$ )

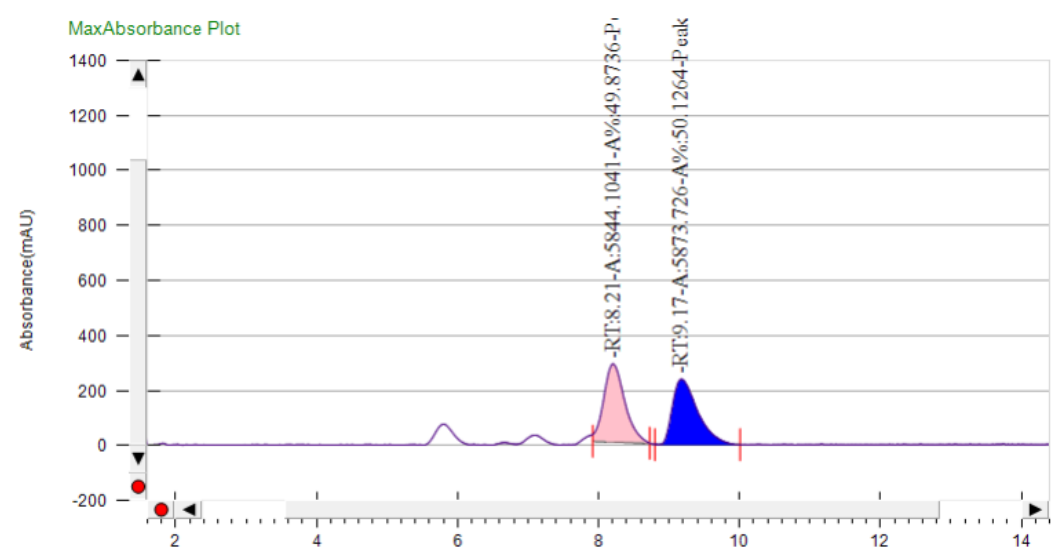

Peak Information

\begin{tabular}{|l|l|l|l|l|l|}
\hline Peak No & \% Area & Area & Ret. Time & Height & Cap. Factor \\
\hline 1 & 49.8736 & 5844.1041 & $8.21 \mathrm{~min}$ & 283.9236 & 8207.2167 \\
\hline 2 & 50.1264 & 5873.726 & $9.17 \mathrm{~min}$ & 238.1279 & 9173.8667 \\
\hline
\end{tabular}

\section{Enantioenriched}

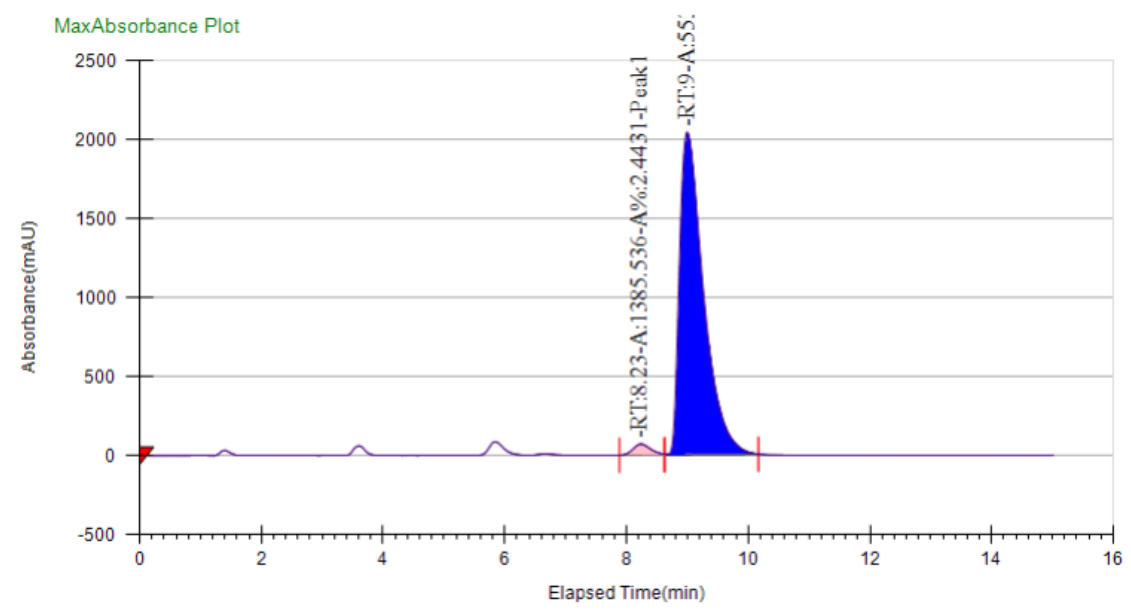

Peak Information

\begin{tabular}{|l|l|l|l|l|l|}
\hline Peak No & \% Area & Area & Ret. Time & Height & Cap. Factor \\
\hline 1 & 2.4431 & 1385.536 & $8.23 \mathrm{~min}$ & 69.7019 & 8232.2167 \\
\hline 2 & 97.5569 & $\begin{array}{l}55327.654 \\
8\end{array}$ & $9 \mathrm{~min}$ & 2040.8461 & 8998.8667 \\
\hline
\end{tabular}




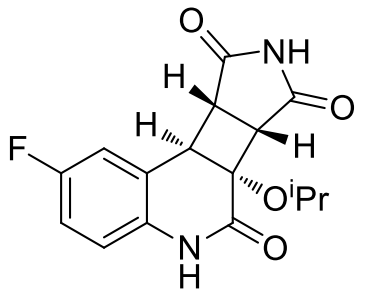

$3 h$

Racemic (Daicel CHIRALPAK ${ }^{\circledR}$ OJ-H, isocratic 20\% MeOH, $3.0 \mathrm{~mL} / \mathrm{min}$ )

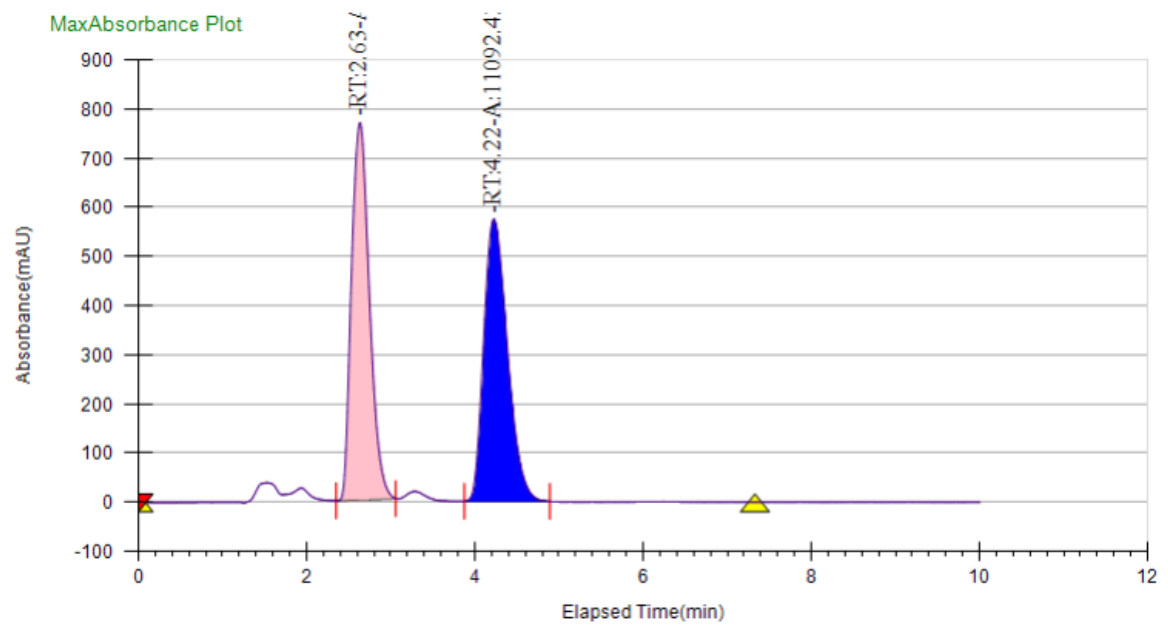

Peak Information

\begin{tabular}{|l|l|l|l|l|l|}
\hline Peak No & $\%$ Area & Area & Ret. Time & Height & Cap. Factor \\
\hline 1 & 50.3347 & $\begin{array}{l}11241.958 \\
1\end{array}$ & $2.63 \mathrm{~min}$ & 767.9485 & 2632.3 \\
\hline 2 & 49.6653 & $\begin{array}{l}11092.439 \\
2\end{array}$ & $4.22 \mathrm{~min}$ & 574.149 & 4223.95 \\
\hline
\end{tabular}

\section{Enantioenriched}

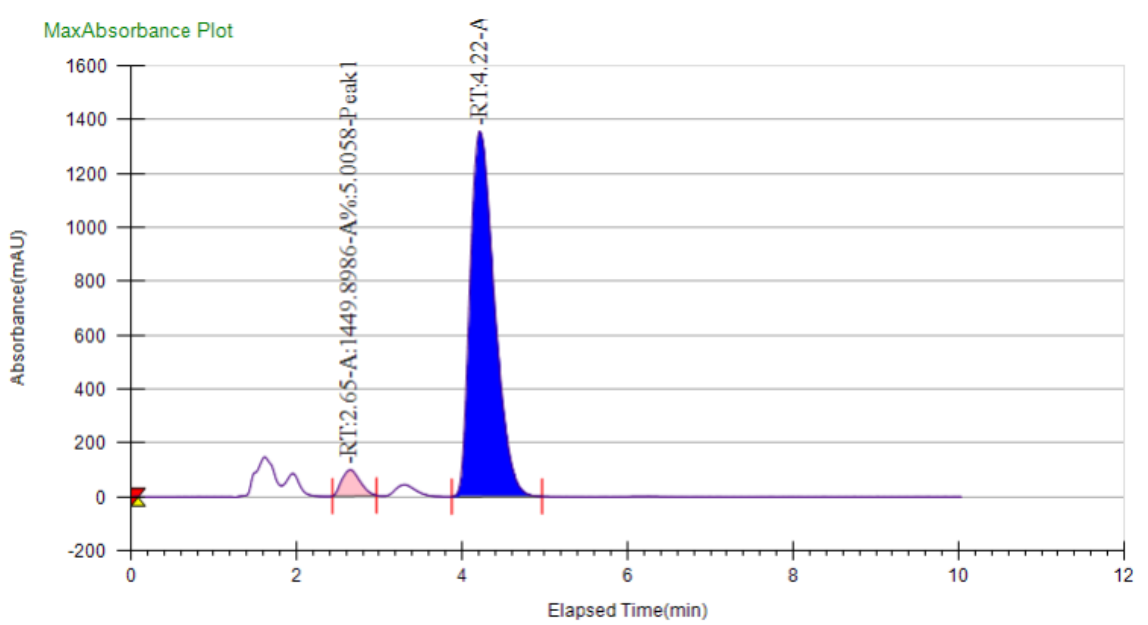

Peak Information

\begin{tabular}{|l|l|l|l|l|l|}
\hline Peak No & \% Area & Area & Ret. Time & Height & Cap. Factor \\
\hline 1 & 5.0058 & 1449.8986 & $2.65 \mathrm{~min}$ & 96.6928 & 2648.9667 \\
\hline 2 & 94.9942 & $\begin{array}{l}27514.440 \\
9\end{array}$ & $4.22 \mathrm{~min}$ & 1355.8644 & 4215.6167 \\
\hline
\end{tabular}




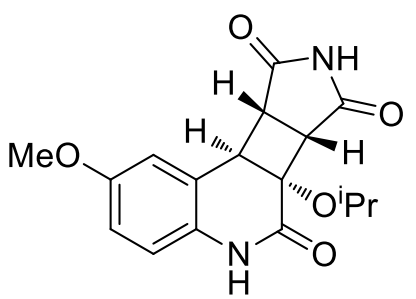

$3 \mathbf{i}$

Racemic (Daicel CHIRALPAK ${ }^{\circledR} \mathrm{OJ}-\mathrm{H}$, isocratic $20 \% \mathrm{MeOH}, 3.0 \mathrm{~mL} / \mathrm{min}$ )

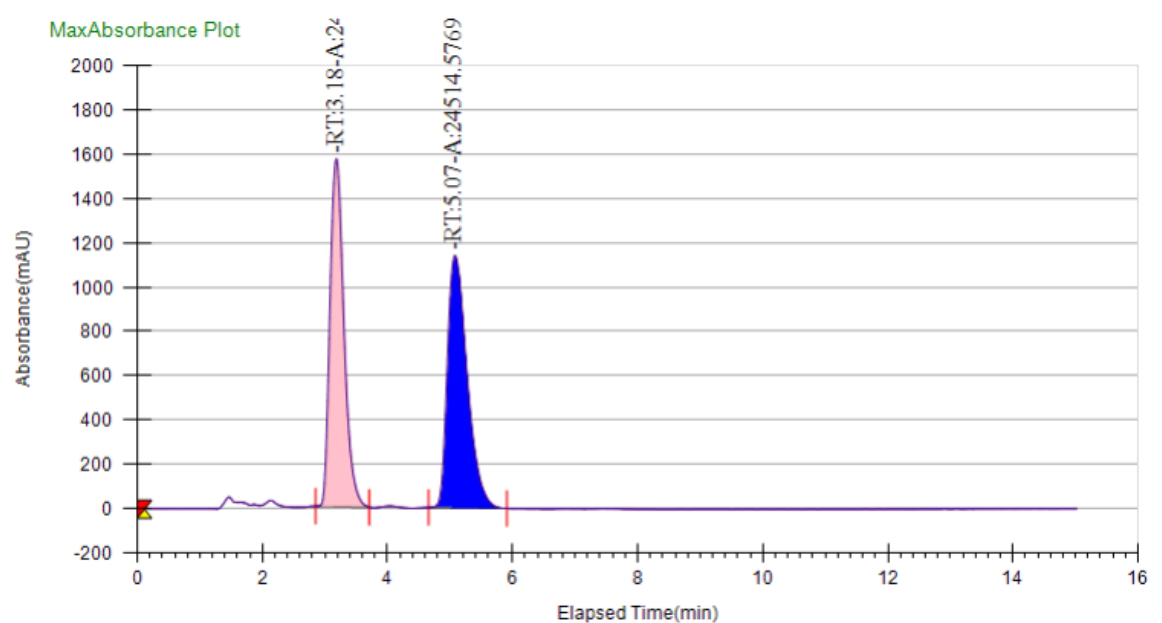

Peak Information

\begin{tabular}{|l|l|l|l|l|l|}
\hline Peak No & \% Area & Area & Ret. Time & Height & Cap. Factor \\
\hline 1 & 49.5496 & $\begin{array}{l}24076.912 \\
5\end{array}$ & $3.18 \mathrm{~min}$ & 1570.3162 & 3182.3 \\
\hline 2 & 50.4504 & $\begin{array}{l}24514.576 \\
9\end{array}$ & $5.07 \mathrm{~min}$ & 1137.2063 & 5073.9333 \\
\hline
\end{tabular}

\section{Enantioenriched}

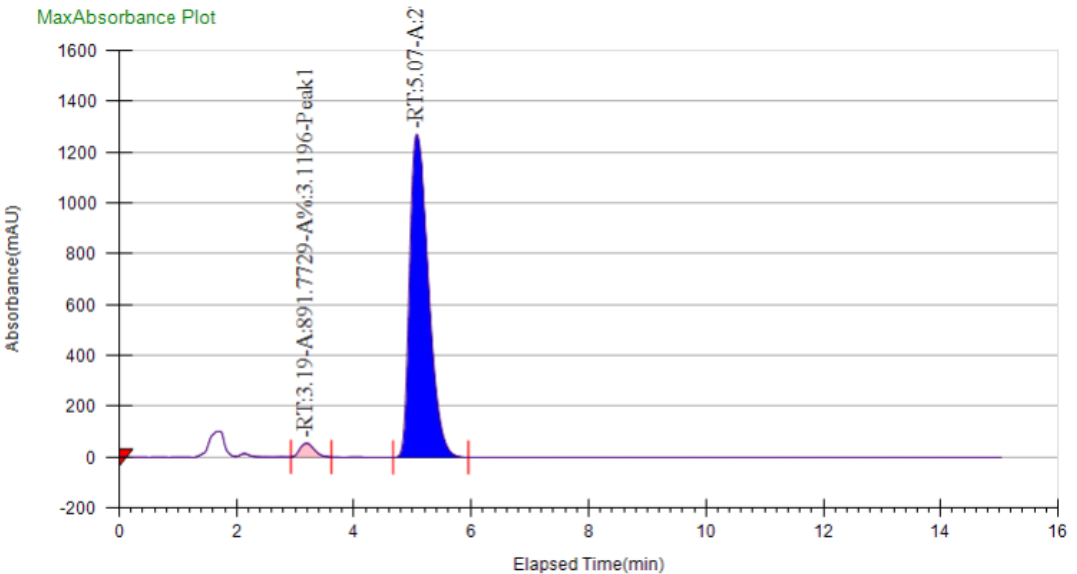

Peak Information

\begin{tabular}{|l|l|l|l|l|l|}
\hline Peak No & $\%$ Area & Area & Ret. Time & Height & Cap. Factor \\
\hline 1 & 3.1196 & 891.7729 & $3.19 \mathrm{~min}$ & 54.1131 & 3190.6167 \\
\hline 2 & 96.8804 & 27694.437 & $5.07 \mathrm{~min}$ & 1271.028 & 5065.6 \\
\hline
\end{tabular}


<smiles>[2H][C@]12C(=O)Nc3cc(Br)ccc3[C@H]([C@@H]3C(=O)NC(=O)[C@@H]31)[C@@H]2C(F)(F)F</smiles>

Racemic (Daicel CHIRALPAK ${ }^{\circledR}$ OD-H, isocratic 20\% MeOH, $3.0 \mathrm{~mL} / \mathrm{min}$ )

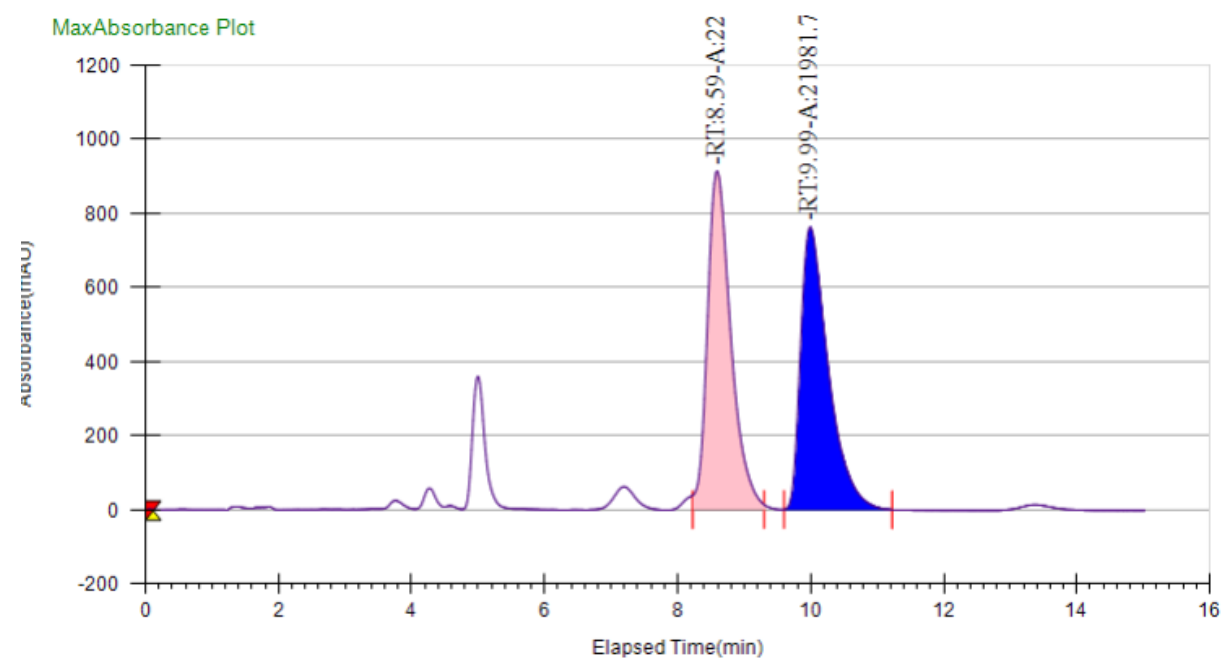

Peak Information

\begin{tabular}{|l|l|l|l|l|l|}
\hline Peak No & \% Area & Area & Ret. Time & Height & Cap. Factor \\
\hline 1 & 50.4107 & $\begin{array}{l}22345.918 \\
2\end{array}$ & $8.59 \mathrm{~min}$ & 914.967 & 8590.55 \\
\hline 2 & 49.5893 & $\begin{array}{l}21981.797 \\
4\end{array}$ & $9.99 \mathrm{~min}$ & 762.7972 & 9990.5167 \\
\hline
\end{tabular}

\section{Enantioenriched}

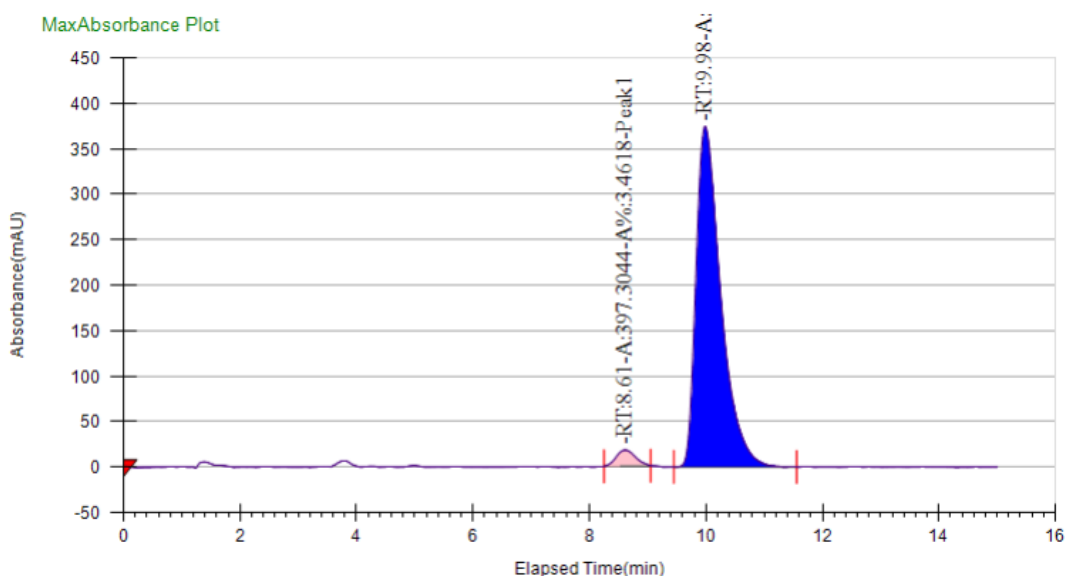

Peak Information

\begin{tabular}{|l|l|l|l|l|l|}
\hline Peak No & $\%$ Area & Area & Ret. Time & Height & Cap. Factor \\
\hline 1 & 3.4618 & 397.3044 & $8.61 \mathrm{~min}$ & 17.593 & 8607.2167 \\
\hline 2 & 96.5382 & $\begin{array}{l}11079.441 \\
3\end{array}$ & $9.98 \mathrm{~min}$ & 374.6226 & 9982.1833 \\
\hline
\end{tabular}


<smiles></smiles>

3k

Racemic (Daicel CHIRALPAK ${ }^{\circledR}$ OD-H, isocratic 20\% MeOH, $3.0 \mathrm{~mL} / \mathrm{min}$ )

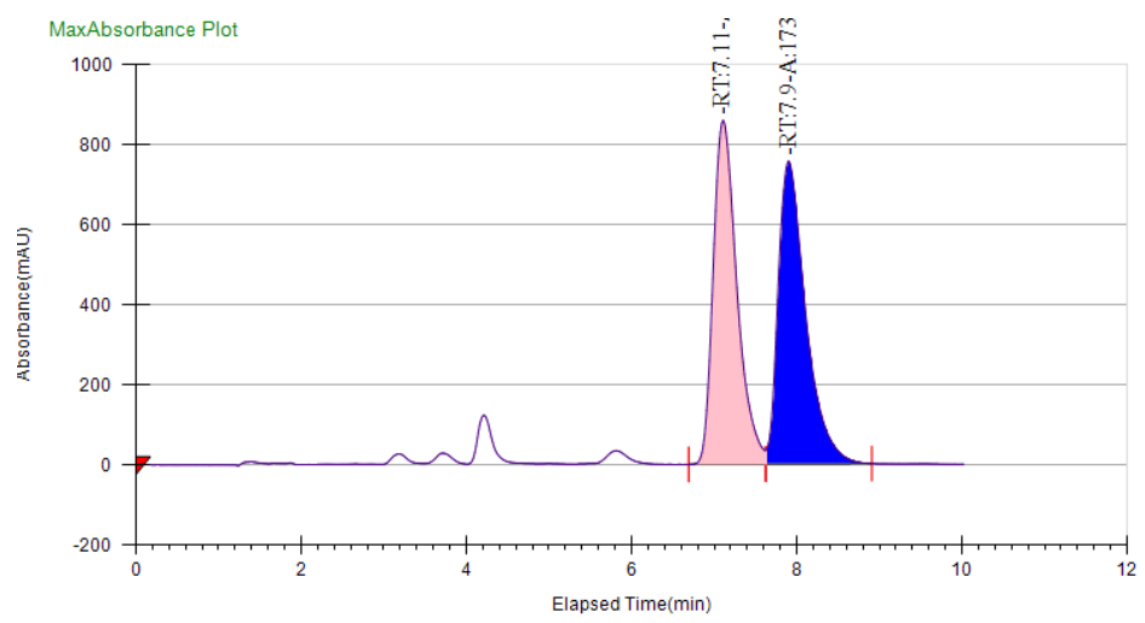

Peak Information

\begin{tabular}{|l|l|l|l|l|l|}
\hline Peak No & $\%$ Area & Area & Ret. Time & Height & Cap. Factor \\
\hline 1 & 49.5784 & $\begin{array}{l}17040.643 \\
8\end{array}$ & $7.11 \mathrm{~min}$ & 859.1194 & 7107.2333 \\
\hline 2 & 50.4216 & $\begin{array}{l}17330.432 \\
4\end{array}$ & $7.9 \mathrm{~min}$ & 756.1548 & 7898.8833 \\
\hline
\end{tabular}

\section{Enantioenriched}

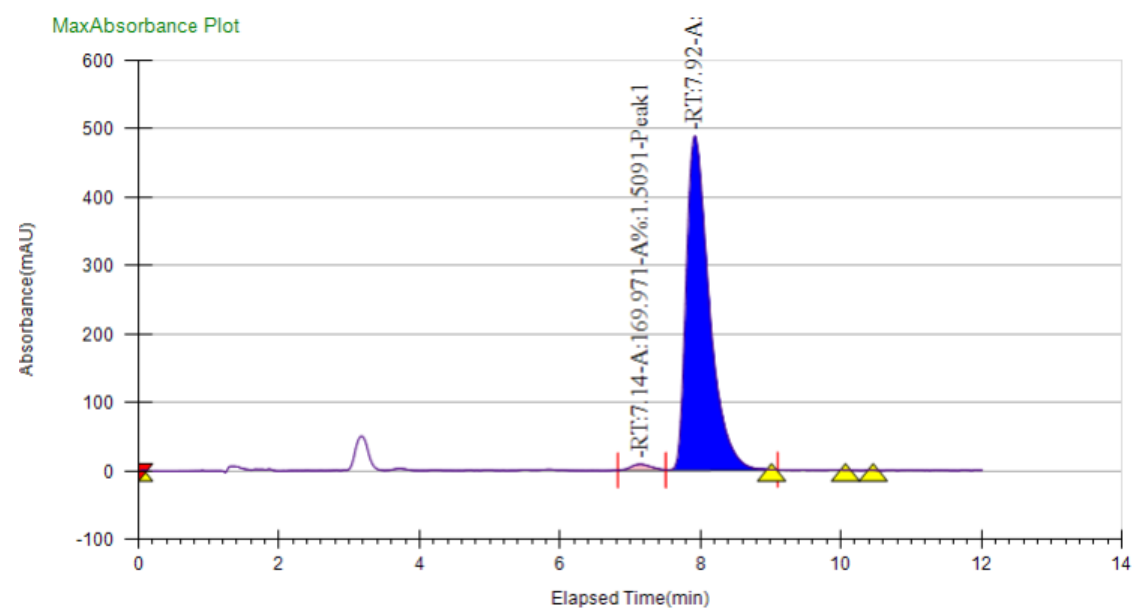

Peak Information

\begin{tabular}{|l|l|l|l|l|l|}
\hline Peak No & \% Area & Area & Ret. Time & Height & Cap. Factor \\
\hline 1 & 1.5091 & 169.971 & $7.14 \mathrm{~min}$ & 8.7985 & 7140.5667 \\
\hline 2 & 98.4909 & 11093.091 & $7.92 \mathrm{~min}$ & 488.4023 & 7915.55 \\
\hline
\end{tabular}




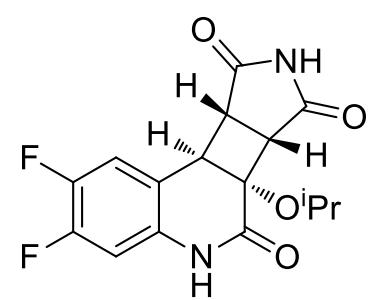

3I

Racemic (Daicel CHIRALPAK ${ }^{\circledR}$ OD-H, isocratic 20\% MeOH, $3.0 \mathrm{~mL} / \mathrm{min}$ )

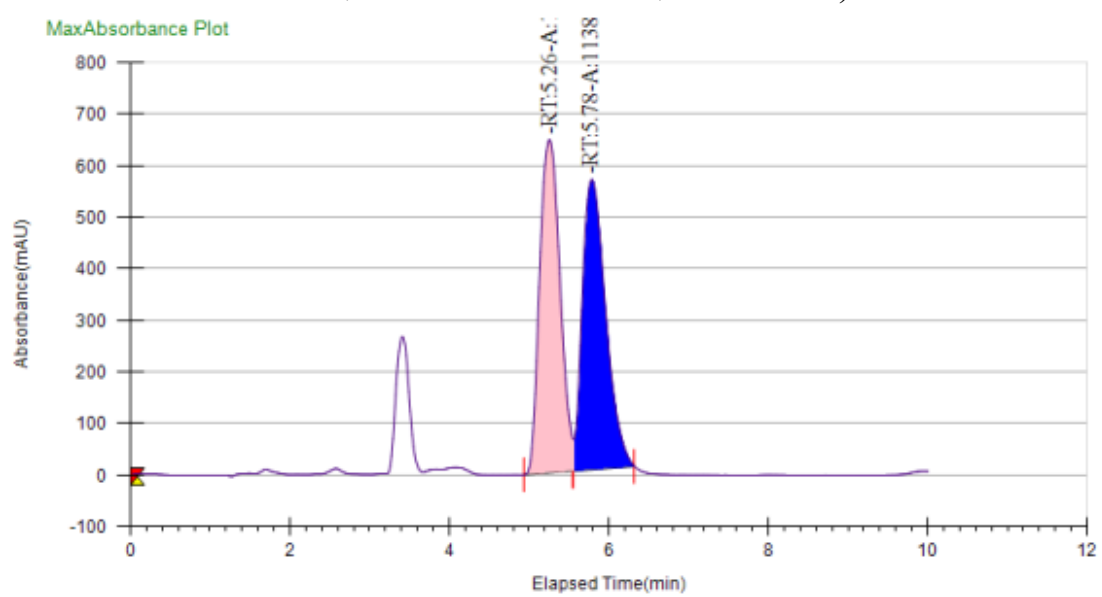

Peak Information

\begin{tabular}{|l|l|l|l|l|l|}
\hline Peak No & $\%$ Area & Area & Ret. Time & Height & Cap. Factor \\
\hline 1 & 49.9079 & $\begin{array}{l}11341.708 \\
3\end{array}$ & $5.26 \mathrm{~min}$ & 646.1867 & 0 \\
\hline 2 & 50.0921 & $\begin{array}{l}11383.584 \\
2\end{array}$ & $5.78 \mathrm{~min}$ & 562.7244 & 0 \\
\hline
\end{tabular}

\section{Enantioenriched}

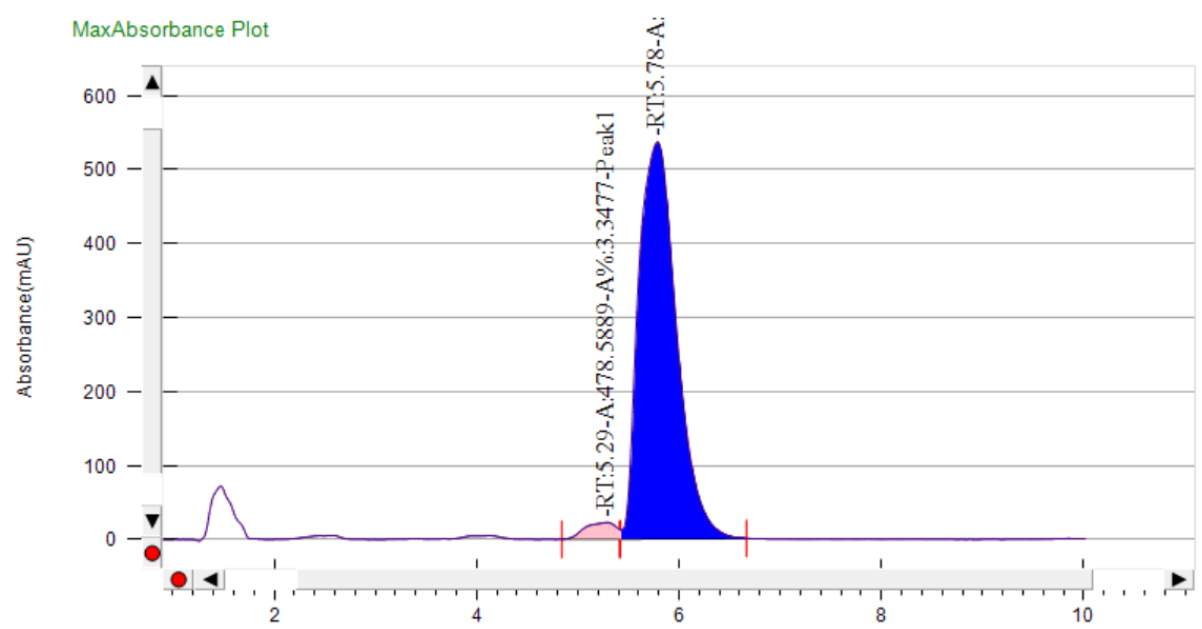

Peak Information

\begin{tabular}{|l|l|l|l|l|l|}
\hline Peak No & \% Area & Area & Ret. Time & Height & Cap. Factor \\
\hline 1 & 3.3477 & 478.5889 & $5.29 \mathrm{~min}$ & 22.3257 & 0 \\
\hline 2 & 96.6523 & 13817.576 & $5.78 \mathrm{~min}$ & 536.4022 & 0 \\
\hline
\end{tabular}


<smiles>[2H][C@@]12c3c(Cl)cccc3NC(=O)[C@@H]1C(=O)N[C@H]1C(=O)NC(=O)[C@H]12</smiles>

$3 m$

Racemic (Daicel CHIRALPAK ${ }^{\circledR}$ OD-H, isocratic $20 \% \mathrm{MeOH}, 3.0 \mathrm{~mL} / \mathrm{min}$ )

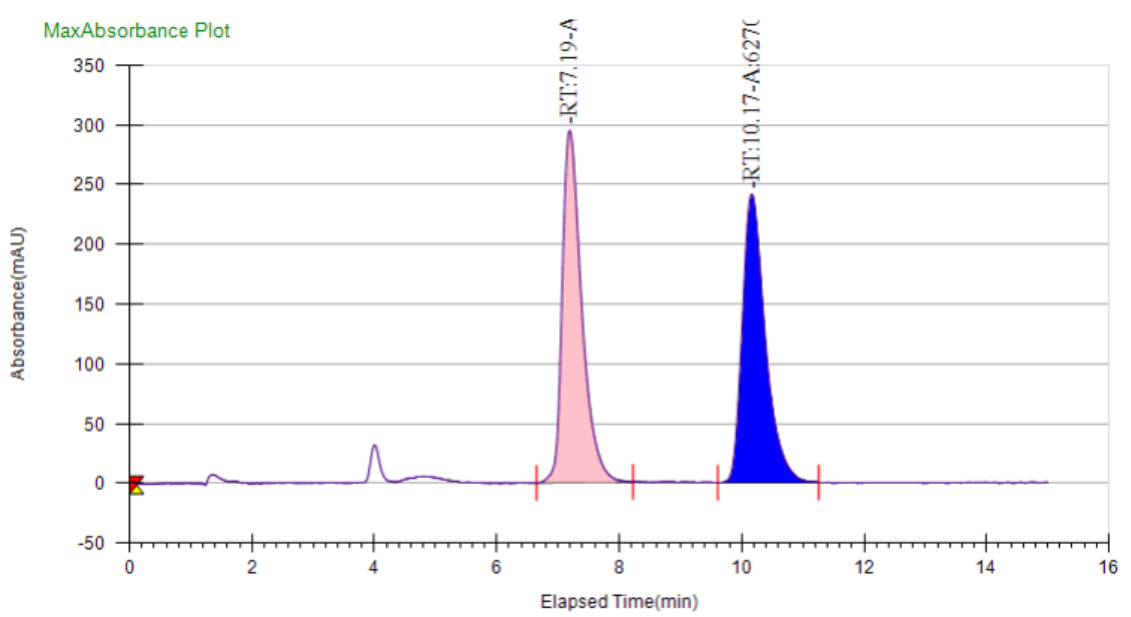

Peak Information

\begin{tabular}{|l|l|l|l|l|l|}
\hline Peak No & \% Area & Area & Ret. Time & Height & Cap. Factor \\
\hline 1 & 50.9116 & 6503.5032 & $7.19 \mathrm{~min}$ & 295.0747 & 7190.5667 \\
\hline 2 & 49.0884 & 6270.6008 & $10.17 \mathrm{~min}$ & 241.7064 & 10165.5167 \\
\hline
\end{tabular}

\section{Enantioenriched}

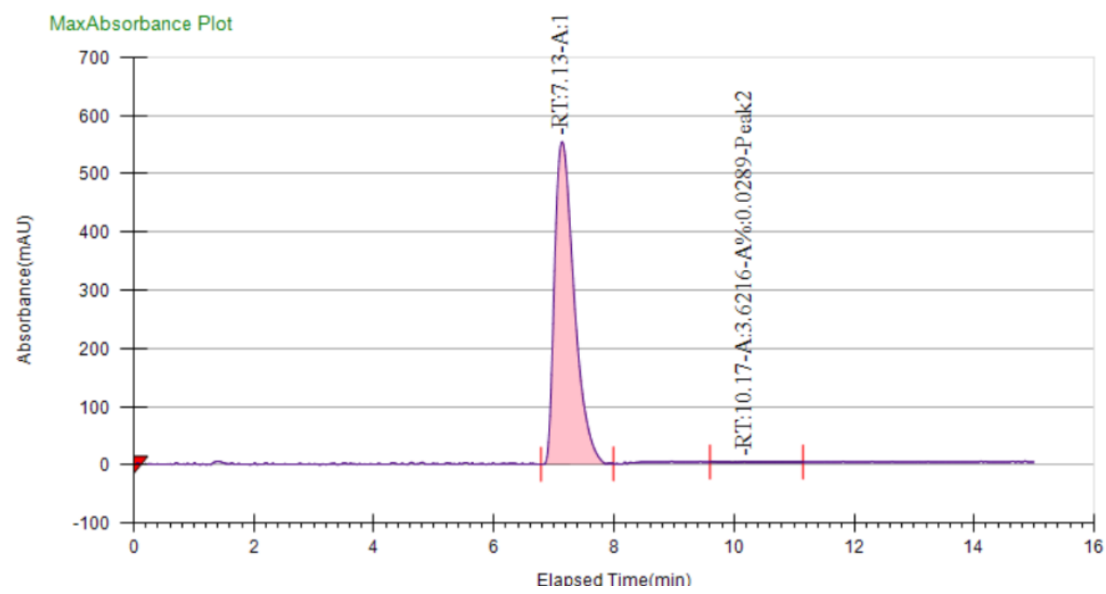

Peak Information

\begin{tabular}{|l|l|l|l|l|l|}
\hline Peak No & $\%$ Area & Area & Ret. Time & Height & Cap. Factor \\
\hline 1 & 99.9711 & $\begin{array}{l}12525.719 \\
2\end{array}$ & $7.13 \mathrm{~min}$ & 554.1727 & 0 \\
\hline 2 & 0.0289 & 3.6216 & $10.17 \mathrm{~min}$ & 0.9375 & 0 \\
\hline
\end{tabular}




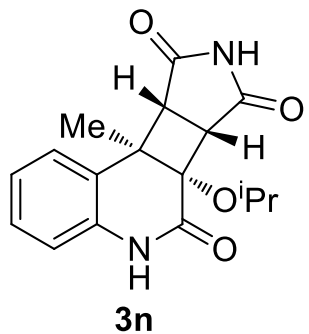

Racemic (Daicel CHIRALPAK ${ }^{\circledR}$ AD-H, isocratic 20\% MeOH, $3.0 \mathrm{~mL} / \mathrm{min}$ )

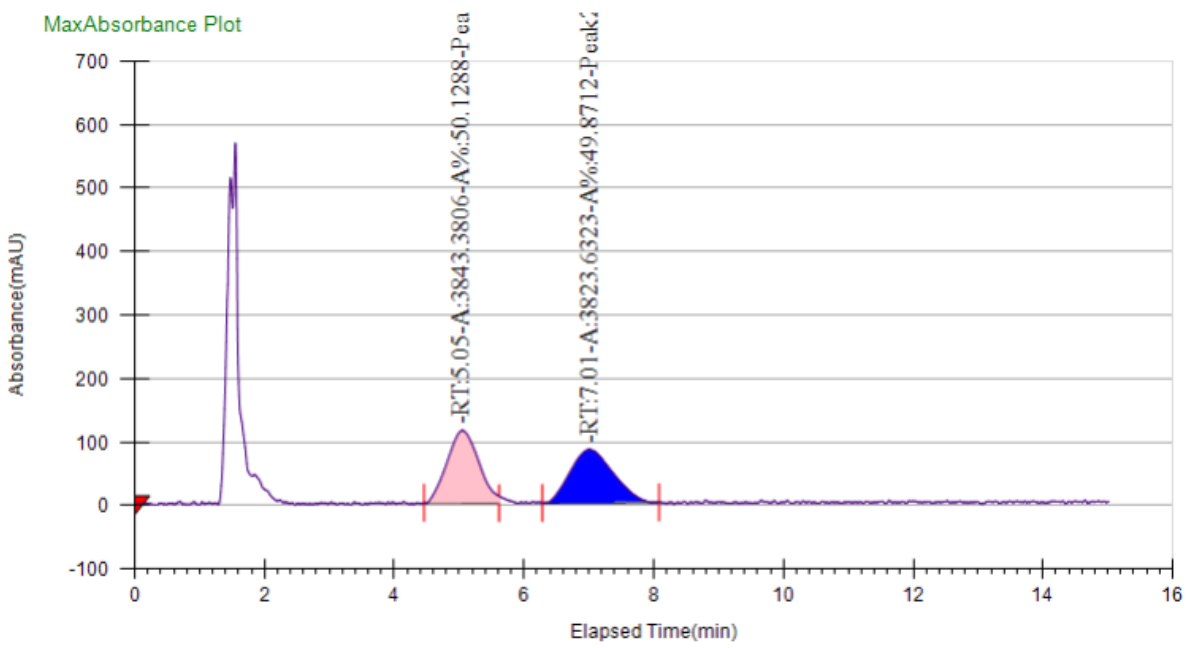

Peak Information

\begin{tabular}{|l|l|l|l|l|l|}
\hline Peak No & \% Area & Area & Ret. Time & Height & Cap. Factor \\
\hline 1 & 50.1288 & 3843.3806 & $5.05 \mathrm{~min}$ & 114.3053 & 5048.9333 \\
\hline 2 & 49.8712 & 3823.6323 & $7.01 \mathrm{~min}$ & 83.9513 & 7007.2333 \\
\hline
\end{tabular}

\section{Enantioenriched}

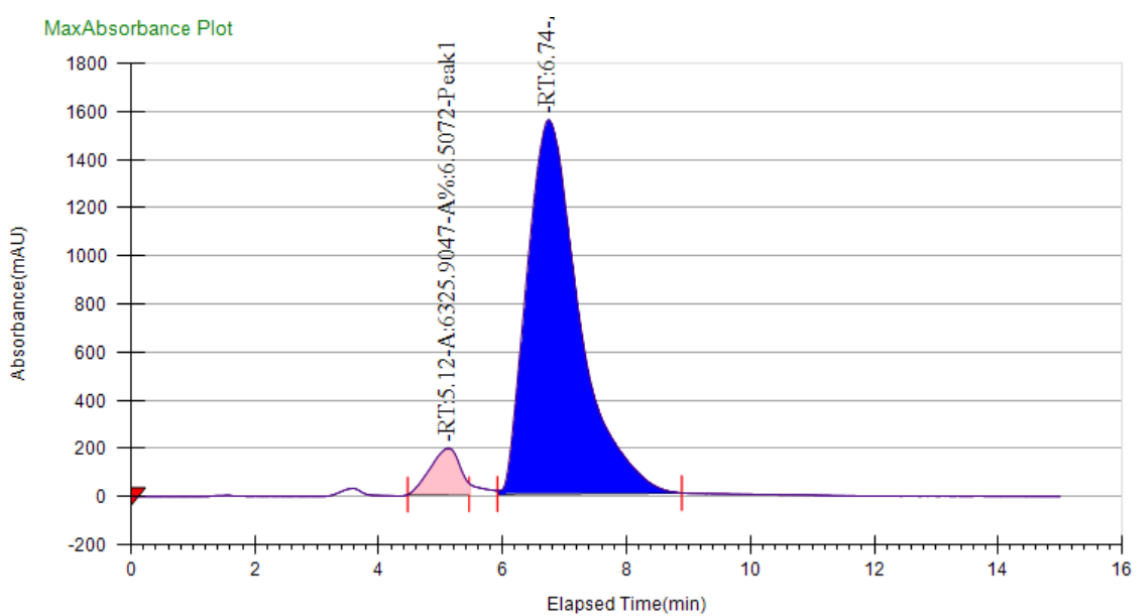

\section{Peak Information}

\begin{tabular}{|l|l|l|l|l|l|}
\hline Peak No & \% Area & Area & Ret. Time & Height & Cap. Factor \\
\hline 1 & 6.5072 & 6325.9047 & $5.12 \mathrm{~min}$ & 191.0788 & 5123.9333 \\
\hline 2 & 93.4928 & $\begin{array}{l}90888.478 \\
1\end{array}$ & $6.74 \mathrm{~min}$ & 1553.0837 & 6740.5667 \\
\hline
\end{tabular}




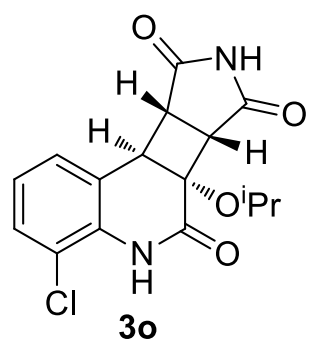

Racemic (Daicel CHIRALPAK ${ }^{\circledR}$ OD-H, isocratic $20 \% \mathrm{MeOH}, 3.0 \mathrm{~mL} / \mathrm{min}$ )

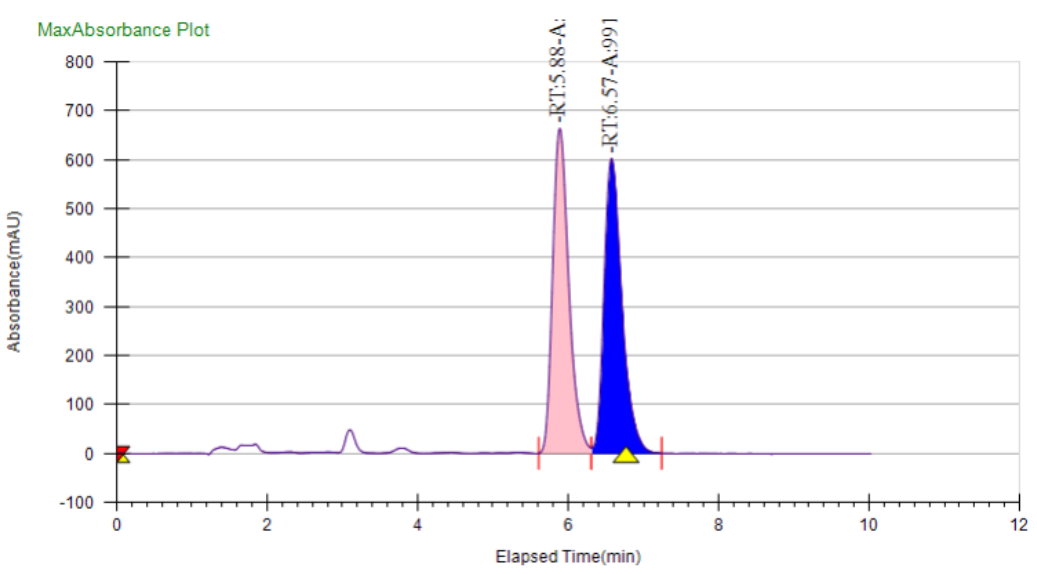

Peak Information

\begin{tabular}{|l|l|l|l|l|l|}
\hline Peak No & \% Area & Area & Ret. Time & Height & Cap. Factor \\
\hline 1 & 49.901 & 9872.46 & $5.88 \mathrm{~min}$ & 663.1358 & 0 \\
\hline 2 & 50.099 & 9911.6356 & $6.57 \mathrm{~min}$ & 602.386 & 0 \\
\hline
\end{tabular}

\section{Enantioenriched}

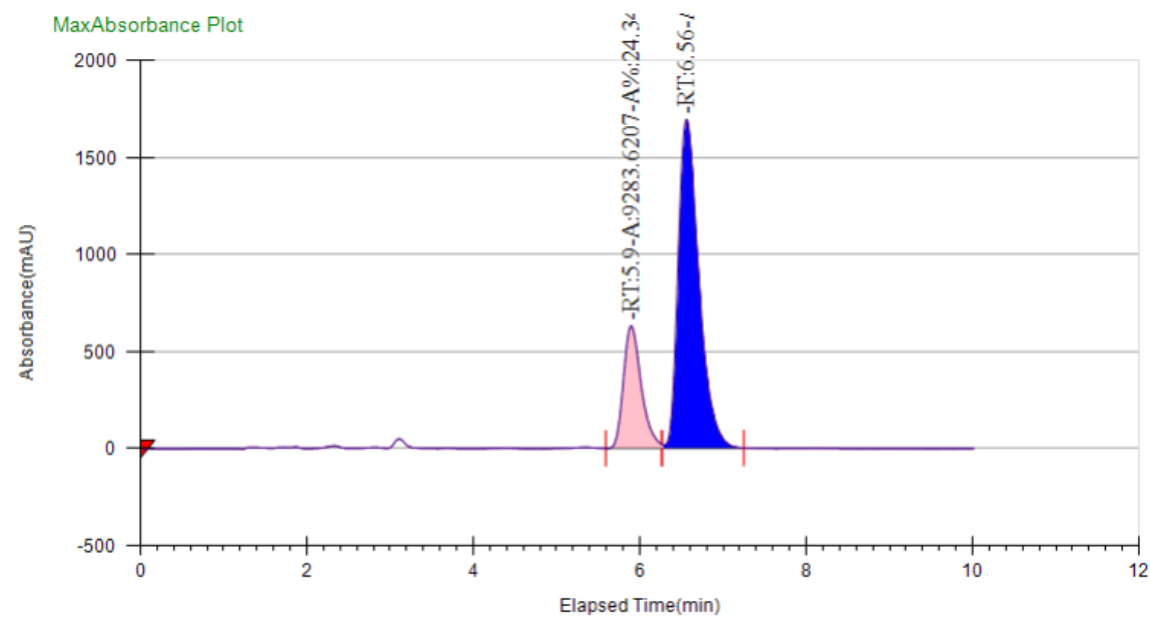

Peak Information

\begin{tabular}{|l|l|l|l|l|l|}
\hline Peak No & \% Area & Area & Ret. Time & Height & Cap. Factor \\
\hline 1 & 24.3493 & 9283.6207 & $5.9 \mathrm{~min}$ & 629.5085 & 5898.9167 \\
\hline 2 & 75.6507 & $\begin{array}{l}28843.205 \\
7\end{array}$ & $6.56 \mathrm{~min}$ & 1692.4888 & 6557.2333 \\
\hline
\end{tabular}




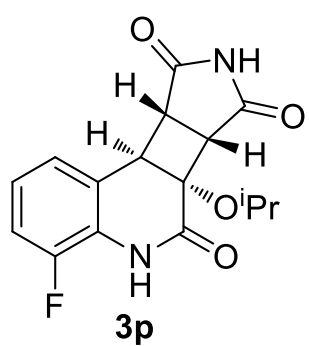

Racemic (Daicel CHIRALPAK ${ }^{\circledR}$ OD-H, isocratic $20 \% \mathrm{MeOH}, 3.0 \mathrm{~mL} / \mathrm{min}$ )

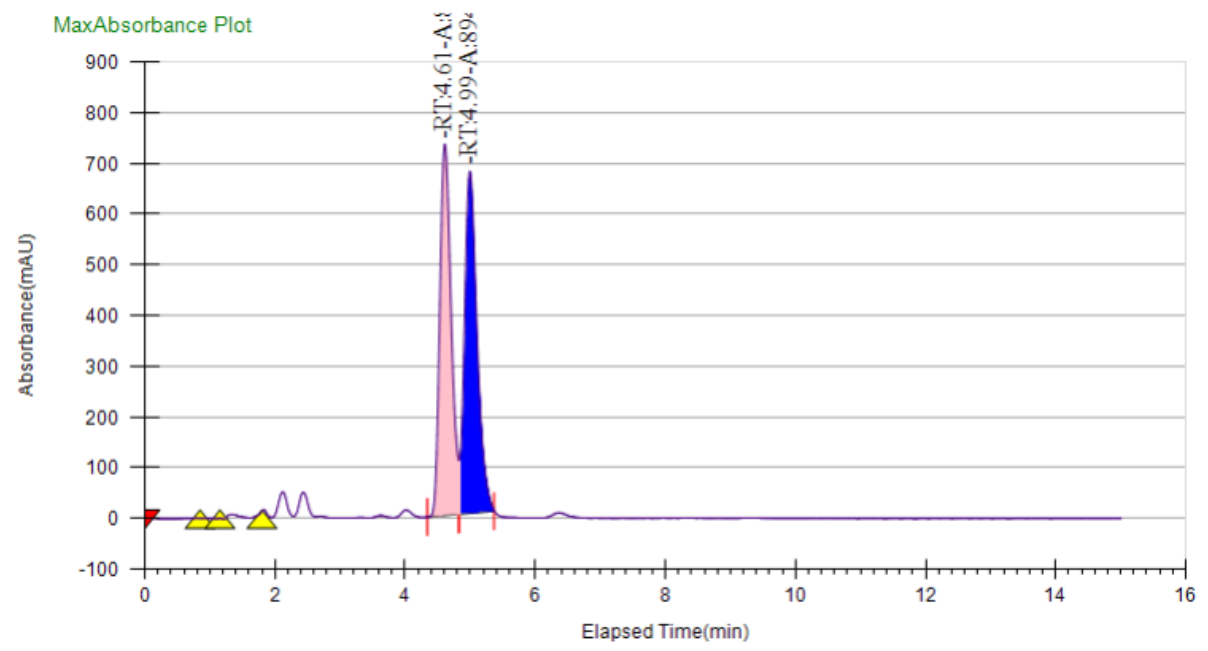

Peak Information

\begin{tabular}{|l|l|l|l|l|l|}
\hline Peak No & \% Area & Area & Ret. Time & Height & Cap. Factor \\
\hline 1 & 49.3293 & 8711.6116 & $4.61 \mathrm{~min}$ & 732.0155 & 4607.2667 \\
\hline 2 & 50.6707 & 8948.4957 & $4.99 \mathrm{~min}$ & 674.6575 & 4990.6 \\
\hline
\end{tabular}

\section{Enantioenriched}

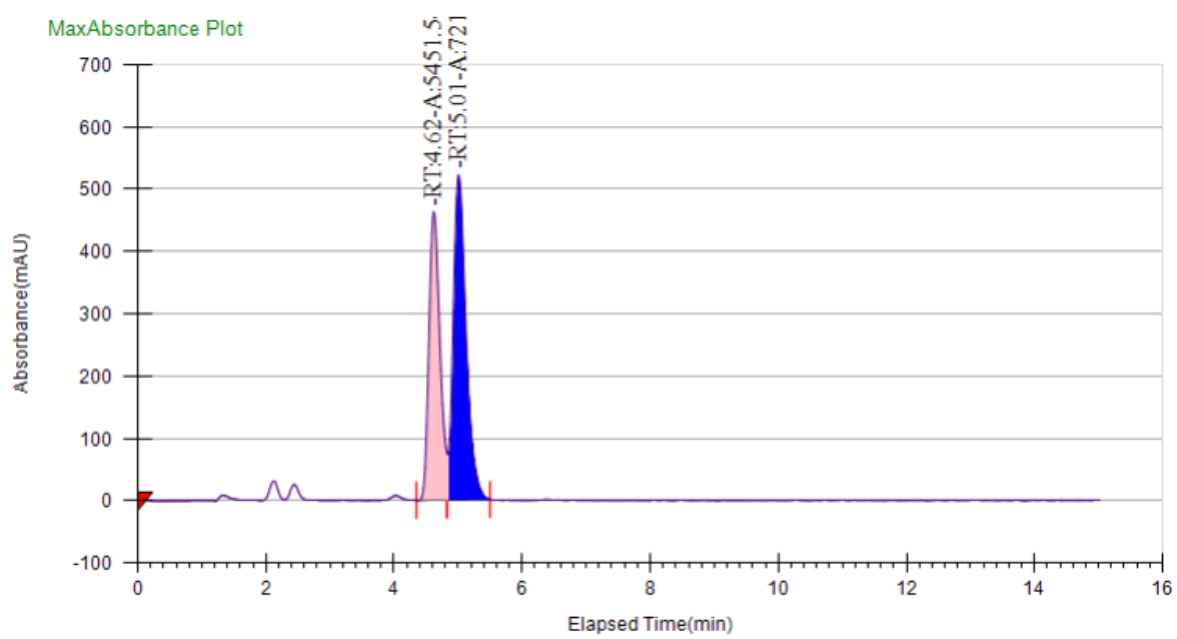

Peak Information

\begin{tabular}{|l|l|l|l|l|l|}
\hline Peak No & \% Area & Area & Ret. Time & Height & Cap. Factor \\
\hline 1 & 43.0234 & 5451.5485 & $4.62 \mathrm{~min}$ & 462.7003 & 4623.9333 \\
\hline 2 & 56.9766 & 7219.5699 & $5.01 \mathrm{~min}$ & 521.8705 & 5007.2667 \\
\hline
\end{tabular}


<smiles>O=C1NC(=O)[C@H]2[C@H]1[C@@H]1C(=O)Oc3ccccc3[C@H]21</smiles>

$3 q$

Racemic (Daicel CHIRALPAK ${ }^{\circledR}$ OD-H, isocratic $20 \% \mathrm{MeOH}, 3.0 \mathrm{~mL} / \mathrm{min}$ )

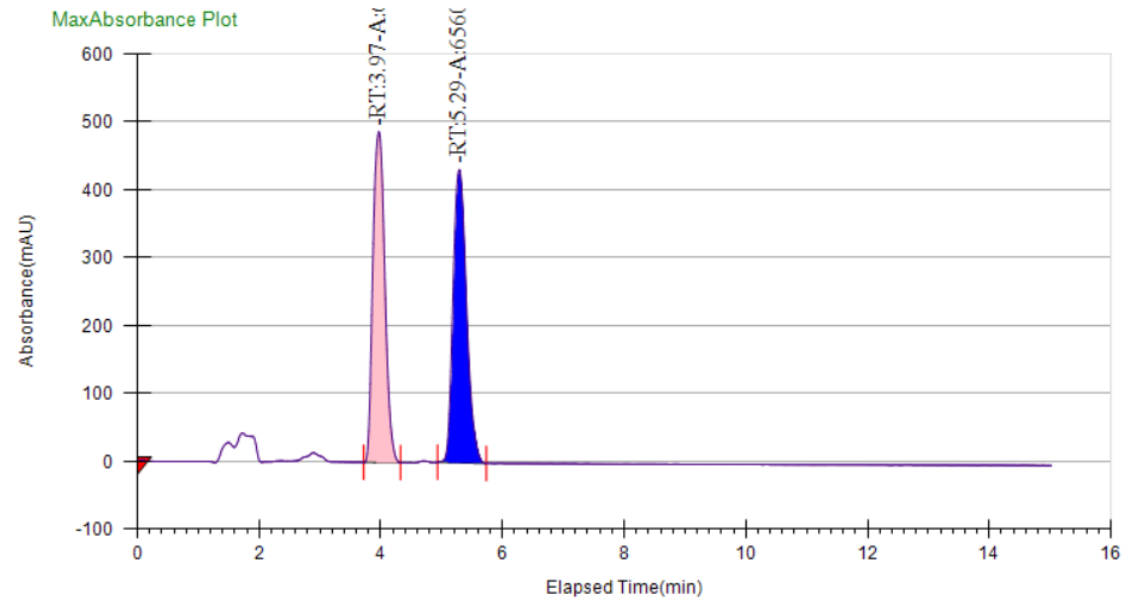

Peak Information

\begin{tabular}{|l|l|l|l|l|l|}
\hline Peak No & \% Area & Area & Ret. Time & Height & Cap. Factor \\
\hline 1 & 49.9619 & 6550.5045 & $3.97 \mathrm{~min}$ & 487.7592 & 0 \\
\hline 2 & 50.0381 & 6560.5032 & $5.29 \mathrm{~min}$ & 432.034 & 0 \\
\hline
\end{tabular}

\section{Enantioenriched}

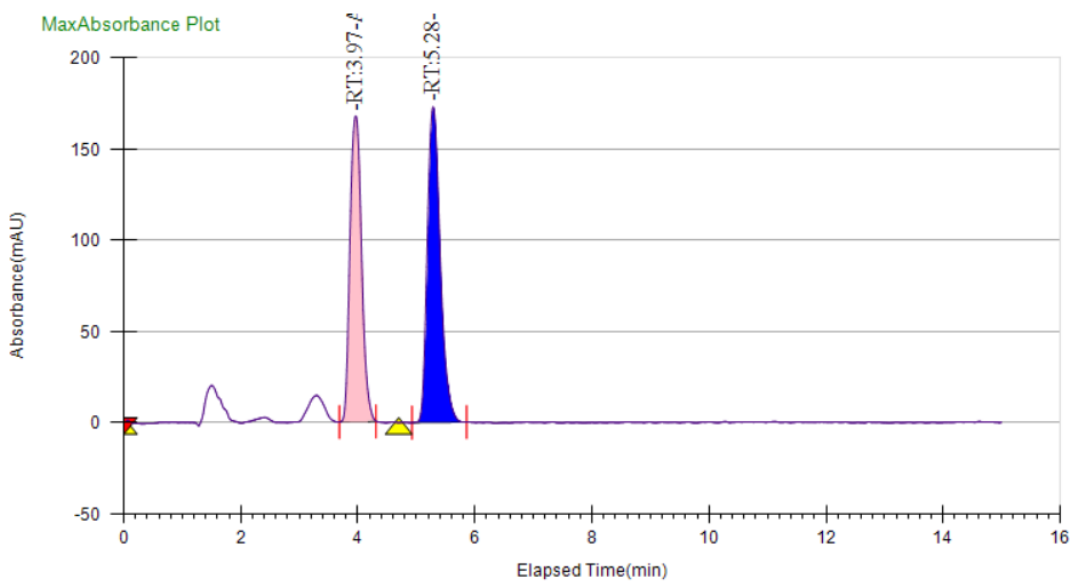

Peak Information

\begin{tabular}{|l|l|l|l|l|l|}
\hline Peak No & $\%$ Area & Area & Ret. Time & Height & Cap. Factor \\
\hline 1 & 46.4422 & 2271.4897 & $3.97 \mathrm{~min}$ & 167.4073 & 0 \\
\hline 2 & 53.5578 & 2619.5099 & $5.28 \mathrm{~min}$ & 172.7807 & 0 \\
\hline
\end{tabular}




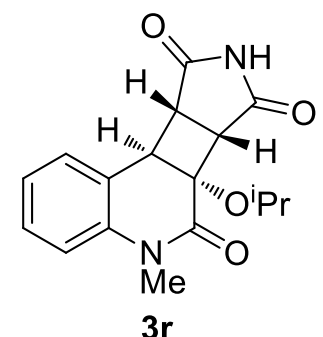

Racemic (Daicel CHIRALPAK ${ }^{\circledR}$ OD-H, isocratic 20\% MeOH, $3.0 \mathrm{~mL} / \mathrm{min}$ )

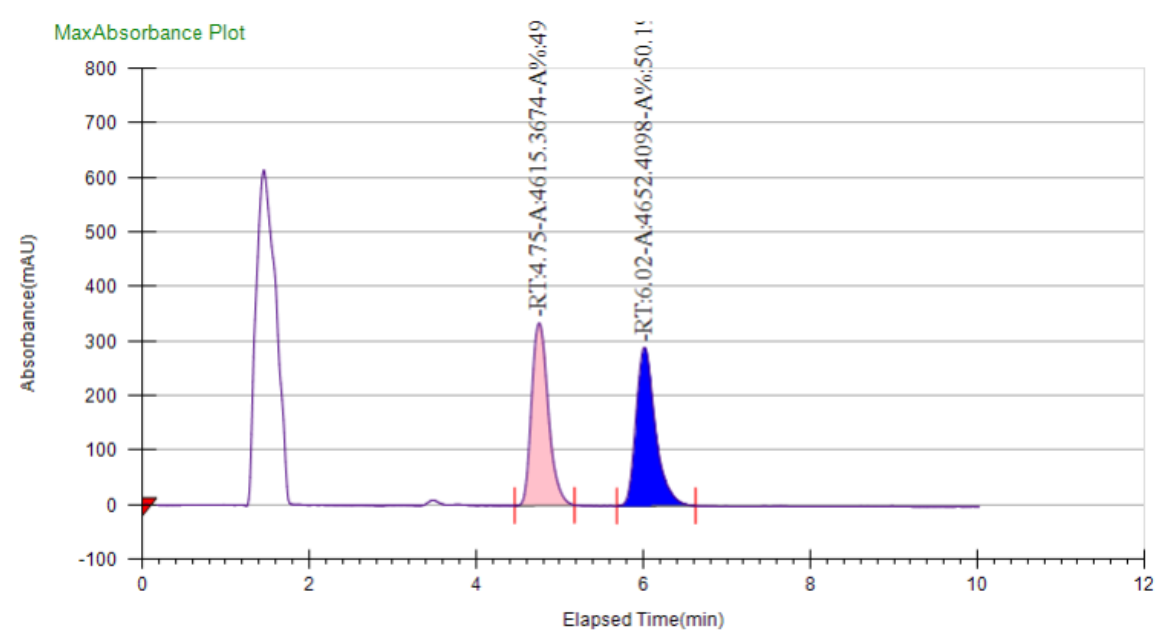

Peak Information

\begin{tabular}{|l|l|l|l|l|l|}
\hline Peak No & \% Area & Area & Ret. Time & Height & Cap. Factor \\
\hline 1 & 49.8002 & 4615.3674 & $4.75 \mathrm{~min}$ & 334.2987 & 4748.9333 \\
\hline 2 & 50.1998 & 4652.4098 & $6.02 \mathrm{~min}$ & 290.982 & 6015.5833 \\
\hline
\end{tabular}

\section{Enantioenriched}

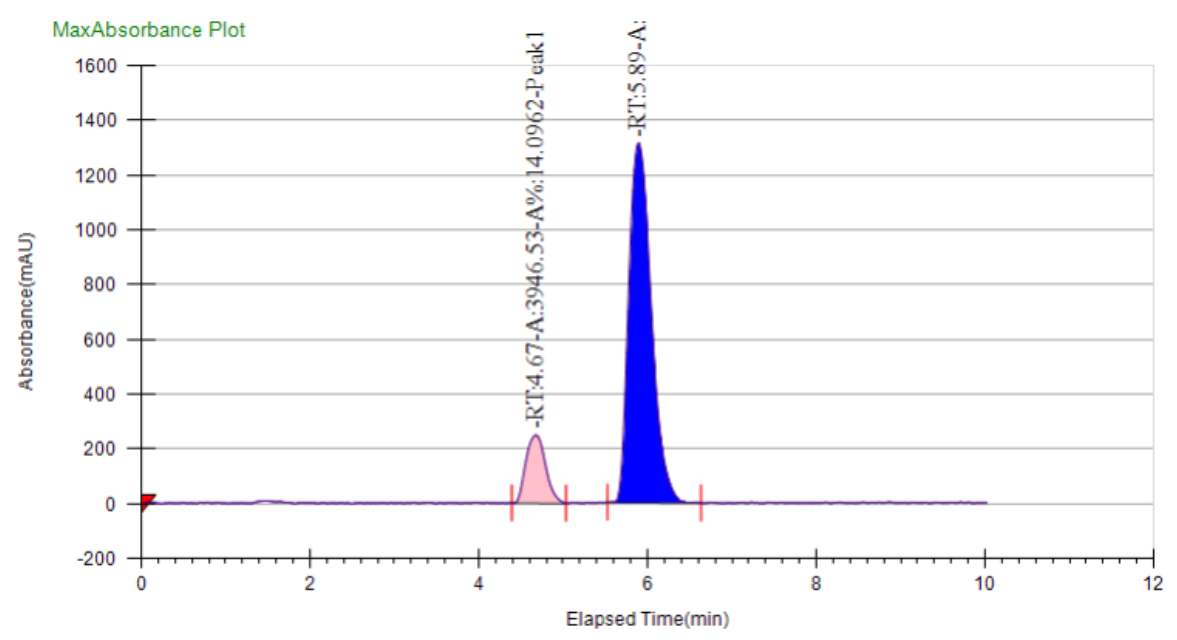

\section{Peak Information}

\begin{tabular}{|l|l|l|l|l|l|}
\hline Peak No & \% Area & Area & Ret. Time & Height & Cap. Factor \\
\hline 1 & 14.0962 & 3946.53 & $4.67 \mathrm{~min}$ & 247.9067 & 4673.9333 \\
\hline 2 & 85.9038 & $\begin{array}{l}24050.551 \\
9\end{array}$ & $5.89 \mathrm{~min}$ & 1312.9553 & 5890.5833 \\
\hline
\end{tabular}




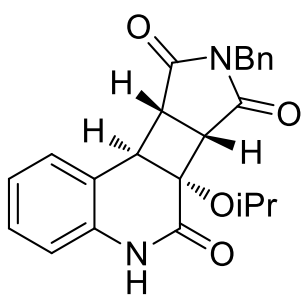

3s

Racemic (Daicel CHIRALPAK ${ }^{\circledR}$ OD-H, isocratic $20 \% \mathrm{MeOH}, 3.0 \mathrm{~mL} / \mathrm{min}$ )

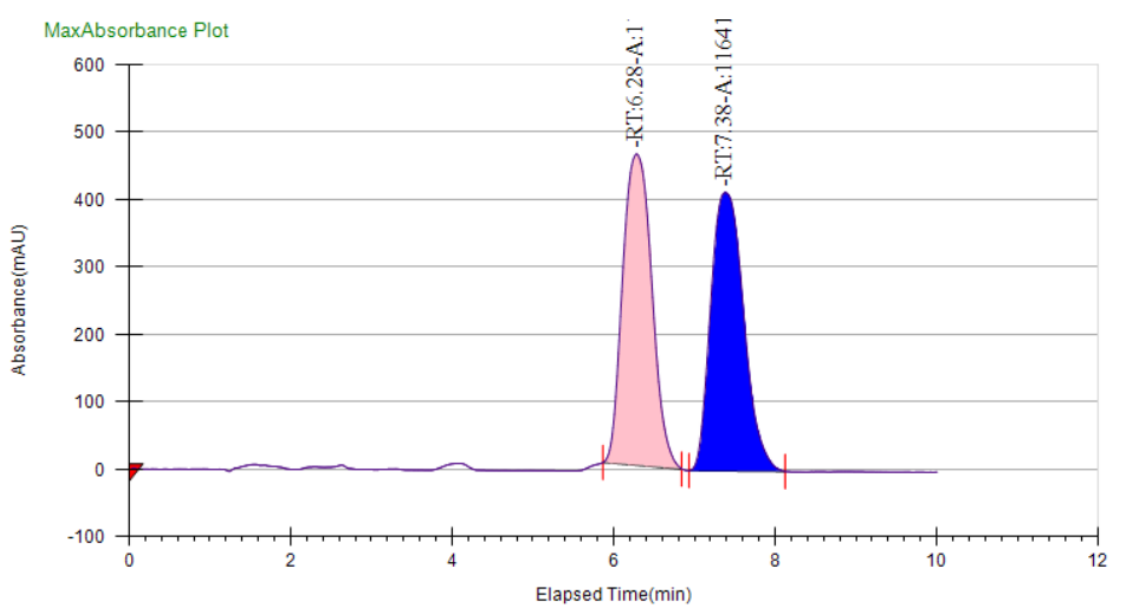

Peak Information

\begin{tabular}{|l|l|l|l|l|l|}
\hline Peak No & $\%$ Area & Area & Ret. Time & Height & Cap. Factor \\
\hline 1 & 49.6997 & 11502.515 & $6.28 \mathrm{~min}$ & 461.9003 & 6282.25 \\
\hline 2 & & 7 & & & \\
\hline
\end{tabular}

\section{Enantioenriched}

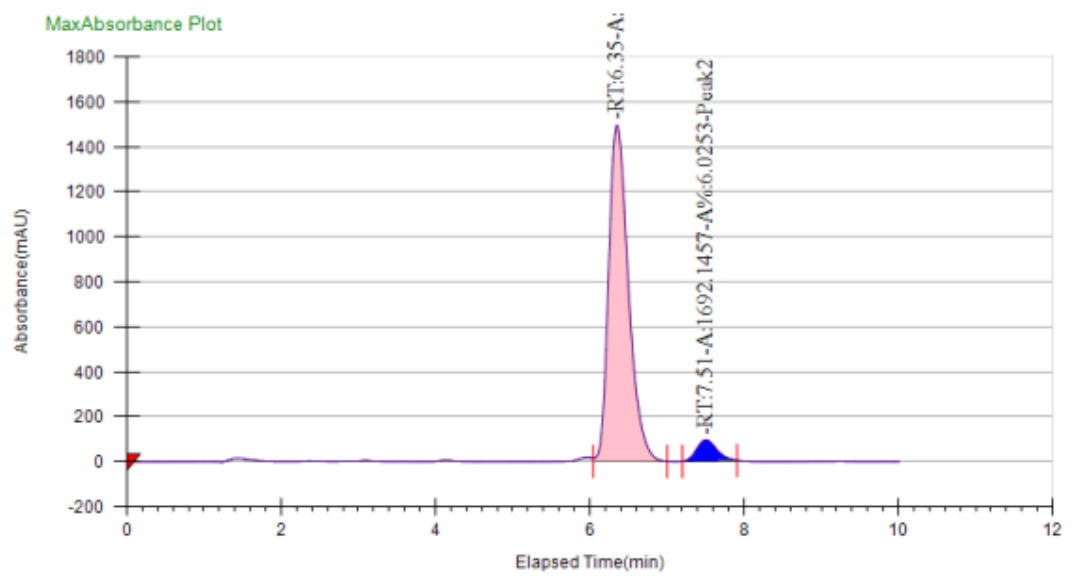

Peak Information

\begin{tabular}{|l|l|l|l|l|l|}
\hline Peak No & \% Area & Area & Ret. Time & Height & Cap. Factor \\
\hline 1 & 93.9747 & 26391.952 & $6.35 \mathrm{~min}$ & 1494.3521 & 6348.9167 \\
\hline 2 & 6.0253 & 1692.1457 & $7.51 \mathrm{~min}$ & 93.9479 & 7507.2333 \\
\hline
\end{tabular}




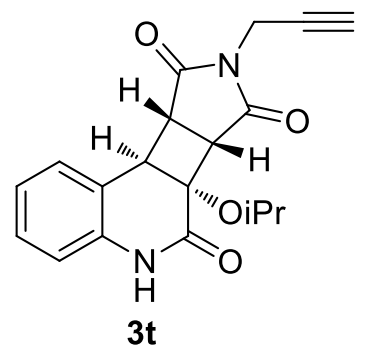

Racemic (HPLC, Daicel CHIRALPAK ${ }^{\circledR}$ AD-H, Gradient 5\% iPrOH (95\% Hexane) to $40 \%$ iPrOH (60\% Hexane), $1.0 \mathrm{~mL} / \mathrm{min}$ )

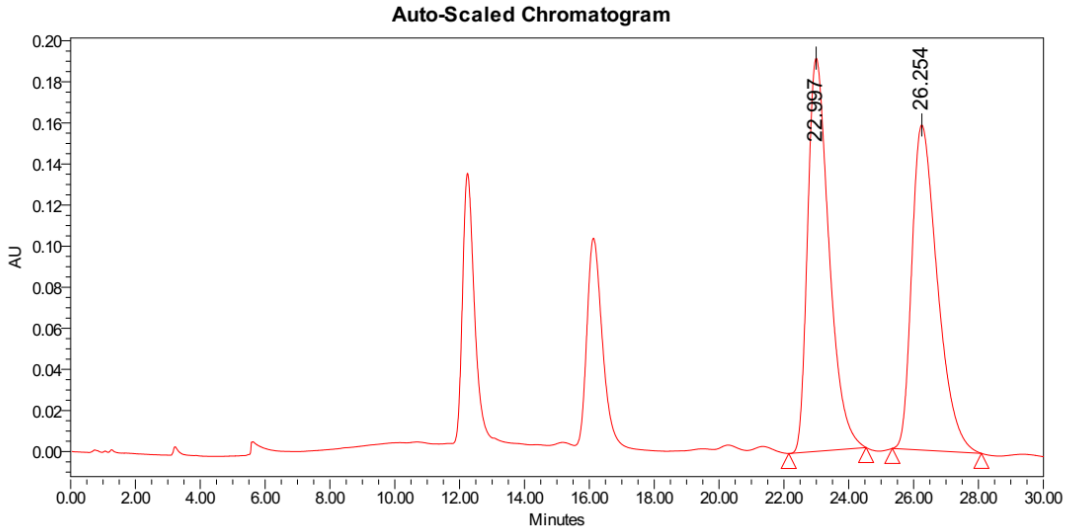

Peak Results

\begin{tabular}{|c|c|c|c|r|}
\hline & RT & Area & Height & \% Area \\
\hline 1 & 22.997 & 8781325 & 191488 & 50.29 \\
\hline 2 & 26.254 & 8679006 & 158274 & 49.71 \\
\hline
\end{tabular}

\section{Enantioenriched}

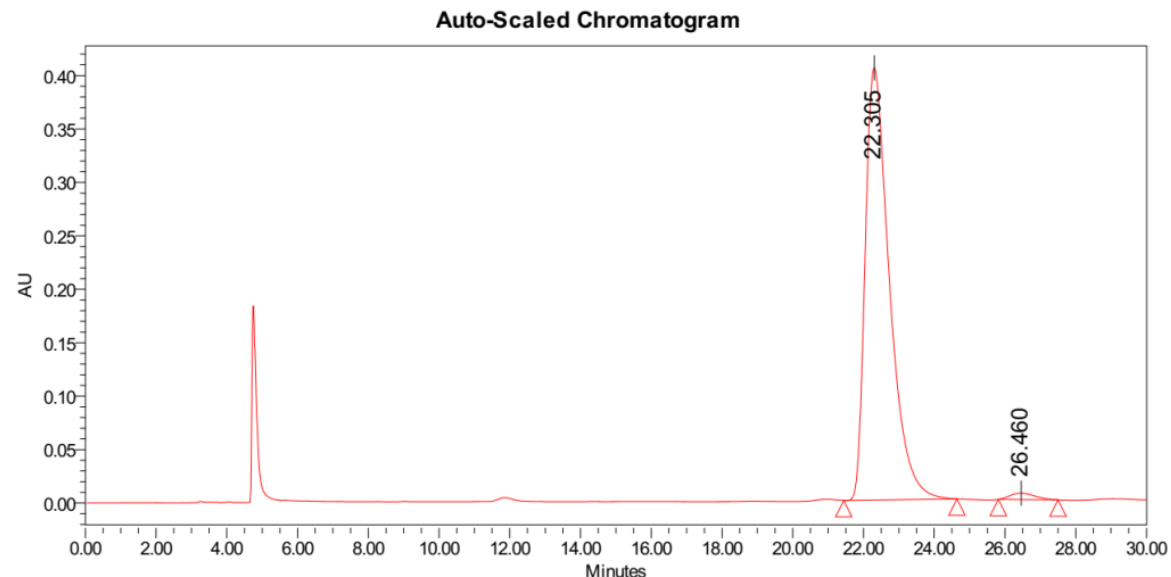

Peak Results

\begin{tabular}{|r|c|r|r|r|}
\hline & RT & \multicolumn{1}{|c|}{ Area } & Height & \% Area \\
\hline 1 & 22.305 & 19213782 & 404446 & 98.48 \\
\hline 2 & 26.460 & 296053 & 6030 & 1.52 \\
\hline
\end{tabular}




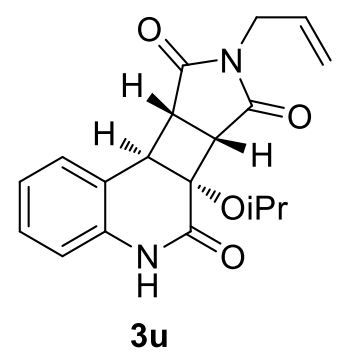

Racemic (Daicel CHIRALPAK ${ }^{\circledR}$ OD-H, isocratic $20 \% \mathrm{MeOH}, 3.0 \mathrm{~mL} / \mathrm{min}$ )

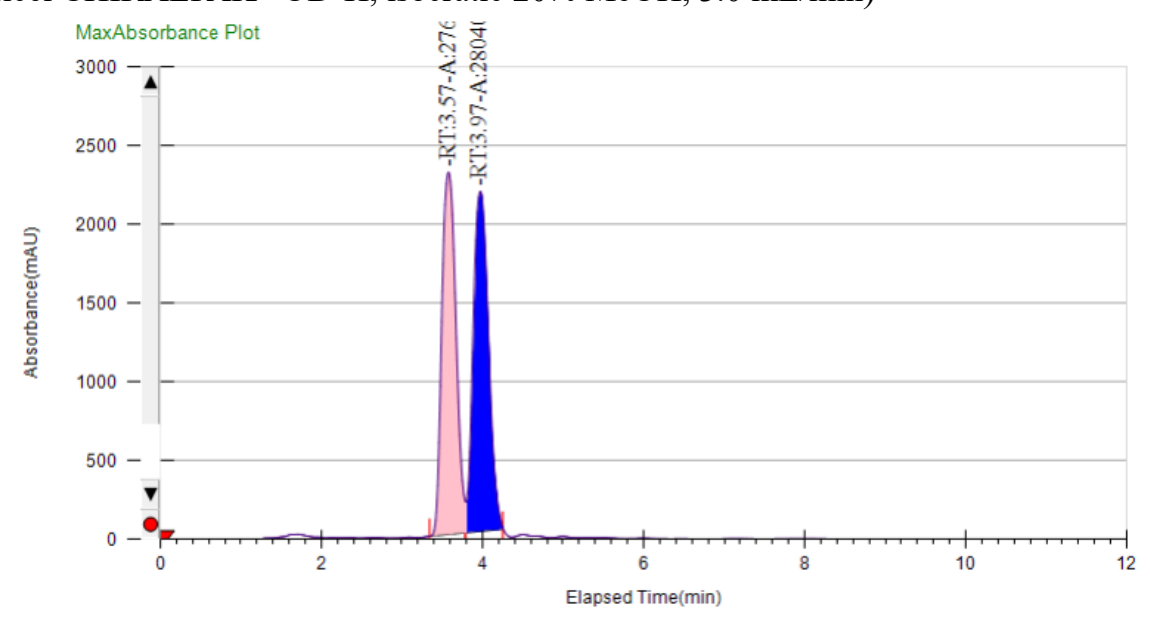

Peak Information

\begin{tabular}{|l|l|l|l|l|l|}
\hline Peak No & \% Area & Area & Ret. Time & Height & Cap. Factor \\
\hline 1 & 49.6657 & $\begin{array}{l}27668.408 \\
3\end{array}$ & 3.57 min & 2299.687 & 3573.95 \\
\hline 2 & 50.3343 & 28040.904 & 3.97 min & 2156.4579 & 3973.95 \\
\hline
\end{tabular}

\section{Enantioenriched}

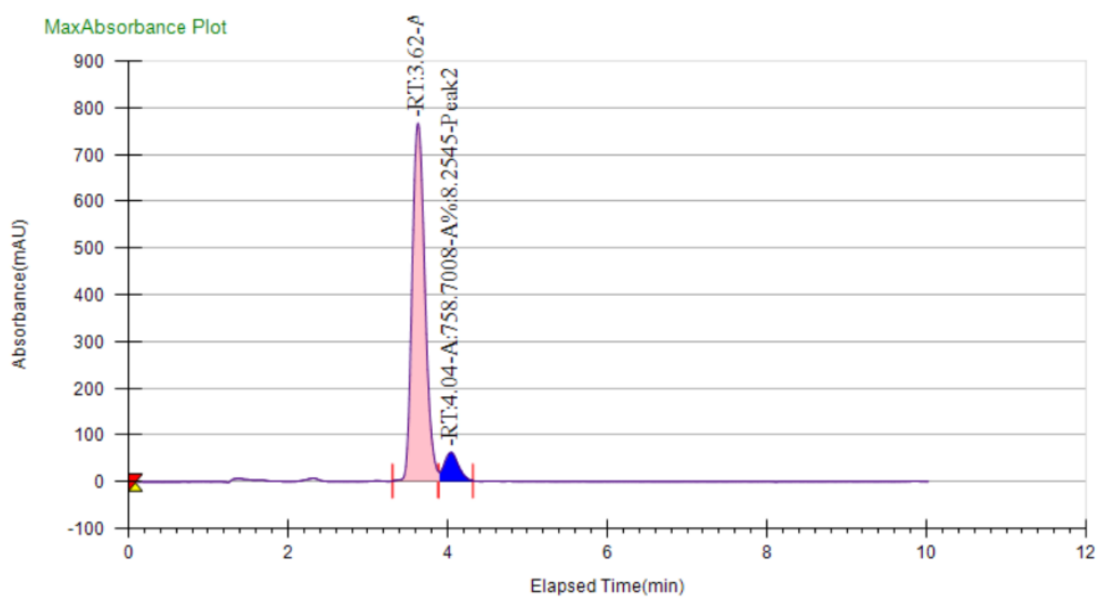

\section{Peak Information}

\begin{tabular}{|l|l|l|l|l|l|}
\hline Peak No & $\%$ Area & Area & Ret. Time & Height & Cap. Factor \\
\hline 1 & 91.7455 & 8432.6091 & $3.62 \mathrm{~min}$ & 765.2103 & 3623.95 \\
\hline 2 & 8.2545 & 758.7008 & $4.04 \mathrm{~min}$ & 60.7419 & 4040.6167 \\
\hline
\end{tabular}


<smiles>[2H][C@]12C(=O)Nc3ccccc3[C@H]([C@@H]3C(=O)N(C(=O)OC)C(=O)[C@@H]31)[C@@H]2C(C)C</smiles>

Racemic (Daicel CHIRALPAK ${ }^{\circledR}$ OD-H, isocratic $20 \% \mathrm{MeOH}, 3.0 \mathrm{~mL} / \mathrm{min}$ )

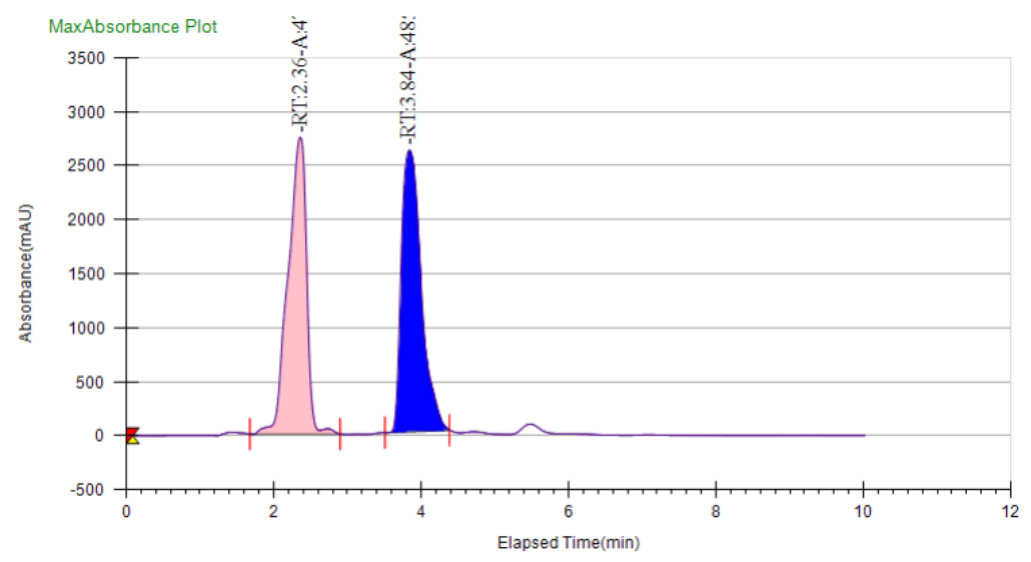

Peak Information

\begin{tabular}{|l|l|l|l|l|l|}
\hline Peak No & $\%$ Area & Area & Ret. Time & Height & Cap. Factor \\
\hline 1 & 49.5785 & $\begin{array}{l}47777.321 \\
4\end{array}$ & $2.36 \mathrm{~min}$ & 2749.646 & 2357.3 \\
\hline 2 & 50.4215 & $\begin{array}{l}48589.759 \\
2\end{array}$ & $3.84 \mathrm{~min}$ & 2609.6966 & 3840.6167 \\
\hline
\end{tabular}

\section{Enantioenriched}

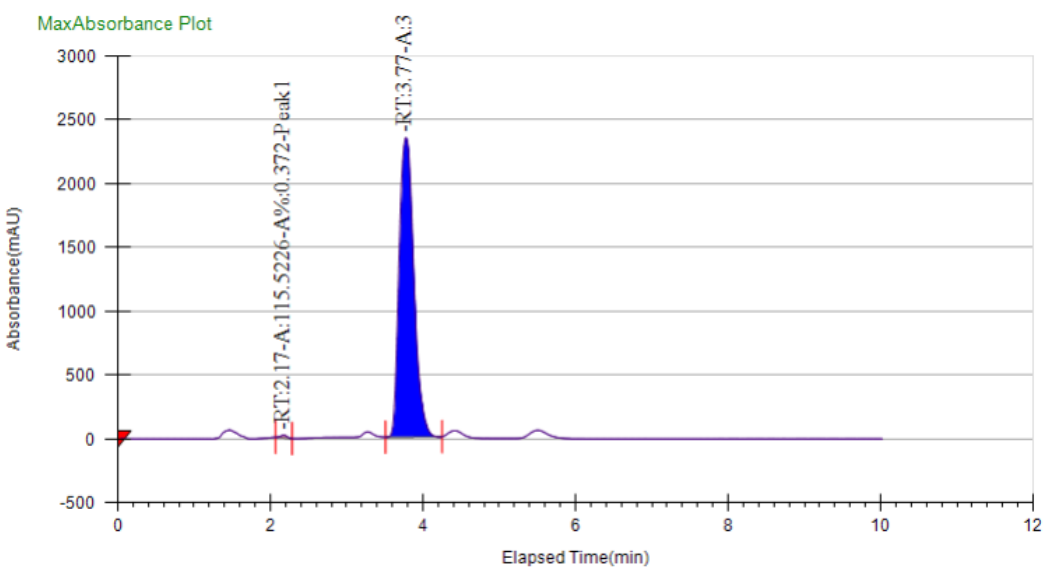

Peak Information

\begin{tabular}{|l|l|l|l|l|l|}
\hline Peak No & $\%$ Area & Area & Ret. Time & Height & Cap. Factor \\
\hline 1 & 0.372 & 115.5226 & $2.17 \mathrm{~min}$ & 20.5205 & 2173.9667 \\
\hline 2 & 99.628 & $\begin{array}{l}30940.504 \\
4\end{array}$ & $3.77 \mathrm{~min}$ & 2346.5626 & 3773.95 \\
\hline
\end{tabular}


<smiles>CCOC(C)=O</smiles>

$3 w$

Racemic (Daicel CHIRALPAK ${ }^{\circledR}$ OD-H, isocratic $20 \% \mathrm{MeOH}, 3.0 \mathrm{~mL} / \mathrm{min}$ )

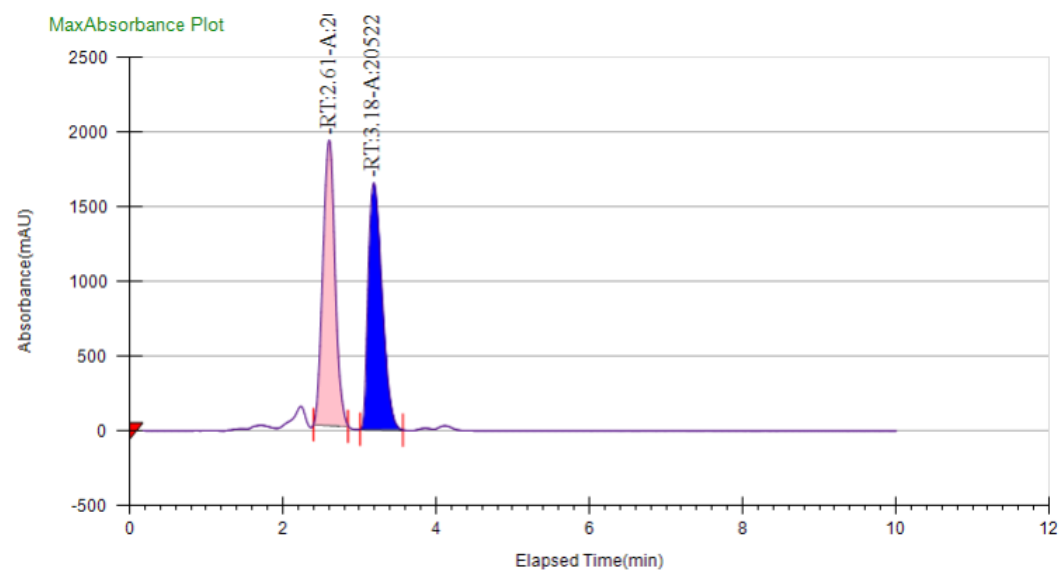

Peak Information

\begin{tabular}{|l|l|l|l|l|l|}
\hline Peak No & $\%$ Area & Area & Ret. Time & Height & Cap. Factor \\
\hline 1 & 50.3225 & $\begin{array}{l}20788.889 \\
7\end{array}$ & $2.61 \mathrm{~min}$ & 1910.7564 & 2607.3 \\
\hline 2 & 49.6775 & $\begin{array}{l}20522.407 \\
2\end{array}$ & $3.18 \mathrm{~min}$ & 1649.4834 & 3182.3 \\
\hline
\end{tabular}

\section{Enantioenriched}

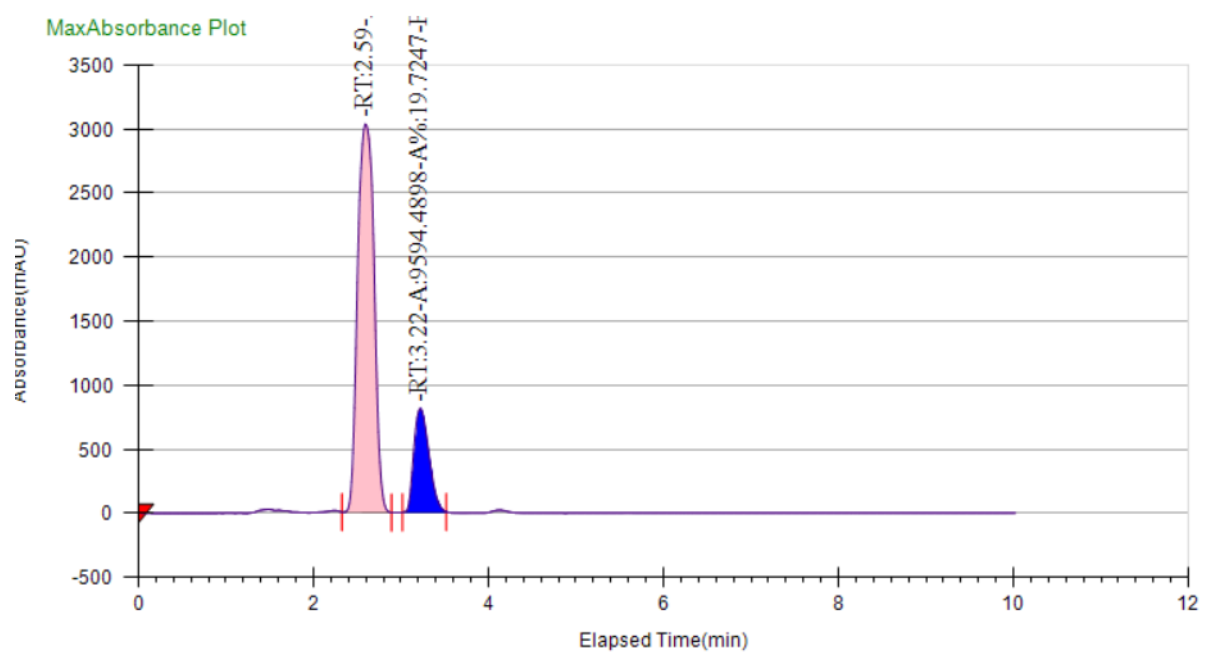

Peak Information

\begin{tabular}{|l|l|l|l|l|l|}
\hline Peak No & $\%$ Area & Area & Ret. Time & Height & Cap. Factor \\
\hline 1 & 80.2753 & $\begin{array}{l}39047.445 \\
9\end{array}$ & $2.59 \mathrm{~min}$ & 3034.5262 & 2590.6333 \\
\hline 2 & 19.7247 & 9594.4898 & $3.22 \mathrm{~min}$ & 809.8666 & 3215.6167 \\
\hline
\end{tabular}


<smiles>[Y19]C1Nc2ccccc2[C@H]2[C@@H]3C(=O)NC(=O)[C@@H]3[C@]3(OCCC=C)C(=O)NC(=O)[C@@H]2[C@H]13</smiles>

Racemic (HPLC, Daicel CHIRALPAK ${ }^{\circledR} \mathrm{OD}-\mathrm{H}$, Gradient $5 \% \mathrm{iPrOH}$ (95\% Hexane) to $50 \% \mathrm{iPrOH}(50 \%$ Hexane), $1.0 \mathrm{~mL} / \mathrm{min}$ )

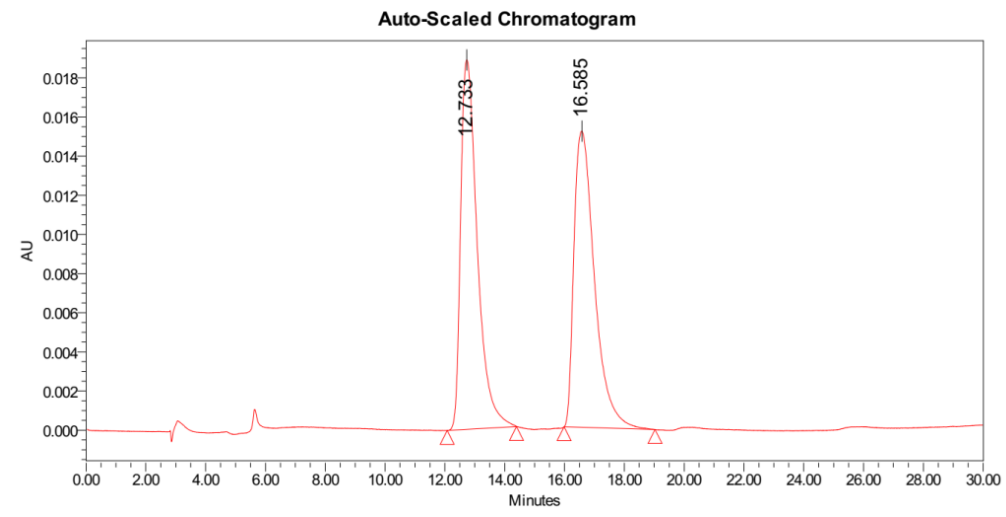

Peak Results
\begin{tabular}{|l|c|c|r|c|r|r|l|}
\hline & Name & RT & $\%$ Area & Area & Height & Amount & Units \\
\hline 1 & & 12.733 & 50.24 & 728494 & 18872 & & \\
\hline 2 & & 16.585 & 49.76 & 721629 & 15128 & & \\
\hline
\end{tabular}

\section{Enantioenriched}

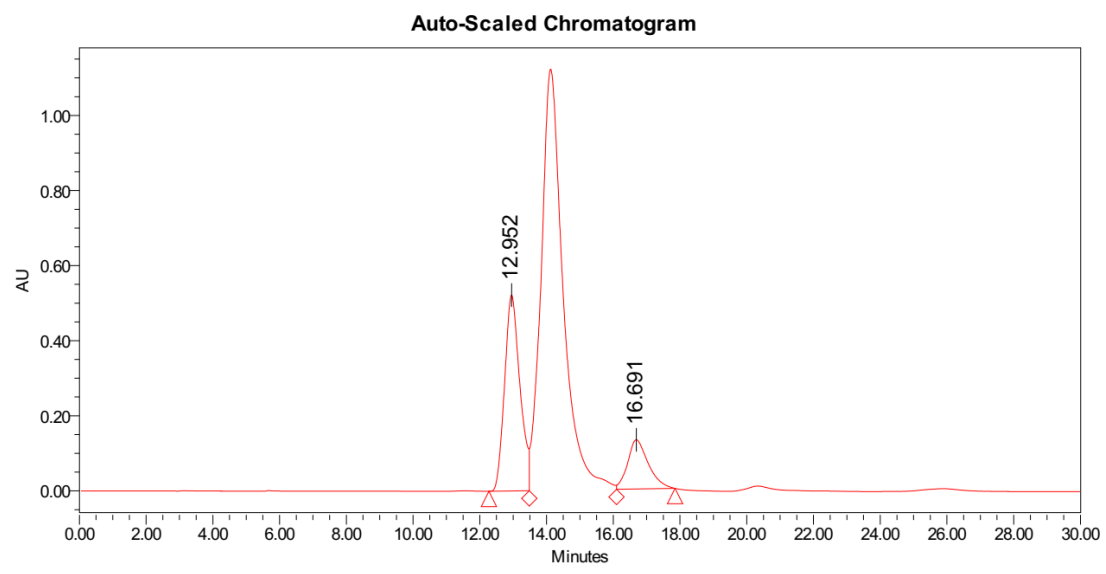

Peak Results

\begin{tabular}{|l|c|r|c|c|c|c|c|}
\hline & Name & $\%$ Area & RT & Area & Height & Amount & Units \\
\hline 1 & & 74.73 & 12.952 & 16663695 & 521729 & & \\
\hline 2 & & 25.27 & 16.691 & 5634896 & 131122 & & \\
\hline
\end{tabular}


<smiles>O=C1NC(=O)[C@@H]2[C@@H]1C[C@@H]2c1ccccc1</smiles>

Racemic (HPLC, Daicel CHIRALPAK ${ }^{\circledR} \mathrm{OD}-\mathrm{H}$, Gradient $5 \%$ iPrOH (95\% Hexane) to $40 \%$ iPrOH $(60 \%$ Hexane), $1.0 \mathrm{~mL} / \mathrm{min}$ )

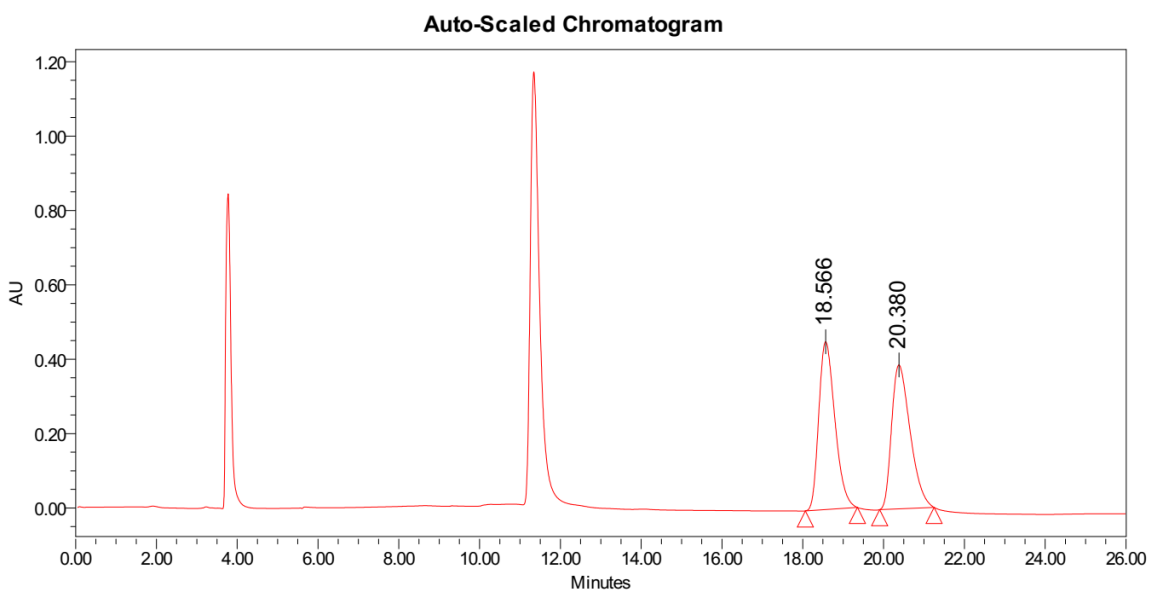

Peak Results

\begin{tabular}{c|c|c|c|c|c|c|r|}
\hline & Name & RT & Area & Height & Amount & Units & \% Area \\
\hline 1 & & 18.566 & 12840413 & 454174 & & & 49.62 \\
\hline 2 & & 20.380 & 13035845 & 392330 & & & 50.38 \\
\hline
\end{tabular}

\section{Enantioenriched}

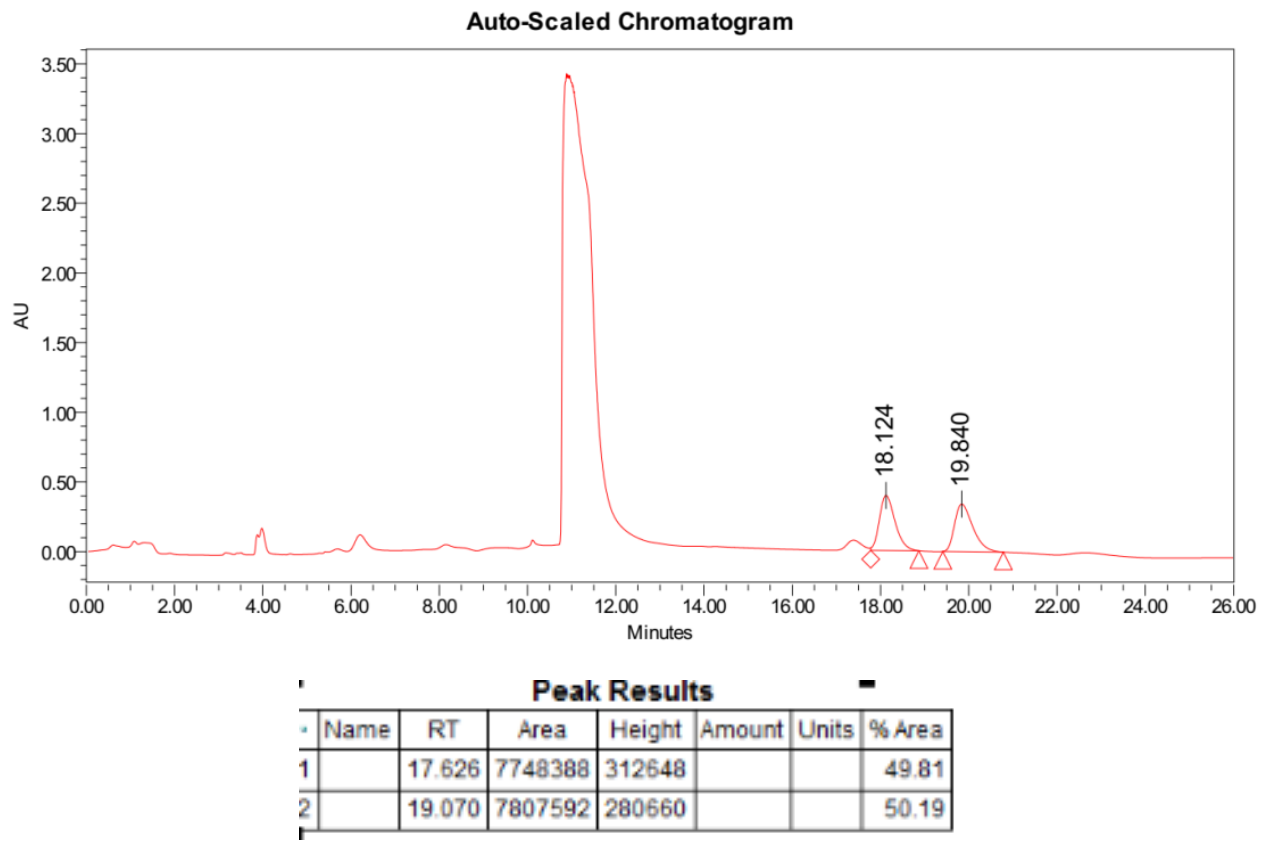




\section{NMR Spectra}

$\underbrace{\underbrace{0}_{0.00}}$
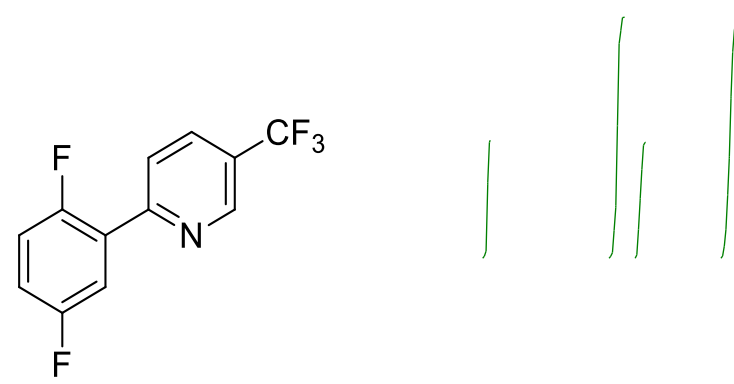

A2, ${ }^{1} \mathrm{H}$ NMR $\left(400 \mathrm{MHz}, \mathrm{CDCl}_{3}\right)$

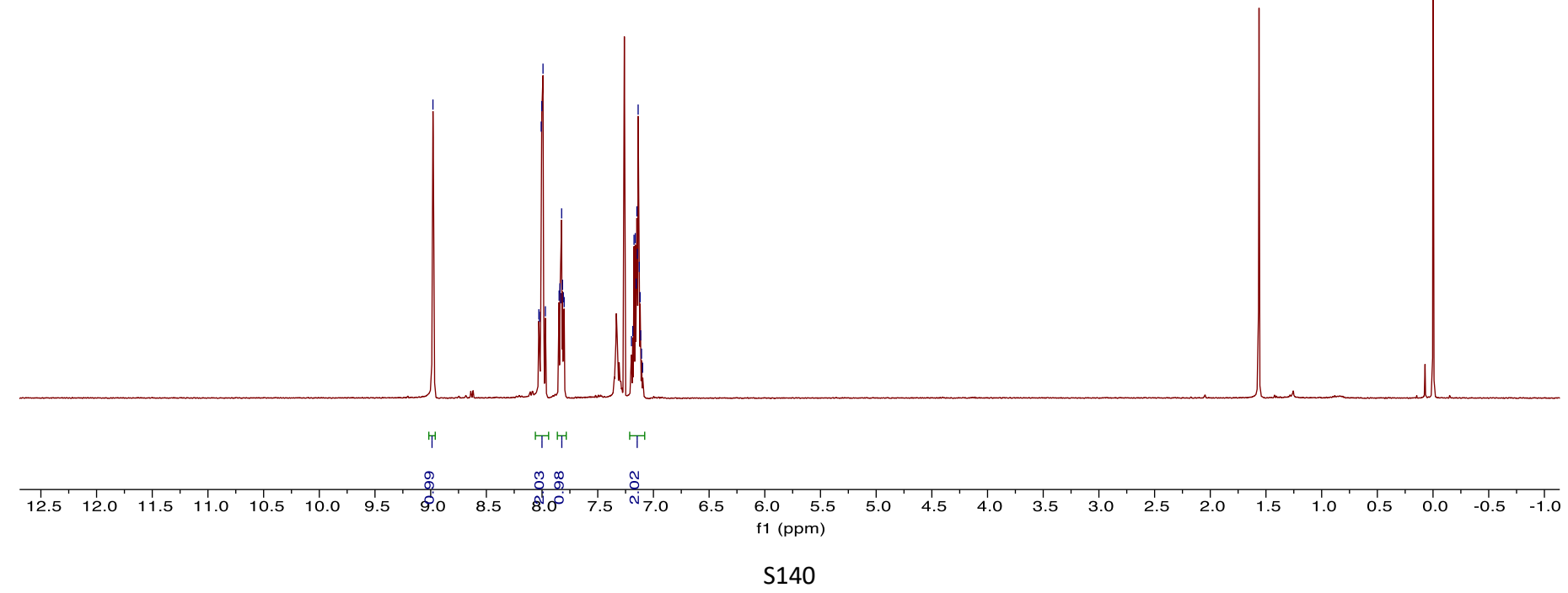




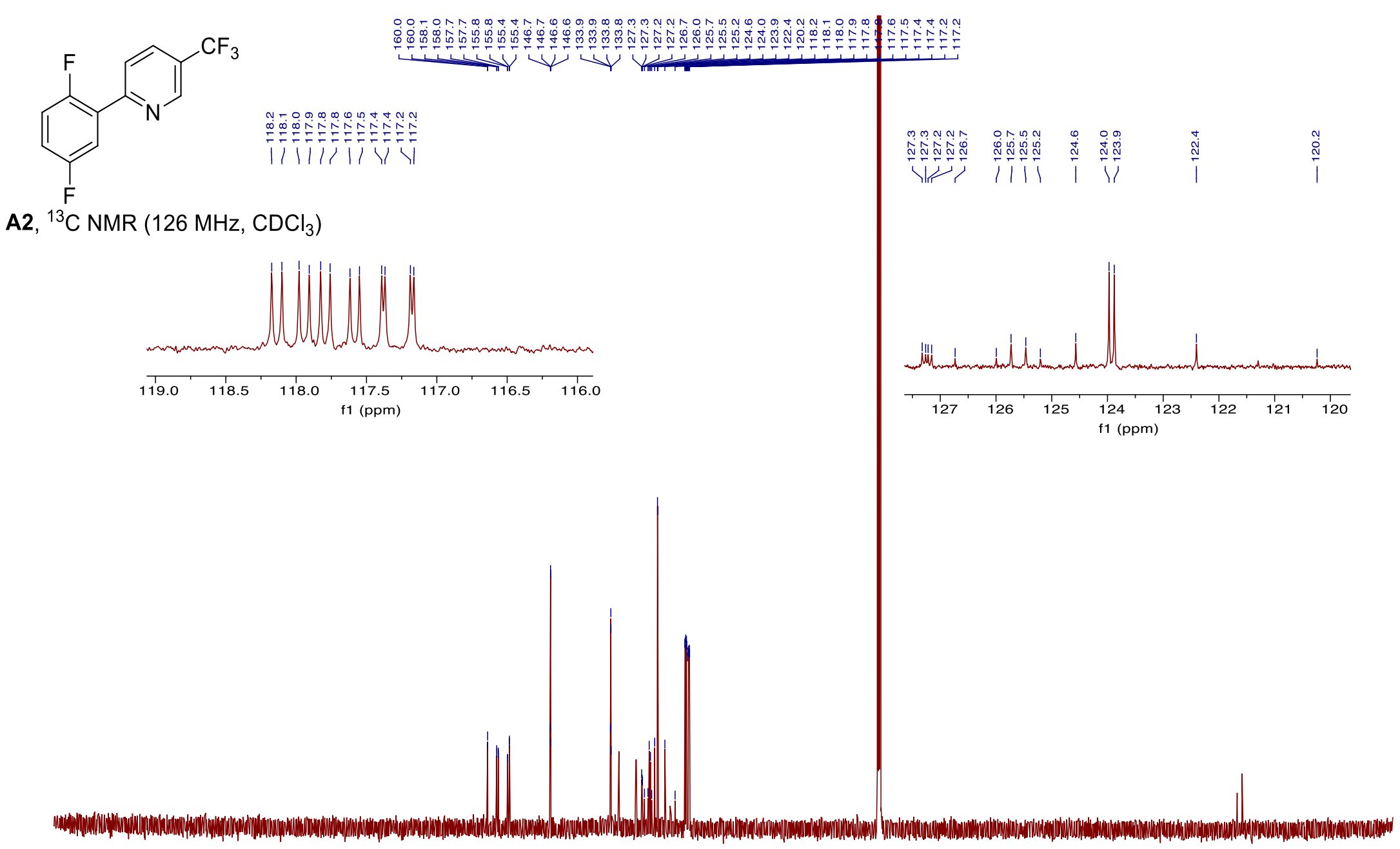

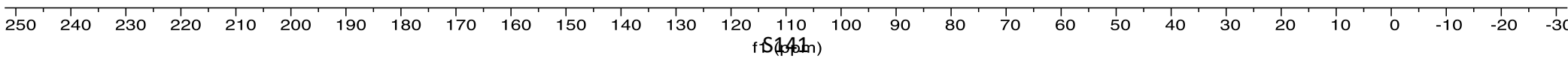




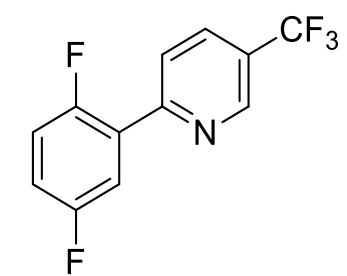

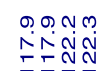

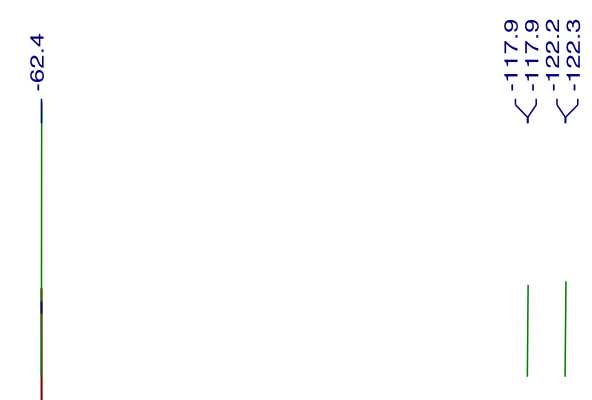

A2, ${ }^{19} \mathrm{~F} \mathrm{NMR}\left(377 \mathrm{MHz}, \mathrm{CDCl}_{3}\right)$

1

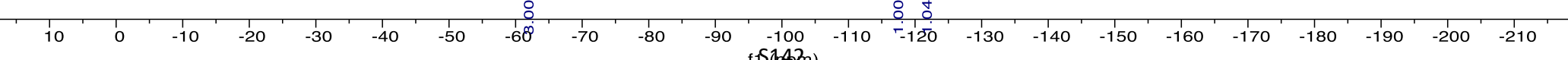




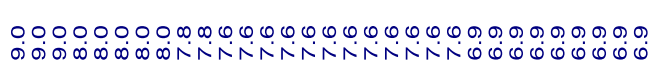

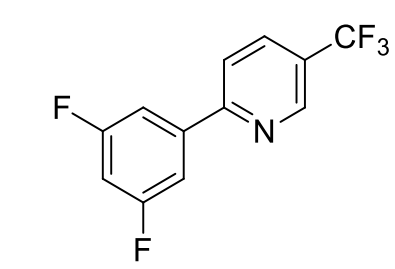

A4, ${ }^{1} \mathrm{H} \mathrm{NMR}\left(500 \mathrm{MHz}, \mathrm{CDCl}_{3}\right)$

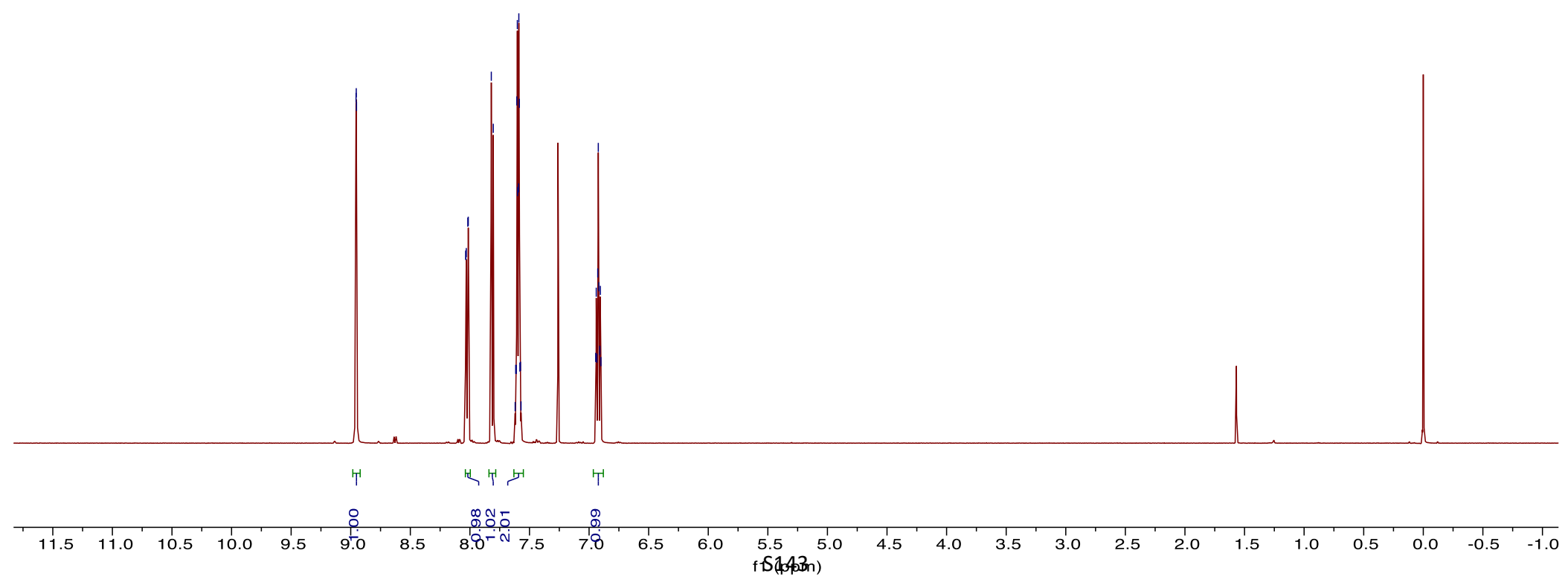



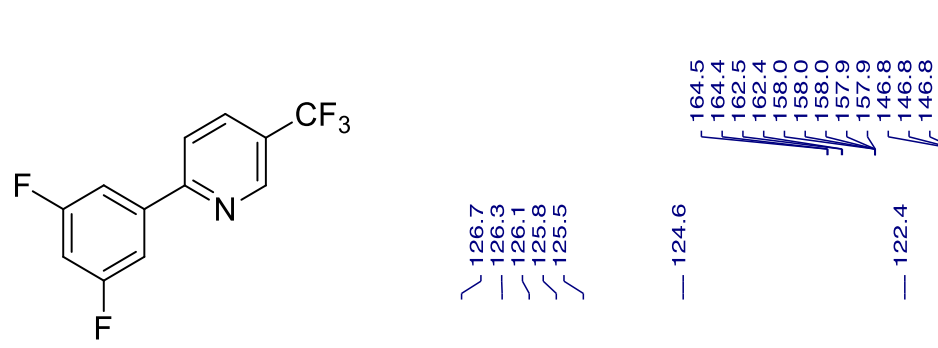

A4, ${ }^{13} \mathrm{C} \mathrm{NMR}\left(126 \mathrm{MHz}, \mathrm{CDCl}_{3}\right)$
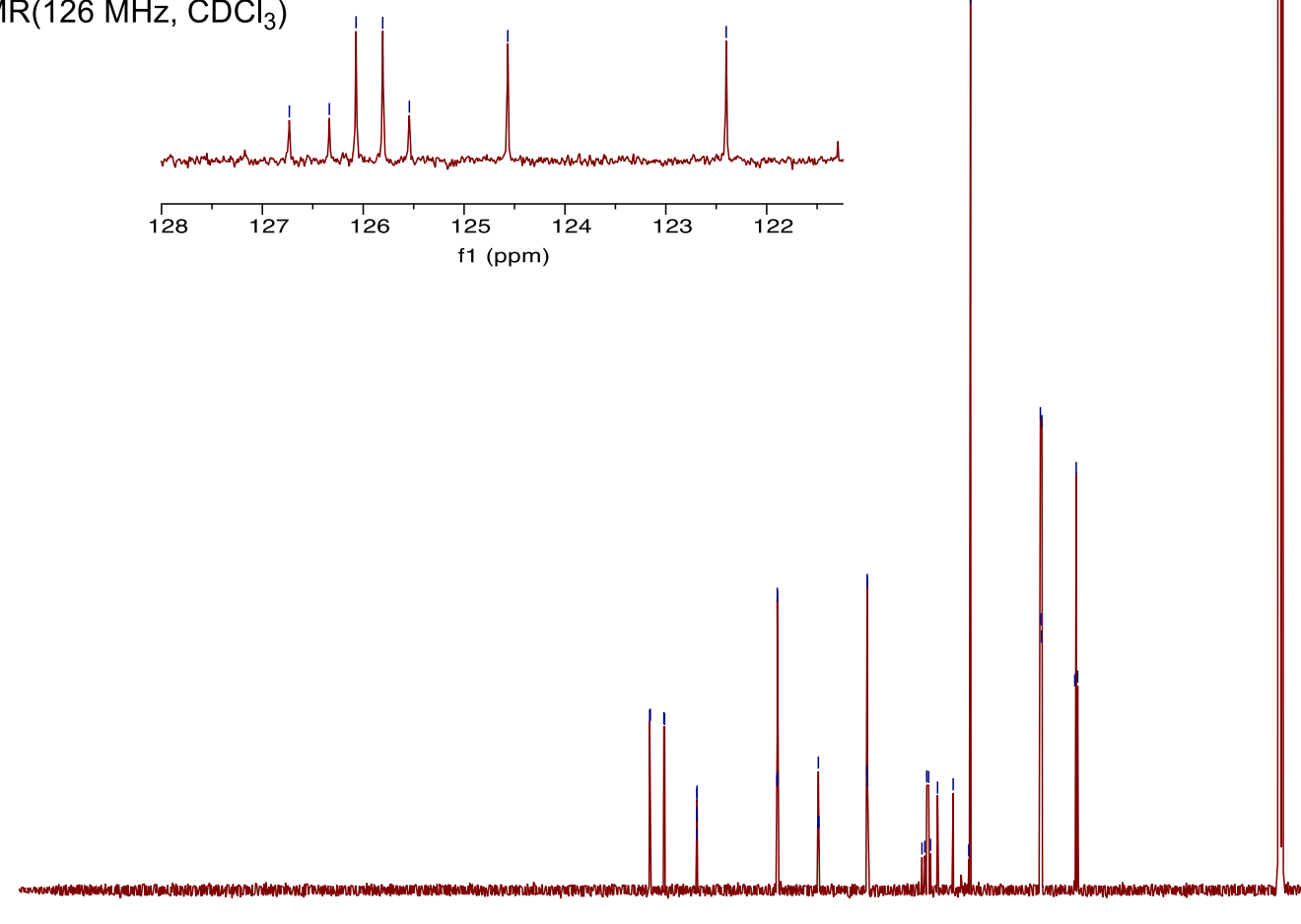

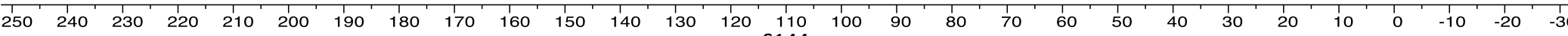
fis (164tom) 


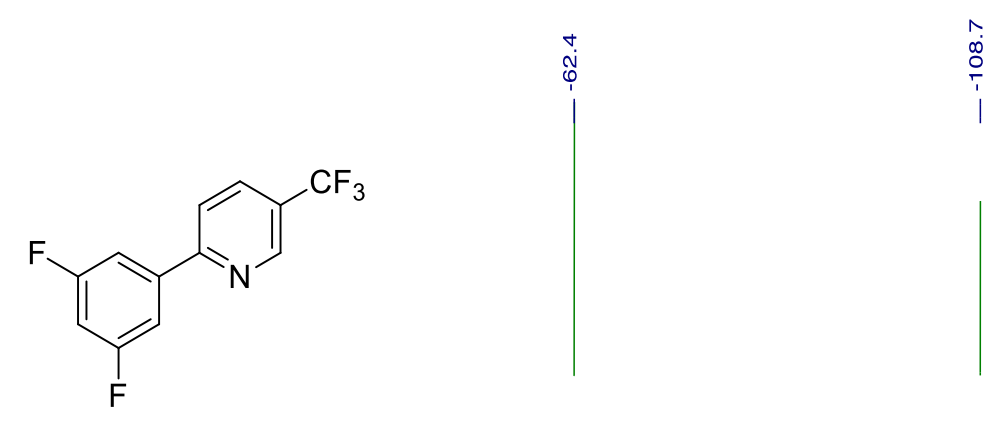

A4, ${ }^{19} \mathrm{~F} \mathrm{NMR}\left(377 \mathrm{MHz}, \mathrm{CDCl}_{3}\right)$

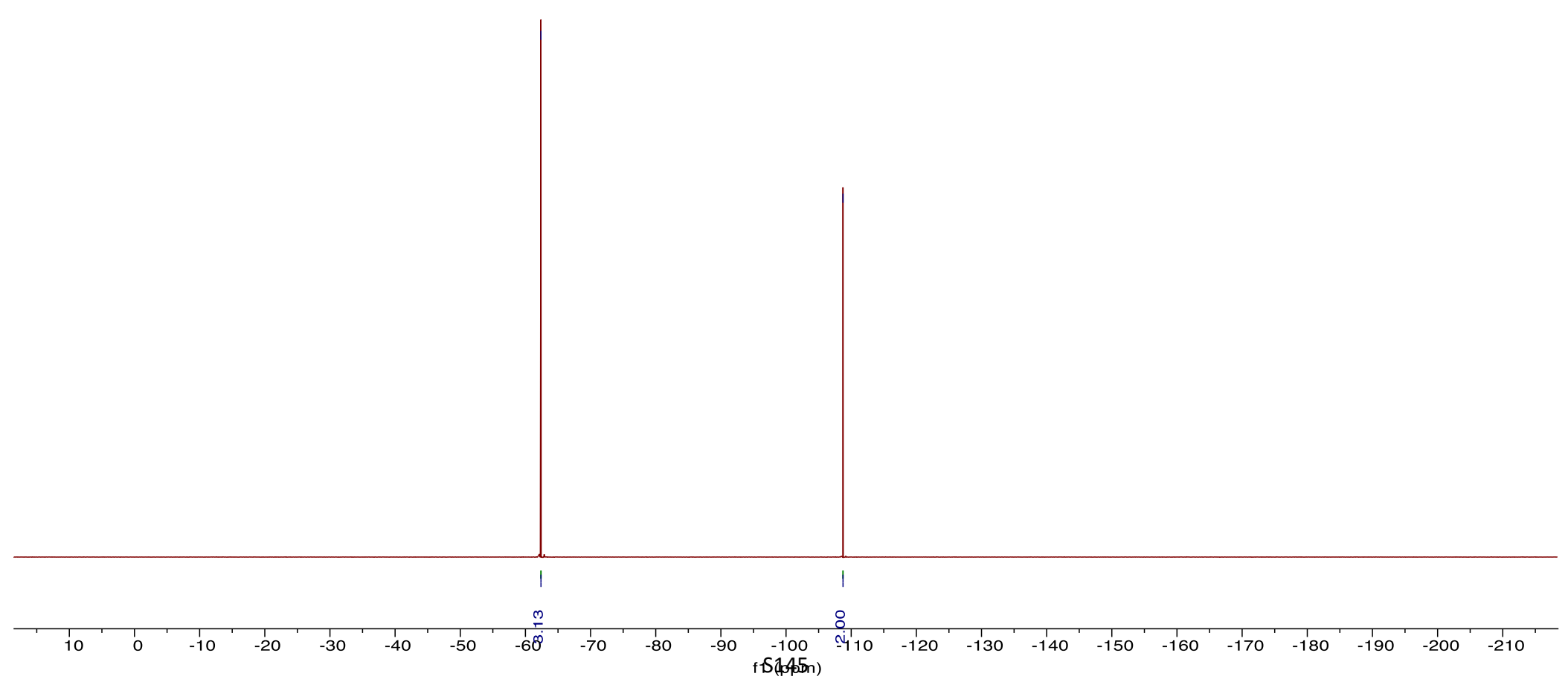




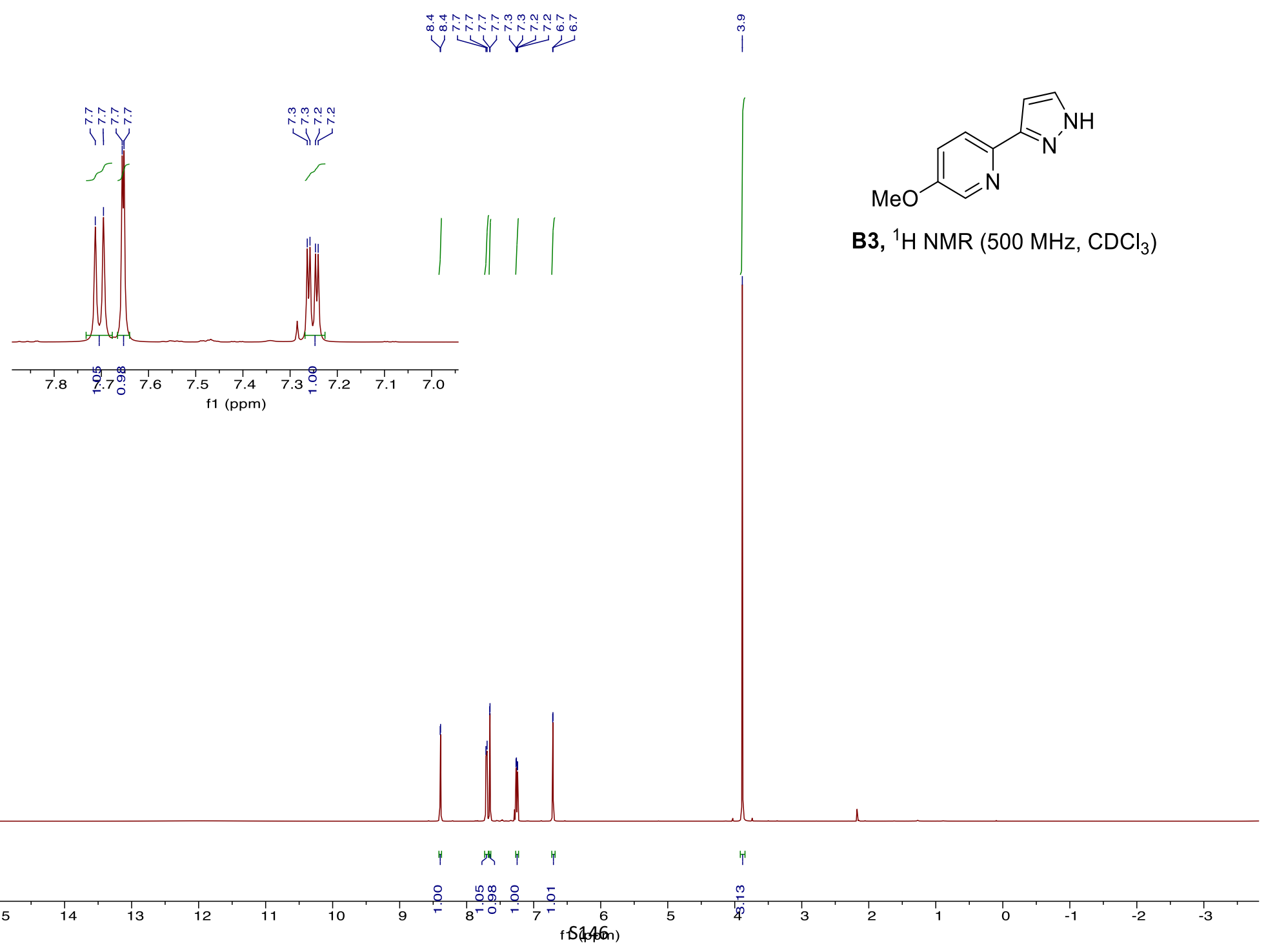




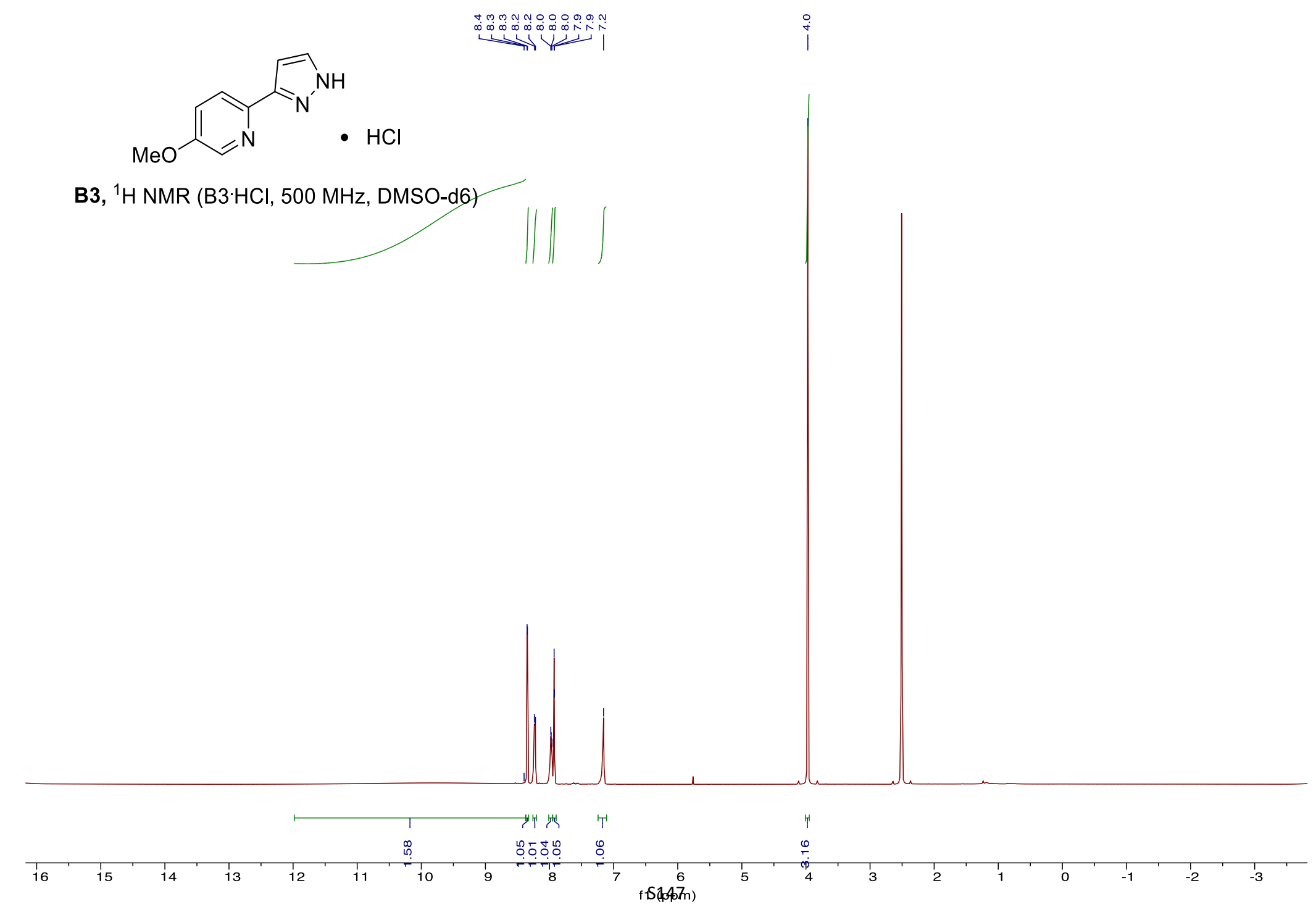




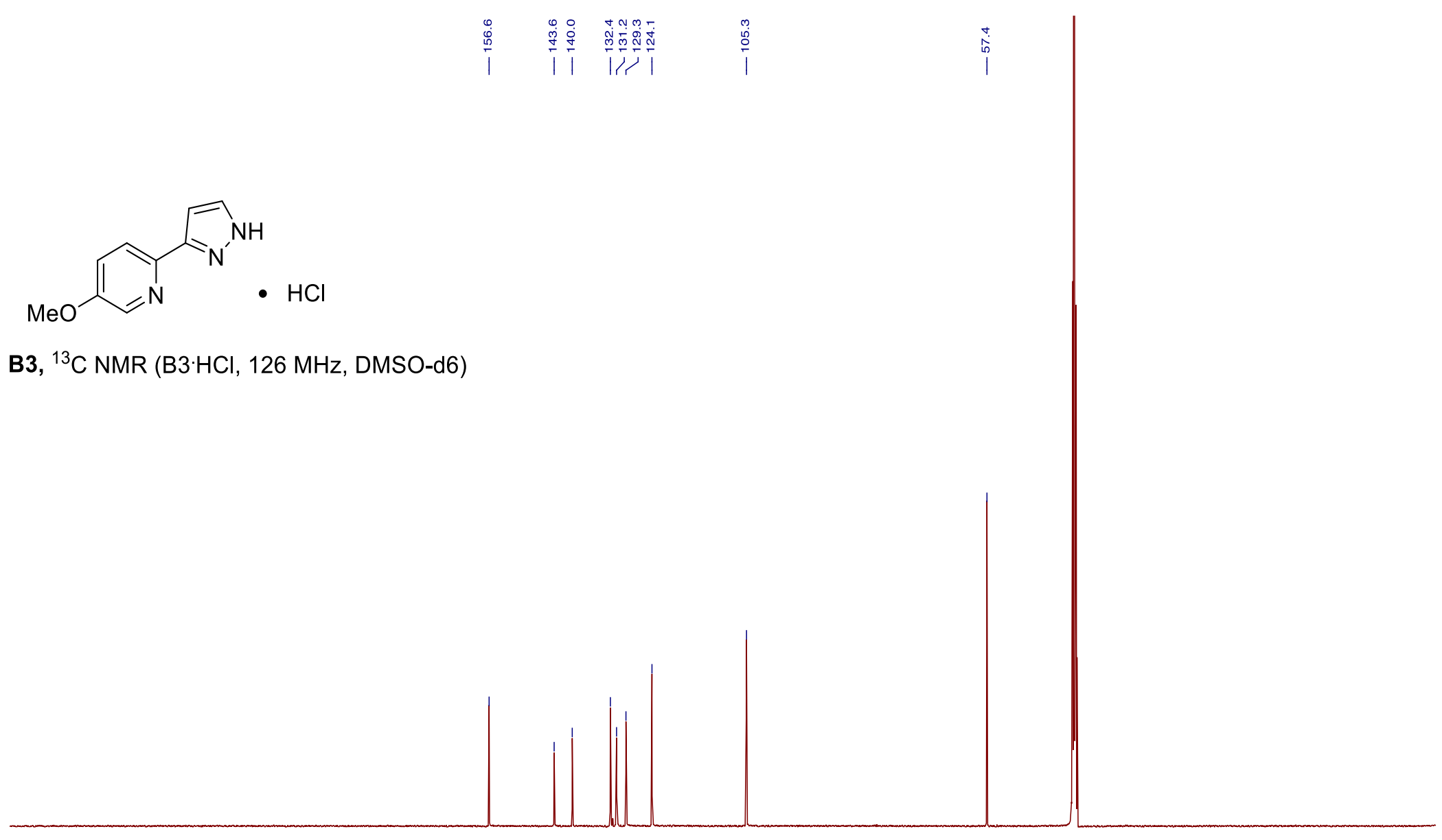

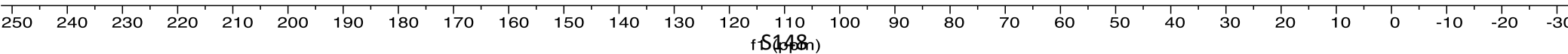




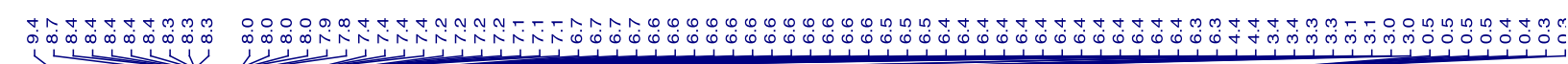

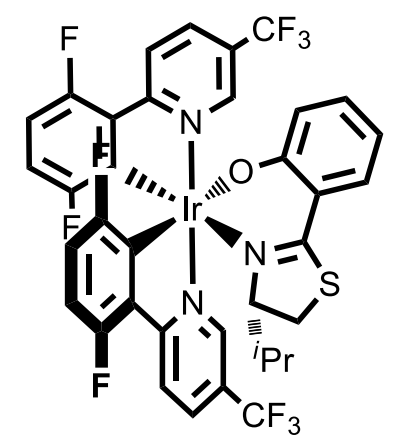

$(\mathrm{S}), \wedge-\left[\operatorname{Ir}\left(\mathbf{A}_{\mathbf{2}}\right)_{2}(\mathrm{PPrHBT})\right]:{ }^{1} \mathrm{H}$ NMR $\left(400 \mathrm{MHz}, \mathrm{CDCl}_{3}\right)$
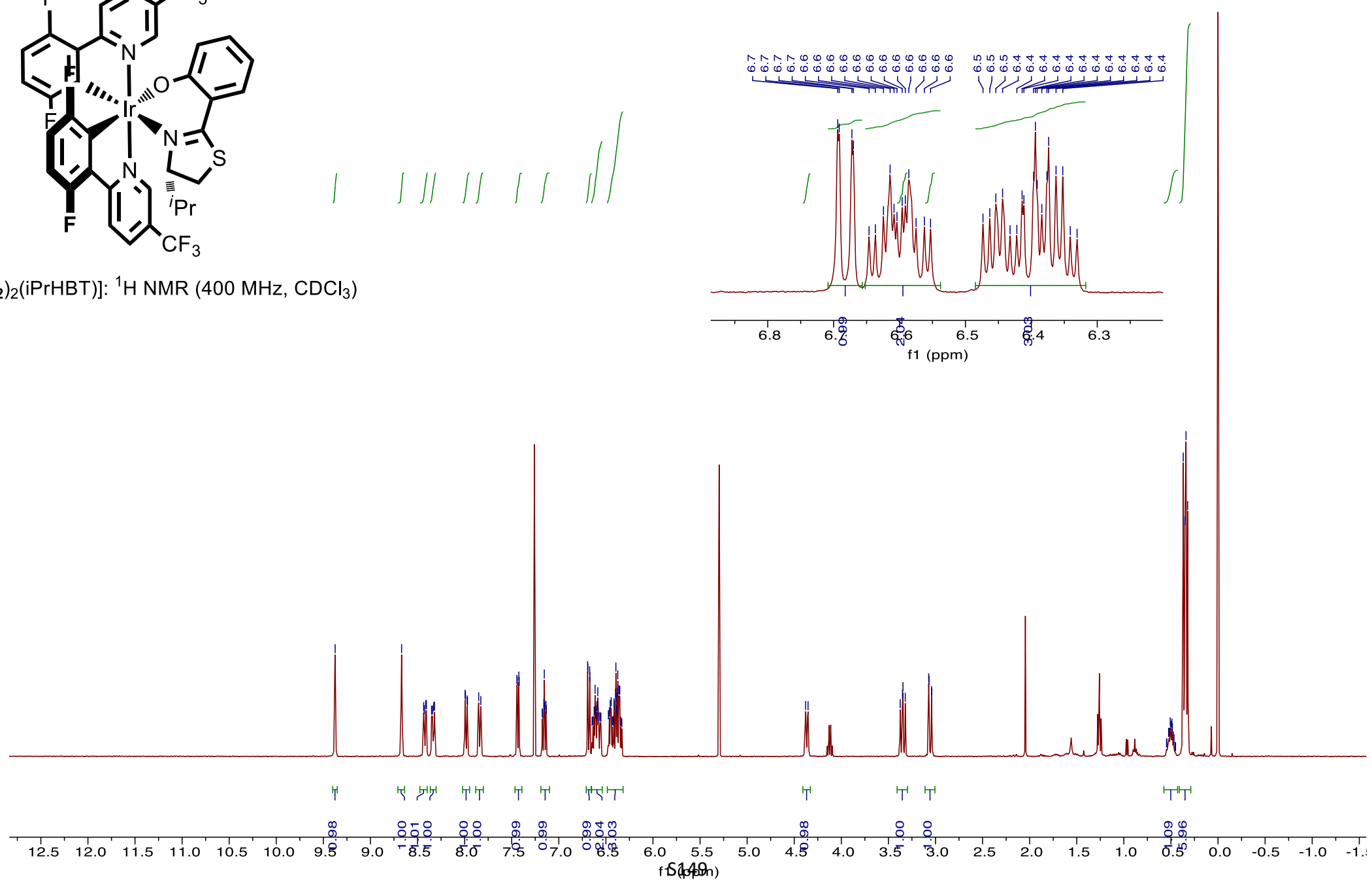


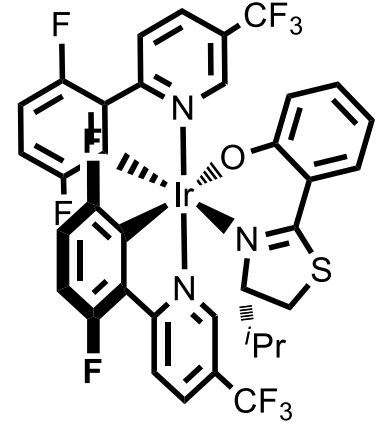

(S), $\wedge-\left[\operatorname{lr}\left(\mathbf{A}_{\mathbf{2}}\right)_{2}(\mathrm{iPrHBT})\right]:{ }^{19} \mathrm{~F}$ NMR $\left(377 \mathrm{MHz}, \mathrm{CDCl}_{3}\right)$
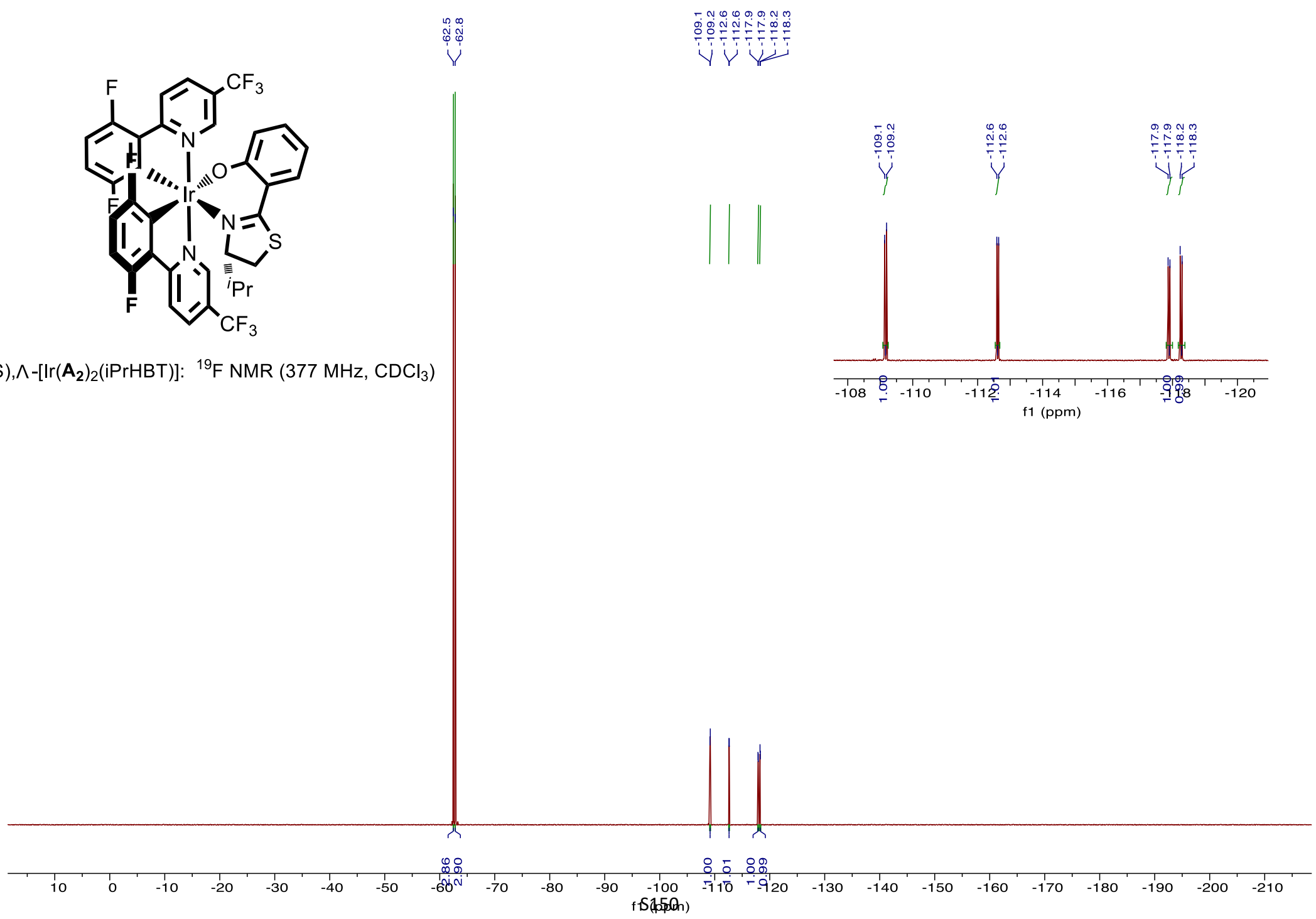
م)

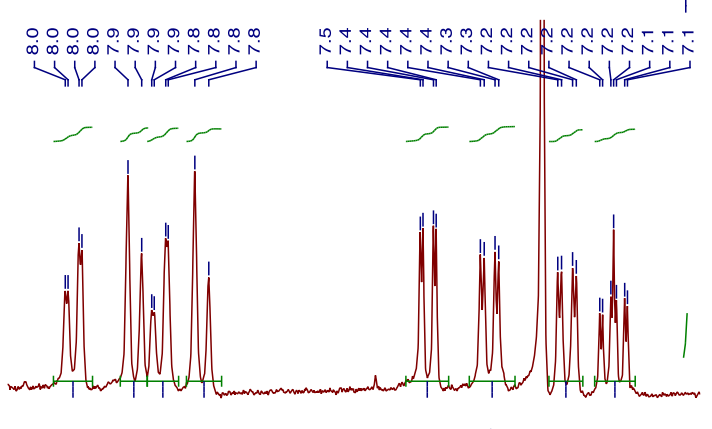

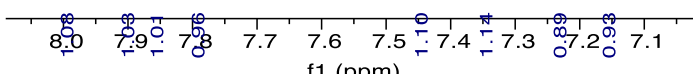

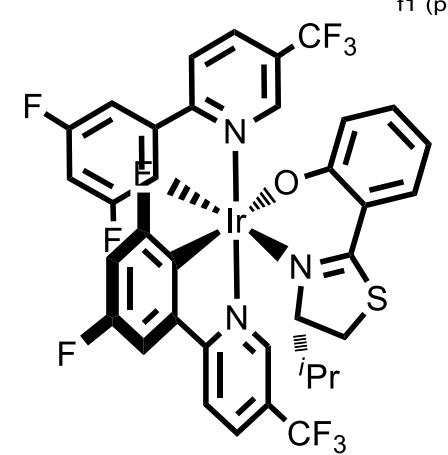

(S), $\wedge-\left[\operatorname{lr}\left(\mathbf{A}_{4}\right)_{2}(\mathrm{PPrHBT})\right]:{ }^{1} \mathrm{H}$ NMR $\left(400 \mathrm{MHz}, \mathrm{CDCl}_{3}\right)$

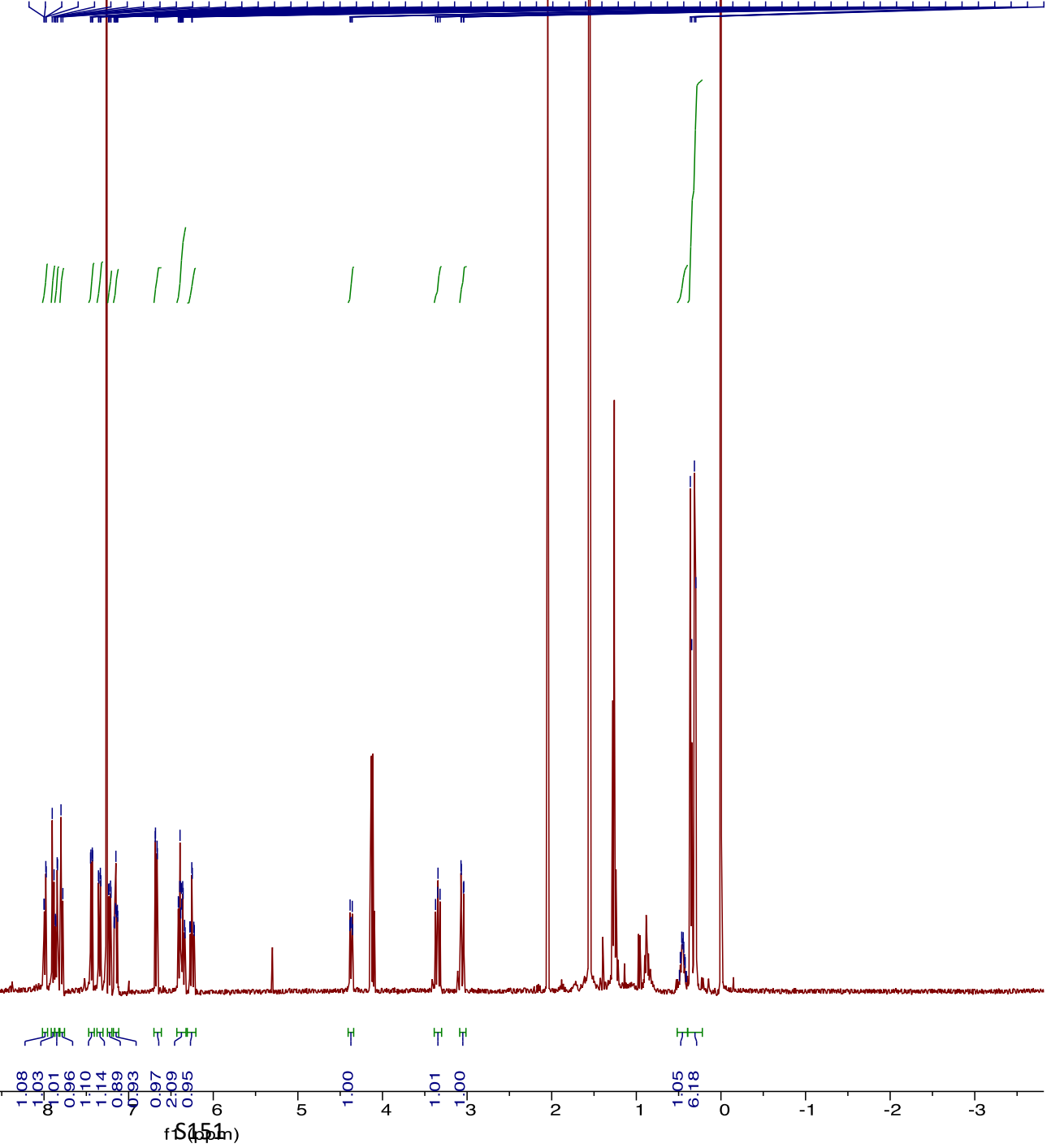




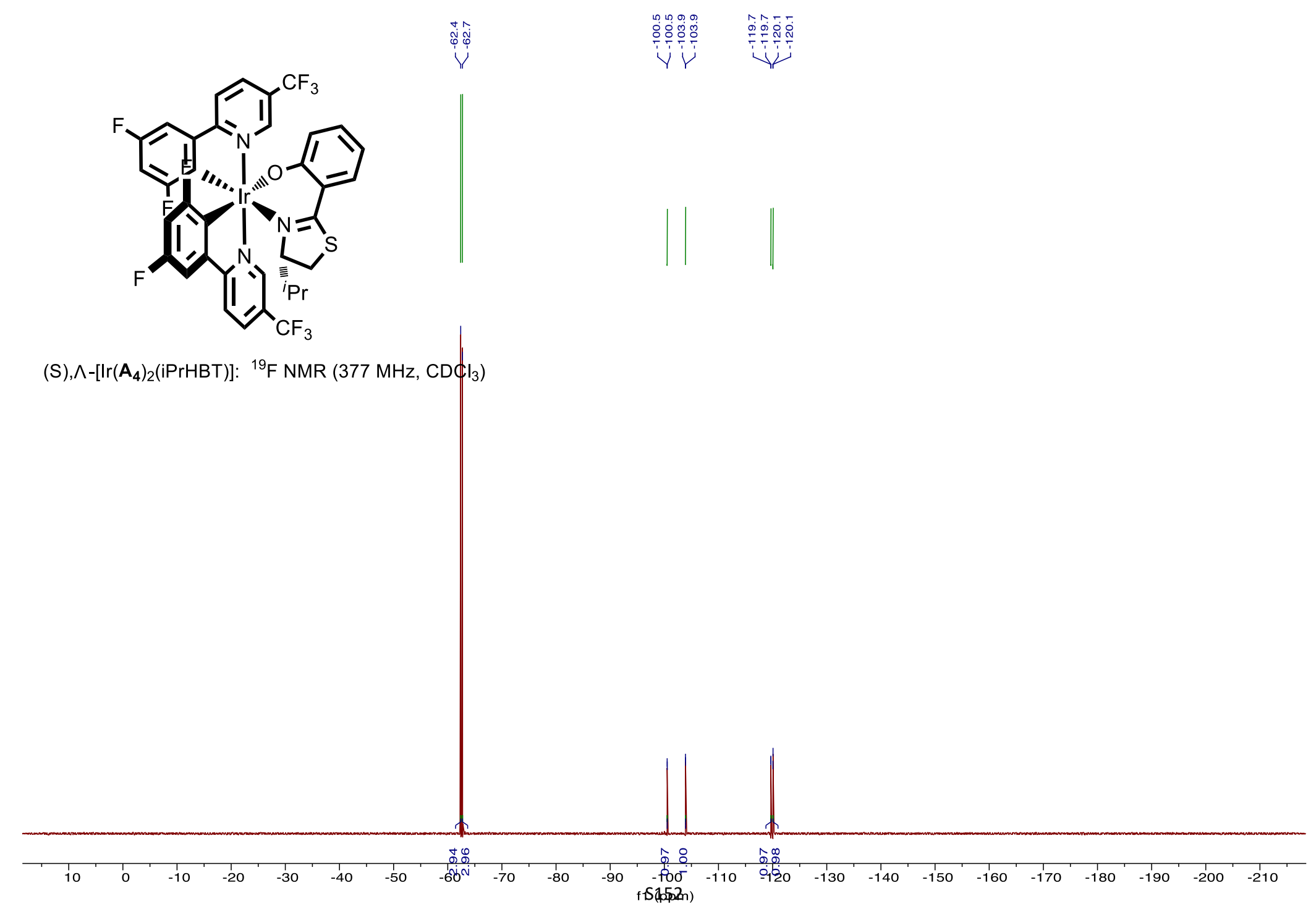




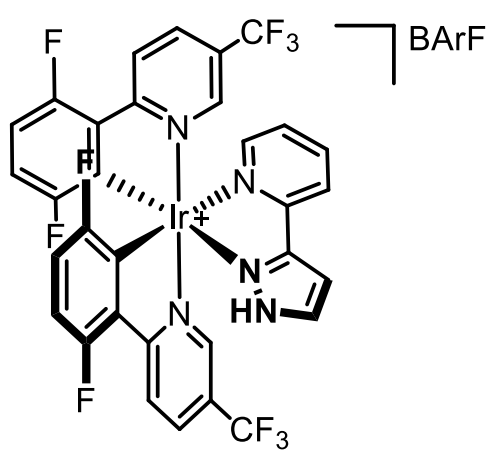

-4b, $\mathrm{H} \mathrm{NMR}\left(400 \mathrm{MHz}, \mathrm{CDCl}_{3}\right)$

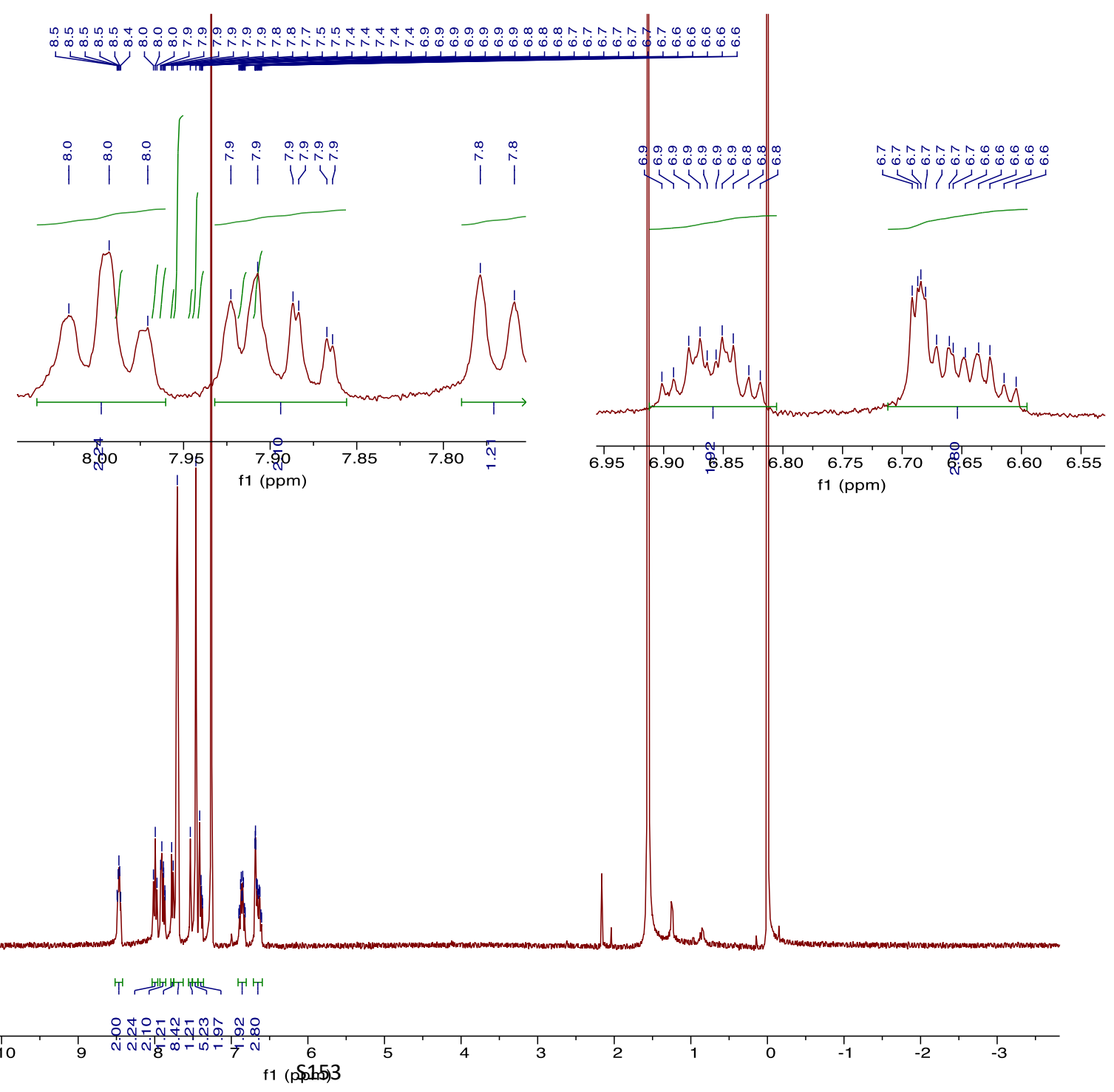




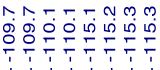

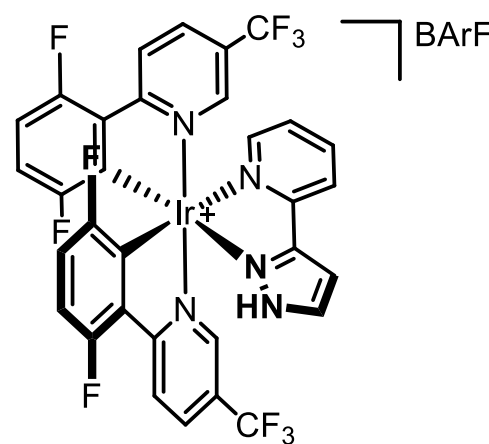

-4b, F NMR ( $\left.377 \mathrm{MHz}, \mathrm{CDCl}_{3}\right)$

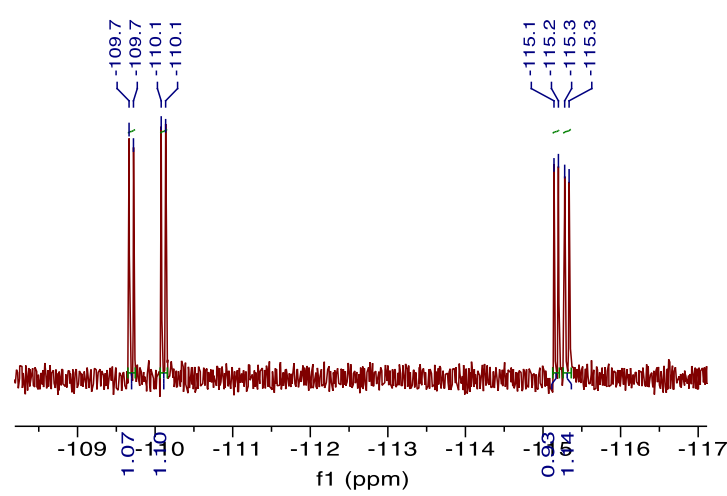




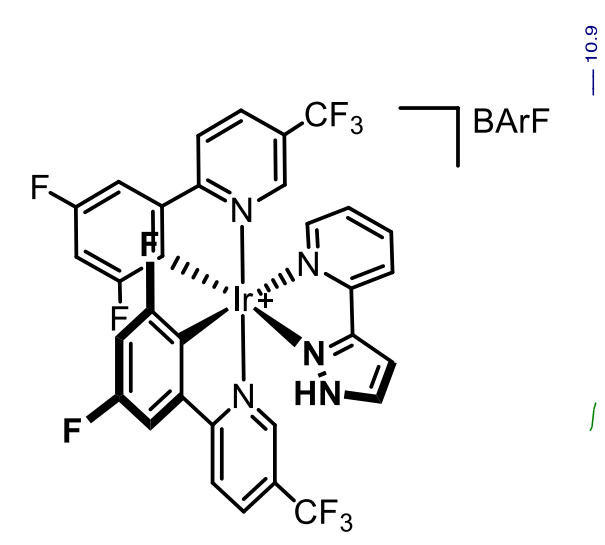

$-4 d, \quad H$ NMR ( $400 \mathrm{MHz}, \mathrm{CDCl}_{3}$ )

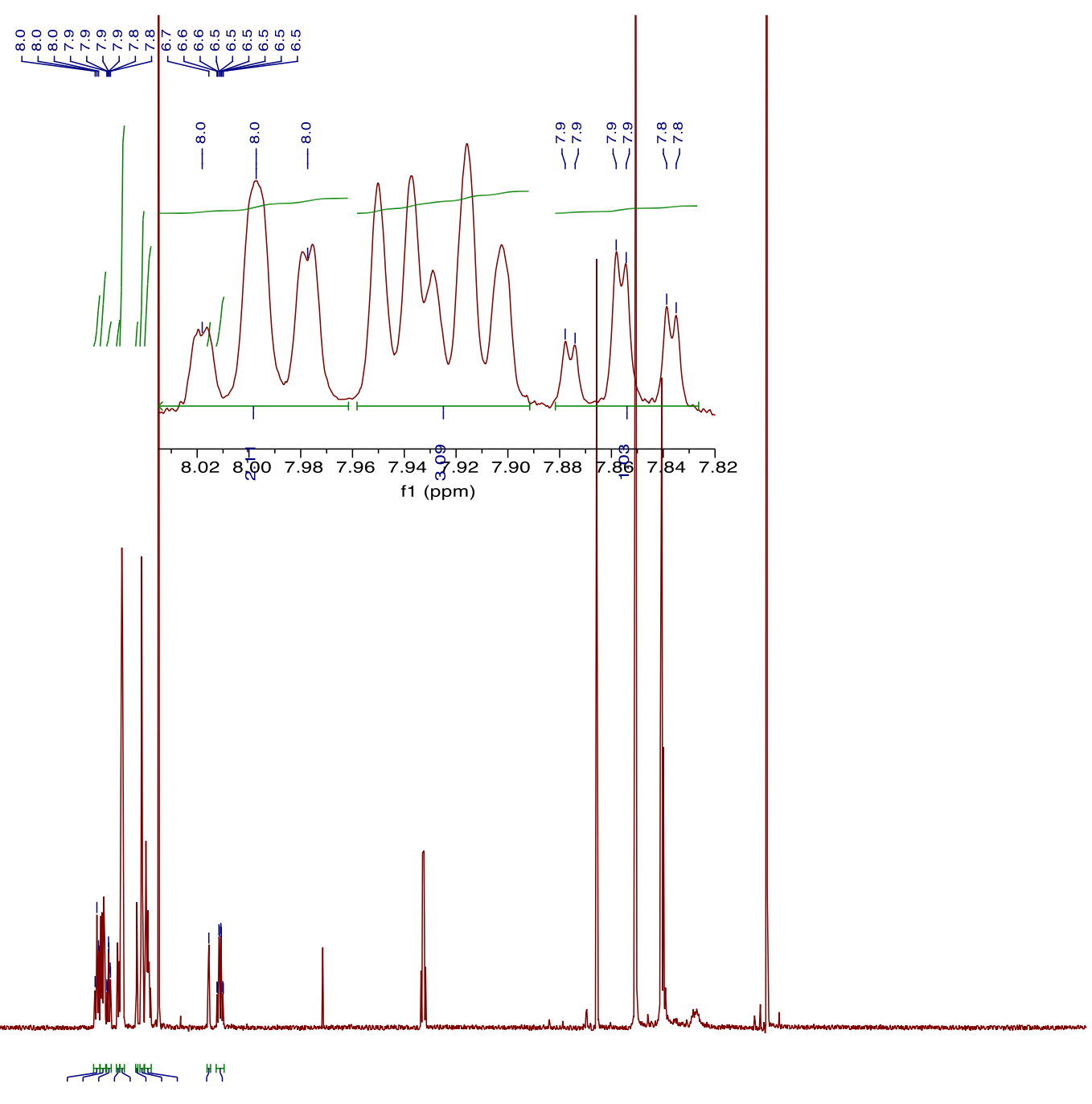

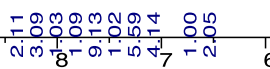

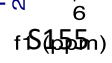



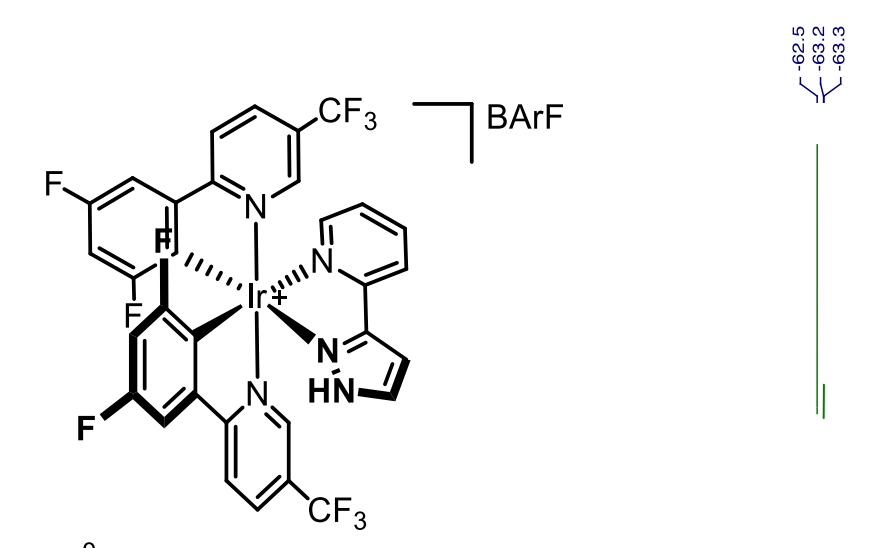

-4d, ${ }^{9} \mathrm{~F} \mathrm{NMR} \mathrm{(} 377 \mathrm{MHz}, \mathrm{CDCl}_{3}$ )

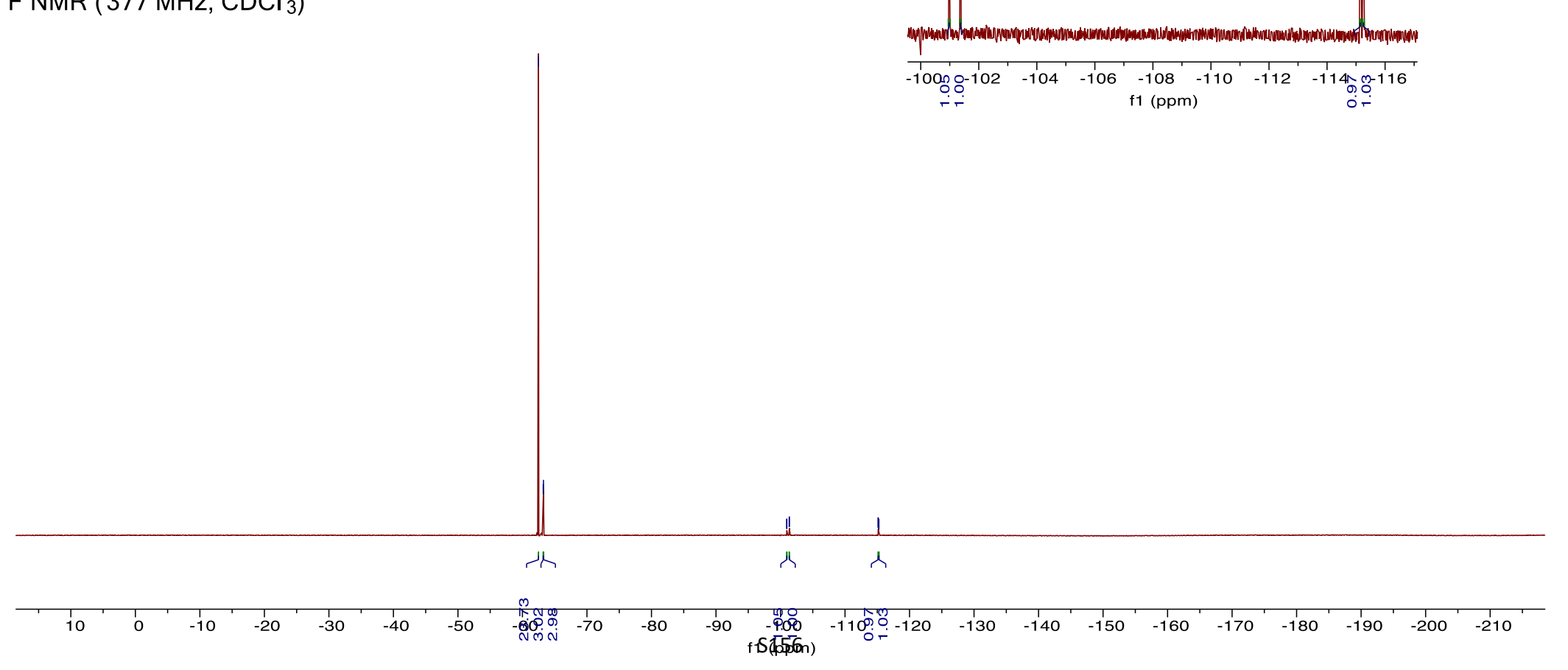




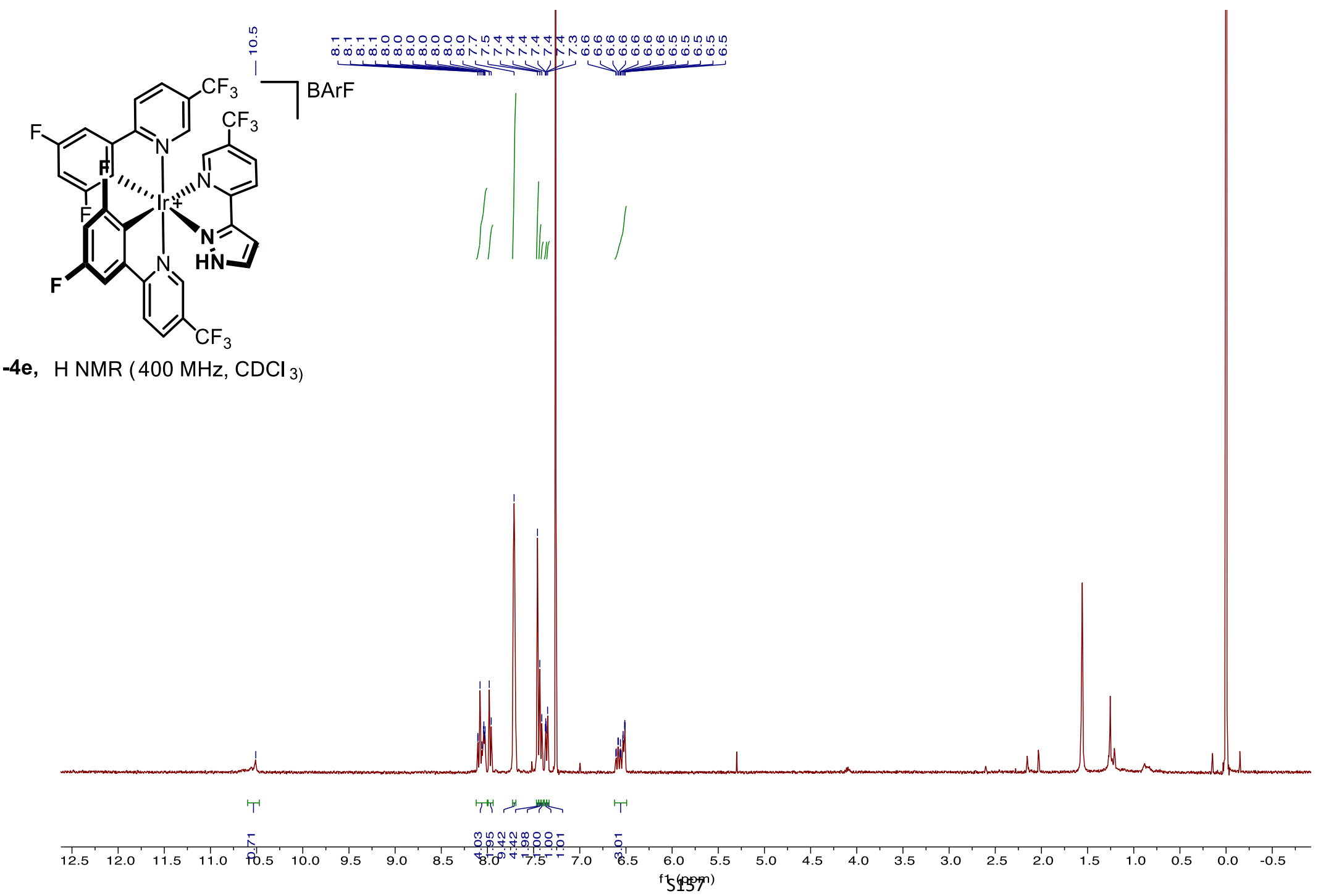



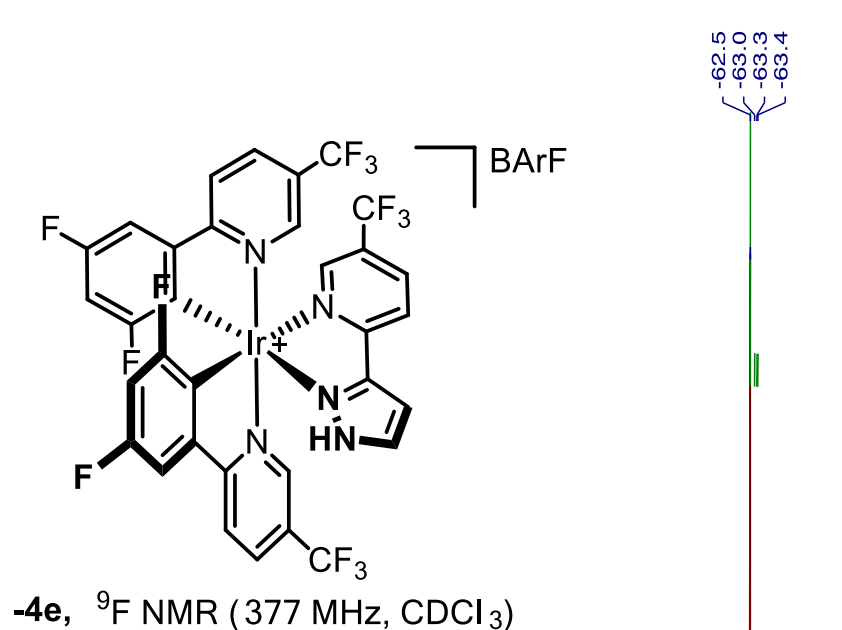

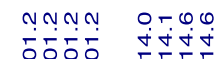

桨

$-4 \mathrm{e},{ }^{9} \mathrm{~F} \mathrm{NMR}\left(377 \mathrm{MHz}, \mathrm{CDCl}_{3}\right)$

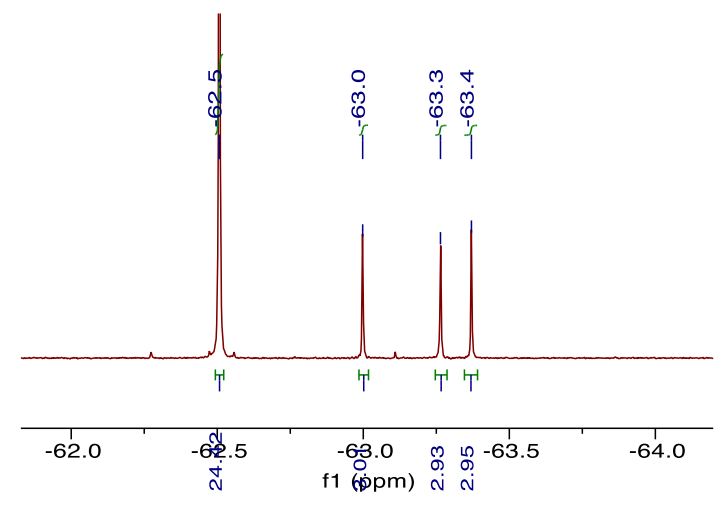

th

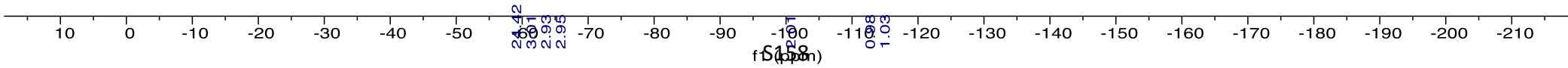




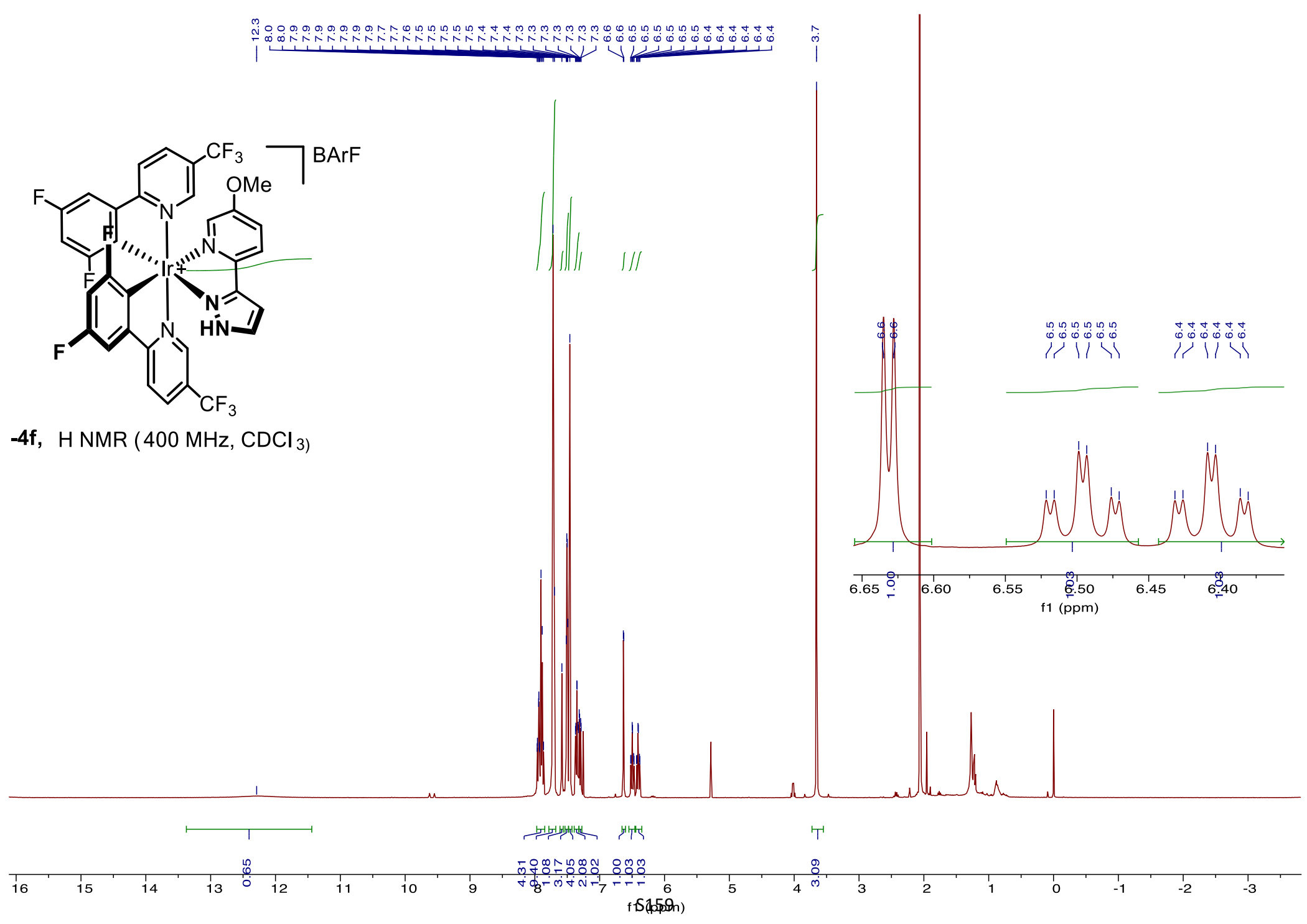




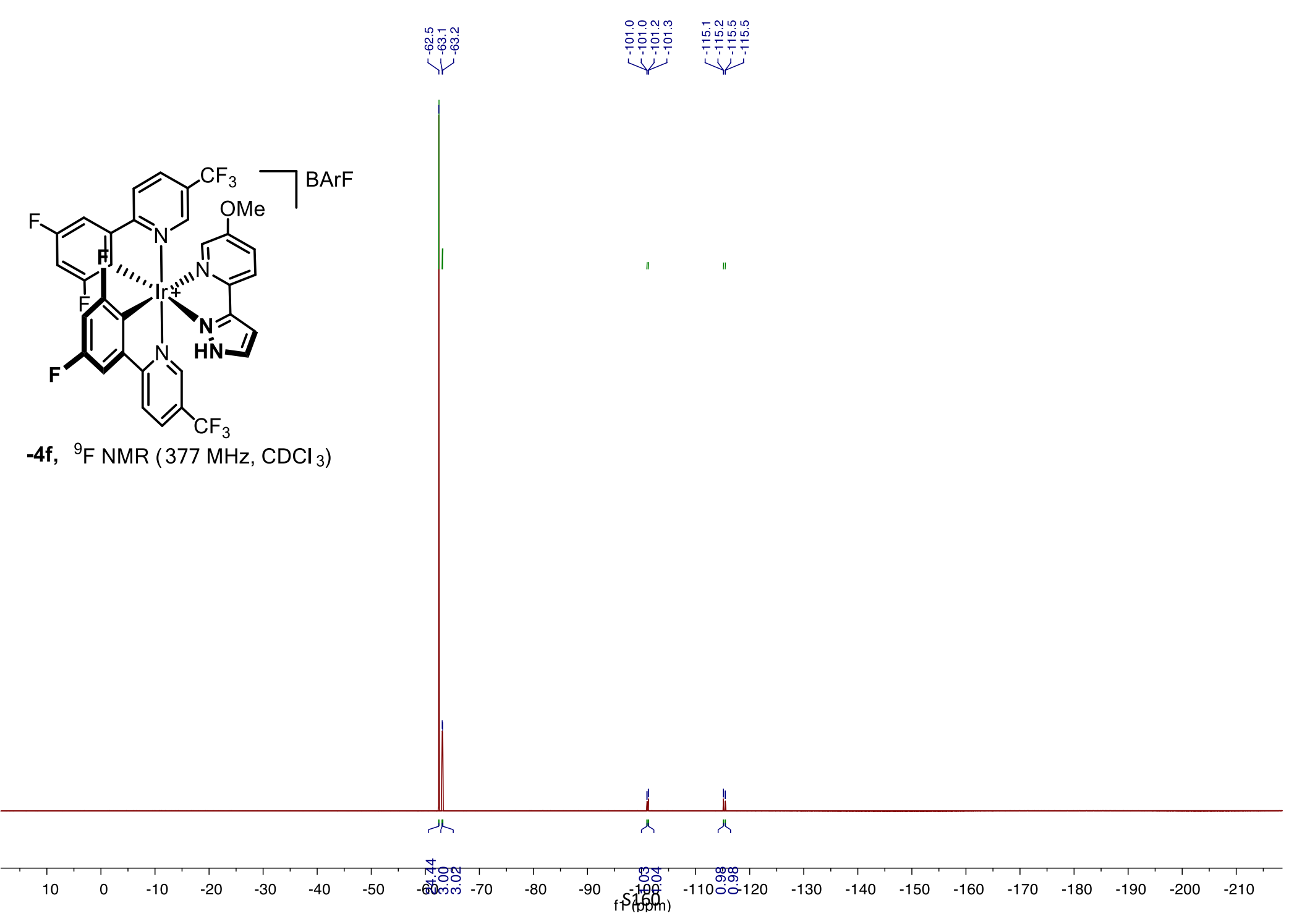




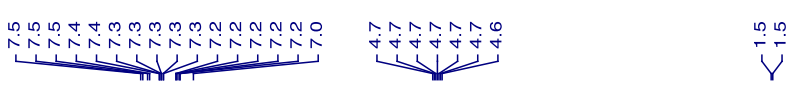

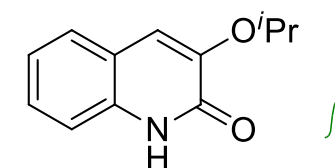

1c, ${ }^{1} \mathrm{H}$ NMR $\left(400 \mathrm{MHz}, \mathrm{CDCl}_{3}\right)$
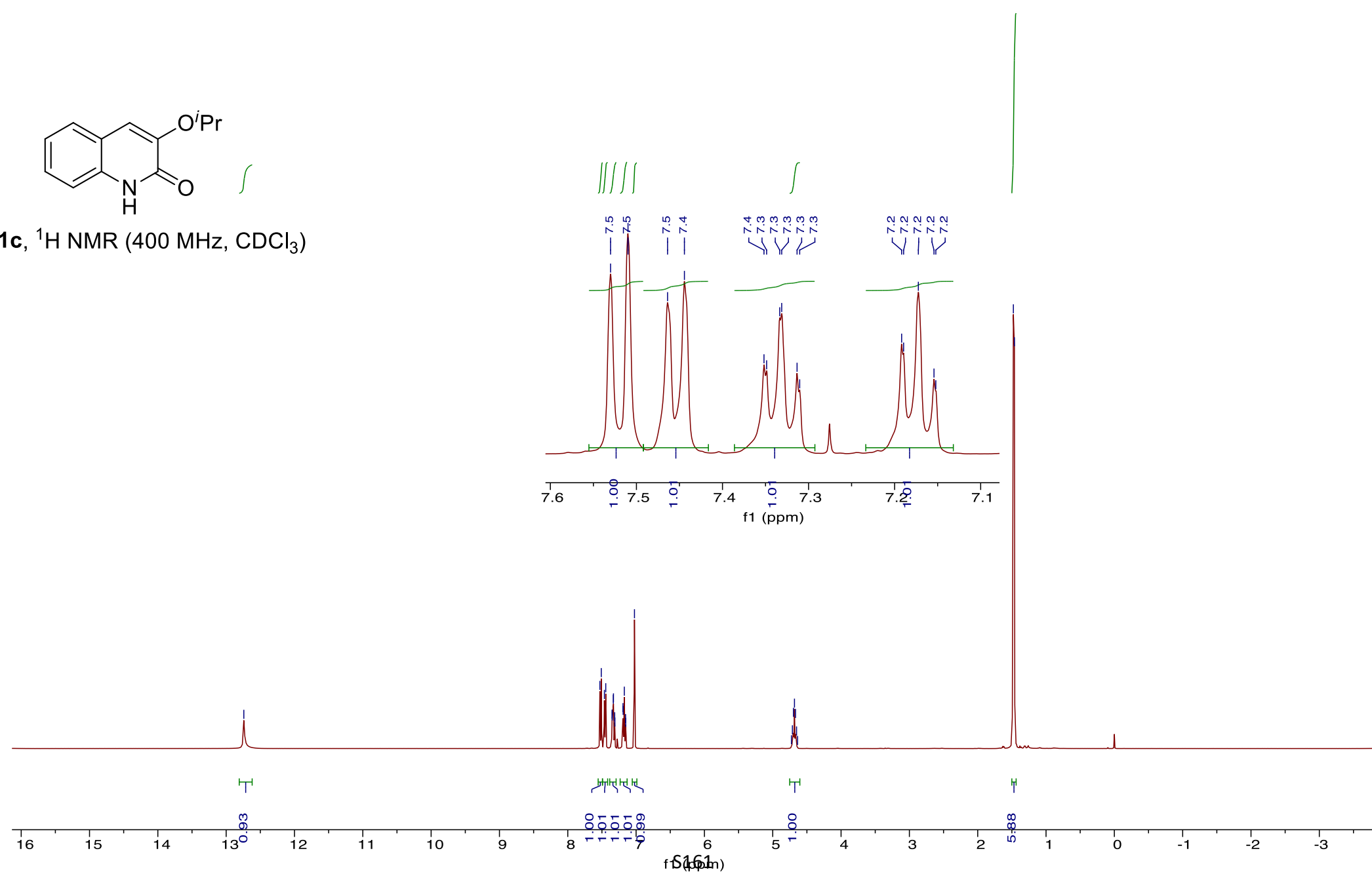

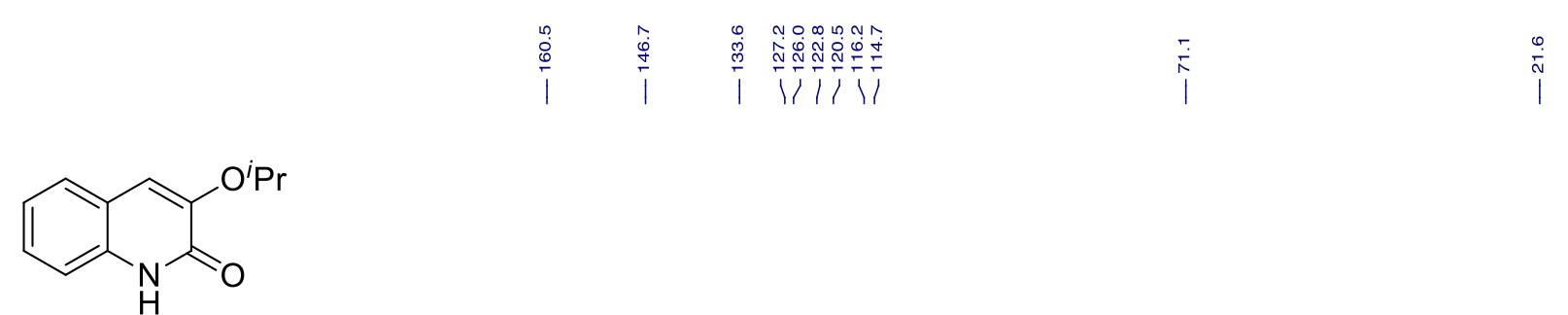

1c, ${ }^{13} \mathrm{C}$ NMR $\left(101 \mathrm{MHz}, \mathrm{CDCl}_{3}\right)$

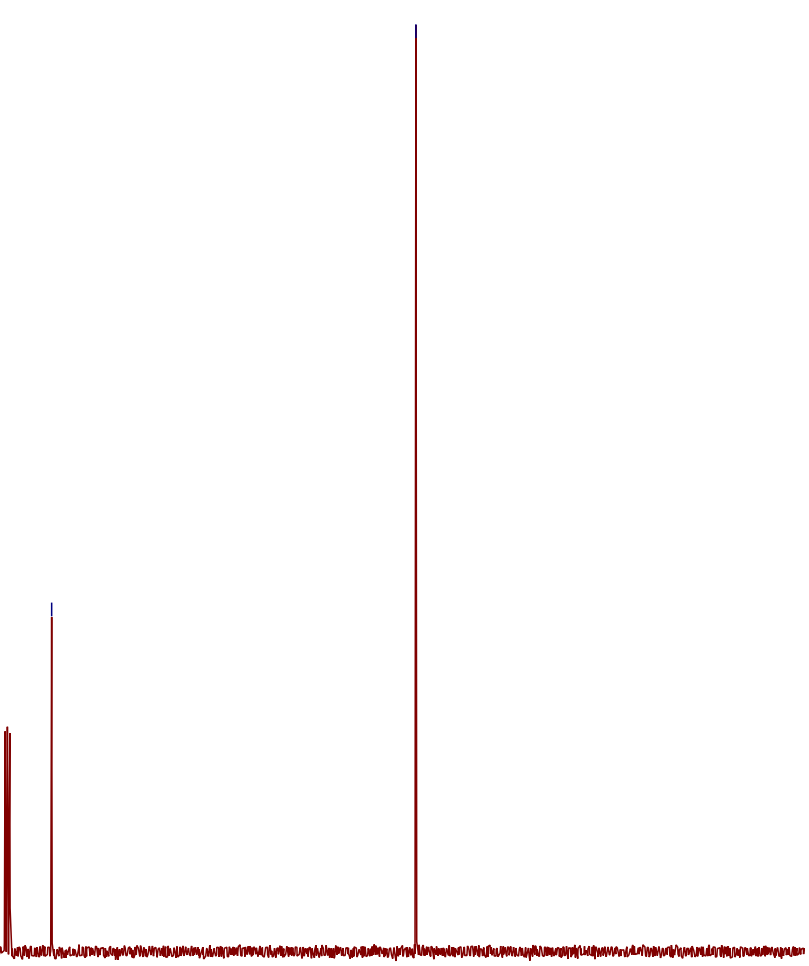

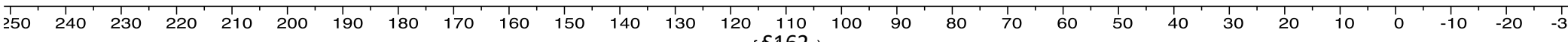

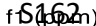




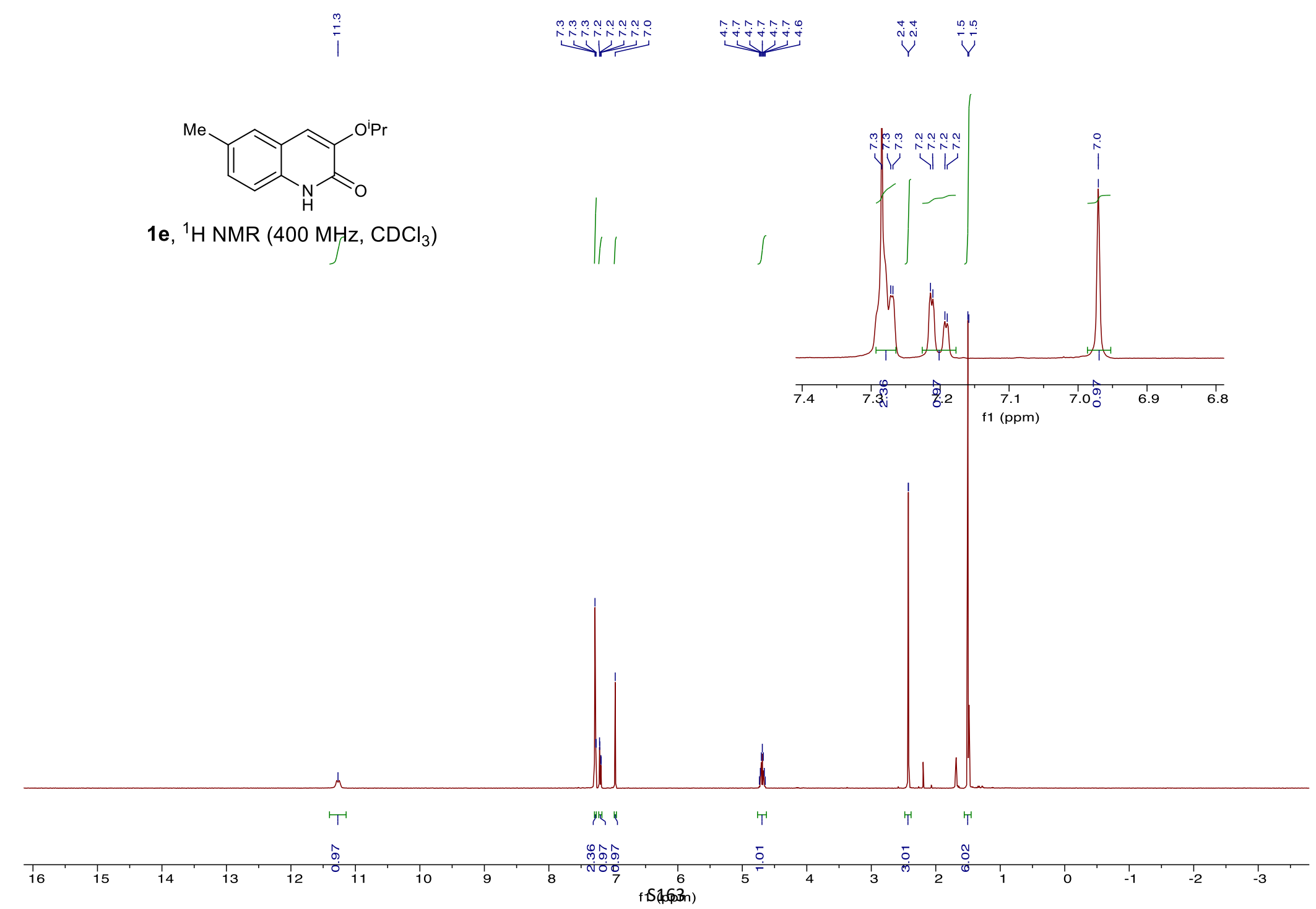




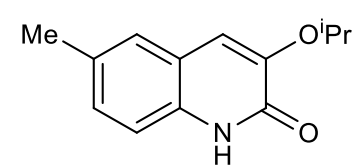

1e, ${ }^{13} \mathrm{C}$ NMR (101 MHz, $\mathrm{CDCl}_{3}$ )

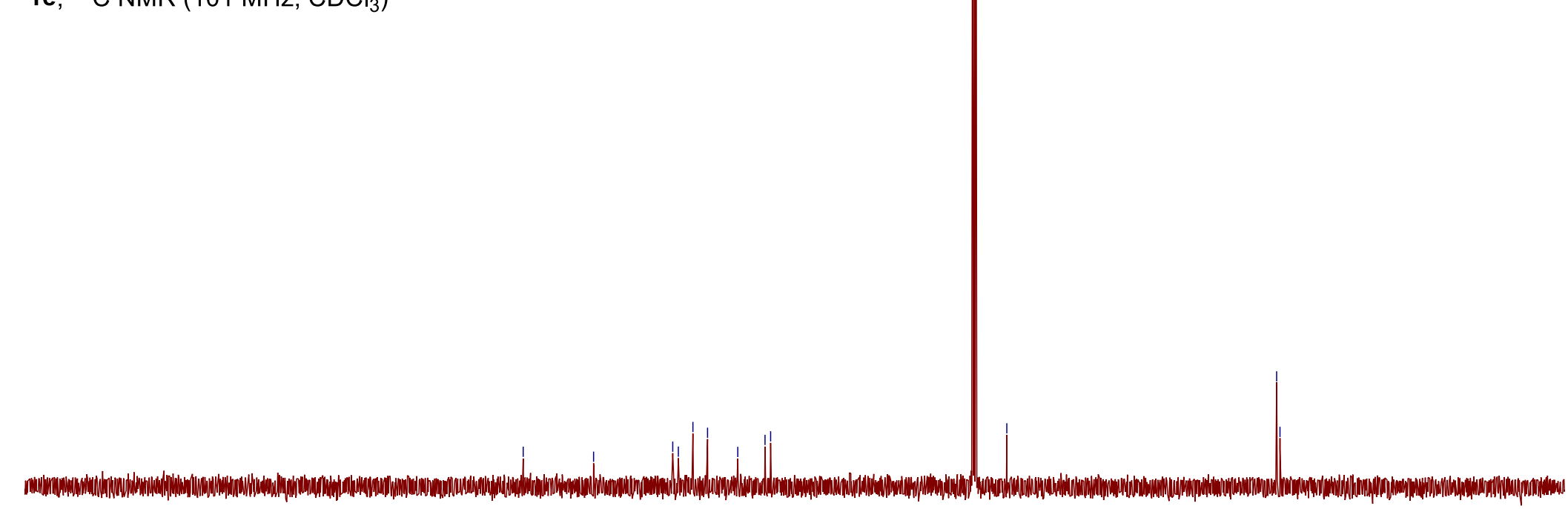

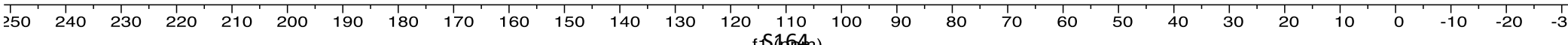

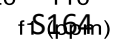




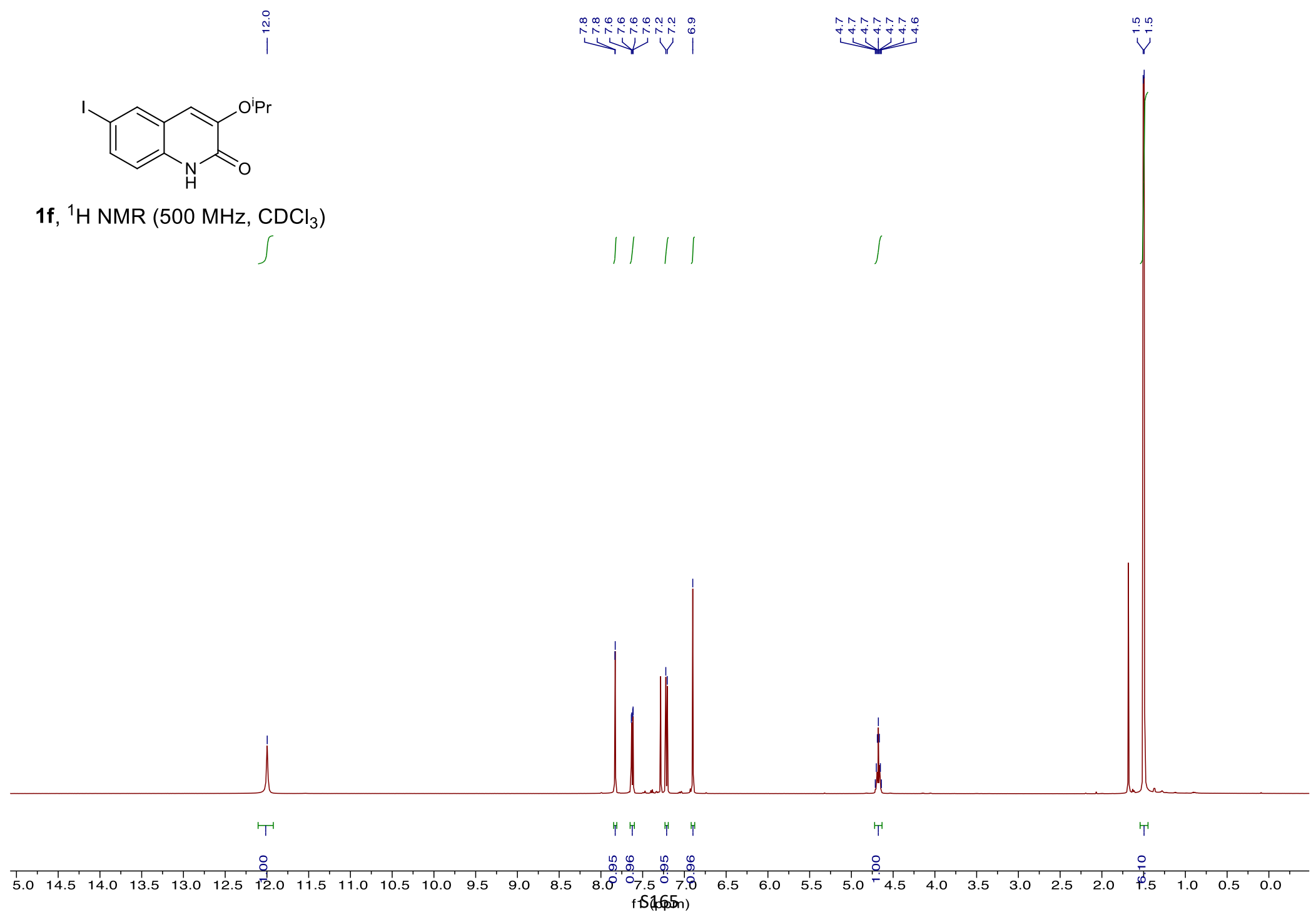




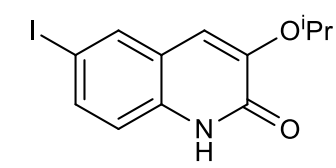

1f, ${ }^{13} \mathrm{C}$ NMR (126 MHz, $\mathrm{CDCl}_{3}$ )

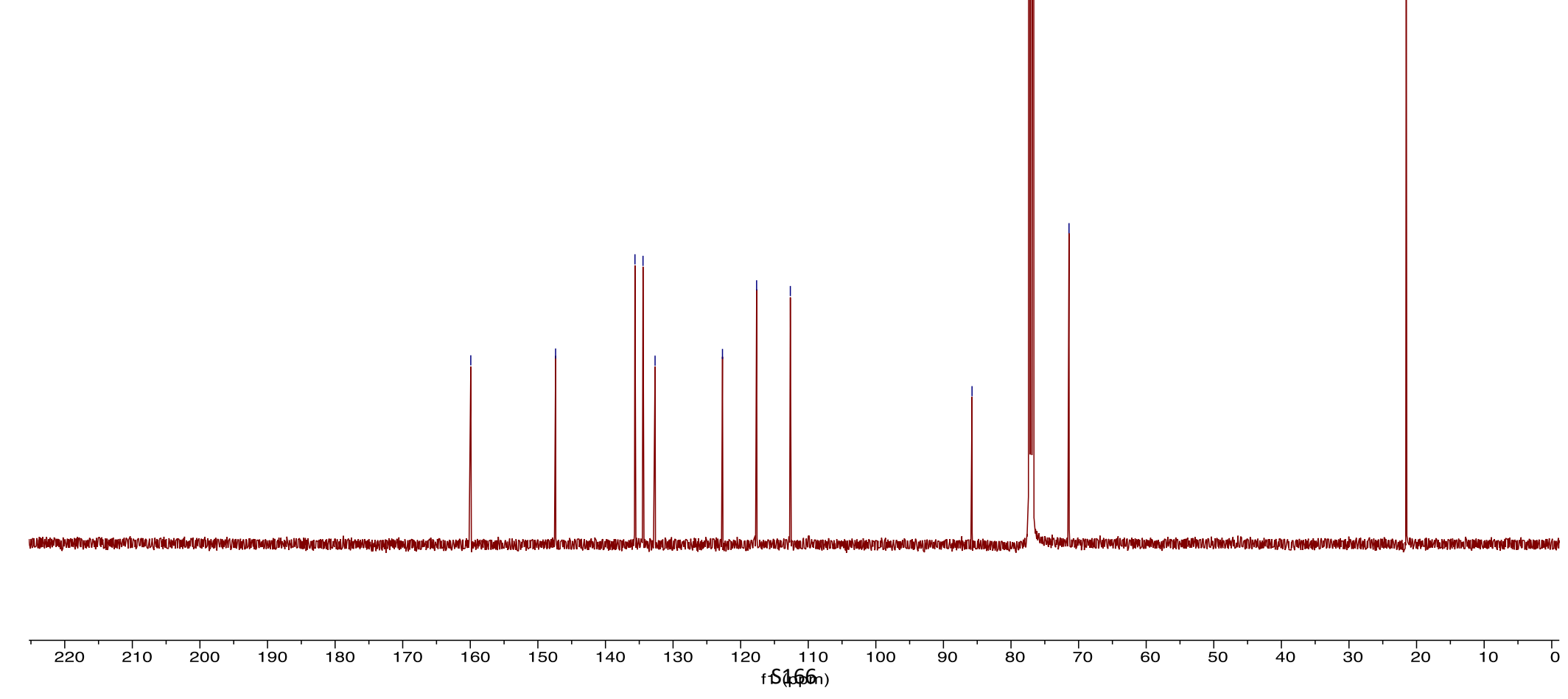


$\sqrt{0.050}$
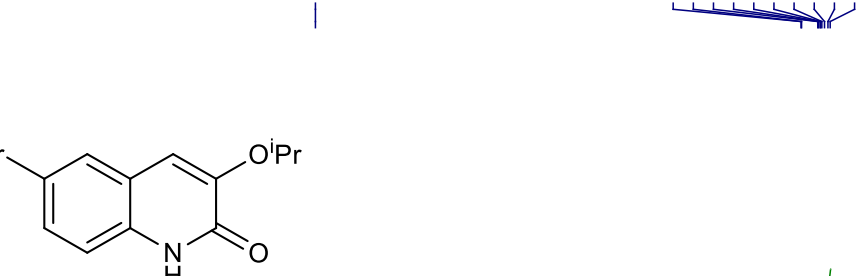

1g, ${ }^{1} \mathrm{H}$ NMR $\left(400 \mathrm{MHz}, \mathrm{CDCl}_{3}\right)$

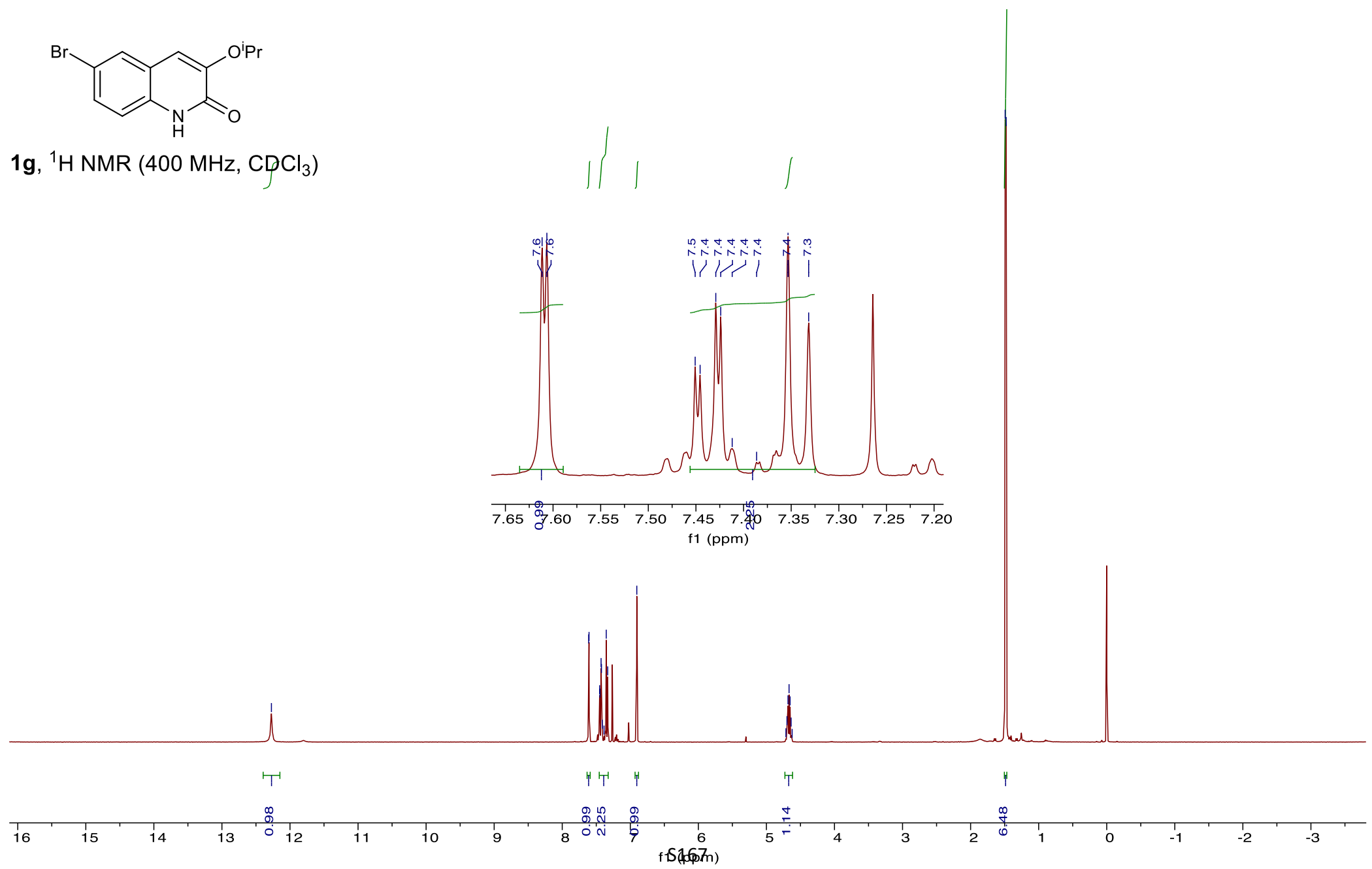

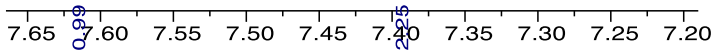
f1 (ppm) 


\section{I}

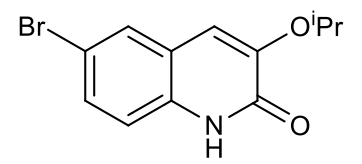

1g, ${ }^{13} \mathrm{C}$ NMR (101 MHz, $\mathrm{CDCl}_{3}$ )

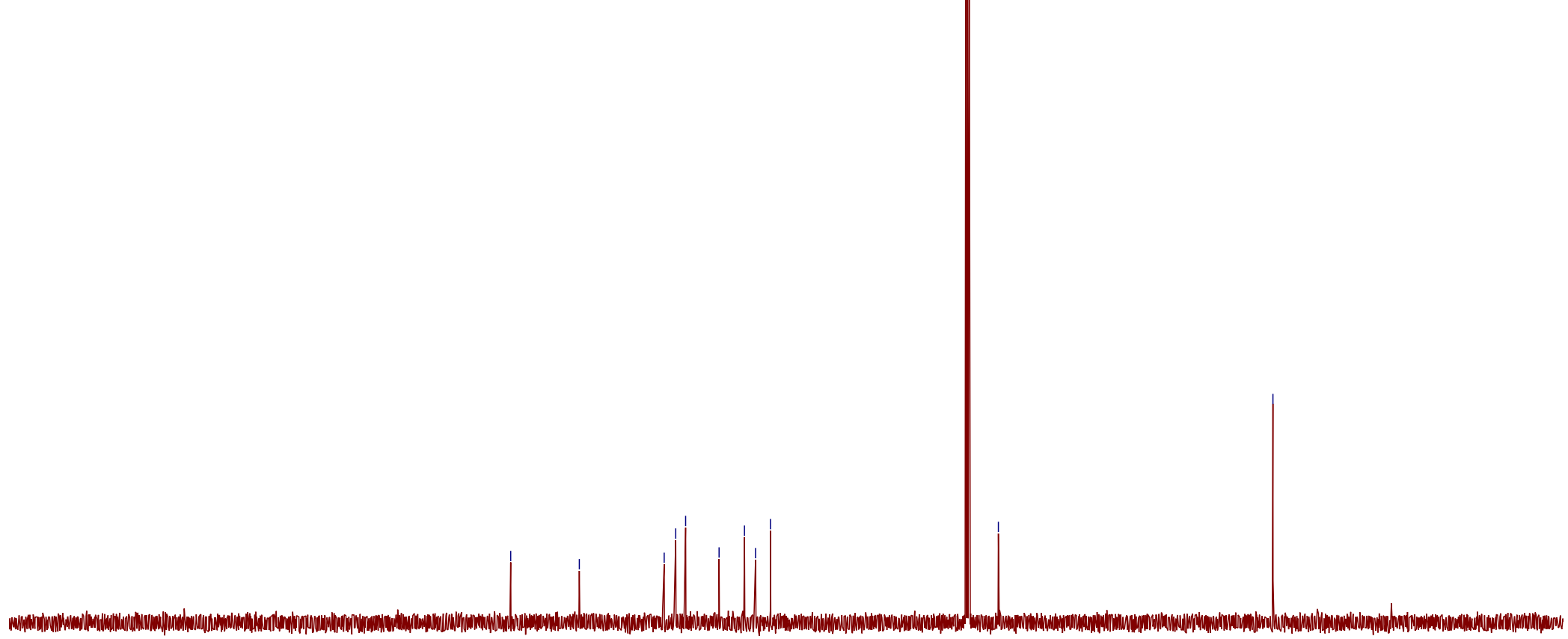

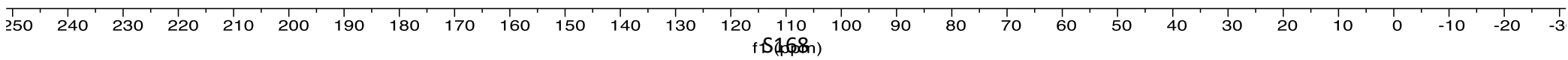




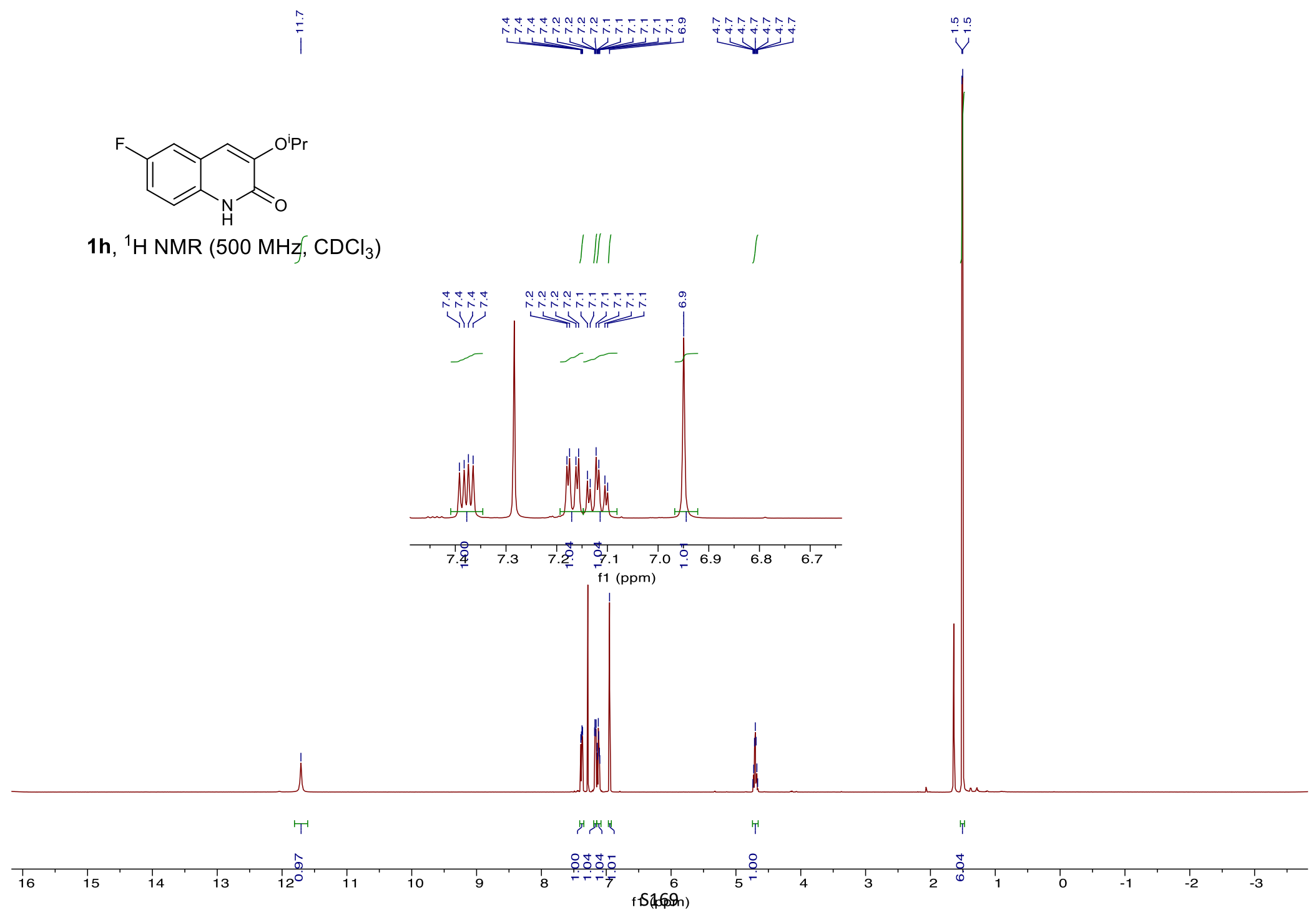


$$
\text { (1) }
$$

1h, ${ }^{13} \mathrm{C} \mathrm{NMR}\left(126 \mathrm{MHz}, \mathrm{CDCl}_{3}\right)$
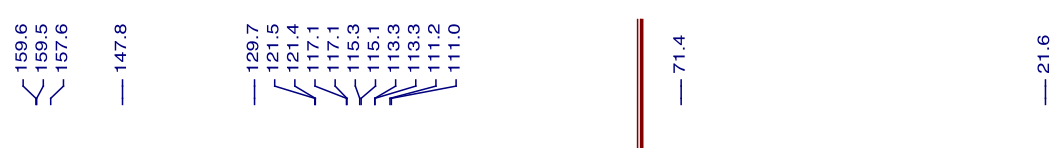


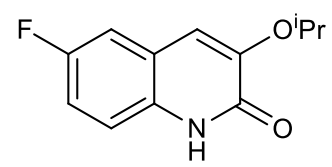

1h, $\left.{ }^{19} \mathrm{~F} \mathrm{NMR} \mathrm{(377} \mathrm{MHz,} \mathrm{CDCl}_{3}\right)$

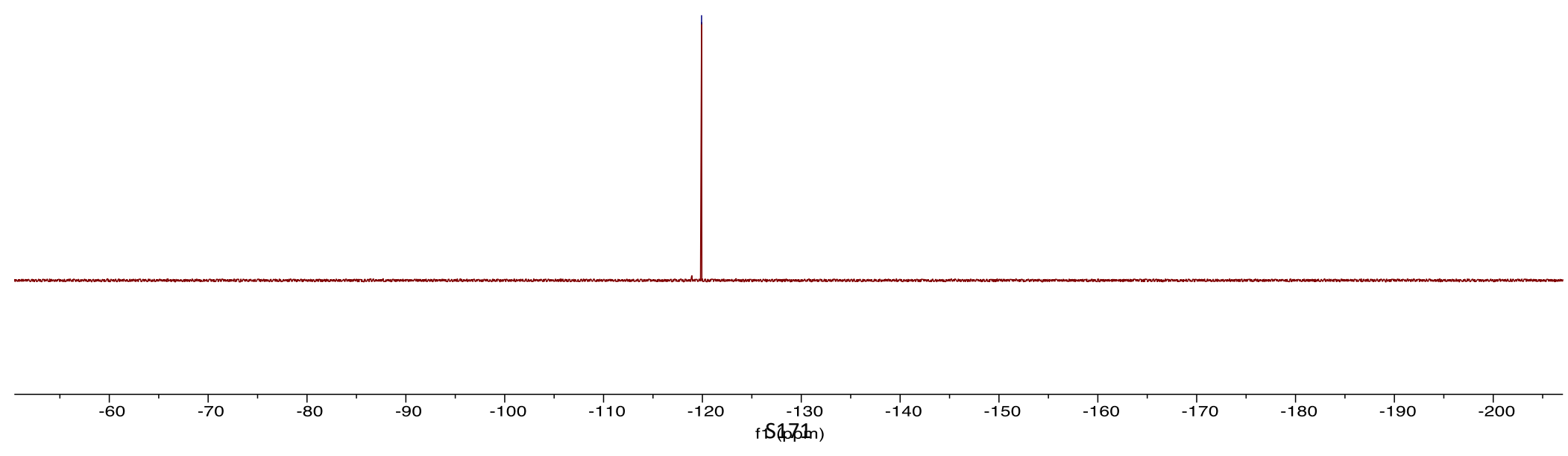


$\mathrm{MeO}$<smiles>CCCOc1cc2cc(I)ccc2[nH]c1=O</smiles>

1i, ${ }^{1} \mathrm{H}$ NMR (400 MHz, $\mathrm{CDCl}_{3}$ )

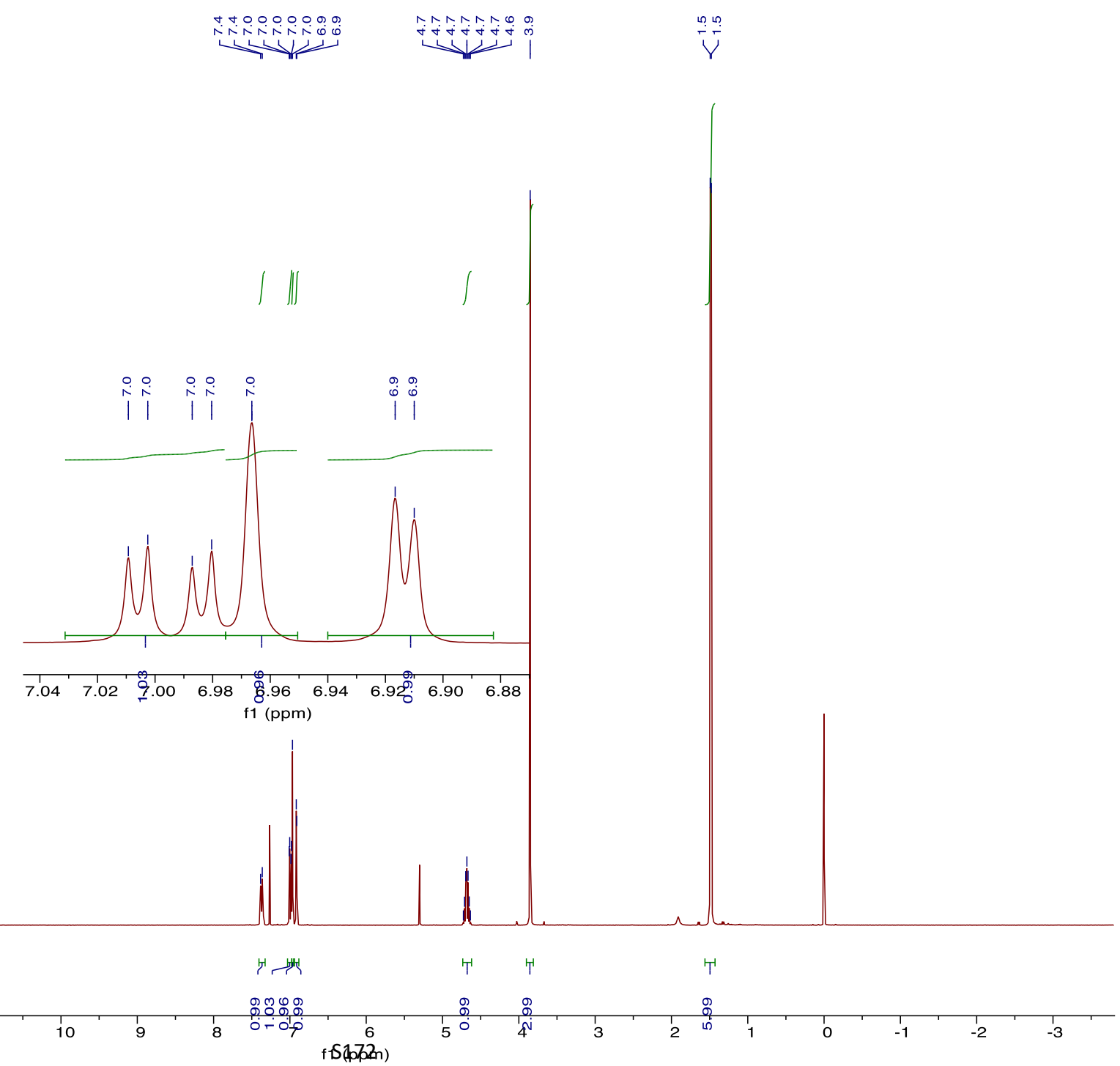




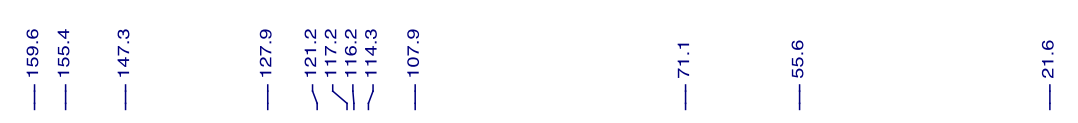

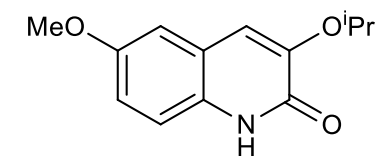

1i, ${ }^{13} \mathrm{C}$ NMR $\left(101 \mathrm{MHz}, \mathrm{CDCl}_{3}\right)$

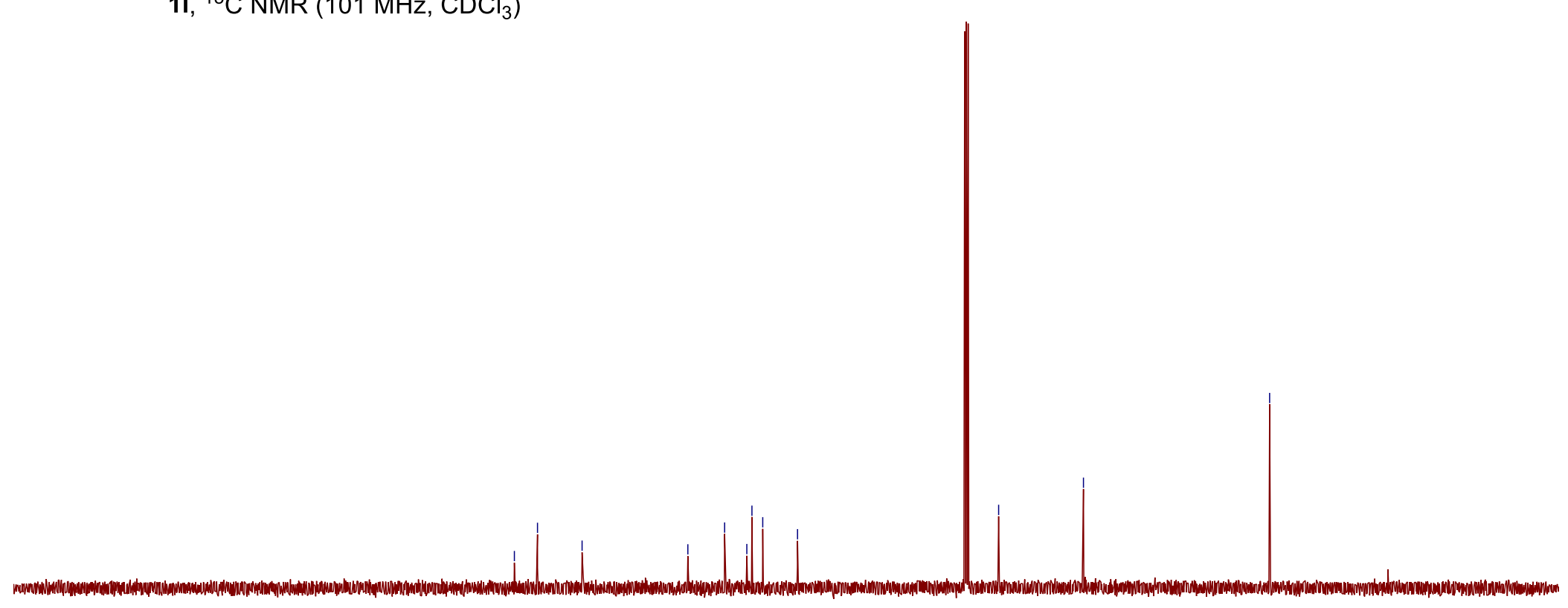

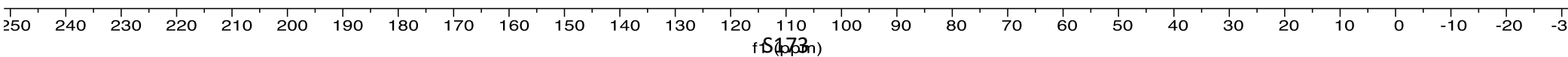




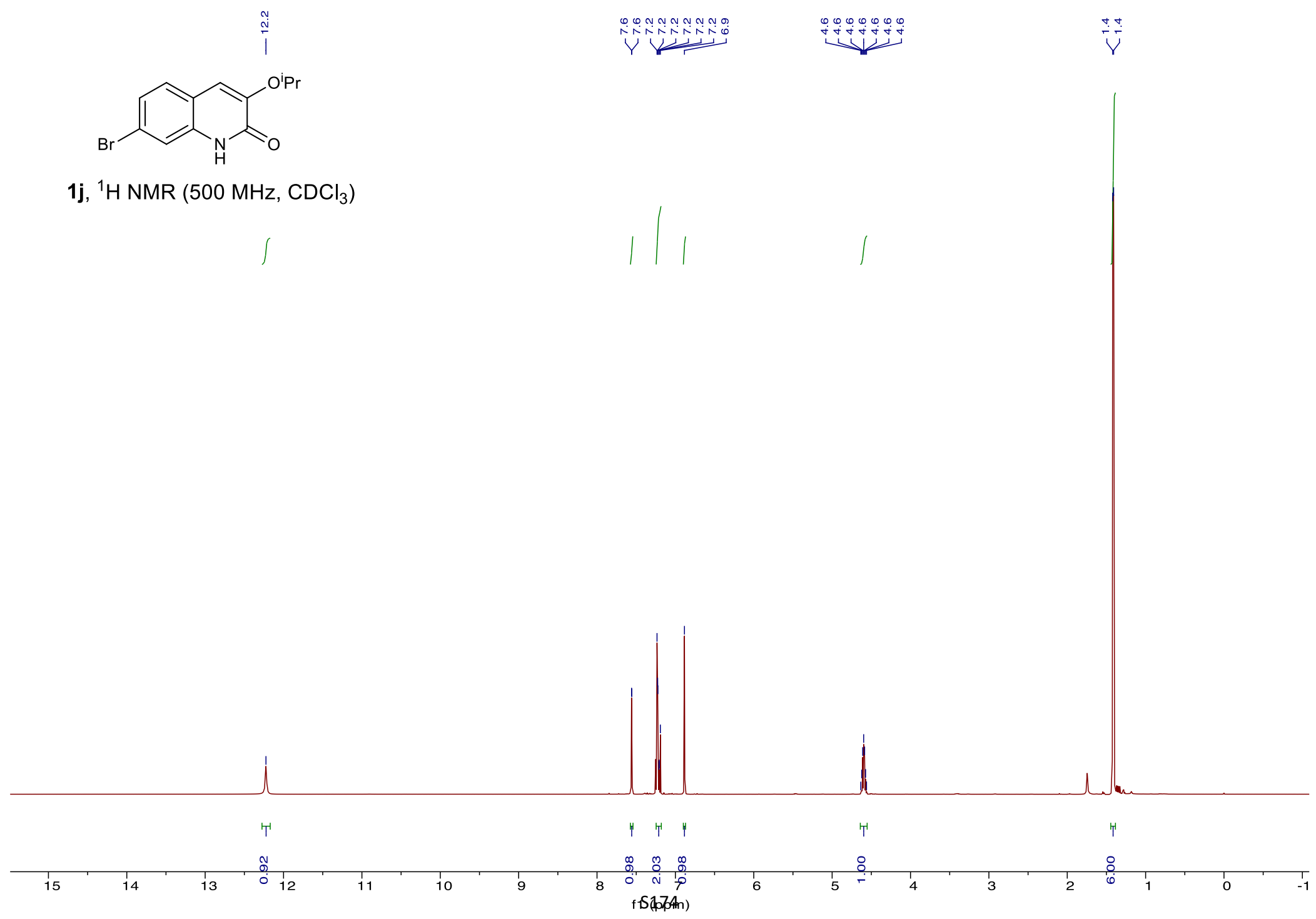




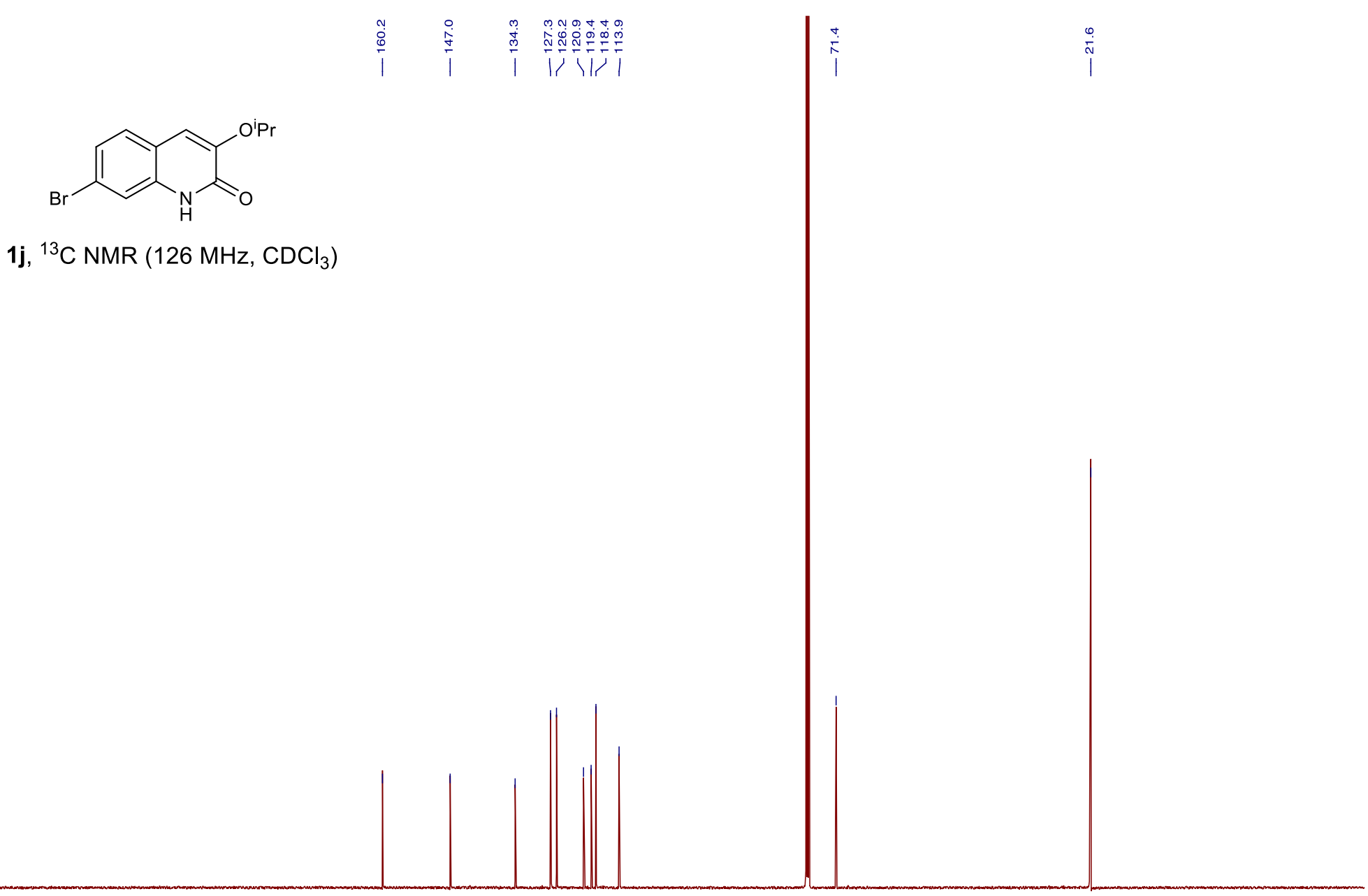

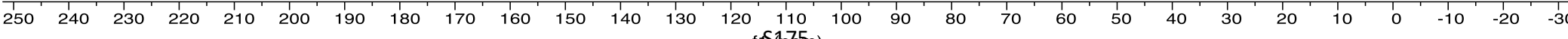
fiStoptopn) 


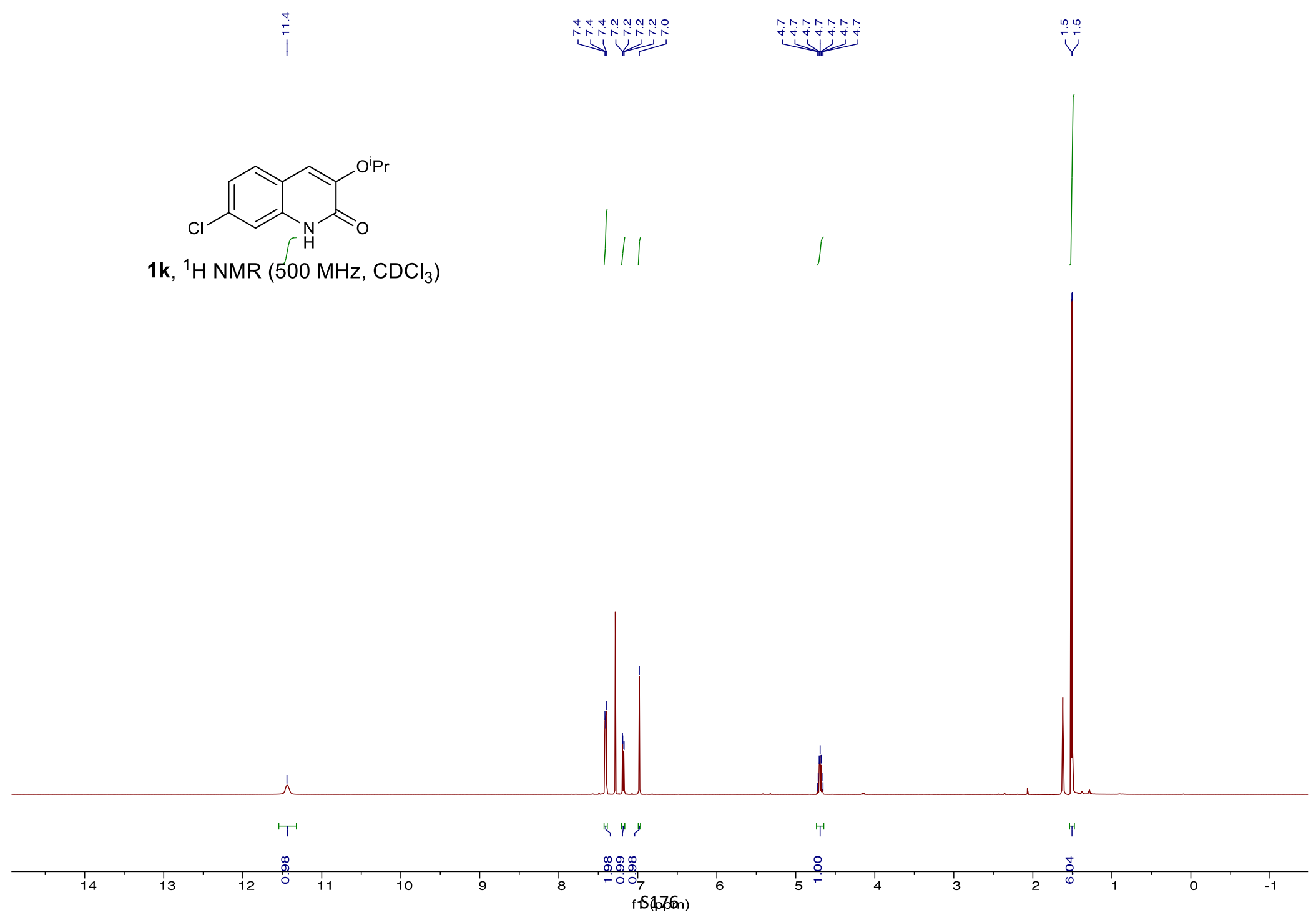




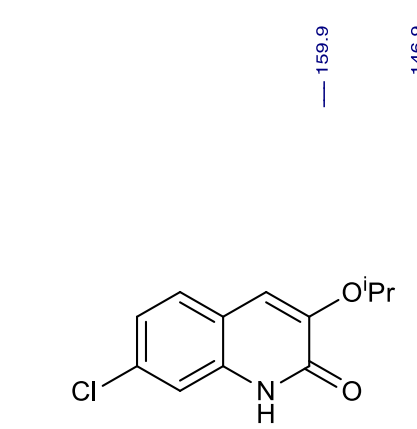

$1 \mathbf{k},{ }^{13} \mathrm{C}$ NMR $\left(126 \mathrm{MHz} \mathrm{CDCl}_{3}\right)$

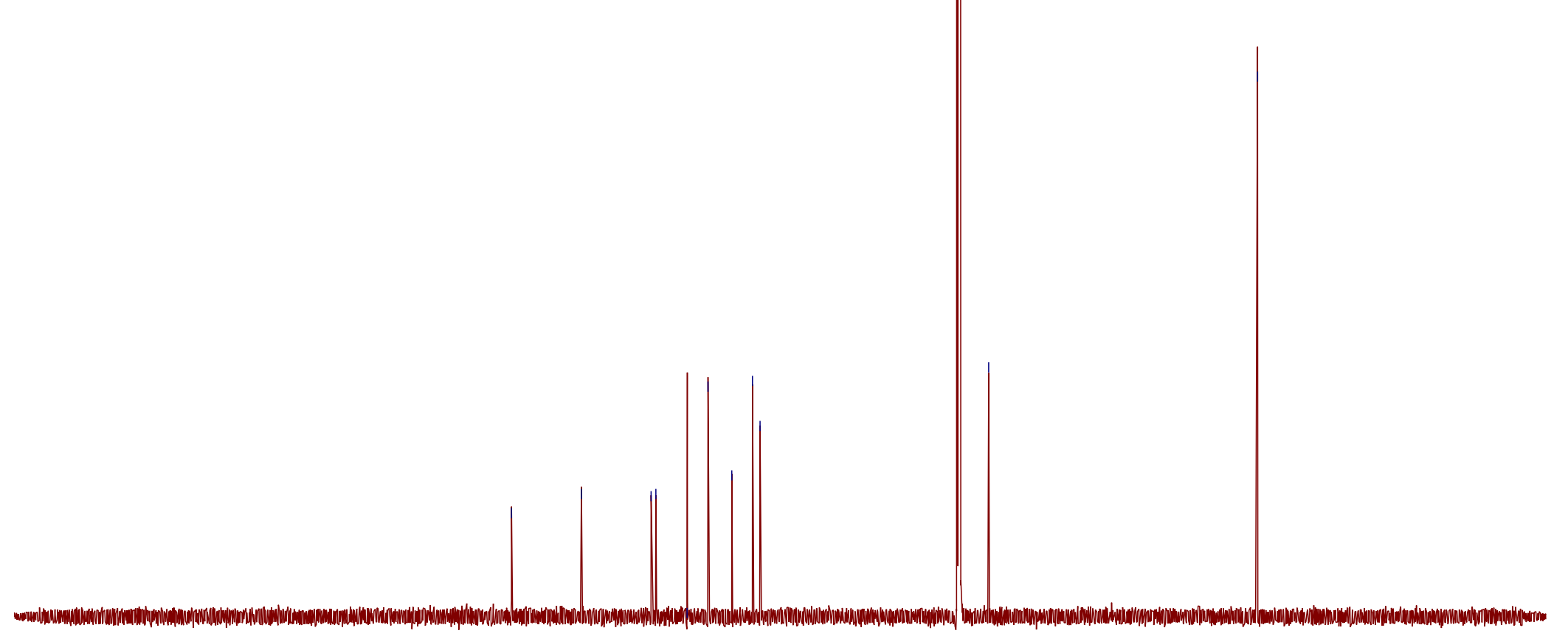

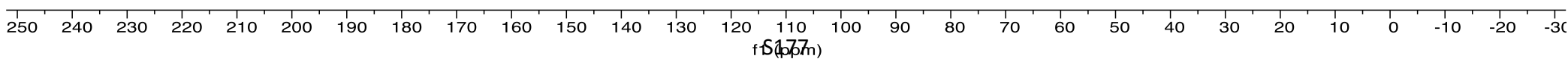




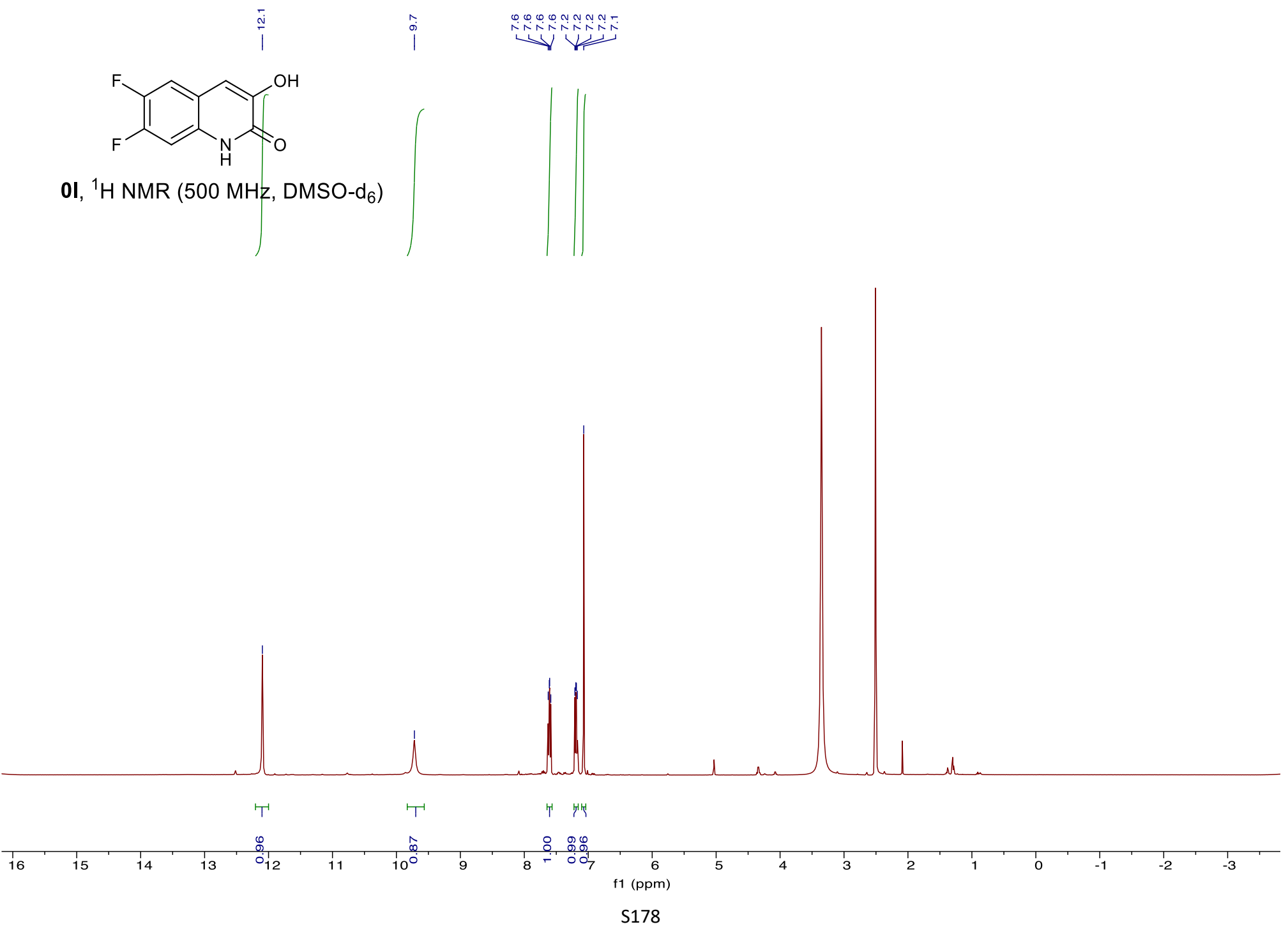




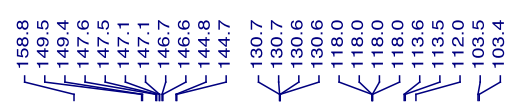

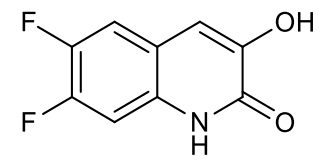

0I, ${ }^{13} \mathrm{C}$ NMR (126 MHz, DMSO-d6)
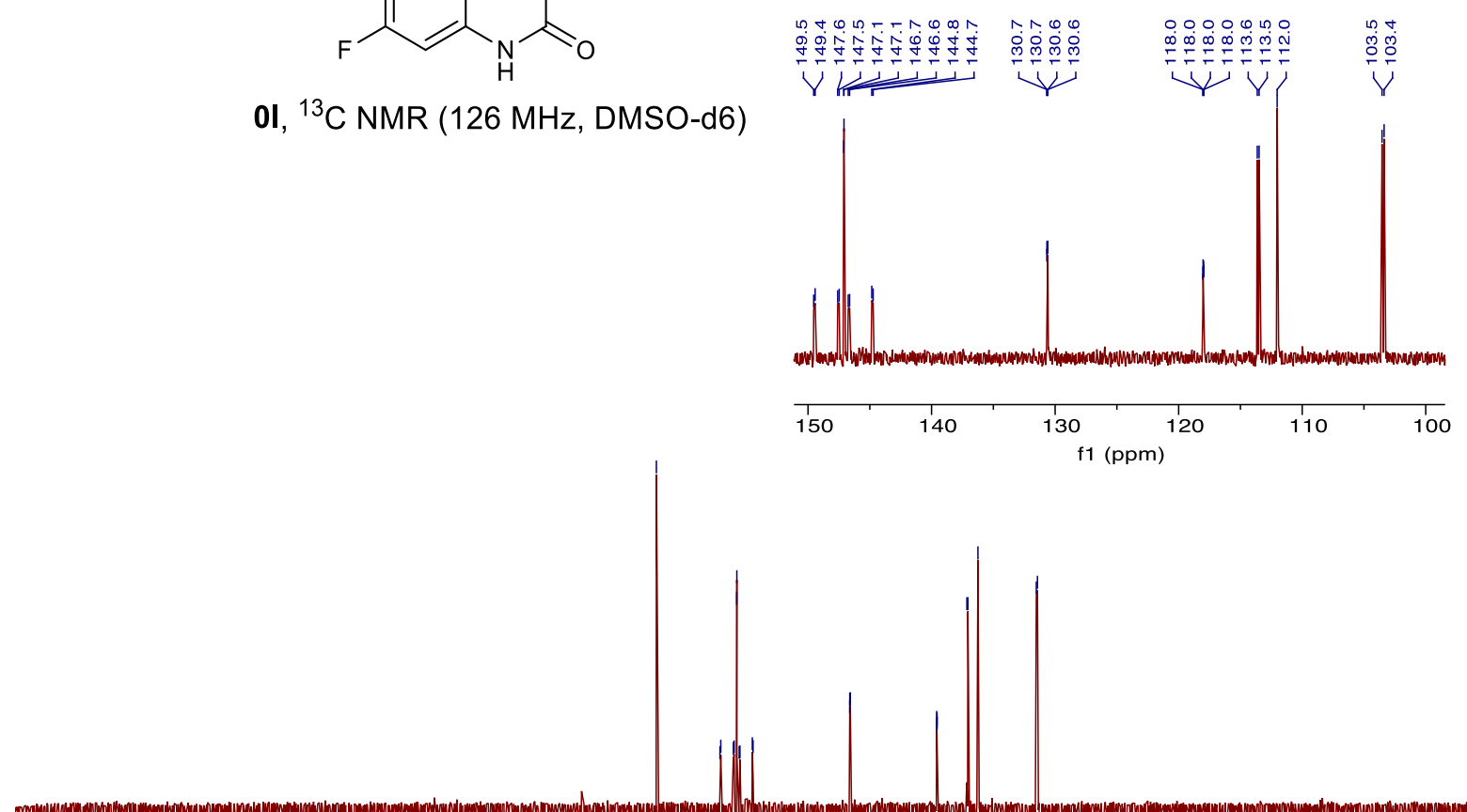

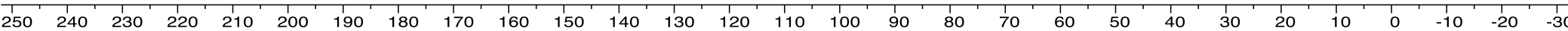

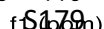




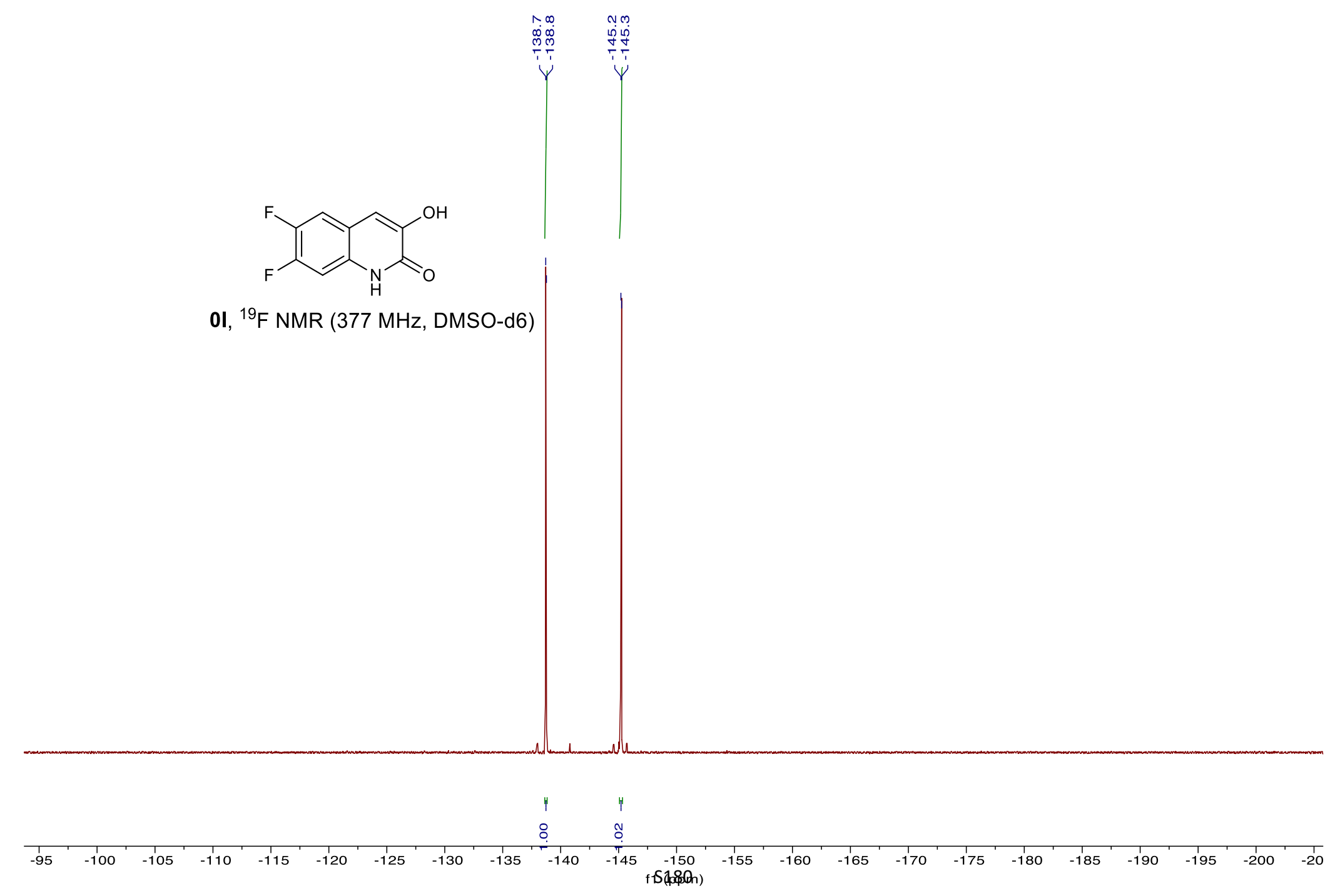




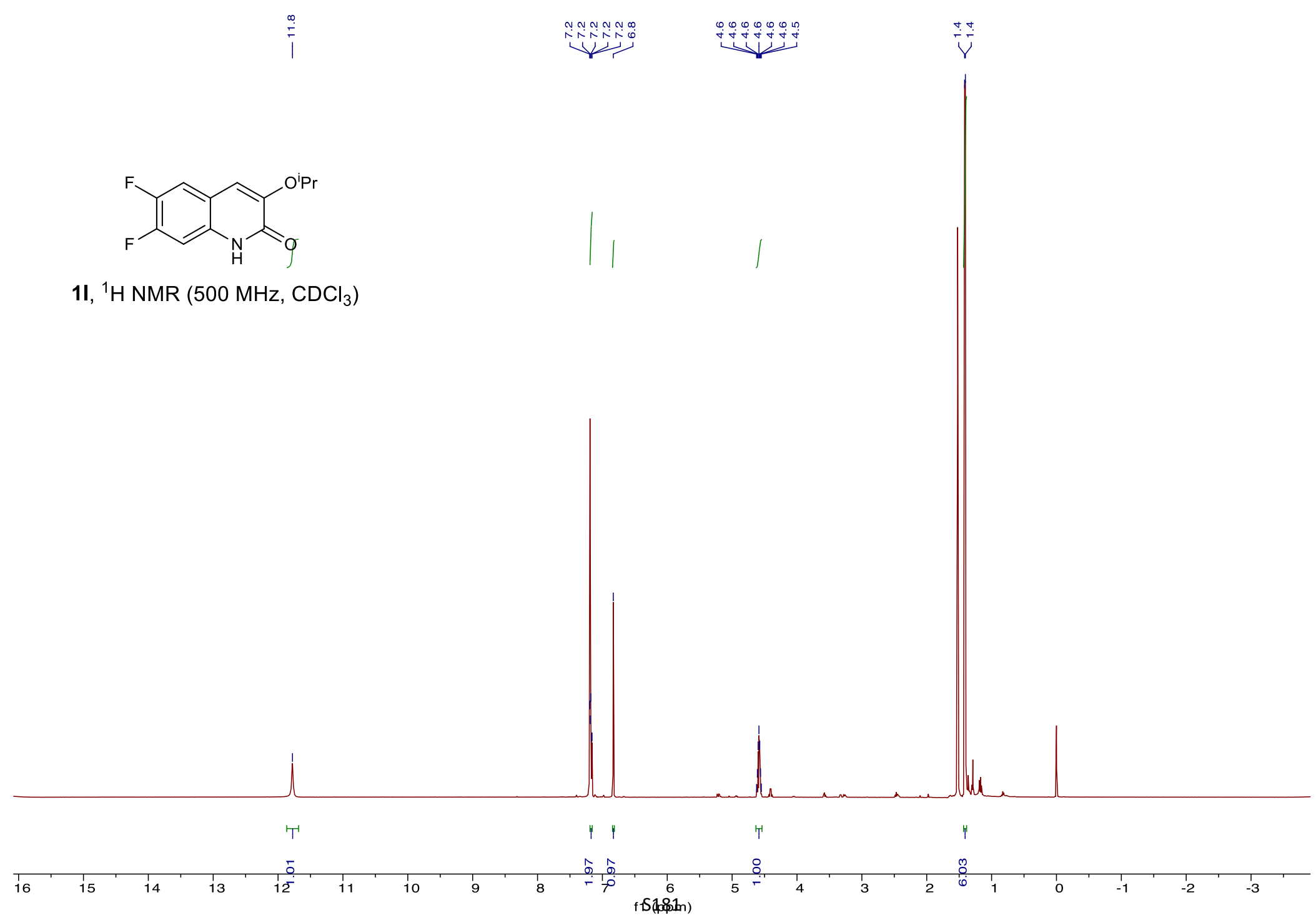




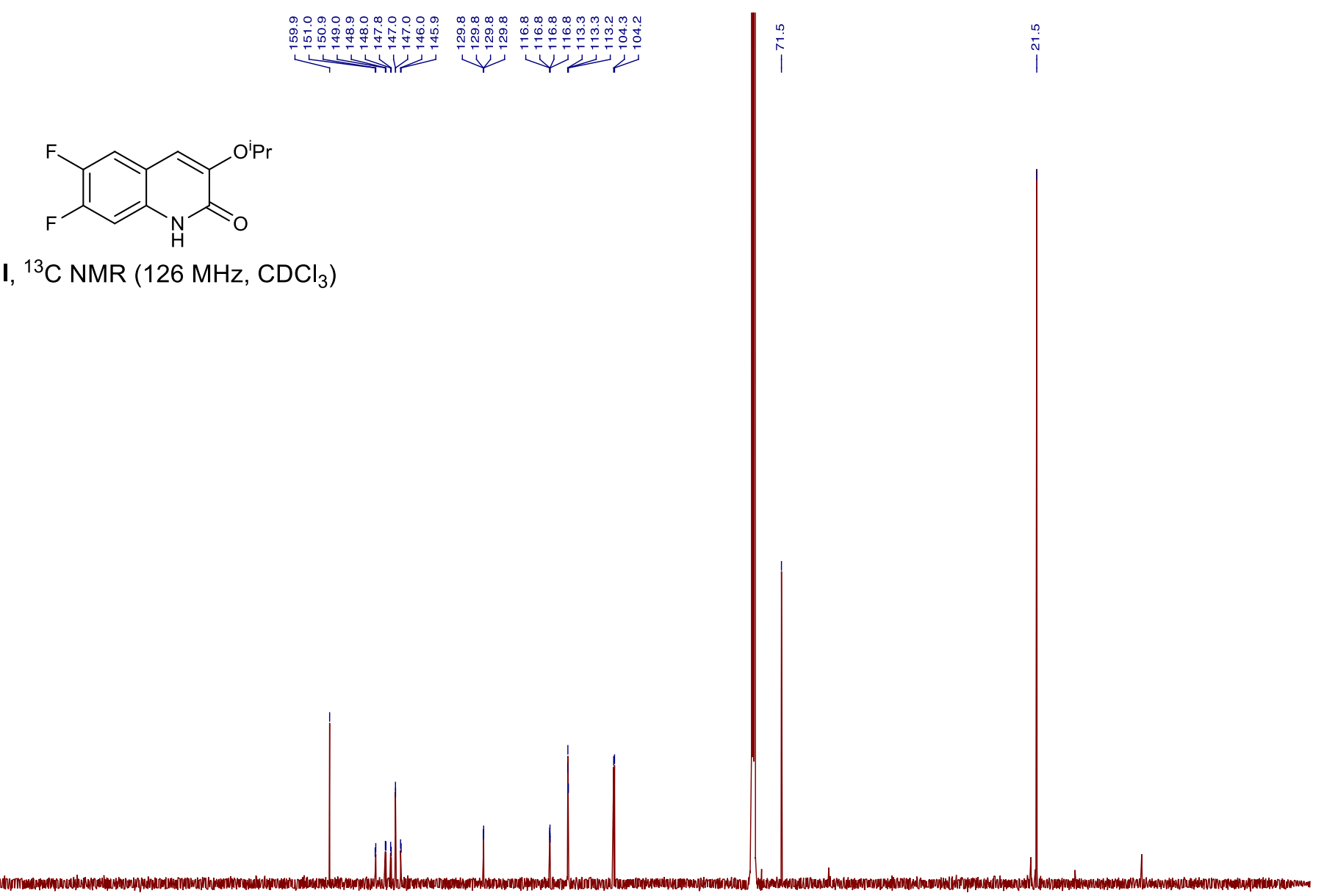

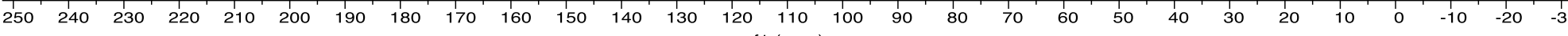
$1(\mathrm{pp})$ 


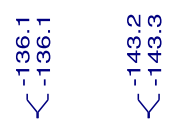

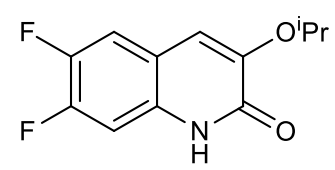

1I, ${ }^{19} \mathrm{~F} \mathrm{NMR} \mathrm{(377} \mathrm{MHz,} \mathrm{CDCl}_{3}$ )

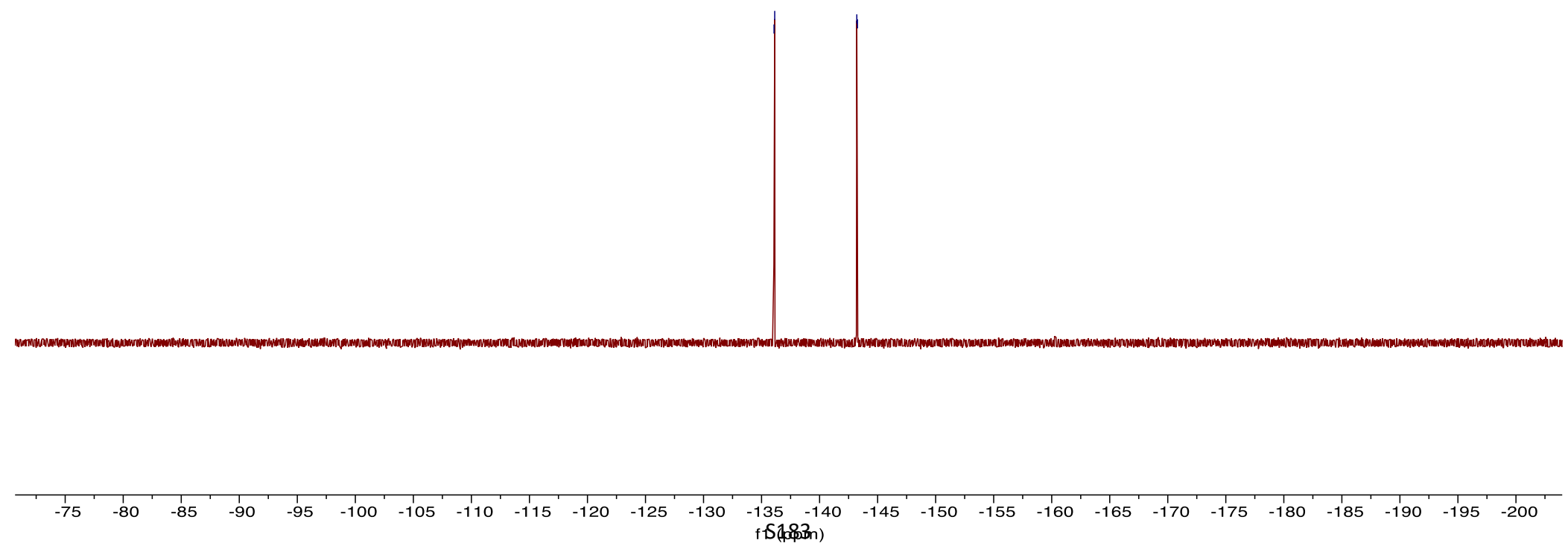



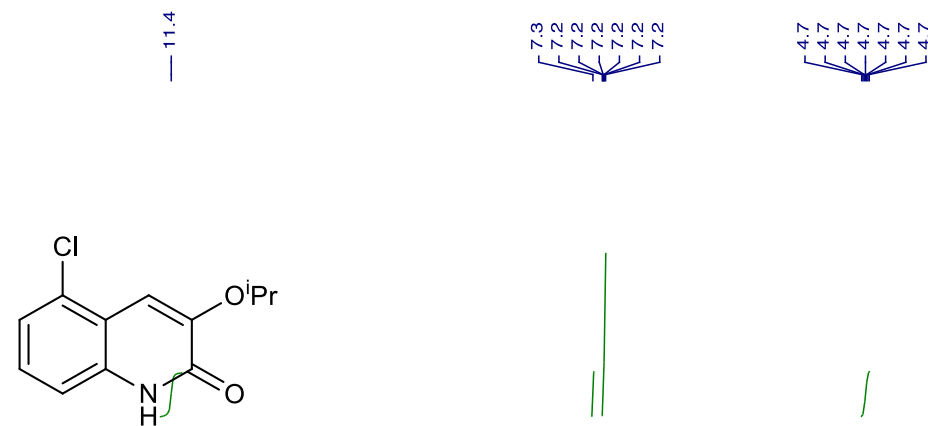

1m, ${ }^{1} \mathrm{H}$ NMR (500 MHz, $\mathrm{CDCl}_{3}$ )

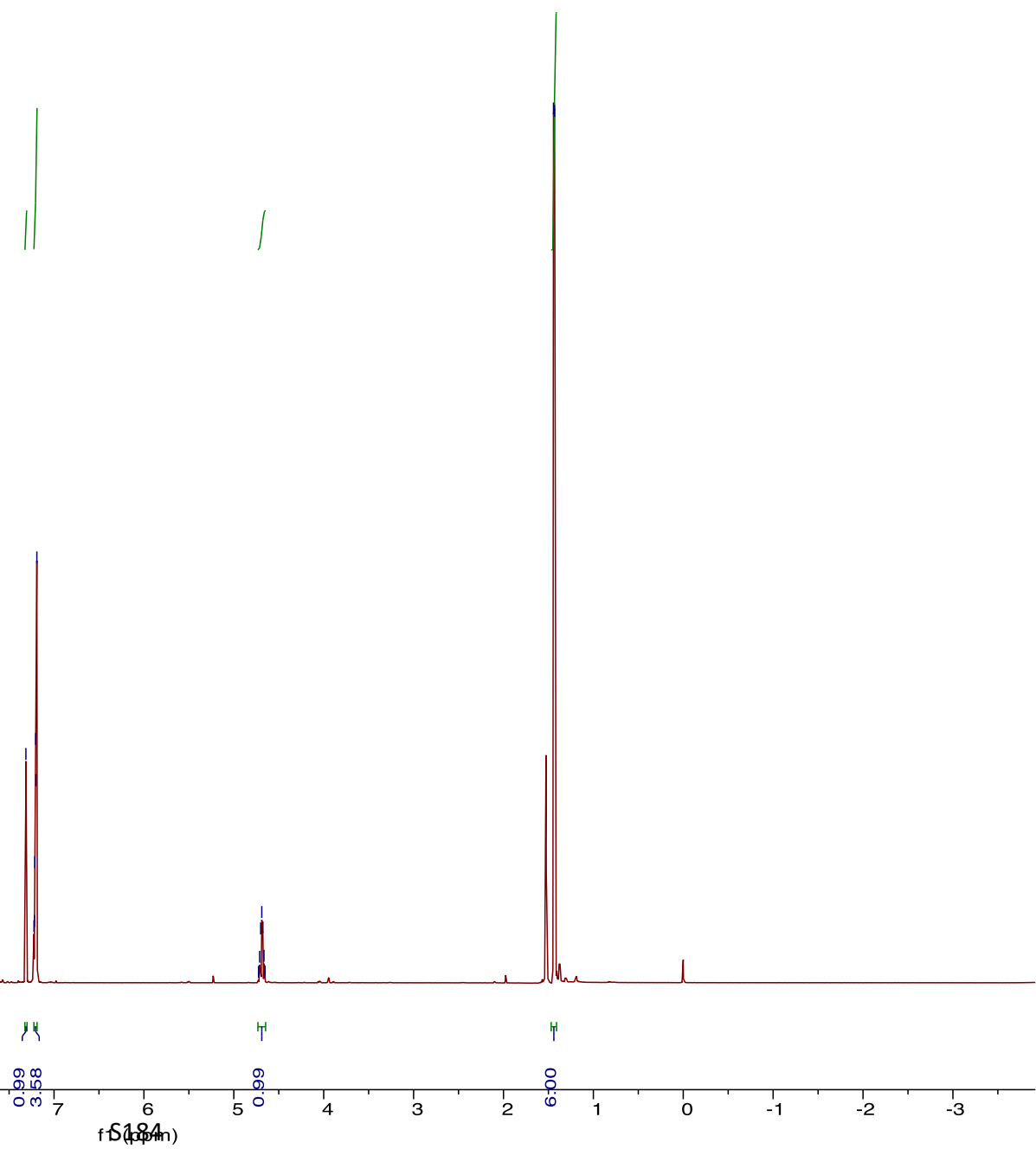




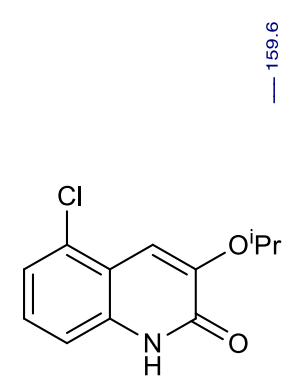

$1 \mathrm{~m},{ }^{13} \mathrm{C}$ NMR $\left(126 \mathrm{MHz}, \mathrm{CDCl}_{3}\right)$

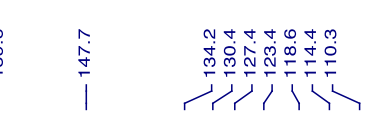




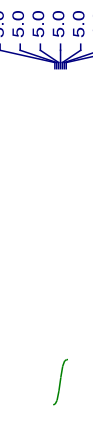

1n, ${ }^{1} \mathrm{H}$ NMR (400 MHz, $\left.\mathrm{CDCl}_{3}\right)$
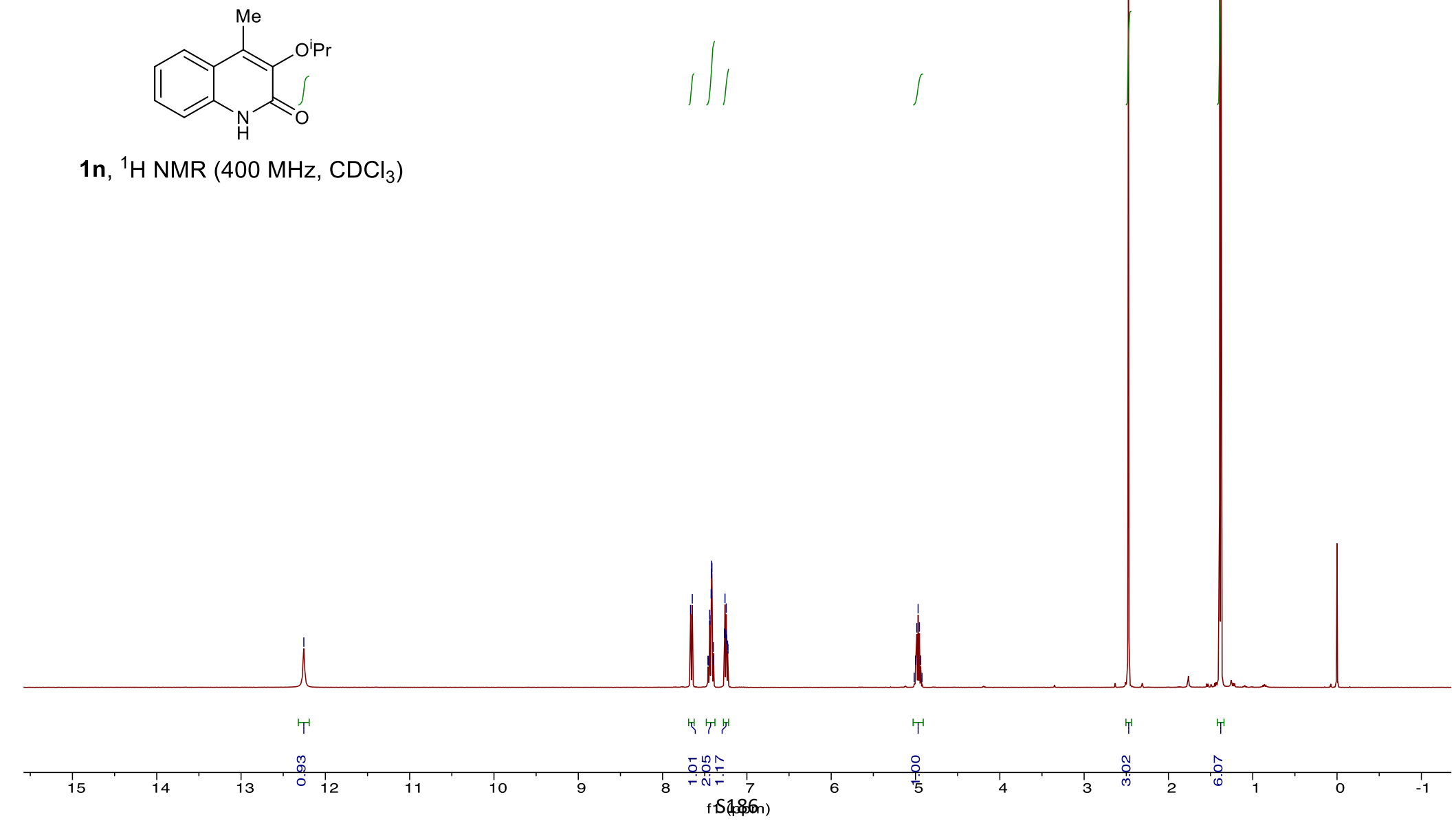


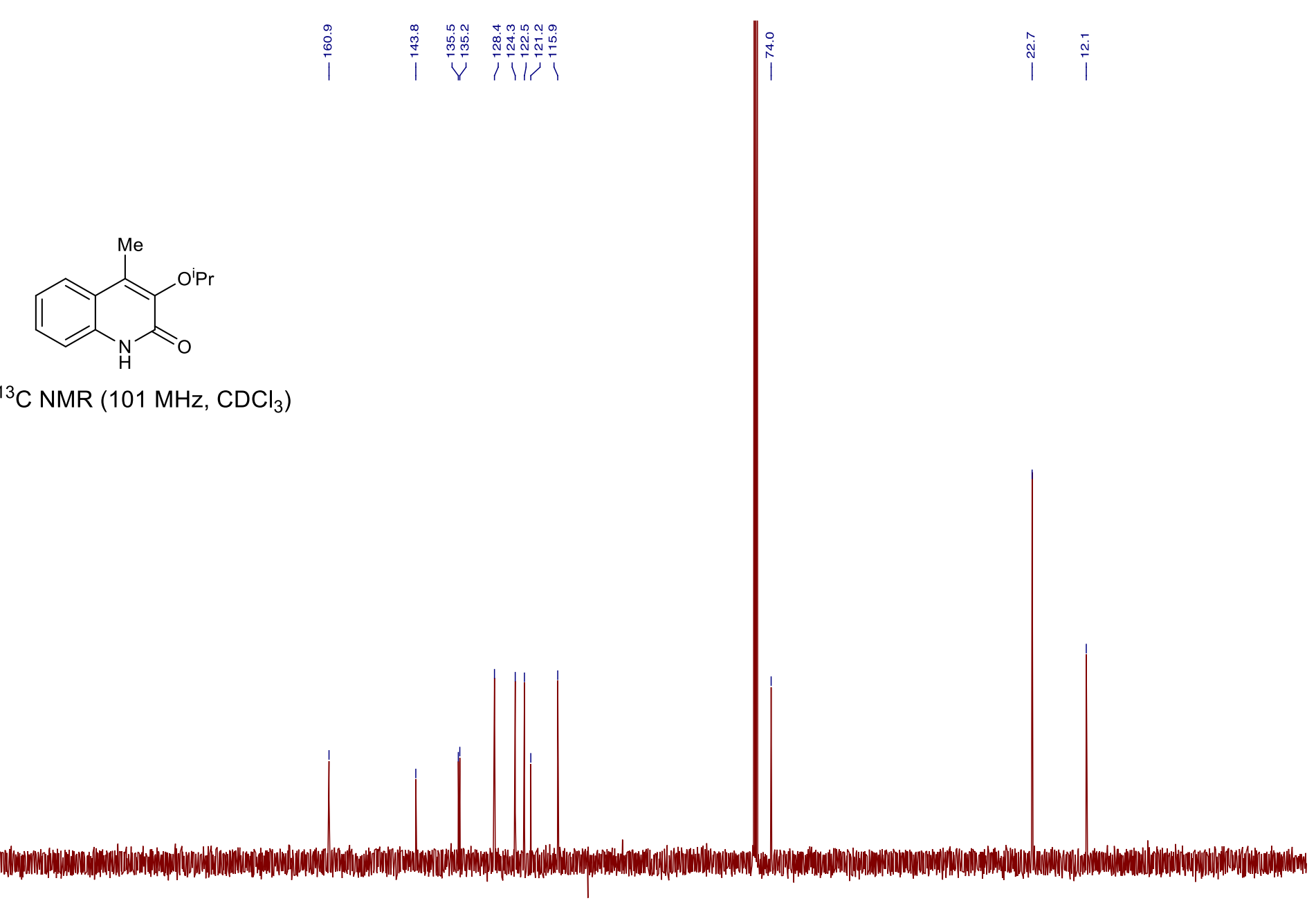

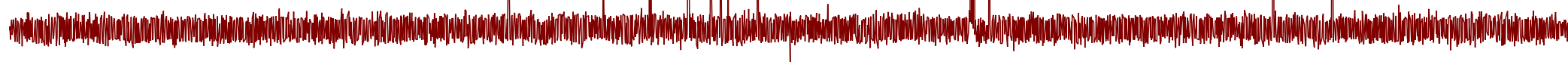

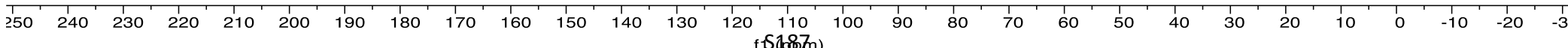




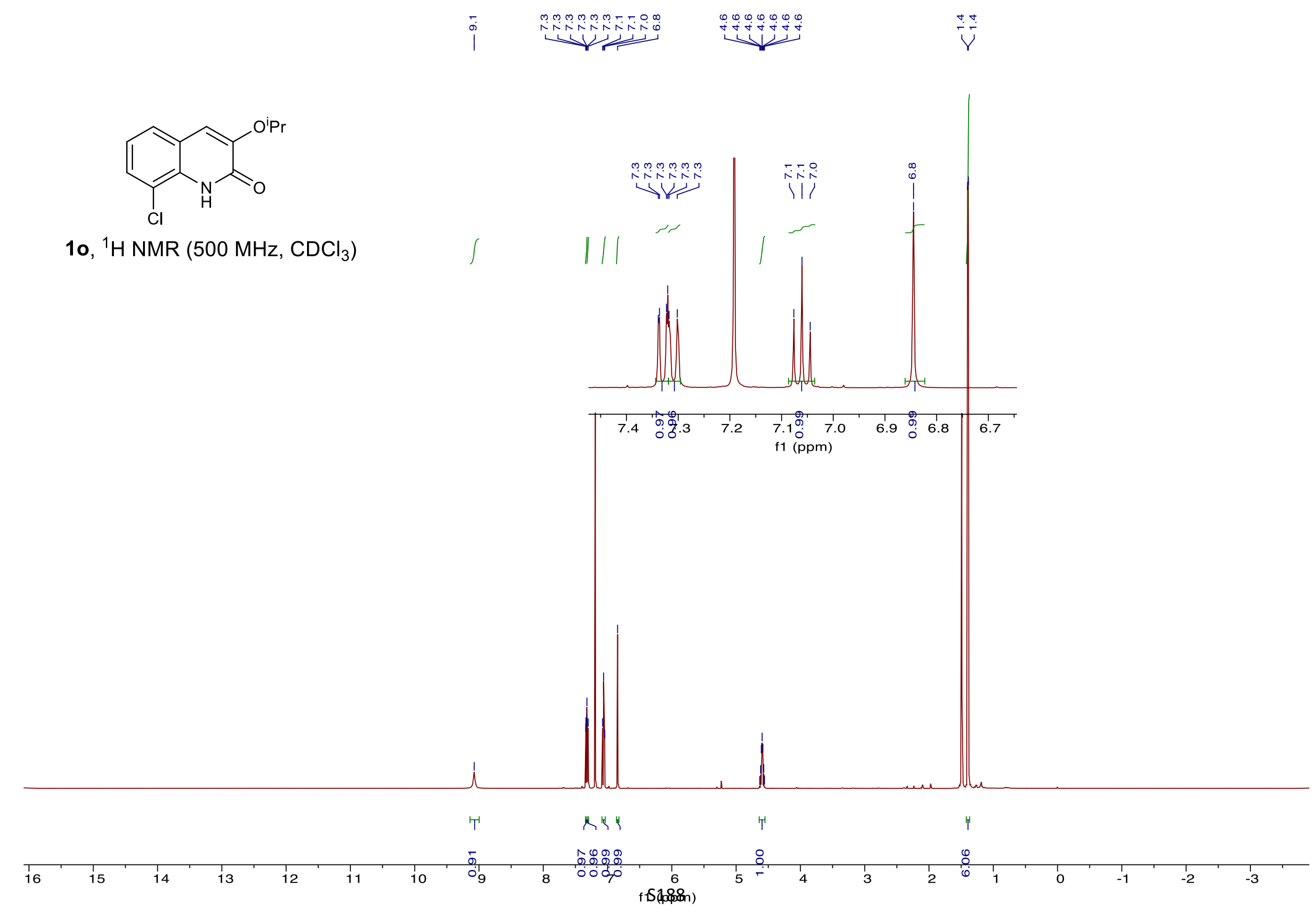




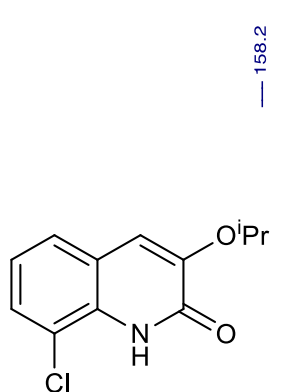

10, ${ }^{13} \mathrm{C}$ NMR $\left(126 \mathrm{MHz}, \mathrm{CDCl}_{3}\right)$

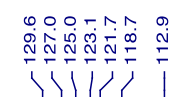

$\mid \stackrel{+}{i}$

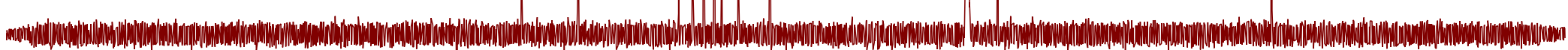

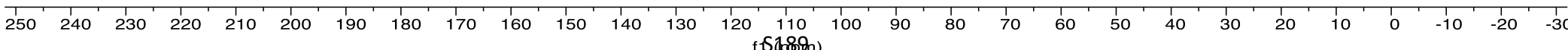





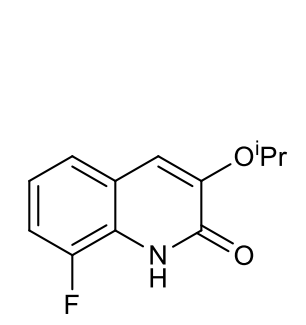

1p, ${ }^{13} \mathrm{C}$ NMR $\left(126 \mathrm{MHz}, \mathrm{CDCl}_{3}\right)$
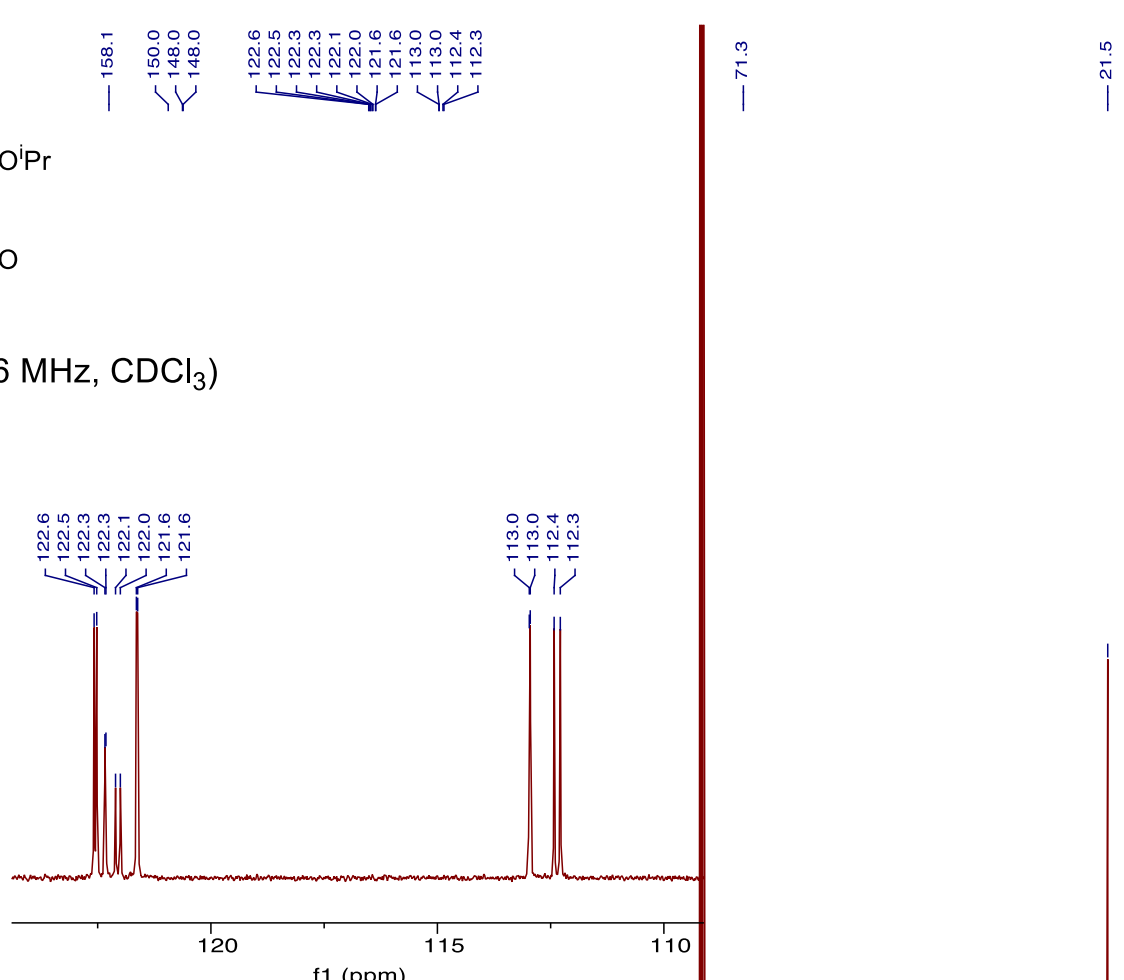


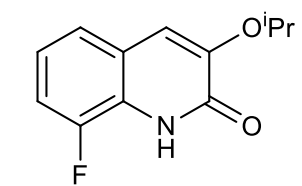

1p. ${ }^{19} \mathrm{~F}$ NMR (377 MHz, $\left.\mathrm{CDCl}_{3}\right)$

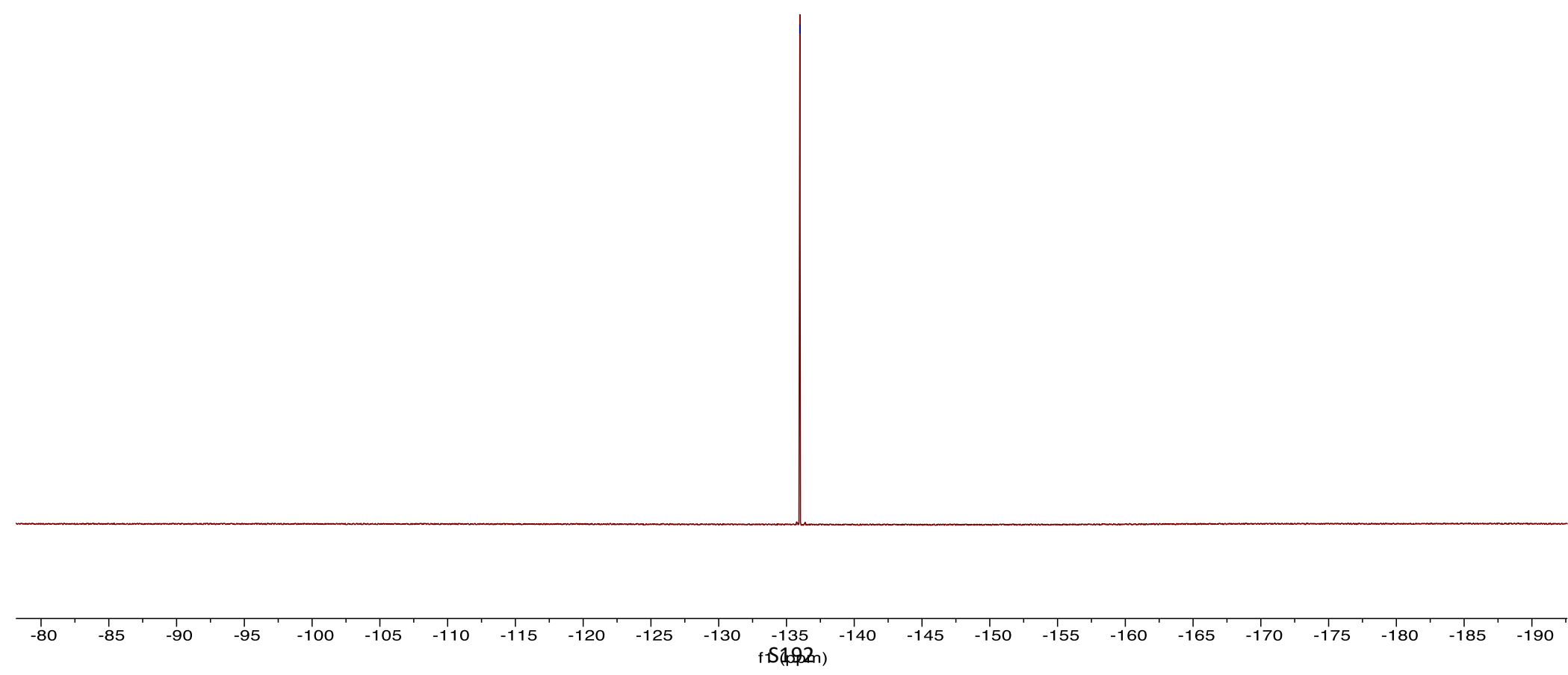




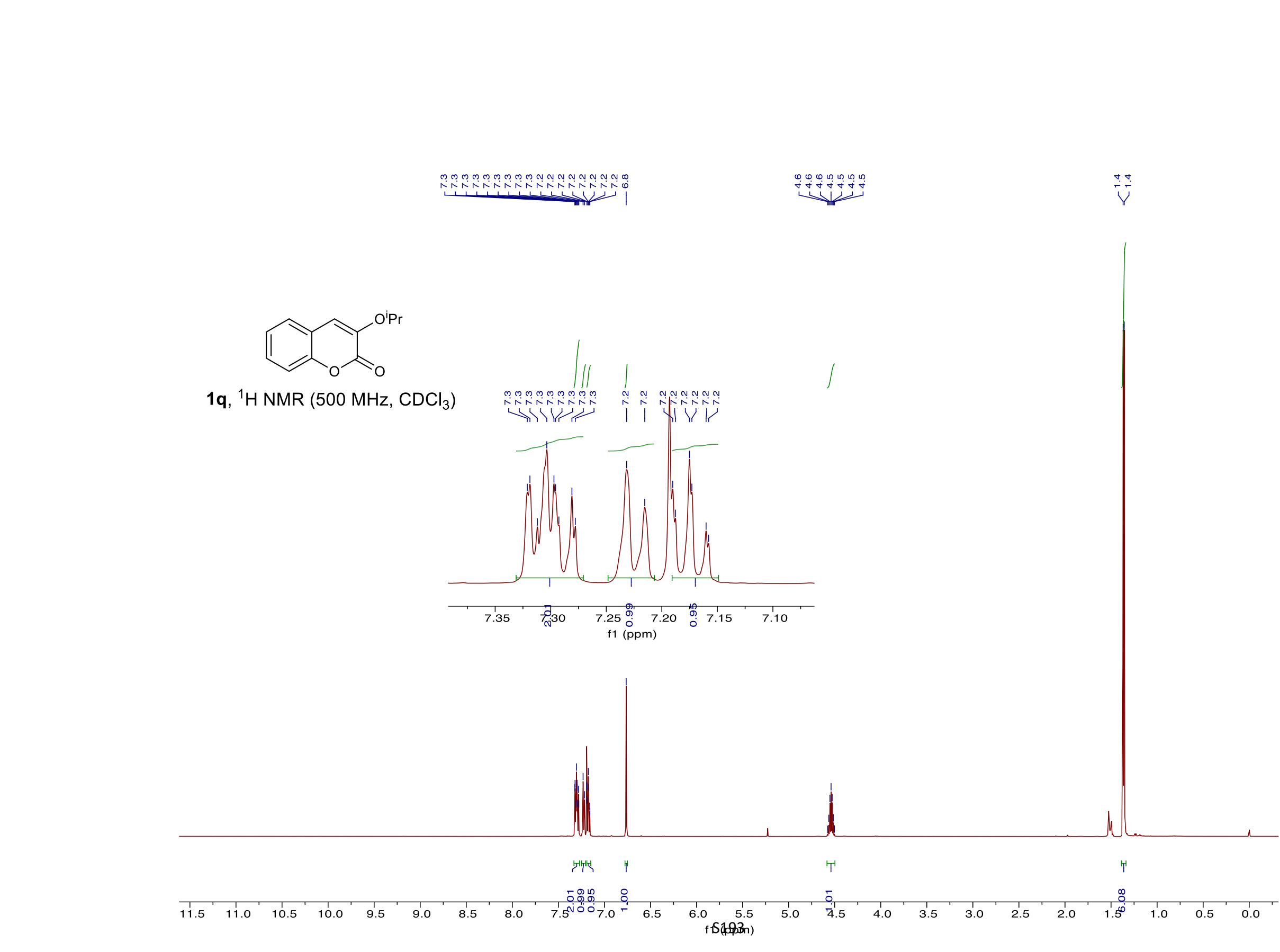




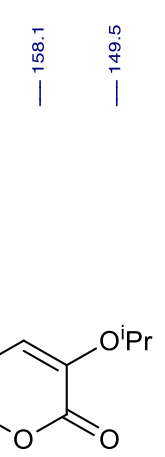

1q, ${ }^{13} \mathrm{C}$ NMR $\left(126 \mathrm{MHz}, \mathrm{CDCl}_{3}\right)$
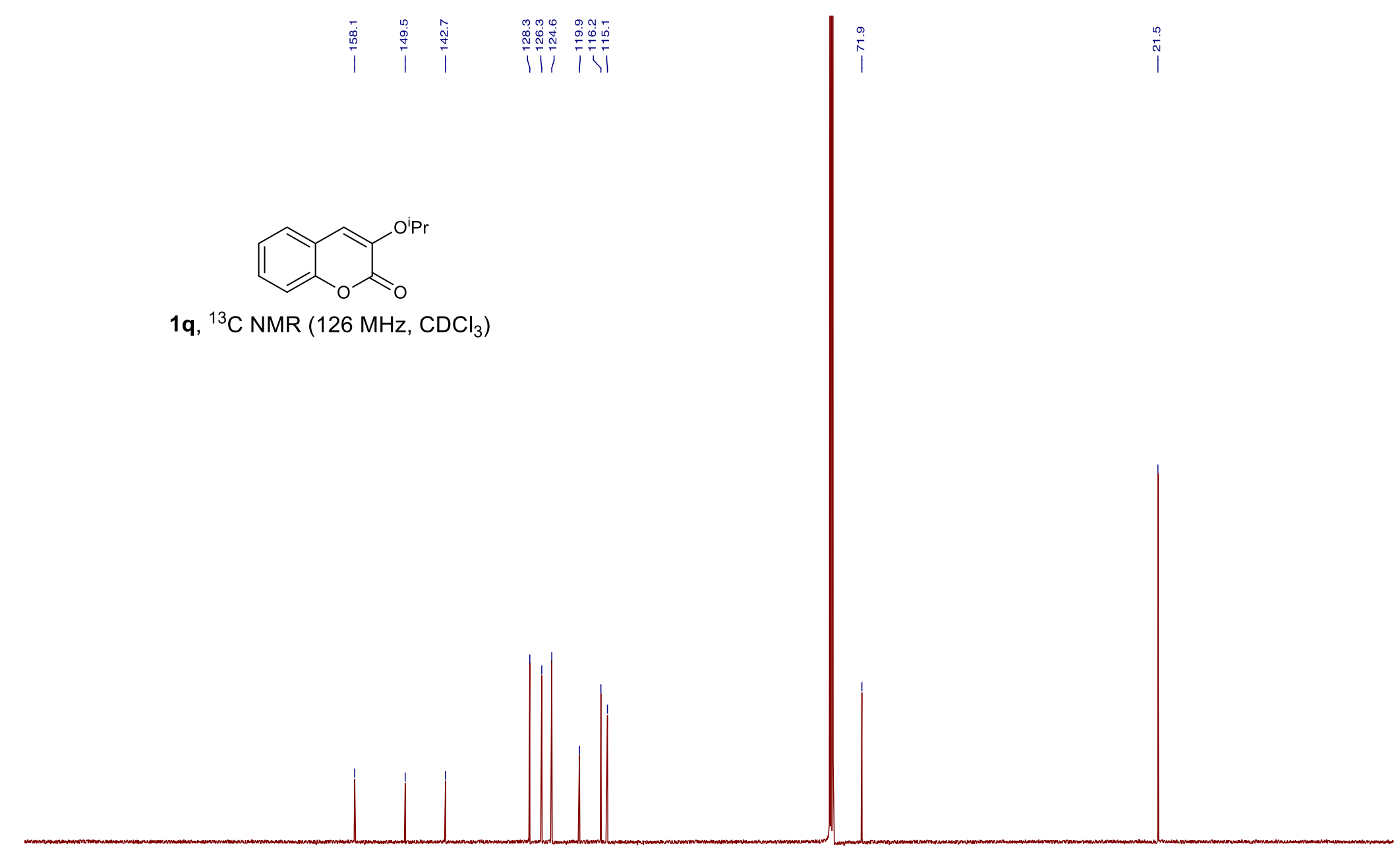

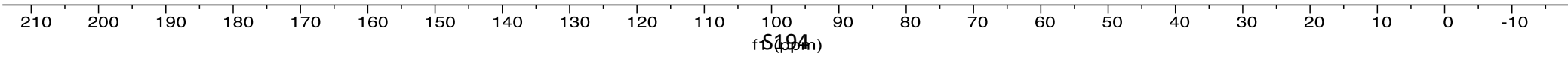




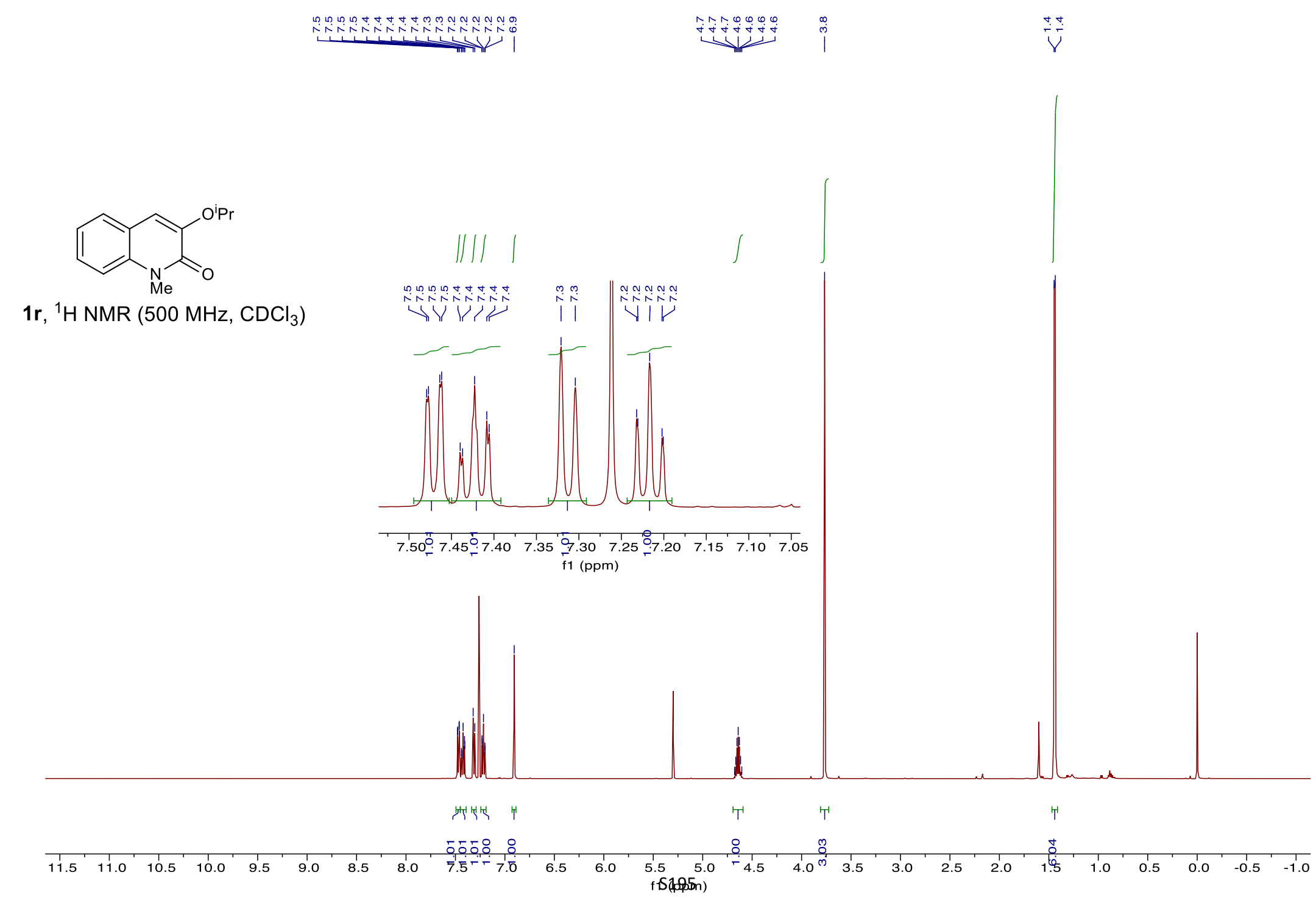




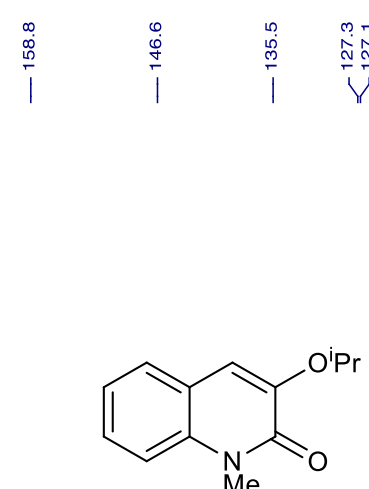

1r, ${ }^{13} \mathrm{C}$ NMR (126 MHz, $\mathrm{CDCl}_{3}$ )

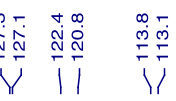

I

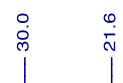

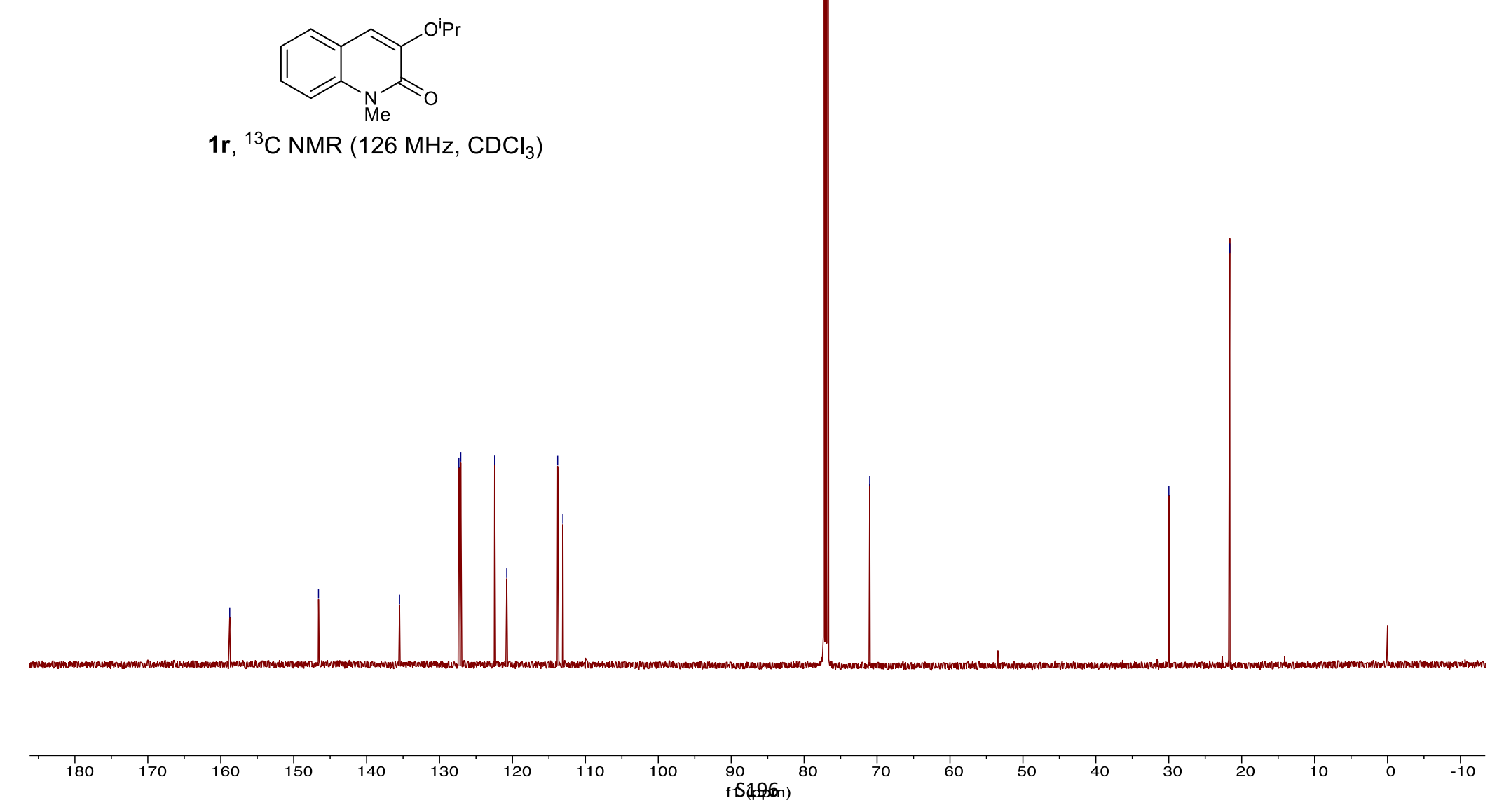


$\underbrace{n}$

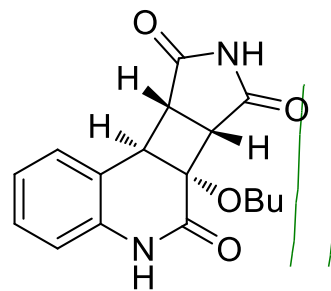

3a, ${ }^{1} \mathrm{H}$ NMR (500 MHz, $\left.\mathrm{CD}_{3} \mathrm{OD}\right)$

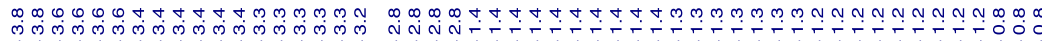
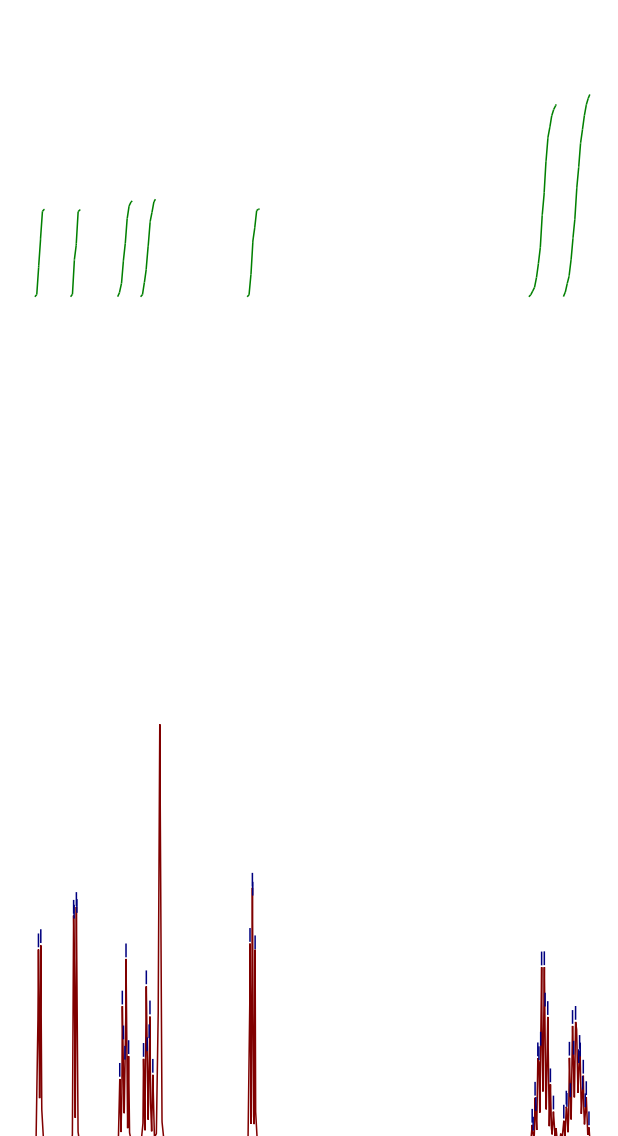

$\mapsto \mapsto$

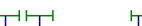

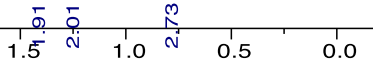

Tr T T T T T

T

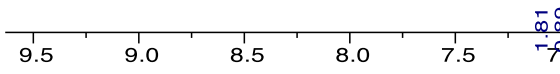

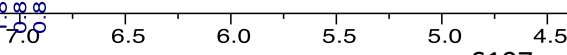

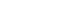




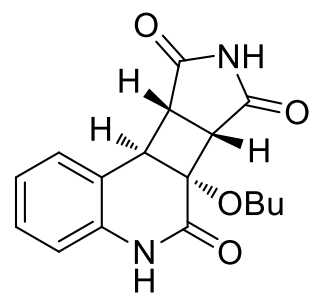

3a, ${ }^{13} \mathrm{C}$ NMR (126 MHz, $\left.\mathrm{CD}_{3} \mathrm{OD}\right)$

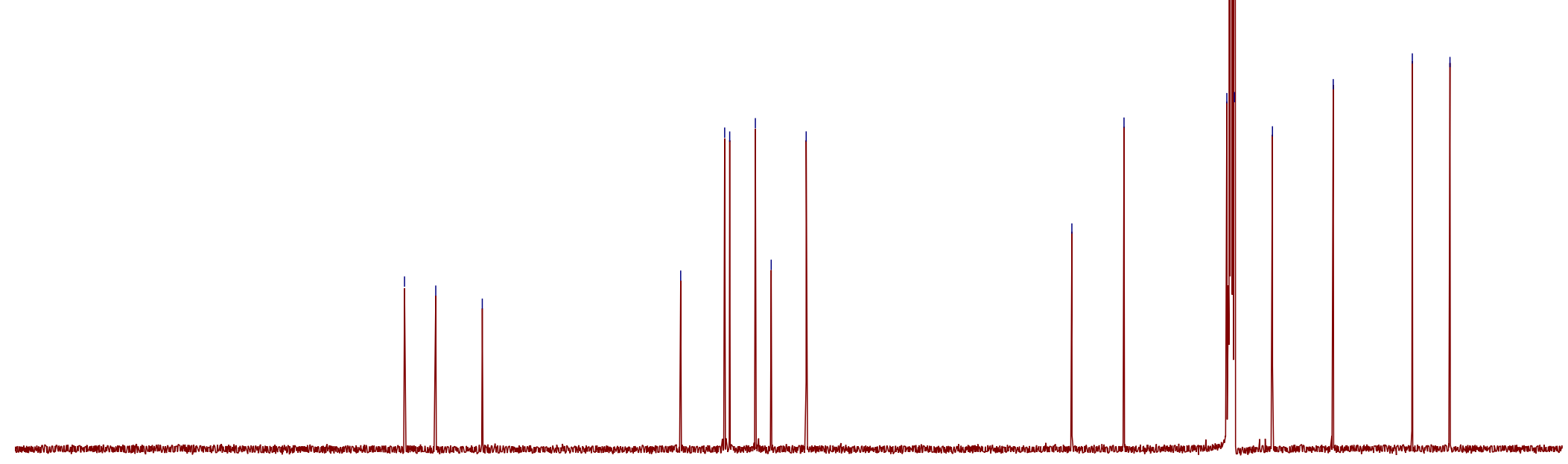

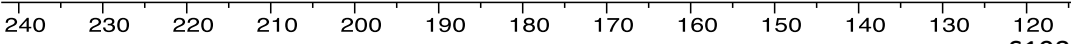




\section{舟}

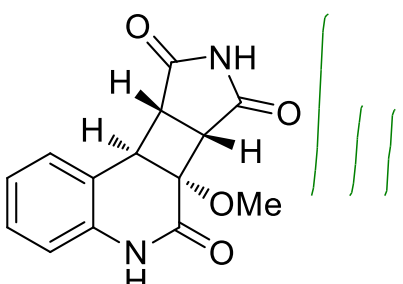

3b, ${ }^{1} \mathrm{H}$ NMR (500 MHz, $\left.\mathrm{CD}_{3} \mathrm{OD}\right)$

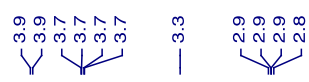

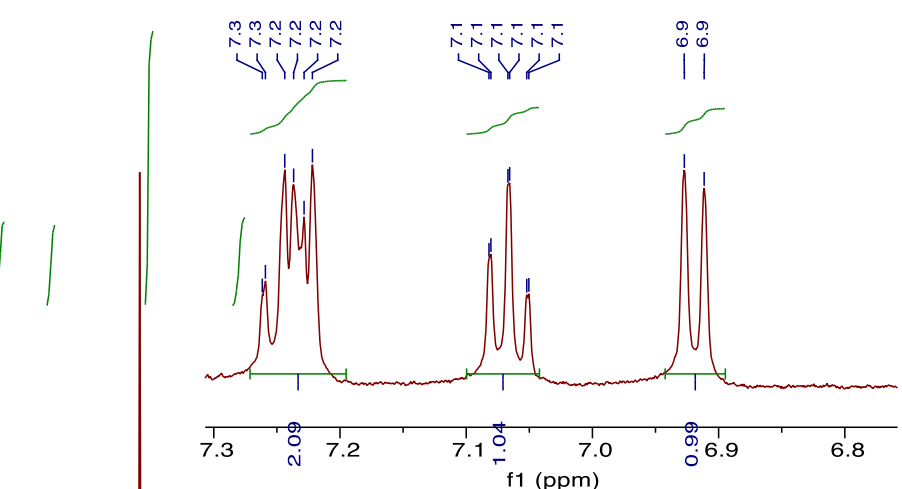

Mll

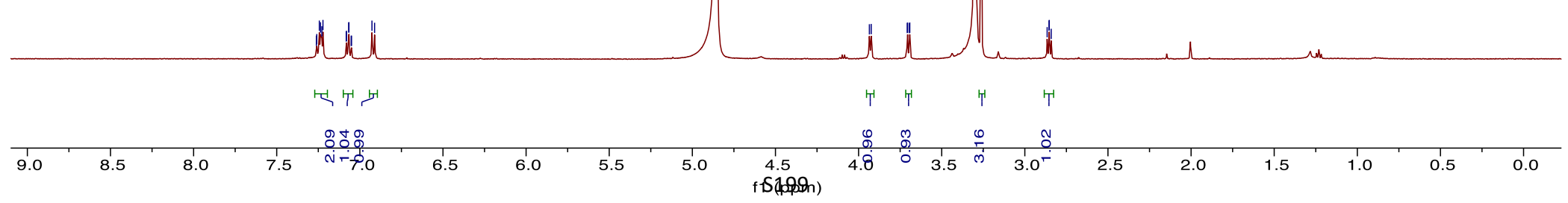




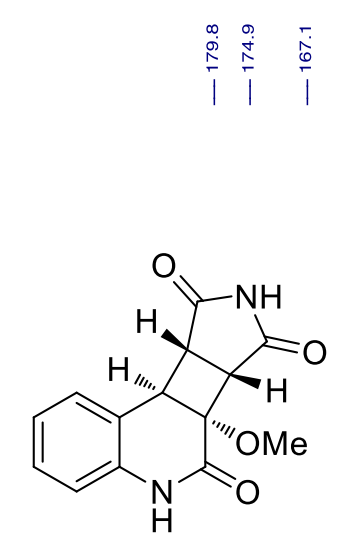

3b, ${ }^{13} \mathrm{C}$ NMR $\left(126 \mathrm{MHz}, \mathrm{CD}_{3} \mathrm{OD}\right)$

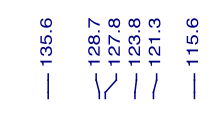

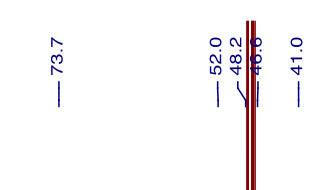

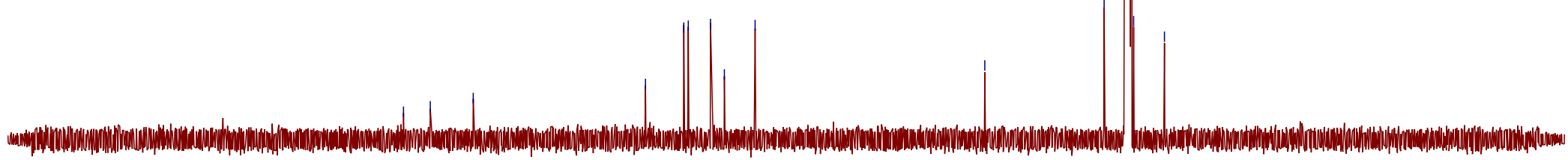

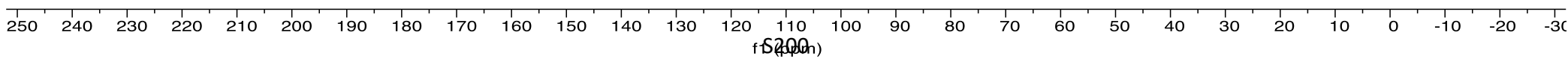




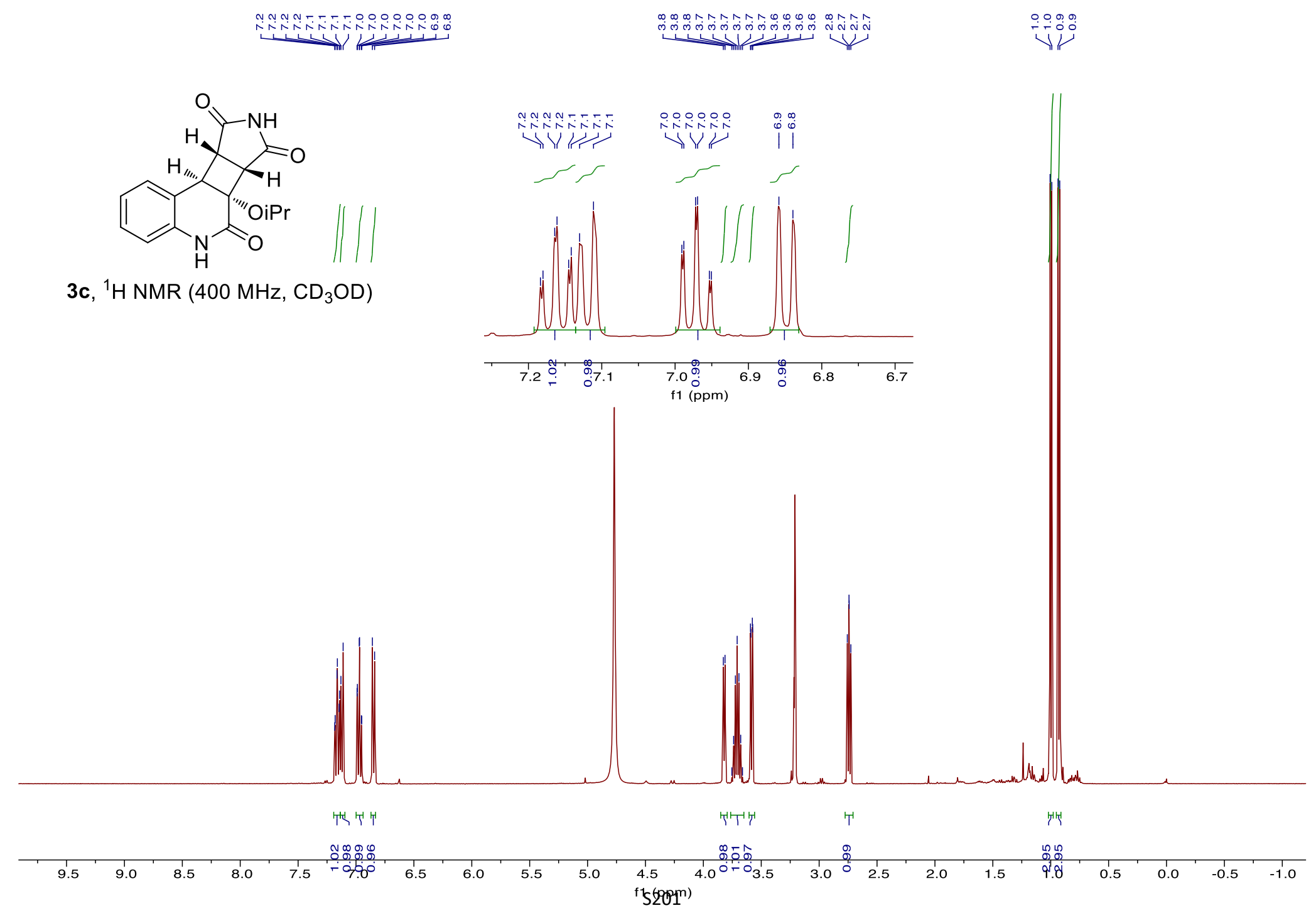




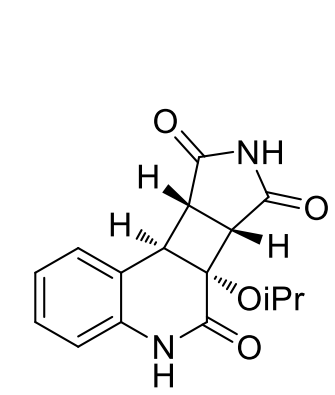

3c, ${ }^{13} \mathrm{C}$ NMR $\left(126 \mathrm{MHz}, \mathrm{CD}_{3} \mathrm{OD}\right)$

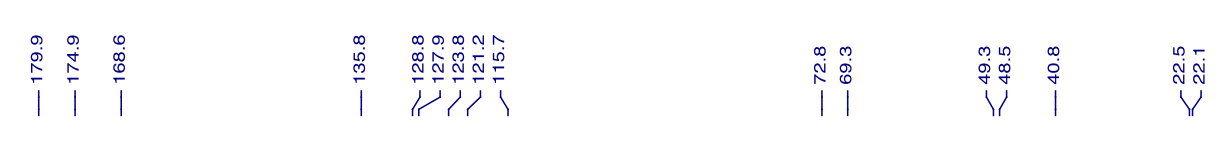

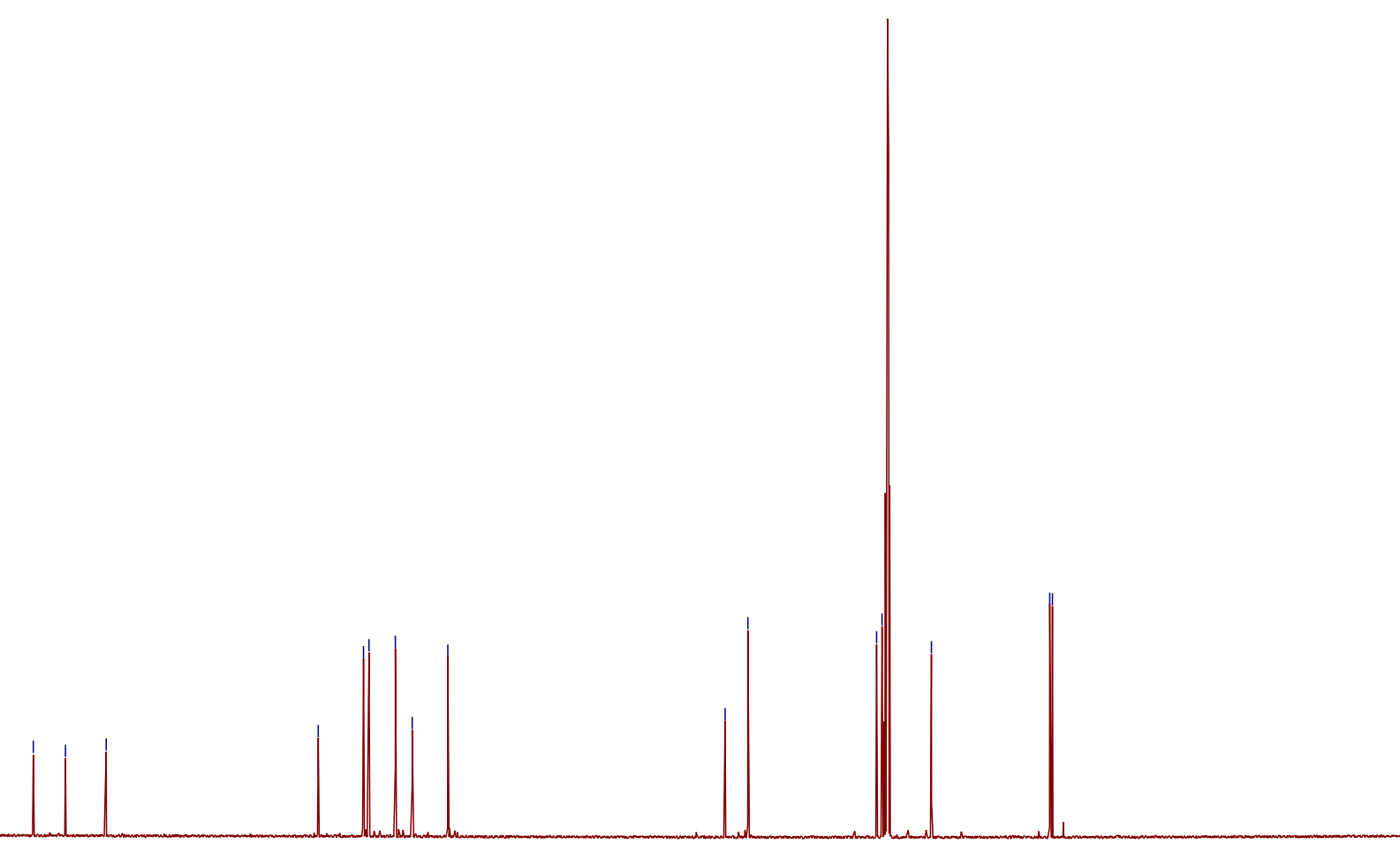

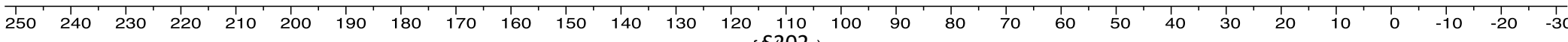
fis 25002 (n) 


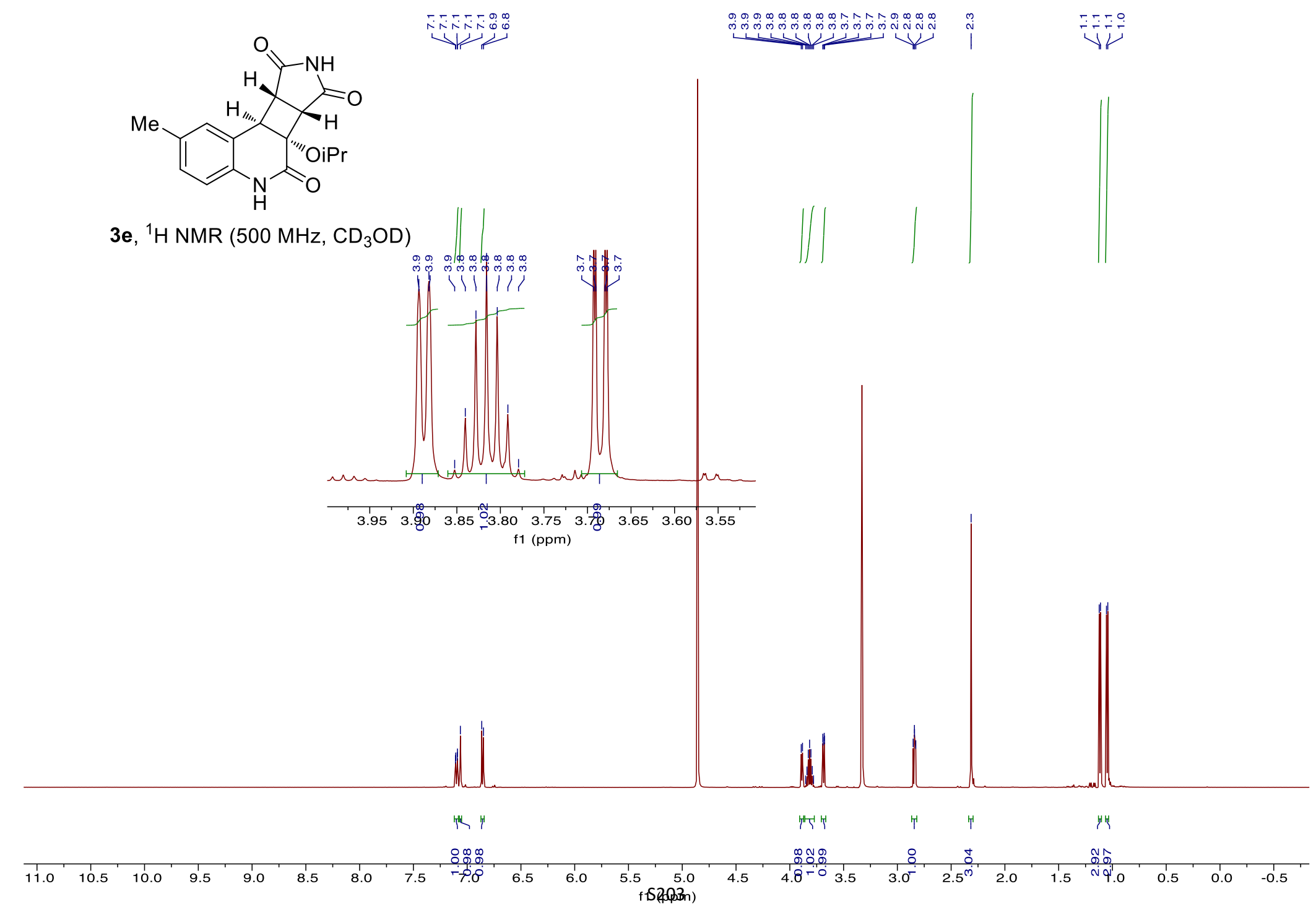




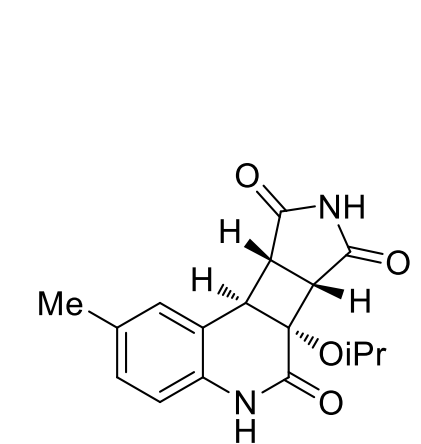

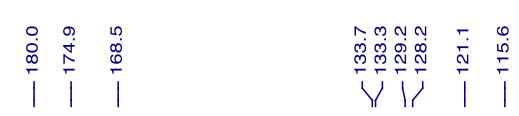

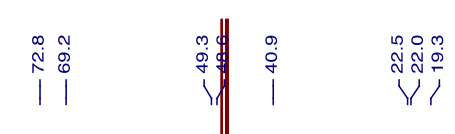

3e, ${ }^{13} \mathrm{C} \mathrm{NMR}\left(126 \mathrm{MHz}, \mathrm{CD}_{3} \mathrm{OD}\right)$

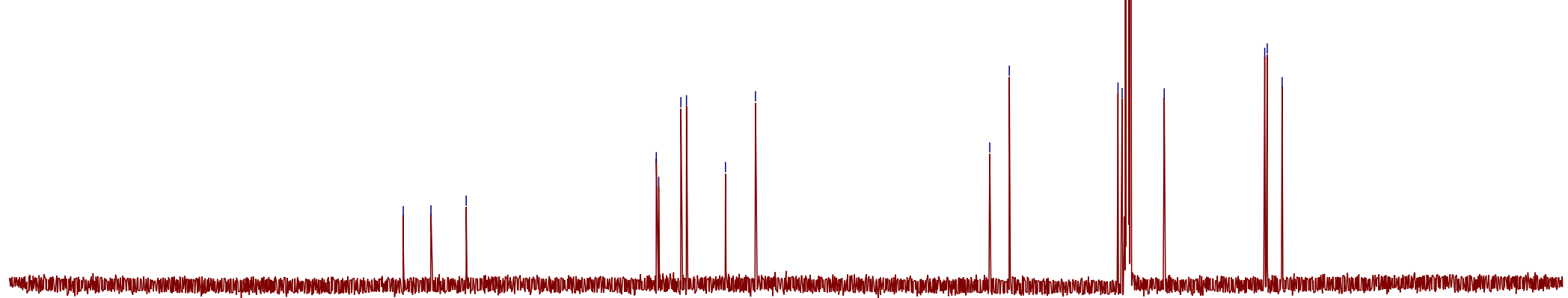

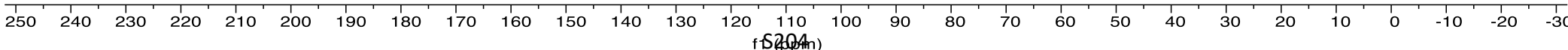




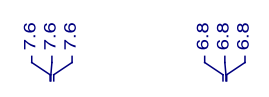

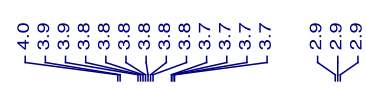
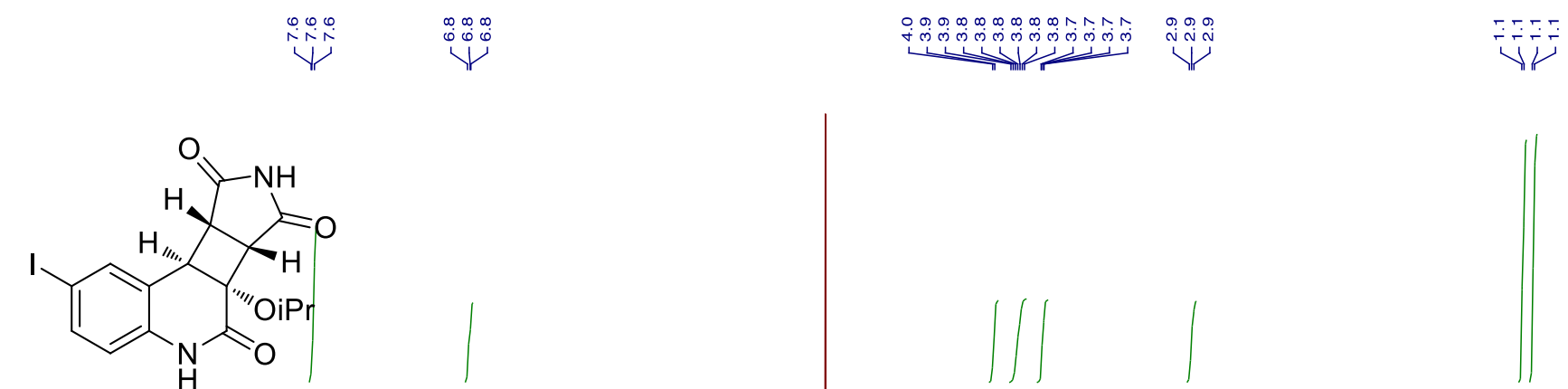

3f, ${ }^{1} \mathrm{H}$ NMR $\left(500 \mathrm{MHz}, \mathrm{CD}_{3} \mathrm{OD}\right)$

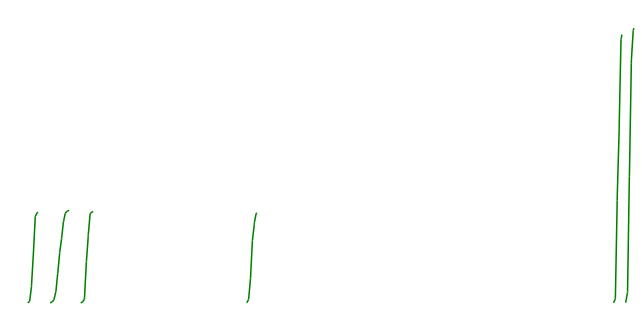

(

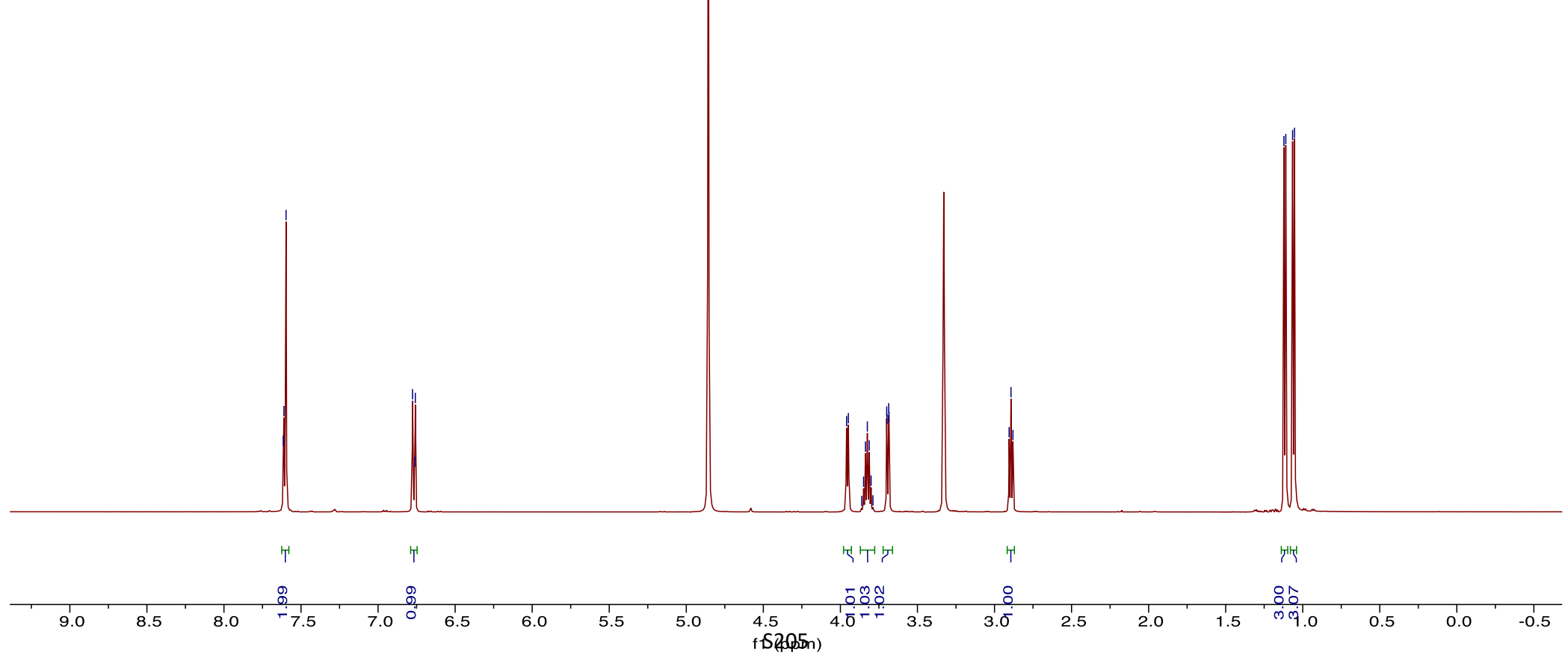




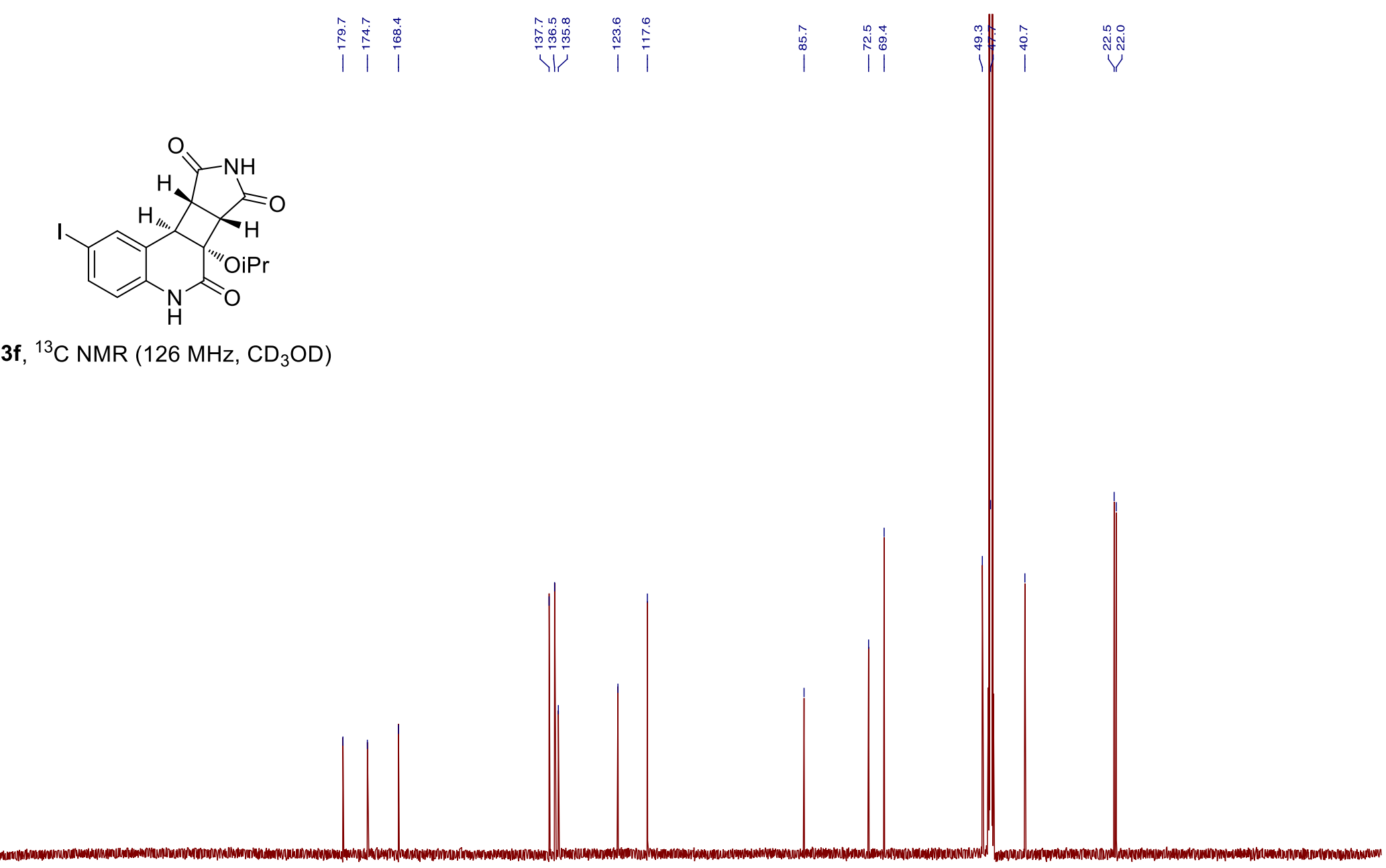

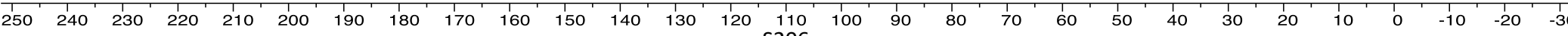
fiSragson) 

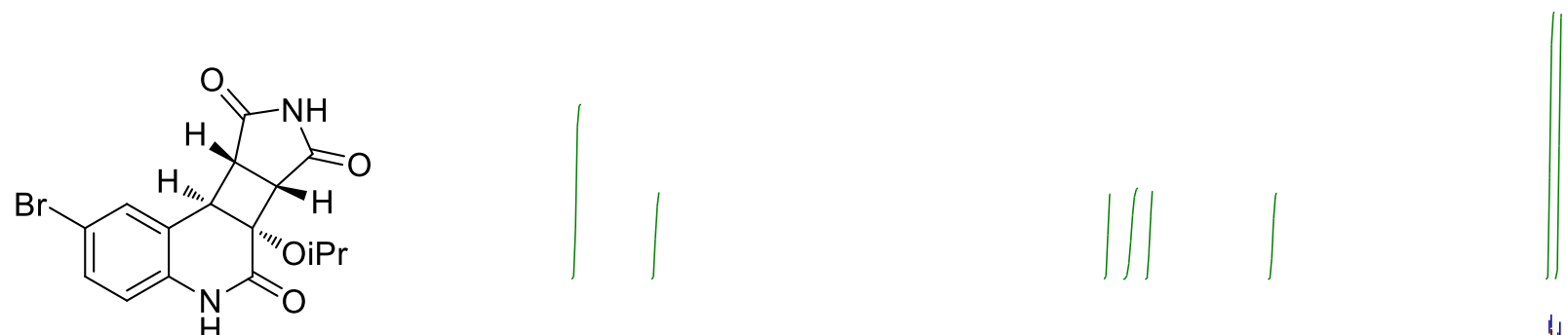

3g, ${ }^{1} \mathrm{H}$ NMR $\left(500 \mathrm{MHz}, \mathrm{CD}_{3} \mathrm{OD}\right)$

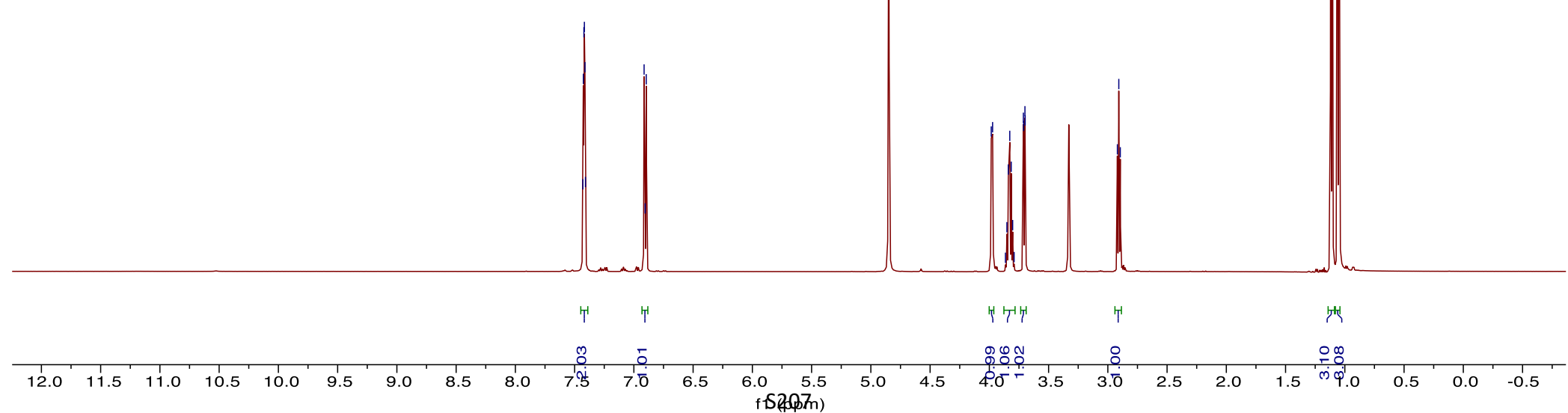




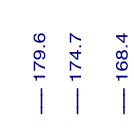

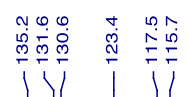

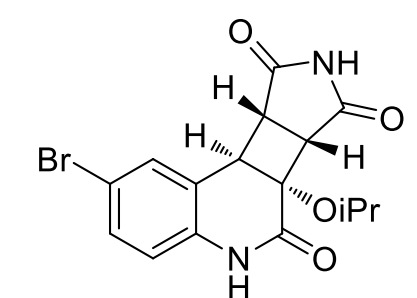

3g, ${ }^{13} \mathrm{C}$ NMR (126 MHz, $\left.\mathrm{CD}_{3} \mathrm{OD}\right)$

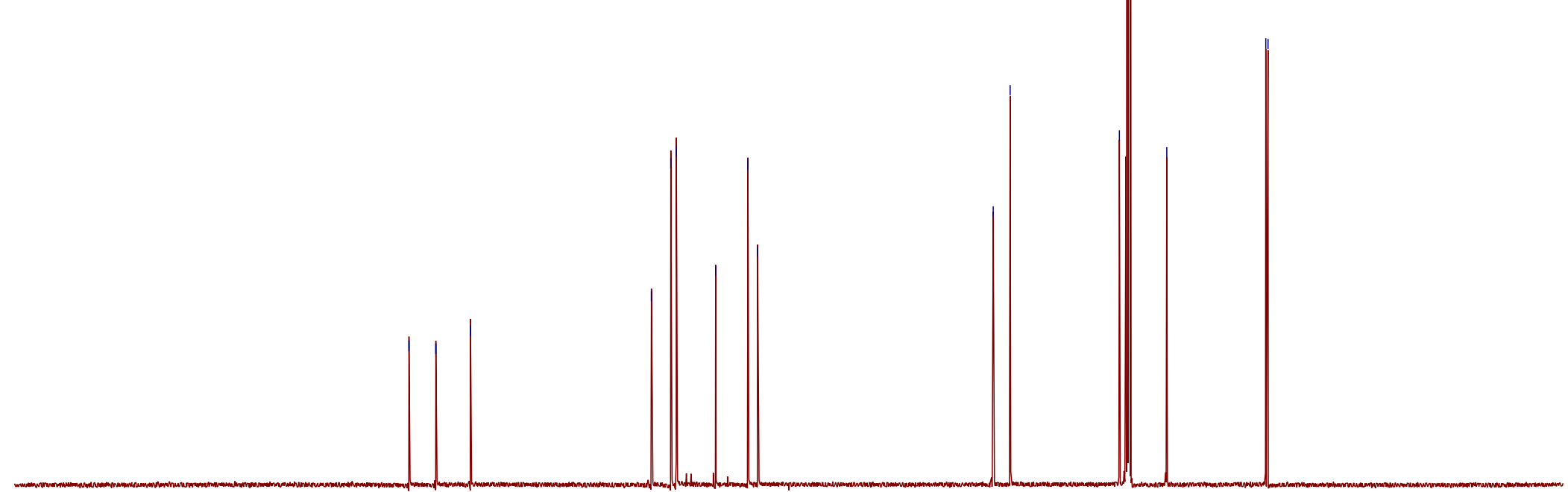

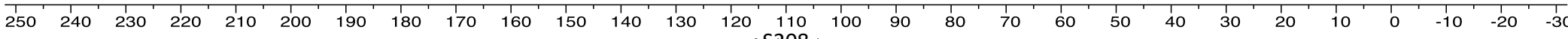
if 15 sopion) 


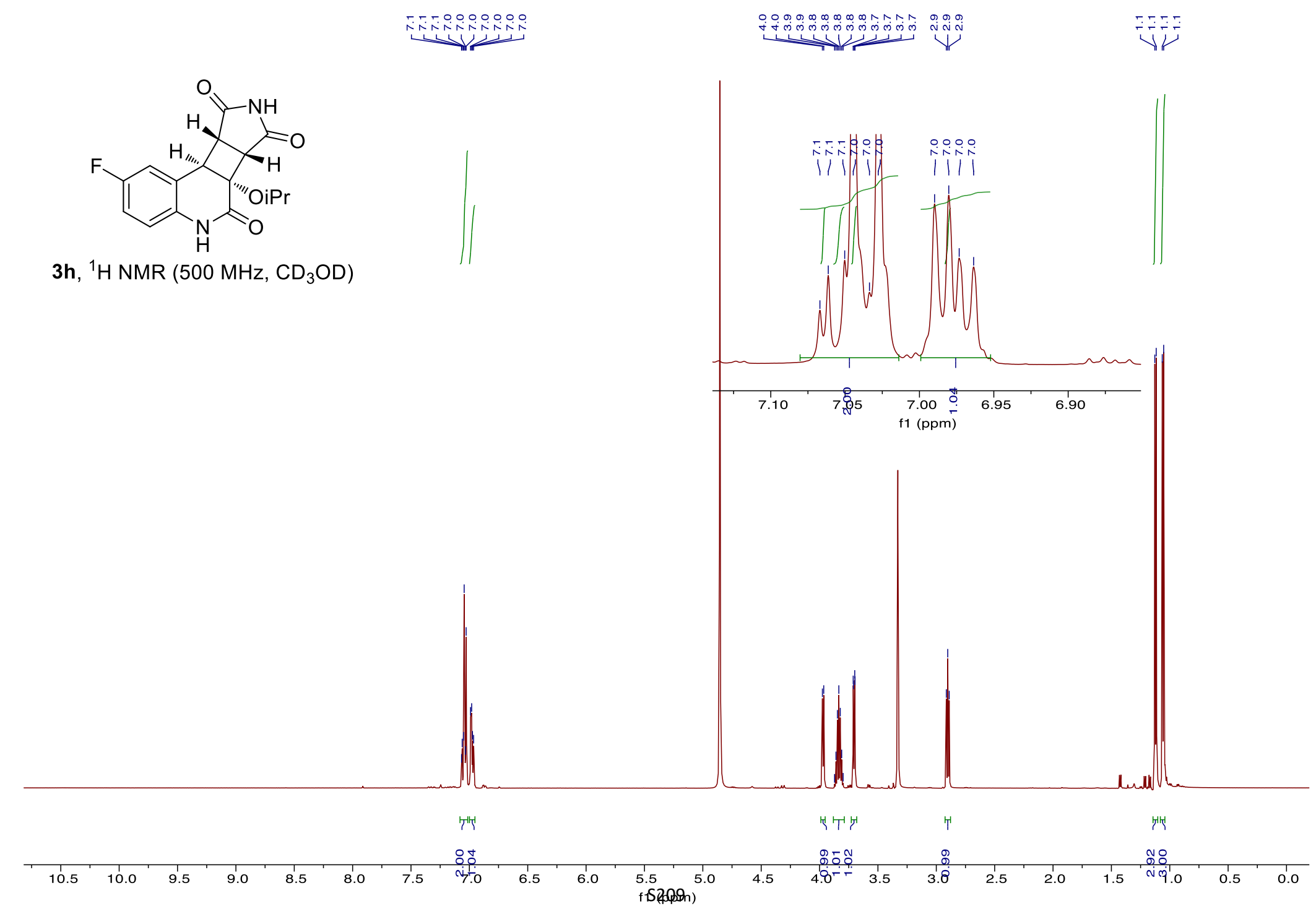




\section{0
$i$
$i$}

3h, ${ }^{13} \mathrm{C}$ NMR $\left(126 \mathrm{MHz}, \mathrm{CD}_{3} \mathrm{OD}\right)$

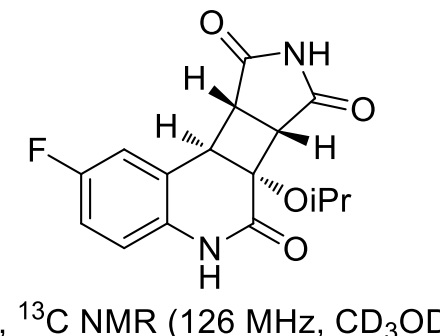

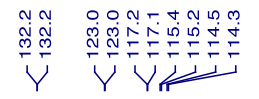

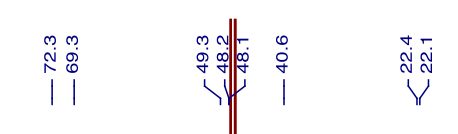

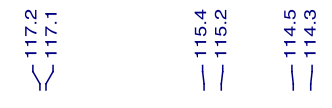

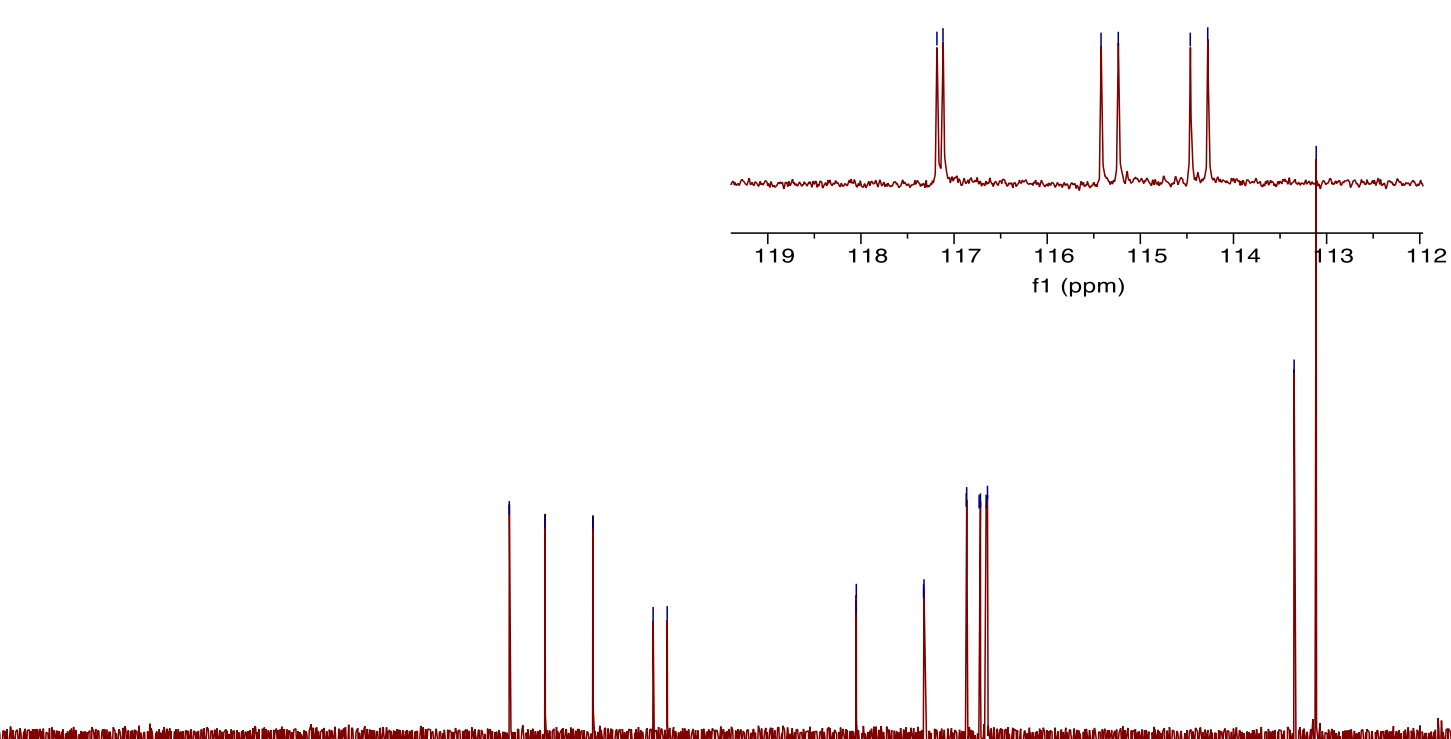

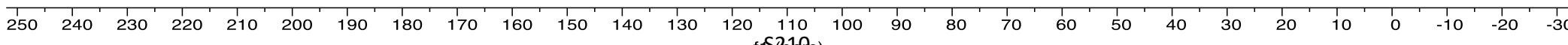
fisfopbon) 


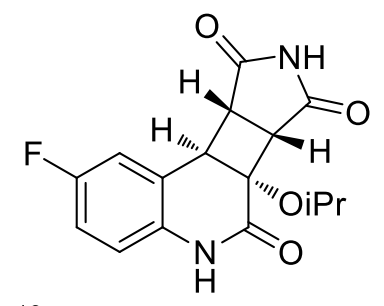

3h, ${ }^{19} \mathrm{~F} \mathrm{NMR}\left(377 \mathrm{MHz}, \mathrm{CD}_{3} \mathrm{OD}\right)$

W.

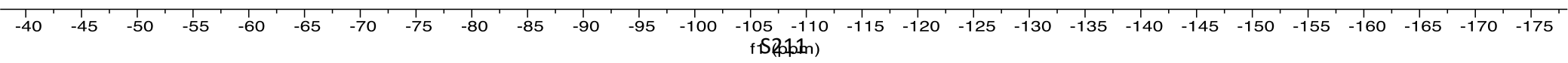




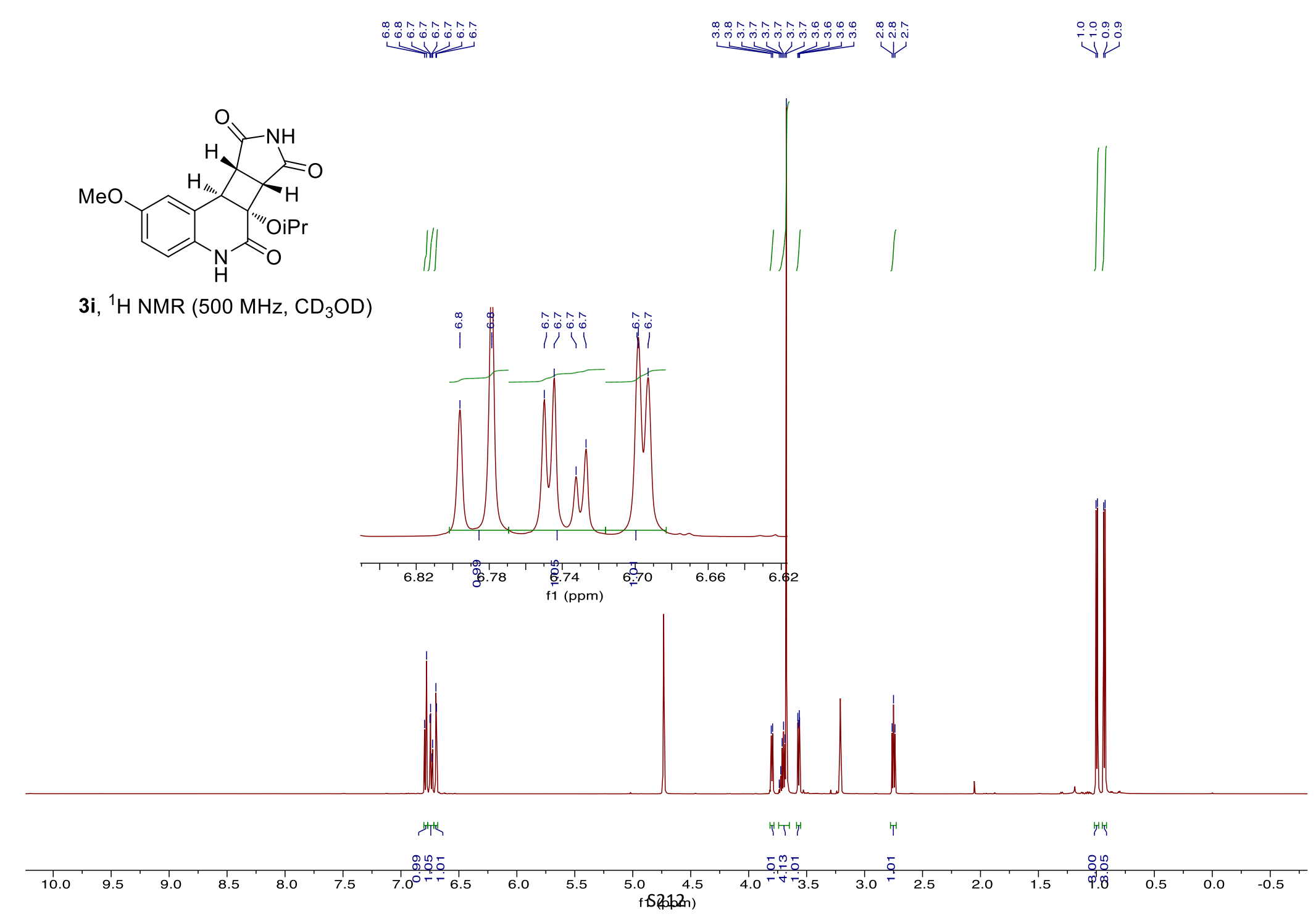




\subsection{0
0}

3i, ${ }^{13} \mathrm{C}$ NMR $\left(126 \mathrm{MHz}, \mathrm{CD}_{3} \mathrm{OD}\right)$

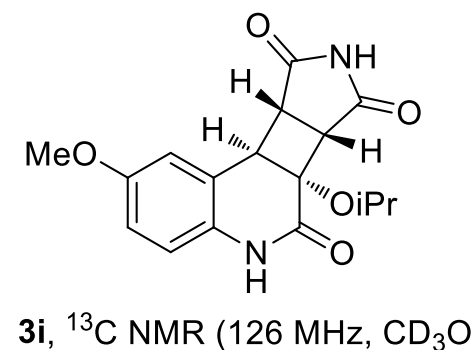

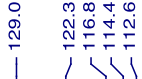

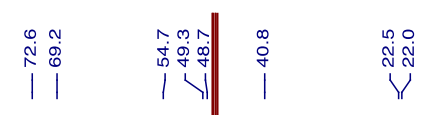

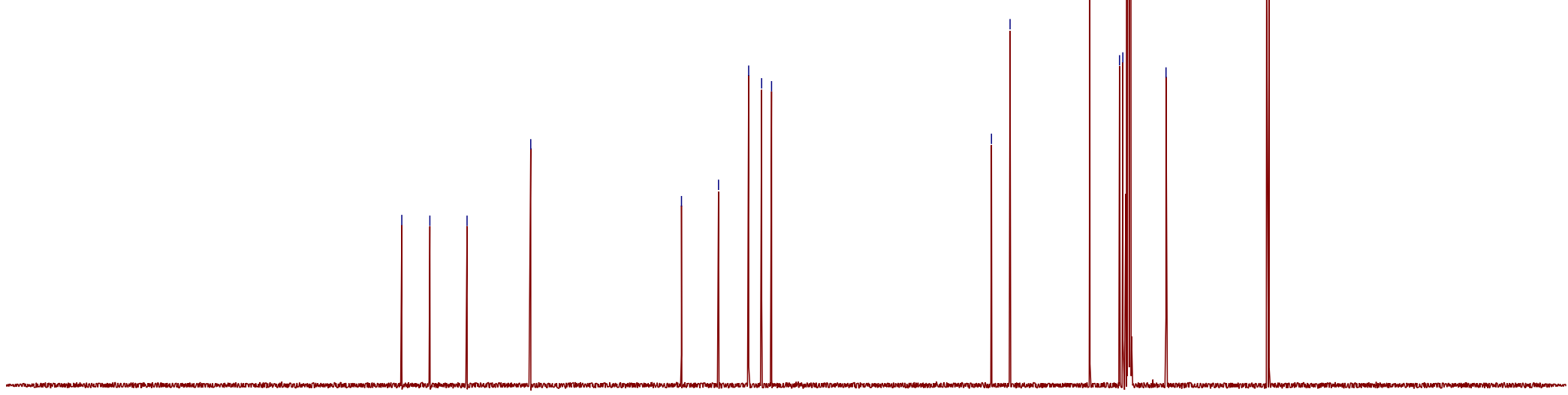

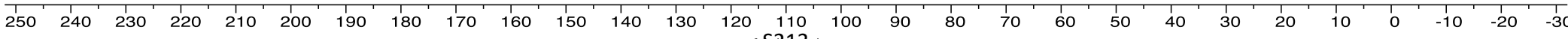
fistopo 


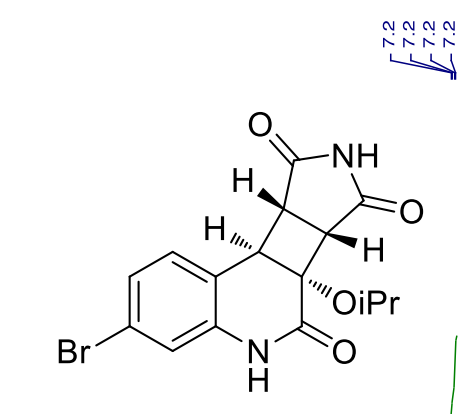

3j, ${ }^{1} \mathrm{H}$ NMR (500 MHz, $\mathrm{CD}_{3} \mathrm{OD}$ )

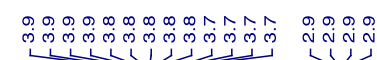
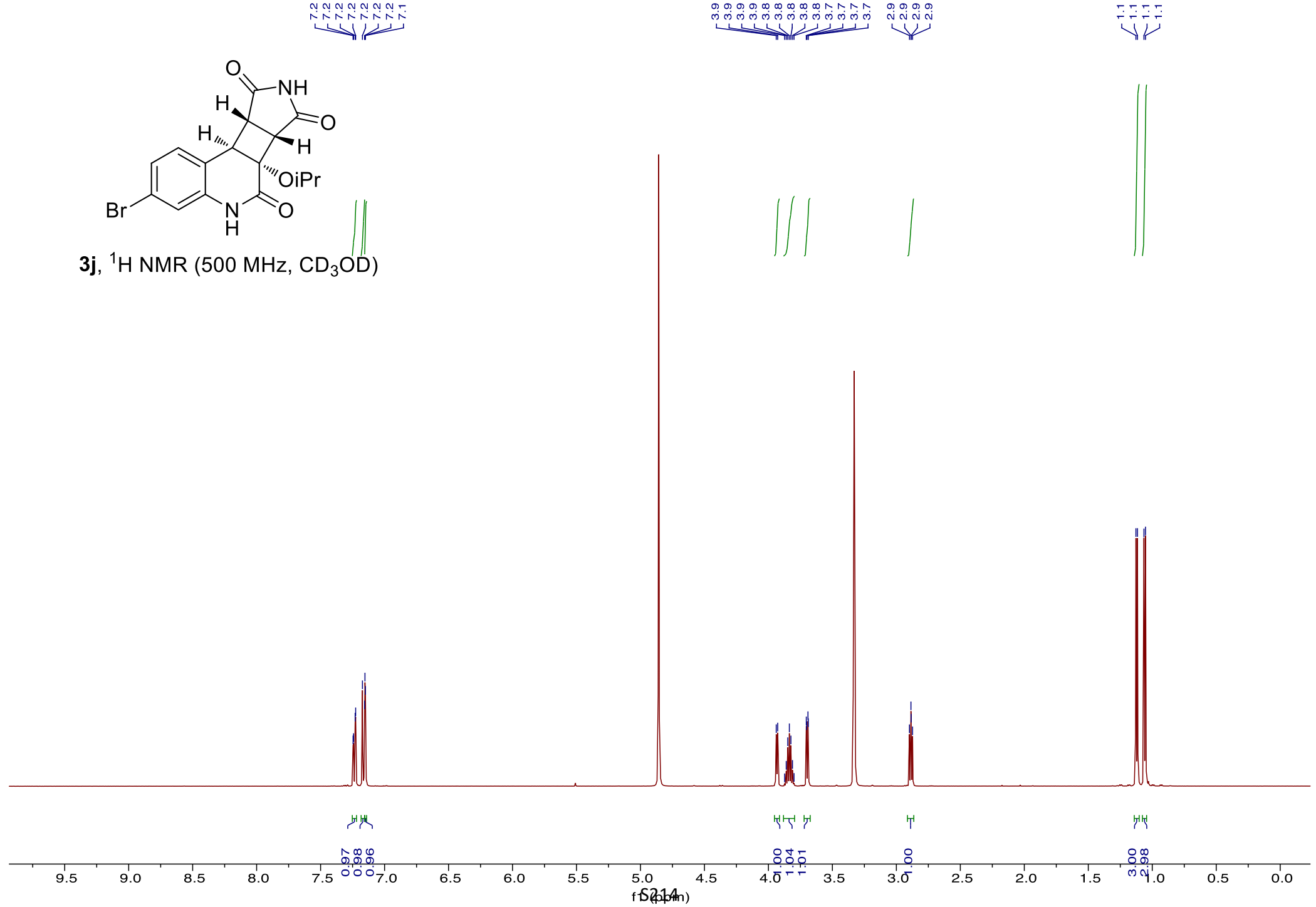


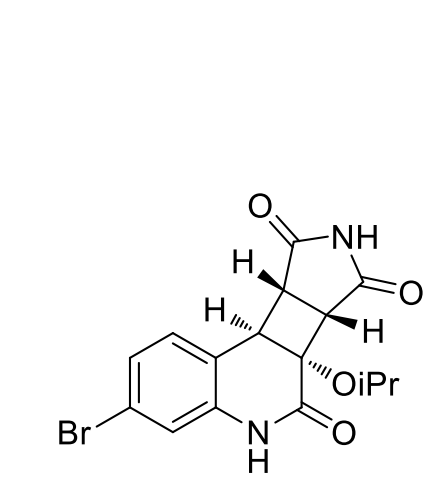

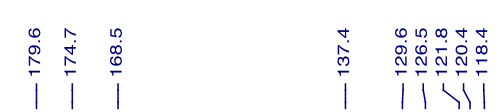

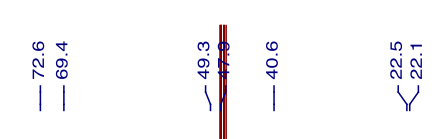

3j, ${ }^{13} \mathrm{C}$ NMR (126 MHz, $\left.\mathrm{CD}_{3} \mathrm{OD}\right)$

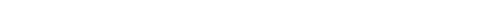

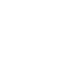

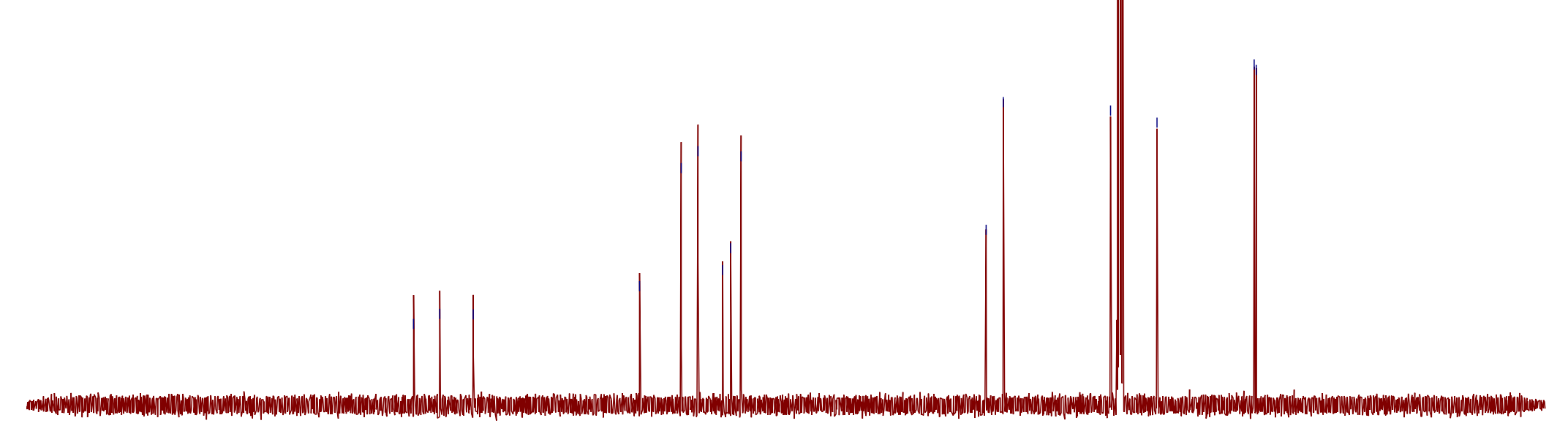

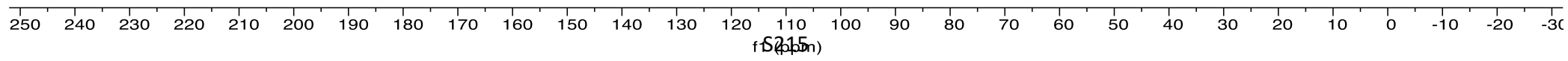




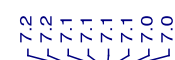

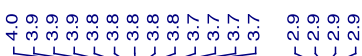
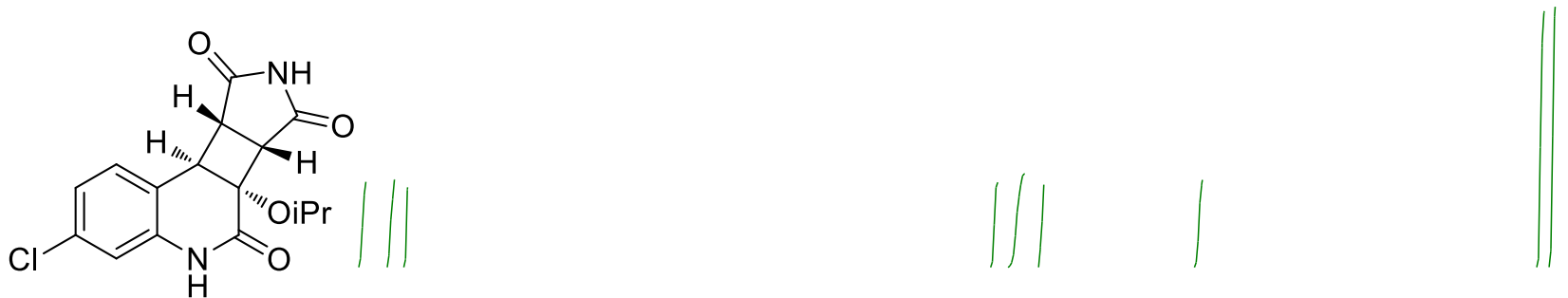

3k, ${ }^{1} \mathrm{H}$ NMR $\left(500 \mathrm{MHz}, \mathrm{CD}_{3} \mathrm{OD}\right)$

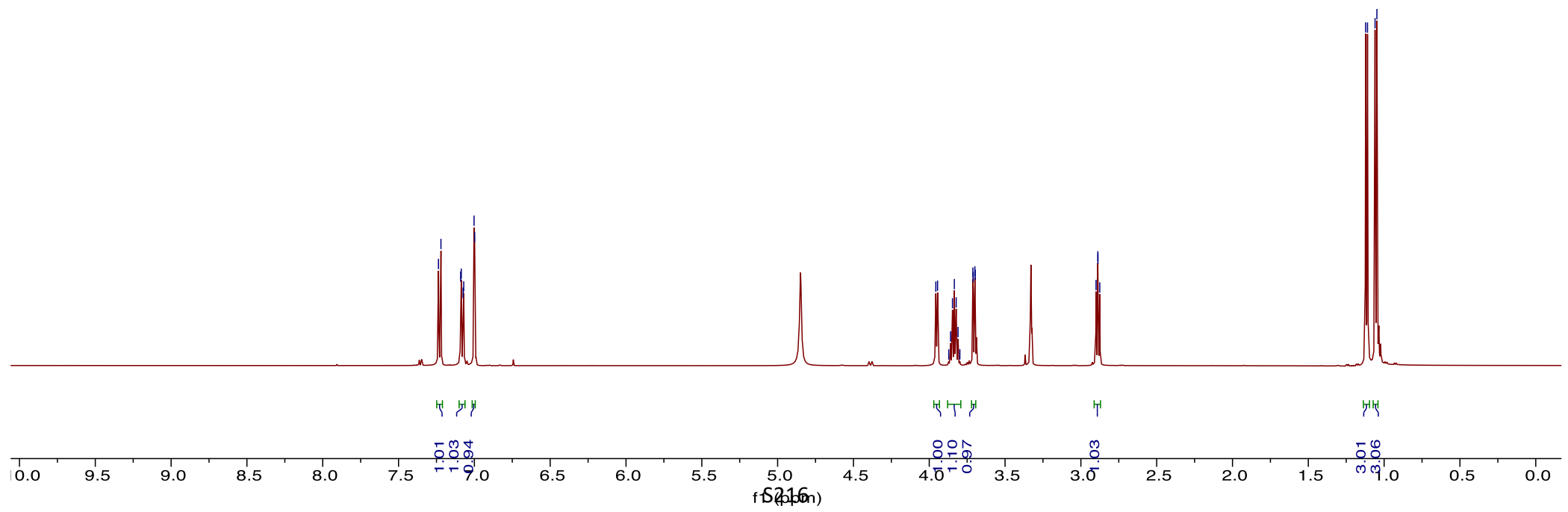


3k, ${ }^{13} \mathrm{C}$ NMR (126 MHz, $\left.\mathrm{CD}_{3} \mathrm{OD}\right)$
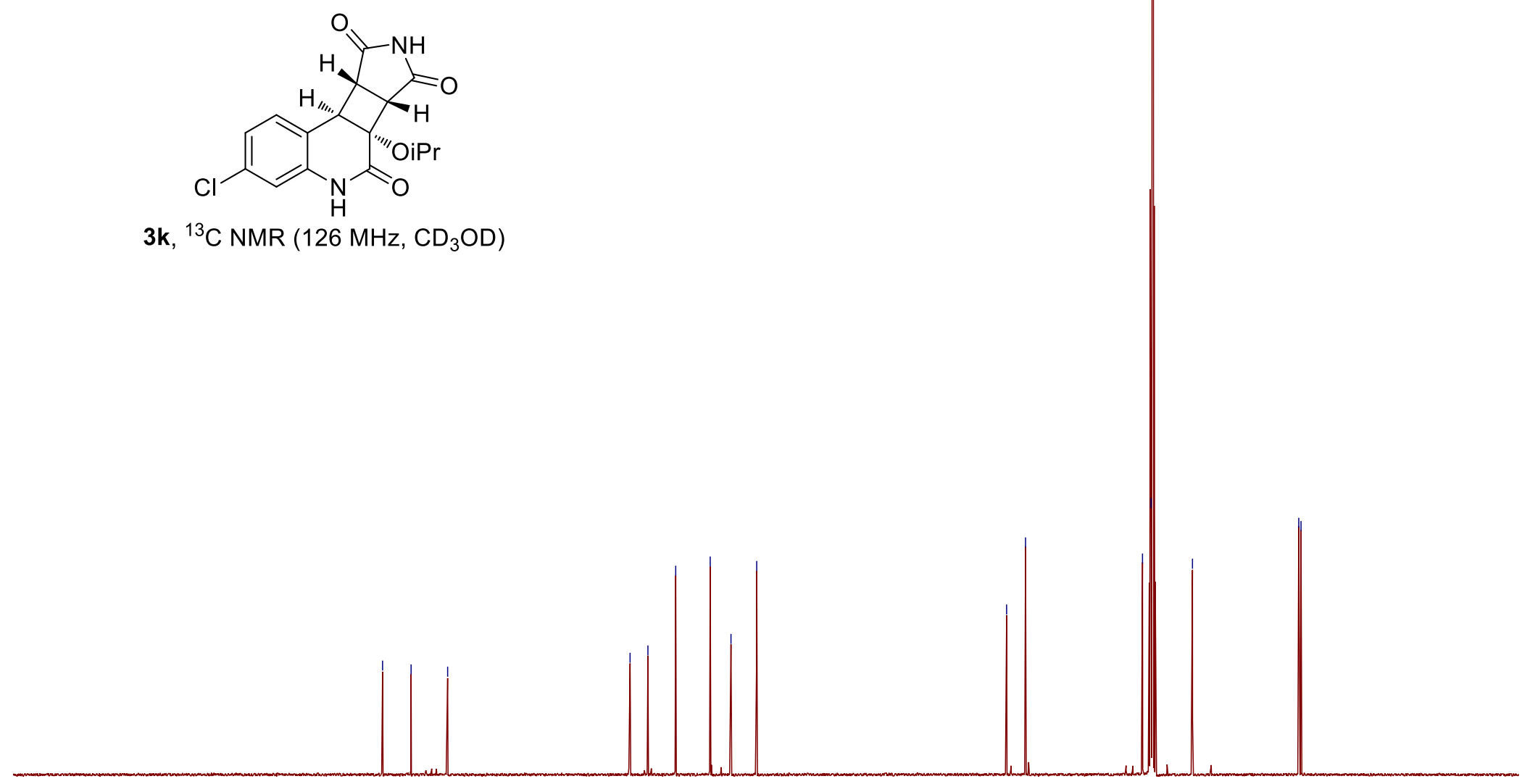

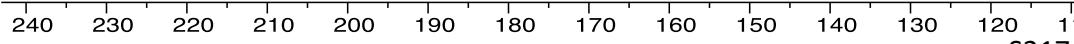




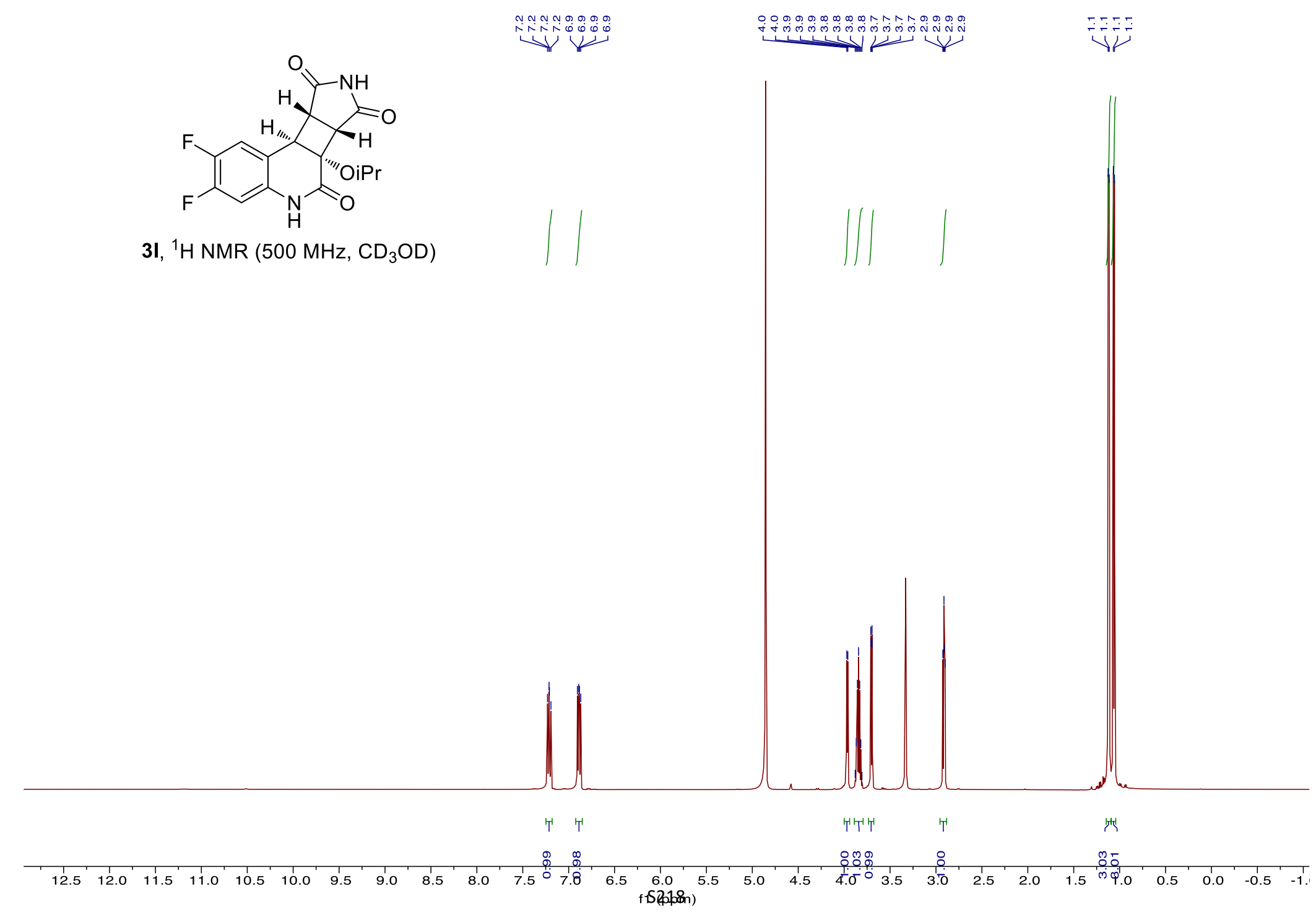




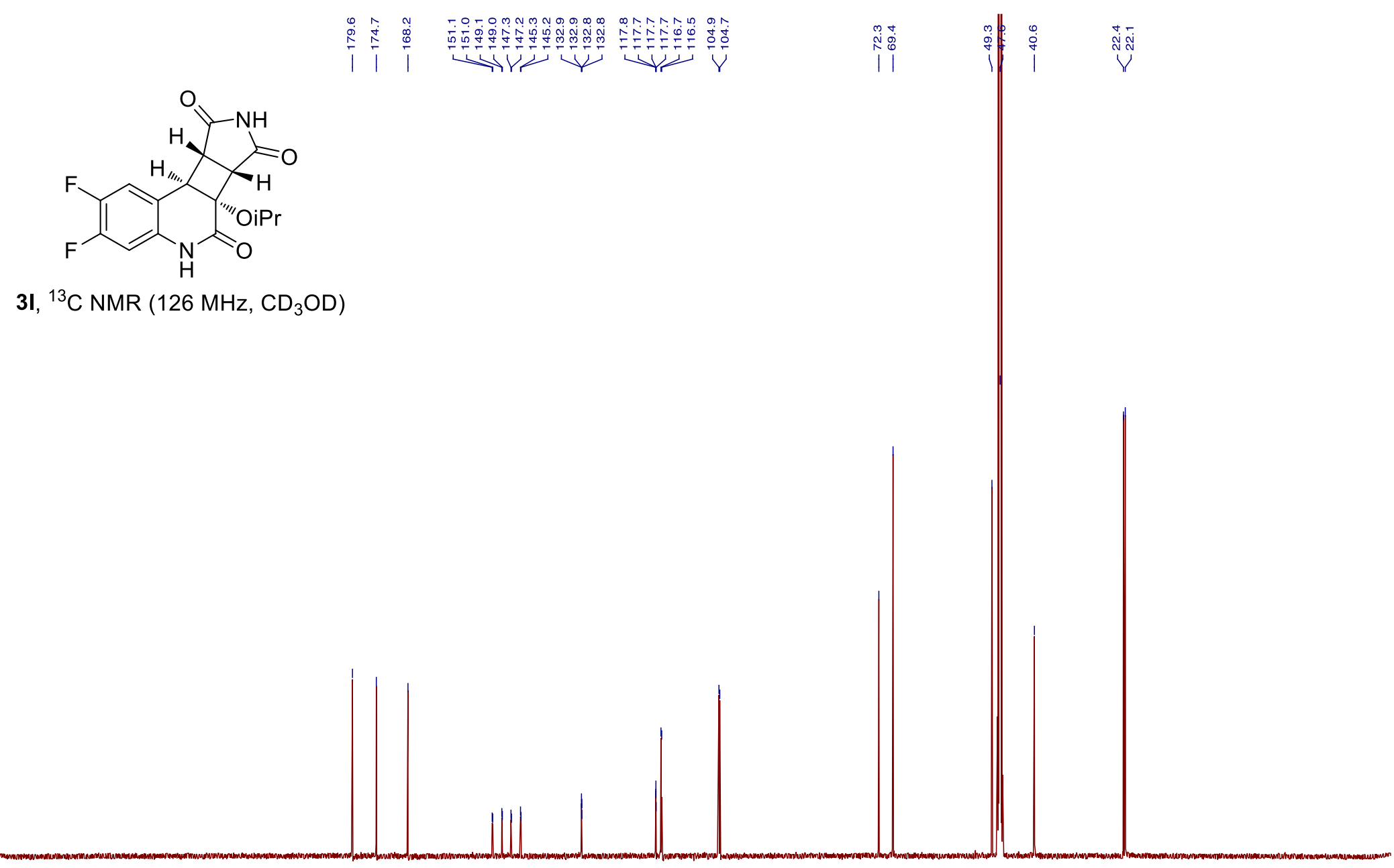

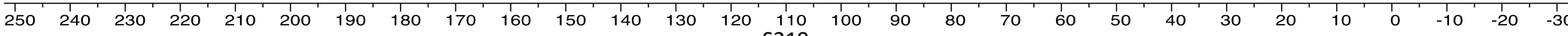
f1S20329h) 


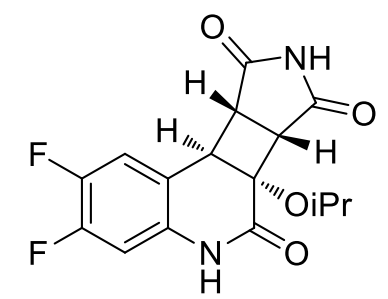

31, ${ }^{19} \mathrm{~F}$ NMR $\left(377 \mathrm{MHz}, \mathrm{CD}_{3} \mathrm{OD}\right)$

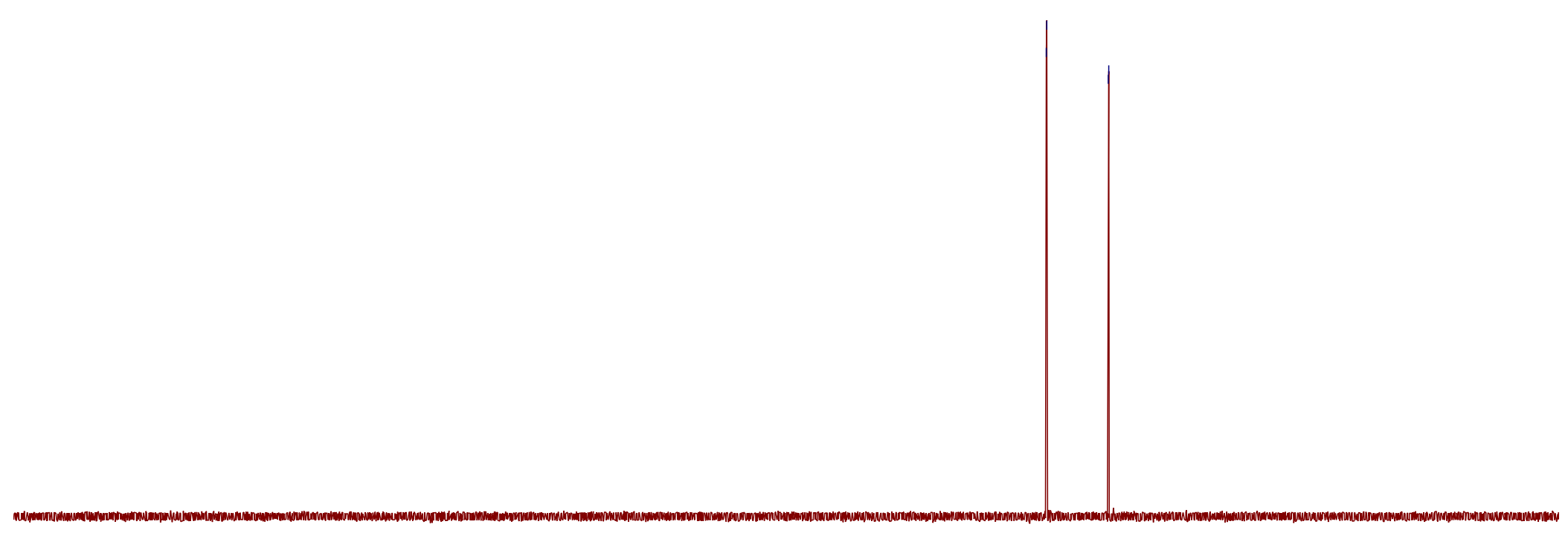

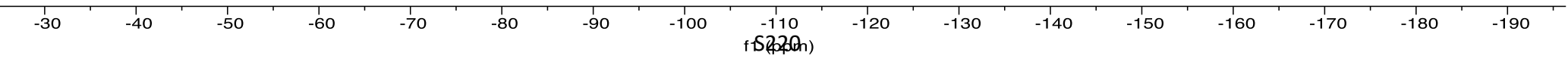




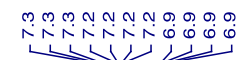

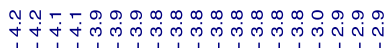

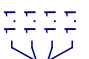

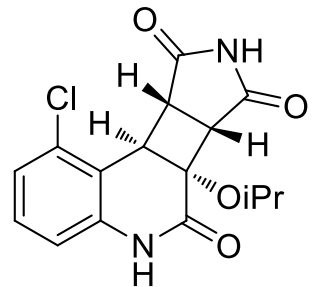

3m, ${ }^{1} \mathrm{H}$ NMR $\left(500 \mathrm{MHz}, \mathrm{CD}_{3} \mathrm{OD}\right)$
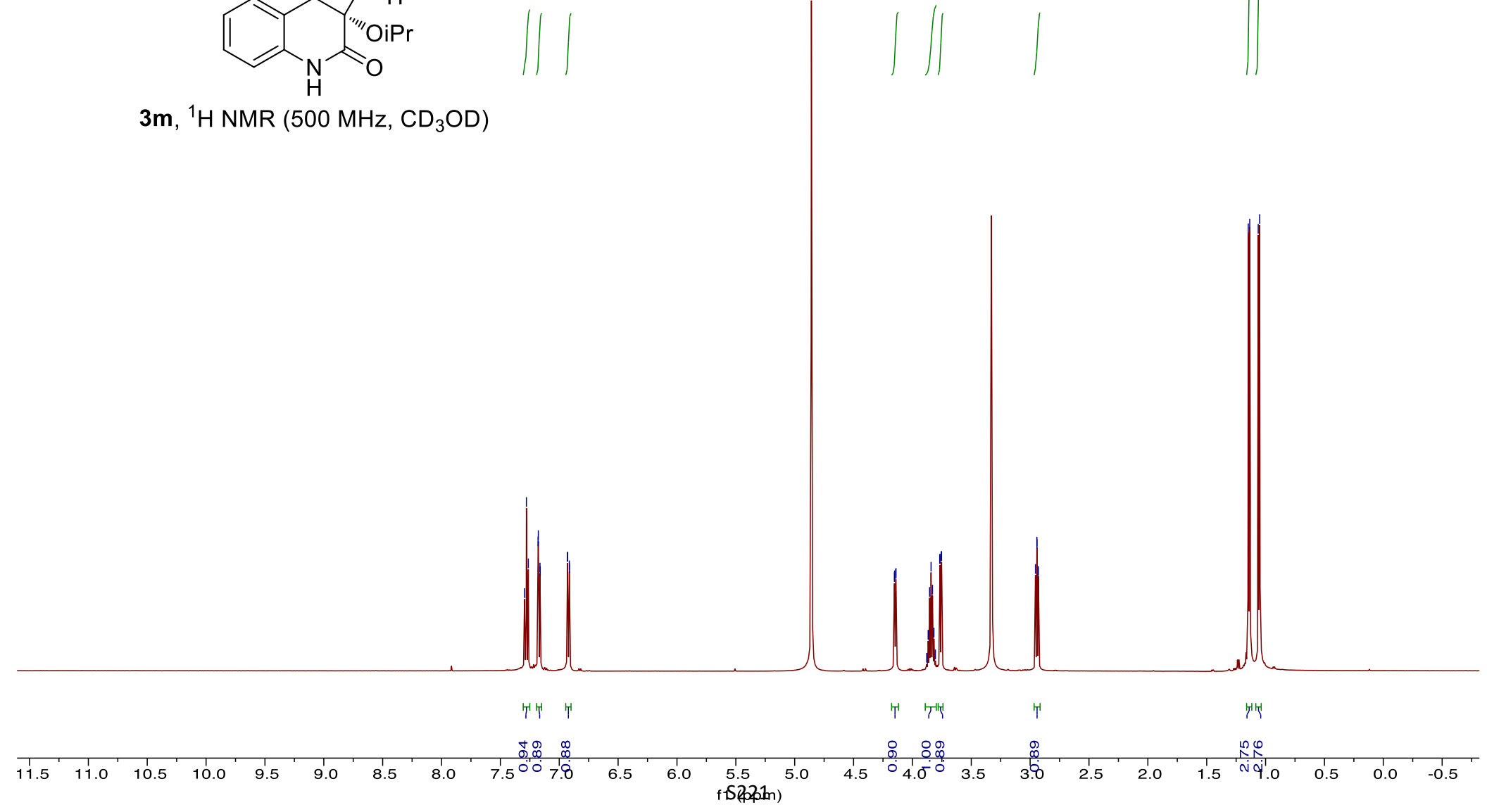


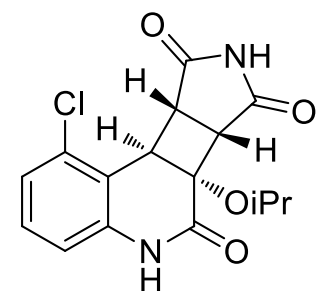

$3 \mathrm{~m},{ }^{13} \mathrm{C}$ NMR (126 MHz, $\left.\mathrm{CD}_{3} \mathrm{OD}\right)$

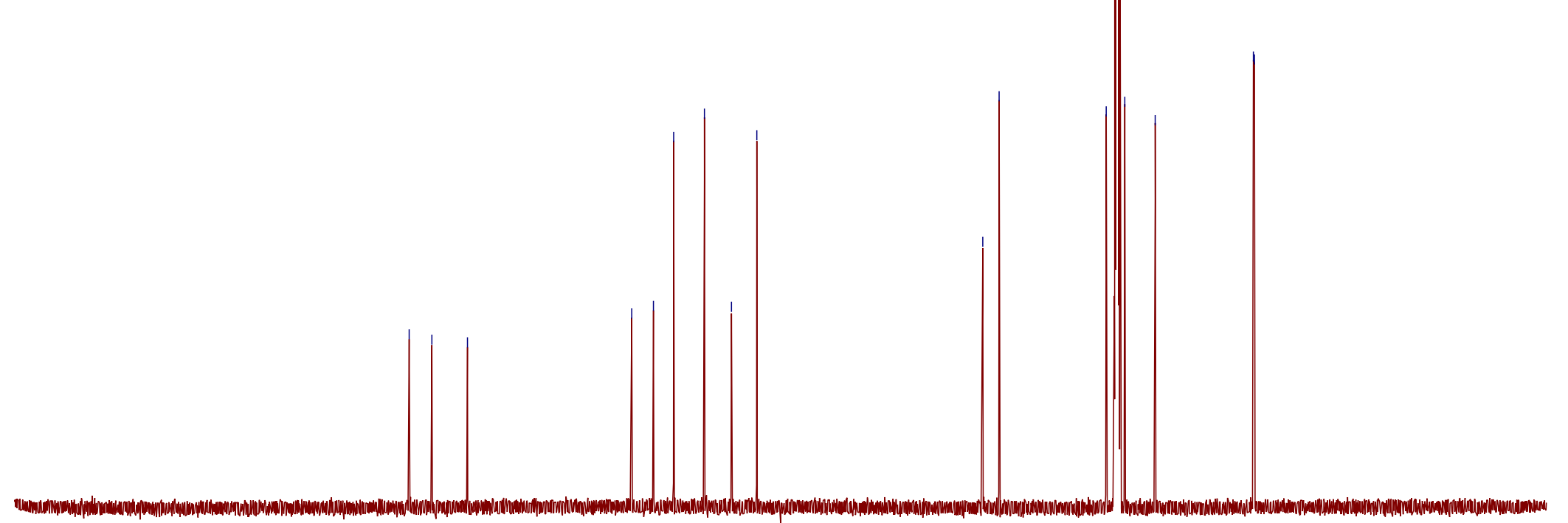

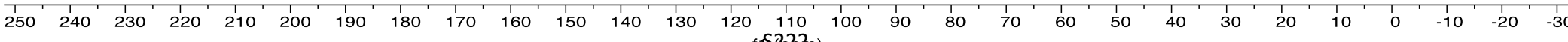
fiS $S(2222 \pi)$ 


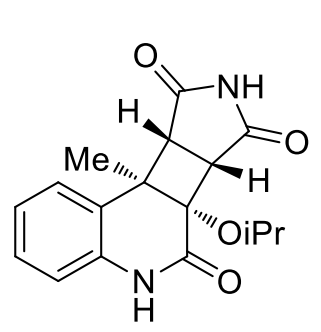

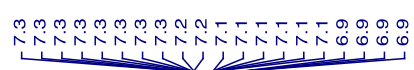

3n, ${ }^{1} \mathrm{H}$ NMR $\left(500 \mathrm{MHz}, \mathrm{CD}_{3} \mathrm{OD}\right)$

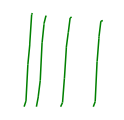

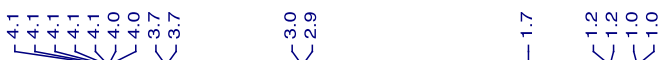
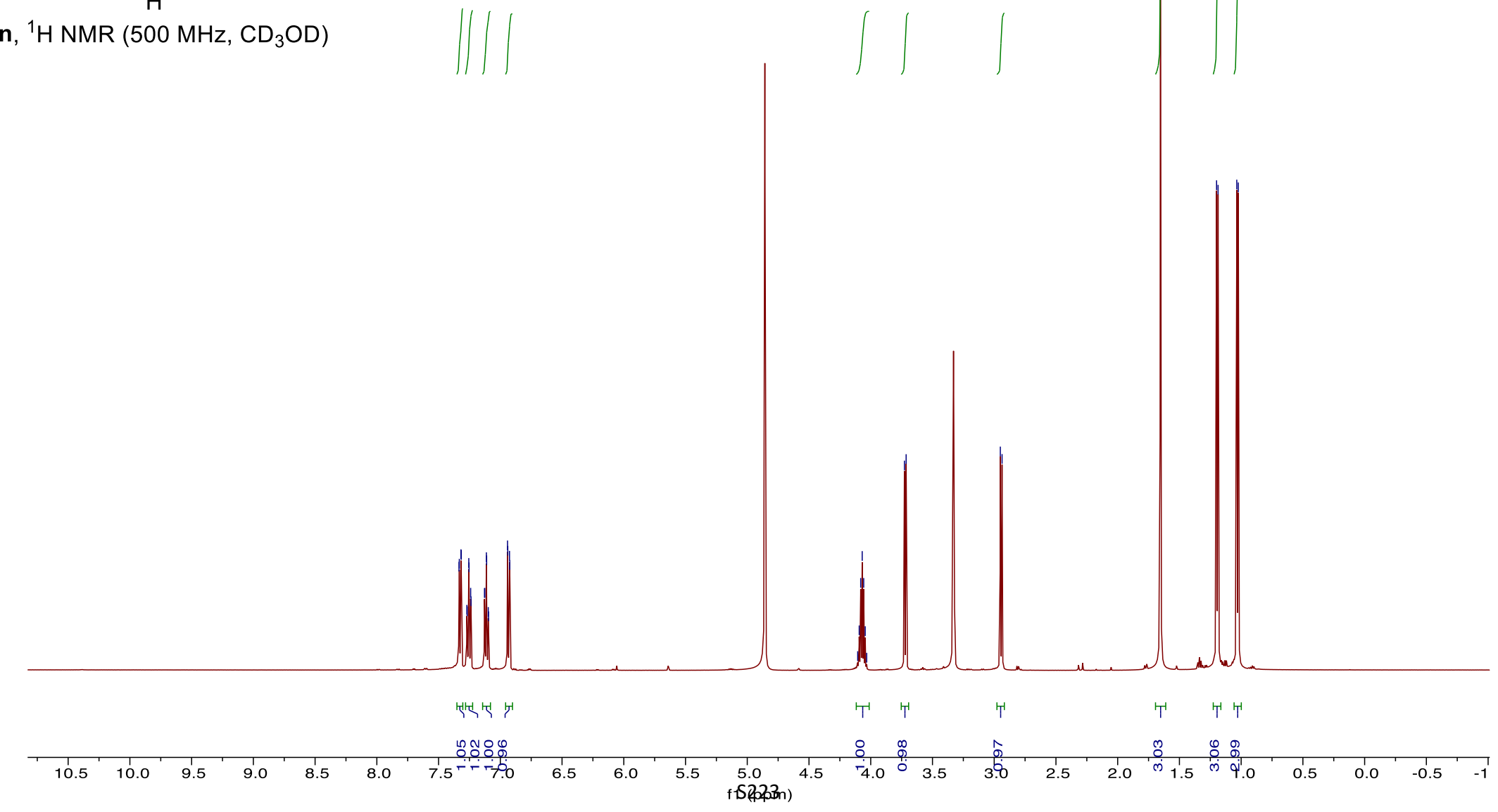

Y V 


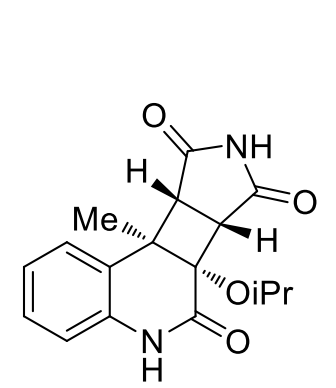

3n, ${ }^{13} \mathrm{C}$ NMR $\left(126 \mathrm{MHz}, \mathrm{CD}_{3} \mathrm{OD}\right)$
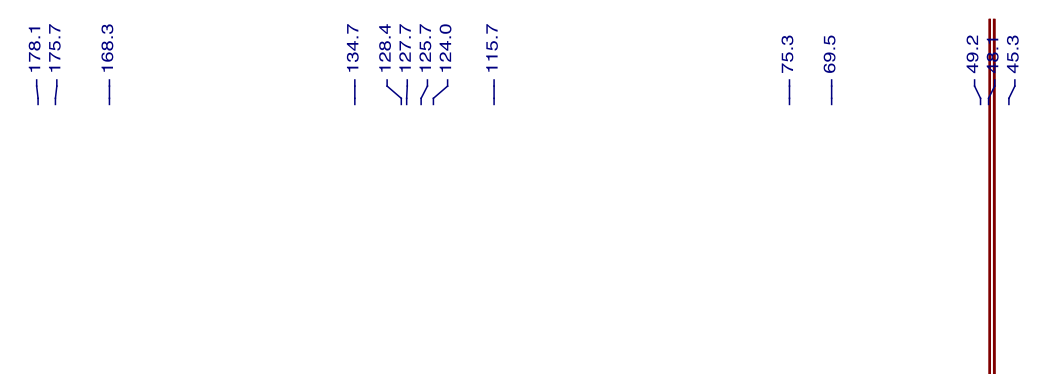


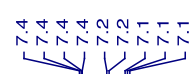

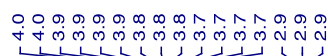

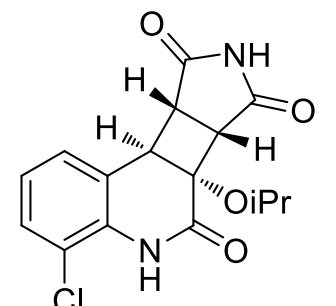

3o, ${ }^{1} \mathrm{H}$ NMR (500 MHz, $\left.\mathrm{CD}_{3} \mathrm{OD}\right)$
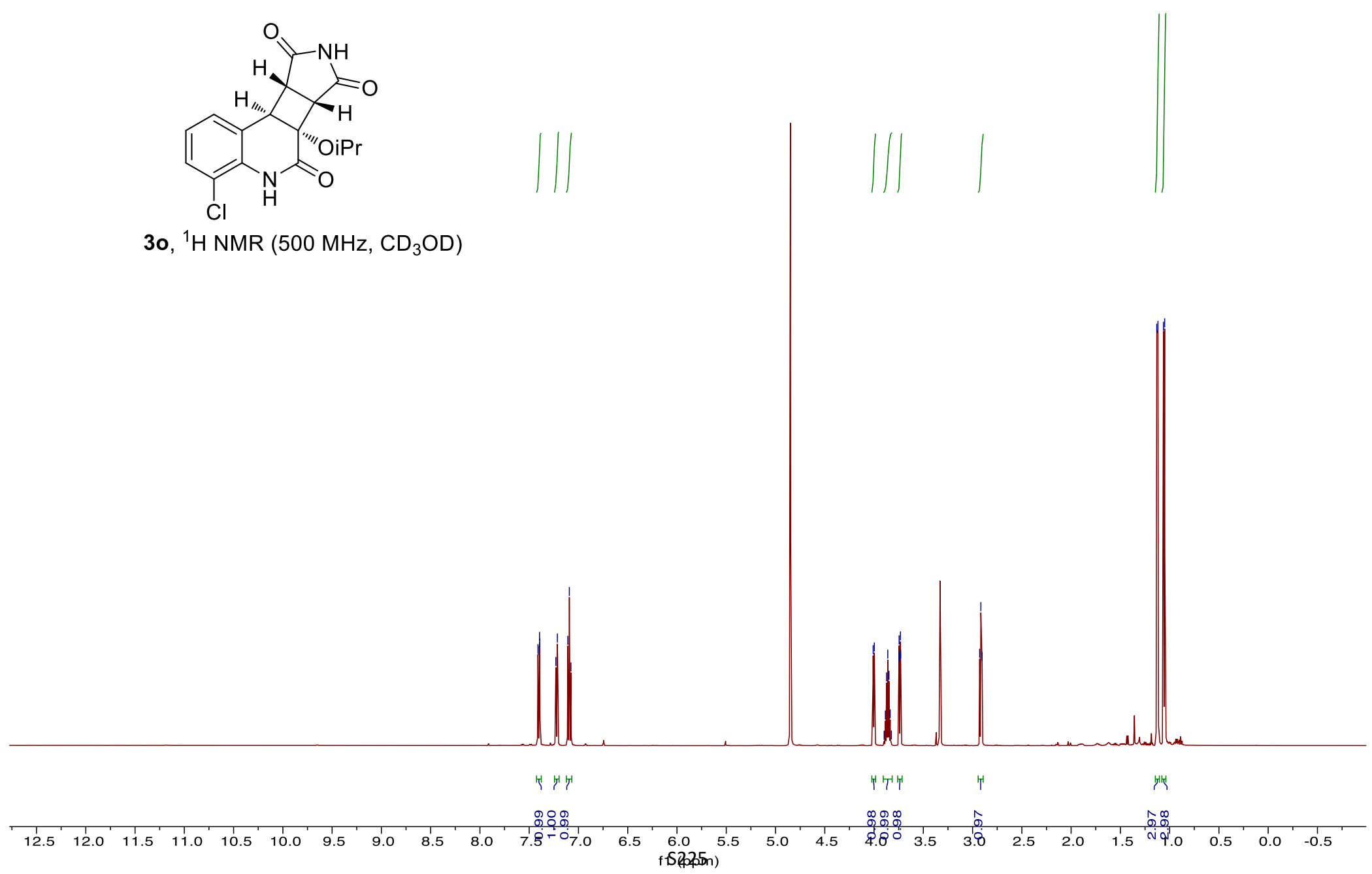


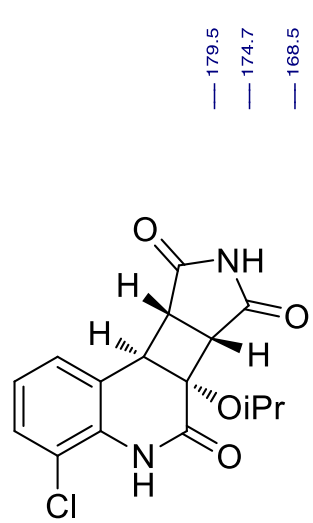

3o, ${ }^{13} \mathrm{C}$ NMR (126 MHz, $\left.\mathrm{CD}_{3} \mathrm{OD}\right)$

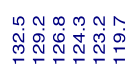

$111 \%$
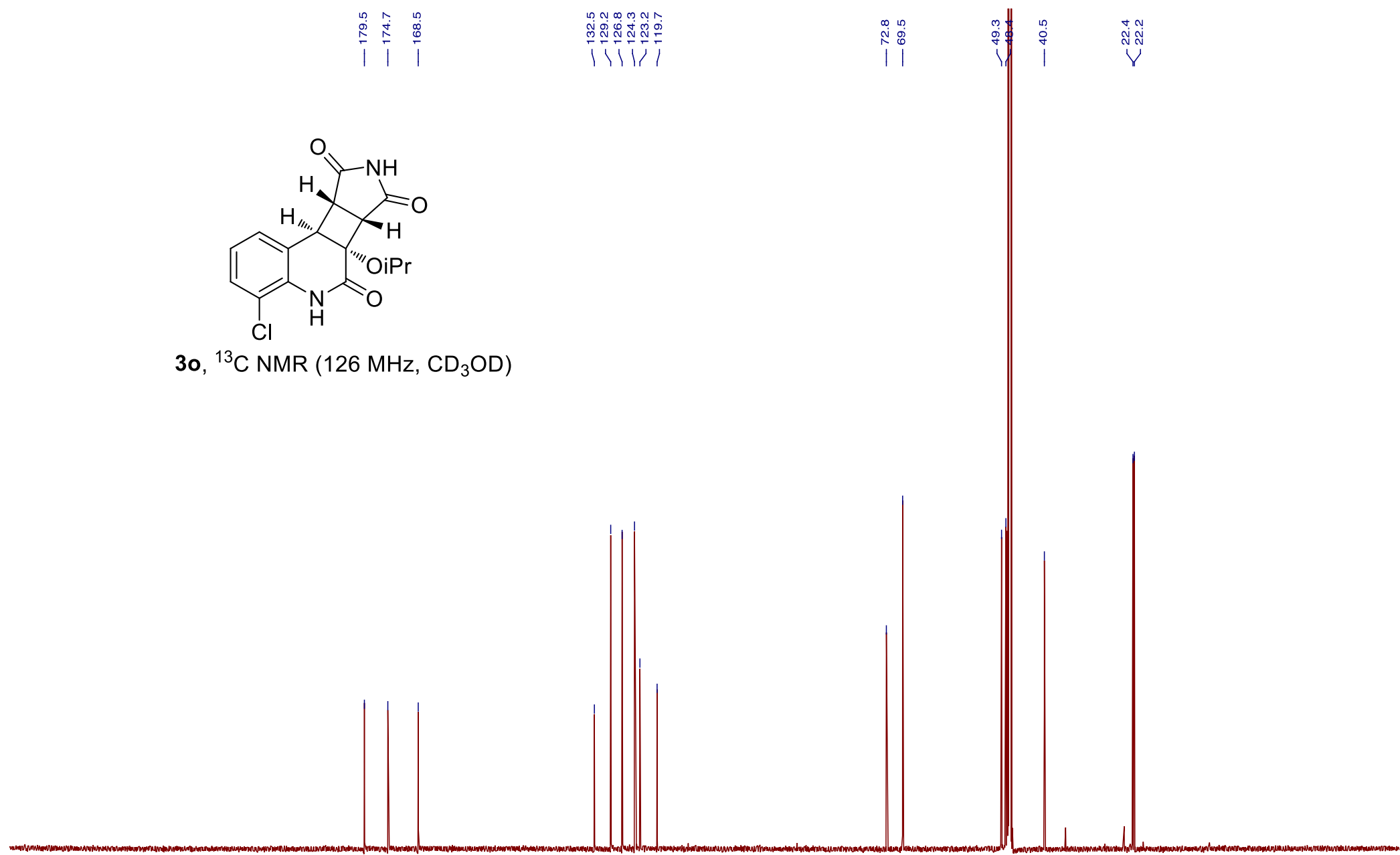

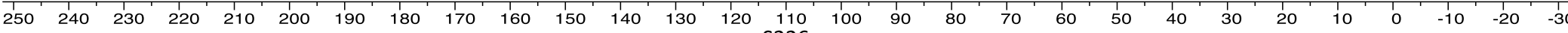
fis 202360 ) 


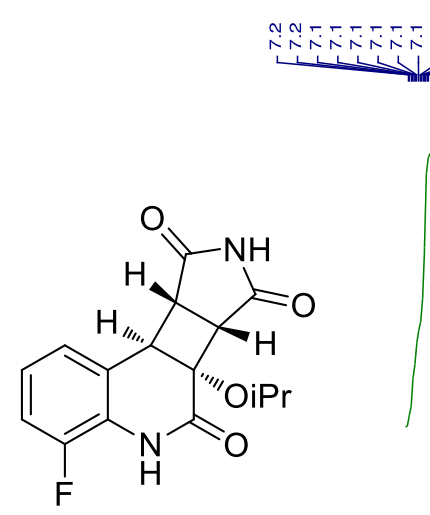

3p, ${ }^{1} \mathrm{H}$ NMR $\left(500 \mathrm{MHz}, \mathrm{CD}_{3} \mathrm{OD}\right)$

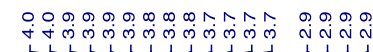

茫
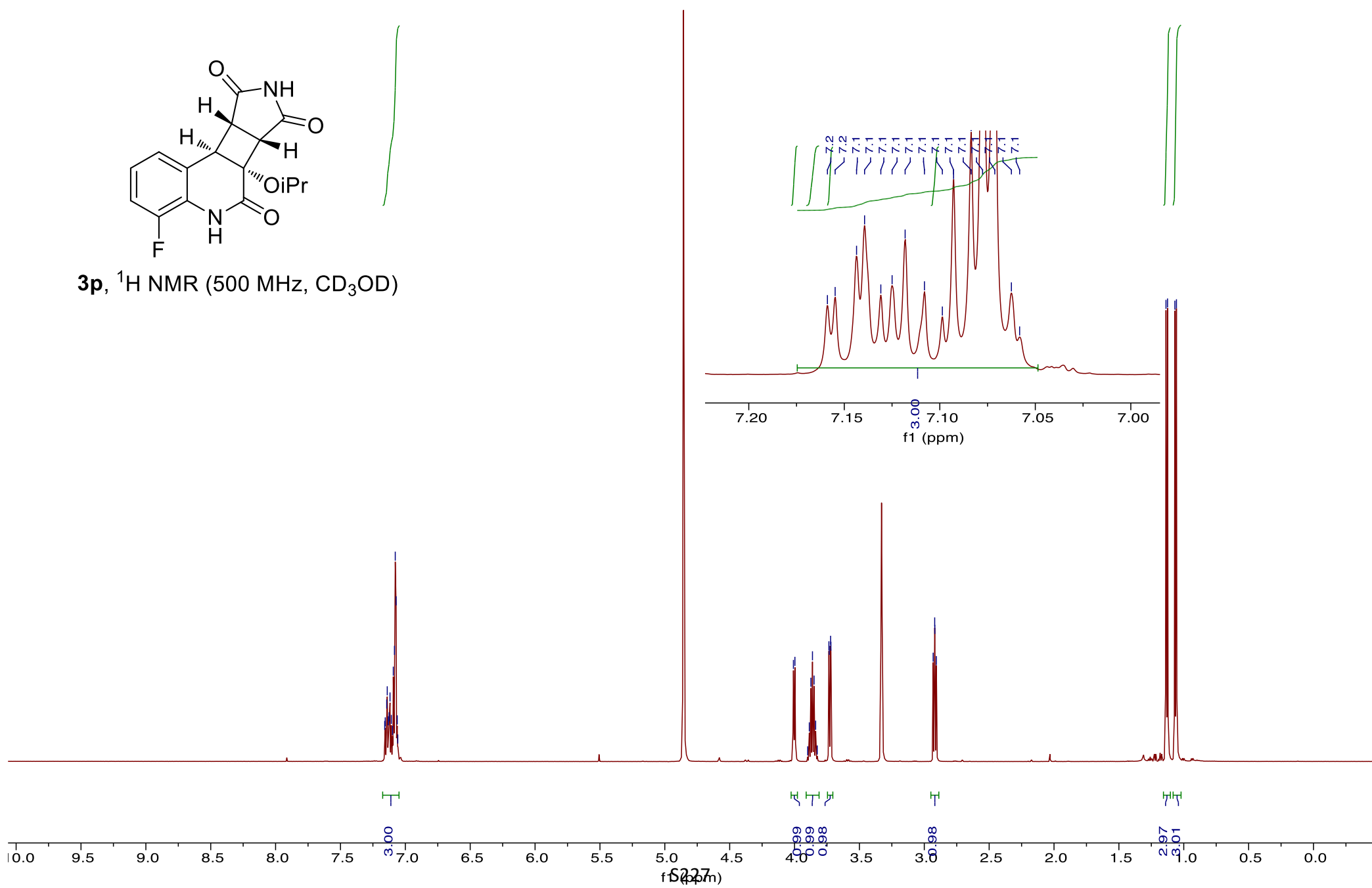

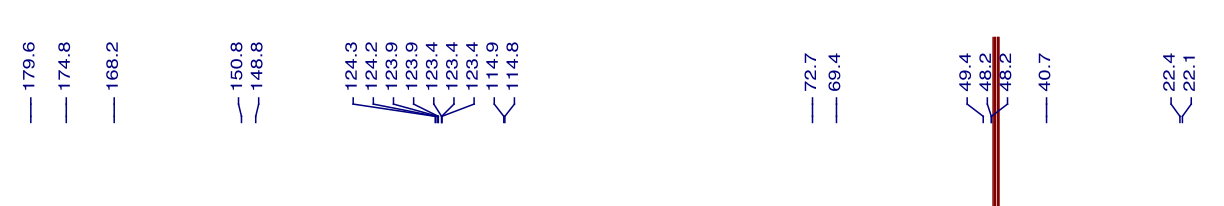

$3 p,{ }^{13} \mathrm{C}$ NMR $\left(126 \mathrm{MHz}, \mathrm{CD}_{3} \mathrm{OD}\right)$
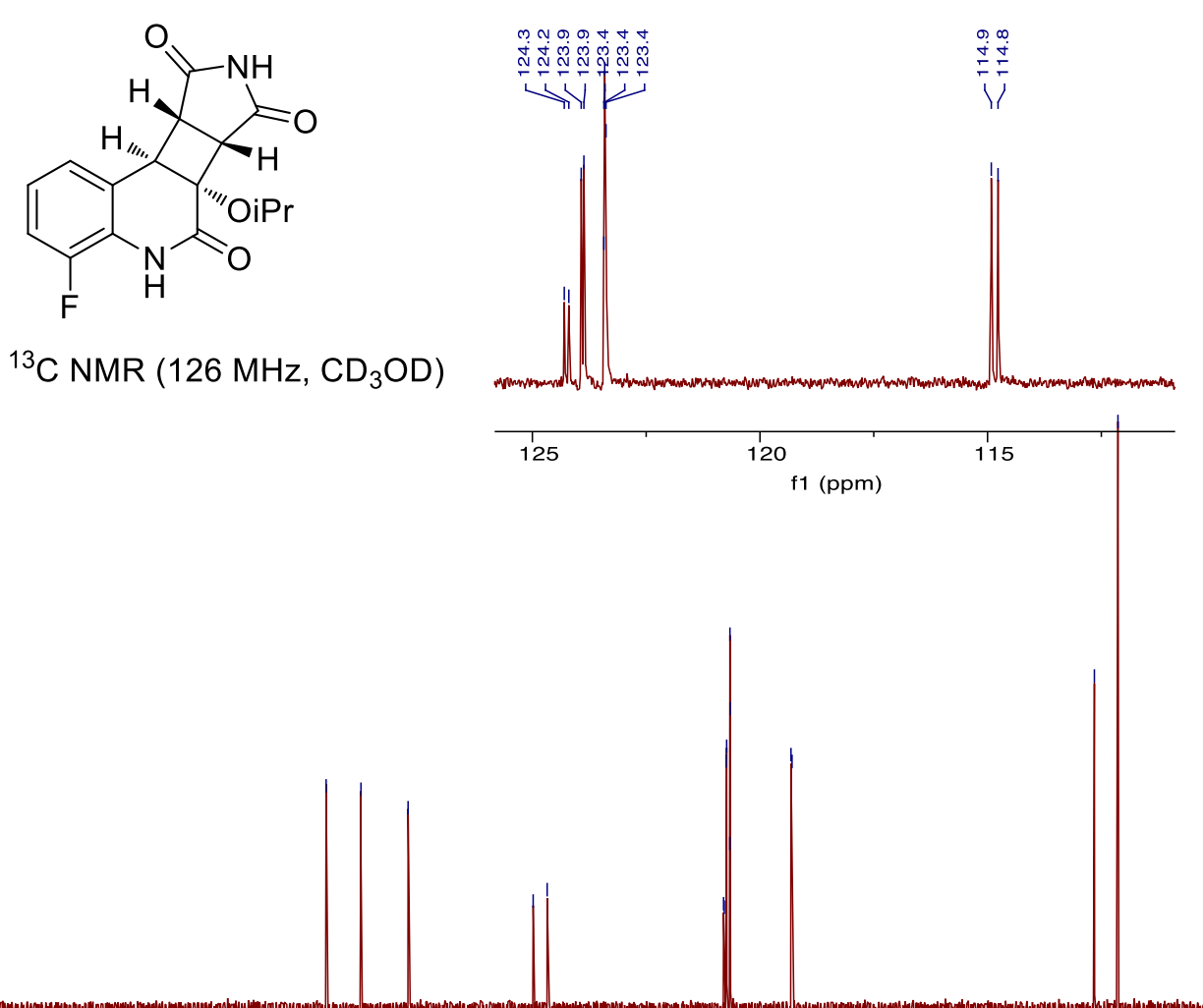

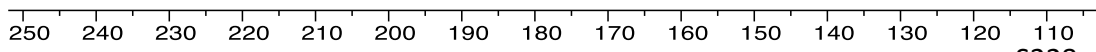




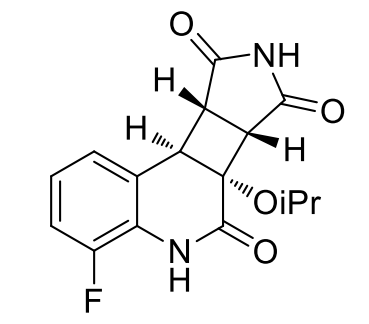

3p, ${ }^{19} \mathrm{~F}$ NMR $\left(377 \mathrm{MHz}, \mathrm{CD}_{3} \mathrm{OD}\right)$ 


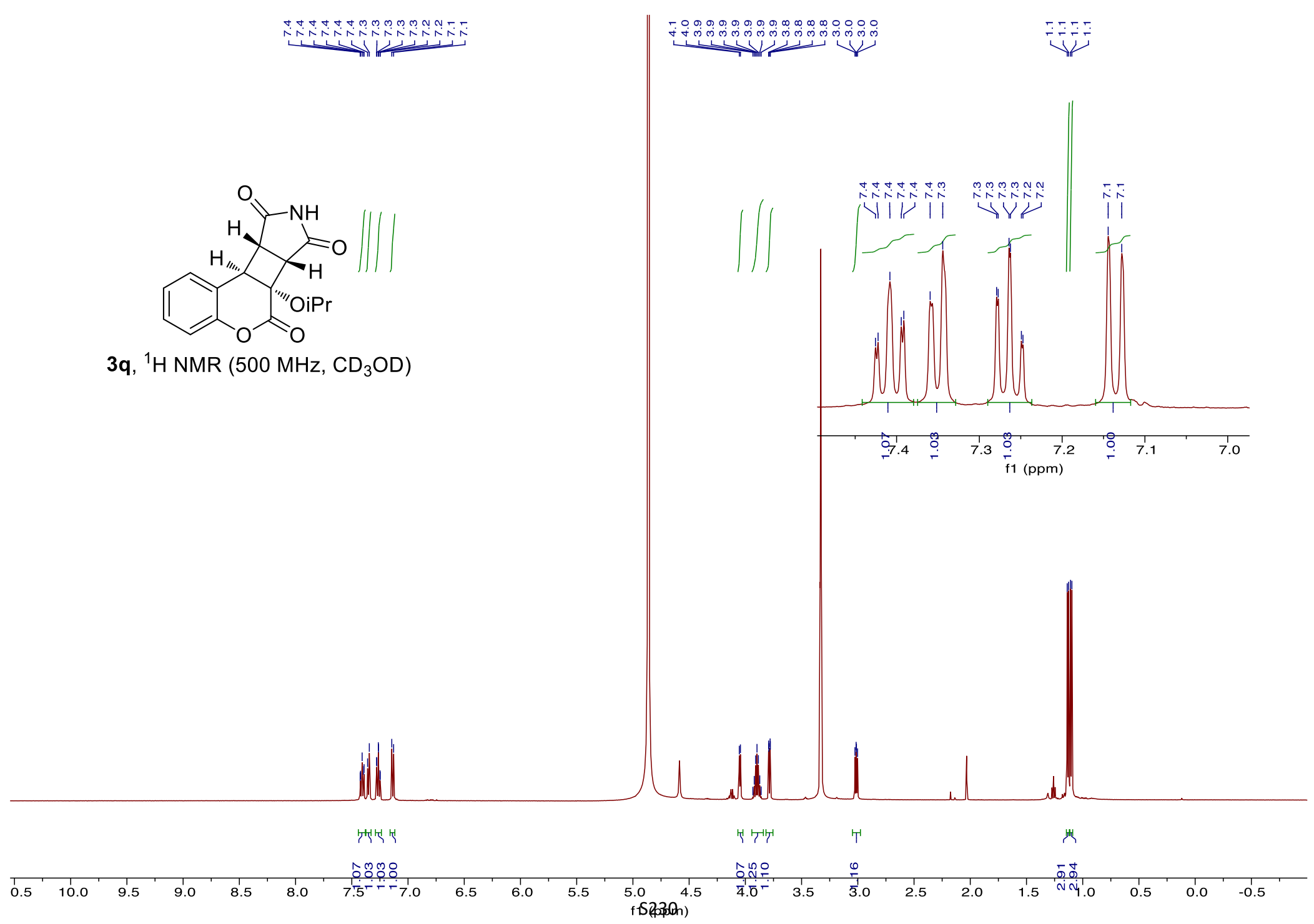




$$
{ }_{\mathrm{O}}
$$

$\left.3 q,{ }^{13} \mathrm{C} \mathrm{NMR} \mathrm{(126} \mathrm{MHz,} \mathrm{CD}_{3} \mathrm{OD}\right)$
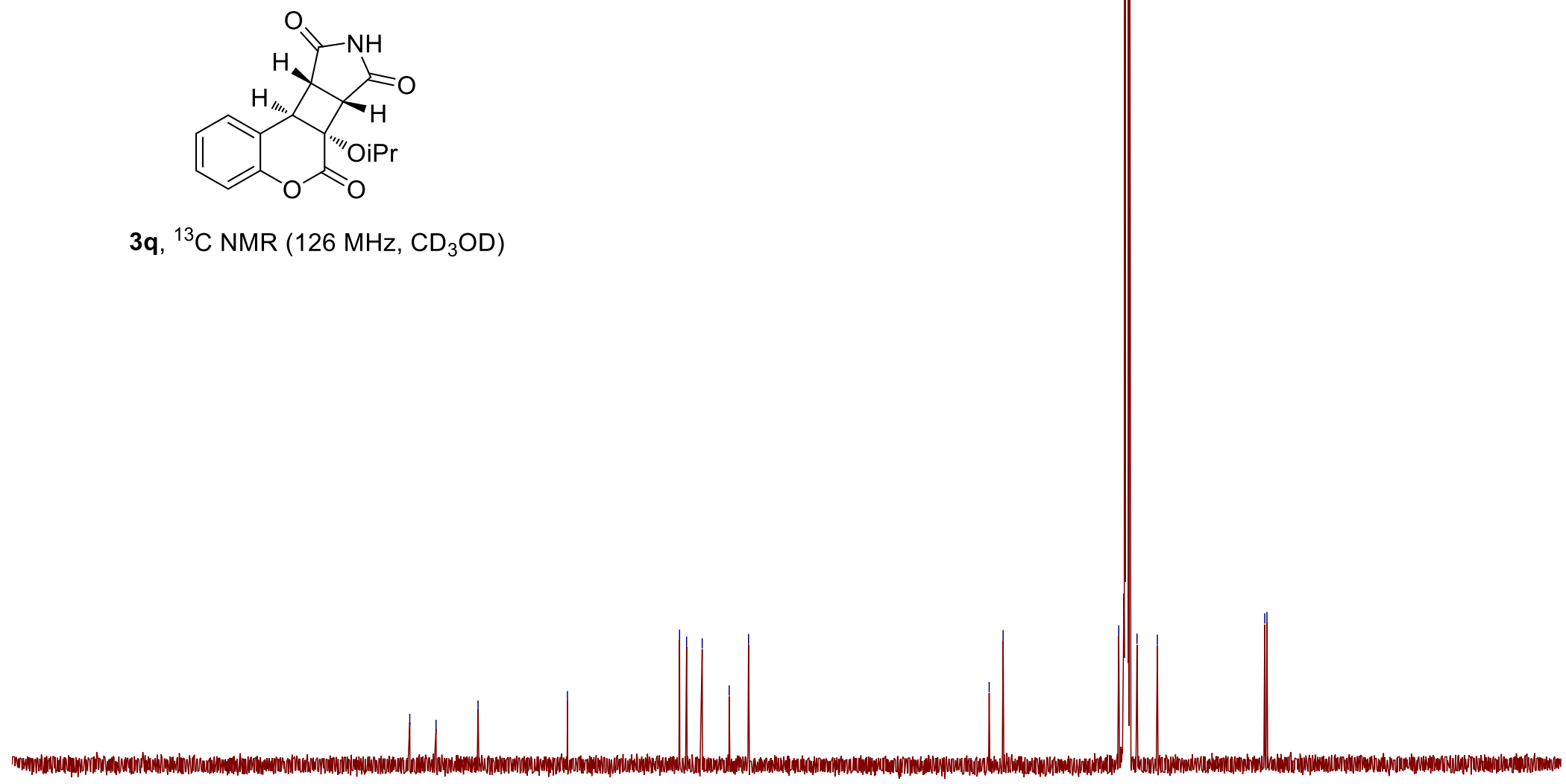

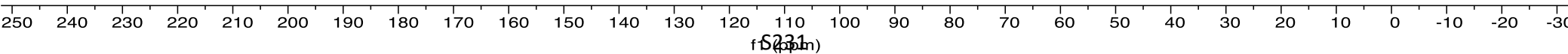



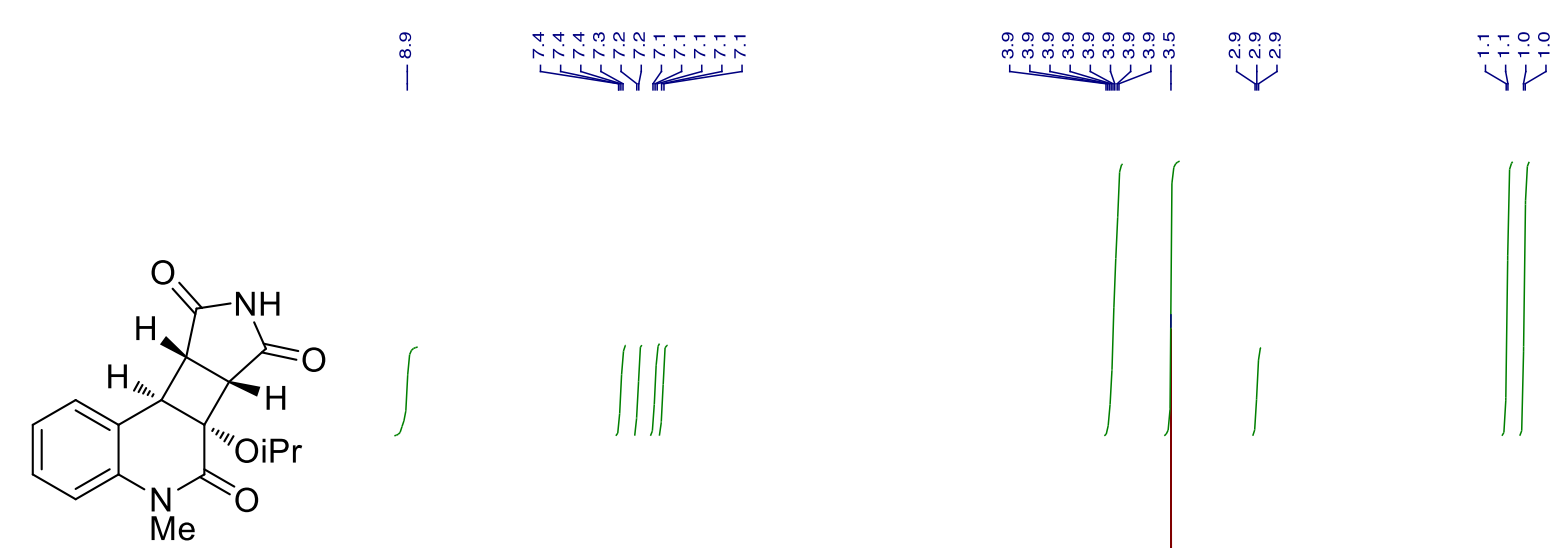

3r, ${ }^{1} \mathrm{H}$ NMR $\left(500 \mathrm{MHz}, \mathrm{CDCl}_{3}\right)$
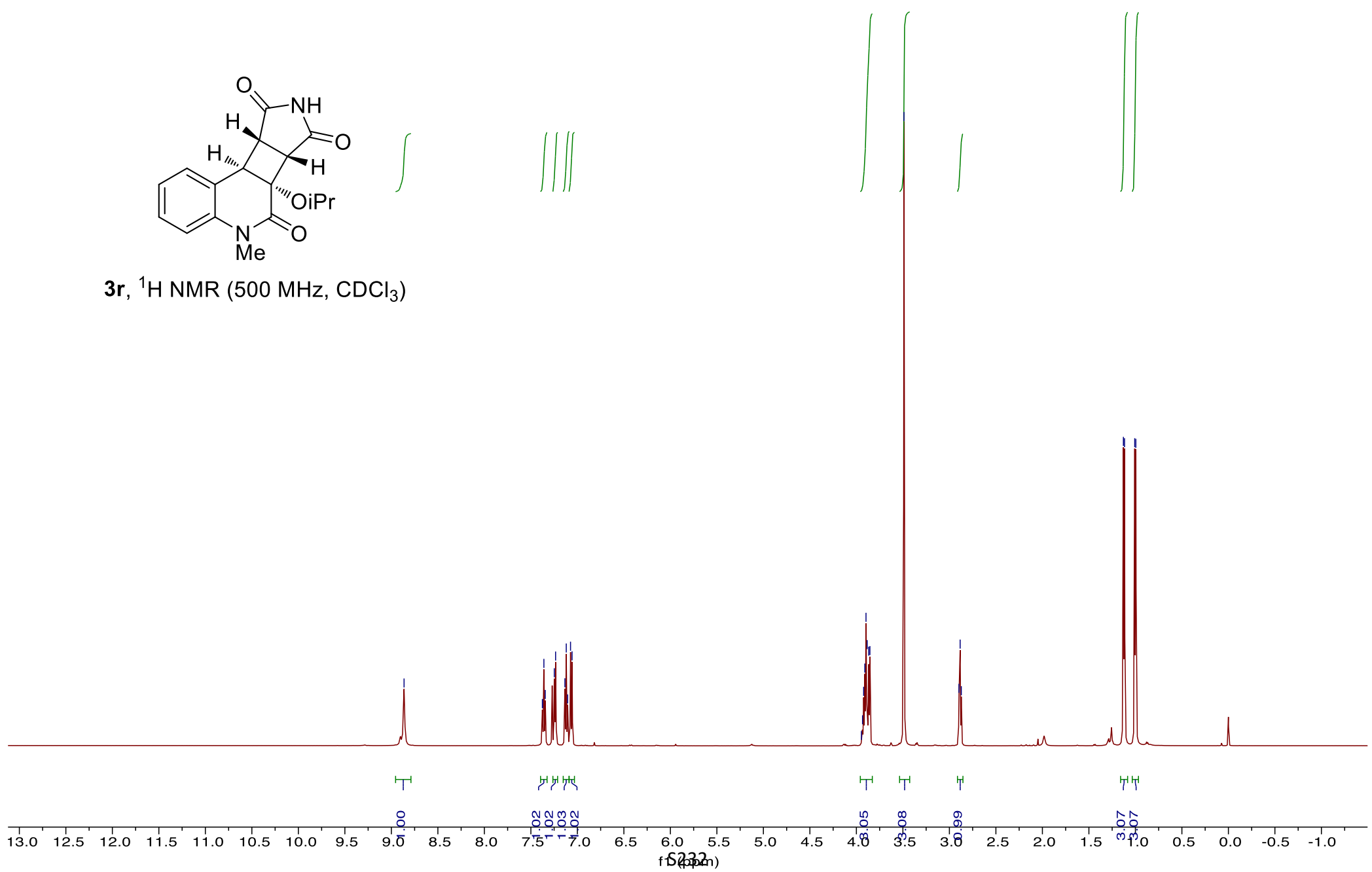


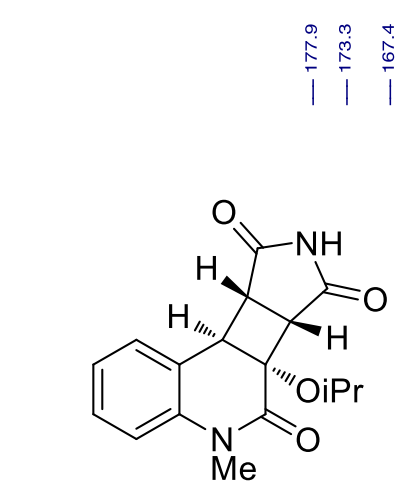

$3 r,{ }^{13} \mathrm{C}$ NMR $\left(126 \mathrm{MHz}, \mathrm{CDCl}_{3}\right)$

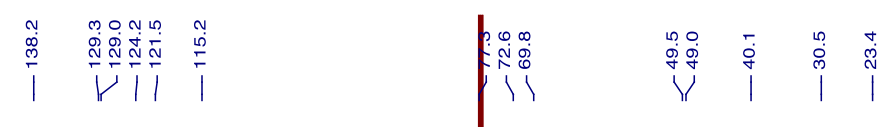

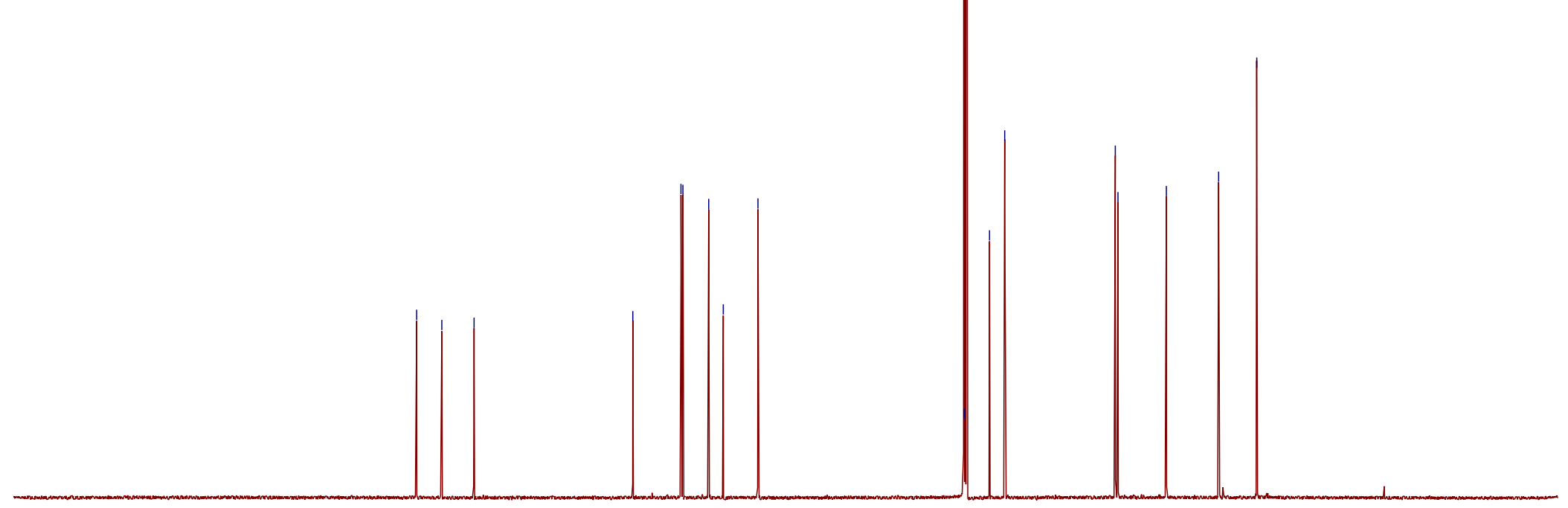

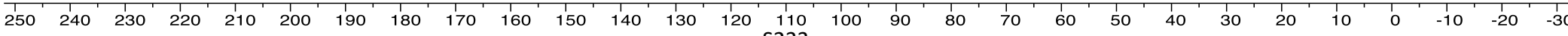

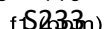




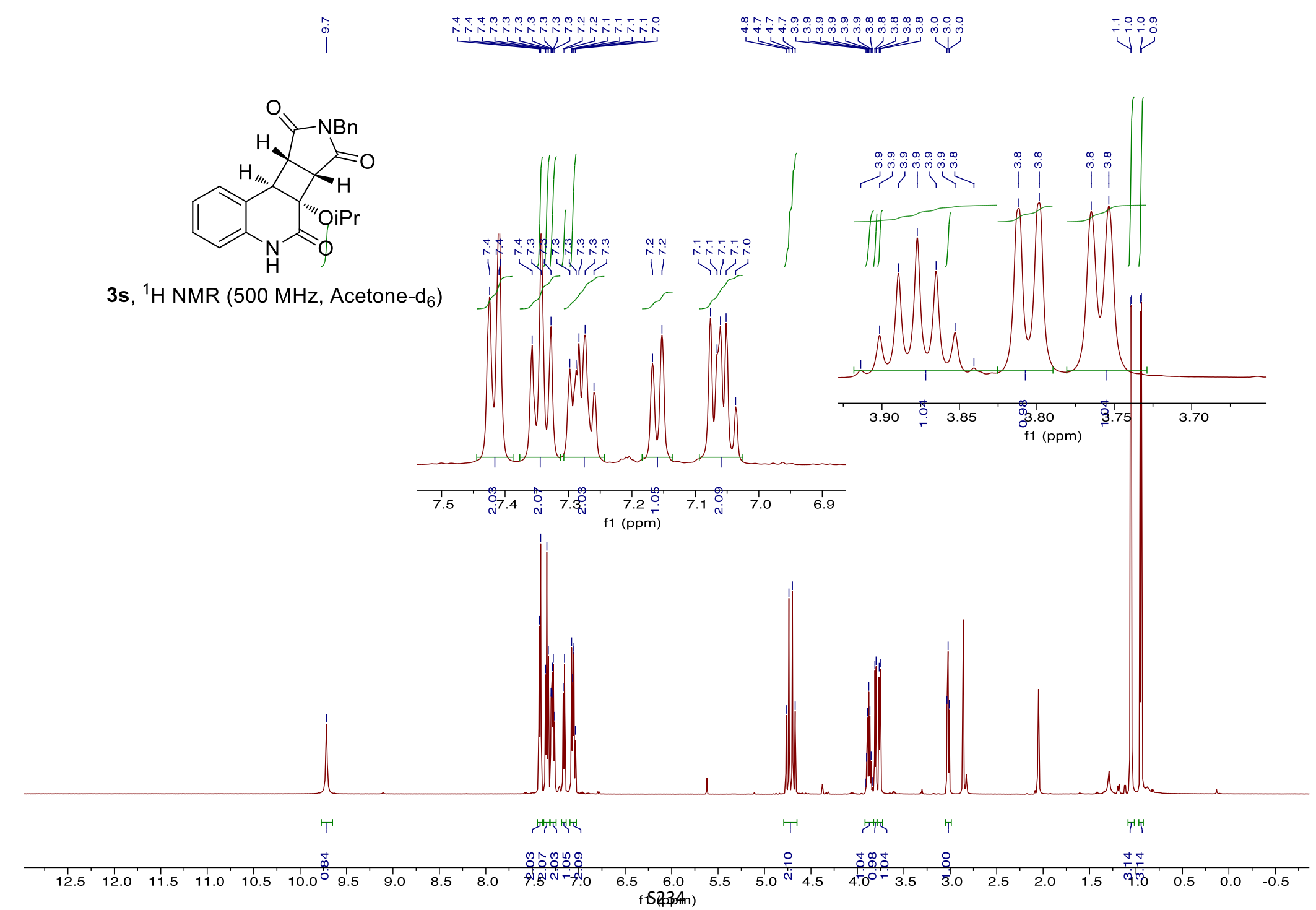




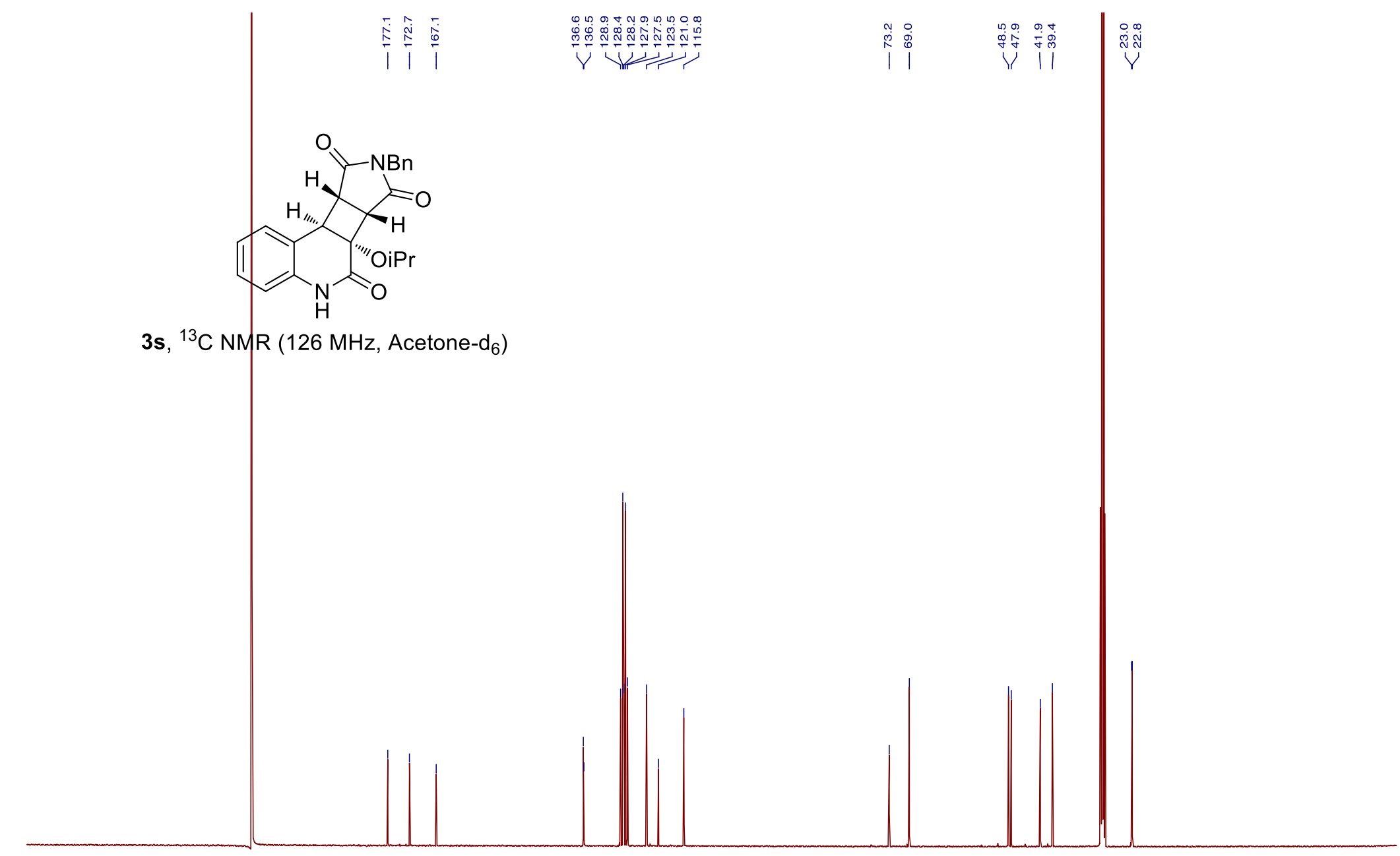

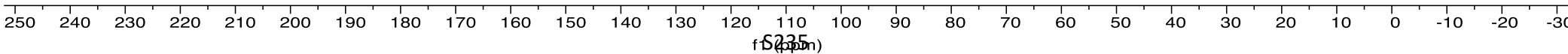




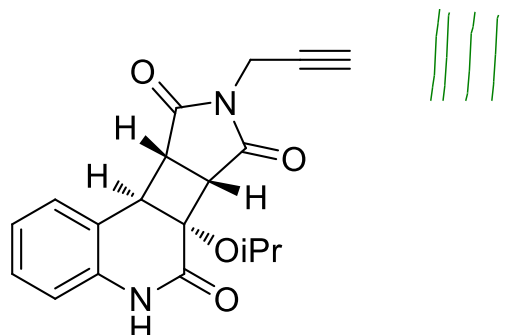

$\infty_{\infty}^{\infty} \sum_{\infty}^{\infty} \infty_{\infty}^{\infty} \infty \infty_{\infty}^{\infty}$

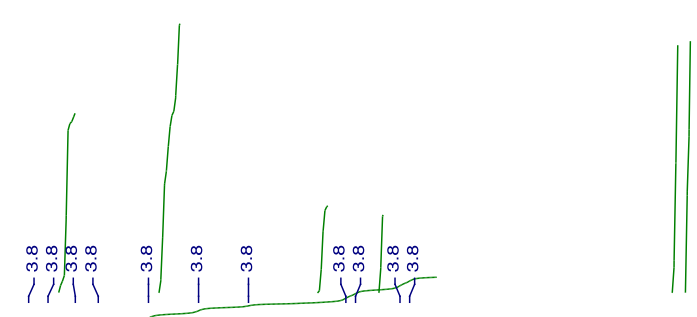

3t, ${ }^{1} \mathrm{H}$ NMR $\left(500 \mathrm{MHz}, \mathrm{CD}_{3} \mathrm{OD}\right)$
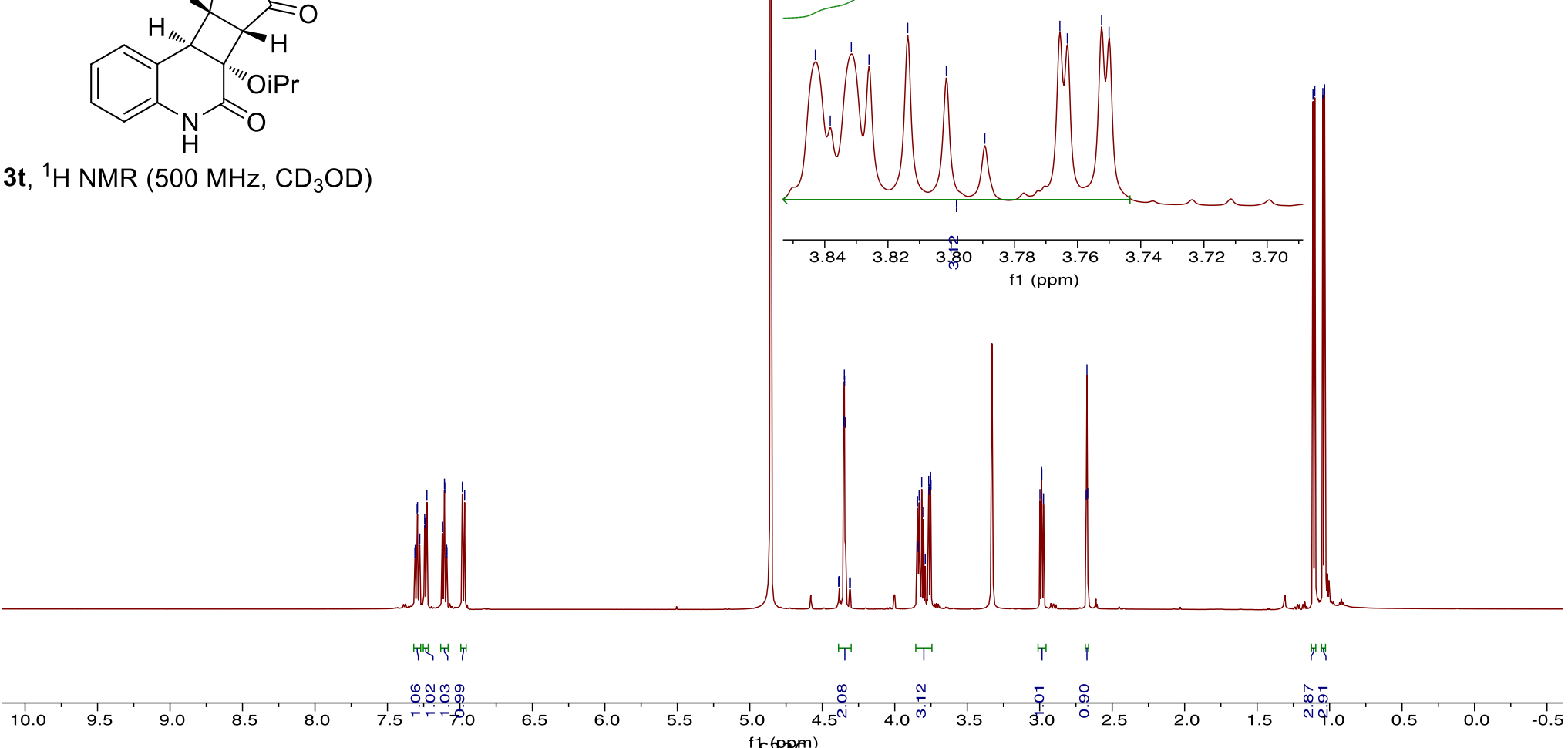


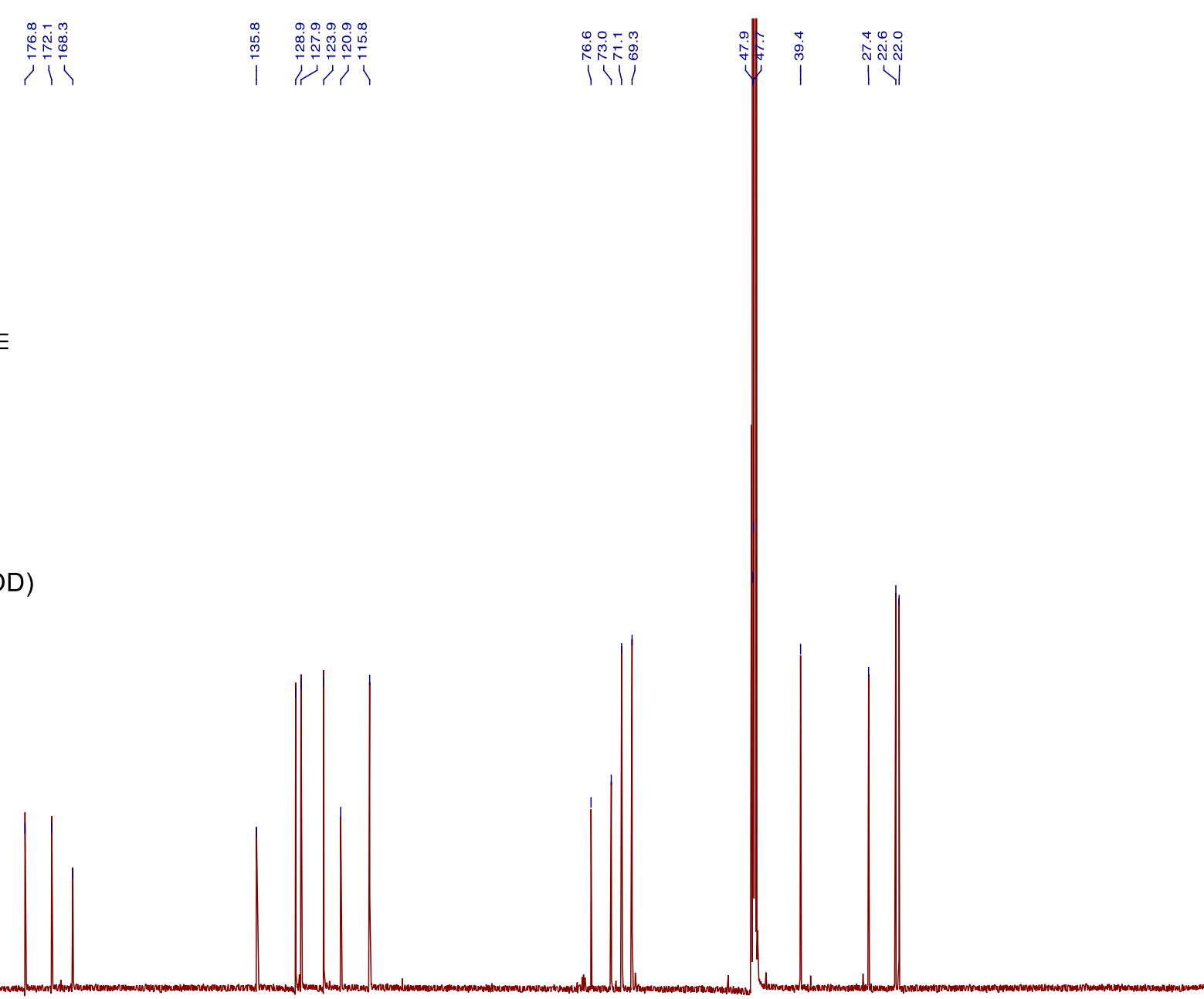

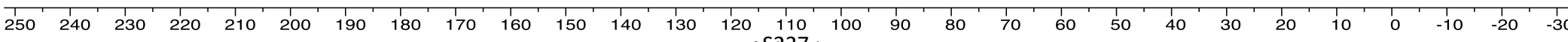
fistaptn) 

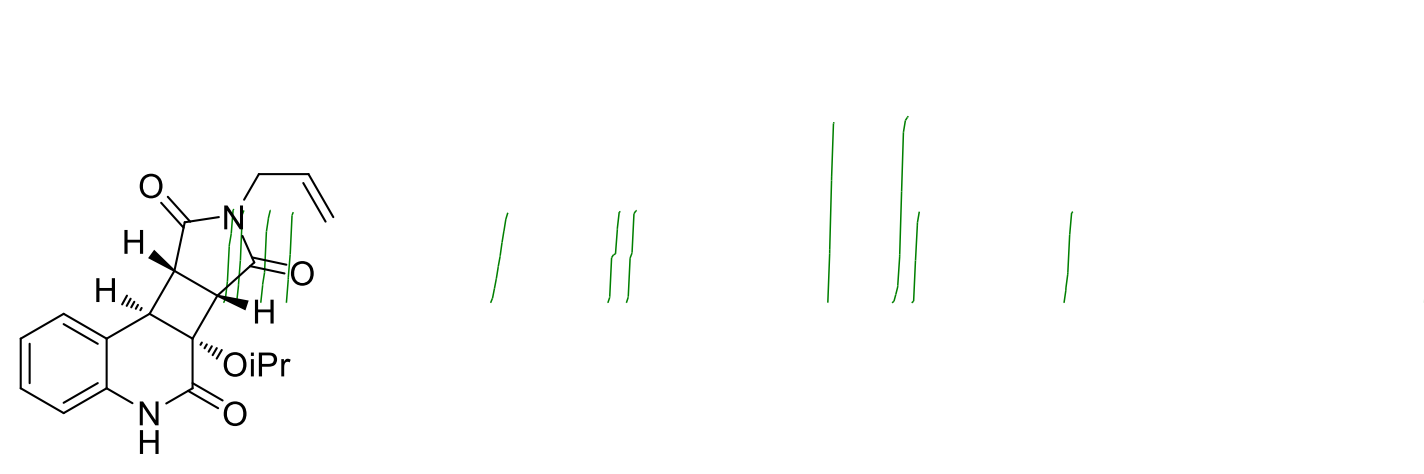

3u, ${ }^{1} \mathrm{H}$ NMR $\left(500 \mathrm{MHz}, \mathrm{CD}_{3} \mathrm{OD}\right)$

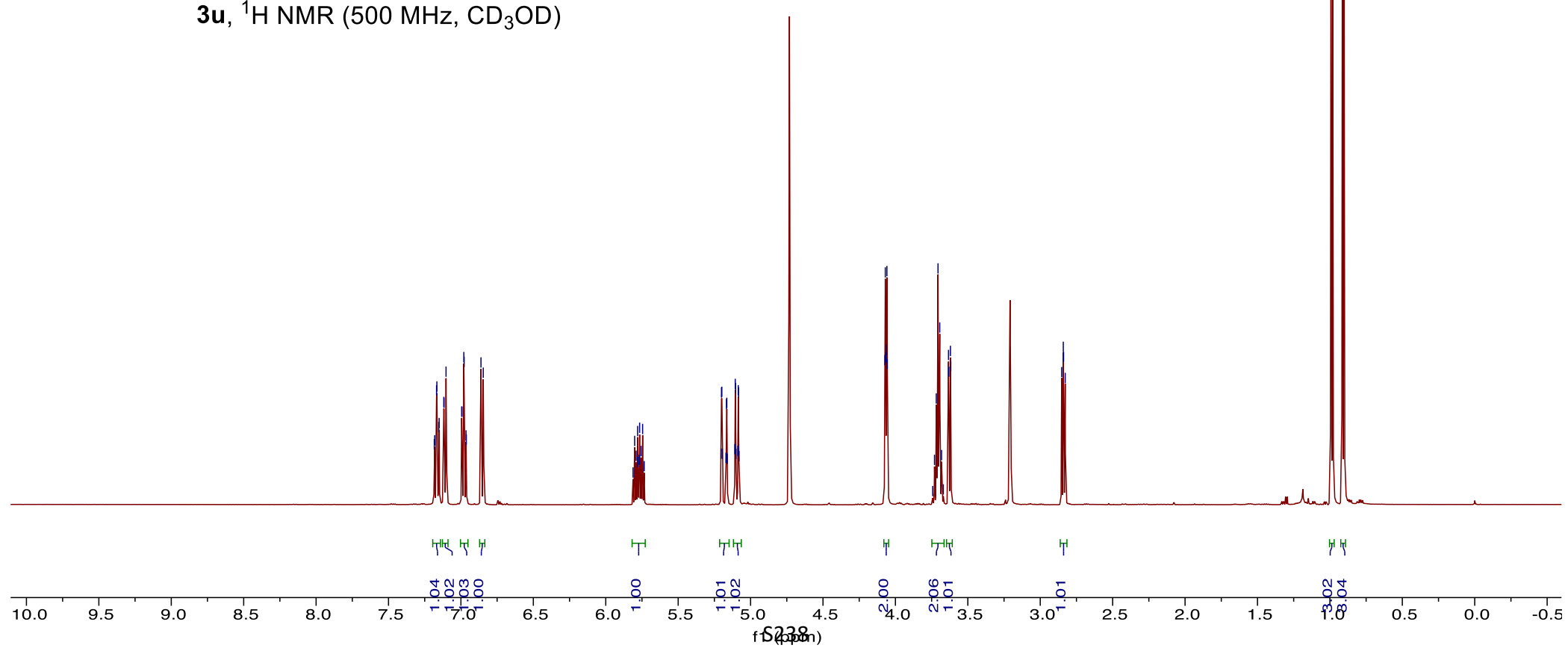




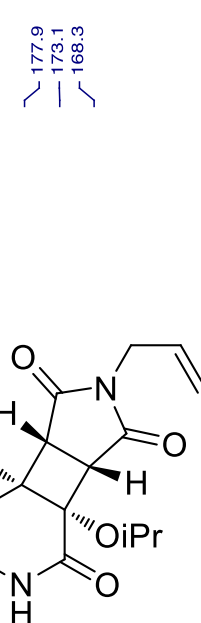

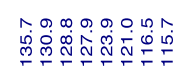

ixiti

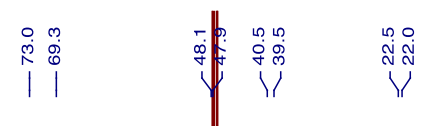

3u, ${ }^{13} \mathrm{C}$ NMR $\left(126 \mathrm{MHz}, \mathrm{CD}_{3} \mathrm{OD}\right)$
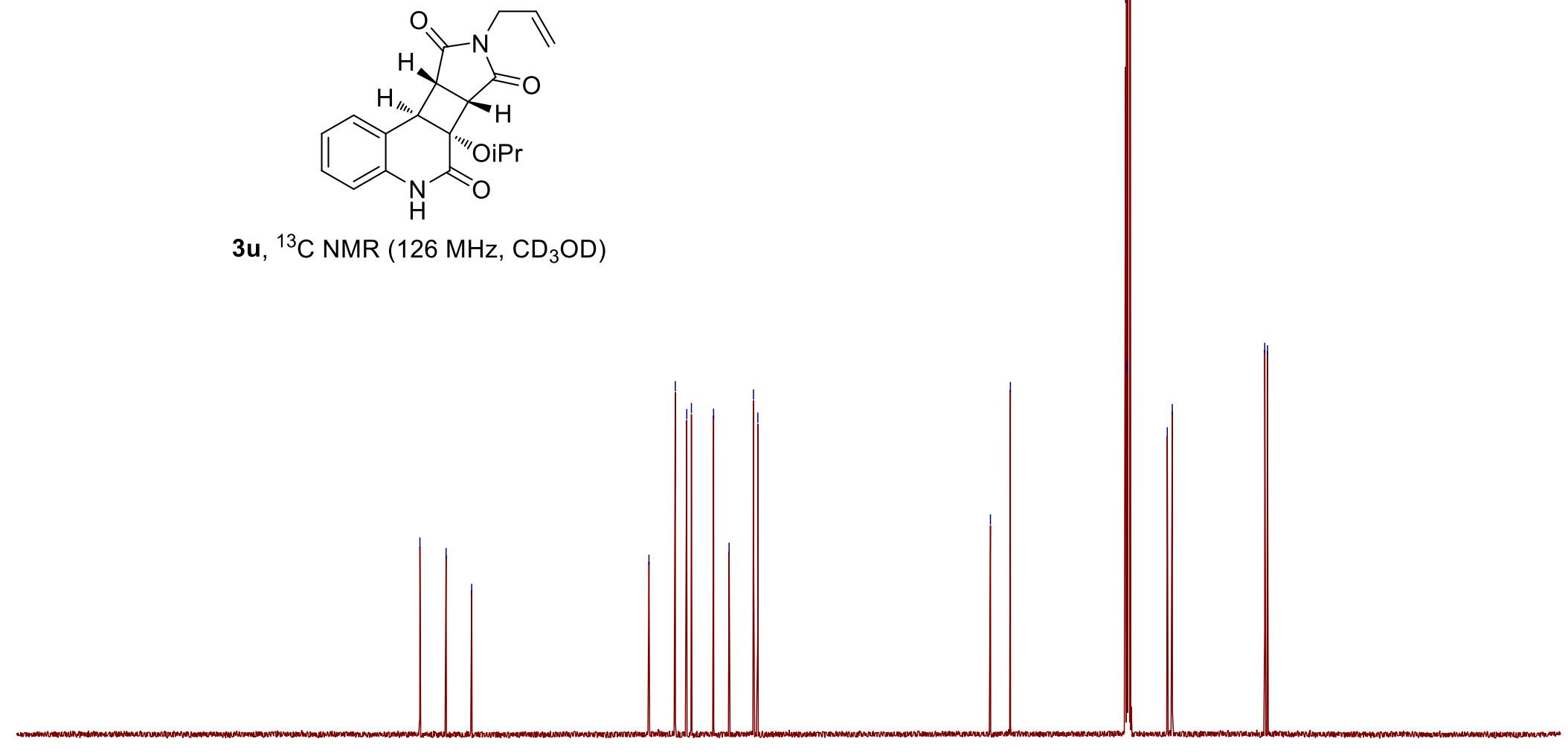

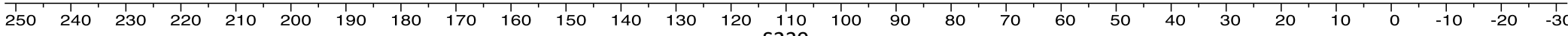

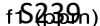




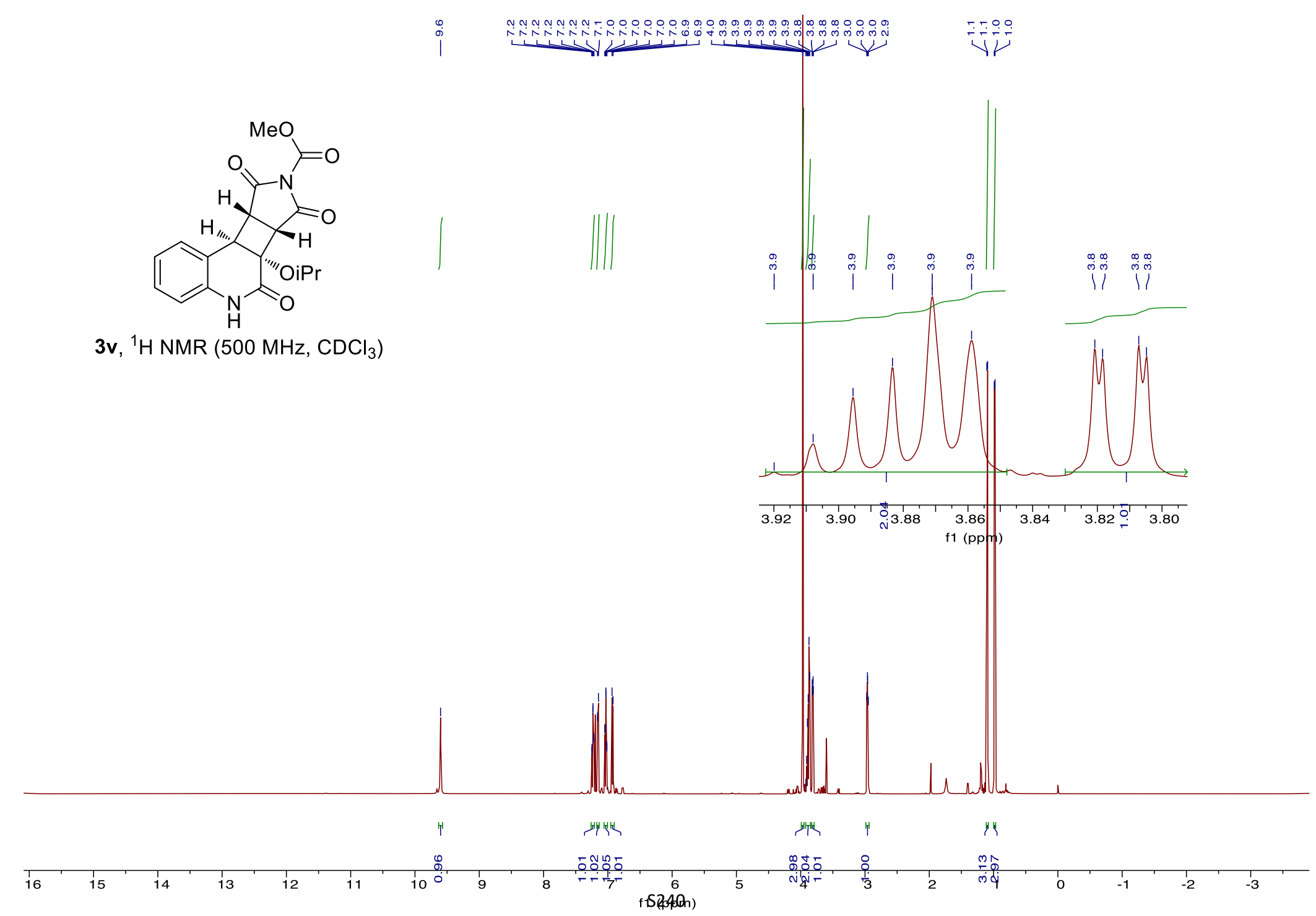




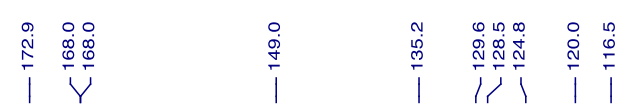

|

3v, ${ }^{13} \mathrm{C}$ NMR (126 MHz, $\mathrm{CDCl}_{3}$ )
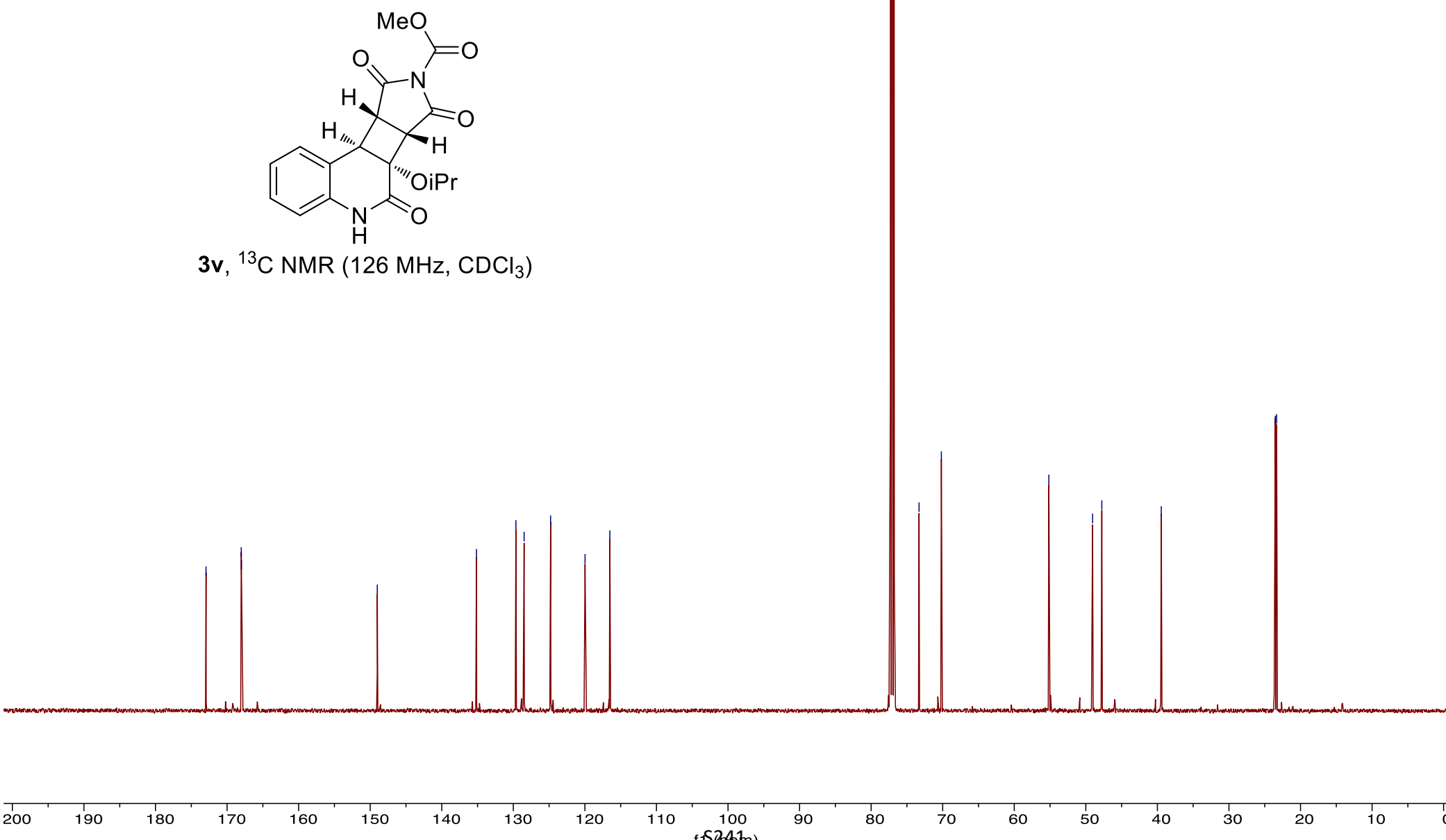


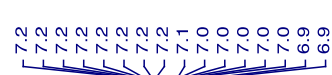

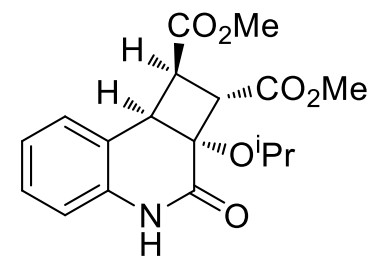

3w, ${ }^{1} \mathrm{H}$ NMR $\left(500 \mathrm{MHz}, \mathrm{CD}_{3} \mathrm{OD}\right)$

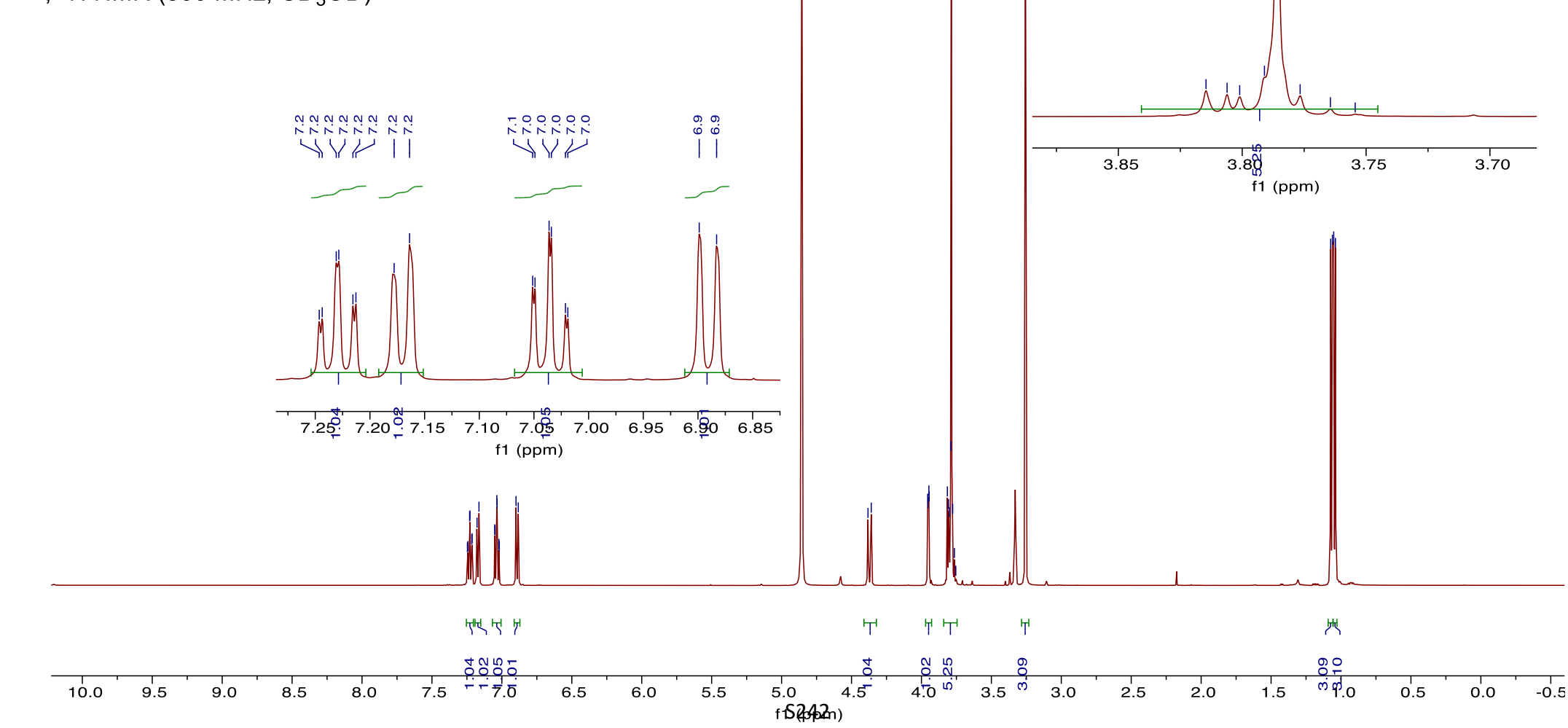




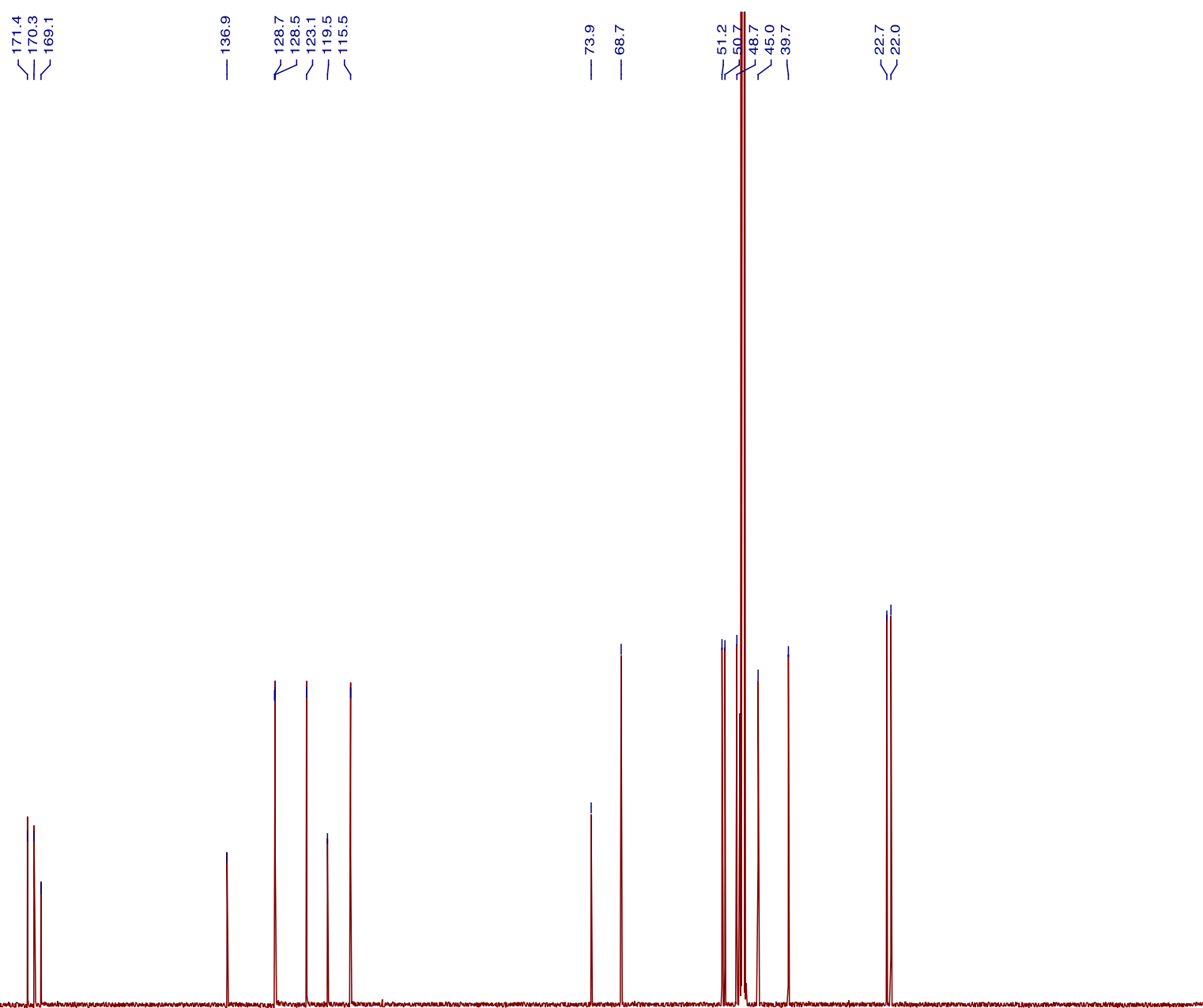

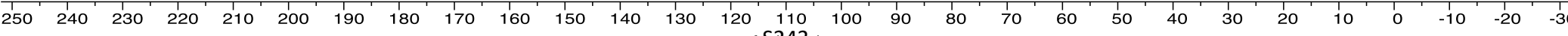
fis 2 s 40 on 


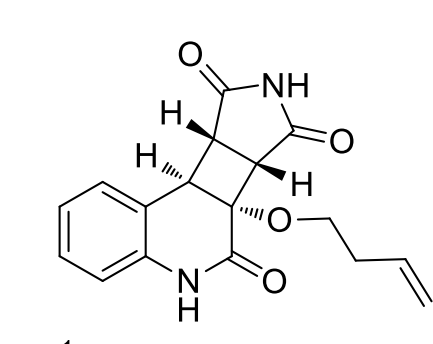

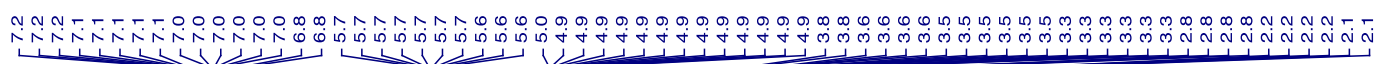

3x, ${ }^{1} \mathrm{H}$ NMR $\left(500 \mathrm{MHz}, \mathrm{CD}_{3} \mathrm{OD}\right)$
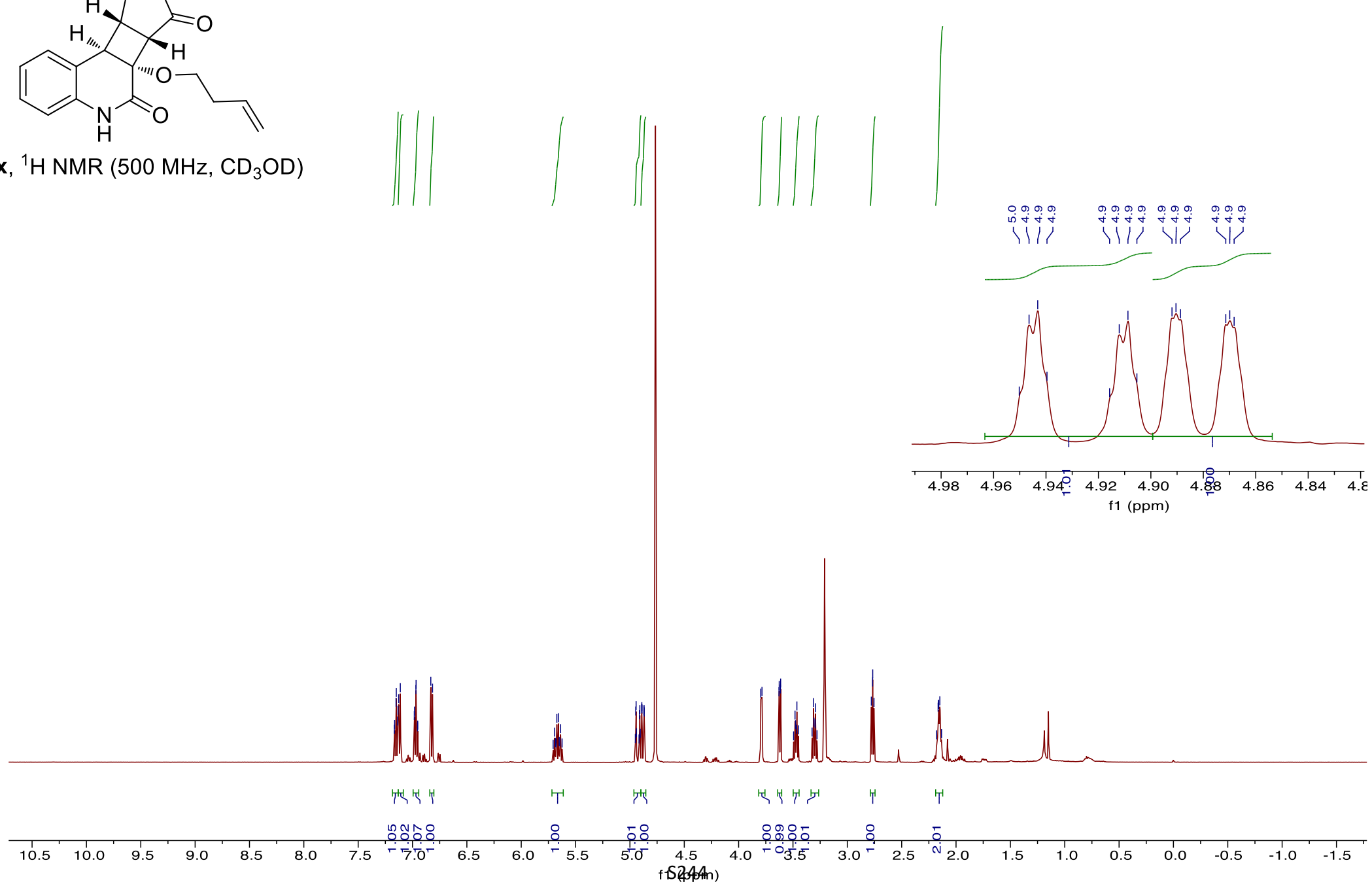


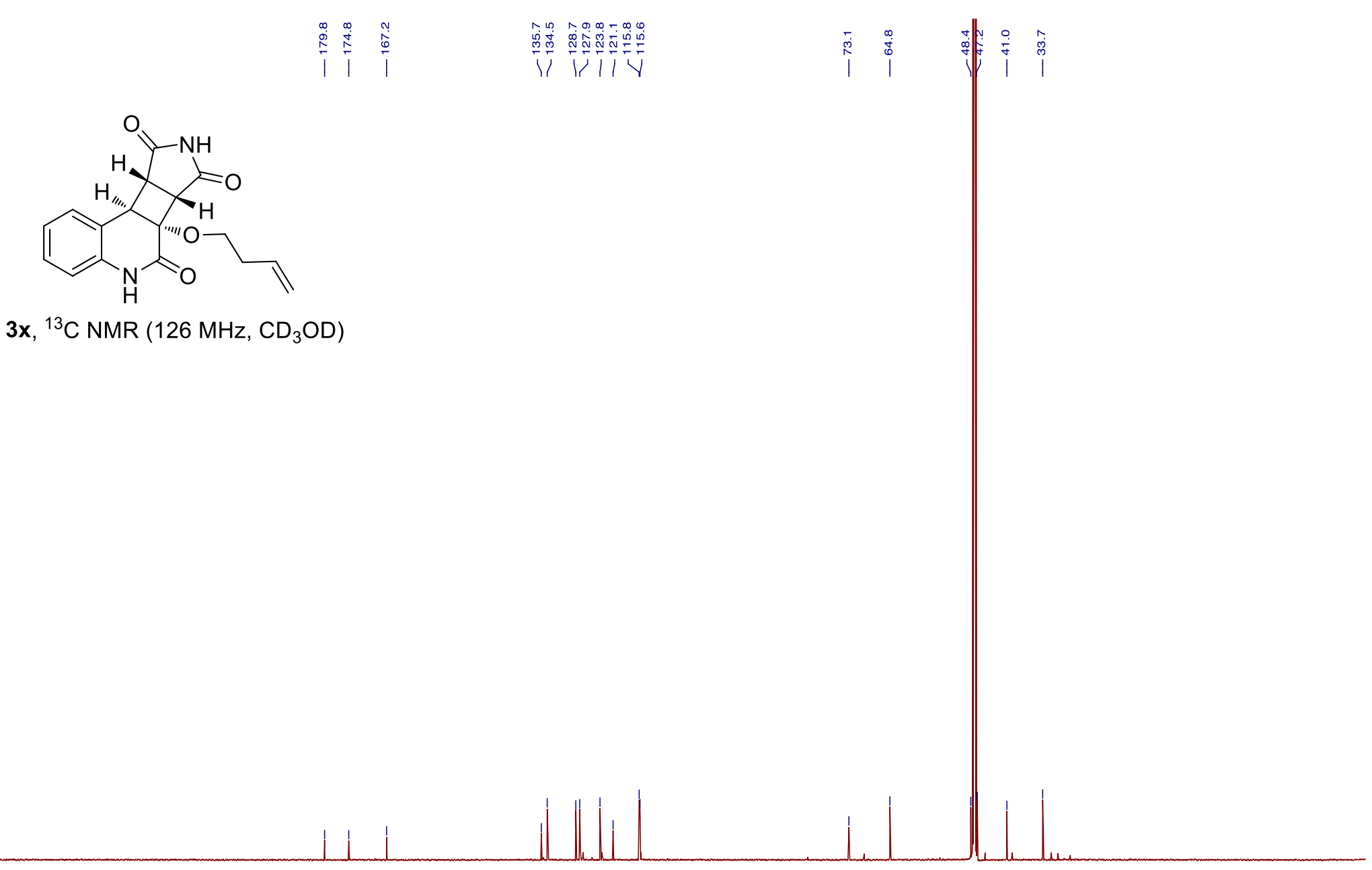

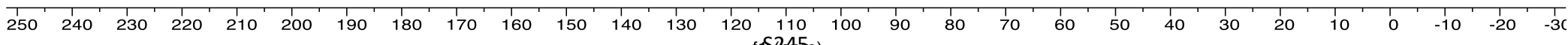
fis 2 s 405 n $)$ 


\section{References}

1 Lowry, M. S.; Goldsmith, J. I.; Slinker, J. D.; Rohl, R.; Pascal, R. A.; Malliaras, G. G.; Bernhard, S. Single-Layer Electroluminescent Devices and Photoinduced Hydrogen Production from an Ionic Iridium(III) Complex. Chem. Mater. 2005, 17, 5712-5719.

2 Skubi, K. L.; Kidd, J. B.; Jung, H.; Guzei, I. A.; Baik, M. H.; Yoon, T. P. Enantioselective Excited-State Photoreactions Controlled by a Chiral Hydrogen-Bonding Iridium Sensitizer. J. Am. Chem. Soc. 2017, 139, 17186-17192.

3 Suzuki, R.; Nozawa, D.; Futamura, A.; Nishikawa-Shimono, R.; Abe, M.; Hattori, N.; Ohta, H.; Araki, Y.; Kambe, D.; Ohmichi, M.; Tokura, S.; Aoki, T.; Ohtake, N.; Kawamoto, H. Discovery and in vitro and in vivo profiles of N-ethyl-N-[2-[3-(5-fluoro-2-pyridinyl)-1H-pyrazol-1-yl] ethyl]-2-(2H-1,2,3-triazol-2-yl) -benzamide as a novel class of dual orexin receptor antagonist. Biorg. Med. Chem. 2015, 23, 1260-1275.

${ }^{4}$ Chen, L.-A.; Xu, W.; Huang, B.; Ma, J.; Wang, L.; Xi, J.; Harms, K.; Gong, L.; Meggers, E., Asymmetric Catalysis with an Inert Chiral-at-Metal Iridium Complex. J. Am. Chem. Soc. 2013, 135, 10598-10601

${ }^{5}$ It should be noted that ${ }^{13} \mathrm{C}$ NMR spectra are not reported for these chiral compounds. Strong couplings with the highly fluorinated ppy ligands resulted in low signal-to-noise ratios and complicated spectra.

6 Maturi, M. M.; Wenninger, M.; Alonso, R.; Bauer, A.; Pöthig, A.; Riedle, E.; Bach, T. Intramolecular [2+2] Photocycloaddition of 3- and 4-(But-3-enyl)oxyquinolones: Influence of the Alkene Substitution Pattern, Photophysical Studies, and Enantioselective Catalysis by a Chiral Sensitizer. Chem..Eur. J. 2013, 19, 7461-7472

7 Duplantier, A. J.; Becker, S. L.; Bohanon, M. J.; Borzilleri, K. A.; Chrunyk, B. A.; Downs, J. T.; Hu, L.-Y.; El-Kattan, A.; James, L. C.; Liu, S.; Lu, J.; Maklad, N.; Mansour, M. N.; Mente, S.; Piotrowski, M. A.; Sakya, S. M.; Sheehan, S.; Steyn, S. J.; Strick, C. A.; Williams, V. A.; Zhang, L. Discovery, SAR, and Pharmacokinetics of a Novel 3-Hydroxyquinolin-2(1H)-one Series of Potent d-Amino Acid Oxidase (DAAO) Inhibitors. J. Med. Chem. 2009, 52, 3576-3585.

8 Manley, P. J.; Bilodeau, M. T. A New Synthesis of Naphthyridinones and Quinolinones: Palladium-Catalyzed Amidation of o-Carbonyl-Substituted Aryl Halides. Org. Lett. 2004, 6, 2433-2435.

9 Yuan, Y.; Yang, R.; Zhang-Negrerie, D.; Wang, J.; Du, Y.; Zhao, K. One-Pot Synthesis of 3-Hydroxyquinolin-2(1H)-ones from N-Phenylacetoacetamide via $\mathrm{PhI}\left(\mathrm{OCOCF}_{3}\right)_{2}$-Mediated $\alpha$-Hydroxylation and $\mathrm{H}_{2} \mathrm{SO}_{4}$-Promoted Intramolecular Cyclization. J. Org. Chem. 2013, 78, 5385-5392

10 Skalenko, Y. A.; Druzhenko, T. V.; Denisenko, A. V.; Samoilenko, M. V.; Dacenko, O. P.; Trofymchuk, S. A.; Grygorenko, O. O.; Tolmachev, A. A.; Mykhailiuk, P. K., [2+2]-Photocycloaddition of N-Benzylmaleimide to Alkenes As an Approach to Functional 3-Azabicyclo[3.2.0] heptanes. J. Org. Chem. 2018, 83, 6275-6289. 
11 Bakowski, A.; Dressel, M.; Bauer, A.; Bach, T., Enantioselective radical cyclisation reactions of 4-substituted quinolones mediated by a chiral template. Org. Biomol. Chem. 2011, 9, 3516-3529

12 Thordarson, P. Determining association constants from titration experiments in supramolecular chemistry. Chem. Soc. Rev. 2011, 40, 1305-1323.

13 Cismesia, M. A.; Yoon, T. P. Characterizing chain processes in visible light photoredox catalysis. Chem. Sci. 2015, 6, 5426-5434.

14 Argazzi, R.; Bignozzi, C. A.; Heimer, T. A.; Castellano, F. N.; Meyer, G. J.,Enhanced Spectral Sensitivity from Ruthenium(II) Polypyridyl Based Photovoltaic Devices. Inorg. Chem. 1994, 33, 5741-5749.

15 Parr, R. G.; Yang, W. Density Functional Theory of Atoms and Molecules. (Oxford University Press: New York, 1989).

16 Bochevarov, A. D.; Harder, E.; Hughes, T. F.; Greenwood, J. R.; Braden, D. A.; Philipp, D. M.; Rinaldo, D.; Halls, M. D.; Zhang, J.; Friesner, R. A. Jaguar: A High-Performance Quantum Chemistry Software Program with Strengths in Life and Materials Sciences. Int. J. Quantum Chem. 2013, 113, 2110-2142.

17 Becke, A. D. Density-functional thermochemistry. III. The role of exact exchange. J. Chem. Phys. 1993, 98, 5648-5652.

18 Lee, C. T.; Yang, W.; Parr, R. G. Development of the Colle-Salvetti correlation-energy formula into a functional of the electron density. Phys. Rev. B 1988, 37, 785-789.

19 Grimme, S.; Antony, J.; Ehrlich, S.; Krieg, H. A consistent and accurate ab initio parametrization of density functional dispersion correction (DFT-D) for the 94 elements H-Pu. J. Chem. Phys. 2010, 132, 154104.

20 Ditchfield, R.; Hehre, W. J.; Pople, J. A. Self-Consistent Molecular-Orbital Methods. IX. An Extended Gaussian-Type Basis for Molecular-Orbital Studies of Organic Molecules. J. Chem. Phys. 1971, 54, 724-728.

21 Hay, P. J.; Wadt, W. R. Ab initio effective core potentials for molecular calculations. Potentials for the transition metal atoms Sc to Hg. J. Chem. Phys. 1985, 82, 270-283.

22 Wadt, W. R.; Hay, P. J. Ab initio effective core potentials for molecular calculations. Potentials for main group elements Na to Bi. J. Chem. Phys. 1985, 82, 284-298.

23 Hay, P. J.; Wadt, W. R. Ab initio effective core potentials for molecular calculations. Potentials for $\mathrm{K}$ to $\mathrm{Au}$ including the outermost core orbitals. J. Chem. Phys. 1985, 82, 299-310.

24 Dunning, T. H., Jr. Gaussian basis sets for use in correlated molecular calculations. I. The atoms boron through neon and hydrogen. J. Chem. Phys. 1989, 90, 1007-1023.

25 Marten, B.; Kim, K.; Cortis, C.; Friesner, R. A.; Murphy, R. B.; Ringnalda, M. N.; Sitkoff, D.; Honig, B. New Model for Calculation of Solvation Free Energies: Correction of Self-Consistent Reaction Field Continuum Dielectric Theory for Short-Range Hydrogen-Bonding Effects. J. Phys. Chem. 1996, 100, 11775-11788.

${ }^{26}$ Edinger, S. R.; Cortis, C.; Shenkin, P. S.; Friesner, R. A. Solvation Free Energies of Peptides: Comparison of Approximate Continuum Solvation Models with Accurate Solution of the Poisson-Boltzmann Equation. J. Phys. Chem. B 1997, 101, 1190-1197. 
27 Friedrichs, M.; Zhou, R. H.; Edinger, S. R.; Friesner, R. A. Poisson-Boltzmann Analytical Gradients for Molecular Modeling Calculations. J. Phys. Chem. B 1999, 103, 3057-3061.

28 Welin, E. R.; Le, C.; Arias-Rotondo, D. M.; McCusker, J. K.; MacMillan, D. W. C. Photosensitized, energy transfer-mediated organometallic catalysis through electronically excited nickel(II). Science 2017, 355, 380-385.

29 Connelly, N. G.; Geiger, W. E., Chemical Redox Agents for Organometallic Chemistry. Chem. Rev. 1996, 96, 877-910.

30 Rehm, D.; Weller, A. Kinetics of Fluorescence Quenching by Electron and H - Atom Transfer. Isr. J. Chem. 1970, 8, 259-271.

31 Ballardini, R.; Varani, G.; Indelli, M. T.; Scandola, F.; Balzani, V. Free energy correlation of rate constants for electron transfer quenching of excited transition metal complexes. J. Am. Chem. Soc. 1978, 100, 7219-7223.

32 McClure, L. J.; Ford, P. C. Ligand macrocycle effects on the photophysical properties of rhodium(III) complexes: a detailed investigation of cis- and trans-dicyano(1,4,8,11-tetraazacyclotetradecane)rhodium(III) and related species. J. Phys. Chem. 1992, 96, 6640-6650.

33 Døssing, A.; Ryu, C. K.; Kudo, S.; Ford, P. C. Competitive bimolecular electron- and energy-transfer quenching of the excited state(s) of the tetranuclear copper(I) cluster Cu4I4py4. Evidence for large reorganization energies in an excited-state electron transfer. J. Am. Chem. Soc. 1993, 115, 5132-5137.

34 Zhu, Q.; Gentry, E. C.; Knowles, R. R. Catalytic Carbocation Generation Enabled by the Mesolytic Cleavage of Alkoxyamine Radical Cations. Angew. Chem., Int. Ed. 2016, 55, 9969-9973.

35 Cismesia, M. A.; Yoon, T. P., Characterizing chain processes in visible light photoredox catalysis. Chem. Sci. 2015, 6, 5426-5434.

36 Hatchard, C. G.; Parker, C. A. Proc. Roy. Soc. (London) 1956, A235, 518-536.

37 Kuhn, H. J.; Braslavsky, S. E.; Schmidt, R. Pure Appl. Chem. 2004, 76, 2105-2146.

38 Monalti, M. et. al. Chemical Actinometry. Handbook of Photochemistry, 3rd Ed; Taylor \& Francis Group, LLC. Boca Raton, FL, 2006, 601-616. 